\title{
Aufbau von Handlungswissen und Kompetenzlernen bei Sportspielen, ein didaktisches Modell der Spielhandlungskompetenzen
}

\author{
Dissertation \\ zur Erlangung des Doktorgrades der Sozialwissenschaftlichen Fakultät \\ der Georg-August-Universität Göttingen \\ vorgelegt von \\ Elkin Arias \\ geboren in Medellín, Kolumbien
}

Göttingen

2012

Dissertationsgebiet: Sportwissenschaften

Betreuer: Prof. Dr. Herbert Hopf 
Meinen lieben Eltern

Maria Aurora und Manuel 


\section{Zusammenfassung}

Die vorliegende Dissertation hat zum Ziel, ein didaktisches Modell für das Lernen und das Training von Sportspielen zu entwerfen. Es berücksichtigt die grundlegenden Prinzipien von solchen Lerntheorien wie Interaktionistischer Konstruktivismus, situiertes Lernen und Handlungstheorie, es berücksichtigt darüber hinaus grundlegende Prinzipien aus den Theorien der Informationswissenschaften und des Wissensmanagements wie Wissenshierarchie und Wissenstreppe. Unter Beachtung der besonderen Bedingungen der Sportvereine, wo die Kinder das Spiel lernen und Spaß haben, aber auch bei Wettspielen Leistung zeigen sollen, wird ein didaktisches Modell der Spielhandlungskompetenzen vorgeschlagen. Dieses ist ein indirektes didaktisches Modell und strebt als Alternative zum traditionellen Direkten Modell die Entwicklung der kognitiven, technischen und psychologischen Spielkompetenz an und übernimmt Elemente aus anderen didaktischen Modellen wie zum Beispiel kooperatives Lernen und individuelles Lernen.

Es wurde ein bestimmtes Forschungsverfahren angewandt, indem das Modell in mehrerenSchrittenentwickelt wurde. Bei jedem Schritt wurde nur eine der vier im Modell vorgeschlagenen didaktischen Strategien mit jungen Fußballspielern überprüft. Dies geschah durch eine partielle Intervention, ohne das gewöhnliche Training zu stören. Die vorgeschlagenen didaktischen Strategien sind: Kleine Fußballspiele mit unterschiedlichen Schwerpunkten (KFSS), Kombinationsspiele, Zweikämpfe und absichtsvolles Üben der technischen Fertigkeiten. Weiterhin wurde das Trainingsinhalt durch eine Befragungsmethode vermittelt. Das Modell wird dann in mehreren Studien überprüft, wobei jede auf eine bestimmte Perspektive und damit auf die Entwicklung einer bestimmten Kompetenz fokussiert ist. Der Hinweis von METZLER (2005b, S.190), verschiedene didaktische Modelle sollten, wegen ihren unterschiedlichen Voraussetzungen, nicht verglichen werden, wurde berücksichtigt. Daher wurde der Vergleich mit anderen didaktischen Modellen vermieden, stattdessen wurden die Ergebnisse und kontextuellen Bedingungen, die die Implementierung des Modells voraussetzt, exploriert.

Die vier Studien zeigen bei der Anwendung des entworfenen Modells eine positive Wirkung auf die Spielkompetenzen der jungen Fußballspieler, allerdings wurden nicht alle Ergebnisse statistisch signifikant. Die Kleinen Fußballspiele mit unterschiedlichen Schwerpunkten ergaben zusammen mit der Befragungsmethode eine positive Wirkung beim Erlernen der Spielprinzipien, jedoch statistisch nicht signifikant. Die Befragungsmethode wies eine signifikant positive Wirkung auf die Entwicklung der spezifischen kognitiven Kompetenz beim Fußballspiel auf. Die Kleinen Spiele mit Schwerpunkten wurden ebenso wirksam wie die Übungen für das Lernen der technischen Fertigkeiten des Fußballs. Die didaktische Strategie der Kombinationsspiele zeigte eine positive, aber statistisch nicht signifikante Wirkung auf die Verbesserung des Offensivspiels.

Das Modell zeigte eine positive Wirkung auf das Lernen der Spielkompetenzen, wenn jede didaktische Strategie in einer unterschiedlichen Studie angewendet wurde. Allerdings sind weitere Studien erforderlich, in denen das gesamte Modell während einer längeren Zeitspanne angewendet wird, um eine Langzeitwirkung bestätigen zu können. Dazu sollten weitere Auswertungsverfahren entworfen und validiert werden, die die Ziele der indirekten Modelle berücksichtigen. Ein Vergleich mit anderen didaktischen Modellen für das Lernen und Training der Sportspiele sollte später unternommen werden. 


\begin{abstract}
The present dissertation aims to design a didactic model for the teaching and training of sports games taking into account the basic principles of learning theories such as Interactionist Constructivism, Situated Learning and the Theory of Action. It also takes into account principles of theories from information science and knowledge management such as the Knowledge Hierarchy and the Knowledge Staircase. Under consideration of the specific conditions of sports clubs, where the game is learned and enjoyed but it is also necessary to perform and to win matches, an instructional model of game action competences is proposed. This is an indirect didactic model and as an alternative to the traditional direct model intends the development of cognitive, technical and psychological game competences. It adapts elements of other instructional models like cooperative learning and individual learning.
\end{abstract}

A research procedure was carried out in which the proposed model was divided in several sets. Every set, containing only one didactic strategy of the four proposed in the model was tested in a study with young football players through partial intervention without disturbing the team's usual training process. The proposed didactic strategies are: Small Games with Different Focal Points, Combination Games, one-against-one exercises and Deliberated Practice of Technical Abilities. Moreover the content was taught through a Questioning Method. The model was then tested in several studies. Each study focused on a particular perspective and consequently on the development of specific competences. The proposal from METZLER (2005b, p. 190), not to compare different didactic models due to their different postulates, was taken in account. Therefore comparison with other models is avoided. Instead the outcomes and contextual factors that determine the implementation of the instructional model are explored.

The four studies show positive effects on the game competences of young football players with the application of the designed model, however not all the results were statistically significant. The small games and a question method showed some positive effects but not statistically significant on the learning of the principles of the game. The question method showed positive significant effects on the development of the specific cognitive competences related to the football game. The small games were as effective as the exercises for the learning of football techniques. The didactic strategy of the combinations games showed some positive but not statistically significant effects on the improvement of offensive play.

The model showed some positive effects on the learning of game competences when every didactic strategy is applied in a different study. To confirm the effects more studies are needed in which the model is implemented during a longer training period. Taking into account the goals of the didactic indirect models, more assessment methods should be designed, refined and validated. A comparison with other didactic models for the learning and training of sport games should be later endeavored. 


\section{Inhaltverzeichnis}

1 Einleitung und allgemeine Problemstellung ................................................ 1

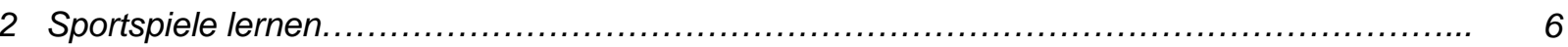

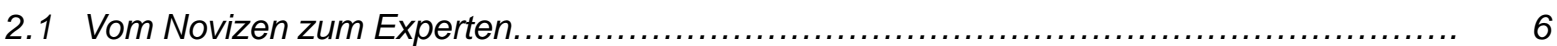

2.2 Traditionelle Ansätze beim Lernen der Sportspiele ........................................ 8

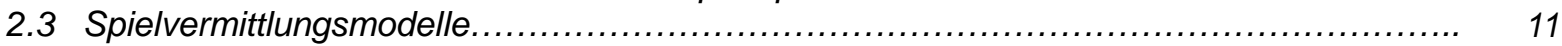

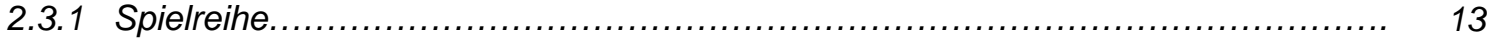

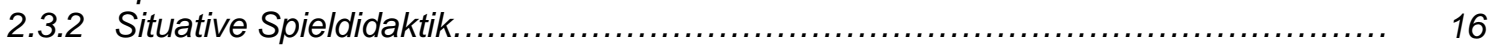

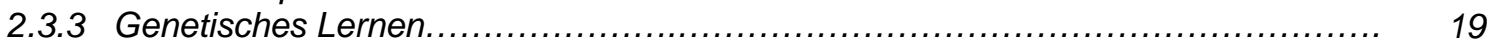

2.3.4 Integratives Modell................................................................. 20

2.3.5 Fußballentwicklungsmodell........................................................ 22

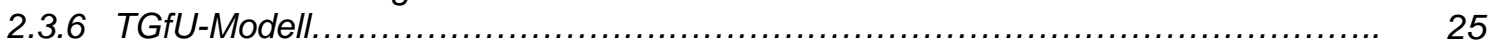

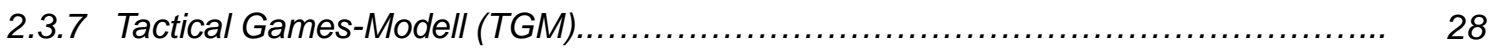

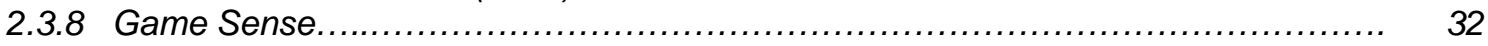

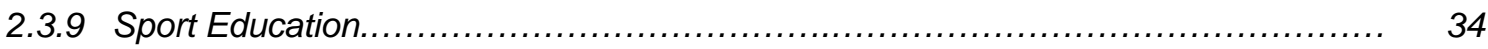

2.3.10 Invasion Game Competences Model (IGCM) .................................... 36

2.3.11 Play Practice ........................................................................ 39

2.3.12 Modell der inzidentellen Inkubation (Heidelberger Ballschule) ..................... 42

2.3.13 Decision Training-Modell....................................................... 46

2.3.14 Situation Model of Anticipated Response Consequences of Tactical Training $($ SMART) ....................................................................... 50

2.3.15 Zusammenfassung der Spielvermittlungsmodelle................................ 53

2.3.16 Stand der Forschung über die Wirksamkeit der Vermittlungsmodelle................. 58

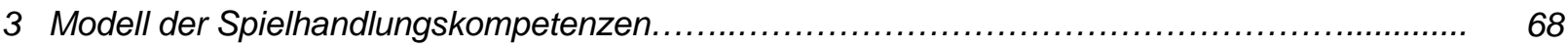

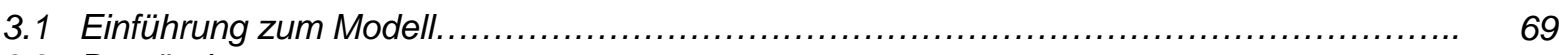

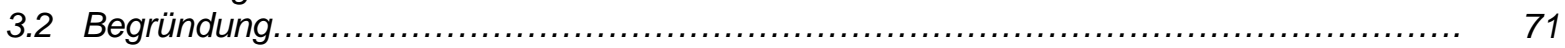

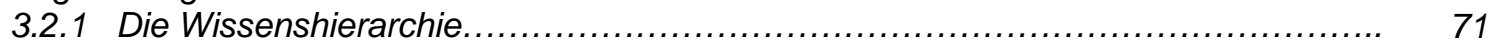

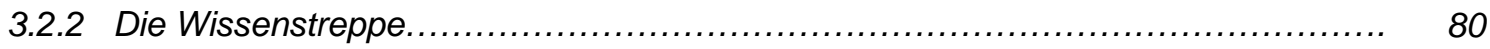

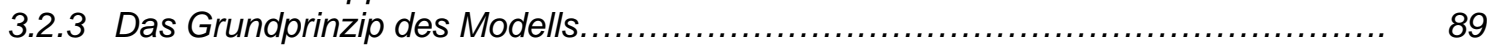

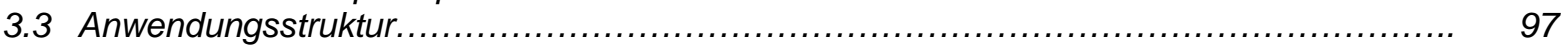

3.3.1 Ziele, Lernmethode und Inhalte.............................................. 91

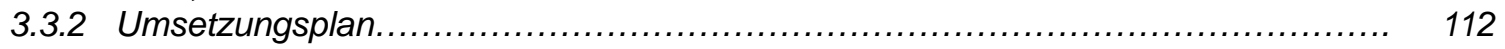

3.3.3 Auswahl der Inhalte und Rolle des Trainers................................... 117

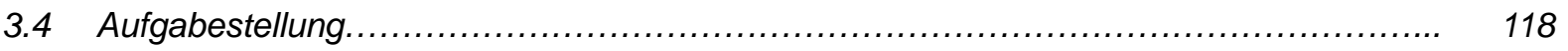

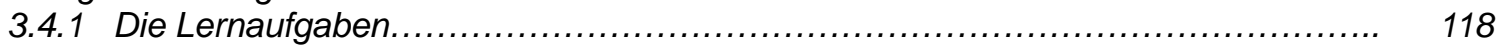

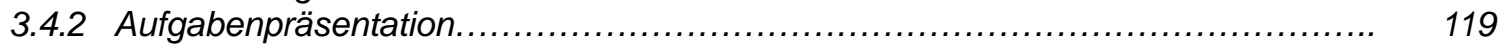

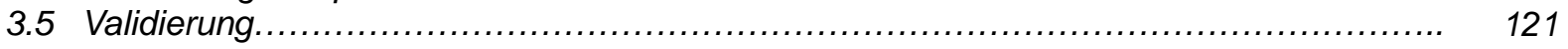

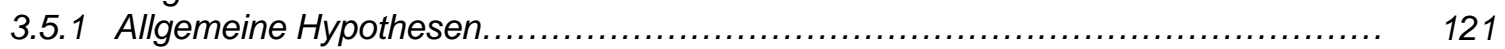

3.5.2 Forschungsstrategien ............................................................ 124

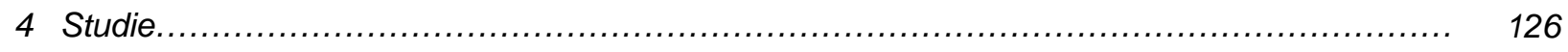

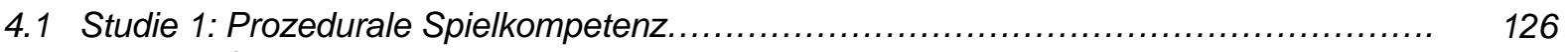

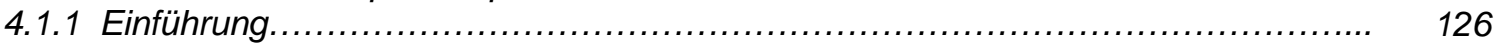

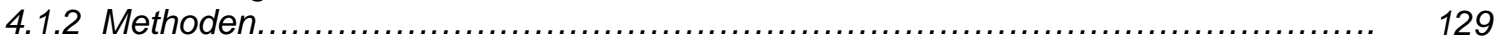

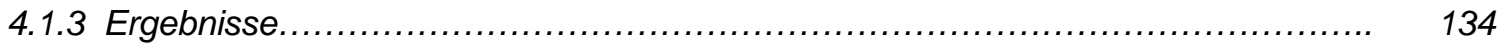

4.1.4 Diskussion und Schlussfolgerung............................................. 136

4.2 Studie 2: Aufbau des präskriptiven Wissens durch eineBefragungsstrategie .................. 138

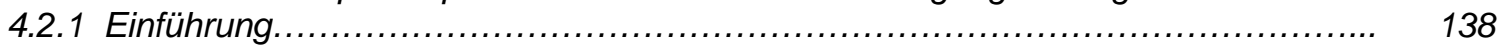

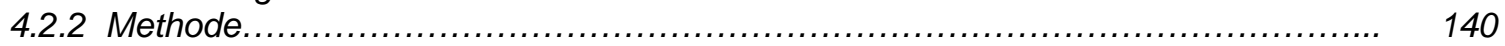

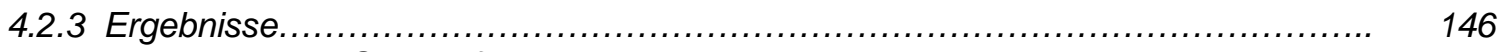

4.2.3 Diskussion und Schlussfolgerung............................................... 148 
4.3 Studie 3: Entwicklung der technischen Kompetenz in Sportspielen durch Kleine

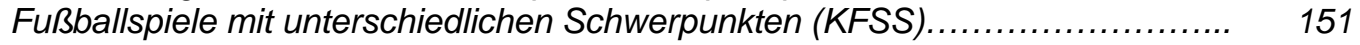

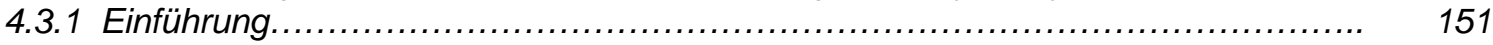

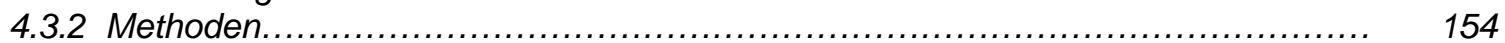

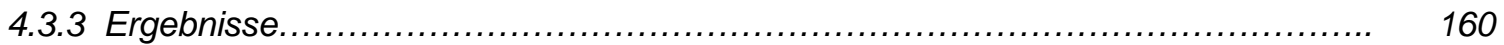

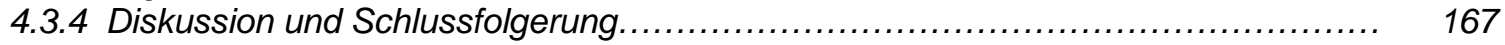

4.4 Studie 4: Förderung der kognitiven Fähigkeiten durch Kombinationsspiele.................. 170

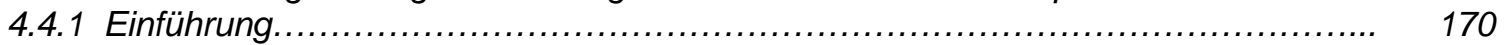

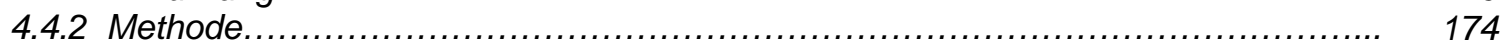

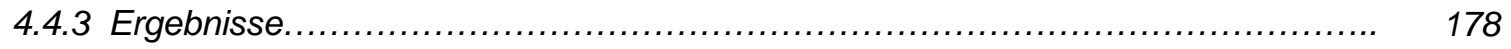

4.4.4 Diskussion und Schlussfolgerung...................................... 181

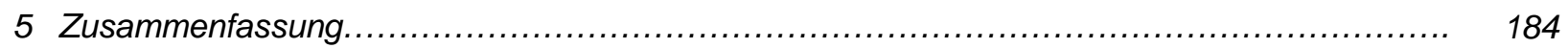

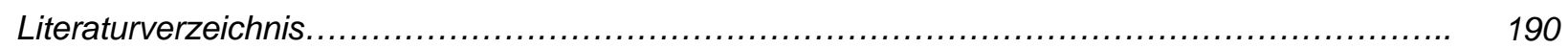

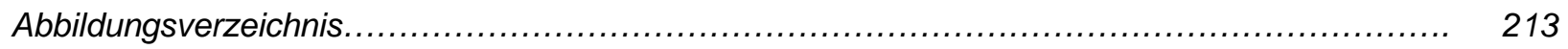

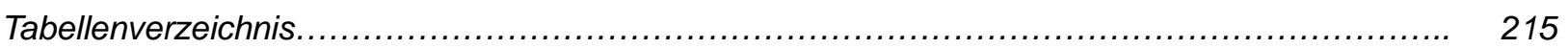

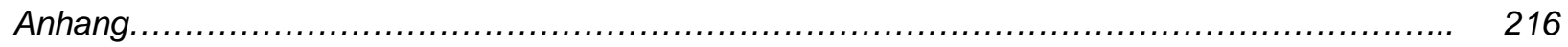

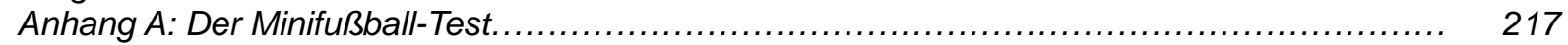

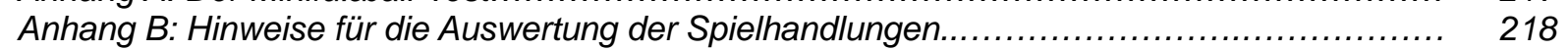

Anhang C: Formblatt: Auswertung der Spielhandlungen....................................... 219

Anhang D: Beispiel Trainingseinheit für die Schulung der Spielkompetenzen: Abwehr........... 220

Anhang E: Beispiel Trainingseinheit für die Schulung der Spielkompetenzen: Angriff............... 221

Anhang F: Schulung der Spielkompetenzen: Beispiel Fragen zum Reflektieren.................. 222

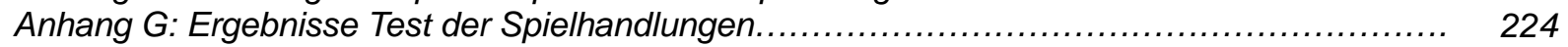

Anhang H: Kolmogorov-Smirnov-Test und Mauchly-Test für die Variablen der Studie 1.......... 227

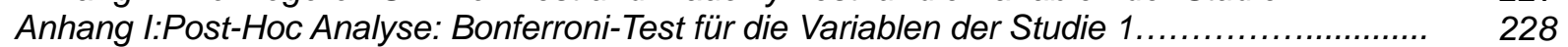

Anhang J:Ergebnisse Auswertungsmethode der Kognitiven Spielkompetenz.......................230

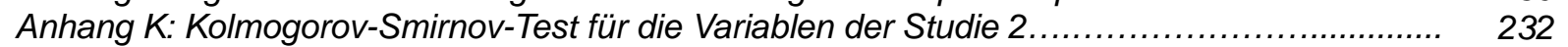

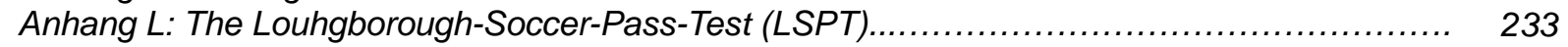

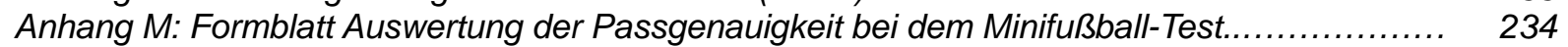

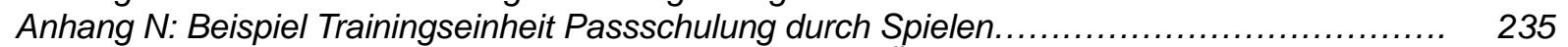

Anhang O: Beispiel Trainingseinheit Passschulung durch Üben.............................. 236

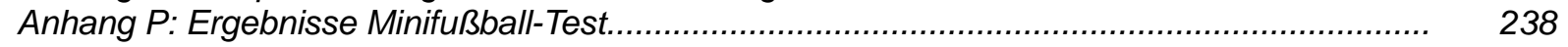

Anhang Q: Ergebnisse Loughborough-Soccer-Pass-Test............................... 243

Anhang R: Kolmogorov-Smirnov-Test und Mauchly-Test für die Variablen der Studie 3........... 248

Anhang S: Formblatt Auswertung des Team-Sport-Assessment-Procedure (TSAP)................ 251

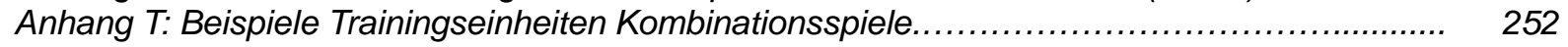

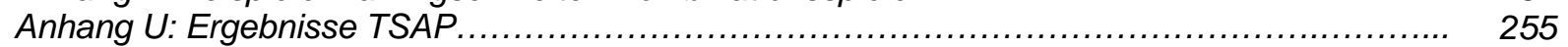

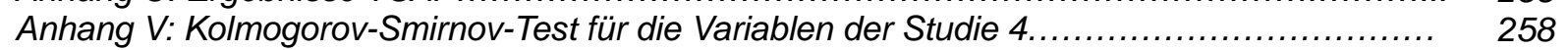

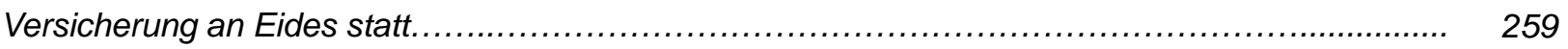

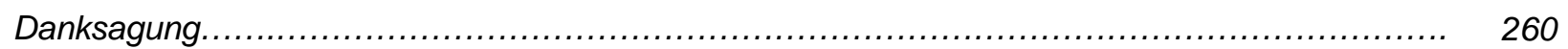

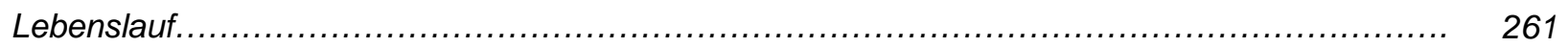




\section{Einleitung und allgemeine Problemstellung}

Ein zentraler Bestandteil des Lehrens und Lernens und damit des Trainings in Sportspielen ist die Entwicklung einer Reihe motorischer Fertigkeiten, die grundlegend für die Leistung der Spieler während des Spiels sein sollen. Mit der individuellen Leistung des ballführenden Spielers nimmt auch die Leistung der Mannschaft zu. Dies wird aus der Logik abgeleitet, dass der Ball das wichtigste Element des Spieles ist und seine Beherrschung und Kontrolle die Leistung beim Spielen gewährleistet. Das hervorragende technische Können einiger der besten Spieler unterstützt die Annahme, dass die Technik $^{1}$ die entscheidende Variable des Erfolgs bei den Sportspielen sei. Im Training richten sich daher viele Bemühungen auf die Entwicklung der sportlichen Technik. Alles geschieht mit dem Ziel, die bestmögliche Beherrschung des Balls zu erreichen. Die Motorikforschung hat hierzu viel beigetragen. Tatsächlich wird die traditionelle Didaktik der Sportspiele auf die Lehre der Technik fokussiert, das heißt, die Didaktik der Sportspiele ist schwerpunktmäßig eine Didaktik der technischen Fertigkeiten mit dem Ball.

Das technische Lernen fordert konkretes Denken, das nach PIAGET (1972, S. 3) zeitlich früher als das abstrakte Denken entwickelt wird. Dieser Logik folgend, sollten während der Spielerausbildung die technischen Fertigkeiten zuerst erlernt werden, die Taktik als kognitiver Stoff und damit als abstraktes Denken wird dann später gelernt. Die technischen Bewegungen können erst nach einer langen Zeitspanne von Üben und Wiederholen automatisiert werden, danach werden sie unbewusst durch untere Strukturen des Zentralnervensystems kontrolliert.

1 Sportliche Technik ist von AUGUSTIN (1998, S. 9) definiert als „praktisch erprobte, charakteristische und effektive Lösungsverfahren für gegebene Bewegungsaufgaben, die aus einer spezifischen Abfolge von effektiven und zweckmäßigen Bewegungen oder Teilbewegungen bestehen“ Technische Fertigkeiten sind nach der Definition von HOHMANN, LAMES und LEZELTER (2002, S. 141) „erprobte, zweckmäßige und effektive Bewegungsfolgen zur Lösung einer definierten Aufgabe in Sportsituationen“. Die Begriffe „sportliche Technik“ und „technische Fertigkeiten“ werden daher wegen ihrer Ähnlichkeit in diesem Sinn Synonym im Text als Bezeichnungen für die Ausführung von reinen Bewegungen ohne Berücksichtigung der Taktik verstanden. Die Abgrenzung der Begriffe „sportliche Technik“ und „sportliche Taktik“ wird in Anlehnung an LOY (2005, S. 200) bestimmt: „Während es sich bei der sportlichen Technik um ein Verfahren zur möglichst optimalen Lösung einer vorgegebenen Bewegungsaufgabe, also um die ökonomische Ausführung eines bestimmten Bewegungsablaufes handelt, hat die Taktik den sinnvollen Einsatz der Technik entsprechend der vorliegenden Spielsituation in einem Wettkampf zum Inhalt“. -Die Trennung zwischen Technik und Taktik entspricht allerdings nicht der Meinung des Autors dieser Arbeit und wird sogar als ein mangelhaftes Merkmal des Direkten Modells kritisiert (siehe S. 8 ff.). 
Die Taktik ist eine wichtige Ergänzung der technischen Fertigkeiten. Wenn es um Kindertraining geht, wird sie allerdings kaum thematisiert. Es wird dabei angenommen, dass die Kinder kein abstraktes Denken besäßen. Bis die Spieler die erforderlichen Voraussetzungen haben, das heißt, die kognitive Entwicklung bis zum abstrakten Denken durchlaufen haben, wird die Taktik im traditionellen Training allenfalls in einer Nebenstelle integriert, indem sie vom Trainer als Information, Hinweis und Rückmeldung vermittelt wird. Die moderne Entwicklung der Sportspiele hat diese Nebenstelle der Taktik in Frage gestellt. In vielen Fällen ist eine gute taktische Stellung auf dem Spielfeld der guten technischen Ausstattung eines Spielers überlegen und kann den Gegenspieler erfolgreich überwinden. Anders gesagt, reicht bei Sportspielen eine gute technische Ausstattung ohne taktisches Können nicht für eine umfassend gute Leistung. Konsequenterweise wird heutzutage bei einigen Spielvermittlungsmodellen sowohl die technische als auch die taktische Ausbildung gleichwertig berücksichtigt als Bestandteile, die wesentlich zur Leistung bei Sportspielen beitragen.

Innerhalb der Didaktik des Schulsports sind didaktische Modelle vorgeschlagen worden, die außer zum technisches Lernen auch zur kognitiven und emotionalen Entwicklung der Schüler beitragen sollen, zum Beispiel Teaching Games for Understanding (BUNKER \& THORPE, 1982), Tactical Games (MITCHELL, OSLIN \& GRIFFIN, 2006), Play Practice (LAUNDER, 2001). Solche didaktischen Modelle zentrieren ihr Interesse auf die Spieler und inr Einwirken auf das Spiel. Das Interesse wird nicht auf den Lehrer als Wissensträger gerichtet, der Informationen für die Lösung der im Spiel vorhandenen kognitiven taktischen Probleme vermittelt und von den Spielern Leistung einfordert, sondern es liegt auf der Entwicklung der Spieler als den einzigen Personen, die durch inr Handeln aktiv auf die Spielkonstellation einwirken können. Folglich wird die Aufmerksamkeit darauf fokussiert, welche Fähigkeit diese Spieler besitzen, Probleme während ihres Agierens innerhalb des Spiels durch eigene Kreativität zu lösen, ohne dabei fertige Rezepte für die Lösung jedes der unzähligen und vielfältigen taktischen Probleme zu besitzen, die ein Spiel beinhaltet. Allerdings wird die wissenschaftliche Validierung dieser Modelle auf den Schulbereich begrenzt, wo die Spieler Anfänger sind und wegen der immer mangelnden Zeit der Lehrinhalt eher aus einer bloßen Einführung in die Sportart besteht.

Im Gegensatz zum Schulsport, wo Sporterziehung, Sportbegeisterung, Gesundheit und sportliche und menschliche Werte die zentralen Ziele des Lernen sind und deswegen alternative Lernmethoden als tauglich angesehen werden (vgl. Ministerium für Schule und Weiterbildung des Landes Nordrhein-Westfalen, 2011), ist auf Vereinsebene noch immer das "Direkte Modell“ das meist eingesetzte. Dort ist die sportliche Leistung das zentrale Ziel des Lernens und man braucht schnelle Ergebnisse und viel Disziplin zur Zielerreichung. Das Direkte Modell scheint diese Endprodukte sicher und effizient zu gewährleisten. Da der ideale Trainer nach der Sichtweise des Direktes Modells über Expertenwissen verfügt und viele Erfahrungen gesammelt hat, soll er die Antwort für alle während des Spiels auftretenden Probleme geben können und die totale Kontrolle über den Trainingsprozess besitzen. Der Lern- und- Trainingsprozess besteht daher bei einem leistungsorientierten Ansatz aus der Vermittlung des Wissens vom Trainer zum Spieler. Mittels der empfangenen Information üben und verfeinern die Spieler die technischen Bewegungen. Die rechtzeitige und geeignete Rückmeldungen des Trainers erlauben ein effektives Lernen und die Automatisierung der technischen Bewegungen. Das Direkte Modell wird daher als das weitaus effektivste für das technische Lernen betrachtet (METZLER, 2005a, S. 208). 
Auf Vereinsebene erreicht das Direkte Modell gute Leistungen im Bereich des technischen Lernens. Beim taktischen Lernen aber sollen die Spieler, die mit dem Direkten Modell lernen, standardisierte Spielzüge üben, in denen kognitive Prozesse wie Wahrnehmung und Verarbeitung von Informationen und die Entscheidung über die beste Handlungsalternative eine Rolle spielen, dies alles in theoretischen Situationen, die während des Spiels entstehen könnten. In dem Direkten Modell werden die Spielzüge unter vereinfachten Bedingungen geübt, das heißt ohne Gegenspieler und bei reduzierter Schnelligkeit. Sie werden als antizipierte Lösungen für die Probleme des Spielkontextes betrachtet. Der Schwierigkeitsgrad wird stufenweise erhöht, dies geschieht in Form eines additiven Prozesses durch die Steigerung der Spielschnelligkeit und das Einfügen von Gegenspielern, die zunächst statisch bleiben, danach semi-aktiv agieren und zum Abschluss aktiv am Spielzug teilnehmen. Nach BREMEN (1987, S. 74-75) wird das Problem mit dieser Art stereotypisierter Spielzüge vor allem in Spielsituationen deutlich, in denen die Anwesenheit von Gegenspielern, deren Anordnung und das eigene Agieren andere Lösungen erfordert als die, die vorher im Training geübt wurden. Die Spielsituationen sind so variabel und vielseitig, dass das vorherige Durchspielen aller Spielkonstellationen im Training faktisch unmöglich ist.

Trotz aller Bemühungen kann man weder präskriptives noch interpretatives noch theoretisches Wissen, nach den Repräsentationsstrukturen des Wissens von WIEMAYER (1994, S. 50 ff.), durch das Direkte Modell vermitteln. WERNER,THORPE und BUNKER (1996, S. 28) sahen darin den Grund für die Entwicklung eines neuen didaktischen Modells: „Our interest in the area grew as we realised that highgly skillful students entering an undergraduate course in Physical Education did not understand the games they played“.BREMER (1987, S. 63) meint folgendes dazu: „Additiv-hierarchische Modelle und systematische Sportspielbeobachtungen erfassen nicht die aus kognitionspsychologischer Sicht fundamentalen intraindividuellen Wahrnehmungs- und Entscheidungsprozesse." Nach der in dieser Arbeit vorgestellten Wissenshierarchie kann man durch das Direkte Modell nur eine Vermittlung der Information erreichen, das Handlungswissen hingegen kann nicht vermittelt werden. Dieses Wissen muss aufgebaut werden, dafür braucht man geeignete didaktische Modelle, die den Lerner zum eigenen Überlegen anregen und auf die Entwicklung der kognitiven Kompetenzen abzielen. Das Direkte Modell allein kann aber dieses Ziel nicht erreichen (WERNER, THORPE \& BUNKER, 1996, S. 28; BREMEN, 1987, S. 63; LAUNDER, 2001, S. 8, BLOMQVIST, VANTTINEN \& LUHTANEN, 2005, S. 151). Ein stufenweiser erfolgender Aufbau des Handlungswissens wird nur durch aktive Teilnahme der Spieler an einem Prozess erreicht, der zum Überlegen während und nach der Bewegung führt. Innerhalb der Lernsituationen wird dieser Prozess vom Trainer erarbeitet und angeleitet. Einige Autoren haben Ansätze vorgeschlagen, die zu den Zielen der Sportvereine beitragen könnten, wie der von HEIL (1970) vorgeschlagene Ansatz „Mit kleinen Spielen zum Großen Spiel“, die „Situative Spieldidaktik“ (BRÜGGEMMAN \& ALBRECHT, 1982); die Modelle „Fußball in Grundsituationen“ oder „Fußball Spielgemäß lernen“ (DIETRICH, 1984), das „Fußballentwicklungsmodell“ (WEIN, 2009), „Invation games Competence“ (TALLIR, MUSCH, LENOIR \& VALCKE, 2004) und das Modell der ,inzidentellen Inkubation“ der Heidelberger Ballschule (ROTH, 1999; MEMMERT, 2000). Letzteres Modell wurde sogar ausführlich empirisch getestet (ROTH \& MEMMERT, 2007) und anhand zahlreicher wissenschaftlicher Publikationen und Anwendungen im Projekt der Heidelberger Ballschule und bei Sportvereinen (z. B. TSG 1899 Hoffenheim) als eine praxistaugliche Alternative zum Direktiven Modell etabliert. Allerdings ist auch dort noch immer eine Trennung zwischen den 
Begriffen Technik und Taktik bei Lehr- und- Trainingsprozessen vorhanden. Sie werden weiterhin als verschiedene Aspekte des Spielsports betrachtet und sollten deswegen durch verschiedene Lehrprozesse und in verschiedenem Alter gelernt werden.

Eine detaillierte Berücksichtigung des Begriffs Handlung bietet eine Verbindung der Begriffe Technik und Taktik als eine Einheit an, zu der beide gehören. Durch die Handlung gelingt es den Spielern, auf die Spielkonstellationen einzuwirken. Je raffinierter das Handlungswissen im Langzeitgedächtnis repräsentiert ist, desto mehr Spielkompetenzen und daher bessere Spielhandlungen können sie ausführen (ERICSSON 2003, S. 78). Dennoch sollten das Handlungswissen und seine Formulierung als Kompetenz innerhalb des Spielkontextes aufgebaut und verbessert werden. Die Handlungstheorie stellt einen theoretischen Rahmen für die Analyse der Spieler in Spielsituationen bereit. Jede Beteiligung am Spiel ist charakterisiert durch kognitive und emotionale Prozesse, die in Beziehung mit den ausgeführten Bewegungen stehen und das Ziel haben, absichtliche Einwirkungen auf das Spiel zu schaffen. Allerdings kritisiert BREMEN (1987, S. 47) die wenigen Anwendungsmöglichkeiten der Handlungstheorie in der Praxis und verlangt die Übersetzung der theoretischen Überlegungen in effektive Methoden für das Erlernen der Sportspiele.

Die vorliegende Arbeit strebt daher an, ein solches didaktisches Modell zu entwerfen und zu testen, das für das Lehren und Trainieren der Sportspiele die Grundprinzipien der Handlungstheorie (nach MUNZERT, 1989, 1995, 1997) berücksichtigt und auf Lerntheorien wie dem interaktionistischen Konstruktivismus (REICH, o.J.a, 2006) und dem Situierten Lernen ${ }^{2}$ (LAVE \& WENGER, 1991) basiert.

Anhand von Strategien aus didaktischen Modellen, die in einem Schulkontext entwickelt und getestet wurden, wird in dieser Arbeit ein Modell entworfen, das auf die Entwicklung der Spielkompetenzen achtet und diesbezüglich einen Schwerpunkt in der Ausbildung und im Trainingsprozess der Spieler setzt. Dieses Modell geht von der Annahme aus, dass Handlungswissen die zentrale Grundlage der Leistung während des Spielens sei und dass dieses Wissen auf einer hohen Hierarchieebene angesiedelt werden müsse, die auch aus Daten und Informationen besteht. Eine reine Information kann direkt vermittelt werden, Handlungswissen hingegen nicht. Es muss durch einen Prozess aktiver Teilnahme und Überlegung aufgebaut werden, der zum Verständnis und zur Ausarbeitung der Aktionsregeln führt. Das Handlungswissen äußert sich in dieser Betrachtung als Kompetenz, wenn es für die Problemlösung angewendet wird. Das vorgeschlagene Modell wird auf die Wissenshierarchie und die Wissenstreppe bezogen, die eine Art Konzeptanordnungen sind, die die Identifizierung, die Erarbeitung und den strukturier-

\footnotetext{
${ }^{2}$ Situated Learning wird auf Deutsch als situiertes Lernen übersetzt. Kersten REICH an der Universität zu Köln veröffentlichte mehrere Texte über diesen didaktischen Ansatz im Rahmen seines,,Methodenpools“, z.B. http://www.uni-koeln.de/hf/konstrukt/reich works/aufsatze/reich 58.pdf und http://methodenpool.uni-koeln.de/-situierteslernen/unterricht.html[20.07.2011]. Auf der digitalen Enzyklopädie Wikipedia wird Situated Learning ebenfalls als situiertes Lernen übersetzt: http://de.wikipedia.org/wiki/Situiertes Lernen[20.07.2011]
} 
ten Ansatz von Konzepten erlaubt. Diese Konzeptanordnungen werden in Kapitel 3 (ab Seite 71) als theoretische Grundlage für das Modell der Spielhandlungskompetenzen ausführlich beschrieben. Sie ermöglichen darüber hinaus eine übersichtliche Betrachtung des Weges, der bei Sportspielen zur Leistung führt. Die effektive Durchführung dieses Weges ist das angestrebte Ziel des in dieser Arbeit vorgestellten Modells der Spielhandlungskompetenzen 


\section{Sportspiele lernen}

Pädagogische und didaktische Anstrengungen haben die Ausbildung von Experten in bestimmten Wissensbereichen zum Ziel. Die Expertiseforschung ${ }^{3}$ stellt eine besondere Forschungsperspektive dar, indem sie von vorne nach hinten vorgeht, d.h. durch die Erforschung der Fähigkeiten und Fertigkeiten von Experten kann man Schlussfolgerungen ziehen, die auf Ausbildungsprogramme anwendbar sind (vgl. ANDERSON, 1996, S. 270). Die wesentlichen Entwicklungen in diesem Bereich tragen in hohem Maße zum Design und zur Verbesserung von didaktischen Verfahren bei, die wirksam und effizient die Ausbildung von Experten steuern sollen. Im Sportbereich hat die Expertiseforschung interessante Schlussfolgerungen ergeben, die für die Entwicklung von didaktischen Modellen wertvoll sind. In folgende Abschnitte werden einige der Schlüsse diskutiert, die die Didaktik der Sportspiele voranbringen könnten.

\subsection{Vom Novizen zum Experten}

Die Expertise von Sportlern ist aus verschiedenen Blickwinkeln untersucht worden mit dem Ziel, daraus Erkenntnisse für didaktische Prozesse, Lehrmethoden und geeignete Rahmenbedingungen zum Lernen und zur Erreichung höherer Leistungen zu gewinnen. Eine Metastudie von ERICSSON und SMITH (1991) lieferte erste Bezugspunkte der Expertiseforschung im Sport.

ERICSSON (2003, S. 77 ff.) schlägt den Forschungsansatz „Expertise Approach“ vor, dem zufolge der grundlegende Unterschied zwischen Experten und Novizen der Umfang des absichtsvollen Übens ist. Der Experte weise einen deutlich größeren Umfang an Übungszeit auf. Entsprechend dieser Auffassung spielt die genetische Ausstattung oder das ererbte Talent keine entscheidende Rolle für die im Idealfall zu erbringenden Leistungen, denn die Merkmale des Experten würden größtenteils im Laufe des Übungsprozesses erreicht. Experten verfügten daher über erworbene Fertigkeiten und Fähigkeiten, die in ihrer Ausprägung und Zusammensetzung für den jeweiligen Anwendungsbereich spezifisch seien (McPHERSON, 1999, S. 370; MCPHERSON \& THOMAS, 1989, S. 200). Damit unterscheiden sie sich von den Novizen. Eine allgemeine, zur Überlegenheit führende Begabung wird hingegen ausgeschlossen. Forschungen, die die Schachexpertise betrafen, sind hierfür in vielfältigen Sportsituationen repliziert

${ }^{3}$ Das digitale Psychologie Lexikon Psychologie 48.com bezeichnet als Expertiseforschung den Forschungsbereich, der sich mit Experten befasst; normalerweise werden bei diesem Forschungsansatz Experten und Novizen gegenübergestellt und verglichen. http://www.psychology48.com/deu/d/expertiseforschung/expertiseforschung.htm[23.07.2011]. Vielfältige Veröffentlichungen im Sport, die sich mit Experten befassen, wenden auch dieses Konzept an, z. B. HAGEMANN, N., TIETJENS, M. und STRAUSS, B. (Hrsg.) (2007). Psychologie der sportlichen Höchstleistung: Grundlagen und Anwendungen der Expertiseforschung im Sport. Göttingen: Hogreffe; NEEB, C. (2008) Expertise. Die aktuellen Entwicklungen und der Stand in der Expertiseforschung mit Bezug auf den Leistungssport. München: Grin. 
worden (JANELLE \& HILLMAN, 2003, S. 28). Übereinstimmend mit dieser Annahme hängen hervorragende Leistungen in Sportspielen eher von erworbenen taktischen und strategischen Fertigkeiten ab, weniger von ererbten koordinativen Fähigkeiten und physiologischen Parametern (GRIFFIN \& PATTON, 2005, S. 7).

Mehrere Studien haben bei Könnern einen größeren Umfang an deklarativen und prozeduralen Wissensbeständen festgestellt (ERICSSON, KRAMPE, \& TESCH-RÖMER, 1993, S. 387). Einige Autoren (KEIL \& PELLEGRINO, 1988, S. 80; EYSENCK \& KEANE, 2000, S. 497) verweisen auf den Umfang erworbenen Wissens als den grundlegenden Unterschied zwischen Experten und Novizen. Von diesem Wissen ausgehend, sind die Experten in der Lage, aus der Umwelt aufgenommene Informationen in ihre Entscheidungen einzubeziehen und damit die spezifischen Anforderungen des gegenwärtigen Zustandes zu bewältigen. Die Experten scheinen das erforderliche breite Wissen zu besitzen, um die Wirksamkeit ihres Entscheidungsprozesses sicherzustellen. Dieses Wissen wurde aufgrund einer längeren Zeitspanne absichtlicher Übung erworben (COTE, BAKER \& ABERNETHY, 2003, S. 101).

Das Wissen über das "Was" und "Wie" spiegelt auf einfache Weise die spezifischen Anforderungen des Sports wider. In jeder Situation soll als Antwort auf die wahrgenommenen Umweltkonstellationen und Handlungsbedingungen nicht nur die geeignete Lösung gefunden werden, sondern sie soll im Anschluss auch genau und korrekt ausgeführt werden. Das erfordert eine Art Koordination des deklarativen und prozeduralen Wissens, die bei Experten als metakognitive Fertigkeit wirksam wird (GREEN \& GILHOOLY, 1992, S. 55). Auf der Basis dieser Fertigkeit treffen die Spieler Entscheidungen, die sie danach auch auf technisch, physiologisch und psychologisch richtige Weise ausführen können.

Zwischen Novizen und Experten liegt dabei ein Lernprozess. „Die fortschreitende Verfeinerung von Expertise wird insbesondere über die reflexive Elaboration von Wissen erreicht." (GRUBER, 2004, S. 21). Im Fall der an Mannschaftsspielen teilnehmenden Spieler finden sich noch Lücken in den spezifischen Ausbildungsmaßnahmen. Viele didaktische Ansätze basieren darauf, dass sie ursprünglich nur für eine einzelne Sportart entwickelt wurden und daher die Besonderheiten der jeweiligen Mannschaftssportart nicht hinreichend berücksichtigen. Die Entwicklung von spezifisch für Sportspiele geeigneten didaktischen Modellen ist daher auch heute noch ein Forschungsdesiderat.

Anschließend werden die Überlegungen über die Didaktik der Sportspiele vorgestellt, die zum Entwurf eines alternativen Modells für die Schulung der Spielkompetenzen geführt haben. 


\subsection{Traditionelle Ansätze beim Lernen der Sportspiele}

Die Mehrheit der Schulsportlehrer und Sportspieltrainer setzt auch heutzutage noch das didaktische Direkte Modell als Lern- und- Trainingsansatz ein. Für lange Zeit ist es sogar das einzige angewandte didaktische Modell auf Vereins- oder Leistungssportebene gewesen, obwohl auch alternative Modelle zur Verfügung standen. Diese wurden jedoch - und werden noch immer -vornehmlich im Schulsport eingesetzt. Das Direkte Modell wird dabei nicht ohne Kritik als ein Ansatz des „der Lehrer/Trainer sagt und die Schüler/Spieler machen“ (METZLER, 2005a, S. 187) bezeichnet.

Das Direkte Modell ist trainerzentriert. Die Trainer denken an bestimmte Lernziele, stellen den Spielern ein Muster der gewünschten Bewegung vor und führen ihnen somit eine wünschenswerte Fertigkeit oder ein Konzept vor. Darauf aufbauend organisieren sie anschließend Trainingsaktivitäten innerhalb einer bestimmten festgelegten Zeitspanne. Während des Übens bieten sie den Spielern außerdem Rückmeldungen und emotionale Förderung an. Die Spieler treffen im gesamten Prozess wenige eigene Entscheidungen, gehorchen vielmehr den Hinweisen oder Anweisungen des Trainers. Das Ziel eines solchen Übens ist eine effektive Ausnutzung der zur Verfügung stehenden Zeit, um möglichst schnelle Ergebnisse beim Lernen zu erzielen. Die Essenz des Modells ist es, den Spielern viele Gelegenheiten zu bieten, die Bewegungen oder Fertigkeiten zu üben. Dadurch kann der Trainer die Übenden kontrollierend beobachten und die korrektiven und emotionalen Rückmeldungen möglichst passgenau und zeitnah liefern (vgl. METZLER, 2005a, S. 189).

Das Direkte Modell enthält somit besondere Prinzipien über Training und Lernen:

- $\quad$ Der Trainer ist die Hauptquelle von Wissen und Entscheidungen, er soll den ganzen Lernprozess führen.

- $\quad$ Der Trainer soll den Inhalt festlegen und diesen in Lernaufgaben umsetzen, meistens in kleine Schritte unterteilt.

- $\quad$ Der Trainer wird als Wissensträger angenommen. Als solcher soll er sein Wissen auf dem effektivsten Weg und mit den effizientesten Medien den Spielern vermitteln.

- $\quad$ Der Trainer soll sein Fachwissen nutzen, um die Lernprozesse zu leiten und zu kontrollieren, er soll auf diese Weise eine effektive Wirkung seines Trainings gewährleisten.

- $\quad$ Lernen ist die Wirkung von Erlebnissen, die nach einem bestimmten Verhalten erfahren werden. Bestärktes Verhalten wird aufrechterhalten, bestraftes Verhalten wird schwächer werden und verschwindet letztlich vollständig.

Im Direkten Modell werden Lehrinhalte, also auch das Spiel, in kleine Schritte untergliedert. Jeder Schritt ist eine technische Fertigkeit, die die Spieler einzeln ausführen sollen, bis sie automatisiert ausgeführt wird. Dies erfolgt so lange, bis die Spieler psychisch reif genug sind, um abstrakte Inhalte zu erfassen und den kognitiven Lehrstoff zu begreifen (METZLER, 2005a, S. 205). Das Ziel der Trainer ist es, auf effiziente Weise nur diejenigen Informationen zu übermitteln, die die Spieler verarbeiten und in Wissen umwandeln sollen. Man hofft, dass die Spieler in der Ausführung der Lerninhalte geschickter werden und dass sie nach dem Training wissen, „was“ sie zu tun haben und „wie“ die Handlung mit hoher Genauigkeit bis hin zur Feinformabläuft. Der Lernfort- 
schritt wird nach biomechanischen Parametern und nach der Reproduktionsgenauigkeit bewertet (BUTLER\& McCAHAN, 2005, S. 40).

Das Direkte Modell basiert auf der Theorie des Behaviorismus. Die Grundannahme lautet, dass Lehrer und Trainer Wissensträger seien. Sie sollen das Wissen durch direkte Methoden an die Spieler übermitteln, die den Lernstoff passiv empfangen und konsequent gemäß den Anweisungen handeln. Die Idee hinter dieser traditionellen Lehr- und Lernvorgehensweise ist, dass ein Spieler, der einmal die erforderlichen technischen Fertigkeiten für eine bestimmte Sportart erlernt hat, von Natur aus in der Lage ist, das Spiel wirksam zu spielen. Der Spieler soll die in Isolation erlernten technischen Fertigkeiten in geeigneter Weise im Spiel anwenden. Die Bewertung ist auf die richtige Ausführung der technischen Fertigkeiten, auf die Geschicklichkeit mit dem Ball und auch auf die richtige Ausführung des taktischen und strategischen Verhaltens gerichtet, das vom Trainer vorgegeben wird.

Die grundlegenden Merkmale des Direkten Modells sind sinnvoll, wenn das ausschließliche Ziel das Lernen von Bewegungsfertigkeiten ist. Diese Merkmale sind:

1. Der Inhalt ist in kleine Schritte unterteilt, die Spieler sollen jeden dieser Teile auf dem Weg zu einem größeren Lernziel beherrschen.

2. Der Trainer liefert den Spielern das Muster, wie sie die richtige Bewegung ausführen sollen. Dies geschieht zusammen mit Vorgaben für die Steigerung der Leistung bei der Ausführung.

3. Die Spieler haben Zeit zu üben und bekommen vom Trainer viele Rückmeldungen.

4. Die Spieler müssen beweisen, dass sie eine Bewegung beherrschen, ehe sie sich mit der nächsten Aufgabe aus einer Reihe von technischen Fertigkeiten und taktischen Inhalten beschäftigen, die zum selben Sportspiel gehören und auch zu lernen sind.

Innerhalb des Modells werden die Inhalte und die Lernaufgaben vom Trainer streng kontrolliert. Die Spieler haben kaum die Möglichkeit, selbst auszuwählen, was und wie sie lernen. Der Informationsfluss im Direkten Modell weist meistens nur in eine Richtung. Der Trainer beginnt mit der Kommunikation, die Spieler hören oder schauen zu. Der Trainer darf Fragen stellen, aber nur um das Begreifen der übermittelte Information festzustellen. Bei Nachfragen sollte er den Sachverhalt erneut erklären. Die Informationen werden meistens verbal vermittelt, bedarfsweise durch Modellierung oder visuelle Lernmittel unterstützt bzw. verdeutlicht.

Eine Trainingseinheit nach traditionellem Muster hat gewöhnlich folgenden Ablauf: Aufwärmen, Ausübung der technischen Fertigkeiten, Spiele auf kleinen Feld ohne und mit Opposition und gegen Ende das eigentliche Zielspiel. Abstrahiert dargestellt folgen also die Bausteine

- Technik-Schulung

- Taktik-Schulung

- Kleines Spiel

- Spiel

aufeinander. 
Ein Beispiel für das Direkte Modell wird im folgenden Exkurs aus einem Pädagogiklehrbuch für Basketball vorgestellt (PEYRO \& SANPEDRO, 1986, S. 203, Übersetzung durch den Autor dieser Arbeit).

\begin{abstract}
„Um eine vollkommene Ausbildung zu schaffen, sollen die Kinder oder die Spieler die größtmögliche Anzahl technischer Fertigkeiten auf eine vernünftige Weise erlernen. Der Spieler soll diese Bewegungen genügend oft wiederholen, bis die angestrebte Leistungsebene erreicht wird. Wenn das Kind ein Problem oder eine Spielsituation lösen muss, kann es infolge dieses Lernprozesses zwischen den möglichen technischen Fertigkeiten die Alternativen erkennen und die geeignetste auswählen. In einigen Fällen wird der Lehrer oder Trainer die Entscheidung treffen, bei anderen werden die Spieler selbst entscheiden. Auf diese Weise können die Spieler ihre Leistung beim Spielen verbessern. Die Progression der technischen Elemente kann in einer Zeitspanne zwischen 4 und 6 Jahren erreicht werden, jeweils abhängig vom Alter und den Vorerfahrungen der Spieler.
\end{abstract}

Die Inhaltstruktur dieses Trainings ist folgende:

a. Beschreibung der Grundlagen des Angriffs:

1. Der wichtigste Punkt für das Lernen.

2. Praktische Übungen und Spiele.

b. Beschreibung der Grundlagen der Verteidigung:

1. Der wichtigste Punkt für das Lernen.

2. Praktische Übungen und Spiele.

c. Spielsituationen im Angriff, individuell und kollektiv:

1. Praktische Übungen.

2. Einführung des Konterangriffs.

d. Spielsituationen in der Verteidigung, individuell und kollektiv:

1. Praktische Übungen.

2. Ausschalten des gegnerischen Angriffs.

e. Realspielsituationen:

1. Im Angriff.

2. In der Abwehr".

Viele pädagogische, didaktische und sportwissenschaftliche Argumente stellen sich dem Direkten Modell entgegen. Es werden dann auch zahlreiche alternative Modelle entworfen und in Lehrbüchern vorgestellt. Häufig wird ihre Wirksamkeit durch empirische Studien bestätigt. Allerdings wird in der Praxis des Schulsports und in Sportvereinen noch immer am häufigsten das Direkte Modell angewendet. LOIBL (2001, S. 25) bemerkt entsprechend, dass „sich die Praxis mit einer bewegungstheoretischen Argumentation gegen alle pädagogisch-didaktischen Erneuerungsvorschläge immunisiert".

Im folgenden Abschnitt werden einige der zur Verfügung stehenden alternativen Modelle näher erläutert. 


\subsection{Spielvermittlungsmodelle}

Die auf das Spiel und die Spieler zentrierte Vorgehensweise "Game and Player Centered Approach" (GPCA) entstand in den achtziger Jahren an der Loughborough Universität in Großbritannien. David BUNKER und Rod THORPE stellten fest, dass Absolventen des Sportspielunterrichts nicht in einer effizienten und erfolgreichen Art und Weise an einem Spiel teilnehmen konnten, obwohl sie eine gute Ausführungsqualität der technischen Fertigkeiten aufwiesen (BUNKER \& THORPE 1986a, S. 17). Die Spieler hatten außerdem Schwierigkeiten bei dem Versuch, die erlernten Fertigkeiten in Spielsituationen einzusetzen. Nach BUNKER und THORPE war dies das Ergebnis des Ansatzes im Sportunterricht, die Aufmerksamkeit auf das Entwickeln der Bewegungsfertigkeiten zu lenken und Taktik, Strategie und „Spielgefühl“ zu vernachlässigen. 1982 publizierten BUNKER und THORPE ihr Modell für Spiellehre: A Model for the Teaching of Games in the Secondary School. Seitdem hat der Ansatz des Modells „Teaching Games for Understanding (TGfU)“ breite internationale Aufmerksamkeit erlangt. Viele Sportwissenschaftler haben in großem Umfang Berichte zu diesem relativ neuen Modell geschrieben $^{4}$.

Vor dem GPCA war es vor allem beim Sportspiellernen üblich, dass die Aneignung von spezifischen Bewegungsfertigkeiten den Schwerpunkt des Sportunterrichts bildete. Die daraus resultierenden Hauptprobleme fassen MANDIGO, BUTLER und HOPPER (2007, S. 14) zusammen:

- Die Spieler wiesen einen Transfermangel von den Bewegungsfertigkeiten zu Spielsituationen auf.

- Die Spieler, die nicht alle Bewegungsfertigkeiten beherrschten, vermuteten, dass sie keinen Erfolg im Spiel erlangen könnten.

- Die Spieler wollten lieber das Zielspiel spielen, statt übermäßig viel Zeit zum Erlernen der Bewegungsfertigkeiten aufzuwenden.

Der GPCA ist lernerzentriert und damit zur Lösung der oben genannten Probleme geeignet. Die Grundidee ist die Schaffung einer optimalen und motivierenden Stimmung, um das Erlernen von exekutiven und kognitiven Handlungsfertigkeiten zu unterstützen. Diese positive Stimmung trägt dazu bei, positive Erfahrungen beim Lernen und Anwenden von Fertigkeiten zur Lösung der Spielprobleme zu machen. Der Schwerpunkt wird auf die Entscheidungsfähigkeit des Spielers in Spielsituationen gelegt. Der Lehrer/Trainer spielt die Rolle eines "Lernerleichterers und Problemschöpfers" (LIGHT, 2005a, S. 173).

GPCA ist eine Vorgehensweise, die den Lernenden zunächst zu verstehen ermöglicht, warum eine technische Fertigkeit ausgeführt werden soll. Erst dann lernen sie, wie die Bewegung optimal ausgeführt wird. Daher betont es vorrangig oder gleichgewichtig mit dem Lernen von technischen Fertigkeiten ein bewusstes taktisches Verständnis

\footnotetext{
${ }^{4}$ HOOPER und LIGHT (2008) verzeichnen 201 wissenschaftliche Artikel zum TGfU und andere GPCA.
} Verfügbar unter http://www.tgfu.org/articles/ReferencesTGfU2007.pdf[12.10.2009] 
(CHOW, DAVIDS, BUTTON, SHUTLEWORTH, RENSHAW, \& ARAÚJO, 2007, 253). THORPE (1990, S. 90) erklärt hierzu, "the basic philosophy of GPCA is that a person can play games with limited techniques and, even with limited techniques be very competitive".

GPCA schafft ein Umfeld, in dem die Spieler ihre eigene Meinung ausdrücken können. Es fördert das kritische Denken und damit die kognitiven Fähigkeiten des Problemlösens. Dieser Ansatz baut die Spielfähigkeiten in einem Schritt-für-Schritt-Prozess auf. Die Spieler haben so von Beginn an mehr Erfolg beim Spielen, noch während sie die komplizierten Spielfähigkeiten im Lernprozess strukturieren. Wenn die Spieler ihre Fähigkeiten bei besonderen Spielaufgaben zu entwickeln beginnen, nimmt die Attraktivität des Spiels zu, was wiederum die aktive Teilnahme und Freude beim Spielen fördert (WEBB \& PEARSON, 2008, S. 3).

Der Erwerb von technischen Fertigkeiten wird am besten mithilfe von Spielen gefördert, die in ihrem Ablauf eine geringe Komplexität aufweisen. Dann können alle Teilnehmer unabhängig von ihrem Leistungsstand daran teilnehmen. Dies umgeht das Hindernis der technischen Fertigkeiten. Die Spieler müssen somit nicht die langsame Entwicklung ihrer Technik abwarten, um erfolgreich am Spiel teilnehmen zu können.

Das Vorgehen nach GPCA ermöglicht die Übertragung der Spielfähigkeit auf andere Spielhandlungen und sogar zu anderen Sportspielen. Daher kann es die Spielkompetenz und die kognitive Kompetenz gleichzeitig mit der Freude am Spiellernen fördern. Den Lehrern/Trainern wird es so möglich, die Kontrolle und die Beteiligung der Spieler am Spiel zu verbessern. Im Idealfall geschieht dies proportional zur Motivation und es entsteht eine optimale Ausgangssituation für erfolgreiches Lernen.

Das GPCA erkennt Unterschiede im Entwicklungsstand der Spieler an und erlaubt ihnen in ihrem eigenen Rhythmus zu arbeiten. Durch situationsgerechte Erfahrungen ermöglicht es dem Lehrer/Trainer, die Wissensstrukturierung zu fördern. Die Spieler gelangen so zu einem vertieften Verständnis der Spielprinzipien und -konzepte. Das GPCA fördert den Aufbau des Handlungswissens, was wiederum das physische und mentale Engagement stärkt (vgl. WEBB \& PEARSON, 2008, S. 3; MANDIGO \& HOLT, 2004, S. 7).

Es geht nicht nur um während das Trainings Spielen an sich, sondern um die Gestaltung Spielqualität. Durch orientierte Problemstellungen führt der Lehrer/Trainer die Spieler zur Verbesserung des Spiels. Er leitet sie von einem chaotischen Spiel von Anfängern zu einem nach rationalen Gesichtspunkten strukturierten und nach den Spielprinzipien organisierten Spiel. Das Handlungswissen wird durch Problemsituationen, denen die Spieler ausgesetzt werden, und ein reflektierendes Spielen aufgebaut. Ihre zunehmende Komplexität erlaubt die Einstufung und zukünftige Beschäftigung mit taktischen und strategischen Problemen, die sich auf höherer Ebene einstellen. Die technische Ausbildung wird, entsprechend eingeordnet, als sinnvoll betrachtet und kann dann gleichzeitig mit den kognitiven Kompetenzen entwickelt werden.

Es wurden im Laufe der Zeit verschiedene Anwendungen, Ansätze, Variationen und Anpassungen des GPCA entwickelt. Auch ohne direkten Bezug zum Ausgangsmodell und sogar noch vor dessen Erscheinen im Jahr 1982 wurden didaktische Ansätze vorgeschlagen, die die Förderung der Spielfähigkeit in den Mittelpunkt stellen. Alle derartigen Ansätze fokussieren sich auf die Spieler und das Spiel und relativieren damit die übertriebene Wichtigkeit der im Direkten Modell im Mittelpunkt stehenden technischen Fer- 
tigkeiten. Im Folgenden werden einige dieser Ansätze anhand des folgenden Organisationsrahmens vorgestellt.

\begin{tabular}{|c|c|c|c|c|}
\hline Einführung & Begründung & Struktur & Aufgabenstellung & Diskussion \\
\hline
\end{tabular}

\subsubsection{Spielreihe}

\section{Einführung}

In den 1960er Jahren erschien die Spielreihe als ein Gegenmodell zu den Lernmethoden, die damals die Sportpädagogik beherrschten. Im Wesentlichen war dies die „Übungsreihe“ als Hauptlernmethode der Sportspiele. Das Konzept der Spielreihe strebte an, die Lernenden so schnell wie möglich in die Zielspiele einzuführen, und setzte dafür die Zuordnung einzelner Spiele in ein Spielkontinuum um.

\section{Begründung}

Insbesondere im Fußball konnte man beobachten, dass Kinder durch die Praxis von adaptierten Variationen der Spiele selbst den Zugang zum Zielspiel schafften. Sie entdeckten dabei immer wieder neue Gestaltungsformen der Wettkampfspiele. Systematisiert man diese kindlichen Spielvariationen, wird die zusammengesetzte Konfiguration von einer weiten Vielfalt von vereinfachten Spielen deutlich, die den Zugang zum Spiel erleichtern (vgl. DIETRICH, 1985, S. 18). Bei einem Prozess aus Umstellung, Wiederaufbau und Wiederholung wurden Phasen einer gewissen Ordnung und Stabilität identifiziert, so dass der von den Kindern selbst vorgenommene Weg in die Didaktik der Spielvermittlung integriert werden konnte. Dabei wurde von der Logik ausgegangen, dass eine Reihe von bestimmten Spielen, die einem Kind den Zugang zum Wettspiel erlaubten, wahrscheinlich in identischer Form auch für andere Kinder als Vermittlungszugang geeignet seien (DIETRICH, 1984, S. 20). Wenn solche Spielreihen in einer systematisch zunehmenden Komplexität angeordnet werden, kann man schrittweise die Spielfähigkeit entwickeln.

Die Spielreihe wurde als geeignete Lernsituation für das Erreichen der folgenden Ziele betrachtet (vgl. DIETRICH, 1985, S. 19):

- Einführung in die Spielregeln und Verständnis ihrer Rolle im Spiel.

- Erfahrung in der Lösung von grundlegenden Situationen von Angriff und Abwehr.

- Taktisches Nachdenken als Teil der Übung verschiedener Handlungsalternativen.

- Schaffung von Spielerfahrungen bei erleichterten, aber ähnlichen Bedingungen wie im Zielspiel.

- Entwicklung der motorischen Fertigkeiten durch ihre Anwendung. 
Um die Ziele zu verwirklichen, werden Spielerlebnisse und bestimmte Lernsituationen erzeugt, die wirksame Handlungsweisen schulen können. Dies geschieht mit der Absicht, sie später in Wettkampfsituationen umsetzen zu können (vgl. DIETRICH, 2007, S. 23).

\section{Struktur}

Die sequentielle Anordnung der Spiele ist charakteristisch für Spielreihen. Es wird mit Kleinen Spielen mit niedrigen technischen Anforderungen angefangen, die eine neue Stellung der partiellen Formen der Zielspiele erlauben. In einem fortschreitenden Prozess werden Bestandteile eingeführt, die das Wettkampfspiel progressiv gestalten. Das Hinzufügen von Bestandteilen impliziert eine Steigerung der Komplexität in kleinen Schritten. Jedes Spiel bildet eine Vorstufe der nächsten, so sind die ersten Spiele durch niedrige Komplexität und viele Umstellungsmöglichkeiten charakterisiert, die sukzessive Erweiterung von Spieleranzahl, Regeln, Spielraum und künstlichen Beschränkungen erfolgt mit Blick auf die Annäherung an das Wettkampfspiel (vgl. KERN \& SÖLL, 2005, S. 257).

Überwiegend im deutschen Sprachraum kann man in Büchern und Zeitschriften zahlreiche Spielreihen finden, die als Spielvermittlungsmodelle dargestellt werden. Nach KERN und SÖLL (2005, S. 256) werden diese Spielreihen als „methodische Hauptstraßen" für das Lernen der Sportspiele betrachtet und werden durch Übungsreihen als "methodische Nebenstraßen“ ergänzt. Im Folgenden werden einige dieser Spielreihen kurz exemplarisch vorgestellt und diskutiert.

\section{Aufgabenstellung}

Mit Kleinen Spielen zum Großen Spiel: Überzahlspiel (HEIL, 1970):

Diese Spielreihe wurde für das Sportspiel Handball entwickelt. Sie kann aber leicht auch bei anderen Invasionsspielen wie Basketball und Fußball angewendet werden. Sie wurde vor allem durch die Broschüre des DFB „Mit kleinen Spielen zum großen Spiel“ (1970) sehr populär gemacht. Die zur Verfügung stehenden Spielern werden so organisiert, dass viele Überzahlsituationen entstehen. Es werden nach und nach Spieler hinzugefügt, bis die Gleichzahl erreicht ist. Die Reihe beginnt mit einem Spiel mit Schießen auf nur ein Tor mit Rollenwechsel von „Angreifern und Torleuten“. Im Anschluss wird ein Überzahlspiel 3:1 mit Mannschafts- und- Positionsvariationen in Angriff und Abwehr durchgeführt. Durch das schrittweise Hinzufügen von weiteren Spielern wird das Spiel auf 4:2 erweitert. Schließlich wird es zum 3:3 und dann zum 3+1:3+1 mit der Teilnahme von Torwarten. Es wird dabei angenommen, dass die Überzahlsituationen mehr Gelegenheiten für das Angriffsspiel ermöglichen, als Gleichzahlsituationen das können. Dabei entstehen viele Schuss- und Wurfgelegenheiten. Durch die Erfahrung in Überzahl haben die Spieler das Erlebnis, bei erleichterten Spielbedingungen zu spielen, was anschließend den Übergang in ein Gleichzahlspiel motivational erleichtert. Die Schulung des Abwehrspiels wird bei dieser Spielreihe nicht berücksichtigt. 
Das Gleichzahlspiel auf ein Tor (KERN \& SÖLL, 2005):

Diese Spielreihe gehört zum so genannten Straßenfußball. Ausgehend von einer 1:1Situation bis zum Spiel 5:5 wird auf nur ein Tor gespielt. Der Torwart agiert neutral, Angriffs- und- Abwehrrolle werden nach einer bestimmten Zeitspanne oder anhand besonderer Regeln getauscht, zum Beispiel nach jedem Tor. Das Spiel auf nur ein Tor stellt einige Vorteile für die Abwehrspieler bereit, was die Spieler im Angriff zwingt, besondere Angriffsstrategien zu entwickeln. So werden technische Spielfertigkeiten wie Dribbeln, Passen und Schießen und taktische Spielfertigkeiten wie Freilaufen, Anbieten und Individualabwehr geschult. Am Anfang können Schwierigkeiten in der Ballbeherrschung entstehen, ebenso, wenn das Spiel maßgeblich von geschickten und experimentierfreudigen Spielern kontrolliert wird.

Das Kombinationsspiel (EMRICH, 2007):

Auch diese Spielreihe wurde ursprünglich für das Handballspiel entworfen. Sie beginnt mit einer Gleichzahlsituation, der Schwerpunkt ist das Kombinationsspiel innerhalb der Mannschaft. Es wird auf einem begrenzten Feld ohne Tore gespielt, das Ziel ist das Halten des Balls und das Spielen von möglichst vielen Pässen, ohne dabei den Ballbesitz zu verlieren. Die Wände der Sporthalle werden als Tore genutzt. Folglich wird die Komplexität durch bestimmte Einschränkungen gesteigert. Zum Beispiel ist ein Tor nur dann gültig, wenn der Ball einen bestimmten Punkt an der Wand berührt, oder man darf nur aus einer bestimmten Entfernung zum Tor werfen. Das Kombinationsspiel kann speziell für Anfänger mühsam sein. Die Wand als ein großes Tor nutzen zu können, ermöglicht aber die Vereinfachung des Spielziels und gewährt den Spielern mehr Freiheiten, die richtige Stellung auf dem Spielraum zu finden.

Fußball in Grundsituationen (DIETRICH, 1984, 1985):

Die letzte Version dieses Ansatzes wurde im Jahr1985 publiziert. Diese Spielreihe zerlegt Fußball, Basketball und Handball in drei Grundsituationen:

- Grundsituation 1: Torschießen - Torabwehr: Es geht um zahlreiche Formen von Torschussspielen. Torschütze und Torwart wechseln einander ab.

- Grundsituation 2: Herausspielen von Torschusssituationen auf ein großflächiges Tor, diverse Varianten.

- Grundsituation 3: Aufbauen und Stören des Angriffspiels. Zwei Mannschaften führen Spielzüge von 3 gegen 3 bis sieben gegen sieben, ggf. sogar elf gegen elf Spieler durch, gespielt wird auf Tore mit oder ohne Torwart.

Das besondere Kennzeichen dieses Modells ist die Einbeziehung von Formen und Verhaltensweisen, die sich im Straßenfußball beobachten lassen.

\section{Diskussion}

Es haben sich nicht alle Erwartungen der jeweiligen Autoren von Spielreihen in der Praxis erfüllt. Eine Reihe, die sich bei der einen Gruppe als erfolgreich erwiesen hat, könnte bei der anderen wenig oder nicht wirksam sein. Die Spielreihen können nicht automatisch die Entwicklung der Spielfähigkeit hervorbringen, die Besonderheiten der Gruppe und einzelner Spieler erlauben es nicht, eine standardisierte, allgemeine Spielreihe zu 
gestalten, die bei jedem Spiellernprozess wirksam ist (vgl. DIETRICH, DÜRRWÄCHTER, \& SCHALLER, 2007, S. 19).

Es ist nicht immer möglich, die pädagogischen Ziele mit denjenigen des Spiels zu vereinbaren, da das Spiel Mittel und Ziel der Spielreihe zugleich ist (vgl. DIETRICH, 1985, 18-19). Gerade dann, wenn ein Spiel erfolgreich und flüssig läuft und die Lernenden dabei Spaß haben, wird es durch den Trainer unterbrochen. Er will an dieser Stelle eine komplexere Spielsituation einführen, um entsprechend seiner pädagogischen Absicht das Spiel weiterzuentwickeln. Sein Vorgehen entspricht zwar der Natur des Spiels und auch dem Wunsch der Kinder, im Zielspiel erfolgreich zu spielen, widerspricht aber der situativen Motivation. In der Realität ist es daher nicht immer möglich, das pädagogische Vorhaben (das Spiel aktiv zu lernen) mit der Spielidee zu vereinbaren.

Spielreihen sind nicht wissenschaftlich erprobt worden. Sie wurden als praktische didaktische Werkzeuge für die Schulung der Sportspiele entworfen, ihre Anwendbarkeit, Wirksamkeit und Vergleichbarkeit ist aber bis heute nicht Forschungsobjekt gewesen. Es wurde außerdem bislang nicht versucht, ein festes theoretisches Fundament für den Nachweis ihrer didaktischen Wirksamkeit zu schaffen.

\subsubsection{Situative Spieldidaktik}

\section{Einführung}

BRÜGGEMANN und ALBRECHT (1982) analysierten die Leistungsfaktoren des Fußballs und deren Beiträge zum Spielverhalten. Sie schlugen die Situative Spieldidaktik als als Rückbesinnung auf den Straßenfußball vor. Darin organisierten die Kinder das Spiel selbst und erreichten selbstständig Spielfähigkeit, Kenntnisse und Spielerfahrung. Dieses Modell unterscheidet sich von der Spielreihe dadurch, dass es einen Zusammenhang mit der wirklichen Spielumgebung herstellt. Durch die Regeln wird der Schwerpunkt auf das Training von Technik, Taktik und Kondition gelegt.

\section{Begründung}

Das Fußballspiel ist von ständig wechselnden Situationen geprägt. Diese erfordern genaue Wahrnehmung und Beurteilung des Spielgeschehens, um eine geeignete Entscheidung treffen zu können und durch die Ausführung einer technischen Fertigkeitoder eines taktischen Manövers einen Wettbewerbsvorteil zu erreichen. Während des situativen Spielens sammelt der Spieler gleichsam die Erfahrung von Erfolg und Misserfolg und gewinnt daher schrittweise Lernkontrolle (vgl. BRÜGGEMANN \& ALBRECHT, 1982, S. 13-16).

Die Situative Spieldidaktik betrachtet das Spielverhalten in verschiedenen Situationen als ein ganzheitliches Lernelement, innerhalb dessen die Leistungsfaktoren des Fußballs gemeinsam auftreten. Dies geschieht jedoch in verschiedener Ausprägung und Intensität. Die Situative Spieldidaktik setzt das Spiel als „Spielübung“ ein, in der kognitive Fähigkeiten gefördert werden. Nach diesem Konzept ist es nicht so wichtig, wie „richtig“, gemessen am Lehrbuch, eine technische Fertigkeit ausgeführt wird, sondern wie 
erfolgreich diese Bewegung für die Lösung der Spielsituation ist, ohne für den Gegner vorhersagbar zu sein.

Die eingesetzten Spiele ähneln denen des Straßenfußballs. Mit bestimmten Regeln und unterbrechungsfreiem Spielverlauf bieten sie optimale Möglichkeiten für die Anwendung des Prinzips des entdeckenden Lernens. Nach diesem Lernprinzip findet durch die selbstständigen Versuche und Erfindungen ein Lernprozess statt und das Gelernte wird dann im wirklichen Spielwettbewerb angewendet. Wenn der Straßenfußball unter allen didaktischen Voraussetzungen im Training umgesetzt wird, kann man Verbesserungen im Spielverhalten durch die folgenden Merkmale hervorrufen (vgl. BRÜGGEMANN \& ALBRECHT, 1982, S. 15):

- „Häufige Wiederholung bestimmter Bewegungsabläufe und Verhaltensweisen innerhalb ihrer situativen Anwendung.

- Möglichkeit zur alternativen Situationslösung als individuelle Verhaltensfreiheit.

- Keine Überforderung des Anfängers durch komplexes Lernen und Spielverhalten mit seinen vielfältigen Herausforderungen“.

Nach der Situativen Spieldidaktik kann der Straßenfußball als Übungsform zur Entwicklung der technischen Fertigkeiten, der verfügbaren taktischen Mittel und auch der konditionellen und kognitiven Fähigkeiten beitragen. Außerdem fördert er die Kreativität der jungen Fußballspieler, die ansonsten durch die häufige Anwendung von nicht-situativen Übungsreihen eingeschränkt wird.

\section{Struktur}

Das Ziel der Situativen Spieldidaktik ist das Erlernen des Spielverhaltens in Wettbewerbssituationen. Das Spielverhalten wird von zwei Gruppen von Faktoren bestimmt:

a) Faktoren, die nicht verändert werden können, wie die körperlichen und physiologischen Merkmale der teilnehmenden Spieler.

b) Technische, taktische, konditionelle und kognitive Faktoren, die durch Training beeinflusst werden können.

Beim Lernen des Spielverhaltens müssen alle Leistungsfaktoren in jeder Lernaufgabe vorhanden sein, die nicht-veränderbaren Faktoren stellen dabei die Anfangsbedingungen. Die veränderbaren Faktoren werden durch Spiele auf kleinen Feld beeinflusst. Diese Spiele stellen einen Ausschnitt aus dem großen Spiel dar und alle möglichen Lösungsalternativen werden dabei ausgeschöpft. Die taktischen Mittel werden variiert, um auf bestimmte Schwerpunkte hinzuwirken, die das Spielverhalten gestalten. Es werden drei grundlegende Änderungen der Spielregeln implementiert (BRÜGGEMANN \& ABRECHT, 1982, S. 29-30):

- Die Provokationsregeln fördern die Anwendung einer bestimmten technischen Fertigkeit oder einzelner taktischer Mittel. Diese werden durch viele Wiederholungen während des Spiels geübt. Beispiel: Ein Tor ist nur gültig, wenn es nach einem Doppelpass fällt oder mit dem linken Fuß geschossen wurde.

- Die Fortsetzungsregeln vermeiden ständige Spielunterbrechungen nach Torschuss oder Ballaus. Durch die Anwendung dieser Regeln wird eine konstante Aufmerksamkeit und Konzentration gefördert, was die kognitiven Faktoren stärkt. Beispiel: Nach einem Torschuss wird der Ball zum Angreifer zurückgegeben, der kann das Angriffsspiel fortsetzen und weiter Torgelegenheiten suchen. 
- Die Korrekturregeln sind zusätzliche Regeln, die mit dem Zweck in das Spiel eingeführt werden, unerwünschtes Spielverhalten zu korrigieren oder die Schwierigkeit des Spiels künstlich zu steigern. Durch diese Regeln werden lange Spielunterbrechungen und lange verbale Instruktionen des Trainers vermieden. Beispiel: Wenn das Angriffsspiel zu häufig im Zentrum des Spielfeldes stattfindet, kann eine Regel eingeführt werden, die vor dem Torschuss einen Querpass vorschreibt. Auf diese Weise wird das Spiel über die Flügel gefördert.

\section{Aufgabenstellung}

BRÜGGEMANN und ABRECHT (1982, S. 31) betrachten die Spiele als Übungsformen. Die Auswahl den zu trainierenden Schwerpunkt und das zu verbessernde Spielverhalten bestimmt die Gestaltung eines Übungsspiels. Der Trainer sollte die Organisationsformen, Aufgaben und Regeln systematisch planen. Dabei sind Leistungsniveau, Spieleranzahl, der zur Verfügung stehende Raum sowie die verfügbare Zeit und die Lernmaterialien zu berücksichtigen. Die Gestaltung eines Übungsspiels erfolgt dann schrittweise:

Tabelle 1: Gestaltung eines Übungsspiels bei der Situativen Spieldidaktik (BRÜGEMANN \& ALBRECHT, 1982, S. 31)

\begin{tabular}{|c|}
\hline Erster Schritt: Der Trainer bestimmt das Lernziel \\
\hline Zweiter Schritt: Der Trainer prüft den „Ist-Zustand" seiner Spieler \\
\hline Dritter Schritt: Der Trainer bereitet die Übungsvoraussetzungen vor \\
\hline Vierter Schritt: Der Trainer reglementiert die Kriterien des Parteispiels \\
\hline Fünfter Schritt: Der Trainer legt die Übungsinhalte durch Aufgaben und Regeln fest \\
\hline
\end{tabular}

\section{Diskussion}

Die Situative Spieldidaktik ist ein Modell, das das Spiel überwiegend als Lernmittel anwendet. Deswegen kann man es den spielzentrierten Ansätzen zuordnen. Allerdings beinhaltet es eine exzessive Abhängigkeit vom Trainer. Er bestimmt Lernziele, Bedingungen, Lernaufgabe, Progression und Auswertungsverfahren. Es wird kaum die Beteiligung der Spieler an der Gestaltung des didaktischen Prozesses angestrebt, daher sind die Kritikpunkte von DIETRICH (1985, S. 18) über die Spielreihe auch für die Situative Spieldidaktik gültig.

BRÜGGEMANN und ALBRECHT entwickelten in „Fußballhandbuch, modernes Fußballtraining“ (1982) eine Reihe von Anwendungen und Empfehlungen und eine grundlegende theoretische Basis. Es findet sich jedoch bisher in der sportwissenschaftlichen Literatur keine empirische Überprüfung der Wirkungen der Situativen Spieldidaktik auf das Erlernen des Fußballspiels. 


\subsubsection{Genetisches Lernen}

\section{Einführung}

DIETRICH (1985, S. 19) hat im Spielreihenkonzept das Problem erkannt, dass der Übergang von einer Spielform zur nächsten durch den Trainer allein bestimmt wird. Dieser legt die Abfolge fest und entscheidet, wann das gegenwärtige Spiel enden soll und wann die Kinder weiterspielen sollen. Der Trainer bestimmt auch, wie die Endform des Spiels aussehen soll. Im Gegensatz dazu werden im freien Spiel die Spielformen und der Übergang von den Spielern selbst bestimmt. Sie identifizieren die Spielprobleme und bemühen sich, diese selbstständig zu lösen. Das erfordert nicht nur kognitive, sondern auch soziale Prozesse. Die technischen und taktischen Fähigkeiten werden auch als Mittel und Zweck der Lösung thematisiert. Im Verlauf dieses Prozesses entstehen neue Spielhandlungen. Sie werden durch das wiederholte Analysieren, Denken, Sprechen, Handeln und Ausprobieren stabilisiert. So werden kognitive Muster entwickelt und ausgeprägt, es wirkt ein „kognitives Lernen“ (vgl. LOIBL, 2001, S. 19).

\section{Begründung}

Genetisches Lernen trägt zur Rekonstruktion eines Spiels aus seinem Ursprung heraus bei. Dadurch findet der Spieler das Spiel für sich selbst. Dieser Vorgang stellt eine besondere Form des Problemlösens dar. Drei Prinzipien charakterisieren das Modell (WAGENSCHEIN, 1991, S. 67):

- Genetisch: Die Spieler arbeiten am ursprünglichen Problem und finden selbstständig Lösungen dafür.

- $\quad$ Sokratisch: Die Fragen der Spieler werden an sie zurückgegeben und der Lernprozess durch Fragen unterstützt und bestätigt.

- $\quad$ Exemplarisch: Der Prozess wird an ausgewählten Beispielen durchgeführt, deren Wirksamkeit sich erwiesen hat.

\section{Struktur}

Der erste Schritt ist, das Problem bewusst zu machen und bei den Spielern das Interesse an seiner Lösung zu wecken. Das Problem soll ein Problem des Lernenden sein, durch die Exposition des Phänomens sollen die Spieler selbst neue Probleme aufwerfen. Die Spieler reproduzieren also nicht die vom Trainer vermittelte vorgefertigte Lösung, sondern sie suchen selbstständig Lösungen für neue Probleme, die während des Spiels entstehen. Durch das Ausprobieren bestätigen oder lehnen sie Lösungen ab, dadurch wird das Verständnis gefördert und die Kreativität entwickelt. Der Trainer unterstützt den Vorgang durch Fragen nach dem sokratischen Prinzip. Es können für dasselbe Problem unterschiedliche Lösungen gefunden werden, dazu kommt unterschiedliches Erproben bis zur Optimierung der Lösung. Lernen durch das Frage-Antwort-Spiel wurde von LEIST (1993, S. 224, zitiert nach LOIBL, 2001, S. 21) als „hypothesengeleitetes Handeln" bezeichnet.

Die Struktur des Modells kann folgendermaßen dargestellt werden: 


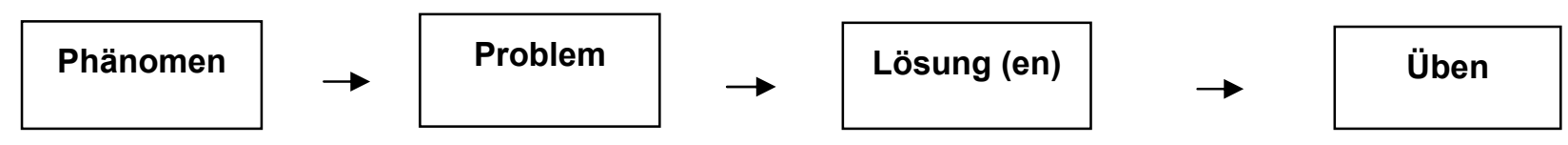

Abbildung 1: Struktur des Spielvermittlungsmodells Genetisches Lernen (LOIBL, 2001, S. 20)

\section{Aufgabenstellung}

Die Spielreihe dient als grundlegende Form des Genetischen Lernens, die Spiele werden durch Regeländerungen als Probleme dargestellt. Die Befragung durch den Trainer provoziert und unterstützt das Problematisieren und die Auseinandersetzung mit dem Problem. Das Erproben der Lösungen schließlich erlaubt die Bestätigung oder Ablehnung der Lösungen und dabei „hypothesengeleitetes Handeln“.

\section{Diskussion}

Zahlreiche Vorschläge für die Umsetzung des Modells sind als Lehrbücher veröffentlicht worden, z. B. SCHMIDT (1991) für Fußball, JANALIK (1982) und BIETZ (1994) für Handball, LOIBL $(1991,2001)$ für Basketball usw. Es finden sich allerdings bisher keine wissenschaftlichen Veröffentlichungen, die die Annahmen des Modells empirisch bestätigen würden. Ein Heft der Fachzeitschrift Sportpädagogik (1/2008) berichtet über zahlreiche praktische Umsetzungserfahrungen des Genetischen Lernens im Schulsportkontext.

\subsubsection{Integratives Modell}

\section{Einführung}

Trotz einer intensiven Schulung der technischen Fertigkeiten und intensiver Spielpraxis durch Spielreihen schaffen es die Lernenden häufig nicht, ein dynamisches, kreatives und wirksames Spiel aufzuziehen. LOIBL (2001, S. 8-9) merkt diesbezüglich an, dass die Spieler nach einer traditionellen Spielschulung zwar einige Bewegungen befriedigend ausführen können, diese aber nicht anwenden und schon gar nicht ihr ganzes Repertoire ausschöpfen. Einige Bewegungen, die noch bei standardisierten Übungen und Tests gut ausgeführt wurden, tauchen während des Spiels schlicht nicht mehr auf. Aus taktischer Sicht bilden die Spieler genau das Spielverhalten nach, auf das der Trainer sie hingewiesen hat. Dieses ist aber leicht zu durchschauen und zu neutralisieren. Die Spieler schaffen keine neuen, improvisierten Spielhandlungen, die den Gegner überrumpeln und neue, im Training bisher nicht vorgestellte Wege zum gegnerischen Tor öffnen könnten. Von dieser Überlegung ausgehend, formuliert LOIBL die Notwendigkeit einer Handlungs- bzw. Spielschulung anstelle einer Technikschulung. Ziel ist die 
Förderung von Bewegungshandlungen bzw. Spielhandlungen als Lösung für die im Zielspiel vorhandenen Spielaufgaben anstelle des Erlernens der technischen Fertigkeiten. Das integrative Modell beansprucht die Integration der Modelle Spielreihe und Genetisches Lernen. Im Gegensatz zu diesen zielt es aber auf die spielsportübergreifende Schulung der Basisfähigkeiten und -fertigkeiten für die Entwicklung einer allgemeinen Spielfähigkeit ab.

\section{Begründung}

Durch eine überzeigende Argumentation gegen die klassische Technikorientierung favorisiert das integrative Modell ebenso wie die Spielreihe oder das Genetische Lernen das Spielen von Anfang an. Es motiviert die Kinder nahezu von selbst und erleichtert somit die Vermittlung von Spielidee und Spielstruktur durch das Spiel selbst. Das Grundprinzip ist dabei, die Spielsituationen und Zusammenhänge zu erkennen, Spielprobleme selbstständig zu lösen und mit dem Spielgerät geschickt umzugehen. Alles das wird im Spiel so erarbeitet, dass es später auf andere Spielsituationen und Sportspiele übertragen werden kann.

Als theoretischen Hintergrund stellt LOIBL (2001, S. 12-15) anthropologische und soziokulturelle Gegebenheiten vor. Der Mensch versuche durch eine subjektive Auseinandersetzung objektive Aufgaben zu lösen, die die Umwelt ihm stelle. Er lege selbst die Ziele fest, vermeide Fehler und organisiere seine Bewegungshandlungen so, dass er die für sich selbst optimalen Lösungen für jede Situation finden könne. Es sei daher notwendig, eine Lernumwelt zu gestalten, die im Gegensatz zu einer Wettbewerbsumwelt eine entspannte Situation bereitstelle und Fehler als wesentliche Bestandteile des Lernens erlaube. LOIBL begründet das Modell außerdem durch eine eingehende Diskussion über ein erfahrungsorientiertes Lehren und Lernen und versucht dabei eine „Erfahrungstheorie" zu entwerfen (S. 32-39).

\section{Struktur}

Beim integrativen Modell wird ein zweistufiges Vorhaben umgesetzt. Zunächst erfolgt eine „didaktische Reduktion“ durch die Vereinfachung der Aufgabe. Diese vereinfachte Spielsituation begünstigt das Erlernen der technischen Bewegungen. Der Zweck ist jetzt nicht Tore zu schießen oder Punkte zu erzielen, sondern die Bewegung selbst. Beim Basketball wird beispielsweise der richtige Ballwurf der Zweck der Übung und nicht das Erzielen des Korbes (LOIBL, 2001, S. 41). Im zweiten Schritt werden in vereinfachten Spielsituationen Spielerfahrungen gesammelt, wobei das Erlernen von Spielhandlungen das Ziel ist. LOIBL sagt dazu, „wenn Spielen heißt, Spielsituationen zu lösen, dann muss Spielen lernen heißen, Spielsituationen lösen zu lernen“ (S. 42). Die Vereinfachung der Spielsituationen erfolgt durch Lockerungen und Anpassung der Spielregeln, „denn die Regeln des Spiels schreiben die Schwierigkeit der Techniken fest" (S. 43).

Im folgenden Raster sind die Alternativen der didaktischen Reduktion dargestellt: 
Tabelle 2: Struktur des integrativen Modell, Alternativen der didaktischen Reduktion, nach LOIBL (2001, S. 43)

\begin{tabular}{|c|c|}
\hline \multicolumn{2}{|c|}{ komplizierte Technik } \\
\multicolumn{2}{|c|}{ komplexe Spielsituation } \\
\hline Technikorientierung & Erfahrungsorientierung \\
\hline komplizierte Technik \\
in vereinfachter Situation üben
\end{tabular}$\quad \begin{gathered}\text { mit vereinfachter Technik } \\
\text { komplexe Spielsituationen lösen }\end{gathered}$

\section{Aufgabenstellung}

Bei verschiedenen Basisspielen wird die Ballkontrolle vereinfacht, so dass die Aufmerksamkeit auf den Raum sowie die Gegner und Mitspieler gerichtet werden kann, es entsteht dann ein vereinfachtes Spiel. Es werden auch vielfältige Übungs- und Spielformen in drei Stufen zugeordnet (LOIBL, 2001, S. 23):

1. „Stufe: allgemeine koordinative und antizipative Fähigkeiten im Umgang mit dem Ball, Ballantizipation und Ballgefühl,

2. Stufe: Schulung der Basistechniken, Werfen und Fangen, Passen, An- und Mitnehmen des Balles sowie das Prellen/Dribbeln und Ballführen.

3. Vermittlung von methodischen Übungsreihen und komplexen Spiel- und Übungsformen sowie von sportspielspezifischen Techniken und Taktiken."

\section{Diskussion}

LOIBL berichtet in seinem Buch: „Basketball, genetisches Lehren und Lernen“ (2001) über breit angelegte Unterrichtsversuche zur Umsetzung seines Modells mit Spielern aus den verschiedensten Altersklassen. Das integrative Modell wurde erfolgreich umgesetzt, die gewünschten Lernergebnisse wurden bei technischen und taktischen Spielhandlungen bestätigt. Ein Transfer zu anderen Sportarten, z. B. Fußball und Vereinssport, hat sich als machbar erwiesen. Wissenschaftliche Untersuchungen, die die Wirksamkeit des integrativen Modells und systematische Vergleiche mit anderen Spielvermittlungsmodellen anstreben, sind bisher jedoch nicht publiziert worden.

\subsubsection{Fußballentwicklungsmodell}

\section{Einführung}

Ein spielerisch intelligenter Spieler weiß, wie und wann die inm zur Verfügung stehenden technischen Fertigkeiten anzuwenden sind, um dem Gegner überlegen zu sein. $\mathrm{Er}$ 
nimmt die Spielhandlungen der Mitspieler und Gegner und die Möglichkeiten und Risiken seiner eigener Handlungen wahr, erkennt sofort die Lösungen für verschiedene problematische Spielsituationen und kann sie anwenden. Ein intelligenter Spieler trifft die besten Entscheidungen zum richtigen Zeitpunkt. Für die Förderung dieser Spielintelligenz schlagen WEIN und SCHREINER (2004; WEIN, 2009) ein didaktisches Modell vor, das auf dem Spiel selbst basiert und das sie als Minifußball bezeichneten. Es geht um kleine Fußballspiele auf verkleinertem Feld und mit adaptierten Regeln. Es findet ein sich stetig steigernder Prozess statt, während dessen Verlauf sich die Anzahl der Spieler erhöht, von 3 gegen 0 bis 4 gegen 4. WEIN und SCHREINER (2004, S. 23-30) schlagen auch ein Auswertungssystem für die Spielfähigkeit vor und diskutieren die Notwendigkeit, die Spieleranzahl bei Wettkampfspielen an die Möglichkeiten der Kinder anzupassen. Auf diese Weise könnte man die Wettkämpfe mit einer steigenden, altersabhängigen Spieleranzahl organisieren, von 3 gegen $3 \mathrm{im}$ frühen Alter bis 11 gegen 11 in der obersten Stufe der Ausbildung.

\section{Begründung}

Mehr als $50 \%$ der Fehler im Fußball werden auf die Entscheidung eines Spielers zurückgeführt und nicht auf die fehlerhafte Ausführung der technischen Fertigkeiten (vgl. WEIN \& SCHREINER, 2004, S. 6). Das isolierte Trainieren der je technischen, taktischen und konditionellen Bestandteile hat deswegen keinen Sinn, wenn nicht jeder dieser Faktoren erfolgreich während des Spiels angewendet werden könnte. Wenn die Trainer die technischen Fertigkeiten durch unendliche Wiederholungen schulen, lassen sie etwas sehr Wichtiges außer Betracht: die Spieler lernen auf diese Weise nicht, wie und wann diese Bewegungen für die Auflösung der Spielsituation anzuwenden sind.

Beim Fußballentwicklungsmodell findet das Lernen während des Spiels statt. Die technischen, taktischen, kognitiven und konditionellen Anforderungen an die Spieler werden mit steigendem Schwierigkeitsgrad gemeinsam trainiert. Da dies auch für das Zielspiel zutrifft, ist das Modell, einen Training ohne spielen überlegen. Die Anpassung des Wettbewerbs an die körperlichen, kognitiven und emotionalen Merkmale der Kinder weckt die Wettkampfmotivation wie in der Vergangenheit der Straßenfußball.

\section{Struktur}

Es geht um ein Modell, das eine umfassende Ausbildung des Fußballspielers anstrebt und das in hierarchischer Weise in verschiedenen Entwicklungsstufen organisiert wird, die dem Alter und der Entwicklungsphase entsprechen. Jede Stufe beinhaltet definierte Ziele und Nebenziele, die den psychischen und physischen Entwicklungsstand der Spieler berücksichtigen. Mit den Spielern werden Übungen abgehalten, die an ihre Interessen, Fähigkeiten und Erwartungen angepasst sind. Nach WEIN $(2009,19)$ sind es nicht die Kinder, die sich dem Fußball anpassen müssten, sondern die Methoden und Aufgaben müssten das Alter und die Besonderheiten der Kinder berücksichtigen. Da die Übungen und Übungsspiele ähnliche Merkmale aufweisen wie der Wettkampf, gelingt der Transfer vom Training zum Wettkampfspiel problemlos. 
Tabelle 3: Stufe des Fußballentwicklungsmodells nach WEIN (2009, S. 17-18)

\begin{tabular}{|c|} 
Erste Fußballentwicklungsstufe \\
(für Mädchen und Jungen ab 7 Jahren) \\
Spiele für grundlegende Fertigkeiten und Fähigkeiten \\
Zweite Entwicklungsstufe \\
(für Mädchen und Jungen ab 8 Jahren) \\
Spiele für Minifußball \\
Dritte Entwicklungsstufe \\
(für Mädchen und Jungen ab 10 Jahren) \\
Spiele für Fußball 5:5 und 7:7 \\
Vierte Entwicklungsstufe \\
(für Mädchen und Jungen ab 13 Jahren) \\
Spiele für Fußball 8:8 \\
Fünfte Entwicklungsstufe \\
(für Mädchen und Jungen ab 14 Jahren) \\
Spiele für das offizielle Spiel
\end{tabular}

\section{Aufgabenstellung}

Das motorische und das kognitive Lernen sollen einander gegenseitig ergänzen. Bei der Schulung des Fußballs soll man also das Erlernen der technischen Fertigkeiten und die Spielintelligenz in komplexen Spielsituationen fördern. Der Trainer soll dafür Lernaufgaben stellen, in denen der Spieler mit solchen Situationen konfrontiert wird, in denen eine schnelle Entscheidung notwendig ist und die Lösung als eine technische Fertigkeit implementiert werden soll. Durch gezieltes Fragen in Spielsituationen beabsichtigt der Trainer, das zu fördern, dass die Spieler selbstständig eine Lösung finden. Auf diese Weise kann sich die Spielintelligenz ohne ständige Hinweise des Trainers entwickeln. WEIN und SCHREINER (2004, WEIN, 2009) schlagen in ihren Lehrbüchern praktische Spielanwendungen für das Erlernen des geeigneten Spielverhaltens und die dazugehörigen Fragestellungen vor.

\section{Diskussion}

Das Fußballentwicklungsmodell könnte als eine Anwendung des genetischen Lernens auf den Fußball eingeordnet werden. WEIN bewirbt außer in seinen Lehrbüchern sein Modell auch durch Vorträge in vielen Ländern und durch seine Tätigkeit beim Spanischen Fußballbund. Berichte künden davon, dass die Umsetzung des Modells bei der 
Nachwuchsausbildung des Fußballsportvereins TSG 1899 Hoffenheim erfolgreich gelungen sei (WEIN, 2009, S. 9; TSG HOFFENHEIM, 2008, S. 39). Bisher liegen jedoch in der sportwissenschaftlichen Literatur keine Studien vor, in denen das Modell empirisch getestet wurde.

\subsubsection{TGfU-Modell}

\section{Einführung}

BUNKER und THORPE (1982, 1986a, b, c; THORPE, 1989) betrachten das Spiel als Aktivität, in der die Spieler fortwährend Probleme lösen müssen. Aus diesem Grund sollten sie intelligente Entscheidungen treffen können, um effektiv am Spiel teilzunehmen. Daher seien die Anforderungen an die Spieler eher von taktischen als von technischen Kompetenzen geprägt. Die Autoren schlagen vor, den Lernenden ein vereinfachtes Spiel vorzustellen, in dem die technischen Anforderungen gemindert seien. Dies ermögliche das Spielen und darüber die Entwicklung der kognitiven Fähigkeiten. Da der Spieler während des Spielens viele Entscheidungen zu treffen habe, sei das Wissen, „was" „wie“ zu machen sei gleich wichtig wie das Wissen, wann es zu machen sei (vgl. BUNKER \& THORPE, 1986a, S. 7).

Das TGfU-Modell wurde 1982 von BUNKER und THORPE vorgeschlagen und von BUTLER (2002, BUTLER \&McCAHAN, 2005) modifiziert. Dieses Modell kehrt die Reihenfolge der Progression des traditionellen Direkten Modells um. Das Hauptelement jeder Lernsitzung ist das Spiel. Es steht im Zentrum und der größte Teil der verfügbaren Zeit ist inm gewidmet. Das TGfU-Modell „enables students to learn about the game and practice the technique within the context of a game rather than separate from it" (THORPE, 2001, S. 29).

\section{Begründung}

Für das TGfU-Modell wurden zwei Begründungen vorgeschlagen, die Entscheidungsfähigkeit und der Lerntransfer. Der erste Grund ist von der Überlegung hergeleitet, dass Kinder in der Lage sein müssen, gute Entscheidungen zu treffen, wenn sie kompetente Spieler werden wollen (BUNKER \& THORPE, 1986a, S. 9). Die Entscheidungsfähigkeit wird als die taktische Komponente beim Sport betrachtet, sie berücksichtigt den damit verbundenen kognitiven Bereich (BUNKER \& THORPE, 1986a, S.9; MITCHELL \& OSLIN \& GRIFFIN, 1997, S. 504; TURNER \& MARTINEK, 1992, S. 17; KIRK \& MACPHAIl, 2002, S. 177). Richtige Entscheidungen sind erforderlich, um erfolgreich zu spielen. BUNKERundTHORPE (1986b, S.16) haben auch darauf hingewiesen, dass es bei „Invasion games“ notwendig sei, eigenen Spielraum zu schaffen und den des Gegners einzuschränken. Spieler müssen Entscheidungen darüber treffen, wann und wie der Pass erfolgen muss, und die Mitspieler darin unterstützen, bei jedem Spielzug die beste Position zu finden (LEE, 2004, S. 4). Zusätzlich zu diesen Situationen müssen die Spieler technische Fertigkeiten und Laufwege wählen, das Gegnerverhalten richtig antizipieren und ihr eigenes Gesamtverhalten dem Spielkontext anpassen. 
Das TGfU-Modell wurde entwickelt, um den Lernenden das Verständnis der taktischen Komplexität des Spiels zu erleichtern, weil es ein wichtiger Bestandteil des Spiels ist (vgl. BUNKER \& THORPE, 1986c, S. 25, OSLIN \& MITCHELL, 1998, S. 33; SIEDENTOP, HASTIE, \& VAN DER MARS, 2004, S. 9; KIRK \& MACPHAIL, 2002, S. 177). Darüber hinaus argumentieren BUNKER und THORPE (1986c, S. 25), dass man die Entscheidungsfähigkeit von der einen zur anderen Sportart übertragen könne. Die tatsächliche Übertragung des Lernens stützt sich auf die Annahme der annähernden Gleichheit der taktischen Probleme in ähnlichen Sportarten. ALMOND (1986a, S. 71-72) klassifiziert die Sportspiele nach ihren Gemeinsamkeiten in die vier Kategorien Netz/Wand, Invasion, Feld und Zielerreichung.

\section{Struktur}

Es geht darum, gut strukturierte Spiele zu entwickeln, die die Spieler mit besonderen Anforderungen und mit Änderungen der „sekundären Regeln“ (ALMOND, 1986b, S. 7374) konfrontieren. Sekundäre Regeln sind beispielsweise Größe des Spielfelds, Teilnehmerzahl, Größe und Position der Tore, Kriterien für den Gewinn von Punkten usw. In den Spielen sind die Spieler gefordert, Entscheidungen zu treffen, was die Lernsitzung in eine Art Übungspraxis, Entscheidungen zu treffen, umwandelt. Dadurch wird zugleich das Spielverständnis erhöht und dastaktische Bewusstsein gefördert.

Das TGfU-Modell von BUNKER und THORPE (1982, 1986a, b, c) ist ein Leitfaden, der es Lehrern und Trainern ermöglicht, durch einen graduellen Prozess die Spieler zu einer höheren Kompetenzstufe zu führen. Das Modell schließt die folgenden pädagogischen Prinzipien ein:

1. Spielbeobachtung: Exploration von verschiedenen Sportspielen, um den Spielern die Gelegenheit zu geben, Unterschiede und Ähnlichkeiten in Bezug auf taktische Aspekte zu vergleichen.

2. Repräsentation des Wettkampfs: durch vereinfachte Spiele mit der gleichen taktischen Struktur wie das offizielle Spiel.

3. Übertreibung: durch das Manipulieren der sekundären Regeln werden taktische Probleme akzentuiert.

4. Taktische Komplexität: das Spiel soll dem Entwicklungsniveau und den Spielmöglichkeiten der Spieler angepasst sein. Im Laufe des Lernprozesses soll die taktische Komplexität erhöht werden.

BUTLER (2002, BUTLER \& McCAHAN, 2005) führt einige Umstellungen am Modell durch. In der Essenz werden das Spiel und seine Handlungen die zentralen Themen des Lernens, die technischen Fertigkeiten werden noch stärker innerhalb des Spielkontextes zusammen geübt, weit eher als von inm getrennt. Das TGfU-Modell beginnt mit den Etappen 3 und 4 des Direkten Modells, d.h. mit dem Spiel, im Gegensatz dazu erfolgt das TGfU in umgekehrter Reihenfolge. In der Abbildung 2 wird das Modell vorgestellt. 


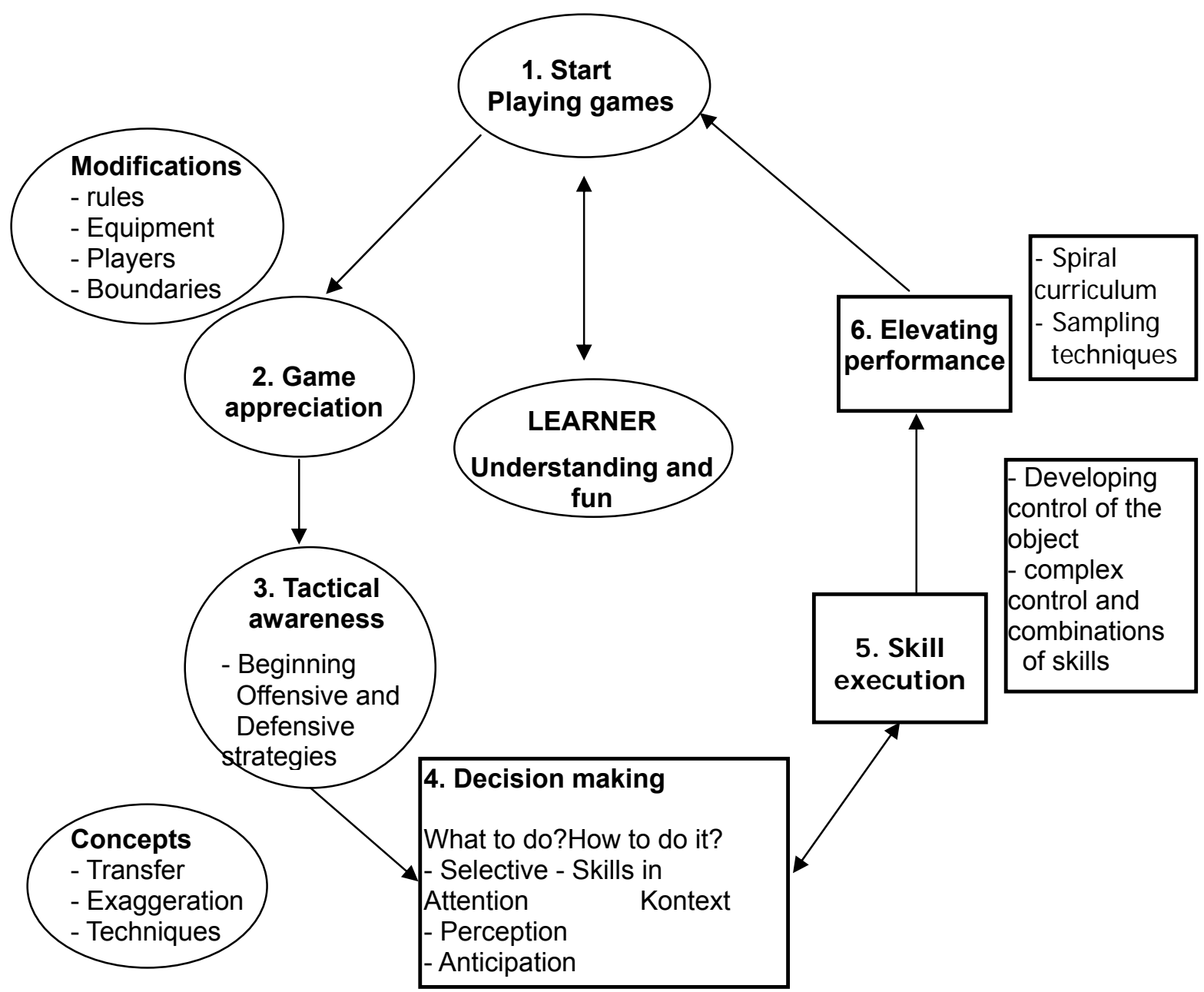

Abbildung 2: Das Teaching Games for Understanding Modell (BUTLER \& McCAHAN, 2005, S. 49)

\section{Aufgabenstellung}

Zentrale Einzelaspekte des TGfU-Modells sind (vgl. BUTLER \& McCAHAN, 2005, S. 50):

1. Spiel: Alle Lernenden können das Spiel spielen. Jeder kann ohne Rücksicht auf seine technischen Fertigkeiten teilnehmen. Das Spiel wird durch die Modifizierung von sekundären Regeln ihrem Können angepasst.

2. Spielaufwertung: Spieler lernen, die Probleme zu identifizieren, die innen durch die modifizierten Spiele gestellt werden.

3. Taktische Bewusstsein: Die Spieler verstehen das Spiel, indem sie geeignete Lösungen zu Problemen finden, die in jeder Spielhandlung vorhanden sind. 
4. Treffen von Entscheidungen: Die Spieler lernen durch das Praktizieren der Grundelemente, richtige Entscheidungen zu treffen. Die Grundelemente der Entscheidungsprozesse sind: Aufmerksamkeit auf essentielle Spielhandlungen richten, Antizipation der Handlungen der Gegenspieler und Auswahl von geeigneten eigenen Handlungen.

5. Technische Fertigkeiten: Die Spieler finden sinnvoll das Üben der technische Fertigkeiten, wenn sie sich ihrer Notwendigkeit für ein besseres Spielen bewusst sind und die gelernte Techniken innerhalb eins Spielkontextes anwenden können.

6. Leistung: Das Leistungsniveau der Spieler nimmt zu, wenn der Prozess zyklisch durchgeführt wird.

\subsubsection{Tactical Games-Modell (TGM)}

\section{Einführung}

Fünfzehn Jahre nach Einführung des TGfU erstellten GRIFFIN, MITCHELL und OSLIN ein anderes Modell, das auf denselben theoretischen Rahmenüberlegungen basiert. Es wird als Tactical Game Approach (TGM) bezeichnet (MITCHELL, OSLIN \& GRIFFIN, 2006). Es wurde bisher häufig im Sportunterricht in der Schule eingesetzt (METZLER, 2005a, S. 405).

\section{Begründung}

Das TGM wird als eine modifizierte Version des TGfU angesehen (vgl. GRIFFIN, MITCHELL \& OSLIN, 2006, S. 13; METZLER, 2005a, S. 402; OSLIN \& MITCHELL, 2005, S. 224). Die Entwickler des TGM stimmen der Überlegung zu, dass das primäre Ziel der Lehre im Sportunterricht die Ausbildung der Entscheidungsfähigkeit der Spieler sei. Die Instruktion solle das taktische Bewusstsein betonen, so dass die Spieler in der Lage seien, das Spiel und ihre Rolle als Spieler zu verstehen (vgl. HOLT, STREAN \& BENGOECHEA, 2002, S. 103). Das TGM ist ebenfalls ein auf die Spieler zentrierter Ansatz (KIRK \& McPHAIL, 2002, S. 184, MITCHELL, OSLIN \& GRIFFIN, 2006, S. 8). Die Lehrer spielen die Rolle des Lernerleichters. Daher sollen sie den Spielern Fragen stellen, um das taktische Bewusstsein zu fördern. Auch hier ist die Grundannahme, dass die Spieler sich jederzeit in einem spielerisch problematischen Kontext zurechtfinden müssten.

Insgesamt ist das TGM dem TGfU-Modell sehr ähnlich. Es existieren jedoch einige wichtige Unterschiede. Das TGM schlägt bestimmte Inhalte vor. Die Lernenden beginnen mit einfachen grundlegenden taktischen Problemen, und die Komplexität wird allmählich gesteigert. Das TGfU-Modell hingegen bringt das generalisierte Konzept des taktischen Bewusstseins hervor, schlägt aber keine bestimmten Inhalte vor. Das TGM beinhalt explizitere Anwendungen in Alltagslehrsituationen.

Die Grundlagen des TGM beinhalten zwei Aspekte: Motivation und spielzentrierter Unterricht. Durch die ständige Spielerfahrung führt das Modell den Lernenden in die takti- 
schen Kenntnisse ein und fördert die aktive Teilnahme der Lernenden während des Lernprozesses. Die TGM hebt das Spiel als eine wirksame, motivierende Maßnahme für das Lernen hervor. MITCHELL, OSLIN \& GRIFFIN (2006, S. 8) fanden einen Mangel an Interesse und am Reiz bei jenen traditionellen Lehr-Ansätzen, die sich auf die Technik konzentrieren. Sie argumentieren, dass die geschickter Lernenden sich von diesen Ansätzen gelangweilt fühlten, da die Eintönigkeit der isolierten Übungspraxis von technischen Fertigkeiten sie im Lernprozess beschränke.

Zu Beginn des Unterrichts stellt der Lehrer den Schülern ein Spiel vor, um die Teilnahme am Spielsport unter Berücksichtigung der der kognitiven Komponenten des Spielsports zu fördern. Danach dient das erworbene taktische Bewusstsein als Motivation für das Üben der technischen Fertigkeiten. Die Wahrnehmung der Unterschiede zwischen dem „Soll-Zustand“ und dem „Ist-Zustand“ des Spiels provoziert die Notwendigkeit, die technische Leistungsfähigkeit im Sport zu verbessern. Die Spieler erkennen diesen Zusammenhang nur bewusst, wenn sie wissen, dass sowohl die taktische als auch die technische Komponente für den Erfolg notwendig sind.

\section{Struktur und Aufgabestellung}

Ein auf das Spiel zentriertes Modell bedeutet, dass Spielen eine primäre und eine zentrale Rolle beim Lernen einnimmt. Ein Spiel ist nicht nur ein Produkt des Lernens, sondern auch ein Mittel für die Erreichung der Lernziele. Im TGM gilt Ähnliches wie im TGfU. In diesem Modell werden drei Komponenten der sechs Lehrschritte des TGfU abgeändert. Die Hauptmerkmale des TGM können als drei Abschnitte zusammengefasst werden:

a) Mit Kleinen Spielen wird die Aufmerksamkeit auf ein spezifisches taktisches Problem gelenkt. Dies setzt eine Lernumgebung voraus, die zum taktischen Bewusstsein beiträgt.

b) Durch Fragestellungen wird das kritische Denken angeregt. Die Orientierung trägt zur Lösung der taktischen Probleme bei.

c) Die Übung der technischen Fertigkeiten soll zur Lösung der taktischen Probleme durch richtig ausgeführte Technik beitragen. Die Kluft zwischen der Taktik (was zu tun ist) und der Technik (wie es zu tun) wird dadurch verringert. Das inspiriert den Wunsch der Lernenden, die Techniken dieses Sports zu verbessern. 


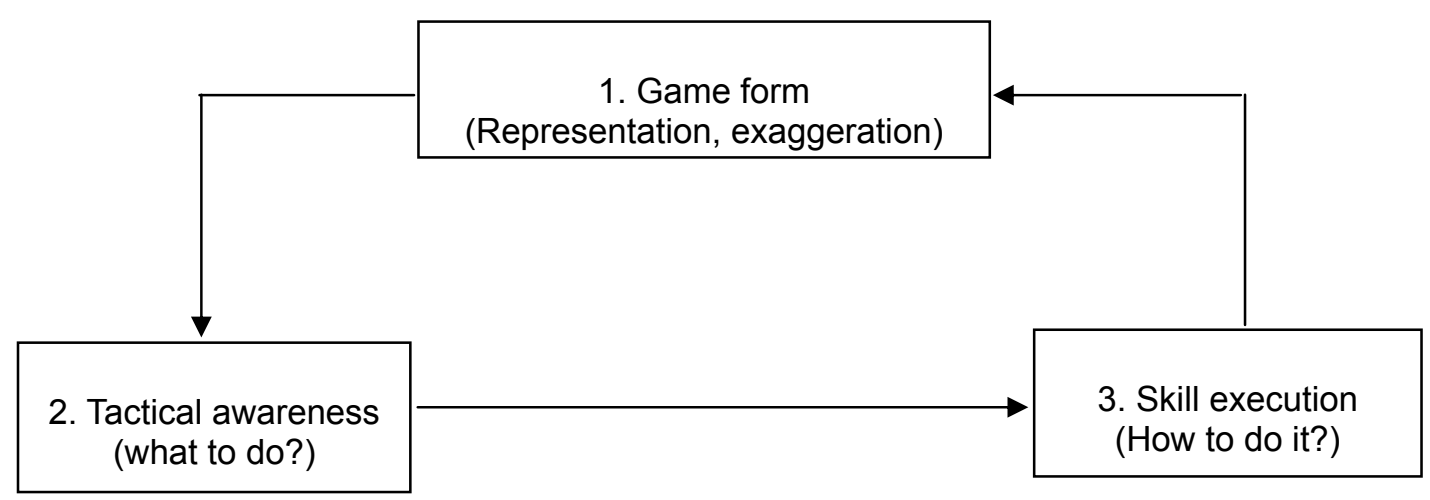

Abbildung 3: Das Tactical Games Modell, (MITCHELL, OSLIN \& GRIFFIN, 2006, S. 13)

\section{Diskussion}

Die empirischen Belege für das TGfU und das TGM haben die Aufmerksamkeit vieler Forscher auf sich gezogen (z.B. ALISON \& THORPE, 1997; CHANDLER \& MITCHELL, 1990; MITCHELL, FRENCH, WERNER, RINK, TAYLOR, HUSSEY, \& JONES, 1996; GRIFFIN, OSLIN \& MITCHELL, 1995; TURNER \& MARTINEK, 1995, 1999). Diese beiden Spielansätze wurden abwechselnd in der Literatur diskutiert. Aufgrund der Ähnlichkeiten zwischen TGfU und TGM haben Forscher häufig beide Ansätze zusammen überprüft und in den meisten Fällen wurden beide Ansätze in einem zusammengemischt (HOLT, WARD \& WALLHEAD, 2006; RINK, FRENCH, \& TJEERDSMA, 1996; HARVEY, 2006).

Die Forschung bezüglich beider Modelle ist zumeist auf den Vergleich ihrer Auswirkungen mit denen des traditionellen Direkten Modells fokussiert. Die vorliegenden Forschungsergebnisse decken dabei eine Vielzahl von Aspekten ab. Forscher analysierten die Auswirkungen verschiedener Lehr-Bedingungen in den Altersgruppen des Grundschul-, Sekundarstufen- und College-Sportunterrichts. Überprüft wurden die Ansätze in verschiedenen Sportarten wie Badminton, Basketball, Hockey, Fußball, Tennis und Volleyball (ALISON \& THORPE, 1997; GRIFFIN, MITCHELL \& OSLIN, 1997; MITCHELL, GRIFFIN \& OSLIN, 1995; TURNER \& MARTINIEK, 1995,1999). Drei Bereiche der Lernleistungen (Psychomotorik, Kognition und Emotion) werden herkömmlich gemessen. Die Studien haben sich in erster Linie auf den motorischen und kognitiven Bereich konzentriert (TURNER \& MARTINEK, 1995, 1999; FRENCH, WERNER, TAYLOR, HUSSEY \& JONES, 1996; FRENCH, WERNER, RINK, TAYLOR, \& HUSSEY, 1996).

Die meisten Studien haben darauf hingewiesen, dass es keine signifikanten Unterschiede bezüglich der Verbesserung der technischen Leistung zwischen TGfU oder TMG und dem Direkten Modell gibt (FRENCH et al., 1996a; FRENCH et al., 1996b; McPHERSON \& THOMAS, 1989; TURNER \& MARTINEK, 1995). Eine Ausnahme von diesem Forschungsergebnis entstammt einer Studie von TURNER und MARTINEK (1999), in der der TGfU-Ansatz bessere Ergebnisse für die Ausführung der Fertigkeiten des Passens, Schießens und Dribbelns im Spiel hervorbringt als der traditionelle, auf die Technikvermittlung ausgerichtete Unterricht. Zusammengenommen sprechen die Ergebnisse der Forschung zu TGFU und TGM dafür, das Urteil zu unterstützen, dass 
diese Ansätze der traditionellen Unterrichtsmethode nicht überlegen sind, wenn es um das Lernen technischer Fertigkeiten des Sports geht (HOLT, WARD, \& WALLHEAD, 2006; RINK, FRENCH, \& TJEERDSMA, 1996; SILVERMAN, 1997). Jedoch liefern viele Studien positive Ergebnisse für TGfU- und TMG-Gruppen bei der Auswertung in Bezug auf das deklarative und prozedurale Wissen der Schüler (ALISON \& THORPE, 1997; McPHERSON, 1999). Die Studienergebnisse zeigen, dass die Lernenden der taktisch ausgerichteten Gruppen überlegene Leistungen bei der Bewertung des Wissens über Regeln, Feldaufstellungen und Entscheidungsfähigkeit besitzen, obwohl sie im Vergleich noch schlechtere Leistungen bezüglich der technischen Fertigkeiten aufweisen. Allerdings haben GRIFFIN, OSLIN und MITCHELL (1995); LAWTON (1989), und TURNER und MARTINEK (1992) in den Lernergebnissen ihrer Studien keine signifikanten Unterschiede bei der kognitiven Leistung nachweisen können. Zusammenfassend kann die Forschung bislang also noch nicht bestätigen, dass TGfU und TGM einen besseren Weg darstellen, hohe Leistungen beim Lernen der Sportspiele zu erzeugen (HOLT, WARD, \& WALLHEAD, 2006; RINK, FRENCH, \& TJEERDSMA, 1996).

In der Praxis sind TGfU und TGM für die Lehrer und Trainer schwieriger zu meistern als das Direkte Modell. Dies geht zurück auf den großen Umfang von Kenntnissen, die mit der Verwendung von kritischen Fragestellungen im Training verbunden sind und den Zweck haben, das taktische Bewusstsein der Spieler zu fördern. Sowohl das TGfUModell als auch das TGM setzen Fragen des Lehrers ein, die nach der Einführung der modifizierten Spiele gestellt werden (MITCHELL, OSLIN \& GRIFFIN, 2006). Die Lehrer müssen mit ihren Fragen in der Lage sein, die Identifizierung der taktischen Probleme und deren Lösungen zu erleichtern. Die Qualität der Fragen ist demnach entscheidend für die Erreichung der Lernziele wie Identifizierung der Spielprobleme und taktisches Bewusstsein (DYSON, GRIFFIN \& HASTIE, 2004, S. 24). Die Lehrer müssen daher die taktischen Anforderungen der jeweiligen Situation so gut kennen, dass sie den Schülern angemessene Fragen stellen können. Entsprechend sind Inhalt und Zeitpunkt der Fragen sehr eng mit den Kenntnissen der Lehrer verbunden. Diese Variable ist von entscheidender Wichtigkeit für den Gesamterfolg des Lernens.

Im Anschluss an die Durchführung der modifizierten Spiele verwenden sowohl TGFU als auch TGM die traditionelle Weise für die Schulung der technischen Fertigkeiten. Die technische Schulung wird in einem deutlich aus dem Spielkontext herausgehobenen Zustand präsentiert, was möglicherweise die kognitive Beteiligung wenig fördert oder sogar behindert. KIRK und McPHAIL (2002, S. 190) haben daher darauf hingewiesen, dass keiner der Ansätze bei der Einführung der technischen Fertigkeiten einen wirklichen Unterschied zum Direkten Modell aufweist. Beide Modelle schlagen die Schulung der Technik nach demselben traditionellen Konzept vor.

Eine weitere weit verbreitete Kritik an TGfU und TGM betrifft die Präsentation des Spiels zu Beginn des Unterrichts. Der Erfolg bei der Bewältigung der taktischen Probleme während des Spiels basiert auf der Annahme, dass die Spieler von Anfang an in der Lage sind, sich auf die Taktik zu konzentrieren. Aus einer traditionellen Sicht ist aber die Beherrschung der technischen Fertigkeiten notwendige Voraussetzung für das Erlernen von Taktiken. Nach dieser Meinung könnten vor allem gering qualifizierte Lernende, die kaum den Ball beherrschen, nicht darüber nachdenken, wie, wo und mit wem ihr taktisches Zusammenspiel stattfinden soll. Gemessen an den taktischen Anforderungen des Zielspiels, wäre es also utopisch ein organisiertes Spiel von den Anfängern zu verlangen. RINK, FRENCH, und TJEERDSMA (1996, s. 410) legen daher Wert darauf, dass 
die Einführung der Techniken immer als erster Schritt notwendig ist, bevor die Kinder taktisches Denken entwickeln können. Kinder könnten sich nicht auf mehr als eine Aufgabe gleichzeitig konzentrieren, solange sie noch in der prä-operatorischen Phase des Lernens sind.

\subsubsection{Game Sense}

\section{Einführung}

In der Mitte der neunziger Jahre entwickelt, ist Game Sense eine Anpassung des vor allem für den Schulsport entwickelten TGfU-Modells an das Leistungstraining. Als Alternative zum traditionellen Trainingsmodell des Mannschaftssports, in dem die technischen Fertigkeiten spiralförmig wiederholt werden und ihre Beherrschung als eine Voraussetzung für das taktische Training betrachtet wird, wird bei Game Sense das Spiel zum Zentrum des Trainings. Die Spieler werden herausgefordert zu durchschauen, was sie tun und warum sie so handeln. Das Ziel ist es, den Spielern ein umfassendes Spielverständnis zu ermöglichen.

\section{Begründung}

Game Sense basiert auf den Grundlagen des TGfU-Modells. Die Spieler entwickeln taktisches Bewusstsein durch das Spiel und die Fragestellungen des Trainers. Die kognitiven Anforderungen fördern die kognitive Einbeziehung und damit das Verständnis im Lernprozess. Die Methode des entdeckenden Lernens löst ein lebenslanges Lernen aus, so dass der Spieler bei späteren Lernphasen seiner Entwicklung bereits erreichte Erkenntnisse gewinnbringend einsetzen kann und sogar auf andere Sportspiele und Lebensdimensionen transferieren kann.

\section{Struktur}

Das modifizierte Spiel ist der Schwerpunkt des Modells. Durch entdeckendes Lernen entwickelt der Spieler sein Spielverständnis. Der Trainer beobachtet das Spiel und bietet Rückmeldungen an, die als Fragestellungen kognitive Herausforderungen darstellen. Der Trainer versucht durch diese Fragestellungen auch, das Bewusstsein der Spieler auf die richtige Anwendung der technischen Fertigkeiten in bestimmten Situationen zu lenken. So fördert er bei den Spielern die Erkenntnis, dass die Verbesserung der technischen Fertigkeiten eine Notwendigkeit ist. 


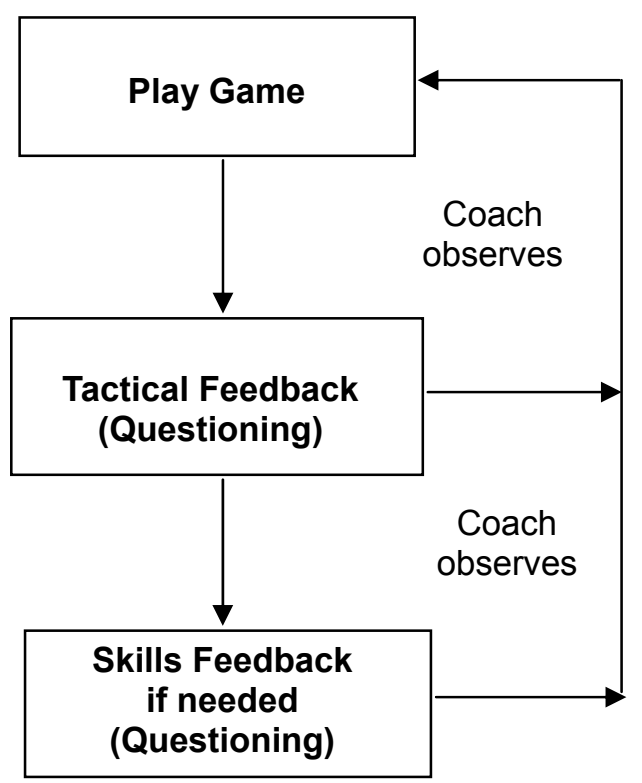

Abbildung 4: Das Game Sense Modell (nach den DUYN, 1997, S. 3)

\section{Aufgabenstellung}

Der Trainer modifiziert die Regeln des Spiels, um bestimmte taktische Aspekte zu betonen bzw. gezielt zu übertreiben. Beispielsweise kann er den Verteidigern eine zusätzliche Aufgabe auferlegen - etwa um ein Hütchen außerhalb des Spielraums herumzulaufen - bevor sie ihre defensiven Aufgaben im Spiel erfüllen. Die Angreifer finden in dieser Situation eine räumlich zerstreute Abwehr vor. Im Verlauf der Übung werden die Angreifer ein besseres Verständnis darüber gewinnen, wie man gegen eine zerstreute Abwehr effektiv spielen kann und welche unterschiedlichen Alternativen es gibt, um Stellungsfehler der Gegenspieler unmittelbar auszunutzen. Der Trainer setzte zusätzlich Fragestellungen ein, um die Spieler dazu zu bringen, über die gemachten Erfahrungen nachzudenken. Die Antworten sind dabei selten eindeutig. Normalerweise gibt es mehrere richtige Antworten für jede Frage. Der Trainer hebt die taktischen Aspekte der Antworten durch weitere Fragen hervor und hebt dabei Aspekte von

- Zeit: „Wann würdest du das machen?“

- Raum: „Wo...?“

- Risiko: „Welche...?“

genauer hervor. Im Anschluss stellt er genügend Spielzeit für die Realisierung der neu gewonnenen Erkenntnisse im Spiel bereit und nimmt sich Zeit, um zu beobachten, wie die Umsetzung gelingt. Wenn das Spiel gut läuft, kann der Trainer eine Steigerung der Anforderungen anleiten, indem er die Regeln verändert und so den Schwierigkeitsgrad erhöht. Davor soll der Trainer das Verständnis der Spieler für die alte Situation bestätigen und auf die individuellen Unterschiede achten. 


\section{Diskussion}

Das Game Sense Modell wurde auf unterschiedlichen Leistungsniveaus umgesetzt, vor allem im Training professioneller Rugbyvereine in Australien und Neuseeland. Game Sense hat sich auch als eine wirksame didaktische Strategie im Schulsport erwiesen, wie mehrere empirische Studien berichten (z. B. LIGHT \& GEORGAKIS, 2005). Game Sense profitiert vom großen Umfang empirischer wissenschaftlicher Studien rund um das TGfU-Modell, da Game Sense vom TGfU abgeleitet wurde und sich beide Modelle sehr ähnlich sind.

\subsubsection{Sport Education}

\section{Einführung}

Es handelt sich um ein Modell, das eine Ausbildung der Sportkultur durch die Sportspiele fördern soll. Sein Ziel ist es, kompetente Personen auszubilden, die Spaß am Sport haben und die sozialen Werte annehmen, die aus seiner Praxis entstehen. Die Förderung des Sports und die Begeisterung für die Sportkultur, die für ein lebenslanges Sporttreiben motivieren, werden als Hauptwirkungen des Modells angestrebt (SIEDENTOP, HASTIE \& VAN DER MARS, 2004, S. 8). SIEDENTOP (1994, S. 4), der das Modell entworfen hat, glaubte an die Sportspiele und ihr Potenzial für die Erreichung der Erziehungs- und- Sozialisationsziele von Kindern und Jugendlichen. Auf diese Weise erhält auch der Schulsport seine Legitimation im Schulcurriculum.

\section{Begründung}

Sport Education wurde entworfen, um die Erfahrung von Sporttreibenden zu bereichern (SIEDENTOP, HASTIE \& VAN DER MARS, 2004, S. 7). Die Übungspraxis umfasst mehr als den reinen Sportinhalt. Andere Erlebnisse werden betont, so sollen die Kinder Mitglieder einer Mannschaft werden, an Wettkämpfen teilnehmen, eine Dokumentation von Ergebnissen und die Erstellung eines Ablaufplans für Spiele erlernen usw. Diese Erlebnisse erweitern das Lernen und die Erfahrungen, die durch die Praxis des Sports gesammelt werden. Während der Teilnahme haben die Spieler die Gelegenheit, auf das Spielgeschehen als Kapitän, Trainer, Schiedsrichter, als Organisator von Ligen, Turnieren und Schlussfeiern usw. einzuwirken. Mithilfe des Sport Education Modells kann man auch über die oberflächlichen Lernergebnisse hinausgehen, die geschaffen werden, wenn man im Sportunterricht viele Sportarten und sportliche Lerninhalte in geringer Zeit unterbringen möchte, mithin der Normalfall im Schulsport. Die Autoren des Modells meinen jedoch, dass ein langfristiges Engagement in nur einem Sportspiel ein tieferes Lernen bewirkt. Die Spieler hätten die Möglichkeit, bessere technische und taktische Spielfertigkeiten zu erlernen. Indem sie über eine längere Zeit eine einzige Sportart betreiben und erweiterte Rollen im Kontext des Spielgeschehens einnehmen. Letztlich werden sie also kompetenter. Nach SIEDENTOP (1994, S. 11) besitzt ein kompetenter Spieler ausreichende Fertigkeiten, um effektiv an einem Spiel teilnehmen zu können. Er ist auch fähig, die komplexen taktischen und strategischen Elemente zu verstehen und auszuführen, die charakteristisch für diesen Sport sind. Die traditionellen Lerninhalte 
des Schulsports werden hingegen häufig außerhalb des Kontextes gelehrt. Durch Sport Education könnte man ein kontextuelles, effektives und progressives Lern- und- Entwicklungsprogramm durch den Wettkampf erreichen.

\section{Struktur und Aufgabenstellung}

Das SportEducation-Modell unterstützt die Übung der Sportspielarten als Grundinhalt der Schulsportprogramme und umfasst die folgenden Merkmale (ZHANG, 2008, S. 5052):

1. Eine lange Zeitspanne der Praxis eines einzigen Spiels. Im Gegensatz dazu wird im üblichen Sportunterricht ein Wechsel mehrerer Sportarten in relativ kurzer Zeit gelehrt. Sport Education braucht diese längeren Zeitspannen zur Zielerreichung. Die Schüler haben durch längere Zeitspannen in der Beschäftigung mit nur einem einzigen Spiel mehr Gelegenheit, Zusammenhänge zu erkennen, zu üben und umfassende Erfahrungen in Beziehung mit der Sportart zu sammeln.

2. Die ausgewählten Mannschaften bleiben eine ganze „Saison“ zusammen. Die Spieler haben so mehr Zeit zum gegenseitigen Kennenlernen. Sie können sich mit der Mannschaft identifizieren und ein Zugehörigkeitsgefühl zu einer Gruppe entwickeln. Die Teilnahme an einem Turnier oder ein Preis am Ende der Saison verstärkt den Wettkampfgeist.

3. Der vielgestaltige Wettkampf innerhalb der Schule ist ein essentieller Bestandteil des Sport Education Modells. Die Lernenden haben die Möglichkeit an Ligen und Turnieren teilzunehmen und sich dafür vorzubereiten.

4. Eine Schlussveranstaltung und eine Schlussfeier stellt das allerletzte Ziel des Sportlernens dar. Bei einer Schlussveranstaltung können die besten Mannschaften und Spieler identifiziert werden. Ein Schlussturnier und eine Schlussfeier sind in allen Sportarten üblich und sollen als Ziel in jedem Lernprogramm Sport Education umgesetzt werden.

5. Der Datensatz über Spielergebnisse in immer gleichen Mannschaften trägt als Rückmeldung eine besondere Bedeutung und begünstigt das Lernen. Die Erstellung eines Datensatzes während des ganzen Lernprogramms ermöglicht die Kontrolle des eigenen Lernens und der Verifizierung der eigenen Fortschritte durch die Lernenden selbst. Die Lernenden können die Datenreihe der Ergebnisse in mehreren Durchgängen nutzen, um ihre Fertigkeiten am Anfang des Programms zu diagnostizieren und daraus neue Lernziele zu erstellen.

6. Alle Teilnehmer wirken an der Schlussveranstaltung mit und feiern die Schlussveranstaltung gemeinsam. Solche Feierlichkeiten sind Bestandteile des Sports, wie speziell am Beispiel der Olympischen Spiele und bei Fußballweltmeisterschaften deutlich wird. Sie ermöglichen, dass die Teilnehmer ihre Beteiligung als besondere Erlebnisse in Erinnerung behalten und Leistungen und Fairplay einen besonderen Stellenwert erhalten.

Das Aufsummieren dieser sechs Bestandteile schafft ein echtes Lernen, in dem alle Teilnehmer Verständnis für den Sport erwerben und Wege dafür erkennen, wie sie sich am besten daran beteiligen können. Außerdem lernen sie, wie ein Wettkampf organisiert wird und wie sie mit Erfolg daran teilnehmen können. 


\section{Diskussion}

Die Forschung bezüglich Sport Education weist darauf hin, dass die Anwendung des Modells die emotionalen Wirkungen des Lernens der Sportspiele erheblich verbessert. Die Spieler verbessern ihre Interaktion und Kooperation mit den Mitspielern durch die Teilnahme und durch ihre Rolle als Spieler, Trainer, Schiedsrichter, Organisatoren, Helfer usw. (WALLHEAD \& O'SULLIVAN, 2005; GRANT, 1992). Auch die kommunikativen Fähigkeiten verbessern sich in hohem Maße (HASTIE \& SHARPE, 1999). Der Eindruck der Schulsportlehrer stimmt mit den Ergebnissen der Forschung überein. Auch sie meinen, dass Sport Education die personalen und sozialen Fertigkeiten der Spieler verbessert (WALLHEAD \& NTOUMANIS, 2004). Allerdings gibt es bisher keine ausreichenden Beweise für die Bestätigung von Sport Education als ein Modell zur Steigerung der Spielleistungen. Es haben sich keine Unterschiede zwischen diesem Modell und dem traditionellen Modell im Ergebnis des Spielverständnisses gezeigt. Das Modell könnte demnach auch unwirksam für die Verbesserung der technischen Fertigkeiten sein (WALLHEAD \& NTOUMANIS, 2004; O'DONOVAN, 2003). Die verschiedenen Rollen könnten die effektive Spiel und damit Übungszeit verringern. Das Modell vertraut zu viel auf das kooperative Lernen, die mangelnde Vorbereitung der Spieler könnte der Qualität des Trainings schaden, wenn sie selbst die Rolle des Trainers spielen (WALLHEAD \& O'SULLIVAN, 2005). Diese Unfähigkeit, die Entwicklung der technischen und taktischen Kompetenzen zu fördern, ist die große Schwäche des Modells.

\subsubsection{Invasion Game CompetencesModel (IGCM)}

\section{Einführung}

Das Modell der Kompetenzen für Invasionsspiele (IGCM) stammt aus der Universität Gent in Belgien (MERTENS \& MUSCH, 1990; MUSCH \& MERTENS, 1991) und wurde später durch eine Gruppe von Sportwissenschaftlern aus Belgien, den Niederlanden, Portugal und Tschechien (MUSCH, MERTENS, TIMMERS, MERTENS, GRAÇA, TABORSKY, REMY, DE CLERCQ, MULTAEL, \& VONDERLYNCK, 2002) weiterentwickelt. Das Modell wurde ursprünglich für Basketball umgesetzt, die europäische Gruppe erarbeitete drei didaktische CD-ROMs mit Hinweisen für das Lehren des Basketballs unter Anwendung des IGCMs.

\section{Begründung}

Die Autoren des IGCMs wurden von den Ideen der Modelle Sport Education (SIEDENTOP, 1994) und Teaching Games for Understanding beeinflusst. Das Modell wurde dafür entworfen, die Vorteile der beiden Modelltypen miteinander zu verbinden. Die Spieler sollten nicht nur mit Erfolg am Spiel teilnehmen, sondern auch eine andere Rolle bei der Organisation der Spielsportpraxis in der Schule spielen. Zwei Gruppen von Kompetenzen sollen geschult werden: Spielkompetenz und Organisations- und Führungskompetenz. 


\section{Struktur}

\section{Spielkompetenz:}

Die Entwicklung der Spielkompetenz soll die soziale, emotionale, kognitive und motorische Bereiche berühren. Dies gelingt durch die Förderung der Fähigkeiten zu Teamarbeit, Wahrnehmung und Entscheidungsfähigkeit sowie die Schulung der technischen Fertigkeiten. Ein Beispiel hierfür ist es, einen Ball zu einem Mitspieler mit niedrigem Fertigkeitsniveau zu passen, der sich aber in einer günstigeren Feldstellung befindet als man selbst (GRACA, MUSCH, MERTENS, TIMMERS, MERTENS, TABORSKY, REMY, DE CLERCQ, MULTAEL \& VONDERLYNCK, 2003, S. 8). Bei dieser Handlung würden alle drei Lerndomänen integriert und in Einklang gebracht. Es ist notwendig, dass der Spieler am Ball die Fähigkeit hat, die Passmöglichkeiten zu identifizieren, die Vorteile der Stellung des Mitspielers zu erkennen und bereit ist, auf andere Spielmöglichkeiten zu verzichten und die Verantwortung dem schwächeren Mitspieler anzuvertrauen. Es ist auch notwendig, dass der Spieler am Ball über die physischen und motorischen Voraussetzungen für die Ausführung eines richtigen Passes verfügt. Er soll den optimalen Pass wählen und ihn unter Berücksichtigung der Umstände des Passannehmers ausführen; dessen Stellung, Bewegungen, seine Verteidiger und die allgemeine Entwicklung des Spielzuges sind zu berücksichtigen. Diese Zusammenhänge machen überdeutlich, dass ein Pass weit mehr ist als nur eine technische Fertigkeit.

\section{Organisationskompetenzen:}

In Anlehnung an das Modell Sport Education wird beim IGCM das Lernen von Kompetenzen für andere Rollen angestrebt, die sich um das Spielgeschehen herum anordnen, so etwa die Rolle des Schiedsrichters und des Trainers. Die Entwicklung der Kompetenz für das Spielgeschehen unterstützt und ermöglicht auf diesem Weg die Selbstorganisation der Praxis, was die Verantwortung, Autonomie und Selbstregelung des Lernens fördert. Das Einnehmen dieser Rollen fördert den Erwerb von Kenntnissen und Fertigkeiten, die für die Unterstützung des Spiels gebraucht werden. Es gilt zum Beispiel, das Engagement als Mannschaftskapitän, Schiedsrichter, Trainer oder die Verantwortung für das Spielfeld und die didaktischen Lernmaterialen zu übernehmen. Die Herausforderungen, die diese dem Spiel inhärenten Aufgaben stellen, werden als Stimuli für den Erwerb eines tieferen Verständnisses des Spiels betrachtet. Die verschiedenen Rollen als Nicht-Spieler sind eine Gelegenheit für die Entwicklung der Organisations- und Entscheidungsfähigkeiten und die Autonomie der Kinder und Jugendlichen.

\section{Aufgabenstellung}

Beim IGCM kann man drei Aufgabenarten unterscheiden:

\section{Grundspielaufgaben:}

Die Grundspielaufgaben sind modifizierte Versionen des Wettkampfs. Sie sind dem Spielniveau der Gruppe angepasst und haben die Absicht des Erwerbs und des Übens der motorischen, kognitiven und sozialen Kompetenzen. Jedes Spiel hat die Merkmale eines Wettkampfs, da es bei diesem Modell wichtig ist, dass die modifizierten Spiele als 
wirkliche Wettkampfspiele anerkannt werden. Die Grundspielaufgaben haben daher die folgende Charakteristik:

1. Das Ziel ist dasselbe wie in der vollen Version der Invasionsspiele (d.h. Tore/Punkte schießen bzw. erzielen, Tore/Punkte verhindern).

2. Jede Spielaufgabe beinhaltet die vollständige Struktur des Invasionsspiels.

3. Die Handlungen von Angriff und Verteidigung stehen in Beziehung zu einander (Opposition).

4. Es gibt einen natürlichen Übergang von Angriff zu Verteidigung und umgekehrt (Gewinnen oder Verlieren des Balles).

5. Die Lernaufgaben sind problematische Situationen (die Information darüber, was zu tun ist, wie und wann es geschehen muss, ist aber nicht bei jeder Spielhandlung vorhanden).

Partielle Spielformen:

Durch das Spiel allein werden nicht alle Lernziele der Invasionsspiele erreicht. Im Modell werden daher auch partielle Spielformen für die Übung von besonderen Situationen angewendet. Dabei geht es um simplifizierte Situationen, die die Beziehung von Angriff und Verteidigung und das Ziel des Spiels aufrechterhalten, die aber auf besondere Spielprobleme eingeschränkt werden. Dies können zum Beispiel das Herausspielen von Torgelegenheiten bzw. das Vermeiden von Torgelegenheiten des Gegners sein. Man versucht, günstige Spielsituationen zu schaffen, in denen sich die Spieler auf besondere Aspekte konzentrieren können, ohne die kontextuellen Merkmale des Spiels aus den Augen zu verlieren. Auf diese Weise haben die Spieler mehr Gelegenheiten, die Spielprobleme zu identifizieren und die Spielherausforderungen zu überwinden. Im Unterschied zur Grundspielaufgabe gibt es bei den partiellen Spielformen nicht unbedingt eine Gleichsetzung oder einen natürlichen Übergang von Abwehr zu Angriff. Nur eine dieser Situationen wird mit den entsprechenden Lösungen gezielt geübt.

\section{Aufgabenbasierte Spiele:}

Diese Aufgaben sind Mittel für die Konkretisierung der Lösungen für Spielprobleme. Sie beschränken die Auswahlmöglichkeiten und stellen eindeutige Lösungen vor. Dadurch werden erstmals gezielt die technischen Ausführungen betont. Dies geschieht innerhalb eines simplifizierten Kontextes, aber mit klarem Verweis auf die eigentlichen Spielsituationen. Auf diese Weise wird der Transfer von Lernsituationen auf Spielsituationen erleichtert. Es wird dann die integrierte Verbesserung der technischen Fertigkeiten versucht, wo sich die Wahrnehmung, taktische Entscheidungsfähigkeit und technische Ausführung kombinieren lassen und gleichzeitig aktiviert werden müssen.

\section{Designkriterium der Aufgaben:}

Wegen des Fehlens offizieller Wettkämpfe in der Schule sind die Sportlehrer im Gegensatz zu Trainern besonders frei von Stress und externem Leistungsdruck. Sie sollen daher jedem Spieler dieselbe Spielzeit und eine geeignete Lernumwelt gewährleisten. Sie sollen die Faktoren beachten, die die Ausgrenzung einzelner Spieler oder den Selbstausschluss hervorrufen. Sie sollen Spiel- und Übungssituationen schaffen, die 
Möglichkeiten für die Entwicklung der Spielfähigkeiten bieten und gleichzeitig die Wahrscheinlichkeit verringern, aus der Teilnahme am Lernprozess verdrängt zu werden. Der Lehrer soll auf die Bildung von Mannschaften und Arbeitsgruppen achten, bei Kleinen Spielen sollten zum Beispiel die Mannschaften sowohl aus schwächeren als auch aus stärkeren Spielern bestehen, damit alle ihre Spielleistung verbessern können.

Die Anpassung des Schwierigkeitsgrads der Spiele an die Spiel- und Handlungsfähigkeit der Spieler kann durch die Anpassung der Ausführungsbedingungen und das Einfügen von erleichterten Regeln erreicht werden. Zum Beispiel könnte der Trainer/Lehrer besondere Regeln beim Basketball einführen, mit denen das Spiel in der Abwehr langsamer abläuft und der Angriff so erleichtert wird, dass den Angreifern mehr Zeit zur Verfügung steht, um über die Spielprobleme nachzudenken und sie effektiv zu lösen.

\section{Diskussion}

Das IGCM wurde in Vergleichsstudien eingesetzt. TALLIR, LENOIR, VALCKE und MUSCH, (2007) testeten die kognitive Wirkung des Modells bei einer Experimentalgruppe und verglichen die Ergebnisse mit einer Kontrollgruppe, in der ein traditionelles Modell angewendet wurde. In der Experimentalgruppe wurde eine Effizienz steigerung gegenüber der Kontrollgruppe festgestellt, d. h. bei einer Zwischenevaluation wurde eine schnellere Verbesserung der Entscheidungsfähigkeit in der Experimentalgruppe gefunden als bei der Kontrollgruppe. Im Gegensatz dazu war die Kontrollgruppe effizienter beim Lernen von Erinnerungsaufgaben. Allerdings wurden keine signifikanten Unterschiede zwischen den Gruppen am Ende der 12-wöchigen Intervention festgestellt. Beide Gruppen verbesserten gleichmäßig alle Variablen der Entscheidungsfähigkeit und Erinnerung.

\subsubsection{Play practice}

\section{Einführung}

Der australische Sportlehrer Alan LAUNDER brachte das Modell Play Practice 2001 durch die Veröffentlichung seines „Buches Play Practice, the games approach to teaching and coaching sports" in die Diskussion.

\section{Begründung}

LAUNDER kritisierte die traditionellen Modelle für das Lernen der Sportspiele und ordnete sie drei Gruppen zu.

Das ganze Spiel (full game): Die Spielergruppe wird in zwei Mannschaften unterteilt und es wird unmittelbar gespielt. Der Lehrer/Trainer fügt nur selten technische oder taktische Inhalte hinzu, aber die Spieler haben ausreichende Zeit zu spielen und angeblich viel Spaß dabei. Allerdings wird das Spiel von den stärkeren und erfahrenen Spielern beherrscht. Die Anfänger haben wenige Gelegenheiten, am Spiel teilzunehmen, was Frustrationsgefühle und Demotivation für das gesamte Sporttreiben hervorruft. 
Die Minispiele (minor games): Dies sind Spiele mit einigen Charakteristika des Sports, sie stellen aber den Spieler nicht in einen Kontext die ähnlich zu diesem des Zielspieles ist. Zwischen den Minispielen und dem eigentlichen Spiel gibt es mehr Unterschiede als Ähnlichkeiten, die Teilnehmer schaffen es nicht, ein Verständnis über technische und taktische Elemente zu gewinnen und lernen keine Spielprinzipien. Ein Beispiel wären die Staffelspiele bei denen die Übung der technischen Fertigkeiten angestrebt wird. Die Lernenden haben aber kaum Gelegenheiten, den Ball zu berühren, und bleiben sehr viel Zeit einfach stehen, während sie auf ihren Staffeleinsatz warten. Durch diese Spiele ist es nicht möglich, einen Lerntransfer zum eigentlichen Spiel zu fördern.

Das Direkte Modell: Grundsätzlich ist es auf das Lernen technischer Fertigkeiten ausgerichtet. Der Trainer widmet den detaillierten Erklärungen der Bewegungen viel Zeit und die Lernenden üben in isolierten Situationen. Es wird wenig gespielt, nur am Ende der Lernsitzung bleibt einige Zeit dafür übrig. Nach LAUNDER (2001, S. 29) beschäftigen sich die Trainer zu viel mit Stereotypen, während das eigentliche Spiel flexible Antworten erfordert. Ein Lerntransfer ist auch von diesem Modell nicht zu erwarten.

\section{Struktur}

LAUNDER schlägt ein Modell vor, das Lernsituationen durch das Spiel und den Wettkampf in den Mittelpunkt stellt. Das Play Practice-Modell strebt damit an, Spielkompetenz, Kenntnisse für die Sportpraxis und Spaß beim Lernen ohne Berücksichtigung des Leistungsniveaus zu erbringen. Es will auch erreichen, dass Kinder in der Sportpraxis engagiert bleiben, sogar noch nach der Schulzeit.

Play Practice berücksichtigt die Entwicklung der Sportfertigkeiten, was sowohl technische Fertigkeiten als auch die Entscheidungsfähigkeit beinhaltet. Der Spieler soll lernen, „was“ er in verschiedenen Spielsituationen machen muss und „wie“ er handeln soll. Folglich soll das Lernprogramm sich auf die technische und taktische Schulung und den Transfer zum Spiel konzentrieren, da das Hauptziel des Lernens die Entwicklung der Spielkompetenzen ist. Für LAUNDER sind Technik und Taktik verschiedene Elemente, die aber nicht getrennt berücksichtigt werden sollten. Beide sollten gleichzeitig gelernt werden, die Beziehung zwischen ihnen ist die Grundlage des Play Practice-Modells (ZHANG, 2008, S. 73).

LAUNDER meint, dass das Spiel sehr wichtig für das effektive Lernen sei und rät Lehrern und Trainern dazu, die Mehrheit der Lernaufgaben als Spiel zu verpacken. Das Spiel trägt dabei einen doppelten Gewinn in sich. Einerseits fördert es die Motivation und andererseits den Lerntransfer, wenn der Trainer Spielaufgaben stellt, die sich am Lernen der Sportfertigkeiten orientieren. Die Lernaufgaben werden als Spiele eingeführt und beeinflussen die Lernergebnisse. Im Idealfall maximieren sie den Lernerfolg.

Der Entwurf von LAUNDER beschränkt sich nicht auf Sportspiele. In seinem Buch schlägt er die Umsetzung seines Modells auch für Individualsportarten wie Leichtathletik und Golf vor. Das Grundprinzip, nach dem die Lernaufgaben als Spiel verpackt werden sollen, könne auch bei diesen Sportarten wirksam werden. 


\section{Aufgabenstellung}

Das Spiel und der Wettkampf sind die Hauptmerkmale des Play Practice-Modells. Das Ziel ist es, die Teilnahme zu fördern und eine effektive Lernumwelt zu kreieren, in der sich der Lernende gut aufgehoben fühlt und Spaß an den Herausforderungen hat, die die Lernaufgabe stellen. Das Modell besteht aus drei Stufen: focusing play, shaping play und enhancing play.

Focusing play ist die erste Stufe des Modells. Sie bezieht sich auf das Lernen der technischen Fertigkeiten. Die Lernaufgaben sollen als Spiel gestellt werden. Solche Spiele üben bedeutsam die technischen Fertigkeiten. Ein Beispiel ist das Spiel mit Überzahl beim Fußball, 3 vs. 2, in dem die Spieler in Überzahl von den Regeln verpflichtet werden, eine besondere technische Fertigkeit anzuwenden. Auf diese Weise bekommen sie die Gelegenheit, die technischen Fertigkeiten unter erleichterten Bedingungen in einer Spielsituation zu üben.

Shaping play ist die zentrale Stufe des Modells, die den Erfolg des Lernens determiniert. Shaping ist ein Verfahren der Spielmodifizierung, worin der Trainer Modifizierungen zu sekundären Regeln einfügt. Er verfolgt damit das Ziel, das Üben von besonderen Spielelementen zu intensivieren. Die folgenden Variablen bieten sich dabei für Modifikationen an:

- Punkte für Tore: Punkte auf das gewünschte Verhalten anrechnen, z. B. Tore mit dem schwachen Fuß werden mit Doppeltor belohnt.

- Materialien: Durch die Modifizierung der Lernmaterialien kann man das Spiel vereinfachen.

- Spielraum: Die Verkleinerung des Spielfeldes bietet den Spielern mehr Beteiligungsgelegenheiten an.

- Anzahl der Spieler: Eine kleinere Spieleranzahl begünstigt das taktische Bewusstsein.

- Beschränkung der technischen Fertigkeiten: Fokussierung auf die Fertigkeit, die es zu lernen gilt.

Enhancing play ist die dritte Stufe des Modells. Die Steigerung der Komplexität findet durch das Erhöhen der Anzahl der Spieler, die Erweiterung des Feldes und das Variieren der Regeln statt. Nach LAUNDER (2001, S. 57) sollte der Trainer sicherstellen, dass die Spieler die technischen Fertigkeiten und die taktischen Elemente des Spiels beherrschen. Deswegen sollte er das Spektrum der Lernaufgaben erweitern und progressiv die Komplexität steigern.

Das Play Practice Modell ist eine Alternative für das Lehren und Training des Sports durch Spielen. Der Trainer soll bei diesem Modell den Übungsprozess auf besondere technische und taktische Ziele fokussieren, hierzu Spielaufgaben entwerfen und auf diesem Weg die Lernziele erreichen.

\section{Diskussion}

Die Forschung hat sich bislang wenig mit Play Practice beschäftigt. Einige wissenschaftliche Arbeiten erweitern die theoretischen Grundlagen des Modells (LAUNDER 
\&PILTZ, 2006), prüfen aber nicht dessen Praxiswirkung. HOLT, WARD und WALLHEAD (2006) studierten die Wirkungen des Modells auf den Lerntransfer beim Fußball. Die Ergebnisse zeigen, dass das Modell wirksamer für fortgeschrittene Spieler eingesetzt werden kann als für Anfänger. Sie unterstützen damit nicht die Absicht von LAUNDER, ein Modell vorzuschlagen, das angeblich Anfängern das Lernen erleichtert und besonders für den Schulsportkontext geeignet ist. ZHANG (2008) überprüfte die Wirkung des Play Practice-Modells auf das Lernen technischer Fertigkeiten im Tischtennis mit erwachsenen Studenten. Die Ergebnisse legen im Vergleich mit einer Kontrollgruppe, die nach einem traditionellen Modell lernte, ein besseres Ergebnis nahe.

Die ursprüngliche Intention von LAUNDER war es, ein Modell vorzuschlagen, das den Anfängern das Lernen des Sports erleichtert und sich für die Anwendung im Schulsport eignet. Um diese Eignung zu überprüfen, sind weitere Studien sowohl im Anfänger- als auch im Fortgeschrittenenbereich notwendig. Ebenfalls werden Studien benötigt, die erforschen, wie sich das Modell kognitiv und emotional bei der Sportausübung auswirkt. Erst dann kann die Wirksamkeit des Play Practice Modells bestätigt oder verneint werden.

\subsubsection{Modell der inzidentellen Inkubation (Heidelberger Ballschule)}

\section{Einführung}

KRÖGER und ROTH (1999, S. 9) gehen von der Überlegung aus, dass heutzutage die Kinder mangelnde Spielmöglichkeiten hätten. In der Vergangenheit spielten sie häufig auf der Straße und hatten keine Frühspezialisierung, nun aber trainieren sie in sehr jungem Alter in einer bestimmten Sportart bei einem Sportverein. KRÖGER und ROTH warnen vor dieser Gefahr der Frühspezialisierung, die dem pädagogischen Prinzip der multilateralen Sportausbildung widerspreche. Sie schlagen vor, die Straßenspielkultur wiederzubeleben. Diese könnte in den Schulsport und in den Sportverein eingefügt werden. So würde eine allgemeine Grundausbildung vor der Phase der Sportartspezialisierung entstehen. Aus diesem Ansatz heraus wurde eine „Sportartübergreifende Ballschule" vorgeschlagen. Sie ist ausgerichtet auf vielfältige Erfahrungen bei besonderen taktisch und technisch orientierten Spielen und Übungen. Diese bilden in gesammelter Form einen methodischen Weg für die Bildung einer festen Basis, die die spätere Sportspezialisierung unterstützt. Die Autoren schlagen konkrete Übungs-, Spiel- und Trainingsformen dafür vor.

\section{Begründung}

Das Modell der Ballschule strebt den Aufbau einer „effektiven antizipativen Verhaltenssteuerung" an (KRÖGER \& ROTH, 1999, S. 16). Das Sammeln von Spielerfahrungen hat als Ergebnis, dass die Kinder an Sicherheit gewinnen und die Wirkungen ihrer eigenen Handlungen vorauszusehen lernen. Dies geschieht nach dem Modell von HOFFMANN (1993, S. 41-42) zum Aufbau verhaltenssteuernder Antizipationen. Nach HOFFMANN wird eine Handlung immer von Antizipationen und Ergebnissen begleitet. Die Antizipationen beinhalten auch die Erwartungen des Kindes an die Ergebnisse. Diese werden mit den wirklichen Handlungsergebnissen verglichen und das Kind lernt zu 
identifizieren, welche Handlungen die erwarteten Ergebnisse bewirken und welche nicht. Progressiv werden Kenntnisse über die geeigneten Handlungen erworben, die für die Auflösung der Spielsituationen gebraucht werden. Die Antizipationen werden dabei ständig nach der Folge des Verhaltens während des Spiels korrigiert und adaptiert.

Das späte Schulkindalter ist für die Entwicklung der koordinativen Fähigkeiten bestens geeignet. Die Sportwissenschaften liefern für diese Behauptung in der wissenschaftlichen Diskussion zahlreiche Beweise. Die noch nicht vollständige Entwicklung des Zentralnervensystems und der Reifungsprozess ermöglichen einen hohen Spielraum für das Training der Koordination im Kindes- und Jugendalter. Allerdings sind die wissenschaftlichen Ansätze bezüglich der Strukturierung der koordinativen Fähigkeiten vielfältig und in Teilen sogar widersprüchlich. Es existiert bisher kein wissenschaftlicher Konsens darüber, was genau koordinative Fähigkeiten sind und wie sie gezielt beeinflusst werden können. Das Modell der Ballschule entscheidet sich demgemäß für die Einbeziehung verschiedener Koordinationskonzepte. Aus diesem Grund wird im Rahmen dieses Modells nicht mehr das Konzept koordinativer Fähigkeiten genutzt, sondern es ist die Rede von „allgemeinen koordinativen bzw. motorisch informationellen Anforderungsklassen“ (KRÖGER \& ROTH, 1999, S. 21). In der Ballschule werden also allgemeine koordinative Anforderungen umgesetzt. Dies geschieht nicht auf konventionelle Weise, vielmehr werden sie als eine „Ballkoordinationsschulung“ durch das „Bewältigen von informationell-motorischen Anforderungsbausteinen geübt“ (S.22).

Die Idee eines fertigkeitsorientierten Ansatzes, vorgeschlagen als eine dritte Stufe der Ballschule, wurde aus der „Modularitätshypothese“ abgeleitet. Ihr zufolge werden die menschlichen Handlungen durch kognitive Zentralsysteme kontrolliert, die aufgabenorientiert arbeiten. Solche Zentralsysteme werden als Module organisiert und stellen eine Abbildung über die „Anordnung der Dinge in der Welt“ dar. Sie erleichtern die Übersetzung der Handlungsabsichten in motorische Handlungen (vgl. KRÖGER \& ROTH, 1999, S. 29). Die Module befassen sich mit spezifischen Aspekten der Aufgabe und arbeiten automatisch, ein bewusster Zugriff darauf ist beschränkt. Die aus der Kognitionspsychologie stammende Modularitätshypothese wurde in Arbeiten von HOSSNER (1995) für den Sport umgesetzt. Ihr liegt die Annahme einer aktiven Konstruktion von Wissen zugrunde. Dieser Prozess soll durch die Ballschule gefördert werden.

\section{Struktur}

Die Ballschule besteht aus einem „ABC für Anfänger“, das drei methodische Vorgaben beinhaltet: einen spielerisch-situationsorientierten Pfeiler $(A)$, eine fähigkeitsorientierte Ballschule $(B)$ und den fertigkeitsorientierten Zugang (C). Diese drei Bereiche widersprechen sich nicht, sondern ergänzen einander. Das folgende Raster veranschaulicht die Zusammenhänge. 
Tabelle 4: Zugang, Ziele, Inhalte und Methoden der allgemeinen Ballschule (KRÖGER \& ROTH, 1999, S. 13)

\begin{tabular}{|l|l|l|}
\hline \multicolumn{1}{|c|}{ Zugang } & \multicolumn{1}{|c|}{ Ziele } & \multicolumn{1}{c|}{ Inhalte und Methoden } \\
\hline A: Situationsorientiert & Spielen lernen & $\begin{array}{l}\text { „Reines“ Spielen in sportspiel- } \\
\text { übergreifenden Taktikbausteinen }\end{array}$ \\
\hline B: Fähigkeitsorientiert & $\begin{array}{l}\text { Verbesserung der Ballkoordina- } \\
\text { tion }\end{array}$ & $\begin{array}{l}\text { Üben von sportspielübergreifen- } \\
\text { den informationellmotorischen } \\
\text { Anforderungsbausteinen }\end{array}$ \\
\hline C: Fertigkeitsorientiert & $\begin{array}{l}\text { Verbesserung grundlegender } \\
\text { Ballfertigkeiten }\end{array}$ & $\begin{array}{l}\text { Üben von sportspielübergreifen- } \\
\text { den Technikbausteinen }\end{array}$ \\
\hline
\end{tabular}

Die situationsorientierten Spiele sind auf den Ausgleich der unzureichenden Spielerfahrungen ausgerichtet, worunter die Kinder heutzutage wegen des Wegfalls der Straßenspielkultur leiden. Darin geht es deshalb nicht darum, eine bestimmte Leistung zu erreichen. Im Mittelpunkt steht vielmehr das Explorieren, Ausprobieren und Spielen lassen unter dem Motto „zu spielen lernt man durch spielen“. Die Kinder sollen in erster Linie lernen, frei zu spielen und dadurch die Fähigkeiten für die Wahrnehmung von taktischen Situationen entwickeln, und lernen, mit Spielregeln umzugehen. In dieser Phase werden auch Übungen zur Entwicklung der sensomotorischen Fähigkeiten als Vorbereitung für das spätere Lernen technischer Fertigkeiten bereitgestellt. Dieser fähigkeitsorientierte Ansatz strebt die Bildung einer allgemeinen Basis der Faktoren der technischen Leistung an. Die Ballschule beabsichtigt in dieser Phase die Entstehung der koordinativen Voraussetzungen als allgemeine Grundlage der hohen technischen Fertigkeitsleistungen des Sports. Es besteht eine Analogie zum IQ, der die Hauptgrundlage für hohe kognitive Leistungen darstellt (vgl. KRÖGER \& ROTH, 1999, S. 12).

Der fertigkeitsorientierte Zugang zielt auf die Entwicklung einer Reihe von Technikbausteinen für die Gestaltung der spezifischen technischen Fertigkeiten ab. Es geht im Einzelnen um eine Schulung der allgemeinen technischen Elemente, die allen Sportspielen gemeinsam sind und die die Basis für die allmähliche Gestaltung der technischen Fertigkeiten bilden. Diese Technikbausteine sind unspezifisch und deswegen zwischen verschiedenen Spielen transferierbar. HOSSNER (1995, S. 22) bezeichnet diese Technikbausteine deshalb als „Baumaterial“ für verschiedene „Bewegungsgebäude“ und schlägt ihre frühzeitige Ausbildung vor (vgl. KRÖGER \& ROTH, 1999).

\section{Aufgabenstellung}

Aus einer Literaturrecherche und eigenen Befragungen entwickelten KRÖGER und ROTH sieben allgemeine offensive Taktikbausteine, die durch leichte Modifikationen auch in defensive Bausteine umgewandelt werden könnten, wenn sie in die Gegenrichtung umgesetzt werden (z. B. „ins Ziel treffen verhindern“, statt „,ins Ziel treffen“). Durch diese Bausteine sollen die Kinder übergreifende Spielfähigkeiten und taktische Kompe- 
tenzen erwerben. Für die Taktikbausteine werden autonome Spielformen vorgeschlagen, die der Förderung der allgemeinen Spielfähigkeit dienen sollen. Dies sind:

- ins Ziel treffen

- den Ball zum Ziel bringen

- einen Vorteil herausspielen

- das Zusammenspiel

- eine Lücke erkennen

- die Gegnerbehinderung umgehen

- sich Anbieten und Orientieren.

Mit diesem Ansatz einer fähigkeitsorientierten Ballschule strebt das Modell die Verbesserung der allgemeinen Ballkoordination an. Dies geschieht durch das Üben von einfachen Ballfertigkeiten mit einer Vielfalt von afferenten und efferenten Anforderungen und durch eine Aufgabe, die unter verschiedenen Druckbedingungen ausgeführt werden muss.

Der fertigkeitsorienterte Zugang besteht aus dem Erwerb von verschiedenen Technikbausteinen, die in unterschiedlichen Strukturen organisiert werden können. Diese Technikbausteine sind ebenso wie die Taktikbausteine auf einer allgemeinen, übertragbaren Fertigkeitsebene angesiedelt, die unterhalb der spezifischen Spielfertigkeiten liegt. Die Technikbausteine werden nicht durch das Spielen gelernt, sondern als Übungen gestellt. Sie können isoliert oder kombiniert mit anderen Fertigkeiten geübt werden und je nach der angewendeten Lehrmethode variiert werden. KRÖGER und ROTH (1999) beziehen sich mit ihrem Ansatz auf die Arbeiten von HOSSNER und KORTMANN (1995, S. 43). Diese haben 21 Technikbausteine für das Volleyballtraining benannt, die für die Allgemeinheit der Sportspiele adaptiert und umgesetzt werden. Die entstehenden Technikbausteine sind:

- Den Winkel steuern (Schlag, Schuss, Wurf)

- den Krafteinsatz steuern (Schlag, Schuss, Wurf)

- den Spielpunkt des Balles bestimmen

- Laufwege und -tempo zum Ball festlegen

- $\quad$ sich verfügbar machen

- Zuspielrichtung und -weite vorwegnehmen

- Abwehrposition vorwegnehmen

- Laufwege beobachten

\section{Diskussion}

Das Modell schlägt ein Konzept vor, das für die Schulung der Koordination und die Vorbereitung für die spätere Übungspraxis der Sportspiele einen konzeptuell und theoretisch gut begründeten Weg für das frühe Lernalter darstellt. Das Modell bietet eine vernünftige Auflösung des Problems der Vielfältigkeit von Ansätzen, Konzepten und Vor- 
schlägen bezüglich der Gliederung und praktischen Umsetzung koordinativer Fähigkeiten. In Bezug auf die spezifische Schulung der Sportspiele leidet das Modell aber darunter, dass es hierfür nicht definiert ist. Dies ist dem Anspruch der Autoren geschuldet, einen sportartübergreifenden Ansatz zu entwerfen.

Die Entwickler des Modells der Ballschule haben zahlreiche Lehrbücher, theoretische Bearbeitungen und einige empirische Forschungen vorgelegt (z. B. KRÖGER \& MEMMER, 2002; ROTH; MEMMERT \& SCHUBERT, 2006; ROTH \& MEMMERT, 2007; MEMMERT \& ROTH, 2003). Allerdings wurde das Modell unzureichend getestet, um seine Wirksamkeit zu überprüfen oder es mit anderen didaktischen Modellen zu vergleichen. Es fehlt an experimentellen Arbeiten über das Modell, um die theoretischen Ansätze mit empirischen Ergebnissen zu untermauern. SINNIG (2008) kritisiert die Ballschule wegen der geringen Einbindung der Lernenden in die Gestaltung des Lernprozesses und der fehlenden Berücksichtigung ihrer individuellen Bedürfnisse. In diesem Sinne ist die Ballschule eher ein spiel- und aufgabenzentriertes als ein spielerzentriertes Modell. Es berücksichtigt die pädagogischen Situationen und Lernziele des Schulsports nur unzureichend. Aus anderer Sicht betontSINNIG die mangelnden Hinweise und die fehlende Transparenz des Modells für einen Übergang vom allgemeinen Vorspiel zum spezifischen Spiel. Es ist ihrer Ansicht nach nicht ersichtlich, wie das ABC konkret zum spezifischen Sportspiel übertragen werden soll. Die Übertragung wird vielmehr der Intuition überlassen. „Eine eindeutige Positionierung, wann gespielt und geübt [wird,] bzw. wie und warum sich die Phasen abwechseln, wird ebenso nicht klar erkennbar." (SINNIG, 2008, S. 45).

\subsubsection{Decision Training Modell}

\section{Einführung}

In ihrem Buch „Perception, Cognition, and Decision Training: The Quiet Eye in Action“ (2007) schlägt Joan VICKERS ein Modell für das Sporttraining vor, das für die Verbesserung der Entscheidungsfähigkeit entworfen wurde. VICKERS hat ihr Modell auf eine Reihe von Individualsportarten und Sportspielen übertragen. Ihr Ziel ist die Ausbildung von intelligenten Sportlern, die Entscheidungsfähigkeit im richtigen Augenblick des Wettkampfs präsentieren. VICKERS zweifelt an den traditionellen „behavioristischen Modellen", in denen die Sportler ständig wiederholt isolierte technische Fertigkeiten ausführen. Langfristig würden so zwar technische Verbesserungen erreicht, dieses Lernen würde aber nicht auf Wettkampfbedingungen transferierbar sein, da die Sportler unter Zeit- und- Gegnerdruck unfähig wären, das Gelernte umzusetzen.

\section{Begründung}

Das Modell Decision Training betrachtet das technische und physiologische Training zwar als wichtig, beachtet aber auch die Bedeutung des kognitiven Trainings und fokussiert deshalb die Entwicklung der kognitiven Fähigkeiten der Sportler. Diese haben einen hohen Anteil an sportlichen Leistungen und müssen daher berücksichtigt werden, ohne die technischen und physiologischen Anteile dabei zu vernachlässigen. VICKERS (2007, S. 23) schließt deshalb die repetitive Übung nicht aus. Sie glaubt aber daran, 
dass Trainer auch die Entscheidungsfähigkeit der Sportler trainieren können, indem sie die Sportler kognitiv am Trainingsprozess beteiligen. Dafür sind Information von hoher Qualität notwendig. Für die Praxis schlägt das Decision Training Modell komplexe Trainingsaufgaben vor, die das Üben der Entscheidungsfähigkeit während des Trainings betonen und dann auf Wettkampfsituationen transferiert werden. Langfristig würden die Sportler auf diesem Weg dazu befähigt, die Auflösung neuer Spielsituationen im Wettkampf zu leisten, die vorher nicht geübt werden.

Das Modell bekräftigt die Bedeutung der Entwicklung der kognitiven Fähigkeiten in einem spezifischen Spielkontext (RAAB, 2007, S. 9). Solche Fähigkeiten sind Aufmerksamkeit, Antizipation und Erinnerungsvermögen. Sie können im Training verbessert werden, indem der Trainer Aufgaben bereitstellt, die ein "hohes Niveau von kognitiver Anstrengung" erfordern (VICKERS, REEVES, CHAMBERS, \& MARTELL, 2004, S. 224). Der Trainer bietet explizite Informationen über Konzepte und Strategien an und kontrolliert ihr Verständnis durch das Stellen von Fragen.

\section{Struktur}

Das Decision Training Modell besteht aus drei Schritten für das Trainieren der Entscheidungsfähigkeit (VICKERS, 2007, S. 166 ff.):

1. Bei jeder Entscheidung und die ihr zugrunde liegenden kognitiven Fähigkeiten bei bestimmter Spielsituation identifizieren. Jede Entscheidung sollte eine der folgenden sieben kognitiven Fähigkeiten mit einem taktischen Element einer spezifischen Sportart verbunden werden:

- Anticipation: Fähigkeit des Sportlers, schon während der Handlungsvorbereitung den Verlauf des Geschehens vorherzusagen.

- Attention: Fähigkeit, aus der Informationsmenge die vor der Handlung zur Verfügung steht, die richtige Hinweise herauszufiltern

- Concentration: Fähigkeit, ständig die richtige Information annehmen und keine Aufmerksamkeit auf irrelevante Ereignisse schenken.

- Pattern recognition: Fähigkeit des Sportlers, bedeutende Hinweise annehmen, während er in einer komplexen Wettkampfumwelt eine Handlung ausführt.

- Memory: Fähigkeit, schnell eine Lösung aus dem Langzeitgedächtnis zu ziehen um sich ständig auf wechselnden Sportsituationen anpassen zu können.

- Problem solving: Fähigkeit, gegebene problematische Situationen erfolgreich zu lösen, wenn keine eindeutige Lösung zur Verfügung steht.

- Decision Making: Fähigkeit, die beste aus mehreren möglichen Handlungsalternativen auszuwählen.

2. Eine geeignete Lernaufgabe für das Üben dieser Fähigkeit entwerfen. Jede Lernaufgabe sollte ein "cognitive trigger" beinhalten. Ein „cognitive trigger" lässt während der Handlungsausführung den Sportler, und auch den Trainer wissen, ob die getroffene Entscheidung richtig ist. Sieben "cognitive triggers" werden für diesen Schritt identifiziert: 
- Object cues: Der Sportler hat die Aufgabe seine Aufmerksamkeit auf einen spezifischen Gegenstand zu richten und ihn vor der Handlung identifizieren, z.B., die Zahlen und Buchstaben auf dem Ball ausrufen vor inn annehmen und weiter passen.

- Localization cues: der Sportler sollten der Sportler sollte seine Aufmerksamkeit auf ein bestimmten Platz richten und ein Geschehen vorhersagen, z.B. einen bestimmten Platzt innerhalb des Tores ausrufen und in diese Richtung schießen.

- Quiet-eye-cues: Durchführung von Aufgaben und Übungen mit Hilfe von eyetracking-Technologien. Bei diesen wird eine Videokamera auf eine Brille gestellt, mit solchen Geräte kann man das Blickverhalten beobachten, messen und analysieren, dadurch werden Schlüsse gezogen über die genaue Stelle wo der Sportler seinen Blick fixiert bevor er eine Entscheidung trifft.

- Reaction-time cues: Bei dieser Aufgabe soll der Sportler in einer sehr kurzen Zeit eine bekannte Lösung für eine Situation aus dem Gedächtnis ziehen und sie auch sehr schnell ausführen.

- Memory cues: Aufgabe die die Fähigkeit für einen schnellen Zugang zum Langzeitgedächtnis trainieren soll. Der Sportler wird angefordert, ein bestimmtes Element erinnern und die Aufmerksamkeit auf diesem Gedanken bewahren, gleichzeitig versucht er, sich an ein anderes, verschiedenes Element zu erinnern.

- Kinesthetic cues: Aufgabe wo der Sportler angefordert wird, ein besonderes Gefühl für eine Position, Technik oder Ereignis zu entwickeln.

- Self-coaching cues: die Sportler sollen bei dieser Aufgabe Entscheidungen über ihr eigenes Trainingsprogramm treffen, sie sollen ein Problem bei ihrer Leistung identifizieren, die Ursache finden und eine Trainingsstrategie für die Lösung dieses Problems vorschlagen. Alle diese 3 Verfahren sollen sie auch vernünftig begründen.

3. Eine der folgenden sieben Werkzeuge für das Training der Entscheidungsfähigkeit in unterschiedlichen Kontexten auswählen:

- Variable pratice: Variation der Situation, wenn nur eine technische Fertigkeit gelernt wird.

- Random practice: Variation der Situation, wenn gleichzeitig mehrere Bewegungen gelernt werden.

- Bandwidth feedback: Verzögerung und Reduktion der Rückmeldungen, dabei werden die Rückmeldungen nur bei bestimmten Ereignissen gegeben.

- Questioning: Überprüfung des Verständnisses der getroffenen Entscheidung.

- Videofeedback: Selbstanalyse der Leistung.

- Hard-first tactical instruction and Modelling: Frühzeitiges Lernen der technischen und taktischen Konzepte im Lernprozess.

- External focus of instruction: Veranschaulichung der Anwendung der technischen und taktischen Fähigkeiten für die Leistungssteigerung.

Das Modell erhält damit die folgende Struktur: 


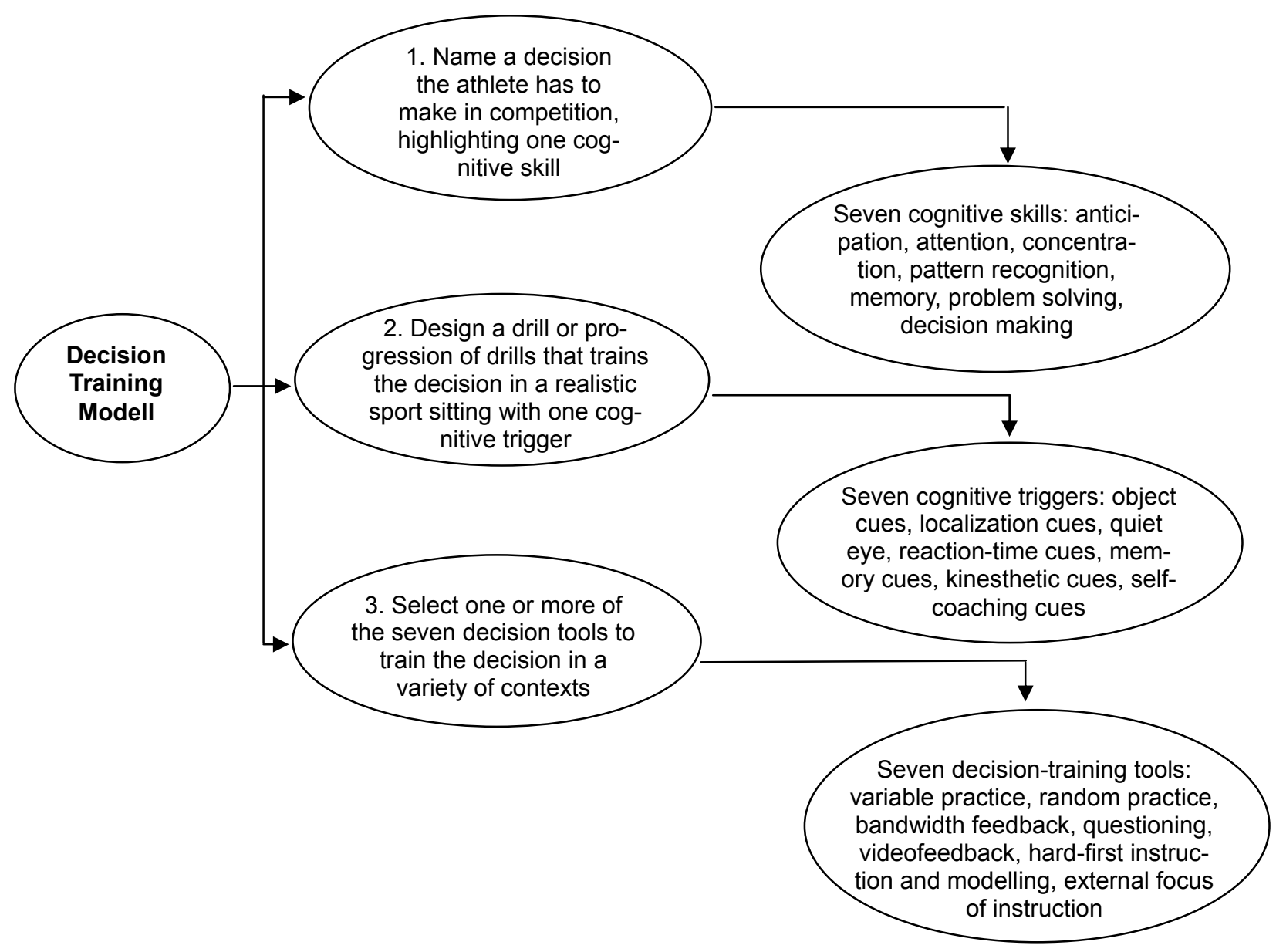

Abbildung 5: Das Decision Training Modell, (nach VICKERS, 2007, S. 167)

\section{Aufgabenstellung}

Ein Beispiel dafür, wie das Decision Training umzusetzen ist, wird von GRANTHAM (2008) vorgestellt. Ein Fußballspieler soll die Wahrnehmungsfähigkeit verbessern, um den Ball ständig verfolgen zu können:

"The first step is to identify the decision(s) that need to be made in a competitive environment. In this case, the cognitive skill to be developed is attention (tracking the ball). Step two requires the coach to design a drill with a cognitive cue to train the decision identified in step one. A cognitive trigger allows both the coach and athlete to know if the correct decision has been made whilst performing the skill or tactic.

To improve a football player's attention, an object cue will be used as the cognitive trigger, to see if the player can track the ball effectively. The player will be asked to call out numbers or letters written on the ball before receiving a pass and taking a shot on goal. In step three, one or more of the decision training tools are used to train the decision highlighted in step 
one within the context of step two. In this example, the training session could be a random practice using smart combinations (the drills simulate conditions similar to those found during a match) of receiving a pass and taking the shot".

\section{Diskussion}

VICKERS, REEVES, CHAMBERSund MARTELL, (2004) stellen viele Beispiele und Anwendungen des Modells in einer Reihe von Sportarten vor, darunter Tischtennis, Baseball, Basketball, Badminton, Golf, Schwimmen, Radfahren, Biathlon, Schlittschuhlaufen und Freestyle Ski-Jumping. Mit der Verwendung von eye-tracking-Technologien haben VICKERS und Mitarbeiter mehrere Forschungsarbeiten durchgeführt, in denen die Anwendbarkeit des Modells für das Lernen der technischen Fertigkeiten bestätigt wurde. In der Anwendbarkeit des Modells kann hingegen für das Lernen der taktischen Fähigkeiten und Spielkompetenzen nur geringer Erfolg beobachtet werden. Das Modell ist daher eher zur technischen Perfektionierung bei Hochleistungssportlern geeignet als zum grundlegenden Erlernen einer Spielsportart. Man kann es daher eher als ein Modell des motorischen Lernens als ein Spielvermittlungsmodell bezeichnen.

\subsubsection{Situation Model of Anticipated Response Consequences of Tactical Training (SMART)}

\section{Einführung}

Das SMART-Modell (RAAB, 2000, 2001, 2007) erklärt die impliziten und expliziten Lernprozesse der Sportspiele. Das implizite Lernen wäre demnach nur in Situationen mit geringer Komplexität effektiv, hohe Komplexität einer Situation erfordere hingegen ein explizites Lernen. Das Modell begründet auch den Lerntransfer zu ähnlichen Situationen mit identischen Ursache-Wirkungs-Strukturen, die zu denselben Ergebnissen führen. Die Erklärung lautet hier, dass die Strukturen und Lösungsmöglichkeiten per Bildung von Wenn-dann-Regeln im Gedächtnis abgebildet werden. Der Lerntransfer wird dann nicht auf die Ähnlichkeit der Spielsportart zurückgeführt, wie andere Vermittlungsmodelle dies behaupten, sondern auf die Ähnlichkeit der abstrakteren Wenn-dannRegeln hinter der konkreten Sportart.

\section{Begründung}

Die Entwicklung der Entscheidungsfähigkeit im Sport erfolgt, wenn das Erkennen der Beziehungen zwischen Spielsituation gelingt, die durch Bewegungen ausgelöst wurden und deren Wirkung in der Umweltsituation sichtbar wird. Das Lernen dieser Beziehungen führt zur Verbesserung der Entscheidungsfähigkeit bei ähnlichen Situationen während des Spiels oder sogar bei einer ähnlichen Situation in einem anderen Sportspiel. Das Erkennen der Situation und die Wirkungen die mit einer bestimmten Entscheidung verbunden werden, befähigt also dazu, Entscheidungen zu treffen, die die gewünschte Wirkung in der jeweiligen Spielsituation nach sich ziehen. Das SMART-Modell legt auch bei taktischen Schulungen Wert darauf, dass in Form von Wenn-dann-Regeln gelernt wird. Diese Regeln werden in einem Doppelprozess gebildet. Zunächst findet ein impli- 
zites bottom-up statt, das die Wahrnehmungsanteile abbildet. Anschließend erfolgt über ein explizites top-down die Verknüpfung mit dem kognitiven Ziel. Beide Prozesse interagieren in Abhängigkeit der Komplexität des Lerninhalts mehr oder weniger miteinander.

\section{Struktur}

In einer Spielsituation mit Optionsgenerierung und Auswahlmöglichkeiten werden Rückmeldungen von unterschiedlichem Maß erzeugt. Diese machen die Wirkungen der Entscheidung auf die Umwelt erkennbar. Ein gutes Erkennen der Wirkungen der eigenen Entscheidung führt so langfristig zu einer besseren Auswahlbei einer späteren ähnlichen Spielsituation. Das Modell der Wenn-dann-Regeln ist situationsspezifisch, nicht aber sportartspezifisch. Das implizite Erkennen der Situation erfolgt in Bezug auf eine erweiterte Fähigkeit zur Optionsgenerierung und Auswahl bei ähnlichen impliziten Situationen und umgekehrt.

Zum Beispiel kann beim Tennis ein Spieler im Laufe eines Spiels bewusst oder unbewusst die Variationen des Aufschlags erproben (mit oder ohne Topspin, unterschiedliche Schlagwinkeln und Kraftdosierung usw. [option generation]), bei einer bestimmten Spielsituation eine Aufschlagart wählen (choice), die Ergebnisse wahrnehmen (effect of choice) und auf diese Weise allmählich implizit oder explizit Situationen erkennen (implicit or explicit recognition), wo bestimmte Aufschlagarten bestimmte Wirkungen auslösen. Mit diesen Kenntnissen lernen die Spieler, bessere Entscheidungen bei zukünftigen, ähnlichen Situationen zu treffen.

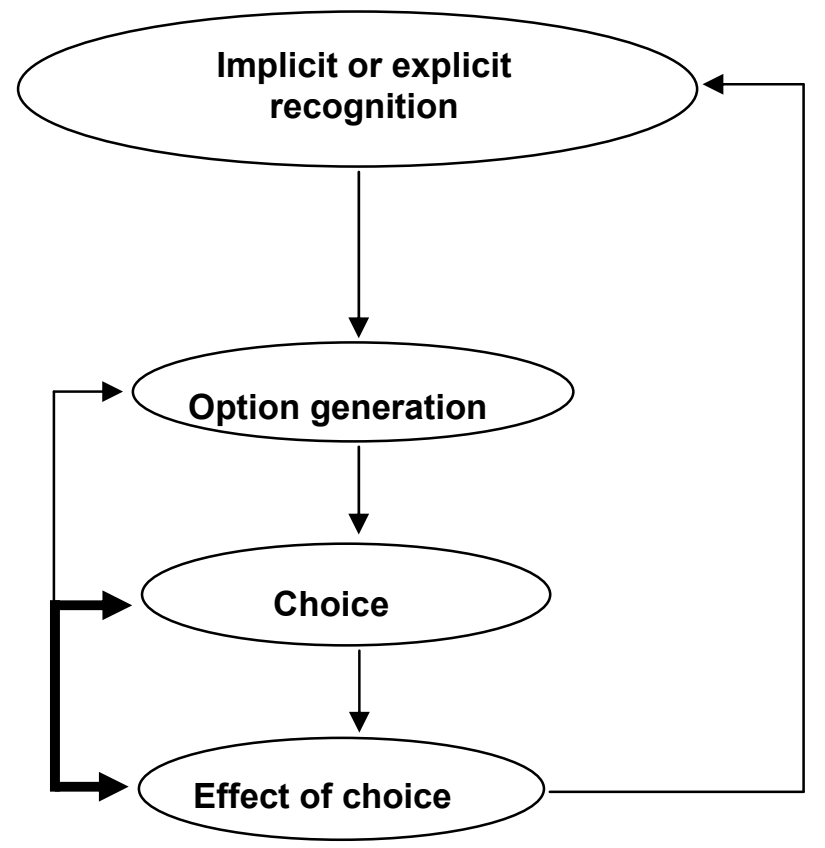

Abbildung 6: SMART: Situation Model for Anticipated Response consequences of Tactical training. Die Rückmeldung der Wirkung einer Entscheidung kann die Prozesse in unterschiedlichem Ausmaß ändern (nach RAAB, 2007, S. 11) 


\section{Aufgabenstellung}

Das SMART-Modell setzt einen Zweiphasenprozess um. Wahrnehmungsanteile (bottom-up) und kognitive Anteile (top-down) finden Berücksichtigung in der Spielsituation.

Bottom-up sind Prozesse, bei denen durch ein gezieltes Training der Wahrnehmungsfähigkeit die Aufmerksamkeit verbessert wird. Dabei wird gelernt, die relevanten Eigenschaften einer Spielsituation von den nicht relevanten zu unterscheiden. Damit ist ein implizites Lernen gemeint. Top-down sind Prozesse, wo Instruktionen vermittelt werden. Der Trainer wies den Spielern darauf hin, welche Konsequenzen eine bestimmte Entscheidung zur Folge haben würde. Damit ist ein explizites Lernen gemeint.

RAAB (2000, S. 203) stellt eine Reihe von insgesamt fünf Prinzipien für das Taktiktraining auf. Damit wird versucht, das SMART-Modell auf die "Trainersprache"zu übersetzen. Sie werden aus der theoretischen und empirischen Gestaltung des Modells abgeleitet und als Empfehlungen für das Taktiktraining dargestellt.

Suche $=$ Trainiere Bottom-up-Prozesse.

Möglichkeiten= Trainiere Top-down-Prozesse.

Anforderungen $=$ Trainiere komplexitätsspezifisch.

Regeln= Trainiere erst inzidentell dann intentional.

Taktiktraining= Trainiere divergente und konvergente Lösungen.

\section{Diskussion}

RAAB (2000) weist durch mehrere Experimente sowohl im Labor als auch im Feld bei den Sportarten Basketball, Volleyball und Handball nach, dass das inzidentelle oder implizite Lernen zur Anerkennung von impliziten Wenn-dann-Regeln führt. Wenn diese Wenn-dann-Regeln verbalisiert werden, wird ein absichtliches (explizites) Lernen erreicht. Bei seinen Experimenten strukturiert RAAB eine "taktische Grammatik" mit der die bottom-up- und top-down-Prozesse repräsentiert werden. Weiterhin werden computerisierte Modularisierungen dieser Grammatik gebildet mit dem Ziel, eine Beschreibung der Entscheidungsprozesse sowohl bei impliziten als auch bei expliziten Lernsituationen zu erzeugen. Das SMART Modell stellt allerdings keine praktische Umsetzung dieser Erkenntnisse bereit, die zum besseren Lernen und Training der Entscheidungsfähigkeit beitragen. Es versucht lediglich, mit seinem theoretischen und experimentellen Einsatz zu verdeutlichen, welchen Prozessen das Entscheidungstreffen im Sport zugrunde liegen. Das Modell strebt also ausschließlich eine theoretische Beschreibung der Entscheidungsprozesse im Sport unter Berücksichtigung der Wahrnehmungs- und Informationsanteile an (RAAB, 2000, 2001, 2007). Es ist damit kein didaktisches, sondern ein theoretisches Modell zur Entwicklung der Entscheidungsfähigkeit und der Untermauerung der theoretischen Annahmen in diesem Bereich. Dennoch versucht RAAB methodische Anwendungen aus dem Modell ableiten und stellt eine "Prinzipienliste“ vor. Berichte über die partielle Umsetzung dieser Prinzipien liegen in mehrere Veröffentlichungen vor (z.B. RAAB, 1999; RAAB \& GWODZ, 1997, zitiert nach RAAB, 2000, S. 201). 


\subsubsection{Zusammenfassung der Spielvermittlungsmodelle}

Die Spielvermittlungsmodelle entstanden in verschiedenen Zeitspannen und ihre Entstehung folgt keiner bestimmten Reihenfolge, allerdings bestehen einige von ihnen aus Modifizierungen oder Ergänzungen von früher entwickelten Modellen. Seit dem Erscheinen des TGfU wurden mehrere Modelle entwickelt, die auf denselben Prinzipien basieren, besonders im englischen Sprachraum. Aber vor dem TGfU waren schon einige Modelle in Deutschland publiziert worden, viele haben ihren Ursprung in den sechziger Jahren, ihre Basis wurde später wieder für den Entwurf von anderen herangezogen. Die deutschen Modelle berücksichtigen bereits früher entstandene deutsche, aber nicht in Bezug auf TGfU und umgekehrt. Diese getrennte Entwicklung der Spielvermittlungsmodelle ist auffällig, aber dafür wird in der Literatur keine Erklärung gefunden. In jüngster Zeit wurden technische Geräte wie das „Eye Tracking“ und die computerisierte Modellierung für die Entwicklung von weiteren Modellen angewendet. Keines der Spielvermittlungsmodelle wurde ausführlich untersucht, einige wurden durch empirische Studien nicht bestätigt.

Die Spielvermittlungsmodelle können daher in 3 große Gruppen unterteilt werden:

- Spielvermittlungsmodelle aus deutscher Herkunft,

- Spielvermittlungsmodelle rund um TGfU überwiegend im englischen Sprachraum,

- Spielvermittlungsmodelle, die aus technologischen Umsetzungen entstanden sind.

Tabelle 5 enthält eine Synopse der in dieser Arbeit kommentierten Spielvermittlungsmodelle. 
Tabelle 5: Synoptische Darstellung der Spielvermittlungsmodelle

\begin{tabular}{|c|c|c|c|c|}
\hline \multicolumn{5}{|c|}{ Spielvermittlungsmodelle aus deutscher Herkunft } \\
\hline & Begründung & Struktur & Aufgabestellung & Bemerkungen \\
\hline $\begin{array}{l}\text { Spielreihe, } \\
1970\end{array}$ & $\begin{array}{l}\text { Systematisierung der } \\
\text { selbst-organisierten } \\
\text { Spiele der Kinder, } \\
\text { Förderung von Spiel- } \\
\text { erlebnissen und tak- } \\
\text { tischem Nachden- } \\
\text { ken. }\end{array}$ & $\begin{array}{l}\text { Sequentielle Anord- } \\
\text { nung der Spiele mit } \\
\text { steigender Komplexi- } \\
\text { tät. }\end{array}$ & $\begin{array}{l}\text { Zahlreiche Vorschlä- } \\
\text { ge für spezifische } \\
\text { Sportspiele. }\end{array}$ & $\begin{array}{l}\text { Ihre Anwendbarkeit } \\
\text { und Wirksamkeit } \\
\text { wurde nicht empirisch } \\
\text { untersucht. }\end{array}$ \\
\hline $\begin{array}{l}\text { Situative } \\
\text { Spieldidaktik, } \\
1982\end{array}$ & $\begin{array}{l}\text { Förderung von } \\
\text { Wahrnehmungs-, } \\
\text { Beurteilungs- und } \\
\text { Entscheidungsfähig- } \\
\text { keit durch das Prinzip } \\
\text { des entdeckenden } \\
\text { Lernens. }\end{array}$ & $\begin{array}{l}\text { Kleine Fußballspiele } \\
\text { mit Veränderung der } \\
\text { Regeln. }\end{array}$ & $\begin{array}{l}\text { Der Trainer bestimmt } \\
\text { das Lernziel, prüft } \\
\text { den „Ist-Zustand“ } \\
\text { der Spieler, bereitet } \\
\text { die Voraussetzungen } \\
\text { und Regeln vor und } \\
\text { stellt die „Spiel- } \\
\text { Übungen“ dar. }\end{array}$ & $\begin{array}{l}\text { Mehr auf das Spiel- } \\
\text { lernen als auf das } \\
\text { Techniklernen fokus- } \\
\text { siert. } \\
\text { Das Modell istin ei- } \\
\text { nem Lehrbuch publi- } \\
\text { ziert worden. } \\
\text { Keine empirische } \\
\text { Überprüfung. }\end{array}$ \\
\hline $\begin{array}{l}\text { Genetisches } \\
\text { Lernen, } 1991\end{array}$ & $\begin{array}{l}\text { Die Spieler identifi- } \\
\text { zieren die Spielprob- } \\
\text { leme und bemühen } \\
\text { sich, diese selbst- } \\
\text { ständig zu lösen, was } \\
\text { ein „kognitives Ler- } \\
\text { nen“ fördert. }\end{array}$ & $\begin{array}{l}\text { Exposition des Phä- } \\
\text { nomens, Identifizie- } \\
\text { rung des Problems, } \\
\text { Lösungsalternative } \\
\text { vorschlagen und } \\
\text { ausprobieren. }\end{array}$ & $\begin{array}{l}\text { Darstellung der Spie- } \\
\text { le durch Regelände- } \\
\text { rungen als Probleme. }\end{array}$ & $\begin{array}{l}\text { Zahlreiche Lehrbü- } \\
\text { cher und Umset- } \\
\text { zungserfahrungen } \\
\text { sind über dieses Mo- } \\
\text { dell publiziert worden. } \\
\text { Keine empirische } \\
\text { Bestätigung. }\end{array}$ \\
\hline $\begin{array}{l}\text { Integratives } \\
\text { Modell, } 2001\end{array}$ & \begin{tabular}{|l|} 
Begründung durch \\
anthropologische und \\
soziokulturelle Ge- \\
gebenheiten so wie \\
durch eine Erfah- \\
rungstheorie. Lösung \\
von objektiven Auf- \\
gaben durch subjek- \\
tive Auseinanderset- \\
zung.
\end{tabular} & $\begin{array}{l}\text { Zweistufiges Vorha- } \\
\text { ben: } \\
\text { Didaktische Redukti- } \\
\text { on. } \\
\text { Spielerfahrungen in } \\
\text { vereinfachten Spielsi- } \\
\text { tuationen. }\end{array}$ & $\begin{array}{l}\text { Basisspiele mit ver- } \\
\text { einfachter Ballkon- } \\
\text { trolle. } \\
\\
\text { Vielfältige Spiel- und } \\
\text { Übungsformen in drei } \\
\text { Stufen geordnet. }\end{array}$ & $\begin{array}{l}\text { Ein Lehrbuch über } \\
\text { Basketball veröffent- } \\
\text { lichtet und Berichte } \\
\text { über ihre Umsetzung } \\
\text { bei anderen Sport- } \\
\text { spielen. } \\
\text { Keine empirische } \\
\text { Überprüfung. }\end{array}$ \\
\hline
\end{tabular}




\begin{tabular}{|c|c|c|c|c|}
\hline & Begründung & Struktur & Aufgabestellung & Bemerkungen \\
\hline $\begin{array}{l}\text { Fußballent- } \\
\text { wicklungs- } \\
\text { modell, } 2004\end{array}$ & $\begin{array}{l}\text { Gemeinsames Trai- } \\
\text { ning der technischen, } \\
\text { taktischen, kognitiven } \\
\text { und konditionellen } \\
\text { Dimensionen durch } \\
\text { das Spiel. Anpas- } \\
\text { sung des Wettbe- } \\
\text { werbs an die Mög- } \\
\text { lichkeiten der Kinder. }\end{array}$ & $\begin{array}{l}\text { Hierarchische Orga- } \\
\text { nisation in verschie- } \\
\text { denen Entwicklungs- } \\
\text { stufen der gesamten } \\
\text { Ausbildung des Fuß- } \\
\text { ballspielers. }\end{array}$ & $\begin{array}{l}\text { Komplexe Spielsitua- } \\
\text { tionen, wo schnelle } \\
\text { Entscheidungen not- } \\
\text { wendig sind und } \\
\text { gezielte Fragestel- } \\
\text { lungen für die Förde- } \\
\text { rung von selbständi- } \\
\text { gen Lösungen. }\end{array}$ & $\begin{array}{l}\text { Lehrbücher und zahl- } \\
\text { reiche Vorträge in } \\
\text { vielen Ländern. Be- } \\
\text { richte über erfolgrei- } \\
\text { che Umsetzung bei } \\
\text { Fußballsport- } \\
\text { vereinen. } \\
\text { Keine empirische } \\
\text { Überprüfung. }\end{array}$ \\
\hline $\begin{array}{l}\text { Modell der } \\
\text { inzidentellen } \\
\text { Inkubation, } \\
1999 \\
\text { (Heidelberger } \\
\text { Ballschule) }\end{array}$ & $\begin{array}{l}\text { Das Sammeln von } \\
\text { Spielerfahrungen } \\
\text { wirkt als antizipative } \\
\text { Verhaltens- } \\
\text { steuerung. Ein fertig- } \\
\text { keitsorientier-Ansatz } \\
\text { als Alternative zum } \\
\text { koordinativen Fähig- } \\
\text { keiten-Ansatz. }\end{array}$ & $\begin{array}{l}\text { ABC für Anfänger, } \\
\text { das aus drei metho- } \\
\text { dischen Vorhaben } \\
\text { besteht: } \\
\text { A. Situationsorien- } \\
\text { tiert. } \\
\text { B. Fähigkeits- } \\
\text { orientiert. } \\
\text { C. Fertigkeits- } \\
\text { orientiert. }\end{array}$ & $\begin{array}{l}\text { Sieben allgemeine } \\
\text { offensive und defen- } \\
\text { sive Bausteine für die } \\
\text { allgemeine Spielfä- } \\
\text { higkeit. } \\
\text { Technikbausteine als } \\
\text { Übungen. }\end{array}$ & $\begin{array}{l}\text { Vernünftige Lösung } \\
\text { des Problems der } \\
\text { Vielfältigkeit der Kon- } \\
\text { zepte über } \\
\text { koordinative Fähig- } \\
\text { keiten. Zahlreiche } \\
\text { Lehrbücher und theo- } \\
\text { retische Bearbeitun- } \\
\text { gen. Einige empiri- } \\
\text { sche Bestätigungen. } \\
\text { Fehlende Hinweise } \\
\text { für den Übergang } \\
\text { zum spezifischen } \\
\text { Spiel. }\end{array}$ \\
\hline \multicolumn{5}{|c|}{ Spielvermittlungsmodelle rund um TGfU überwiegend im englischen Sprachraum } \\
\hline & Begründung & Struktur & Aufgabestellung & Bemerkungen \\
\hline TGfU, 1982 & $\begin{array}{l}\text { Förderung des Ver- } \\
\text { ständnisses der tak- } \\
\text { tischen Komplexität } \\
\text { des Spiels und da- } \\
\text { durch die Entschei- } \\
\text { dungsfähigkeit und } \\
\text { Transfer zu anderen } \\
\text { Sportspielen. }\end{array}$ & $\begin{array}{l}\text { Umsetzung einer } \\
\text { umgekehrten Rei- } \\
\text { henfolge des Direk- } \\
\text { ten Modells. } \\
\text { Spiele mit veränder- } \\
\text { ten sekundären Re- } \\
\text { geln. } \\
\text { Beobachtung von für } \\
\text { das Modell entwickel- } \\
\text { ten pädagogischen } \\
\text { Prinzipien. }\end{array}$ & $\begin{array}{l}\text { Reihenfolge der fol- } \\
\text { genden Einzelaspek- } \\
\text { te: } \\
\text { - Spiel. } \\
\text { - Spielaufwertung. } \\
\text { - Taktische Bewusst } \\
\text { sein. } \\
\text { - Treffen von Ent } \\
\text { scheidungen. } \\
\text { - Leistung. }\end{array}$ & $\begin{array}{l}\text { Am meisten empi- } \\
\text { risch überprüftes } \\
\text { Modell. Zahlreiche } \\
\text { Studien zum Ver- } \\
\text { gleich des TGfU mit } \\
\text { dem Direkten Modell. } \\
\text { Keine Bestätigung } \\
\text { der Überlegenheit } \\
\text { des TGfU. }\end{array}$ \\
\hline
\end{tabular}




\begin{tabular}{|c|c|c|c|c|}
\hline & Begründung & Struktur & Aufgabestellung & Bemerkungen \\
\hline $\begin{array}{l}\text { Tactical Ga- } \\
\text { mes, } 1995\end{array}$ & $\begin{array}{l}\text { Modifizierte Version } \\
\text { des TGfU. Bestimmte } \\
\text { Inhalte vorgeschla- } \\
\text { gen für alltägliche } \\
\text { Anwendung, allmäh- } \\
\text { lich Steigerung der } \\
\text { Komplexität. Motiva- } \\
\text { tion und Spielzen- } \\
\text { trierter Unterricht. }\end{array}$ & $\begin{array}{l}\text { Spiele auf kleinen } \\
\text { Feld lenken die Auf- } \\
\text { merksamkeit auf ein } \\
\text { taktisches Problem. } \\
\text { Durch Fragestellun- } \\
\text { gen wird das kriti- } \\
\text { sche Denken ange- } \\
\text { regt. } \\
\text { Isoliertes Techniktrai- } \\
\text { ning. }\end{array}$ & $\begin{array}{l}\text { Spiel mit veränderten } \\
\text { Regeln. } \\
\text { Fragen und Antwor- } \\
\text { ten. } \\
\text { Techniktraining. }\end{array}$ & $\begin{array}{l}\text { Häufig wissenschaft- } \\
\text { lich geprüft und mit } \\
\text { ähnlichen Ergebnis- } \\
\text { sen wie das TGfU. } \\
\text { Schwer zu imple- } \\
\text { mentieren wegen } \\
\text { großer Anforderun- } \\
\text { gen an die Kenntnis- } \\
\text { se der Lehrer. Die } \\
\text { Technik wird traditio- } \\
\text { nell unterrichtet. }\end{array}$ \\
\hline $\begin{array}{l}\text { Game Sense, } \\
1997\end{array}$ & $\begin{array}{l}\text { Anpassung des TGfU } \\
\text { an den Leistungs- } \\
\text { sport. Kognitive An- } \\
\text { forderungen an die } \\
\text { Spieler gestellt, da- } \\
\text { her Förderung des } \\
\text { Verständnisses. }\end{array}$ & $\begin{array}{l}\text { Modifizierte Spiele. } \\
\text { Fragestellungen. }\end{array}$ & $\begin{array}{l}\text { Gezielte Modifizie- } \\
\text { rung der Sekundären } \\
\text { Regeln. } \\
\text { Fragen und Antwor- } \\
\text { ten. } \\
\text { Nachdenken. } \\
\text { Umsetzung der ge- } \\
\text { wonnenen Kenntnis- } \\
\text { se. } \\
\text { Steigerung des } \\
\text { Schwierigkeitsgrads. }\end{array}$ & $\begin{array}{l}\text { Breite Umsetzung im } \\
\text { Rugby. } \\
\text { Profitiert von empiri- } \\
\text { schen Studien rund } \\
\text { um TGfU. } \\
\text { Erfolgreiche Umset- } \\
\text { zung auch im Schul- } \\
\text { sport, dokumentiert } \\
\text { durch empirische } \\
\text { Studien. }\end{array}$ \\
\hline $\begin{array}{l}\text { Sport Educa- } \\
\text { tion, } 1994\end{array}$ & $\begin{array}{l}\text { Langfristiges Enga- } \\
\text { gement in nur einer } \\
\text { Spielsportart in der } \\
\text { Schule, wirksamer } \\
\text { als mehrere Spiele in } \\
\text { kurzer Zeit. } \\
\text { Durch kontextuelles } \\
\text { Sporttreiben wird das } \\
\text { Spiel kompetenter } \\
\text { erlernt als durch iso- } \\
\text { lierte Übungen. } \\
\text { Vielfältige Rollen } \\
\text { rund um das Spiel } \\
\text { als Schiedsrichter, } \\
\text { Organisator und } \\
\text { Trainer besorgen } \\
\text { erzieherische Erleb- } \\
\text { nisse. }\end{array}$ & $\begin{array}{l}\text { Vorbereitung und } \\
\text { Teilnahme an Wett- } \\
\text { kämpfen innerhalb } \\
\text { der Schule während } \\
\text { einer relativ langen } \\
\text { Zeitspanne. } \\
\text { Beteiligung in ver- } \\
\text { schiedenen Rollen: } \\
\text { als Spieler, Trainer, } \\
\text { Schiedsrichter und } \\
\text { Organisator. } \\
\text { Schlussveranstaltung } \\
\text { und Feierlichkeiten. }\end{array}$ & $\begin{array}{l}\text { Aufteilung der Spieler } \\
\text { in Mannschaften, die } \\
\text { die ganze Saison } \\
\text { zusammenbleiben. } \\
\text { Gemeinsame Vorbe- } \\
\text { reitung des Wett- } \\
\text { kampfs und Ab- } \\
\text { schlussturnier. } \\
\text { Ständige Unterstüt- } \\
\text { zung durch den Trai- } \\
\text { ner. }\end{array}$ & $\begin{array}{l}\text { Empirische Studien } \\
\text { bestätigen die Wirk- } \\
\text { samkeit des Modells } \\
\text { in der sozialen und } \\
\text { emotionalen Kompe- } \\
\text { tenz. Die Spielkom- } \\
\text { petenz verbessert } \\
\text { sich in geringem } \\
\text { Maße. } \\
\text { Kein besseres Spiel- } \\
\text { verständnis als durch } \\
\text { das Direkte Modell. }\end{array}$ \\
\hline
\end{tabular}




\begin{tabular}{|c|c|c|c|c|}
\hline & Begründung & Struktur & Aufgabestellung & Bemerkungen \\
\hline $\begin{array}{l}\text { Invasion } \\
\text { Game Com- } \\
\text { petences } \\
\text { Model } \\
\text { (IGCM), } 2002\end{array}$ & $\begin{array}{l}\text { Verbindung der Mo- } \\
\text { delle TGfU und Sport } \\
\text { Education, um beider } \\
\text { Vorteile auszunut- } \\
\text { zen. } \\
\text { Schulung von Spiel- } \\
\text { kompetenz und Or- } \\
\text { ganisations- und } \\
\text { Führungskompetenz. }\end{array}$ & $\begin{array}{l}\text { Entwicklung der } \\
\text { Spielkompetenz } \\
\text { durch modifizierte } \\
\text { Spiele und Fragestel- } \\
\text { lungen in Anlehnung } \\
\text { an das TGfU. } \\
\text { Entwicklung von } \\
\text { Organisationskompe- } \\
\text { tenz durch das Spie- } \\
\text { len von verschiede- } \\
\text { nen Rolle als } \\
\text { Schiedsrichter, } \\
\text { Mannschaftskapitän, } \\
\text { Organisator usw. }\end{array}$ & $\begin{array}{l}\text { Grundspielaufgaben: } \\
\text { Spiele auf kleinen } \\
\text { Feld als problemati- } \\
\text { sche Situationen dar- } \\
\text { gestellt. } \\
\text { Partielle Spielformen: } \\
\text { Übung von besonde- } \\
\text { ren Spielsituationen. } \\
\text { Aufgabenbasierte } \\
\text { Spiele: Betonung der } \\
\text { technischen Fertigkei- } \\
\text { ten im Spielkontext. }\end{array}$ & $\begin{array}{l}\text { Empirische Studien } \\
\text { bestätigen schnelle- } \\
\text { re Verbesserung der } \\
\text { Entscheidungs- } \\
\text { fähigkeiten im Ver- } \\
\text { gleich zum Direkten } \\
\text { Modell. } \\
\text { Effizienter, aber nicht } \\
\text { wirksamer als das } \\
\text { Direkte Modell für } \\
\text { das Erlernen der } \\
\text { Spielkompetenz. }\end{array}$ \\
\hline $\begin{array}{l}\text { Play Practice, } \\
2001\end{array}$ & $\begin{array}{l}\text { Die traditionellen } \\
\text { Modelle werden stark } \\
\text { kritisiert, sie ermögli- } \\
\text { chen keinen Lern- } \\
\text { transfer, die Trainern } \\
\text { vermitteln Stereoty- } \\
\text { pen, Anfänger haben } \\
\text { wenig Gelegenheit, } \\
\text { am Spiel teilzuneh- } \\
\text { men, was Demotiva- } \\
\text { tion bewirkt. }\end{array}$ & $\begin{array}{l}\text { Die meisten Lernauf- } \\
\text { gaben werden als } \\
\text { Spiel verpackt. }\end{array}$ & $\begin{array}{l}\text { Drei Stufe: } \\
\text { - Focusing Play. } \\
\text { - Shaping Play. } \\
\text { - Enhancing Play. }\end{array}$ & $\begin{array}{l}\text { Wirksamer für Fort- } \\
\text { geschrittene als für } \\
\text { Anfänger. } \\
\text { Einige empirische } \\
\text { Studien bei Studen- } \\
\text { ten bestätigen ihre } \\
\text { Wirksamkeit }\end{array}$ \\
\hline \multicolumn{5}{|c|}{ Spielvermittlungsmodelle die aus technologischen Umsetzungen entstanden sind } \\
\hline & Begründung & Struktur & Aufgabestellung & Bemerkungen \\
\hline $\begin{array}{l}\text { Decision } \\
\text { Training Mo- } \\
\text { dell, } 2007\end{array}$ & $\begin{array}{l}\text { Die Entwicklung der } \\
\text { kognitiven Fähigkei- } \\
\text { ten in einem spezifi- } \\
\text { schen Spielkontext } \\
\text { verbessert die Ent- } \\
\text { scheidungsfähigkeit. }\end{array}$ & $\begin{array}{l}\text { Drei Schritte: } \\
\text { Die Entscheidung } \\
\text { identifizieren. } \\
\text { Die geeignete Lern- } \\
\text { aufgabe entwerfen. } \\
\text { Eines von sieben } \\
\text { Werkzeugen für das } \\
\text { Training der Ent- } \\
\text { scheidungsfähigkeit } \\
\text { wählen und umset- } \\
\text { zen. }\end{array}$ & $\begin{array}{l}\text { Identifizierung der } \\
\text { zugrunde liegenden } \\
\text { kognitiven Fähigkeit. } \\
\text { Entwurf einer Lern- } \\
\text { aufgabe. } \\
\text { Umsetzung von Vor- } \\
\text { haben für das Trai- } \\
\text { ning der Entschei- } \\
\text { dungsfähigkeit. }\end{array}$ & $\begin{array}{l}\text { Zahlreiche Anwen- } \\
\text { dungen bei verschie- } \\
\text { denen Sportarten mit } \\
\text { Hilfe von Technolo- } \\
\text { gien. } \\
\text { Bestätigung ihrer } \\
\text { Wirksamkeit für die } \\
\text { Perfektionierung der } \\
\text { Technik bei Hochleis- } \\
\text { tungssportlern. }\end{array}$ \\
\hline
\end{tabular}




\begin{tabular}{|c|c|c|c|c|}
\hline & Begründung & Struktur & Aufgabestellung & Bemerkungen \\
\hline $\begin{array}{l}\text { Situation } \\
\text { Model of An- } \\
\text { ticipated Re- } \\
\text { sponse Con- } \\
\text { sequences of } \\
\text { Tactical Train- } \\
\text { ing (SMART), } \\
2000\end{array}$ & $\begin{array}{l}\text { Die Bildung von } \\
\text { „wenn-dann“-Regeln } \\
\text { erleichtert das Er- } \\
\text { kennen der Bezie- } \\
\text { hungen, die inner- } \\
\text { halb der Spielsituati- } \\
\text { on entstehen und } \\
\text { daher zur Entwick- } \\
\text { lung der Entschei- } \\
\text { dungsfähigkeit bei- } \\
\text { tragen. }\end{array}$ & $\begin{array}{l}\text { In Spielsituationen } \\
\text { gestalten die Rück- } \\
\text { meldungen nach den } \\
\text { Spielaktionen die } \\
\text { „wenn-dann“-Regeln } \\
\text { und lassen die Wir- } \\
\text { kung der eigenen } \\
\text { Entscheidungen er- } \\
\text { kennen. }\end{array}$ & $\begin{array}{l}\text { Die beide Phasen } \\
\text { eines Prozesses, die } \\
\text { die Handlung auslö- } \\
\text { sen: Bottom-up und } \\
\text { Top-down, werden } \\
\text { systematisiert. Die } \\
\text { „wenn-dann“-Regeln } \\
\text { werden als eine „tak- } \\
\text { tische Grammatik“ } \\
\text { strukturiert und am } \\
\text { Computer modulari- } \\
\text { siert. }\end{array}$ & $\begin{array}{l}\text { Als Spielvermitt- } \\
\text { lungsmodell vorge- } \\
\text { stellt, ist aber ein } \\
\text { theoretisches Modell, } \\
\text { das die Verbesserung } \\
\text { der Entscheidungs- } \\
\text { fähigkeit erklärend } \\
\text { vornimmt. Mehrere } \\
\text { empirische Studien } \\
\text { im Rahmen der Pro- } \\
\text { motionsarbeit des } \\
\text { Autors. }\end{array}$ \\
\hline
\end{tabular}

\subsubsection{Stand der Forschung über die Wirksamkeit der Spielvermittlungsmodelle}

Zu Beginn der Erforschung der Wirksamkeit vom,Game and Player Centered Approach“ (GPCA) konzentrierte sich die Forschung auf einen Ansatz, der den Vergleich der Modelle und die Antwort "welche Methode die Besten ist" zum Ziel hatte. Die meisten Forschungsarbeiten wurden dabei mit Bezug zum Modell Teaching Games for Understanding (TGfU) durchgeführt. Es ist dadurch das bestuntersuchte Modell, während weniger Forschungsergebnisse in Bezug auf die Modelle Tactical Games, Game Sense, Inzidentellen Inkubation (Heidelberger Ballschule), Genetisches Lernen und Invasion Games Competence vorliegen. Die anderen Modelle wurden von der Forschung nicht berücksichtigt oder können kaum als "Spielreihe“ angesehen werden. Die Forschungsarbeiten an didaktischen Modellen des Spiellernens berücksichtigen drei Hauptbereiche des Lernens. Dies sind die technischen Fertigkeiten, das deklarative Wissen und die Spielleistung. In der vorliegenden Arbeit werden diese Hauptbereiche mit den für erfolgreiches Spielen notwendigen Kompetenzen verbunden und als technische Kompetenz, kognitive Kompetenzbzw. Wissenskompetenz und prozedurale Spielkompetenz bezeichnet. Eine Auswahl der bedeutendsten Studien in diesen drei Bereichen wird im Folgenden diskutiert.

\section{Technische Kompetenz}

Eine der ersten Studien der Wirksamkeit des TGfU als Lehr- und Lernansatz wurde von LAWTON (1989) im Badminton durchgeführt. Die TGfU-Gruppe zeigte ein größeres Maß an Verbesserung der technischen Fertigkeiten als die ausschließlich technikorientiert übende Gruppe. Der Unterschied wurde allerdings nicht statistisch signifikant. McPHERSON und FRENCH (1991) haben festgestellt, dass sich der Vorhand- und Rückhandschlag im Tennis verbesserte, wenn die Gruppe mit einem technik-orientierten Ansatz lernte, während sich im Volleyball diejenige Gruppe deutlicher verbesserte, die mit einem TGfU-Ansatz lernte. In einem 10-Stunden-Tennisunterricht stellte TURNER (2003) eine Tendenz zu einer besseren Kontrolle, Entscheidung und Ausführung für die Gruppe mit der TGfU-Methode fest. TURNER und MARTINEK (1992) fanden keine sig- 
nifikanten Unterschiede im Lernen von technischen Fertigkeiten, wenn die beiden Ansätze verwendet wurden um Feldhockey zu lehren. Ebenfalls ohne erhebliche Unterschiede zwischen den Gruppen lernten die Probanden acht Stunden im Fußball (MITCHELL, OSLIN \& GRIFFIN, 1995) und in einem Programm von neun Stunden im Volleyball (GRIFFIN, OSLIN \& MITCHELL, 1995).

Ähnliche Ergebnisse wurden für drei Gruppen im Badminton gefunden, die mittels TGfU, technik-orientiertem Training und einer Kombination der beiden Methoden ausgebildet wurden (FRENCH, WERNER, RINK, TAYLOR, \& HUSSEY, 1996; FRENCH, NEVETT, SPURGEON, GRAHAM, RINK \& MCPHERSON, 1996). ALISON und THORPE (1997) stellten fest, dass die technischen Fertigkeiten der beiden Gruppen, die mit einem TGfU-Ansatz lernten, ebenso gut wie die Ergebnisse einer Gruppe mit einem technik-orientierten Ansatz waren, Übungszeit war hier eine Intervention von 12 Wochen. TURNER und MARTINEK (1999) stellten fest, dass die Technik-Gruppe deutlich schneller in der Ausführung technischer Bewegungen im Hockey war als die Kontrollgruppe. Andere Intervention im Badminton (BLOMQVIST, LUHTANEN \& LAASKO, 2001) stelltet fest, dass beide Treatmentgruppen (eine technik-orientierte und eine TGfU-Gruppe mit Videounterstützung) besser waren als die Kontrollgruppe, sowohl die TGfU-Gruppe als auch die technik-orientierte Gruppe zeigten im Laufe der Zeit eine signifikante Verbesserung. Eine Studie von HARRISON, BLAKEMORE, RICHARDS, OLIVER, WILKINSON, und FELLINGHAM (1998) zeigte, dass sowohl die TGfU- als auch die technik-orientierten Gruppen signifikante Verbesserungen in Bezug auf die Ergebnisse im Prätest hatten, die technischen Fertigkeiten des Volleyballs wurden dabei mit Hilfe der Tests der American Alliance for Health Physical Education, Recreation and Dance (AAHPERD) untersucht. ZHANG (2008) stellte ein besseres Lernen der technischen Fertigkeiten des Tischtennis fest durch ein Lernprogramm wo das Play Practice Modell umgesetzt wurde als durch ein Lernprogramm wo ein traditionelles Direktes Modell umgesetzt wurde.

In der vorliegenden Vielfalt untermauern die Studien die Auffassung, dass das „Lernen durch Spielen" die technischen Fertigkeiten nicht verschlechtere. Dies könnte zumindest den Trainern, die behaupten, dass Spieler ihre technischen Fertigkeiten verlören, wenn sie zu viel spielten, ihre Vorbehalte nehmen. Diese Feststellung allein stellt aber keine überzeugende Schlussfolgerung für die Fähigkeit des TGfU-Ansatzes dar, die Technikleistung gezielt zu erhöhen. Allerdings wurden die verwendeten Tests allesamt aus dem Spielkontext herausgelöst durchgeführt, also unter statischen, objektivierten Testbedingungen. Dies kann zur Folge haben, dass die Probanden zwar eine hohe Genauigkeit bei der Ausführung der Kriteriumsaufgaben zeigen, der Einsatz der gelernten Fertigkeiten in einer Spielsituation unter Zeit- und Gegenspielerdruck dabei jedoch unberücksichtigt bleibt. Aus diesem Grund ist es interessant, dass die Forschungsarbeiten von FENOGLIO (2003) mit U9-Fußballspielern der Manchester United Akademie gezeigt haben, dass "Small Sided Football Games“ (SSFG) die Häufigkeit des Einsatzes der technischen Fertigkeiten im Spiel erhöhen. Die Studie ergab, dass SSFG (4 vs. 4) zusätzliche 585 Pässe, 481 Torschüsse, 301 Tore, 525 Zweikämpfe und 436 Dribbelsituationen einbrachten, wenn sie im Vergleich zu 8-vs.-8-Spielen im Training eingesetzt wurden. Eine weitere Studie von OWEN, TWIST und FORD (2004) mit älteren Spielern (mittleres Alter: 17 Jahre) ergab, dass sich die Häufigkeit der technischen Fertigkeiten erhöht, wenn die Spielfeldgröße reduziert wird. Ebenso konnte der gegenteilige Effekt der Frequenzverringerung gezeigt werden, wenn das Spielfeld vergrößert wurde. Dies wird darauf zurückgeführt, dass kleinere Spielflächen den Spielern mehr Möglichkeiten 
geben, Entscheidungen zu treffen und motorische Ausführungen umzusetzen, sie erlangen daher mehr internes Feedback ihren Entscheidungen, egal ob sie gut oder schlecht waren. Die Spieler lernen dabei, ihre guten von schlechten technischen Ausführungen zu unterscheiden, sie lernen also implizit im Kontext des Spiels.

Obwohl diese Studien nicht dokumentieren, ob die technischen Ausführungen der Pässe, des Dribbelns usw. geeignete oder ungeeignete Kriteriumsaufgaben waren, unterstützen sie die Vorstellung, dass die Spieler im Spiel eine große Anzahl von Möglichkeiten haben, technische Fertigkeiten auszuüben und zu lernen. Der Einsatz von Kleinen Spielen und die Unterstützung durch rechtzeitige und angemessene Rückmeldung durch einen kompetenten und gut ausgebildeten Trainer kann die Entwicklung der Spielfähigkeit wie gleichermaßen die der technischen Fertigkeiten der Spieler fördern (vgl. HARRISON, BLAKEMORE, RICHARDS, OLIVER, WILKINSON \& FELLINGHAM, 1998). Zur konkreten Festigung dieser Vorstellung bedarf es aber weiterer gezielter empirischer Forschung.

\section{Kognitive Kompetenz und Wissenskompetenz}

Die wichtigste Quelle zur Beurteilung der Wissensentwicklung der Teilnehmer in Studien der TGfU-Forschung ist die Verwendung von schriftlichen Wissenstests (ALISON \& THORPE, 1997; FRENCH, WERNER, RINK, TAYLOR \& HUSSEY, 1996; FRENCH, NEVETT, SPURGEON, GRAHAM, RINK \& MCPHERSON, 1996; JONES \& FARROW, 1999; TURNER und MARTINEK, 1992, 1999). Das deklarative Wissen wird mit Hilfe dieser Tests ausgewertet. TURNER, ALLISON und PISSANOS, (2001, S. 287) haben sich dafür ausgesprochen, deklaratives Wissen als eine Vorstufe zum prozeduralen Wissen zu betrachten. Als Wissen über Bezeichnungen und sachliche Informationen wie z. B. Aktionsregeln ist es damit Grundlage für die Anwendung der bekannten Aktionsregeln im Rahmen und "constraints“ des Spiels.

Studien mit der Anwendung der genannten schriftlichen Prüfungen ergeben uneindeutige Befunde. Einige Autoren beobachten eine Veränderung des Wissens, andere können diese nicht nachweisen. Tatsächlich fanden HARRISON et al. (1998) keine signifikanten Unterschiede zwischen Prä- und Postleistungen in schriftlichen Wissenstests über Regeln, Strategien und Techniken des Volleyballs. Auch LAWTON (1989) fand keine signifikanten Unterschiede in der Entwicklung des deklarativen Wissens im Laufe der Zeit. Aus der geringen Verbesserung der Gruppe, die über sechs Sitzungen Badminton mit einem technik-orientierten Ansatz lernte, schloss er allerdings, dass ein technik-orientierter Ansatz wenig zur Entwicklung der Intelligenzleistung beiträgt. Tatsächlich machte die TGfU-Gruppe im Vergleich größere Fortschritte im Verständnis von Taktiken und Strategien, als dies der Technik-Gruppe gelang.

TURNER und MARTINEK (1992) fanden keine Beziehung zwischen Wissen und der Fähigkeit des Entscheidungstreffens und fanden auch keine signifikanten Unterschiede in der Entwicklung des deklarativen Wissens im Laufe der Zeit. Allerdings repliziert TURNER (1996a) seine Studie im Hockey noch einmal selbst mit einer längeren Intervention und stellt fest, dass die TGfU-Gruppe sich nun deutlich in Bezug auf ihr deklaratives Wissen verbessert.

RINK, FRENCH, und TJEERDSMA (1996, S. 410) haben nach der überprüfung mehrerer empirischen Studien festgestellt, dass Spieler, die mittels eines TGfU-Ansatzes ge- 
lernt haben, bessere Prüfungsleistungen über taktisches Wissen abliefern als die Spieler, die mittels eines technik-orientierten Ansatzes gelernt haben. Auch ALISON und THORPE (1997) fanden größere Effekte im Wissen und Verständnis bei TGfU-Gruppen im Hockey und Basketball. Studien von MITCHELL, OSLIN und GRIFFIN (1995) im Fußball und GRIFFIN, OSLIN und MITCHELL (1995) im Volleyball zeigen ebenfalls höhere Leistungen in deklarativen Wissenstests für die TGfU-Gruppen im Vergleich zu den technik-orientierten Gruppen. Auch diese Studien unterstützen damit die Annahme, dass die kontextualen Spiele besser auf die Entwicklung des deklarativen Wissens einwirken könnten, als dies den technik-orientierten Ansätzen gelingt. Auch TURNER und MARTINEK (1999) stellten fest, dass die TGfU-Gruppen in Bezug auf ihr deklaratives und auch prozedurales Wissen (als Entscheidungsfähigkeit verstanden) deutlich höher bewertet werden können als die Kontrollgruppe. Eine weitere Studie im Tennis (TURNER, 2003) ergab höhere Wissenswerte über Fertigkeiten für die TGfU-Gruppe, jedoch kein besseres Verständnis für Regeln und Taktiken gegenüber einer technik-orientiert trainierenden Gruppe.

Wegen Zweifeln an der Übertragbarkeit der Ergebnisse der schriftlichen Leistungen auf praktische Leistungen verzichten BLOMQVIST, LUHTANEN, und LAASKO (2001) nicht nur auf einen schriftlichen Wissenstest, sondern prüfen das Spielverständnis beim Badminton auch mit der Darstellung von „problematischen Situationen", die oft im Spiel auftreten. Ihre Ergebnisse zeigen, dass die Treatmentgruppen bessere Leistungen bei den Tests für Spielverständnis liefern als die Kontrollgruppe, aber nur die TGfU-Gruppe zeigte eine signifikante Verbesserung in diesem Test im Laufe der Zeit.

Die weitgehende Uneinheitlichkeit der Auswirkungen auf die Wissensentwicklung bei Einsatz des TGfU-Ansatzes könnte wegen nicht geeigneter Messverfahren und Studiendesigns entstehen. Daher sind alternative Auswertungsmethoden entwickelt worden, z. B. die „Verbal Protocol Analysis“ (McPHERSON \& THOMAS, 1989; HARVEY, 2006), Um zur Förderung der empirischen Basis von didaktischen Modellen beizutragen, müssen ferner geeignete methodische Designs und Instrumente zur Auswertung der kognitiven Kompetenzen entwickelt werden, die sowohl das deklarative als auch die Entscheidungsfähigkeit berücksichtigen (McMORRIS, 1998, S. 73).

TALLIR, MUSCH, LENOIR, und VALCKE (2004) analysierten in einer Studie die Unterschiede zwischen den kognitiven Effekten eines traditionellen Modells und dem Invasion Games Competence Modell (IGCM). Im traditionellen Modell übten die Kinder während 12 Einheiten technische Fertigkeiten des Basketballs und spielten nur am Ende jeder Trainingseinheit. Im IGCM standen Lernaufgaben in kontextspezifischen Spielsituationen des Basketballs im Mittelpunkt, deren Fokus das Spielverständnis war. Die Kinder wurden aufgefordert an die richtige Ausführung der technischen Fertigkeiten und an das Ziel des Spiels zu denken. Tests wurden ausgeführt, um Effekte beider Treatments auf das Langzeitgedächtnis und die Entscheidungsfähigkeit festzustellen. Die Ergebnisse zeigen keine signifikanten Unterschiede zwischen den Auswirkungen der zwei Modelle auf die kognitiven Fähigkeiten. Dennoch wurde eine Wirkung des IGCM auf die Effizienz bei der Entscheidungsfähigkeit gefunden. Die Gruppe, die unter den Bedingungen des IGCM lernte, zeigte eine schnellere aber nicht größere Entwicklung als die Gruppe, die unter den Bedingungen des traditionellen Modells lernte. Die Autoren schlussfolgern daraus, dass das IGCM durchaus als effizienter, nicht jedoch als wirksamer als das traditionelle Vermittlungsmodell betrachtet werden sollte. 


\section{Prozedurale Spielkompetenz}

Die meisten Studien in diesem Bereich setzen ein Spielprotokoll ein (McPHERSON \& FRENCH, 1991; McPHERSON \& THOMAS 1989). Bei diesem Protokoll werden bestimmte Merkmale für das Entscheidungstreffen und die technische Ausführung von Bewegungen während des Spiels kodiert. Sind sie im Spielsinn erfolgreich, werden sie mit 1 kodiert. Mit 0 werden sie kodiert, wenn Spieler im Rahmen des Spiels nicht die richtigen Entscheidungen treffen oder nur ungenügende Bewegungsausführung erreichen. Mit dieser Auswertungsmethode haben McPHERSON und FRENCH (1991) herausgefunden, dass eine Gruppe mit einem technik-orientierten Ansatz im Tennis die Ausführung der technischen Bewegungen im Training und Spiel verbesserte. Keine Fortschritte zeigten sich, wenn Strategien gelernt wurden. Die an einem TGfU-Ansatz beteiligten Spieler verbesserten ihre technischen Fertigkeiten erst nach der Einführung der direkten technischen Übungen. Ebenfalls mithilfe eines Spielprotokolls stellte TURNER (2003) fest, dass eine TGfU-Gruppe signifikant besser in der Schlagauswahl und im Entscheidungstreffen im Tennis wurde.

Durch den Einsatz des „Game-Performance-Assessment-Instrument (GPAI)“ im Fußball stellten MITCHELL, OSLIN und GRIFFIN (1995) fest, dass der TGfU-Ansatz wirksam bei der Verbesserung der Spielleistung eingesetzt werden kann, insbesondere in Bezug auf das Spiel mit Ballbesitz und auf das Entscheidungstreffen. Im Volleyball haben GRIFFIN, OSLIN und MITCHELL (1995) ebenfalls herausgefunden, dass die Spielleistung verbessert wurde. Hier zeigten sich die Fortschritte vor allem in den Bereichen der Positionierung auf dem Spielfeld und im Entscheidungstreffen. Auch in einer Studie im Hockey stellte TURNER (1996b) mit dem oben genannten Auswertungsverfahren fest, dass die TGfU-Gruppe deutlich den Entscheidungsprozess im Spiel verbessern konnte.

In einer Studie haben FRENCH, WERNER, TAYLOR, HUSSEY und JONES, (1996) keine Unterschiede zwischen den Gruppen in Bezug auf das Spiel gefunden. In ihrer Studie stellten FRENCH, NEVETT, SPURGEON, GRAHAM, RINK und McPHERSON (1996) fest, dass die TGfU- und technik-orientierten Gruppen besser waren als die Kombinations- und Kontrollgruppe in der Zwischenauswertung. Die Leistung der Kombinationsgruppe überstieg aber die Leistung der anderen Gruppen ab der sechsten und letzten Woche der Erhebung. Im Bereich Hockey haben TURNER und MARTINEK (1999) festgestellt, dass die TGfU-Gruppe eine deutlich bessere Passkontrolle hatte und auch in Weitergaben und im Entscheidungstreffen überlegen war. ALISON und THORPE (1997) zeigten, dass in den TGfU-Gruppen die Spieler ein besseres Verständnis der Spielkonzepte im Hockey und Basketball hatten.

BLOMQVIST, LUHTANEN, und LAASKO (2001) setzten eine Videoanalyse ein, um die Spielleistung im Badminton zu bewerten. Sie fanden einen Trend zu höheren Leistungen in den Treatmentgruppen, die mittels des GPCA-Vorgehens lernten. Hingegen fanden HARRISON et al. (2004) im Volleyball zwischen den Ansätzen unter den Bedingungen des 6-vs.-6-Spiels keinen signifikant großen Unterschied.

Die vorliegenden Studien haben gezeigt, dass das Spiel verbessert werden kann, wenn im Training durch den Einsatz taktischer Probleme gelernt wird. Tatsächlich argumentieren RINK, FRENCH und TJEERDSMA (1996, S. 414), dass die technischen Fertigkeiten sich erst dann voll entwickeln würden, wenn sie in das Spiel einbezogen wären. Auch McPHERSON und KERNODLE (2003, S. 160) nehmen an, dass es erforderlich sei, die taktischen Fähigkeiten früher zu entwickeln. Ihrer Ansicht nach benötigt die 
Entwicklung dieser Fähigkeiten ebenso lange wie die der technischen Fertigkeiten. Demnach gebe es die Notwendigkeit für die Entwicklung der kognitiven Aspekte, da sie mehr zum geschickten Spiel auf allen Ebenen beitrügen als die technischen Fertigkeiten für die Kontrolle des Balls.

Trotzt ausreichender und vielfältiger Literatur über die Didaktik der Sportspiele sind nur wenige Lernmethoden oder didaktische Innovationen experimentell überprüft worden. Die meisten Lehrbücher sind auf Anekdoten oder Kommentare über die erfolgreiche Wirkung der Methode begrenzt; oder es werden Modelle in denselben Büchern vorgestellt, normalerweise stellen sie ein bestimmtes Modell vor und berichten über die erfolgreiche Wirkungen seiner praktischen Anwendung aber keine Studie wird durchgeführt um diese Wirkungen wissenschaftlich zu bestätigen. Deswegen sind empirische Studien rund um Spielvermittlungsmodelle ein wertvoller Beitrag zum Wissenschaftlichen Bereich der Sportspiele. Allerdings beweisen die widersprüchlichen Ergebnisse der komparative Studien Designprobleme der Forschung, vielleicht wegen der mangelnden Erfahrung und Tradition der Sportspielforschung. RINK, FRENCH und GRAHAM (1996, S. 490-502) analysieren einige dieser Probleme, die in zukünftigen Studien berücksichtigt werden sollten. Sie begründen das wie folgt.

Die Mehrheit der Studien beachtet nicht den Einfluss der Trainer. Die Lehrfähigkeit der Lehrer/Trainer könnte die Wirkung der didaktischen Modelle, die verglichen werden, beeinflussen. Es ist möglich, dass die starke Wirkung des Trainers der schwächeren Wirkung eines Modells entgegenwirkt.

Auch wird bei den Studien nicht die Motivation berücksichtigt, die einer der wichtigsten Lernfaktoren ist. Möglicherweise würden Langzeitstudien einige Unterschiede in Bezug auf die Motivation aufzeigen.

Technisch hochkomplexe Sportspiele wie Tennis oder Volleyball erfordern höchstwahrscheinlich zunächst eine spezifische Schulung der Technik durch ein Direktes Modell, um den Effekt der indirekten Modelle assimilieren zu können. Deswegen sind bei diesen Spielen die Spielvermittlungsmodelle, abhängig von Alter und Erfahrung, unterschiedlich wirksam.

Weitere Forschungsdefizite und Empfehlungen für zukünftige Studien werden im nächsten Abschnitt diskutiert.

\section{Forschungsdefizite und Perspektiven weiterführender Studien}

Große Teile der bisherigen Forschung im Bereich der Didaktik der Sportspiele wird als vergleichende Forschung bezeichnet (KIRK, 2005, S. 216). Es bestehen viele Versuche, die Überlegenheit einer didaktischen Vorgehensweise oder Methode gegenüber den anderen festzustellen. Jede didaktische Innovation wird mit den bereits vorhandenen verglichen. Dies geschieht mit dem Ziel, ihre bessere Leistung zu bestätigen - immer in der Annahme, dass Neues besser als das Alte ist. Dies hat zu einer Vielfalt methodischer Versuche geführt, um jeweils die „beste Vorgehensweise“ zu finden, letztlich trägt dieser Prozess so zur Entstehung des „Versus Paradigmas“ in der Forschungsmethodologie bei (METZLER, 2005b, S. 190, 195).

Trotz einiger positiver Ergebnisse haben die Studien bisher jedoch keine abschließende Präferenz bezüglich jener didaktischer Modelle aufgezeigt, die auf das Spiel und die 
Spieler zentriert sind. Man kann sie in diesem Bereich also nicht als "geeigneter" bezeichnen als das Direkte Modell für das Lehr-Lernen der Sportspiele, sie können nicht bevorzugt berücksichtigt werden. RINK, FRENCH und TJEERDSMA (1996, S. 417) argumentieren allerdings dafür, dass diese nicht eindeutige Tendenz kein inhaltliches Problem sei, sondern viel mehr wegen der Schwierigkeit des Vergleichs auftrete, also aufgrund der Variabilität der Studien in Bezug auf Spielart, Alter der Probanden, Dauer und Natur der Intervention, analysierte Variablen und die Auswertungsmethode.

Ein großer Teil der Forschungsarbeit wird der Beobachtung des Verhaltens der Lehrer oder Trainer sowie der Beeinflussung ihrer Verhaltensweisen und der daraus entstehenden Lernauswirkungen, d. h. ihrer Effektivität gewidmet. Aspekte wie Instruktionen, Feedback, Verbal- und Nonverbal-Interaktion, Vormachen und organisatorische Aspekte sind auf vielfältige Weise und in diversen Kombinationen geprüft worden, immer mit dem Ziel, die Effektivität jedes Ansatzes für ein „Mehr“ des Lernens nachzuweisen.

METZLER (2005a) schlägt den Begriff „instructional models“ als eine Lösung für die Differenzierung der globalen Maßnahmen vor, die vorgenommen werden, um eine Lernumwelt zusammenzustellen. Er enthält Methoden, didaktische Strategien, Lehrtechniken, usw. von verschiedenen Vorgehensweisen, die verschiedene Lernumwelten konfigurieren. Jedes Modell beinhaltet Designspezifikationen und Hinweise für das Verhalten der Trainer und Sportler und erzeugt entsprechend der Bedingungen und Intentionen seines Designs verschiedene Ergebnisse. Folglich wäre es nicht logisch, Ergebnisse im Bereich "Spielverständnis“ vom Direkten Modell zu erwarten, weil dieses Modell in seiner Anlage andere Lernergebnisse erzielt. Es wäre im Umkehrschluss auch nicht logisch, bessere Ergebnisse im Bezug auf technische Ausführung von einem TGfU-Modell zu erwarten, weil dessen Design eher zur Förderung der kognitiven Fähigkeiten und Fertigkeiten als zur genauen Reproduktion technischer Fertigkeiten angelegt ist. Nach dieser Argumentation macht es schon im Ansatz in diesem Bereich keinen Sinn, einen Modellvergleich anzustellen, der das Ziel hat herauszufinden, welches Modell das Beste ist. Die Ergebnisse werden je nach Design, Inhalt und Auswertungsparametern relativiert, nach METZLER (2005b, S. 190) würde ein solcher Versuch schlicht „Orangen mit Äpfeln“ vergleichen. Die Perspektive sollte eine andere sein. „...instead, we should be asking questions to explore how a model can achieve those outcomes for which it is designed and to determine the necessary contextual factors and processes for optimal implementation of each model". Innerhalb jedes Modells sollten dann Forschungsprozesse durchgeführt werden, um Implementierungsparameter zu definieren und Lernergebnisse mit design- und kontextabhängigen Faktoren zu verbinden(S. 195).

Einige Autoren (DODDS, GRIFFIN, \& PLACEK, 2001; ROVEGNO, NEVETT, \& BABIARZ, 2001; KIRK \& McPHAIL, 2002) kritisieren die in früheren Studien eingesetzten Evaluationsverfahren der kognitiven Kompetenzen. Da das der jeweiligen Kompetenz zugrundeliegende Wissen sehr spezifisch ist, sind Verfahren wie schriftliche Evaluationen, Verbalprotokolle oder Interviews häufig außerhalb des Spielkontextes eingesetzt und ausgeführt worden. Sie testen spezifisch das bewusste Wissen, das implizite Wissen innerhalb des Spielkontextes wird hingegen nicht berücksichtigt.

KIRK (2005, S. 223) lädt die Sportwissenschaftler deshalb zur Entwicklung von Auswertungsmethoden ein, die innerhalb des eigentlichen Spielkontextes bleiben. Diese Anforderung einer validen Messung des Lernens erfüllen Beobachtungsverfahren für einzelne Spieler oder auch für Gruppen oder Mannschaften im Bezug auf spezifische Merkmale des Spiels. ROVEGNO, NEVETT, und BABIARZ, (2001, S. 343) haben beispiel- 
haft vorgeschlagen, das Kriterium „erreichbares Abspiel während des Spiels“ einzusetzen und es gegenüber dem „präzisen Pass in einer isolierten Situation“ zu bevorzugen. METZLER (2005b, S. 189-193) führt fünf Hinweise für die auf didaktische Modelle ausgerichtete Forschung auf, um die Widersprüche der bisher ausgeführten Forschungsarbeiten zu lösen.

1. Didaktische Modelle sind umfassende und komplexe Pläne für die Erreichung vielfältiger und vielschichtiger Ergebnisse. Das Studium der Modelle sollte aus verschiedenen Perspektiven durchgeführt werden, um eine breite Übersicht über ihre Anwendbarkeit zu schaffen.

2. In der Sportforschung ist es nötig, auf das „Versus Paradigma“ zu verzichten. Stattdessen sollte man planende Variationen der Modelle mit dem Ziel testen, jeden Aspekt des Designs zu verfeinern und ein besseres Verständnis darüber zu gewinnen, was von den Modellen wie und in welchem Kontext bevorzugt einsetzbar ist.

3. Es sollte eine bessere Interaktion zwischen Ausbilder/ Trainer und Forscher angestrebt werden. Das bedeutet, dass der Forscher bereit ist mit Trainern und Spielern zu interagieren, um eine gute Analyse von wirklichen Alltagssituationen zu gewährleisten.

4. Es ist empfehlenswert, die Wechselwirkung zwischen Design, Inhalt, Kontext und Ergebnissen gleichzeitig zu betrachten. Jedes didaktische Modell erschafft ein dynamisches Umfeld, das nur vollkommen verstanden werden kann, wenn man alle seine Bestandteile studiert.

5. Man braucht eine Kongruenz zwischen den gewünschten Ergebnissen, dem ausgewählten Modell und seinen Implementierungsparametern. Bei pädagogischen und didaktischen Prozessen bedeutet dies, dass „man erlangt was man lehrt/ trainiert".

In Bezug auf den fünften Hinweis stellt KIRK (2005, S. 224) fest, dass die breite Anwendung des Direkten Modells im Sport vor allem deshalb gängig ist, weil seitens der Ausbilder/ Trainer der Wunsch besteht, die Kontrolle, Disziplin und Sicherheit beizubehalten, die eben diese Lehrform innen bietet. Infolge der Forderungen der Verwaltungsund Sozialinstanzen könnte in diesem Fall die Kontrolle aber nicht mehr nur ein Lernmittel sein. Sie wird vielmehr in ein Ziel umgewandelt. KIRK geht davon aus, dass dies geschieht, obwohl das angestrebte ultimative Ziel ist, „besser zu spielen“. Die größte Sorge der Trainer ist jedoch, die Kontrolle und Disziplin zu gewährleisten. Der Trainer zielt somit intuitiv darauf ab, als Wissensträger im Zentrum der Lernprozesse zu stehen. Als solcher sieht er sich durch die Erteilung von Befehlen, Instruktionen, Korrekturen und durch das Treffen des Hauptteils der Entscheidungen bestärkt. Das Training der technischen Fertigkeiten und des taktischen Verhaltens in kontrollierten Situationen des Spiels wäre jedoch die bessere Weise, die Kontrolle langfristig aufrecht zu erhalten.

KIRK weist des Weiteren darauf hin, auch die auf den Trainer wirkenden äußeren Umstände zu studieren und die alltäglichen Ereignisse der Lern- und Trainingssituationen zu berücksichtigen. Das Forschungsdesign sollte auf die besonderen Bedingungen jedes Lernkontextes abgestimmt werden und die Implementierung eines Modells sollte so naturgetreu werden, wie diese Bedingungen es erlauben. Gleiches gilt für die Auswertungsmethode und die Interpretation der Ergebnisse. 
Allerdings stellt METZLER (2005b, S. 191-192) fest, dass die Modellvariationen während der Implementierung dazu führen könnten, dass diese Implementierung aus den Designparametern heraustritt und dann der Lernprozess nicht mehr modellgetreu wäre. Deswegen wird im Rahmen dieser Arbeit weder das TGfU-Modell noch andere der bis heute veröffentlichen Modelle eingeführt, da die meisten für den Schulsportkontext entwickelt wurden und nicht genau an die Bedingungen des Wettkampfsports anpassbar sind. Die Bedingungen des Schulsports sind ganz andere als die von Sportvereinen. In der Schule richten sich die Lernziele auf eine lebenslange Begeisterung für den Sport, das Erleben des Sporttreibens und das Hervorrufen erzieherischer Werte des Sports, somit ist der Wettbewerb, der innerhalb der Schule stattfindet, weniger vom Leistungsstress geprägt. Die Sportlehrer haben größere Freiheiten, neue Methoden und didaktische Innovationen einzuführen, da die Leistung eine sekundäre Rolle spielt. Der Lehrer läuft nicht Gefahr, wie das im Vereinsport der Fall ist, dass seinen didaktischen Innovationen die Schuld an einer Niederlage zugewiesen wird; es gibt auch keine überehrgeizigen Eltern, die den Sieg um jeden Preis fordern. Der Unterrichtsinhalt ist keine Vertiefung in die technische, taktische und physiologische Vorbereitung, da Zeitmangel charakteristisch für den Sportunterricht ist, was nur eine Einführung in den Sport erlaubt. Daher sind Modelle wie TGfU, Inzidentale Inkubation, Genetisches Lernen usw. für ein entspanntes und druckfreies Milieu des Schulsports geeigneter als für den Vereinssport.

Die Modelle, die für den Leistungssport entwickelt wurden, etwa Spielreihe und situative Spieldidaktik, wurden nicht genug empirisch getestet. Außerdem sind die Bedingungen heutzutage andere, die Medien haben den Wert des Leistungssports sehr hoch gelegt, die Kinder werden in sehr frühem Alter an den Leistungsgedanken herangeführt und die Leistungsanbeter, Eltern und Trainer eingeschlossen, haben keine Geduld für didaktische Innovationen. Das Modell Game Sense schlägt keinen spezifischen Inhalt vor, es wird daher nicht klar, wie dieses Modell bei Sportvereinen implementiert werden könnte, denn die Anpassung des TGfU an den Leistungssport ist nicht so einfach. Das Decision Training Modell beschäftigt sich nur mit Hochleistungssport.

Es wird daher versucht, ein neues Modell zu entwerfen und empirisch zu validieren. Dies geschieht in der Absicht, es an die realen Bedingungen des Lernens und die Trainingssituationen der Sportspiele innerhalb der Sportvereine anzupassen. Diese Bedingungen sind:

- Die Beteiligung der Trainierenden an Wettkampf- und Trainingsprozessen bereits im frühen Lebensalter.

- Es ist das Ziel, das primäre Bedürfnis von sozialer und motivationaler Kompetenz zu fördern. Es existiert aber auch das Bedürfnis, prozedurale und kognitive Kompetenz zu entwickeln. Das heißt, die Spielleistung und Spielqualität sind ebenfalls primäre Bedürfnisse der Trainer und Spieler, obwohl sie keineswegs die einzigen sind.

- Die Spieler verfügen bereits über Spielerfahrung, Spielfertigkeiten und Spielwissen. Im TGfU-Modell und einigen daran angliedernden didaktischen Modellen werden für spezifische Anwendung, die für den Schulsport designt wurden, Lerninhalte auf allgemeine Inhalte reduziert, die zwischen verschiedenen Sportspielen transferierbar sind (vgl. BUTLER \& McCAHAN, 2005, S. 48; MITCHELL, 2005, S. 68; HOWARTH, 2005, S. 94). Die spezialisierten Trainingsprozesse auf Sportvereinsebene erfordern aber die Entwicklung eines didaktischen Modells, das spezi- 
fisch ist für ein einzelnes Sportspiel und die Bedürfnisse der Vereine berücksichtigt. Gleichzeitig muss es aber auch die Grundprinzipien der kognitiven Orientierung und die Zentralität auf Spiel und Spieler beibehalten.

- Gewünscht wird eine graduelle Anpassung an ein Milieu, in dem lange Zeit das Direkte Modell vorherrschte und in dem die Gültigkeit neuer Entwicklungen nur durch den Sieg im Wettkampf bestätigt wird. Ein neues Modell muss daher vor dem Hintergrund alter Gewohnheiten mit dem generellen Widerstand von Trainern und Spielern rechnen.

Unter Berücksichtigung dieser Bedingungen wird in dieser Arbeit ein didaktisches Modell für das Lernen und Training der Sportspiele vorgeschlagen, das die Entwicklung der Spielkompetenzen in den Mittelpunkt stellt. Das vorgeschlagene Modell nimmt Elemente anderer Modelle auf und versucht, sie an die besonderen Bedingungen der Sportvereine anzupassen. Das Modell wird in mehreren Studien getestet. Jede von innen wird auf eine Einzelperspektive fokussiert und folglich auf die Entwicklung einer bestimmten Spielkompetenz ausgerichtet sein. In einer Mannschaft, die am Wettkampfbetrieb teilnimmt, ist eine Intervention nur bedingt möglich. Vor allem kann der Forschungsprozess nicht in einer schroffen Weise die existierenden Trainingsgewohnheiten auswechseln und schlagartig das Direkte Modell des Trainers umstellen. Aus diesem Grund wird eine Forschungsstrategie vorgeschlagen, in der nur jeweils eine einzelne Perspektive in jeder Studie getestet wird. Es entsteht so eine partielle Intervention, ohne den gängigen Trainingsprozess der Mannschaft vollständig neu zu gestalten. Innerhalb jeder Studie wird eine kurze Intervention durchgeführt. Während mehrerer Trainingseinheiten wird eine Intervention von jeweils 30 Minuten eingeplant. Auf diese Weise wird angestrebt, das Modell mit der partiellen Implementierung einiger Elemente zu testen, die auf die Förderung punktueller Aspekte der Spielkompetenzen ausgerichtet sind, ohne den Arbeitsplan der Trainer, die normalerweise ein Direktes Modell einsetzen, komplett umzugestalten. 


\section{Modell der Spielhandlungskompetenzen}

Ein Modell wird als ein Schema betrachtet, das wie ein Plan funktioniert. Es hat zum Ziel, etwas aufzubauen oder eine bestimmte Vorgehensweise einzuführen. Das Schema bietet eine Gesamtheit von Prozeduren, Maßgaben und Hinweisen an. Diese sollen den Anwendern behilflich sein, die entstehende Gesamtstruktur zu verstehen. Das Schema erlaubt auch, richtige und effektive Entscheidungen zu treffen (METZLER, 2005a). Nach METZLER (S.28) ist ein didaktisches Modell auch ein kohärenter und übergreifender Lernplan, der die Lernziele, die Erwartungen über das Verhalten von Lehrenden und Lernenden, die Lehrtechniken und didaktische Strategie, die Lernaufgaben und die Auswertungs- und Kontrollmethoden für die Verifizierung der korrekten Anwendung des Modells beinhaltet.

In dieser Arbeit wird das Modell der Spielhandlungskompetenzen vorgeschlagen. Es nimmt die Spielhandlungen an als Bausteine, die die Anfänger lernen und üben sollen bis zur Erreichung einer Kompetenz wie die der Experten, um problematische Spielsituationen erfolgreich zu lösen. Da das Wissen die Grundlage der Kompetenz der Experten ist, wird zunächst ein Wissenskonzept erstellt, dem zufolge die Wissensbeschaffung nur anhand eines Aufbauprozesses stattfindet. Dafür schlägt das Modell die Anwendung von besonderen didaktischen Strategien vor, die aufdie Spielhandlungskompetenz einwirken. Dadurch sollen die Spieler die Spielsituationen interpretieren, geeignete Handlungen ausführen und begründen können; ferner sollen sie das Gelernte in ständig neuen problematischen Spielsituationen umsetzen.

Der Schwerpunkt des Modells ist das taktische Bewusstsein und die Entwicklung von spezifischer Kompetenz für eine erfolgreiche Teilnahme am Spiel. Es wird darauf verzichtet, auf die Schulung technischer Fertigkeiten und die Vermittlung taktischer Hinweise, wie sie bei traditionellen Spielvermittlungsmodellen üblich sind, einzugehen.

In Anlehnung an METZLER (vgl. 2005a, S. 29), der einen Rahmen für die Beschreibung eines didaktischen Modells vorschlägt, wird auch in dieser Arbeit ein Rahmen für die Beschreibung des Modells der Spielhandlungskompetenzen benutzt. Folglich wird das Modell nach dem Raster in Tabelle 6 beschrieben:

Tabelle 6: Schritte zur Beschreibung des Modells der Spielhandlungskompetenzen

\begin{tabular}{|c|c|c|c|c|}
\hline Einführung + & Begründung + & Struktur + & Aufgabeaufstellung + & Validierung \\
\hline & $\begin{array}{l}\text { Theoretische Begründung } \\
\text { Annahmen über das Leh- } \\
\text { ren/Training } \\
\text { Annahmen über das Lernen } \\
\text { Annahmen über die Aufgabe des } \\
\text { Trainers }\end{array}$ & $\begin{array}{l}\text { Anwendungs- } \\
\text { Struktur } \\
\text { Ziele, Methoden und } \\
\text { Inhalte } \\
\text { Umsetzungsplan } \\
\text { Inhalt Auswahl } \\
\text { Die Rolle des Trainers }\end{array}$ & $\begin{array}{l}\text { Lernaufgabe } \\
\text { Aufgabenstruktur } \\
\text { Aufgabe Vorstellen } \\
\text { Inhalt Abfolge }\end{array}$ & $\begin{array}{l}\text { Hypothesen } \\
\text { Studien } 1 \text { bis } 4\end{array}$ \\
\hline
\end{tabular}




\subsection{Einführung zum Modell}

Bei traditionellen Spielvermittlungsmodellen weist der Trainer den Spielern eine Reihenfolge von isolierten technischen und taktischen Fertigkeiten an. Diese werden durch Erklärungen, Korrekturen und Rückmeldungen ergänzt. Die Komplexität wird stufenweise gesteigert, bis die volle Spielversion erreicht wird. Bei dem Modell der Spielhandlungskompetenzen weist der Trainer hingegen zunächst Spielaufgaben zu, in denen Grundprinzipien des wirklichen Spiels enthalten sind. Das heißt, dass Mitspieler, Gegenspieler und Tore vorhanden sind, die sekundären Regeln aber modifiziert werden, um besondere technische oder taktische Zielsetzungen zu erreichen. Es werden besonders die kognitiven Fähigkeiten und Fertigkeiten gefordert und die Spielaufgaben werden als problematische Situationen dargestellt. Obendrein werden auch Kombinationsspiele angewiesen, die zur Förderung der kognitiven Fähigkeiten wie Wahrnehmung, Entscheidungs- und Konzentrationsfähigkeit beitragen. Zweikampfübungen werden auch im Training integriert. In geringem zeitlichem Umfang werden auch Selbstlernaufgaben gestellt. Diese sollen die Spieler zu Hause üben und danach in ihr Spiel integrieren.

Zahlreiche Spielvermittlungsmodelle sind vorher vorgeschlagen worden in der Absicht, die tief verwurzelte Vorstellung der Übungsreihe als Hauptmethode des Spiellernens zu überwinden und das Spiel selbst als Hauptmethode zu etablieren (siehe die Spielvermittlungsmodelle S. 10-57). Die Grundidee „zu Spielen lernt man am besten durch Spielen" wird zurzeit noch immer in der wissenschaftlichen Literatur des Sports und von den zuständigen Ausbildungseinrichtungen (z.B. der Deutsche Fußball Bund, DFB) als Leitfaden für die Entwicklung neuer und wirksamer Lehrtechniken der Sportspiele betrachtet. Aus diesem Grund wird der Hauptteil der verfügbaren Trainingszeit dem Spiel gewidmet, die Trainingseinheit besteht fast ausschließlich aus Spielen. Übungen für technische Fertigkeiten und für die Kondition werden ergänzend als Hausaufgabe angewiesen.

Die Spielhandlungen werden spielnah geschult. Jedes Spiel beinhaltet ein taktisches Problem, das das Lernziel darstellt. Die Spieler sollen das Problem identifizieren, sich mögliche Lösungen überlegen und ausprobieren, auf gegebene Fragestellungen antworten und eigene Schlussfolgerungen anstellen. Auf diesem Weg lässt sich „Verständnis" für eine Situation erreichen (MITCHELL, OSLIN \& GRIFFIN, 1997, S. 8-16). z. B. teilt der Trainer in einer traditionellen Trainingssitzung den Inhalt in technische Fertigkeiten wie Passen, Annehmen, Schießen/Werfen, Dribbeln, etc. auf. Diese werden am Anfang des Trainings und während der meisten Zeit geübt. Schlussendlich wird gespielt und der Trainer erteilt taktische Anweisungen und Korrekturen und motiviert den Spieler

durch laute Äußerungen. Im Modell der Spielhandlungskompetenzen wird hingegen erst einmal gespielt. Es werden Spielhandlungen thematisiert, z. B. "offensive Stellung“, „Verhalten ohne Ball“, „Überzahlverarbeitung“, „Positionierung“, usw. Danach werden Kombinationsspiele und Zweikampfübungen bereitgestellt und schlussendlich wird Zeit für das Üben der technische Fertigkeiten bereitgestellt. In dieser Phase wird ausprobiert und Zeit für eigene Erfahrungen gelassen, das eigentliche Üben wird aus Zeitgründen als Hausaufgabe empfohlen.

Das Modell der Spielhandlungskompetenzen basiert auf einer Reihe von Kleinen Spielen (sogenannte Small Sided Games im englischen Sprachraum), die auf Spielhandlungen und taktische Probleme fokussiert sind. Die Spieler lösen zuerst die Probleme im 
kognitiven Bereich, anschließend im motorischen Bereich. Der Trainer stellt eine Liste der häufigsten Spielhandlungen bereit, die den Lerninhalt bilden. Er entwirft eine Reihenfolge von Spielen, die das Handlungswissen schulen sollen. Gespielt wird immer von Anfang an, der Aufbau erfolgt vom einfachen Spiel hin zur komplizierten Spielhandlung. Der Trainer analysiert das Spielverhalten der Spieler und identifiziert Lücken in ihrem Verständnis der Spielsituation. Durch Fragen versucht er ihr Spielverständnis zu verbessern und innen Anhaltspunkte zu liefern, wie sie problematische Spielsituationen erkennen und durch Handlungen lösen können. Statische Übungen sollten nur angewendet werden, um bestimmte schwere Spielsituationen und ihre Lösung einführend darzustellen.

Die Kleinen Spiele sollen das wirkliche Spiel repräsentieren und durch die Modifizierung der sekundären Regeln auf die gewünschten Schwerpunkte konzentrieren. Die modifizierten Regeln zwingen den einzelnen Spieler zur Beschäftigung mit einer bestimmten Spielhandlung, die anderen werden bei späteren Spielen behandelt. Die problematischen Situationen sind die Grundprinzipien des Spiels. Die Spieler sollen sie verstehen, um geeignete Spielhandlungen bei jeder Spielsituation durchzuführen (DOOLITE, 1995, S. 19). Der Trainer kann die Spieler durch Fragen dazu auffordern, unterschiedliche Lösungsmöglichkeiten auszuprobieren. Das wichtigste Merkmal der eingesetzten Spielaufgaben ist, dass der Schwerpunkt auf dem taktischen Bewusstsein liegt. Dadurch kann man die Entscheidungsfähigkeit und Ausführung der technischen Fertigkeiten anpassen und kontextabhängig entwickeln. Der Trainer muss die Spieler dazu auffordern, an die problematischen Spielsituationen zu denken, die während des Spiels auftreten können, und geeignete Lösungsformen dafür vorschlagen. Nur so können die Spielaufgaben vom reinen „Spielen im Training“ unterschieden werden. 


\subsection{Begründung}

Das im Rahmen dieser Arbeit vorgeschlagene didaktische Modell der Spielhandlungskompetenzen wurde aus einem Informations- und Wissensmanagementansatz, aus einem handlungstheoretischen Ansatz und aus einem Ansatz der Spielkompetenz heraus konzipiert. Im Zusammenhang mit der Diskussion über den Erwerb von Wissen, welches menschliche Leistungen erst ermöglicht, werden im Folgenden zwei Schemata eingeführt. Sie sollen der Verdeutlichung der Zusammenhänge dienen und darüber hinaus bei einer Stellungnahme zum Themenkomplex helfen. Am Beispiel der Wissenshierarchie und der Wissenstreppe wird jedoch keine philosophische Diskussion über Information und Wissen geführt. Vielmehr werden grundlegende Begriffe unterschieden und herausgearbeitet, die von erheblicher Bedeutung für den Aufbau von Wissen und den Wissenstransfer sind.

\subsubsection{Die Wissenshierarchie}

Dass Wissen im Langzeitgedächtnis gespeichert wird, ist als Tatsache wissenschaftlich akzeptiert (KEIL \& PELLEGRINO, S. 56; EYSENCK \& KEANE, 2000, S. 251). Die Art und Weise, wie es dort organisiert und repräsentiert ist, ist jedoch eine in der Kognitionspsychologie häufig untersuchte Fragestellung (KEIL \& PELLEGRINO, 1988, S. 56). Um den Weg des Wissens in das Langzeitgedächtnis zu erklären, greifen wir auf Annahmen aus der Informationswissenschaft und dem Wissensmanagement zurück. Das Wissen wird im Rahmen seiner Nutzung vielfältig verknüpft, ist also funktional organisiert und wird entsprechend verarbeitet. In diesem Sinn unterscheidet es sich von reiner Information und von reinen Daten. Eine konkrete Darstellung bietet das DIKW-Modell.

\section{Das DIKW Modell}

DIKW (Data, Information, Knowledge, Wisdom) ist eine Struktur, die aus vier Schichten besteht. Sie wird anhand bestimmter Attribute der gespeicherten Information aufgebaut, die es erlauben, ein Flussdiagramm oder eine Hierarchie von Begriffen auszugestalten. Das reine Datum ist die unterste Eben. Auf der zweiten Stufe trägt die Information bereits einen Kontext darüber, „was“ gespeichert wird. Darüber dient das Wissen „wie“ etwas abläuft/geschieht dem Einsatz der gespeicherten Information. Diese dritte Ebene wird außerdem ergänzt durch das Wissen „warum“ etwas zu tun ist, enthält also bereits eine sehr stark Bewertungskomponente. Das Verständnis der Gründe warum etwas abläuft/geschieht würde den Aufstieg auf die auf die oberste Ebene erlauben. In dieser obersten Ebene trägt die „Weisheit" das vereinte Wissen um den angemessenen Einsatz des Wissens und aller Möglichkeiten, die aus seinem Einsatz entstehen (ZELENY, 1987, S. 59; COOLEY, 1987, S. 82). Die erfolgreiche Strukturierung dieser Hierarchie würde den zukünftigen Erfolg ermöglichen. 


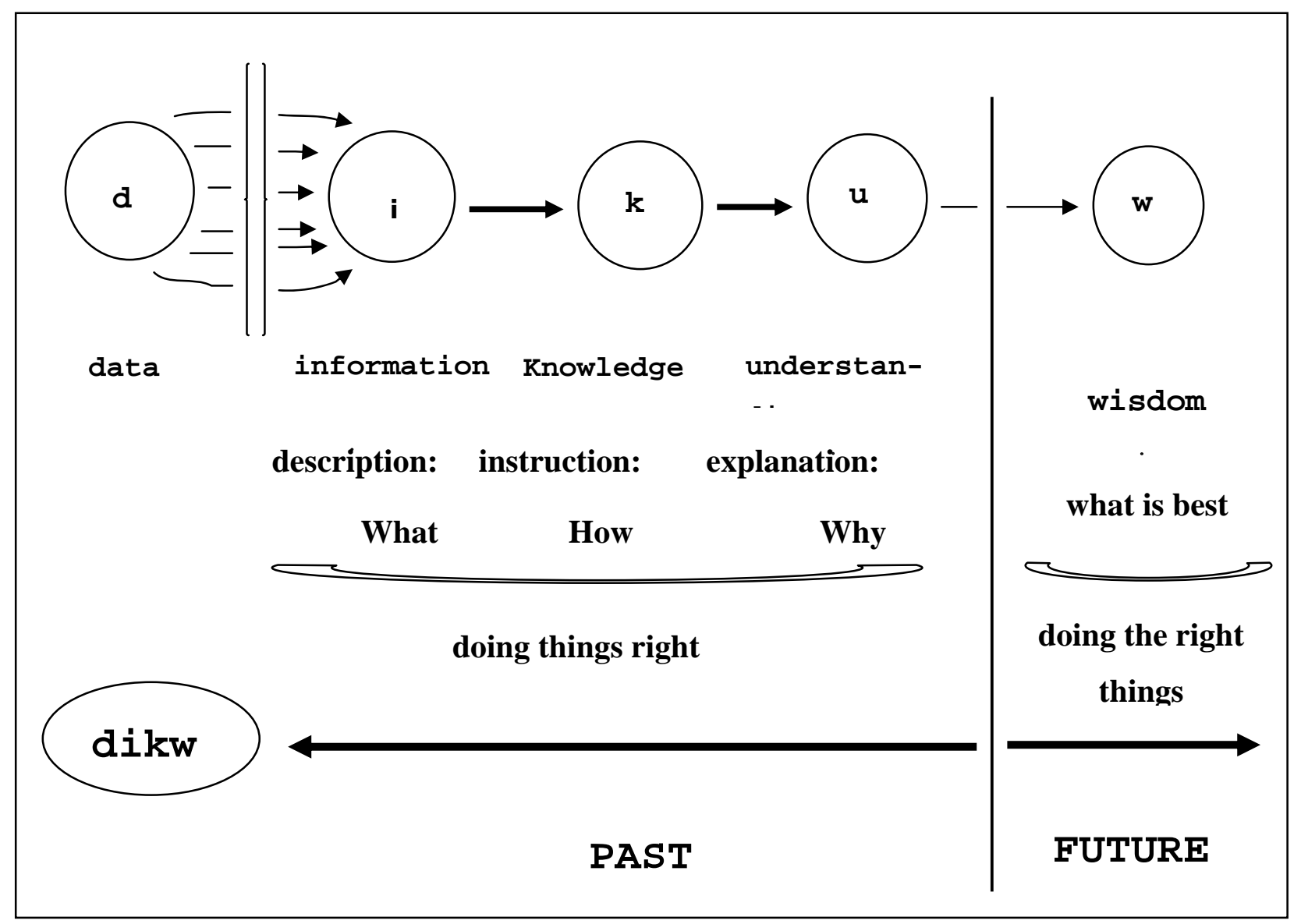

Abbildung 7: Die DIKW Hierarchie (nach Ackoff, 1989, S. 5)

Das DIKW-Modell nimmt also folgende Abgrenzungen von Wissen an:

- Daten sind eine Reihe von Beobachtungen und Messungen.

- Information wird aus analysierten Beziehungen zwischen Daten gezogen.

- Die Information kann Teil einer absichtlichen Vermittlung an einen Empfänger sein und bietet Antworten auf einfache W-Fragen an: „Wer; Was; Wo; Wann“.

- Wissen wird ab der ersten Nutzung von Information im Handeln hervorgebracht.

- Weisheit wird durch die Nutzung von Wissen, durch Reflexion und Kommunikation erlangt. Weisheit erschließt die besondere Ebene von Bewusstheit und wird ausgebildet auf Basis menschlicher Werte wie Wahrnehmung, moralischer Verpflichtung, Ethik, usw. (ACKOFF, 1989, S. 3-9).

Allgemein gesagt, ist Information weniger als Wissen und Daten sind weniger als Information. Daten werden zu Fakten, die nach Strukturierung in Information verwandelt werden, wenn eine Bedeutung zugewiesen wird. Information wird ihrerseits in Wissen 
verwandelt, wenn sie interpretiert und kontextualisiert wird. Es wird angenommen, dass die Daten für sich nur Informationen liefern und erst anschließend, wenn Information vorliegt, das Wissen erzeugt wird. Der Mensch greift dann auf dieses Wissen zurück, um Probleme zu lösen. Schlussendlich wird, wenn menschliche Werte das Handeln leiten, das Verhalten auf Basis der Weisheit bestimmt. In Abgrenzung dazu werden oft beide Konzepte angewendet, so als ob Information und Wissen dasselbe wäre oder zumindest austauschbar verwendet werden könnten. Es ist schwierig, eine Diskussion über Informationen zu führen, die sich nicht auf Wissen beziehen und umgekehrt. Durch dieses methodische Problem wird nicht klar, ob es sich tatsächlich um zwei verschiedene Begriffe handelt.

ROWLEY (2007, S. 167-168) führte eine Analyse der wichtigsten Literatur der Informationswissenschaft und des Wissensmanagements hinsichtlich des DIKW-Modell durch und kommt dabei zu folgenden Ergebnissen:

- Die wesentlichen Elemente sind Daten, Informationen, Wissen und Weisheit.

- Diese Schlüsselelemente sind praktisch immer in der gleichen Reihenfolge aufgeführt, auch wenn einige Modelle zusätzliche Stufen bieten, zum Beispiel „Verständnis“ oder "Erleuchtung“.

- Die höheren Elemente in der Hierarchie lassen sich in Bezug auf die unteren Elemente durch die Identifizierung eines geeigneten Transformationsprozess erklären.

- Die implizite Herausforderung besteht darin, zu verstehen und zu erklären, wie sich Daten in Informationen, Informationen in Wissen und Wissen in Weisheit verwandeln lassen.

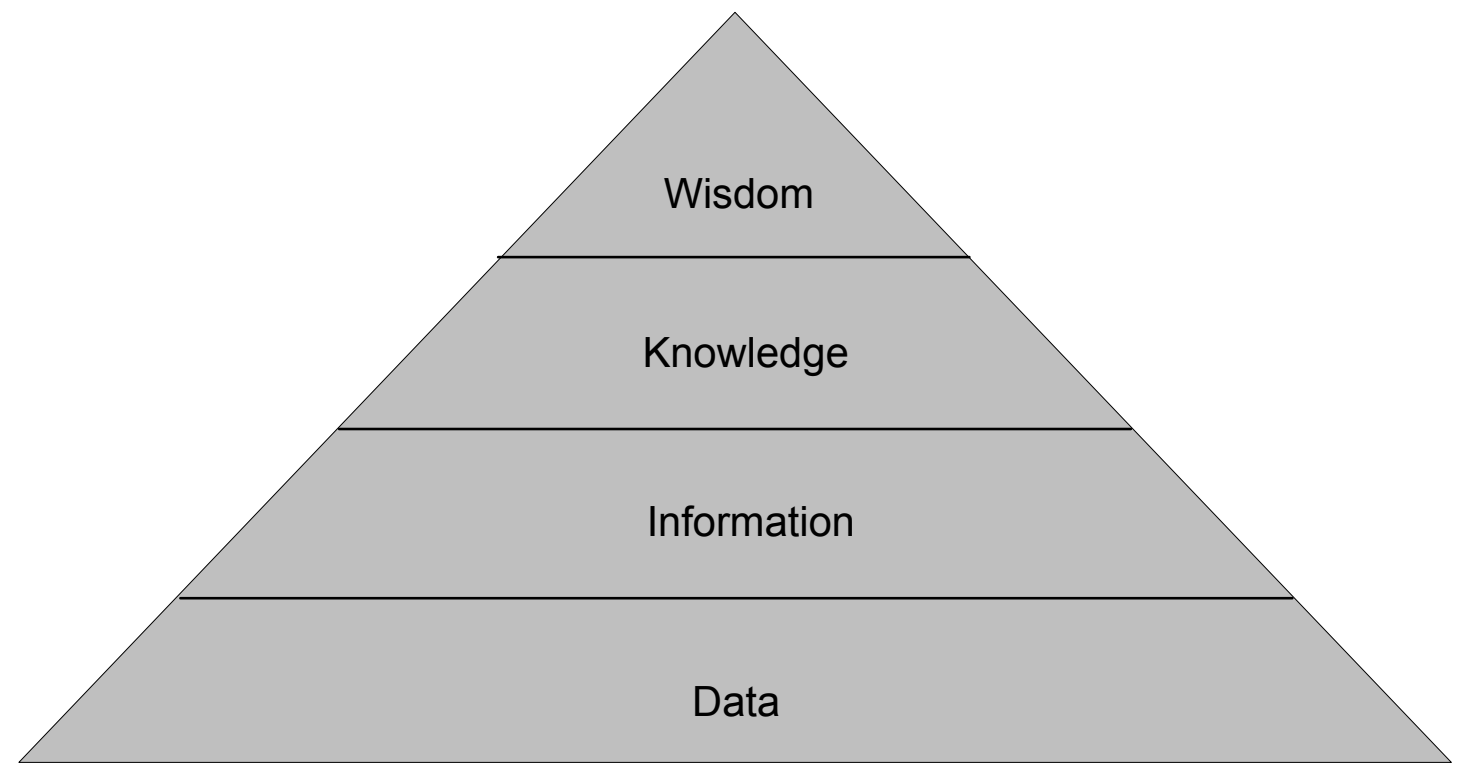

Abbildung 8: Ebene der DIKW Hierarchie (nach Rowley, 2007, S. 164) 
Es besteht Einigkeit darüber, dass Informationen als organisierte oder strukturierte Daten gesehen werden und dass Wissen aus Daten- und/oder Informationen besteht, welche organisiert und verarbeitet wurden. Sie tragen Verständnis, Erfahrungen, akkumuliertes Lernen und „Know-how“, die für aktuelle Probleme oder Aktivitäten verwendet werden können. Es besteht ebenfalls ein wissenschaftlicher Konsens darüber, dass Daten, Informationen und Wissen in Bezug aufeinander definiert sind, auch wenn Daten und Informationen beide als Eingang zum Wissen einwirken können. Dieser Konsens besagt, dass das Konzept einer Hierarchie die Begriffe Daten, Informationen und Wissen verbindet.

Uneinheitlicher sind die Ansichten über die Art der Prozesse, durch die Daten in Informationen und Informationen in Wissen verwandelt werden. ROWLEY (S. 175) fragt sich nach der Art dieser Umgestaltung und der entstehenden Bezüge:

"Is there a sharp divide between data, information and knowledge, or do they lie on a continuum with different levels of meaning, structure and actionability occurring at different levels? So, for example, it is possible to have knowledge with different levels of meaning and actionability?"

Nach BELLINGER (2004) wäre das „Verständnis“ was das Verlassen jeder Ebene ermöglicht und zur nächsten höhere Ebene führt. Daten wären bloße Fakten und hätten zwischen sich selbst keine Verbindungen. Information würde Verstehen von Beziehungen einschließen, eine Art von Ursache und Wirkung. Wissen würde aus einem Verbindungsmuster bestehen, das einen hohen Grad von Vorhersagen der nächsten Geschehnisse ermöglicht. Weisheit umfasst grundlegende und wesentliche Prinzipien des Wissens, die Verarbeitung dieses Konzeptes impliziert eine anspruchsvolle philosophische Diskussion und wird daher in dieser Arbeit nicht mehr berücksichtig, folglich wird die Wissenshierarchie wie in der Abbildung 9 angenommen.

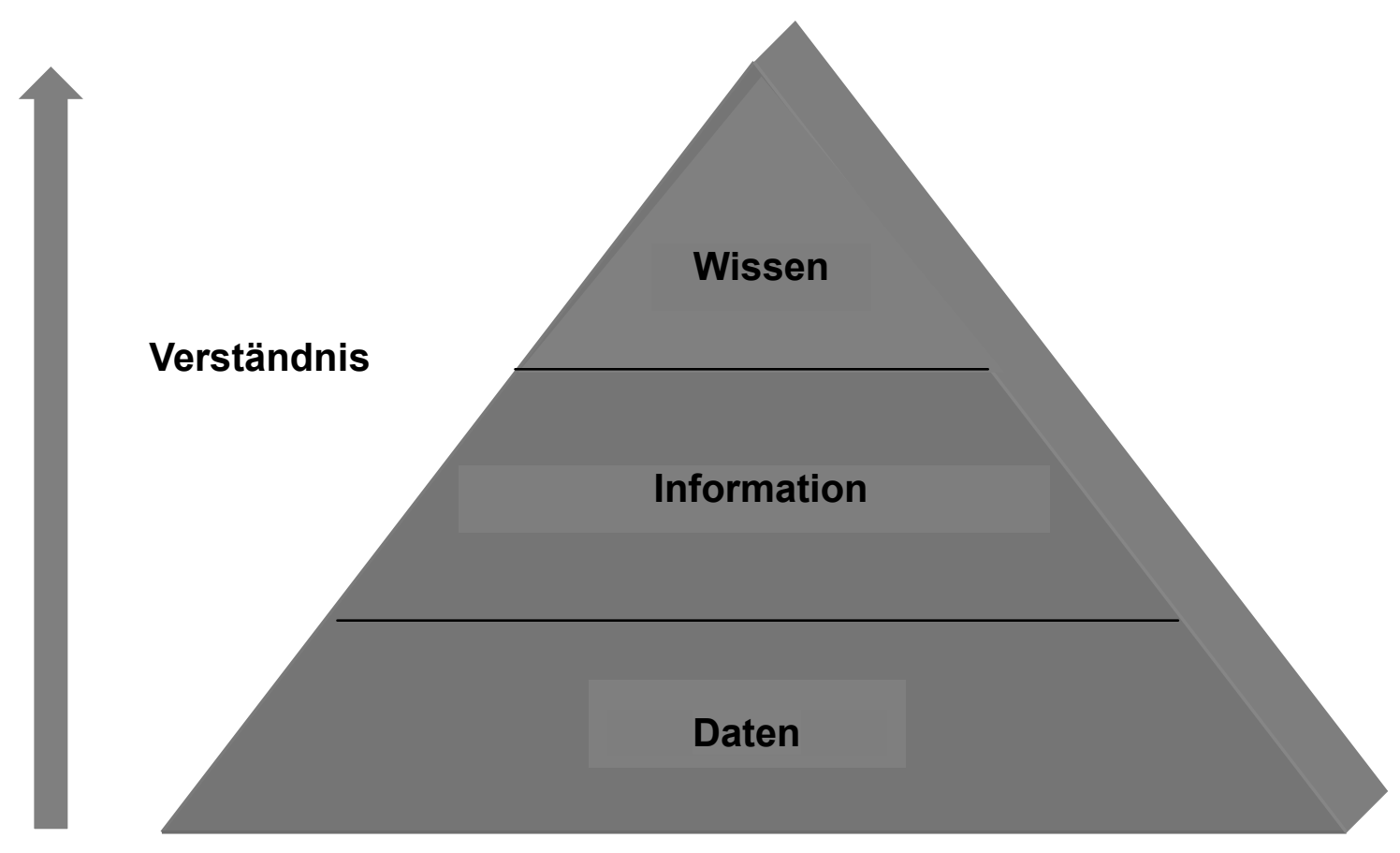

Abbildung 9: Die Hierarchie des Wissens 
Ausgehend von Überlegungen und mit Hilfe der kognitiven Psychologie werde ich einen Entwurf beschreiben, bei dem Wissen aus Daten und Informationen entsteht.

\section{Daten, Information und Wissen}

Daten sind physische Reize, die als Symbole, Töne, Geschmäcker, Gerüche, Berührungen oder Bilder wahrgenommen werden. Sie entstehen also aus Mess- und Beobachtungsverfahren. Sie sind als isolierte Einheiten voneinander unabhängig und haben für sich keinen Selbstwert. Allerdings bilden sie die Basis der Pyramide des Wissens, dessen Erwerb und Entwicklung im menschlichen Gehirn durch Verarbeitungsprozesse vollzogen wird. Die Wahrnehmungsfähigkeit ermöglicht den Erwerb von Daten.

Information entsteht aus dem Zusammenhang von Daten. Auf einer ersten Ebene wird ein Datum mit einem bereits bekannten Inhalt im Langzeitgedächtnis verglichen. Wenn ein Element vorhanden ist, mit dem das Datum eine sinnvolle Beziehung aufnehmen kann, wird die Entstehung von Information stattfinden. Sie wird nach dem dabei durchlaufenen kognitiven Prozess als Kennen bezeichnet (ENGELKAMPF, 2006, S. 5). Die entstandene Information kann durch bewusste Prozesse im Langzeitgedächtnis gespeichert werden. Es ist ebenfalls möglich, Beziehungen zwischen zwei ganz neuen Daten (MILLER, 1993, S. 50) aufzunehmen oder, in unbewusster Weise, könnte aus schon im Langzeitgedächtnis vorhandenen Informationen ein als neu wahrgenommenes Datum erzeugt werden.

Es ist schwer durchführbar, ein isoliertes Datum im Langzeitgedächtnis zu speichern. Damit dies gelingen könnte, wäre es notwendig, einen Typ von Beziehung mit anderen schon im Langzeitgedächtnis vorhandenen Informationen aufzubauen, denn letztendlich sind diese Beziehungen das, was im Langzeitgedächtnis gespeichert ist (KEIL \& PELLEGRINO, 1988, S. 78). Eine Telefonnummer ist z. B. nur schwer im Langzeitgedächtnis zu speichern, bis eine Beziehung zwischen der Nummer und einem Vertreter der Information entsteht, zu der die Nummer passt. Dieser kann beispielsweise ein Freund, ein Geschäftspartner oder eine Institution sein, zu dem oder der diese Nummer gehört. Das isolierte Datum verwandelt sich in sinnvolle Wissenseinheiten, wenn es zu einer Information hinzugefügt wird, die schon im Langzeitgedächtnis vorhanden ist. Sie tritt als ein Rahmen für die Einordnung des Datums in einen Kontext auf und bietet einen „Haken“ an, an dem das Datum verankert ist. Sie trägt damit zur Vermehrung der Information bei. Die aus Erkennungsprozessen entstehende Information wird in verschiedenen Abschnitten des Langzeitgedächtnisses gespeichert und die entstehenden Produkte werden nach der Theorie der Informationsverarbeitung als das Ergebnis der höchsten Leistungen des menschlichen Gehirns bezeichnet. Derselbe Verarbeitungsprozess ist Gegenstand des Studiums dieser Theorie (STENBERG, 1984). Wissen wird aus Beziehungen gebildet, die zwischen den Informationseinheiten entstehen. Es ist im Langzeitgedächtnis angesiedelt und es ermöglicht die Ausführung von Prozessen und Bewertung von Verhältnissen. Das Verfahren von Wissenserwerb ist das Lernen und es wird durch das Verständnis für eine Information ausgelöst. Nur wenn die Verarbeitung der Information zum Verstehen führt, können wir von Wissen sprechen (BELLINGER, 2004.).

Wissen ist nicht statisch. Dadurch unterscheidet es sich von Information. Wissen zu besitzen, bedeutet, über die Fähigkeit zu verfügen, einen Prozess auszuführen, der eine Veränderung der Umwelt bewirken kann, d.h. eine Handlung auszuführen. Im engs- 
ten Sinn ist Wissen nur auf solche Prozesse bezogen zu verstehen, weshalb es sich nicht von Können unterscheidet. Wissen und Können sind in dieser Betrachtungsweise Synonyme (HOTZ, 1995, S. 185). Wissen entsteht nur durch aktive Prozesse von Verarbeitung und Mitmachen. Z. B. kann ich die Information über den Weg zu einem Platz in einer Stadt (Wegbeschreibung) bekommen. Aber nur wenn ich wirklich an diesen Platz käme, würde ich Wissen über die Lage des Platzes und den Weg dorthin erlangen (,ich weiß, wo das ist“).

Hierarchie des Wissens

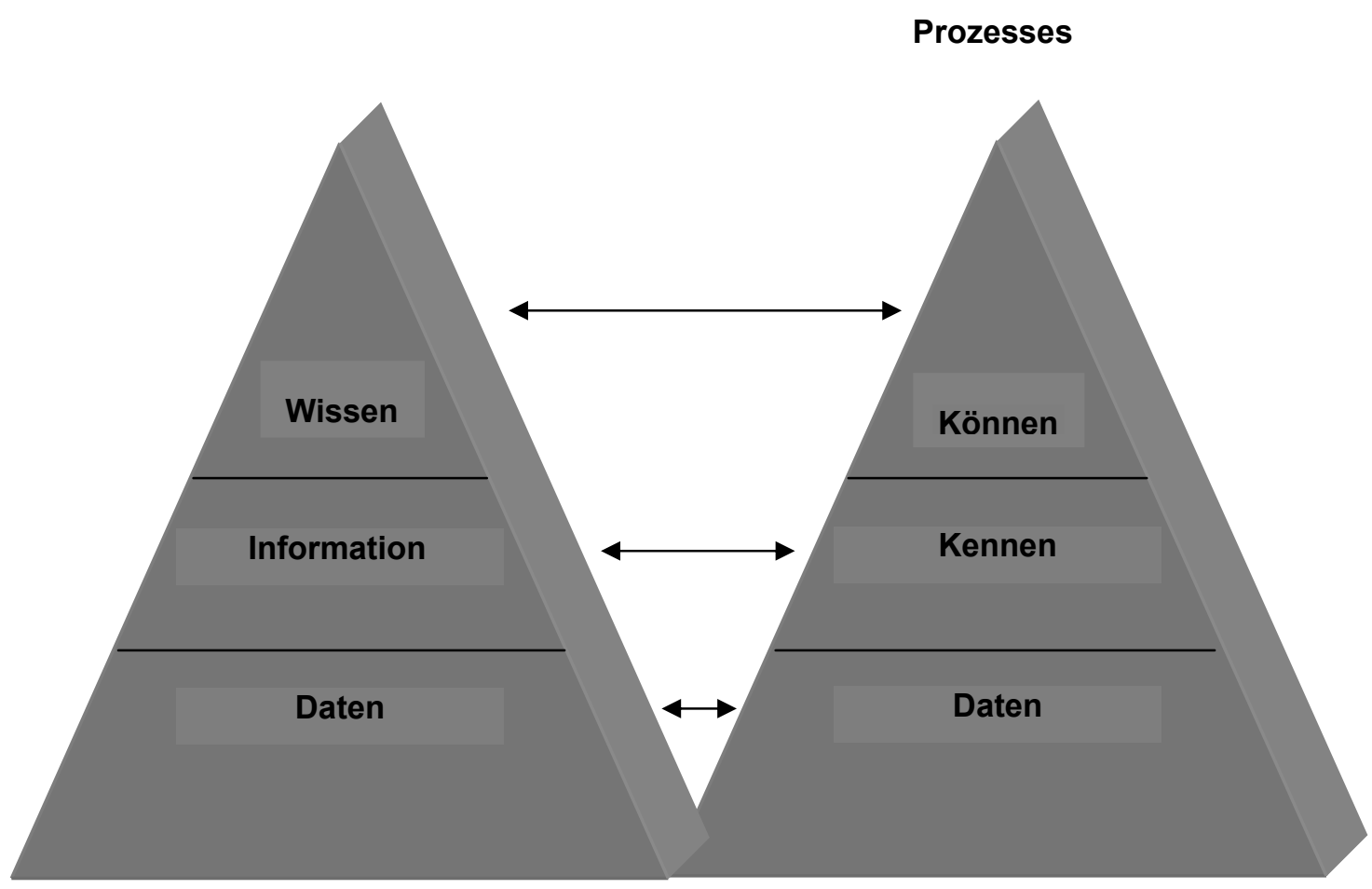

Abbildung 10: Die Hierarchie des Wissens und ihre entsprechenden kognitiven Prozesse

\begin{abstract}
„Weil im Mittelpunkt dieser Informationsverarbeitung die Frage steht, wie ausgehend von Außenreizen Bewusstseinsinhalte entstehen bzw. wie wir Reize in unserer Umwelt erkennen, sprechen wir von 'Informationsverarbeitung im Dienste des Erkennens'. Die Verarbeitung von Reizen beginnt mit der Wahrnehmung oder - wie man auch sagt - mit der Enkodierung des Reizes (periphere Verarbeitung). Es folgt die zentrale Verarbeitung (Kurzzeitspeicher), bei der der Reiz zu bereits im System vorhandenem Wissen (Langzeitgedächtnis) in Beziehung gesetzt wird. Das Ergebnis wird bewusst erlebt. Man spricht von Erkennen (bei einfachen Reizen) oder von Verstehen (bei Reizzusammenhängen). Die Prozesse des Wahrnehmens, Erkennens, Verstehens berühren auf der Verarbeitung aktuell vorhandener Reize. Bezieht sich die zentrale Verarbeitung auf Prozesse der Reaktivation vergangener Reizerfahrungen, dann spricht man von Erinnern oder von Gedächtnis..."
\end{abstract}

(ENGELKAMP, 2006, S. 5).

Wissen wird nach PLATON häufig als „wahre gerechtfertigte Meinung“ (PLATON, zitiert nach BECKERMANN, 2001, S. 574) bestimmt. Etymologisch geht Wissen auf das alt- 
hochdeutsche wizzan zurück, ist also von „ich habe gesehen“ abgeleitet (WALDE, 1938, zitiert nach HOTZ, 1995, S. 181). In der Literatur finden sich demgegenüber viele verschiedene Definitionen für „Wissen“. Hier einige Beispiele:

„Speicherung, Integration und Organisation von Information im Gedächtnis. [...] Wissen ist organisierte Information, es ist Teil eines Systems oder Netzes aus strukturierten Informationen“.

(SOLSO, 2005, S. 242).

„Wissen bezeichnet das Netz aus Kenntnissen, Fähigkeiten und Fertigkeiten, die jemand zum Lösen einer Aufgabe einsetzt“.

(HERBST, 2000, S. 9).

„Gesamtheit der potentiell verhaltenswirksamen Gedächtnisinhalte“

(HOFFMAN, J. 1993, S. 250)

Eine eindeutige Definition von Wissen wurde bislang nicht in der bearbeiteten Literatur gefunden. Zu den großen Bedeutungsunterschieden nach dem jeweiligen Standpunkt der Autoren kommt zusätzlich eine noch nicht abgeschlossene Debatte über die Unterscheidung zwischen Wissen und anderen Begriffe wie Daten, Information, Kennen und Können. Das Problem der Wissensdefinition muss aber nicht unbedingt gelöst sein, um sich mit der Fragestellung des Wissenserwerbs beschäftigen zu können. Einen radikaleren Vorschlag macht Ansgar BECKERMANN (2001, S. 578):

"Meiner Meinung nach liegt die Antwort auf der Hand. Wir sollten etwas mutiger [...] sein und
auf den Wissensbegriff ganz verzichten. Es gibt in der Erkenntnistheorie keine interessante
Frage und keine interessante These, die wir nicht auch ohne diesen Begriff formulieren
könnten. Was ist das Ziel unserer Erkenntnisbemühungen? Wahrheit."

Im Rahmen der vorliegenden Arbeit wird demnach Wissen als mehr als Information oder bloße Ansammlung von Daten betrachtet und ist nicht auf bewusstes Abrufen von Gedächtnisinhalten beschränkt (vgl. WIEMEYER, 1994, S. 10).

Nach ENGELKAMP (2006, S. 2), ist auch die Diskussion über die Unterscheidung zwischen Wissen und Können in der kognitiven Psychologie noch nicht abgeschlossen. Wissen „wie“ etwas funktioniert, wäre nicht dasselbe wie das Wissen „warum“ es funktioniert. Und wiederum abgrenzbar ist das Wissen, „wie man es zum Funktionieren bringt“. So kann eine Testperson z. B. eine Bewegung genau beschreiben und einschätzen (z. B. ein Kampfrichter beim Turnen) - aber sie muss diese Bewegung nicht zwangsläufig selbst ausführen oder unterrichten können. Allerdings habe sich die Psychologie „fast ausschließlich mit dem Menschen als erkennendes Wesen das über Wissen verfügt und es nutzen kann, beschäftigt“ (S. 2). Das „Können“ ist hingegen nicht die entscheidende Frage für die "erkenntnisorientierte“ kognitive Psychologie. ENGELKAMP schlägt deshalb eine „verhaltensorientierte kognitive Psychologie“ vor, die zur Erklärung des Könnens beitragen sollte. ENGELKAMP selbst weist nicht auf Unterschiede zwischen Kennen und Wissen hin und benutzt beide Begriffe austauschbar. In seinem Text (ENGELKAMP, 2006) wird allerdings ein Teil als „Informationsverarbeitung im Dienste des Erkennens“ und ein anderer Teil als „Informationsverarbeitung im Dienste des Verhaltens" bezeichnet. 
Ich gehe in der vorliegenden Arbeit hingegen davon aus, dass der Unterschied zwischen Kennen und Wissen sehr wohl existiert und dass das Kennen aus Information besteht, dass das Wissen aber erst nach der Verarbeitung der Information vorhanden ist und dass die Brücke, die den Übergang von Kennen zum Wissen ermöglicht, das „Verstehen“ ist. Wenn die Umsetzung vom „Kennen“ erfolgreich ist, hat man „Können“ erlangt und darüber hinaus entsteht „Wissen“ (vgl. HOTZ, 1995, S. 189), in diesem Punkt stimmt die Definition von HOTZ mit der Definition von HERBST (siehe Seite 76) und mit der Definition von REINMANN-ROTHMEIER und MANDL (s.u) überein, Wissen ist nur Wissen wenn es für die erfolgreiche Lösung einer Aufgabe benutzt werden kann, vor die Umsetzung von Wissen kann man nur von Kennen sprechen, z.B. „Ich weiß“ bedeute dann „Ich kann“, „Ich kenne“ bedeute hingegen „Ich habe Information darüber“. Das setzt die Annahme voraus, das zwischen Kennen, Können und Wissen - zumindest wenn es um komplexe Verfahren geht - kein direkter Weg liegt. Der Handelnde ist zum Verstehen gezwungen, bevor er Wissen über eine Handlung erzeugen und gut ausführen kann (so z. B. auch bei einer sportlichen Handlung). Deklaratives Wissen würde demnach die Basis von Können sein und umgekehrt. Gemäß explizitem und implizitem Lernen bzw. Gedächtnisinhalt wird das prozedurale Wissen, d. h. das Können ausgebaut (vgl. ANDERSON, 1996, S. 279).

Explizites Wissen entsteht im Rahmen eines Prozesses, in dem der Mensch bewusst über Wissen verfügt und mittels sprachlicher Zeichen außerdem fähig ist, dieses Wissen an andere weiterzugeben. Prozesse von Induktion oder Deduktion erlauben die gezielte Bewusstsein und Äußerung des Wissens. Allerdings nimmt der Empfänger das so dargestellte Wissen als reine Information wahr. Erst nach einem Prozess des Wissensaufbaus kann er aus dieser Information das Wissen sich selbst erwerben. Implizites Wissen entsteht hingegen durch die Ausführung von Handlungen und ist nicht übertragbar, es ist nur durch einen eigenen Aufbauprozess zu erwerben. Nach REINMANNROTHMEIER und MANDL (2001, S. 466) „ist Information [...] der Rohstoff für Wissen. [...] Damit aus Information Wissen wird, muss der Mensch auswählen, vergleichen, bewerten, Konsequenzen ziehen, verknüpfen, aushandeln und sich mit anderen austauschen."

„Erst durch die individuelle Bewertung wird Information zu Wissen. Dabei ist die Gewinnung von Wissen als ein Prozess zu betrachten. Neues Wissen entsteht durch die Kombination von Wissen, durch Vernetzen, ungewöhnliche Entscheidungen und Bewertungen. Der Wert des Wissens entsteht erst durch den Zweck orientierten Einsatz".

(HERBST, 2000, S. 12)

\begin{abstract}
„Wissen (..)eine Form von Information [ist]. Alles Wissen ist Information. Aber nicht alle Information ist Wissen. Information ist eine Menge von Ja- oder Nein-Entscheidungen (...). Information ist wirklich, (...) sie ist messbar (...). Information kann entstehen, und sie kann vergehen, aber auch wieder neu entstehen. (...) Wissen dagegen ist Information, die sich auf Dinge oder Vorgänge in der Außenwelt bezieht. Auf das, was es gibt, und auf das, was es geben könnte (...) Wissen wächst. (...) Es nährt sich aus den Wurzeln der Erfahrung (...)."
\end{abstract}

(DEUTSCH, 1990, S. 187)

Wissen ist allerdings nicht als statische Struktur zu verstehen und sollte nicht als ein stabiler Entwurf repräsentiert werden. Wissen soll dynamisch sein, seine Flexibilität erlaubt es, sich dem Kontext, der Intentionalität und besonderen Bedingungen des Men- 
schen anzupassen. Wissen kann nie isoliert von den Wissensträgern, also den Menschen, betrachtet werden (vgl. VOLLMAR, 2004, S.20). „Wissen erhält Wert, indem es zweckorientiert eingesetzt wird." (HERBST, S. 11, 2000).

Bewusstseinsfähige Wissensrepräsentationen können durch verschiedene Befragungsund Testmethoden erfasst werden (WIEMEYER, 1994, S. 191). Allerdings ist eine interne Wissensrepräsentation des impliziten Wissens unfassbar, gerade weil das Wissen über Handlungen größtenteils unbewusst ist. Hinweise auf den Besitz von Wissen können nur erreicht werden, wenn es zur Lösung einer Situation durch Handeln beobachtbar gemacht wird. Eine direkte Betrachtung ist bisher unmöglich. Das Wissen selbst ist unsichtbar und auch unmessbar.

\begin{abstract}
„Der Erkenntnisgewinnung im Rahmen experimenteller kognitiver Psychologie steht immer das Problem entgegen, dass interne Repräsentationen nicht direkt beobachtbar sind. Der Findigkeit des Experimentators bleibt es überlassen, in geeigneten experimentellen Situationen Indikatoren für Struktur, Inhalt und Entwicklung von Repräsentationen sowie ihrer Beziehung zum manifesten Handeln zu finden“"
\end{abstract}

(KÖRNDLE, 1985, S. 24 Zitiert nach WIEMEYER, 1994, S. 188)

Da Wissen nicht beobachtbar bzw. messbar ist, auch nicht unter Benutzung von modernen Verfahren und Geräten wie z. B. Positronenemissionstomographie (PET) und repetitiver transkranieller Magnetstimulation (rTMS) ${ }^{5}$ (ENGELKAMP, 2006, S. 190), können wir nur das beachten, was der Besitzer mit seinem Wissen ausführen kann. Wir müssen also diejenigen Prozesse betrachten, die durch das Wissen besser oder schneller durchlaufen werden. Solche Prozesse werden bei kognitiven Aufgaben durchgeführt, denen sie ermöglichen, kognitive Leistungen zu erbringen, die sich sowohl auf implizites als auch auf explizites Wissen stützen können. Es lassen sich somit zwei Kernaussagen aus dieser Betrachtungsweise und vielfältige Definitionen von Wissen zusammenfassen:

- Wissen ist nicht messbar. Die Auswertung des Wissens, sowohl in Forschungsansätzen als auch in didaktischen Vorgehensweisen, muss auf die Ergebnisse zurückgreifen, die durch die Wechselbeziehung des Menschen mit dem Kontext entstehen, das heißt auf Ergebnisse aus Handlungen. Ein unmittelbarer Zugang zum Wissen ist (bisher) nicht möglich.

- Wissen ist nicht übertragbar. Die Entstehung des Wissens wird nicht nur durch einen Prozess der Informationsvermittlung möglich, erforderlich ist vielmehr ein Prozess von Wissensaufbau, insbesondere bei prozeduralem Wissen muss ein „implizites Lernen“ stattfinden.

${ }^{5}$ „Die modernen bildgebenden Verfahren wie PET, rMRI und rTMS haben zu einer explosionsartigen Zunahme unseres Wissens über neuronale Prozesse geführt. Trotzdem bleibt unser Wissen noch sehr beschränkt. Beide Verfahren sind auf topologische und korrelative Informationen beschränkt.

Die topologische Beschränktheit bedeutet, dass wir nur den Ort der neuronalen Aktivität kennen, nicht aber die Prozesse. Wir wissen also nur, dass diese Strukturen irgendwie an der Verarbeitung beteiligt sind. Wir kennen nicht die Wissensstrukturen und die Art ihrer Nutzung, und wir wissen noch überhaupt nichts über deren neuronale Realisierung" (ENGELKAMP, 2006, S. 190). 
Ein anderer Ansatz aus dem Wissensmanagement könnte eine Alternative zu diesem ersten Problem der Wissensauswertung liefern: die Wissenstreppe.

\subsubsection{Die Wissenstreppe}

Das Modell der Wissenstreppe aus dem Wissensmanagement könnte uns einen systematischen Zugang zu Leistungen bei Sportspielen anbieten. Von der Hierarchie des Wissens ausgehend, wäre der Entwurf didaktischer Verfahren möglich, deren Wirksamkeit auf Basis der Wissenstreppe überprüfbar wären, um sie beim Lernen von Sportspielen produktiv anwenden zu können (vgl. TSG 1899 HOFFENHEIM, 2008, S. 69).

Die Wissensstreppe (NORTH, 1998) ist eine Art von Weiterentwicklung der Wissenshierarchie. Sie ordnet weitere Konzepte zu, die vom Wissen abgeleitet werden und die sowohl für das Wissensmanagement als auch für die Entwicklung von didaktischen Maßnahmen dienen, die als Ziel den Erwerb und die Umsetzung von Wissen haben.

In der Abbildung 11 wird die Wissensstreppe dargestellt und in den folgenden Textabschnitten (ab S. 80 ff.) werden die ihr zugehörigen Konzepte verarbeitet.

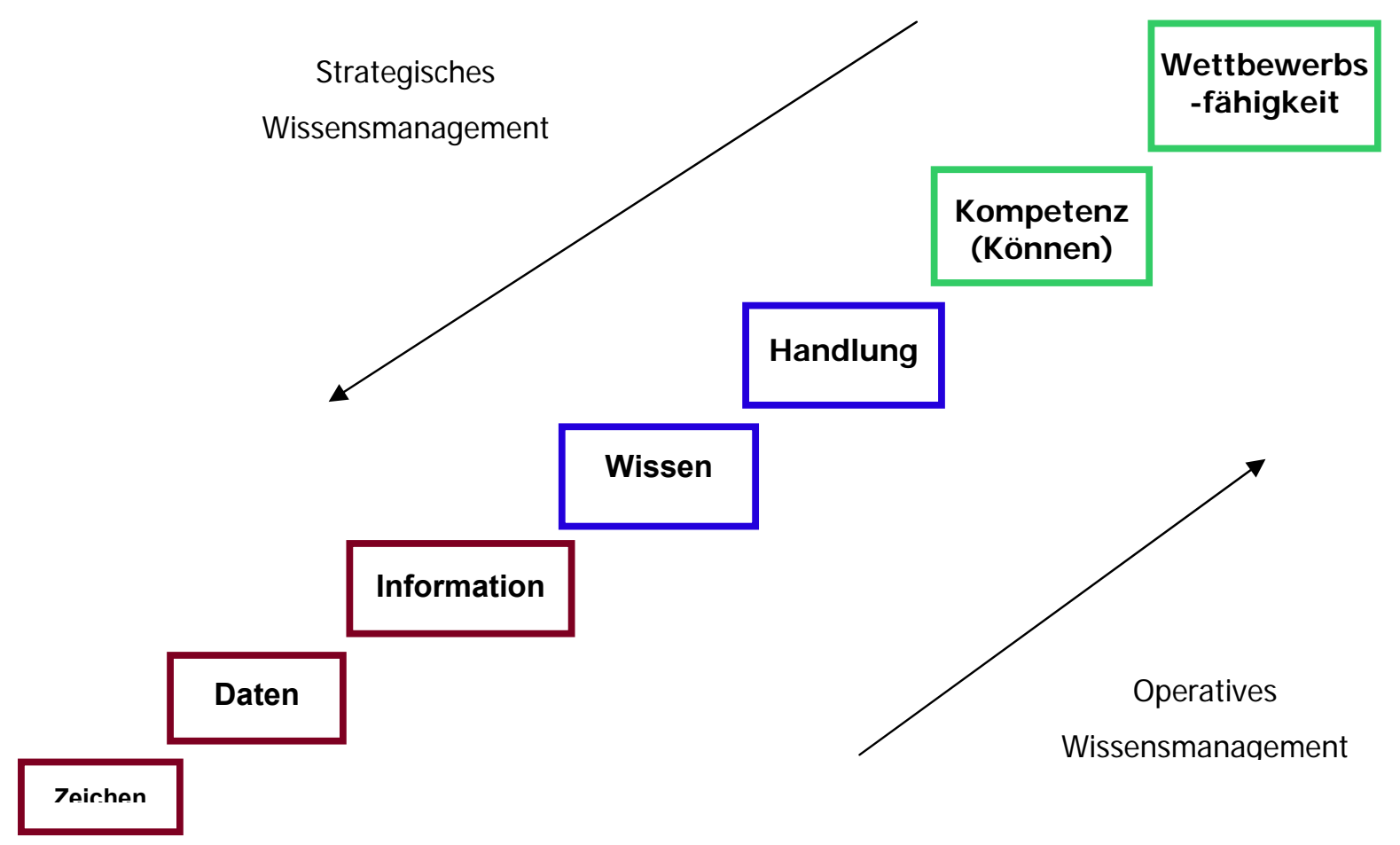

Abbildung 11: Die Wissenstreppe (modifiziert nach NORTH, 1998, S. 39) 
Durch den Durchlauf der Wissensstreppe von oben nach unten in Form eines strategischen Wissensmanagements wird versucht, die Frage zu beantworten, welche Kompetenz und, daraus abgeleitet, welches Wissen benötigt werden, um wettbewerbsfähig zu sein (NORTH, 1998, S. 41).

Durch den Durchlauf der Wissensstreppe von unten nach oben in Form eines operativen Wissensmanagements wird ab „Daten, Informationen und Wissen“ versucht, die Wettbewerbsfähigkeit zu gestalten. „Operatives Wissensmanagement hat daher auch die Aufgabe, Rahmenbedingungen zu schaffen, die Anreize für Wissensaufbau, -teilung und -nutzung bieten". (NORTH, 1998, S. 41).

Über die bereits dargestellten Kategorien der Wissenshierarchie hinaus sind in diesem Schema weitere Ebenen auf dem Weg vom Novizen zur Leistung eines Könners beschrieben.

\section{Handlung}

Die Handlung stellt die Manifestation des Wissens als individuellen Prozess in einem spezifischen Kontext dar und wird für die Stellung von Zielen und Motivation charakterisiert (NORTH, 1998, S. 40). Alle (sportlichen) Bewegungen finden in einer bestimmten Situation statt. Es scheint, dass automatisierte Bewegungen unabhängig von einer spezifischen Situation sein können. Nach ROMBACH (1987, Zitiert nach SEILER, 1995, S. 35) existiert eine solche „Situationslosigkeit“ jedoch nicht. „...Bewegungen entstehen immer in Situationen, werden in Situationen erworben, gelernt, verbessert, ausgeführt und festgestellt..." Die Bewegungen finden immer innerhalb einer Handlung statt, durch Handlung werden Beziehungen zwischen die Menschen und der Umwelt in einer bestimmten Situation wirksam und durch diese Beziehung entsteht das Verständnis der Situation.

\footnotetext{
„Handlung, Action, eine oft komplexe Abfolge von koordinierten und umweltbezogenen Bewegungen, die ein Individuum ausführt. Vom bloßen Verhalten hebt sich $\mathrm{H}$. dadurch ab, dass sie auf die Erreichung eines Zieles gerichtet ist. Das Ziel kann in einer Veränderung der Umwelt oder in einer Veränderung der Situation des Individuums in seiner Umwelt bestehen“
}

(DORSCH, 1982 Zitiert nach SCHAUB, 1993 S. 16)

Die Bewegung kann von außen bestimmt, beobachtet und sogar objektiv nachgemessen werden, aber ihre Intention und Kontrolle können nur subjektiv, d. h., von innen festgestellt werden. Der Ausführende nimmt wahr, schätzt und setzt Ziele nach dem wahrgenommenen Schwierigkeitsgrad und nach der Auswertung seiner Fähigkeiten und Wissen fest. Seine Emotionen, Motivationen, Ängste und Interessen beeinflussen in großem Ausmaß den Ablauf der Handlung. Nach SEILER (1995, S. 32) sind viele psychologische Auffassungen des Handlungsbegriffs vorhanden, die als Schwerpunkt jeweils verschiedene Aspekte haben. Aus diesem Grund sei es nicht möglich von einer homogenen Handlungstheorie zu sprechen. NITSCH und MUNZERT (1991, nach SEILER, 1995 S. 33) schlagen daher vor, von einer "handlungstheoretischen Perspektive“ zu sprechen. Die verschiedenen Auffassungen der Handlungstheorie beziehen sich auf eine besondere Übersicht des Menschen (MUNZERT, 1995, S, 80; NITSCH, 2000, S. 
49) und auf ein wissenschaftliches Verständnis der Handlung als eine hochgradig psychisch organisierte Verhaltensweise (NITSCH, 2000, S. $61 \mathrm{ff}$.).

Nach LEWIN (1926, zitiert nach SEILER, 1995, S. 33) werden Handlungen genutzt, um Ziele zu erreichen. Sie sind deswegen durch diese Ziele kontrolliert und motiviert. Damit unterscheidet sich die "handlungstheoretische Perspektive" (NITSCH, 2000, S. 51; MUNZERT, 1989, S. 1 ff.) von der „Ökologische Aktionstheorien“ (WOLLNY, S. 78 ff.); z.B. das Dynamic-System-Approach (KUGLER; KELSO \& TURVEY, 1982, S. 5 ff.). Nach diesem werden die Bewegungen durch im Kontext vorhandene „Constraints" gesteuert, denn alle bewegten Körperstrukturen müssten sich an die äußere Bedingungen anpassen, die Bewegungen würden also fremd bestimmt und kontrolliert. Im Sportbereich sind viele Ansätze der Handlungspsychologie wiederzufinden. Oft steht dabei der Versuch im Mittelpunkt der Forschung, einen Unterschied zwischen vollständig automatisierten Bewegungen oder Reflexen und einer als "gedankliches Bewegen" bezeichneten Bewegungsausführung (vgl. EBERSPÄCHER, 1987, S. 64) zu finden. Eine Handlung wäre demnach mehr als eine Bewegung. Sie zieht die Ziele, Intentionen und Situationsbezogenheit des Menschen mit in Betracht und hebt sich daher von einer bloßen mechanischen Sicht des Menschen ab.

\footnotetext{
„Die handlungstheoretischen Konzepte stellen nach unserer Einschätzung die vielversprechendsten Ansätze zu einer ganzheitlich integrativen Betrachtung der menschlichen Bewegung dar".
}

(MECHLING, 1984, S.108)

Es ist dennoch schwer, den Begriff „Handlung“ vom Begriff „Bewegung“ zu unterscheiden. Einige Bewegungen beinhalten einen hohen Grad von Stabilität und Effizienz, d. h. sie sind hoch automatisiert und sie können nicht bewusst beschrieben werden. Aus diesem Grund bezeichnen einige Autoren (MEINEL \& SCHNABEL, 1998, S. 36; MUNZERT, 1997, S. 30) die Bewegungen als die motorischen Aspekte der Handlung. Versucht man, die Bewegung ohne künstlich hergestellten Bedeutungszuweisungen zu definieren, so erhält man die Definition aus einem Lexikon.

Hier ist Bewegung (RÖTHIG\&PROHL, 2003, S. 82) aus einer reinen mechanischphysikal perspektive definiert als:

- Die Veränderung der Lage eines Körpers relativ zu einem Bezugssystem.

- Aktive Bewegung umfasst Lage- beziehungsweise Ortsveränderungen, bei denen der Organismus die benötigte Energie selbst aufbringt.

Nach MECHLING (1984, S. 111) wäre diese Definition jedoch zu eng. Sie reduziert den Begriff Bewegung auf ausschließlich physikalische Aspekte und kann deshalb nicht den kognitiven Anteil umfassen. Hingegen erscheint der Begriff Handlung wegen seiner Ausrichtung auf primär psychologische Anteile wiederum zu allgemein. Um Bewegung aus 
handlungsorientierter Sicht zu betrachten, schlägt MECHLING (1984, S. 111) folgerichtig das Konzept der „Bewegungshandlung“6 vor.

Um Klarheit über die Bedeutung des jeweiligen Begriffs zu schaffen, ist an dieser Stelle eine Definition der Beziehung zwischen die Hauptkonzepte Bewegung, Bewegungshandlung, Handlung, Wissen und Verhalten notwendig. GROEBEN (2000, S. 404) schlägt hierfür ein Modell vor, in dem Handeln „die innerlich-bewussten, intentional und kontrolliert ausgeführten Aspekte des menschlichen Agierens umfasst“. Verhalten ist für inn somit der externe, nicht frei kontrollierbare, immer in Bedienung einer speziellen Funktion ausgerichtete Anteil des Agierens. GROEBEN stellt das Tun als eine Mittelkategorie zwischen Handeln und Verhalten. Im Gegensatz dazu betrachtet SEILER (1995, S. 34) das Verhalten als ein umfassendes Konzept, das sowohl Handlungen als auch Bewegungen einschließt.

Davon ausgehend wird im Rahmen dieser Arbeit in Anlehnung an NITSCH Verhalten als ,jede Form der Lebensäußerung“ (1980, zitiert nach SEILER, 1995, S. 34) betrachtet. Handeln wird als die kleinste Einheit des Verhaltens und deswegen als das Untersuchungsobjekt betrachtet, das sowohl von freiwilligen Bewegungen und Fähigkeiten als auch von reflektiertem Denken konstituiert ist. Wissen wird betrachtet als

\begin{abstract}
„die Gesamtheit der Kenntnisse und Fähigkeiten, die Individuen zur Lösung von Problemen einsetzen [gekennzeichnet]. Dies umfaßt sowohl theoretische Erkenntnisse als auch praktische Alltagsregeln und Handlungsanweisungen. Wissen stützt sich auf Daten und Informationen, ist im Gegensatz zu diesen jedoch an Personen gebunden. Es wird von Individuen konstruiert und repräsentiert deren Erwartungen über Ursache-Wirkungs-Zusammenhänge."
\end{abstract}

(PROBST, RAUB \& ROMHARDT, 1998, S. 44)

Das Wissen, das eine Handlungsausführung ermöglicht, wird als Handlungswissen bezeichnet. Es beinhaltet alle die Merkmale, die das Lösen von Problemen innerhalb eines Kontextes erlauben und die zu erbringenden Leistungen gestalten. Der Umfang des vorhandenen Handlungswissens unterscheidet den Könner von einem Novizen. Eine Zuordnung der Konzepte Bewegung, Handlung und Handlungswissen wird in der Abbildung 12 dargestellt. Handlungswissen ermöglicht die Durchführung einer Handlung. Diese könnte man, entsprechend ihrem Kontext, als eine Alltags- Sport- oder, nach MECHLING (s.o) Bewegungshandlung genannt werden. Allerdings bestehen alle Handlungen aus Bewegungen, Intentionen und Ziele.

\footnotetext{
${ }^{6}$ Da nach der Definition von DORSCH (s.o), alle Handlungen Bewegungen umfassen, ist $\mathrm{m}$. E. das Konzept „Bewegungshandlung“ im Sport nicht nötig. Stattdessen sollte vor dem Begriff Handlung das Attribut „sportliche“ gesetzt werden, wenn es um eine Handlung im Sport geht. Man kann diese „sportliche Handlung" aus verschiedenen Perspektiven betrachten, z. B. aus psychologischer, sozialer oder aus physiologischer Sicht. Allerdings werden in anderen Sprachen (z. B. Englisch und Spanisch) Begriffe benutzt, die zur Verwirrung führen könnten. Der Ausdruck: „muscular action“ kann keine Bewegung implizieren, wenn es z. B. um eine statische Kontraktion geht. Im Spanischen kann man ebenfalls keine richtige Gleichwertigkeit des Begriffs Handlung finden.
} 


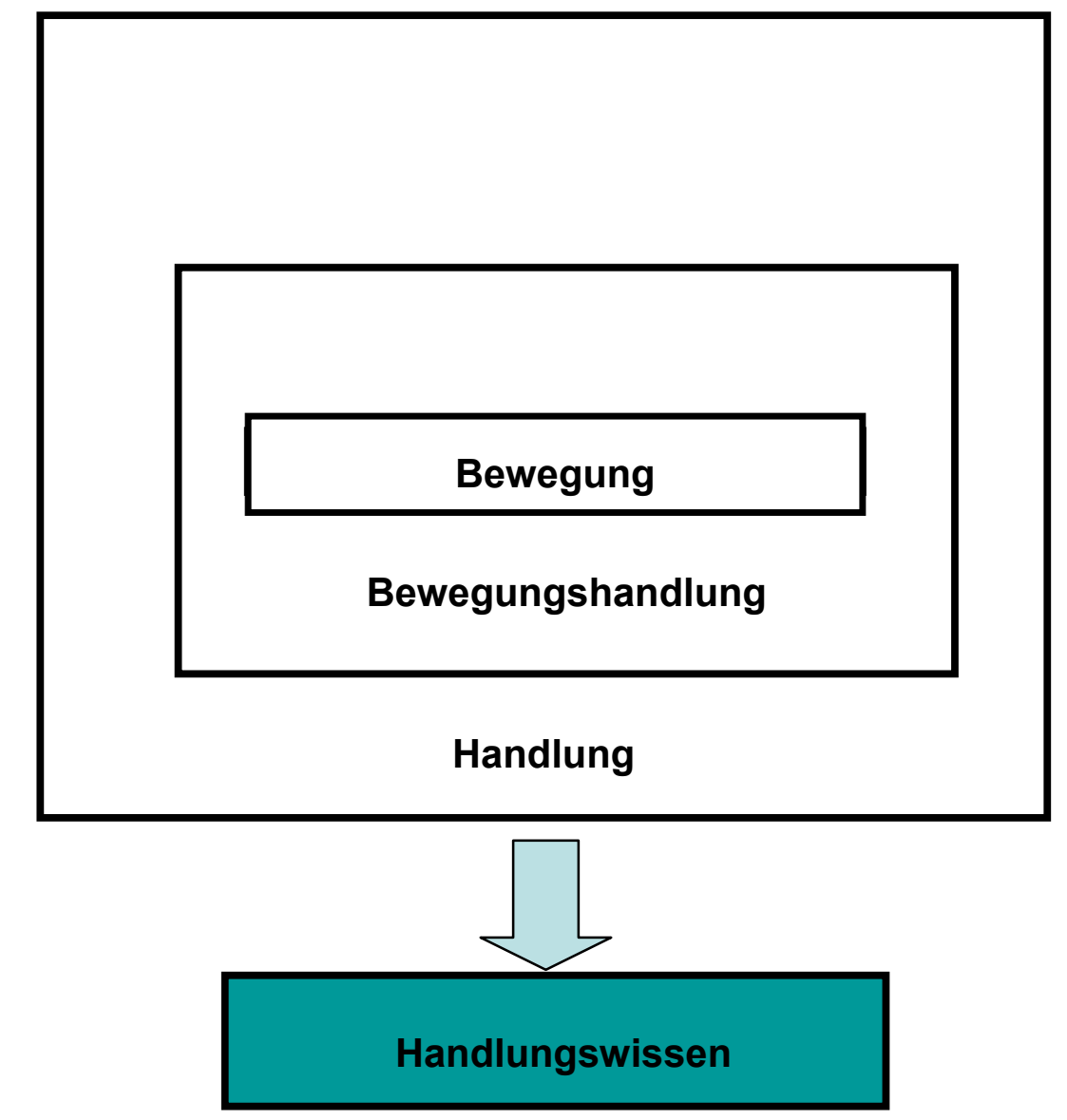

Abbildung 12: Beziehung zwischen Bewegung und Handlung

Hinsichtlich des Handlungswissens schlägt WIEMEYER (1994, S. 50 ff.) ein heuristisches Modell der Wissensrepräsentation mit vier Wissensstrukturen vor:

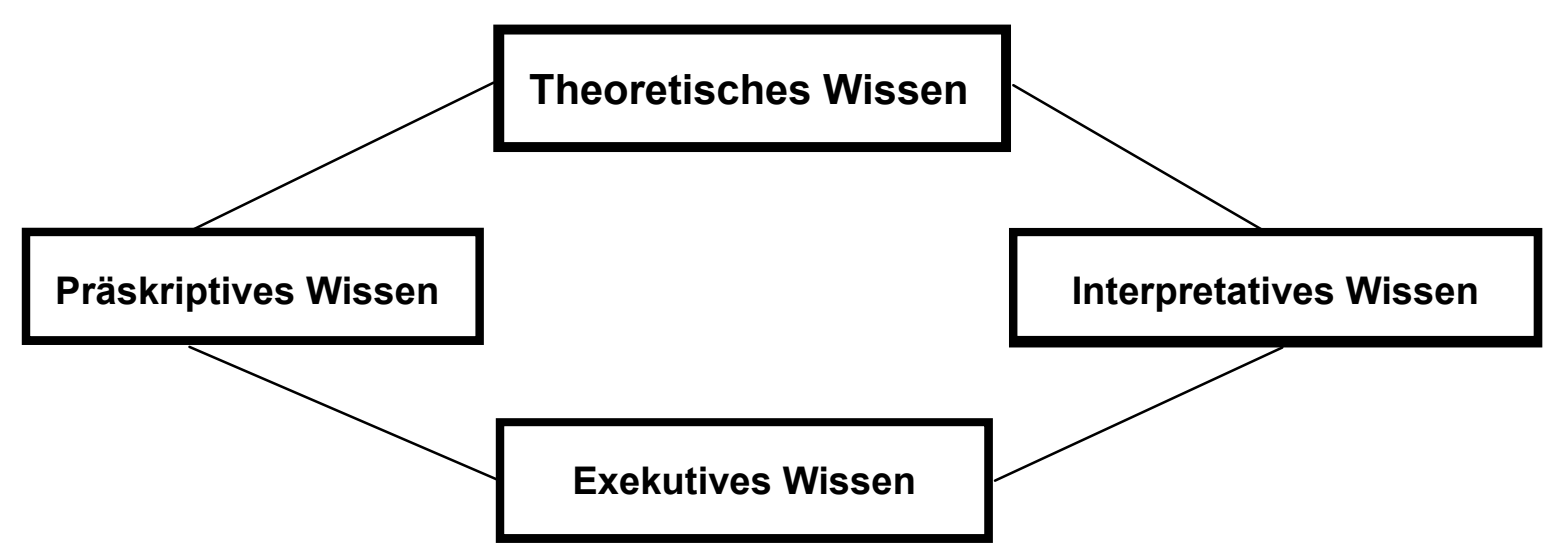

Abbildung 13: Heuristisches Modell der Wissensrepräsentation (modifiziert nach WIEMEYER, 1994, S. 90) 


\section{Theoretisches Wissen}

Als theoretisches Wissen wird auch das deklaratives Wissen bezeichnet. Dieses Wissen kann konkret (z. B. in Form von Bildern) oder abstrakt vorliegen (z. B. In Form von biomechanischen Regeln). Das theoretische Wissen bezieht sich auf eine externe Perspektive, aus der man die Bewegungen aus kinematischem oder kinetischem Blickwinkel beschreiben kann. Auch ihre Ursachen und Wirkungen können auf verschiedenen Ebenen erklärt werden: biomechanisch, neurophysiologisch, psychologisch, usw.

\section{Exekutiv-prozedurales Handlungswissen}

Es entspricht dem motorischen Anteil der Handlungen, zuständig für die Muskelkontrolle und die Bewegungsausführung. Es ist ein unbewusster Bestandteil des Wissens, der sich auf einer unteren Ebene einer kognitiven Hierarchie der Bewegungskontrolle befindet (SCHACK, 2002). Mittels seiner Prozesse können Bewegungen größtenteils automatisiert werden, obwohl sie auch bewusstseins- und willkürfähig sind.

Die Theorie der motorischen Programme (SCHMIDT, 1975, 1988, 1991, 2006) ist die bekannteste und am breitesten diskutierte (WIEMEYER, 1994, S. 52 ff.; MUNZERT, 1989, S. 162 ff.) Theorie für die Erklärung der Bewegungsausführung und -kontrolle. KUGLER, KELSO und TURVEY $(1980,1982)$ schlagen einen „Dynamic Systems approach" vor. Vor allem im deutschsprachigen Raum wird eine handlungstheoretische Perspektive für die Bewegungsausführung vorgeschlagen (NITSCH, 2000, S. 51; MUNZERT, 1997, S. 17; GRÖBEN, 2000).

\section{Präskriptives Handlungswissen}

Diese Struktur beinhaltet indikationale und spezifikationale Aspekte der Handlungen. Sie beziehen sich auf die Fragen des „Was?" und „Wie?" der Handlung. Hier werden vorzeitig die sensorischen Wirkungen der Handlungen vorweggenommen. Der Ausführende antizipiert die spezifischen sensorischen Wirkungen, die gemäß der Situation produziert werden, wenn die Handlung ausgeführt wird. Diese antizipierten Efferenzen könnten mehr oder weniger punktuell sein und können auf verschiedene Aspekte der Handlung bezogen werden, z. B. auf die Endposition der Bewegung oder Eindrücke, die während der Bewegungsausführung wahrgenommen werden. Das präskriptive Bewegungswissen kann verbessert werden, insofern die Handlungserfahrung sich erweitert. Nach jeder Wiederholung werden mit Hilfe des interpretativen Wissens Schlussfolgerungen gezogen, die zum Verfeinern der Vorschritte und Antizipationen der Handlung beitragen.

\section{Interpretatives Handlungswissen}

Durch die Wahrnehmung der eigenen oder fremden Handlungen wird das interpretative Handlungswissen gebildet. Die Wahrnehmung ist subjektiv und es werden subjektive Hypothesen aufgebaut, die in Beziehung mit dem Kontext stehen „...jede Wahrnehmung ist die Bestätigung oder Zurückweisung einer Hypothese, die jemand über die Welt, 
über die Erscheinungsweise oder das Verhalten anderer - oder über sich selbst hat" (PÖPPEL, 1985, S. 67, zitiert nach WIEMEYER, 1994, S. 84).

Das interpretative Wissen wird zeitlich nicht nur nach der Handlung, sondern auch während ihrer Ausführung hervorgerufen. Im Augenblick der Auseinandersetzung mit der Umwelt entsteht ein "Gefühl des Richtigen oder Falschen“, das von den Handlungsergebnissen bestätigt oder verneint wird.

\section{Interaktionsprozesse zwischen den Wissensstrukturen}

Zwischen den Wissensstrukturen finden Prozesse von Induktion und Deduktion statt. Einerseits wird das präskriptive und interpretative Wissen vom theoretischen Wissen beeinflusst (deduktive Richtung). Andererseits wird theoretisches Wissen abhängig vom präskriptiven und interpretativen Wissen aufgebaut (induktive Richtung). Diese Prozesse finden sich $u$. a. bei den folgenden Phänomenen:

- Vor der Ausführung der Handlung, wird präskriptives Wissens aus theoretischem Wissen abgeleitet.

- Während und nach der Ausführung werden sensorische Wirkungen (Feedback) hervorgerufen.

- Nach der Ausführung werden die sensorischen Wirkungen mit dem präskriptiven Wissen verglichen und es wird interpretatives Wissen daraus abgeleitet.

- Die Beziehungen zwischen den Strukturen entwickeln sich als Produktionsregeln „wenn-dann“ (ANDERSON, 1996, S. 279).

Im Lauf des Lernprozesses wird schrittweise das präskriptive Wissens verbessert, es entstehen immer mehr, konkretere und genauere sensorische Wirkungen. Diese Prozesse werden $u$. a. durch ständige Produktion von interpretativen Wissensstrukturen begünstigt und ergeben ihrerseits immer mehr differenzierte, konkretere und spezifischere Aktionsregeln.

\section{Kompetenz}

Kompetenzen beziehen sich im Allgemeinen auf komplexe Fähigkeiten, die verschiedene Entwicklungsebenen besitzen und in vielfältigen Situationen des Lebens zum Ausdruck kommen. Sie sind Hinweise der verschiedenen Entwicklungsgrade in bestimmten Bereichen (ARCOFADER, 2007). Jede Kompetenz ist eine Synthese der Erfahrungen und des Wissens, die die Person im Rahmen ihrer Umweltkonstellationen ausgebildet hat. Nach NORTH (1998, S. 40) unterscheidet "die Kompetenz, Wissen zweckorientiert in Handlungen umzusetzen (sic!)[...] den Lehrling vom Meister".

Im Sport, wo zahlreiche Fähigkeiten konvergieren, bezeichnen die Kompetenzen drei Bereiche der Zulänglichkeit: „Was zu tun ist“ (Wissenskompetenz), „Wie etwas zu tun ist" (prozedurale Kompetenz) und "Wann etwas zu tun ist" (kognitive Kompetenz). Ergänzt werden sie von der Motivation für den Bereich "überhaupt etwas zu tun" (psychologische Kompetenz). „Diese Fähigkeit oder Kapazität wird auch als Kompetenz einer Person oder Organisation bezeichnet. Kompetenzen konkretisieren sich im Moment der Wissensanwendung." (NORTH, 1998, S. 40). Die Kompetenzen werden in bestimmten Handlungssituationen verifiziert. KROG und ROSS (zitiert nach NORTH, 1998, S. 40) 
drücken dieses Merkmal wie folgt aus: „We view competence as an event, rather than an asset. This simple means that competences do not exist in the way a car does; they exist only when the knowledge (and skill) meet the task". Auf diesem Weg wird, wie in Abbildung 14 dargestellt ist, in mehreren Schritten aus Information schlussendlich Kompetenz.

\section{Können}

Können berührt das Umsetzen von Wissen in Fertigkeiten. Es umfasst den Bereich "Wissen wie" als Teil des Wissens (know-how im englischen Sprachraum) und repräsentiert vor allem die Anwendung des impliziten Wissens (vgl. NORTH, 1998, S. 40). Eine Unterscheidung zwischen „Kennen“ und „Können“ scheint hier sinnvoll.

Kennen bezeichnet das theoretische Konstrukt. Es bezeichnet die Prozesse der Informationsverarbeitung, die im Langzeitgedächtnis gespeichert sind. Es wird daher oft auch als theoretisches Wissen bezeichnet. Dieser Fall liegt vor, wenn z. B. ein Trainer Informationen und Hinweise über das richtigen Verhalten in einer Spielsituation übermittelt, die eine erfolgreiche Auslösung dieser Situation durch einen schon geprüften Spielzug erlaubte. Vom richtigen Begreifen der Instruktionen hängt das Wissen der Spieler $\mathrm{ab}$, den Spielzug richtig ausführen. Allerdings wird das Wissen erst dann gefestigt, wenn die Spieler in einer Spielsituation erfolgreich den Spielzug anwenden und die Ruckmeldungen während und nach der Ausführung verarbeiten und Schlüsse aus diesen Rückmeldungen ziehen: der richtige Zeitpunkt der Umsetzung, die möglichen Hindernisse und Missverständnisse, die Auswirkungen der Handlungen der Mitspieler, die möglichen Reaktionen der Gegenspieler usw. Nur nach der erfolgreichen Umsetzung des Spielzuges und dem Erreichen des Zieles kann man bestimmen, dass die Spieler „wissen“, wie man diese Spielsituation auslösen kann, also, dass sie den Spielzug „können“, weil sie über das Wissen verfügen, wie dieser Spielzug erfolgreich umzusetzen ist.

Das Begreifen der Hinweise des Trainers ermöglicht den Spielern, Information zu erwerben. Wenn sie die gewünschten Wirkungen nach der Umsetzung des Spielzuges erreichen, verfügen sie über prozedurales Wissen, also „Können“. Wenn sie nach Anfrage ausführlich verdeutlichen könnten, wie man eine solche Situation lösen kann, verfügen sie über deklaratives Wissen, d.h., über „theoretisches Wissen“, also „Kennen“. Können bezeichnet die Kompetenz, d. h. die Fähigkeit zur korrekten Lösung der entsprechenden Aufgabe und es wird oft als prozedurales Wissen bezeichnet (vgl. BLASER, STUCKE \& WITTE; 1992, S. 117; ANDERSON, 1996, S. 233).

\footnotetext{
„Das Problem des Zusammenhangs zwischen Wissen und Können ist kein akademisches Problem, sondern für die Sportpraxis von eminenter Wichtigkeit. Richtung und Art des Zusammenhangs sind entscheidende Bedingungen dafür, wie motorisches Lernen im Sport organisiert werden muss.

[...] Jedes Lernen besteht grundlegend in einer Einheit von Wissen (Kognition) und Können (Servomotor). So wie einerseits die Aneignung von Wissen zumindest mittelbar auf sensomotorischem Verhalten aufbaut, enthält andererseits jede Aneignung von Können stets kognitive Komponenten
}

[ ...] Es gäbe kein Wissen ohne Können, wie es kein Können ohne Wissen gäbe“

(DAUGS \& BLISCHKE, 1984, S. 381, zitiert nach WIEMEYER, 1994, S. 17) 
Nach meiner hier vertretenen Ansicht wird Wissen nicht nur als kognitiver Inhalt bezeichnet. Kognition ist ein Teil des Wissens. Er kann nicht ohne den sensomotorischen Teil als Wissen bezeichnet werden. Das heißt, dass hinsichtlich der Handlung weder Kennen noch Können alleine Wissensbestände bilden, man benötigt vielmehr beide, um über Handlungswissen zu verfügen.

Wie in der Abbildung 14 dargestellt ist, wird Kennen ab dem Erhalt der Information verstanden. Wenn das Kennen in eine Handlung umgesetzt wird und die Aufgabe erfolgreich gelöst wird, entsteht Können. Das Wissen, das aus Kennen und Können besteht, kann nicht beobachtet werden, es muss sich durch Kompetenz äußern. Die Kompetenzen sind ihrerseits eine Darlegung des richtigen Handelns, das Handlungswissen als unterlegte Basis hat.

Es finden sich in der sportwissenschaftlichen Literatur vielfältige Kompetenzarten, z.B. motorische Kompetenz (HIRTZ, 1998, S. 17), koordinative Kompetenz (GLASSAUER, 2003, S. 37-38), Sozialkompetenz (BORGGREFE, THIEL \& CACHAY, 2006, S. 6). Die unterschiedlichen Kompetenzarten designieren imAllgemeinen die individuellen Eigenschaften, Kenntnisse, Fähigkeiten und Fertigkeiten aus, die ein Mensch besitzt und anwenden kann, um die Anforderungen einer Aufgabe wirksam zu erfüllen und Situationen zu bewältigen.

Bei den Sportspielen werden in der sportwissenschaftlichen Forschung überwiegend drei Kompetenzarten berücksichtigt, die für die Erfüllung der spezifischen Spielanforderungen erforderlich sind. Diese drei Kompetenzarten scheinen angemessen an die individuellen Leistungsvoraussetzungen des Sportspiels zu sein und werden daher bei dem Modell der Spielhandlungskompetenzen auch berücksichtigt:

Kognitive Kompetenz: Verständnis des Spiels und Fähigkeit für die richtige Antwort auf die Frage „Was machen?" während des Spiels. Das theoretische Wissen wird auch von vielen Autoren als kognitive Kompetenz eingeordnet.

Prozedurale Spielkompetenz: richtiges Handeln innerhalb des Spielkontextes. Die Spielkompetenz konkretisiert alle zugehörigen Fähigkeiten und Fertigkeiten der Spieler. Wegen ihrer Öffentlichkeitswirksamkeit werden oft viele davon auf nur technische Fertigkeiten reduziert.

Psychologische Kompetenz: Spielbereitschaft bewusst auf alle Voraussetzungen und Wirkungen gerichtet, die das Spiel verlangt. 


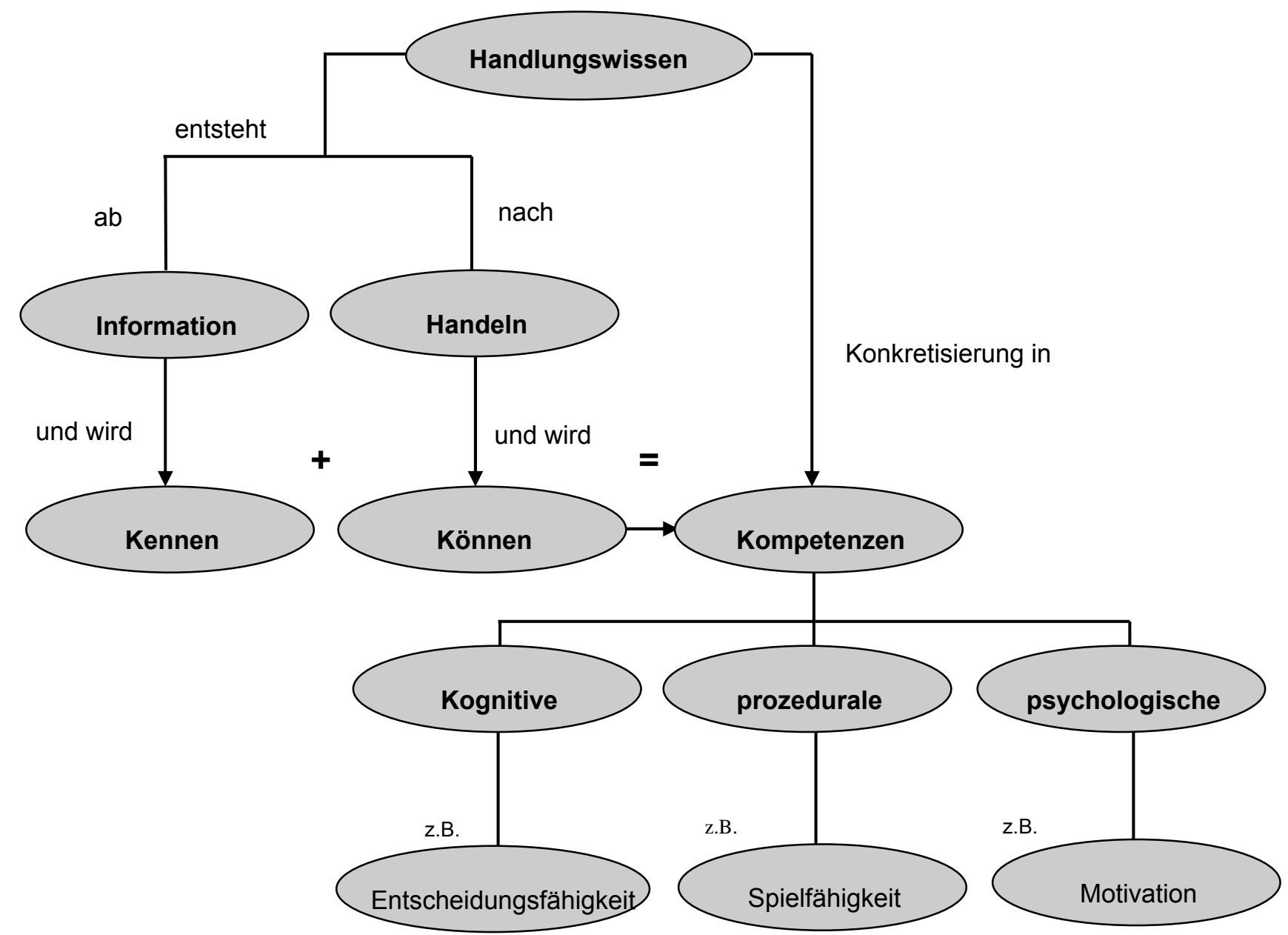

Abbildung 14: Flussdiagramm zur Darstellung der Entstehung und Bestandteile des Handlungswissens und sein Ausdruck als Kompetenzen

\subsubsection{Das Grundprinzip des Modells}

Das Grundprinzip des Modells der Spielhandlungskompetenzen basiert auf der Argumentation, dass der Lernende den Lerninhalt kognitiv verarbeiten muss, bevor er das Wissen durch Bewegungen äußern kann, d.h., der Spieler muss beim Lernen einen Prozess von Verständnis durchlaufen, um Wissen anzueignen und dies auch äußern zu können (siehe die Wissenshierarchie und das DIKW Modell auf Seite $70 \mathrm{ff}$.). Das Modell betont den Prozess vom „Denken und sich danach Bewegen“. Auf diesem Weg versucht man, eine kognitive Verarbeitung des Lehrinhalts und dadurch den Aufbau des Wissens anzuregen. Da die Spieler jederzeit taktisches und technisches Wissen bei Spielsituationen anwenden müssen, wird dieses Wissen auch am besten durch das Spielen aufgebaut. Die Vermittlung von Informationen durch den Trainer eignet sich nicht für die Förderung der kognitiven Fähigkeiten (siehe eine Kritik an das Direkte Mo- 
dell auf Seite $8 \mathrm{ff}$.). Die Spieler sollen ihr Spielverständnis, ihre Fähigkeit, das „Spiel zu lesen" und ihre Entscheidungsfähigkeit verbessern. Damit sollen sie die Abhängigkeit vom Trainer lösen, um ihre eigene Spielgestaltung zu schaffen und dadurch handlungsfähiger zu werden.

In Sportspielen hat der kognitive Bereich vor dem physiologischen Bereich Priorität, denn er limitiert in der Regel die Spielleistung (IGLESIAS, GARCÍA-GONZÁLEZ, GARCÍA-CALVO, LEÓN \& Del VILLAR, 2010, S. 463). Während einer Spielsituation ist es von unumstrittener Relevanz, dass das Wissen darüber wichtig ist, „was“ in dieser Situation zu machen ist und es auch bedeutsam ist, zu wissen, „wie“ es zu machen ist. Allerdings sollte, wenn es um das Spielen geht, das „Was“ an erster Stelle entschieden werden. Die Spieler erkennen und lösen zuerst eine Spielsituation im kognitiven Bereich, was dann die Leistung im motorischen Bereich erleichtert. Jedoch ist die alleinige Entwicklung des kognitiven Bereiches nicht genug. Es muss auch eine Entwicklung des motorischen Bereiches erfolgen, damit die Spieler ihre kognitive Verarbeitung ausführen können. Sie müssen über jede der vier verschiedenen Arten von Wissen verfügen (siehe auf Seite $84 \mathrm{ff}$. die vier Wissensstrukturen von WIEMAYER), um das Hauptziel des Modells zu erreichen, das „kompetente Handeln“. Sobald die Spieler die kognitiven Fähigkeiten entwickelt haben und auch gelernt haben, diese während des Spiels anzuwenden, sind sie bereit, die beiden Bereiche bei einer Spielhandlung interaktiv einzusetzen. Es ist ein einzigartiger Lernprozess, in dem Denken und Bewegung kombiniert werden, um die komplexe Spielsituation erfolgreich lösen zu können. Die Trainer müssen hierfür das Potenzial und die Anforderungen jeder problematischen Situation erkennen.

Innerhalb der Spielsituationen ist es der Spieler selbst, der die Entscheidungen treffen soll. Diese Entscheidungen sollen geeignet sein, um die im Spiel vorhandenen Probleme zu lösen. Der Trainer kann dem Spieler im Spiel nicht immer sagen, was er zu tun hat, wann er es tun muss und wie die Umsetzung zu erfolgen hat. Der Trainer hat also während des Spiels kaum Inzidenz auf die Handlungen der Spieler. Mit Hilfe der Entwicklungen in der Sportpädagogik und der kognitiven Psychologie (RICHARD \& WALLIAN, 2005, S. 20) hat sich die Perspektive beim Lehren und Lernen der Sportspiele geändert. Von einer Fokussierung auf das Wissen des Trainers schwenkte die Perspektive zu einer Fokussierung der Entwicklung der Spieler. Ein Spieler muss nicht die richtige Lösung für alle Probleme des Spiels auswendig lernen, sondern er soll seine Autonomie und Fähigkeiten für das Treffen geeigneter Entscheidungen und das Finden geeigneter Lösungen in komplexen Situationen des Spiels ausbilden. Infolgedessen wurden neue Herangehensweisen an Trainingsprozesse entwickelt und ausprobiert. Herangehensweisen, in denen der Trainer Informationen übermittelt, werden zu Ansätzen umgewandelt, in denen der Spieler sein Wissen aufbauen soll. Allgemein wird das Prozess-Produkt-Paradigma (z.B. die Schema Theorie von SCHMIDT, 1975; 1991; SCHMID \& WRISBERG, 2008) im Sport schrittweise zu Gunsten von Fragestellungen über den Prozess des Wissensaufbaus ersetzt. Aus einer konstruktivistischen Perspektive ist die Frage nicht „was“ die Spielern lernen sollen, sondern „wie“ sie es lernen (BROPHY \& GOOD, 1986, S. 360). Diese Perspektive weist darauf hin, dass das Ziel des Lernens das Verständnis sein sollte. Nach dieser konstruktivistischen Perspektive ist es daher erforderlich, dass die Spieler sich an solchen Handlungen beteiligen, die Denk- und Reflektionsprozesse für das Problemlösen erwecken (RICHARD \& WALLIAN, 2005, S. 21). Die Spieler lernen dann, diese Prozesse auf eine effektivere Weise anzuwenden und Aktionsregeln aufzubauen (vgl. ANDERSON, 1996, S. 279). Im 
Nachhinein sollten die Spieler ihr Verständnis dieser Aktionsregeln durch deren Anwendung zeigen, indem sie neue Spielsituationen lösen.

"Constructivism and inquiry go hand and hand; they are two parts of the same coin. The teacher assesses what students know, and whith this knowledge of the learner, the teacher plans new experiences and knowledge to be gained by the student. Constructivist teaching uses the student's prior knowledge as a building block to integrate new understandings with prior learning. To accomplish this, the teacher plans active experiences to involve students in explorations, theory building, and experimentation so that students can generate and organize data, and communicate with others. As the learner gains new knowledge, previously held beliefs and ideas may change as new interpretations are made"

(LEMLECH, 1998, S. 136)

Eine Reihe von didaktischen Modellen wurde vorgeschlagen, um indirekten Lernstrategien anzuwenden und die taktische Bewusstsein und das Verständnis beim Spiellernen zu fördern. Das TGfU-Modell wurde dabei am gründlichsten erforscht und bietet deshalb die beste theoretische und empirische Begründung für die Anwendung solcher Modelle. Die indirekten didaktischen Modelle fokussieren die Evolution der kognitiven Fähigkeiten der Spieler und ihr Spielverständnis. Dieses wird als ein Prozess charakterisiert, in dem den Spielern problematische Spielsituationen vorgestellt werden und ihnen danach eine Reihe von Fragen gestellt wird, die in Richtung der richtigen Lösung der Situation orientiert sind. Somit haben indirekte Modelle einen mehr zum Konstruktivismus orientierten Bedeutungsumfang als das Direkte Modell. Die indirekten Modelle fördern die Ausbildung von autonomen Spielern durch ihre eigene Aneignung des Handlungswissens fürs Spielen (RICHARD \& WALLIAN, 2005, S. 22).

Entsprechend der Auffassung, dass das Lernen eine Funktion des Kontextes sei, d.h., das Lernen wäre situativ, wurde die Theorie des Situierten Lernens entworfen und durch Beiträge mehrerer Forscher weiterentwickelt (vgl. LAVE \& WENGER, 1991, S. 18). Die Theorie des Situierten Lernens hat ihre Vorläufer in GIBSON (Theory of Affordances), VYGOTSKY (Social Learning) und SCHOENFELD (Mathematical Social Problem).

\footnotetext{
"The theory of situated learning claims that knowledge is not a thing or set of descriptions or collection of facts and rules. We model knowledge by such descriptions. But the map is not the territory: Human knowledge is not like procedures and semantic networks in a computer program. Human knowledge should be viewed as a capacity to coordinate and sequence behaviour, to adapt dynamically to changing circumstances....
}

Because knowledge is not a thing or set of descriptions, we do not learn by transferring facts and rules from one head to another. Understanding how learning is a process of conceiving an activity, and activities are inherently social, puts emphasis on improving learning addressing issues of membership, participation in a community, and identity".

(CLANCEY, 1995, S. 49)

Die zwei grundlegenden Prinzipien des Situierten Lernens sind (LAVE \& VENGER, 1991, S. 22):

1. Das Wissen soll innerhalb eines authentischen Kontextes präsentiert werden, d.h., in Situationen, wo das Wissen einbezogen wird. 


\title{
2. Lernen braucht soziale Interaktion und Zusammenarbeit.
}

KIRK und MACPHAIL (2002) stellen die Perspektive des "Situated Learnig“ in den Sportspielen dar. Diese Perspektive nimmt an, dass sich der Lernende, statt fremde Information zu empfangen, die erst internalisiert werden müssten, aktiv an der Umwelt beteiligen sollte, um sich die Erfahrungen besser anzueignen und so letztlich Verfasser des eigenen Lernens zu werden. Die kontextuelle Interaktion ist dabei das wichtigste Element des Wissensaufbaus. Die fremde Information des Trainers kann als zusätzliche Rückmeldung betrachtet werden, die zur Ausbildung eines Referenzrahmens beiträgt. Sie kann aber nicht die Wahrnehmung der sensorischen Effekte ersetzen, die während der Spielsituationen geliefert werden (GRÉHAIGNE \& GODBOUT, 1995, S. 492).

Die indirekten didaktischen Modelle entsprechen der Theorie der Konstruktivistischen Didaktik. Diese ist eine Anwendung des interaktionistischen Konstruktivismus von REICH (2006), der die Bedeutung der „kulturellen und lebensweltlichen Interaktionen bei der Re/De/Konstruktion von Wirklichkeiten beachtet und analysiert" (REICH, o. J.a, S. 1).

Gemäß der Konstruktivistischen Didaktik ist Lernen ein konstruktiver Prozess, worin die Handlungen immer im Kontext stattfinden. Es basiert auf der Verknüpfung von Handlungsstufen von John DEWEY, die „beim Lehren und Lernen miteinander vermittelt werden müssen" (REICH, o.J.b, S. 180): emotionale Antwort, Definition des Problems, Hypothesenbildung, Testen, Experimentieren und Anwendung.

\begin{abstract}
"Lernende sollen untersuchungsähnliche Beobachtungen, Explorationen, gegenseitigen Austausch, Evaluationen durchführen; sie sollen in einer motivierenden Lernumgebung entdeckendes Lernen praktizieren, wobei der Erwerb neuen Wissens dominant sein soll; ein diskursives Verständnis und eine gemeinsame Wissensaneignung sind erwünscht; Partizipation ist ein Schlüssel zum erfolgreichen Lernen; bei geleiteter Instruktionspraxis muss Anschluss an bisheriges Lernen gehalten werden und neue Situationen müssen neues Lernen provozieren."
\end{abstract}

(REICH, 2006, S. 38)

Die Indirekten Modelle fördern das Aktionsdenken. Dies ist die wichtigste Eigenschaft der konstruktivistischen Perspektive. Nach GRÉHAIGNE, GODBOUT und BOUTHIER (1998, zitiert nach RICHARD \& WALLIAN, 2005, S. 25) kann das "Reflection in Action“ durch einen Prozess erreicht werden, der von folgenden Merkmalen gekennzeichnet ist:

- Die Spieler explorieren lassen. Die Spieler werden mit Spielhandlungen konfrontiert, in denen sie technische und taktische Probleme lösen müssen. Die modifizierten Spielsituationen betonen die zu verbessernden Handlungen und fördern die Generierung von Lösungen für die Problemstellungen, die in dieser Situation auftreten.

- Die Meinungsäußerung fördern und über die vorhandene Spielsituationen reflektieren lassen. Durch offene Fragen führt der Trainer den Sportler zur Identifikation des Problems und zur Generierung vernünftiger Lösungen.

- Diskussionen hervorrufen. Der Trainer versucht eine Diskussion hervorzurufen und moderiert diese durch spezifische zur Lösung orientierte Fragen. 
- Die Lösungen ausprobieren lassen. Sobald die Spieler vernünftige Lösungen erschaffen, soll der Trainer die Spielumwelt vereinfachen und es erlauben, diese Lösungen während des Spielens auszuprobieren.

Die Reflektion während der Aktion und der Austausch von Ideen sind aus konstruktivistischer Perspektive entscheidende Faktoren des Lernens. Der Trainer soll zur Verarbeitung von Aktionsregeln durch die geeignete Interpretation der positiven Elemente des Spiels beitragen. Dazu muss er sich darüber bewusst sein, welche situationsangemessenen Möglichkeiten es gibt, die Spielhandlungen auszuführen. RICHARD und WALLIAN (2005, S. 28) drücken den Mentalprozess während des reflektierenden Spielens wie folgt aus:

\begin{abstract}
„....student's reasoning is based on cause and effect: <If I respond in a certain way, then this will happen>. These connections between causes and effects need to be tested. The only way to test these new strategies is to experiment with them during game play action. This experimentation in action will verify the appropriateness of situations of the same nature. The Debate of Ideas is a never-ending process of reflective thinking and planning of future action (strategies)“.
\end{abstract}

Mit der Anwendung von indirekten Lernstrategien können die Trainer den Spielern eine strukturierte Umwelt zur Verfügung stellen, um sie so zu einer reflektierten Aktion zu führen (in der Aktion reflektieren und über die Aktion reflektieren). Durch die kontinuierliche Lösung derartiger Spielsituationen kann man einen "reflective reflex" entwickeln (COBB, 1986, S. 304).

„....Without reflection, students can only stumble blindly from one trial to another hoping for random success or waiting for an outside observer to tell them what to do next. In either case, no real understanding develops“

(RICHARD \& WALLIAN, 2005, S. 29)

Aus einer konstruktivistischen Perspektive sollten die Spieler die Fähigkeit verbessern, die Spielhandlungen aufmerksam zu beobachten, zu analysieren, sie zu reflektieren, sich angemessene Lösungen für die problematischen Spielsituationen vorzustellen und diese im Spiel auszuprobieren. Sie verarbeiten und lernen Aktionsregeln, wenn sie die sensorischen Effekte während der Ausführung einer Spielhandlung wahrnehmen, die sie als Lösungsform anwenden. All das führt innerhalb eines Spielkontexts zur Schaffung eines "Situated Learning" und damit sowohl explizit als auch implizit zum Aufbau des Handlungswissens für das Spielen.

„... Students will have truly learned if, when faced with a problem that is new but compatible with the resources they have at their disposal, they can suceed, thus demostrating that they have transformed and stabilized their initial behavior and identified and verbalized the actions rules that made their success possible".

(GRÉHAIGNE \& GODBOUT, 1998, zitiert nach RICHARD \& WALLIAN, 2005, S. 30)

\title{
Annahmen über das Lernen und die Aufgabe des Trainers im Sport
}

MEZTLER (2005a) erläutert bei seiner Vorstellung der didaktischen Modelle des Sports zunächst die Annahmen jedes einzelnen Modells über das Lehren und Lernen. In Anlehnung daran werden im Folgenden die Annahmen über das Lernen und Lehren dargestellt, die beim Entwurf des Modells der Spielhandlungskompetenzen berücksichtigt 
wurden. Die folgende Annahme über das Lernen und Lernen wurden aus konstruktivistischen Lerntheorien abgeleitet, die indirekte didaktische Modelle begründen, sie wurden an die Didaktik der Sportspiele angepasst und dienen als Leitfaden des vorgeschlagenen Modells (vlg. z.B. GARRISON, 1993, S. 203; SHUELL, 1987, S. 415; PRATT, 2002, S. 4; METZLER, 2005a, S. 221; REICH, 206, S. 74; JANK \& MEYER, 1991, S. 74; BUTTLER \& McCAHAN, 2005, S. 35; LINDA, GRIFFIN \& PATTON, 2005, S. 9; RICHARD \& WALLIAN, 2005, S. 25).

\section{Annahmen über das Lernen}

Die folgenden Annahmen über das Sportspiellernen haben zur Konzeption des Modells der Spielhandlungskompetenzen geführt, entsprechende didaktische Maßnahmen wurden für jede dieser Annahmen in Modell entwickelt:

- Die Spieler besitzen Vorwissen, welches sie für den Aufbau neuen Wissens nutzen. Das Lernen im kognitiven Bereich ist bei den Sportspielen wichtiger als das Lernen im motorischen Bereich.

Es wird im Modell davon ausgegangen, dass das Spielverständnis am besten durch didaktische Lernstrategien erreicht wird, bei denen die Lernenden versuchen, ausgehend von schon vorhandenem Wissen neues Wissen zu erzeugen. Die meisten Spieler haben schon Spielerfahrung außerhalb des Sportvereins gesammelt. Das selbstorganisierte Ballspiel trägt auch zu implizitem Lernen bei. In dem Modell spielen die Lernenden von Anfang an, verfeinerte technische Fertigkeiten sind keine Voraussetzung dafür.

- Das Lernen im kognitiven Bereich ist bei den Sportspielen wichtiger als das Lernen im motorischen Bereich.

Der Einsatzpunkt des Modells ist das Handlungswissen. Die problematischen Situationen, die innerhalb jedes Spiels vorhanden sind, erfordern die Anwendung von theoretischem, prozeduralem und präskriptivem Wissen.

- Lernen ist im Wesentlichen ein Prozess des Problemlösens. Er wird vollzogen, indem der Lernende Vorwissen nutzt, um Lösungen vorzuschlagen, die er anschließend durch Bewegung, aber auch verbal oder durch visuelle Mittel äußern kann.

Das Hauptziel des Modells der Spielhandlungskompetenzen ist, das Lernen problematischer Spielsituationen zu lösen. Im Zentrum des Lernprozess steht der Lernende als Problemlöser.

- Taktisches Bewusstsein ist wichtiger als Bewegungsfertigkeiten, aber beide werden für die Spielleistung benötigt.

Das Modell berücksichtigt das Training der technischen Fertigkeiten, aber nicht als unabdingbare Voraussetzung für das Spielen. Die Technik wird durch ein selbstständiges Lernen geübt und verfeinert.

- Spieler können taktisches Bewusstsein und Entscheidungsfähigkeiten entwickeln, wenn diese die Hauptziele des Trainings darstellen. 
Der Schwerpunkt des Modells ist nicht die Schulung technischer Fertigkeiten, sondern das taktische Bewusstsein und die geeignete Ausführung, um auf die besonderen Anforderungen der Spielsituation angemessen reagieren zu können.

- Taktisches Bewusstsein und Entscheidungsfähigkeiten sollten unter einer konstruktivistischen Perspektive trainiert werden. Außerdem sollen sie eine geplante Abfolge der auf taktischen Problemen basierenden Lernaufgaben darstellen.

Der Lerninhalt wird aus einer konzeptuellen Sicht definiert, z. B. durch Spielhandlungen wie „Freilaufen“, „sich anbieten“, „Mitspieler begleiten“, „defensive Aufstellung" usw.

- Das Lernen kann unabhängig vom Trainer stattfinden.

Bei Anwendung des Modells werden die Spieler mit einigen Lernmaterialien ausgestattet. Dadurch können sie selbst Aufgaben für das Lernen der technischen Fertigkeiten oder für die Verbesserung der konditionellen Fähigkeiten ausführen.

- Wie alle Arten von Lernen gelingt kognitives Lernen vor allem dann, wenn die Komplexität der Aufgabe mit der kognitiven Entwicklung des Lernenden übereinstimmt.

Es wird bei Anwendung des Modells den Spielern erlaubt, Fortschritte in ihrem eigenen Tempo zu machen. Dies geschieht in Abstimmung mit ihrer Eignung, Lernfähigkeit und den Aufgabeinhalten. Begabtere und erfahrenere Spieler werden darin gefördert, die Aufgaben so schnell wie möglich zu lösen. Weniger erfahrene und unbegabtere Spieler werden aufgefordert, sich so viel Zeit für die Problemlösung zu nehmen, wie sie benötigen.

- Spieler können unterschiedliche Bereitschaft, Motivation, Interessen und Bedürfnisse haben und in verschiedenen Geschwindigkeiten lernen.

Spieler entwickeln sich weiterhin mit individueller Geschwindigkeit, also so schnell, wie sie können oder so langsam, wie sie Zeit benötigen.

- Die meisten Spieler finden es interessanter, motivierender und sehen mehr Bezug zum Wettkampf, wenn im Training gespielt wird. Das reine Üben der Bewegungsfertigkeiten wird zumeist weniger geschätzt.

Während der meisten Zeit der Trainingseinheit widmen sich die Spieler bei Anwendung des Modells dem Spiel mit seinen wesentlichen Bestandteilen, d.h., Ball, Mitspieler, Gegenspieler und Tore.

- Das Lernen findet besser statt, wenn die Lernaufgaben persönliche Bedeutung für die Spieler haben.

Der Lernprozess wird im Modell individuell gefasst. Jeder Spieler ist selbst für das eigene Lernen verantwortlich.

Annahmen über die Aufgabe des Trainers

Auch bezüglich der Rolle des Trainers liegen der Konzeption des Modells einige wesentliche Annahmen zugrunde.Bei Anwendung des Modells sollen die Trainer diefolgendenHinweise beachten: 
- Der Trainer ist ein Spielexperte und Wissensträger. Er dient aber nicht als Vermittler dieses Expertenwissens. Vielmehr nutzt er es, um den Spielern indirekte Lerngelegenheiten anzubieten und den Umgang mit problematischen Spielsituationen zu lernen.

- Der Trainer erleichtert das Lernen der Spieler. Er stellt Fragen, die das Erforschen der Aufgaben und ihrer Lösungen und damit die Kreativität der Spieler fördern.

- Der Trainer stellt direkte und indirekte Strategien zusammen. Direkt sind Strategien, bei denen der Trainer eine Situation vorbereitet und die Spieler durch einen bestimmten Lerninhalt hindurchführt. Indirekt sind die Strategien, mit denen der Trainer die Spieler auffordert, das Spiel durch Spielen zu erforschen, eigenständig zu überlegen und die Spielprobleme selbst zu lösen.

- Die Hauptaufgabe des Trainers ist das Denken der Spieler zu fördern, was auch zur Entwicklung der motorischen Fertigkeiten führt.

- Der Trainer sollte als die Haupthilfsquelle der Spieler während des Trainings dienen, er sollte jedoch keineswegs jede Lernaufgabe zu kontrollieren versuchen.

- Die Fragestellungen sind die Hauptvermittlungsformen des Wissens. Sie müssen an die intellektuellen Fähigkeiten der Spieler angepasst sein.

- Der Trainer soll die Spielprobleme identifizieren und die Trainingsaufgabe so organisieren, dass die Spieler sich auf die Lösung dieser Probleme fokussieren.

- Der Trainer benutzt kleine modifizierte Spiele, um die Lernaufgabe zu gestalten, die die taktische Bewusstsein und motorischen Fertigkeiten entwickeln, welche nötig für die Spielleistung sind.

- Alle Spielaufgaben müssen an die Leistungsebene der Spieler angepasst sein.

- Das Engagement und Lernen der Spieler wird effektiver, wenn sie lange unabhängig vom Trainer arbeiten.

- Es ist möglich und erwünscht, wirklich individuelles Lernen zu ermöglichen.

- Der Trainer sollte die Spieler dahin führen, sich gemäß den besonderen Werten des Sports zu verhalten. 


\subsection{Anwendungsstruktur}

Die Sportspiele werden nach HERNANDEZ (2005, S. 119) als Sportarten von Kooperation und Opposition eingeordnet. Wenn einer dieser Anteile fehlt, wie es normalerweise der Fall ist, wenn traditionelle Lehr- und- Trainingsansätze eingesetzt werden, wird ihre Essenz umgestaltet. Eine Handlung ohne Opposition wird niemals im Spiel vorhanden sein. Ohne Gegnereinfluss trainierte Handlungen sind damit keine Spielhandlungen mehr. Sie können damit nur als isolierte Übungen zugeordnet werden.

Aus traditioneller Sicht werden die Sportspiele nach dem Prinzip vom Leichten zum Schweren untergliedert, um das Lehren im Training zu ermöglichen. Diese analytische Herangehensweise provoziert aber gleichzeitig, dass im Moment der Gestaltung der Teile in ihrer spielnahen Gesamtheit das Ensemble nicht fließend funktioniert. Was isoliert gut klappt, funktioniert nicht in einem Oppositionskontext, wo Gegnereinwirkung und Zeitdruck zentrale Merkmale des Spieles darstellen. Die technischen Fertigkeiten können in isolierten Situationen durchaus hoch präzise sein (z. B. beim Korbwurf ohne Gegenspieler, Dribbeln um Hütchen, Torschuss mit statischem Ball, usw.), aber in Spielhandlungen sind die Wahrnehmungen und Spielanforderungen ganz anders als bei der vormalig isolierten Übung. In den meisten Fällen kann der Spieler mit Hilfe des Trainers die Einzelbestandteile des Spieles ausreichend verarbeiten, d. h. konditionelle und koordinative Fähigkeiten und technische Fertigkeiten hinreichend entwickeln. Allerdings ist der Spieler im Moment des Zusammenfügens während des Spiels überfordert, da er die Einzelstücke nun unter Gegnereinwirkung und erhöhtem Zeitdruck zusammenpassen muss.

In dieser Arbeit wird daher vermieden, das Spiel künstlich zu teilen. Vielmehr soll es simplifiziert werden. Das impliziert ein vereinfachtes Spiel, welches die Essenz von Kooperation und Opposition nicht verliert. Didaktische Strategien werden vorgeschlagen und als ein didaktisches Modell zusammengebracht. Einige dieser didaktischen Strategien wurden bei unterschiedlichen Inhalten innerhalb des Schulsportkontextes ausprobiert, überprüft und validiert (vgl. METZLER, 2005a). Im Vereinssport werden einige Anpassungen nötig, das Prinzip sollte aber dennoch durch dieselben Strategien mit Erfolg für die Ausbildung junger Spieler angewendet werden können. Andere didaktische Strategien wurden entwickelt, um die Bestandteile des Handlungswissens, Kennen und Können, dem die Spielkompetenzen zugrunde liegen, aufbauen zu können

Der Vorschlag besteht aus verkleinerten und vereinfachten Spielen, einer Modifizierung der Small Sided Games (SSG) die im Folgenden als Kleine Fußballspiele mit unterschiedlichen Schwerpunkten (KFSS) bezeichnet werden sollen. Kennzeichnend für diese Art von Spielen sind „Jokerspieler“ (ein Spieler der für beide Mannschaften spielt) und Überzahlspiele (größere Spielerzahl bei einer der Mannschaften). Solche Spiele werden mit Kombinationsspielen (KS), d. h. Spieler-Mitspieler-Ball und mit Eins-gegenEins-Spielen (1:1), d. h. Spieler-Ball-Gegner ergänzt. Hinzu kommen im Bedarfsfall ergänzende oder erläuternde Übungen (Ü), d. h. Situationen der Art Spieler-Ball. Der prozentuale Anteil von KFSS wird größer sein als der von Kombinationsspielen, Zweikämpfen und Übungen. Mit dieser Gestaltung und Kombination wird versucht, den Trainingsschwerpunkt zugunsten der Spiele im Kontext zu verschieben und so das Erwerben und Entwickeln der Spielkompetenzen für den Wettkampf zu ermöglichen.

Die KFSS können gemäß der in einer bestimmten Zeit zu verarbeitenden Kompetenzen angepasst werden. Es geht um eine handlungsorientierte, globale Methode mit fokus- 
sierter Aufmerksamkeit (SANCHEZ, 1986, S. 128). Durch Wissenslernen von manipulierten Spielhandlungen, einhergehend mit zu lösenden Spielaufgaben, wird ein implizites Lernen ausgelöst. Die Spielziele bilden die Orientierung für die Ausführung und Verbesserung der Spielhandlung. Dieser Lernvorschlag basiert auf Ansätzen wie Teaching Games for Understanding (TGfU) (BUNKER \& THORPE, 1986), der Heidelberger Ballschule (ROTH, 1999) und dem Modell des genetischen Lernens (LOIBL, 2001). Im Unterschied zu diesen Ansätzen strebt der Vorschlag jedoch keine allgemeine Schulung der Spielfähigkeit an, d. h. ein Lernen mit Transfermöglichkeit zu vielfältigen Sportspielen, sondern es wird ein spezifisches Lernen des Spiels bei einer bestimmten Sportart beabsichtigt. Das hier vorgeschlagene Verfahren wird dadurch statt an das Spiellernen in der Schule an das Sportspieltraining im Verein in unteren und höheren Altersklassen angepasst. Dies geschieht nach den Konzepten des „Game Sense Approach" (LIGHT, 2005a).

Das Sportspiel kann wie folgt gegliedert und damit vereinfacht werden:

Ebene 1 - Spieler-Ball-Mitspieler-Gegenspieler-Tore.

Ebene 2 - Spieler-Ball-Mitspieler.

Ebene 3 - Spieler-Ball-Gegenspieler.

Ebene 4 - Spieler-Ball.

Die erste Ebene sollte mindestens $60 \%$ der Trainingseinhalte umfassen und kann in mehrere Komplexitätsstufen aufgegliedert werden. Dies gelingt durch das Variieren der Spielmerkmale. Dies sind z. B. Anzahl Mitspieler/Gegner, Größe des Feldes und der Tore und durch die Aufstellung besonderer Regelungen, die die Aufmerksamkeit auf die Entwicklung der gewählten Kompetenzen richtet. Die zweite Ebene kann damit z. B. den Kombinationsspielen zugeordnet werden und sollte $20 \%$ der Trainingseinhalte umfassen. In der dritten Ebene finden Zweikampfübungen statt und diese Ebene sollte 15 $\%$ der Trainingsinhalte umfassen. Die letzte Ebene umfasst $5 \%$ der Trainingsinhalte und besteht aus Übungen für das Training der technischen Fertigkeiten, da die Abwesenheit der Gegenspieler die essentielle Charakteristik der Opposition der Sportspiele aufhebt. Dadurch werden die Spielhandlung, die Koordination und die Technik ausgebildet, die eine ganzheitliche motorische Grundausbildung der Spieler ermöglichen (SCHULZ \& ELSÄßER, 2010, S. 5). Wenn diese Merkmale unter Berücksichtigung der Motivation ausgeformt werden, entstehen Spielkompetenzen, die richtige Spielhandlungen ermöglichen. Über eine lange Zeitspanne betrachtet wird schlussendlich Handlungswissen ausgebildet. Dieses Handlungswissen wirkt sich seinerseits wieder auf die Spielkompetenzen aus und es entsteht ein wiederholter spiralförmiger Prozess, der die Spielfähigkeit steigen lässt und nebenbei die Wettbewerbsfähigkeit der Spieler verbessert.

Im Folgenden werden die Ziele, Inhalte und Lernmethode des Modells näher beschrieben. 


\subsubsection{Ziele, Lernmethode und Inhalte}

Ziele, Lernmethoden und Inhalte sind die wichtigsten Merkmale eines didaktischen Modells (vgl. JANK \& MEYER, 1991, S. 51 ff.). Diese Merkmale prägen das Modell und unterscheiden es von den anderen. Anschließend werden diese Merkmale des Modells der Spielhandlungskompetenzen beschrieben, damit wird angestrebt, einen Leitfaden für die Trainer anzubieten, die das Modell als tauglich für die Spielerausbildung innerhalb der Sportvereine betrachten könnten.

\section{Ziele}

Das Hauptziel des Modells ist der Aufbau von Handlungswissen. Wie im Abschnitt 2.1 (s. Seite 5) behauptet wird, liegt als herausragende Leistung eines Experten das Handlungswissen zugrunde. Die Ausbildung von Sportspielexperten wird dann angestrebt, wenn sie wirkungsvoll die Anforderungen des Spiels erfüllen und schlussendlich auf die Steigerung der Sportspielleistung ihren Mannschaften beitragen.

Die Spielanforderungen verwirklichen sich als Anforderungen auf die Spielfähigkeit, die Koordination und die Technik:

Spielfähigkeit: Die Auseinandersetzung mit Gegenspieler, durch richtiges Handeln zugunsten der eigenen Mannschaft zu entscheiden und dadurch eine vorteilhafte Feldstellung oder eine Überzahlsituation zu schaffen.

Koordination: Präzisionsanforderungen bei engem Spielraum, bei intensiven gegnerischen Einwirkungen und unter Druckbedingungen leisten. ROTH (2000, S. 177) bezeichnet diese Druckbedingungen als Zeitdruck, Präzisionsdruck, Komplexitätsdruck, Organisationsdruck, Belastungsdruck und Variabilitätsdruck. Diese sportspezifische informelle Betrachtungsweise unterscheidet sich von einer fertigkeitsorientierten Betrachtungsweise,welcher im Gegensatz zu einer handlungsorientierten eher eine bewegungsorientierte Argumentation zugrunde liegt und die nach einer sportartübergreifenden allgemeinen Grundausbildung strebt.

Technik: Die technischen Anforderungen der Sportspiele sind nicht zu streng wie diese der einzelnen Sportarten. Eher als die absolut beste Ausführungsform einer Bewegung, wird von den Spielern ein „heuristisches Lösungsprinzip unter Berücksichtigung der jeweiligen Person-, Aufgaben- und Umweltmerkmale“ verlangt (NITSCH \& NEUMAIER, 1995, S. 41). Daher wird beim Modell die folgende Definition von Technik berücksichtigt:

\footnotetext{
„Unter 'Technik' werden Routineverfahren zur situationsspezifischen Lösung sportlicher Bewegungsaufgaben verstanden. Hierin sind sowohl sportartspezifische Bewegungsformen als auch deren situationsabhängige Kombination und Variation eingeschlossen. Grundlage jeder Technik ist die Entwicklung von senso- und psychomotorischen Koordinationsmustern“.
}

(NITSCH \& NEUMAIER, 1995, S. 42)

Das Handlungswissen wird als Kompetenz ausgedrückt, daher wird ein Kompetenzansatz durch das Modell eingeführt, wo sich die unterschiedlichen Anforderungen der Sportspielleistung zuordnen lassen. 
Den drei bei diesem Modell ausgewählten Kompetenzbereichen entsprechen auch die Hauptbereiche des menschlichen Handelns und werden daher am meisten von der Sportspielforschung berücksichtigt (siehe Stand der Forschung über die Wirksamkeit der Spielvermittlungsmodelle auf S. 57).

Die kognitive Kompetenz befähigt den Spieler, richtige Entscheidungen zu treffen, um durch sein Handeln die Frage „was machen in der Spielsituation“ zu beantworten, d.h. wie im Fall der Experten eine geeignete Antwort auf die Umweltkonstellationen und Handlungsbedingungen zu finden (siehe den Absatz von Novizen zum Experten auf S. 7). Die prozedurale Spiel-Kompetenz befähigt den Spieler, die Frage „wie das machen“ zu beantworten und mit der Ausführung von genauen und schnellen Handlungen komplizierte, außergewöhnliche und unvorhergesehene Spielaufgaben zu meistern. Die psychologische Kompetenz befähigt den Spieler, ständig motiviert an dem Spiel teilzunehmen.

Das Zusammenspielen aller drei Kompetenzbereiche bringt spielspezifische Handlungskompetenz zum Ausdruck, um angemessen auf die vielfältigen Spielanforderungen agieren und reagieren zu können. Spielhandlungskompetenzen bedeuten in diesem Zusammenhang, wettbewerbsfähig zu sein. In Abbildung 15 wird diese Aufstellung dargestellt: 


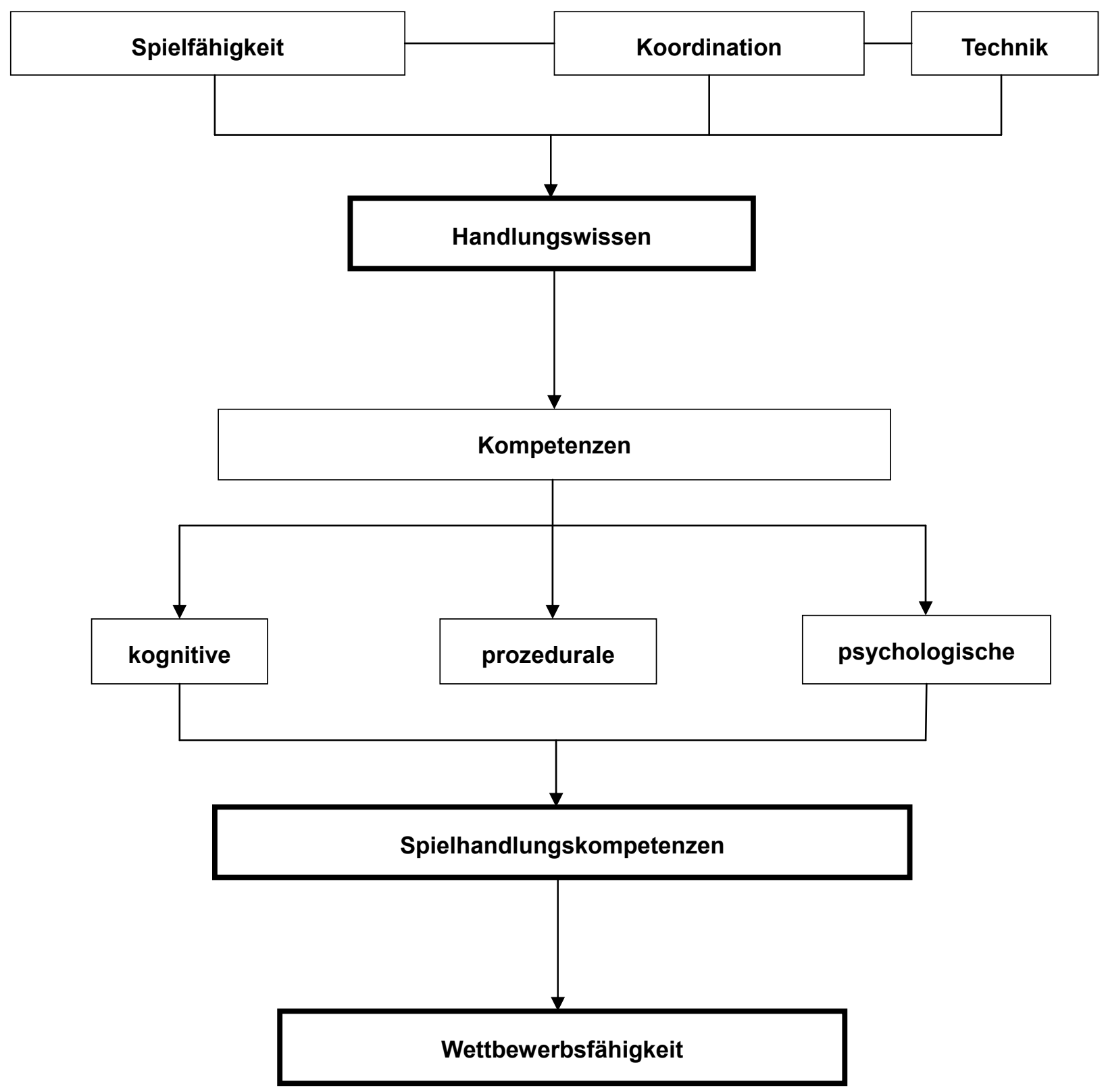

Abbildung 15: Ziele des Modells der Spielhandlungskompetenzen

\section{Lernmethoden}

Eine Lernmethode ist die "Art und Weise des Stoffs Vermittlung“ (JANK \& MEYER, 1991, S. 54). Es geht um ein organisiertes Verfahren, die ausgeführt wird, um ein bestimmtes Lernziel zu erreichen. Im Rahmen seiner Theorie der konstruktivistischen Didaktik ordnet REICH (2007) eine breite Auswahl von Lernmethoden einem „konstruktiver Methodenpool“ zu. Diese Lernmethode könnte bei jenen Ausbildungsmaßnahmen zur Anwendung kommen, die auf diese Theorie basiert werden. Die handlungsorientier- 
ten Lernmethoden eignen sich besonders für die Erreichung der Ziele des in dieser Arbeit vorgeschlagenen Modells, ihre Umsetzung findet durch die Implementierung von didaktischen Strategien statt (s. Inhalte in S. 102). Lernmethode und didaktische Strategien lassen sich beispielweise wie folgt zusammenfügen:

- Bei Anwendung von der Lernmethode „problembasiertes Lernen“ könnte man die Handlungsfähigkeit, die Entscheidungsfähigkeit und die Fähigkeit für ein autonomes Spielen fördern, wenn im Training die Spieler an einem Spiel teilnehmen,welches als Erprobung des Wettkampfspiels dient.

- Bei Anwendung einer „Befragungsmethode“ könnte man durch Fragen das taktische Bewusstsein der Spieler fördern.

- Bei Anwendung der Lernmethode „problembasiertes Lernen“und auch der „Befragungsmethode" könnte man die Spielfähigkeiten durch die Implementierung der didaktischen Strategie der kleinen Fußballspiele mit unterschiedlichen Schwerpunkten (KFSS) fördern.

- Bei Anwendung der Lernmethode „kooperatives Lernen“ könnte man durch die didaktische Strategie der Kombinationsspiele die kognitiven Fähigkeiten und dabei die Koordination der Spieler mit fördern.

- Bei Anwendung der Lernmethode „Partnerarbeit“ könnte man durch Implementierung der didaktischen Strategie der Zweikämpfe die individuelle Taktik gezielt üben.

- Wenn der Trainer die Lernmethode „Freiarbeit“ zuweist, könnten die Spieler selbständig die technischen Fertigkeiten üben.

Viele andere Lernmethoden könnten bei der Implementierung einer dieser didaktischen Strategie zur Anwendung kommen, je nach gewünschten Lernzielen. Unter Umständen könnten auch zunächst die didaktischen Strategien ausgewählt und anschließend die Lernmethode bestimmt werden, z. B. wenn Erziehungsziele wie Kreativität, das Lernen zu lernen, Durchhaltevermögen usw. im Vordergrund stehen.

Die Abbildung 16 stellt den Zusammenhang zwischen Lernmethoden und didaktischen Strategien dar. 


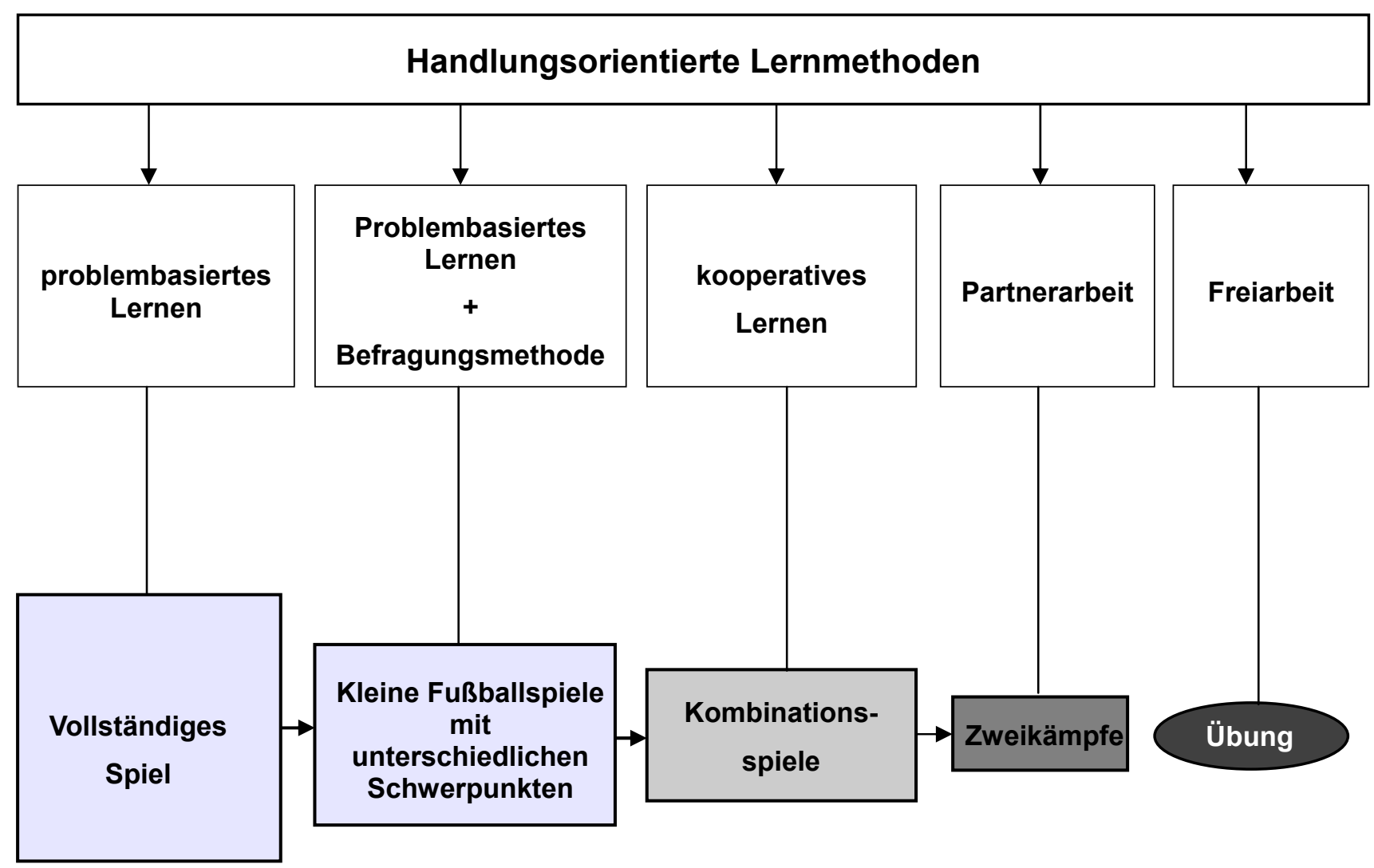

Abbildung 16: Methode des Spielhandlungskompetenzen-Modells

Inhalte

Eine didaktische Strategie ist ein organisiertes Verfahren,welches aus einer Gesamtheit von Aktionen der Lehrer/Trainer und den Lernenden besteht. Die didaktische Strategie ist auf ein bestimmtes Lernziel orientiert und wird von dem Lehrer/Trainer gestaltet und mit der Absicht geplant, das Lernen zu ermöglichen. Im Folgenden werden die didaktischen Strategien des Modells beschrieben.

\section{Kleine Fußballspiele mit unterschiedlichen Schwerpunkten}

Das Modell der Spielhandlungskompetenzen ist auf die taktischen Aspekte des Spiels zentriert und es hebt die intelligente Anpassung der technischen Fertigkeiten an die Spielsituationen hervor. Das Lernziel ist die taktische Bewusstsein der Spieler. Es wird bewusst darauf verzichtet, das Techniktraining als Baustein des gesamten didaktischen Vorhabens in den Mittelpunkt zu stellen. Vielmehr wird die Technik als eine Hilfsgröße zum Erreichen der taktischen Ziele betrachtet, die mit der Intention der Spieler übereinstimmen. Die Schulung der technischen Fertigkeiten wird zwar noch immer als grundlegender Bestandteil des Trainings betrachtet, ihr wird jedoch keine Priorität eingeräumt. Die Technik wird ein Mittel zum Zweck der Zielerreichung der Handlung und wird dieser daher untergeordnet. Die technische Veredlung wird als eine Variable der Leistung angestrebt, die Bewegungen müssen aber dem Spielkontext angepasst werden. 
Weiterhin wird bei der Mehrheit der didaktischen Modelle der Schwerpunkt auf den Angriff gelegt und die Verteidigung wird vernachlässigt. Im Standardfall wenden die Trainer die folgende Oppositionssequenz für die Schulung der Taktik an (LASIERRA, 1990, S. 60; NIEDLICH \& SCHMIDT, 1996, S. 89):
$1: 0$
$2: 0$
$1: 1$
$2: 1$
$2: 2$

Wenn die Verteidiger zum Einsatz kommen, sollen sie sich zunächst passiv verhalten, werden anschließend semi-aktiv und schließlich aktiv. Das impliziert die Berücksichtigung des Angriffs als primär und die Abwehr von gegnerischen Angriffen als sekundäres Ziel, was erst zu einem späteren Zeitpunkt der Spielerausbildung und auf hohem spielerischem Niveau zur Anwendung kommen sollte. Dennoch ist es notwendig, in allen Sportspielen offensive und defensive Stärken im Gleichgewicht zu halten. Jeder offensiven Handlung sollte durch eine defensive Handlung entgegengewirkt werden, die Bedingungen von Kooperation und Opposition schließen mindestens 50\% der Zeit in den defensiven Handlungen ein. Schon in frühem Alter sind beide Situationen immer im Spiel vorhanden, die Kinder spielen gegeneinander und nehmen an Wettkampfspielen teil.

Die Kleinen Fußballspiele mit unterschiedlichen Schwerpunkten (KFSS) stellen pädagogische Situationen bereit, in denen eine synchronisierte Verkettung von offensiven und defensiven Handlungen vorhanden ist. Solche Handlungen entsprechen einer Gegenüberstellung von Interessen und Zielen. Auf diesem Weg wird versucht, die Entwicklung der Entscheidungsfähigkeit durch den andauernden Prozess des Lösens von Spielproblemen zu fördern. Es werden gezielt Lernziele für Angreifer und Verteidiger gesetzt. Jede Spielsituation bezieht daher ein zweifaches Lernziel ein, was die Ausnutzung der zur Verfügung stehenden Lernzeit optimiert. Die Intention dieses Vorgehens ist es, intelligente, entscheidungsfähige, mit Erfahrungen und Spielhandlungswissen ausgestattete Spieler auszubilden.

Die Spielhandlungen werden drei möglichen Spielsituationen zugeordnet, in denen sich die Spieler im Verlauf des Spiels befinden können (HERNANDEZ, 2005, S. 152):

- Der Spieler selbst ist am Ball.

- Der Spieler selbst ist ohne Ball, seine Mannschaft ist jedoch in Ballbesitz.

- Die Mannschaft des Spielers ist nicht in Ballbesitz.

Die gestellten Probleme sind spezifisch für jede Spielsituation. Die KFSS erlauben andauernd und im Spielkontext die Übung der Spielhandlungen. Es werden kontinuierlich die drei Spielsituationen vollzogen, sie ermöglichen ein situiertes Lernen. Es wird davon ausgegangen, dass Lernziele und Lernaufgaben für diese drei Spielsituationen innerhalb des Spielkontextes eingesetzt werden sollen. Die Lernziele sollen dafür je nach Spielsituation an den Übungen der Spielhandlungen orientiert werden.

Um die physiologischen, technischen und taktischen Leistungsvoraussetzungen zu verbessern, sollten die Spieler eine chronologische Exposition von effektiven Trainingssitzungen durchlaufen (REILLY, 2005, S. 20). Sie modellieren ähnliche Anforderungen wie der Wettkampf, berücksichtigen dabei aber die Beschränkungen des Trainingsprogramms durch die zur Verfügung stehende Zeit und die Sportanlagen. Je nach Schwerpunkt kombinierenoder betonen die KFSS die physiologischen, technischen und taktischen Trainingsanregungen (IMPELLIZZERI, RAMPININI, MAFFIULETTI, CASTAGNA, BIZZINI \& WISLOFF, 2008, S. 1). Die bei Erwachsenen gemessene physiologische 
Wirksamkeit legt nahe, dass die physiologischen Anforderungen der KFSS denen im Wettkampf mit reglementierter Spieleranzahl ähnlich sind. Somit eignen sich die KFSS auch für die Entwicklung der konditionellen Fähigkeiten der Spieler (HOFF, 2002; JONES \& DRUST, 2007).

Die US Youth Soccer Association (SNOW \& THOMAS, 2005, S. 9) fasst die Vorteile der KFSS wie folgt zusammen. Die Kleinen Spiele erlauben und fördern folgende Bereiche:

- Den Ball mehrmals berühren.

- Grundlegende taktische Probleme mehrmals Erfahren.

- Erfahrungen bei allen Spielphasen sammeln, da jeder Spieler häufig an Abwehr und Angriff teilnehmen muss.

- Die taktische Bewusstsein verbessern.

- Das Spiel vereinfachen.

- Die Motivation.

- Mehr individuelle Spielbeteiligung, was die Fitness verbessert.

- Die Verantwortung, da jeder Spieler selbst sowohl viele Schussgelegenheiten bekommt, als auch viele Schussgelegenheiten des Gegners zu verhindern hat. Das fördert das Selbstwertgefühl und das Selbstvertrauen.

- Die Meinungsfreiheit, da ein Spieler während des Spielauflaufs jeweils Angreifer, Abwehr und Mittelspieler sein darf. Die Kinder postieren sich in den Spielpositionen, in denen sie sich komfortabel fühlen.

- Das Spiel selbst ist spielerzentriert, der Trainer kann nicht alles kontrollieren.

- Viele Spieler können gleichzeitig spielen, ein normal großes Feld kann in viele kleine Felder unterteilt werden.

\section{Kombinationsspiele}

Die Kombinationsspiele sind Spiele ohne Opposition, die für die Förderung der kognitiven Fähigkeiten wie Aufmerksamkeit, Konzentration und Antizipation entworfen wurden. Jedes Spiel erfordert die gleichzeitige Teilnahme mehrerer Spieler. Sie passen sich den Ball zu und wechseln ihre Feldstellung nach jedem Pass. Nach jedem Wechsel sollen die Spieler ihre Aufmerksamkeit darauf lenken, dass ein neuer Pass sie erreichen kann. Dieser kann, ausgehend von der momentanen Situation, aus verschiedenen Richtungen kommen und kann von einem unbestimmten Mitspieler ausgeführt werden. Jedes Spiel beginnt mit einfachen Pass- und Laufsituationen, die Komplexität wird progressiv gesteigert. Das Hinzufügen eines zweiten Balls erhöht die koordinativen Anforderungen und das Ausführungstempo. Die Richtungswechsel der Pässe und Laufwege provozieren eine kognitive Dissonanz ${ }^{7}$ (BECKMANN, 1984, S. 10 HOWARTH, 2005, S. 102). Die Prozesse von Identifizierung und Auflösung der kognitiven Dissonanzen fördern die kognitive Entwicklung durch die Verbesserung der Aufmerksamkeit, Konzentrationsund Antizipationsfähigkeit (SCHRÖDER, 2001, S. 185). Jedes Spiel beinhaltet Spielmuster, die die spielende Gruppe lösen soll. Es handelt sich um ein spezifisches Muster

${ }^{7}$ Nach FESTINGER (1957, S. 12, Zit. Nach BECKMAN, 1984, S. 10), „..entsteht kognitive Dissonanz dann, wenn zwei Kognitionen nicht zueinander passen oder das Gegenteil des einen Elementes aus dem anderen folgt". 
der Bewegungsrichtung des Balles und ein spezifisches Muster der Laufwege der Spieler, die gleichzeitig durchgeführt werden müssen.

Die Spielmuster werden vom Trainer verändert, sobald sie von den Spielern bewältigt wurden. So gelingt es, den Schwierigkeitsgrad und die Komplexität der Situation fortlaufend zu steigern. Hat er einmal auf die Spielmuster hingewiesen, beschränkt sich der Trainer darauf zu beobachten, das Spielmuster zu verstärken oder zu berichtigen. In das Kombinationsspiel mischt er sich nur ein, um dem Spiel neue Regeln, Laufwege, Richtungswechsel und Bälle hinzuzufügen, das heißt dessen Komplexität zu steigern. Treten Probleme auf, in deren Folge die Spieler die jeweiligen Spielmuster nicht lösen können, sollen die Spieler selbst ohne Hilfe des Trainers die Quelle des Problems identifizieren und effektive Lösungsvorschläge für das Erzielen des geforderten Spielmusters entwickeln. Zwar identifiziert der Trainer die Probleme und kennt deren Lösungen, er trägt aber nicht selbst zu deren Bewältigung bei. Diese Aufgabe überlässt er den Spielern, welche durch die in der Praxis entstehenden Situationen selbst aufgefordert werden, sich kognitiv zu engagieren. Von dieser Anweisung des Nichteingreifens weicht der Trainer nur dann ab, wenn Probleme auftreten, die die Umsetzung des Spiels chaotisch aussehen lassen. In diesem Fall hält der Trainer die Ausführung an und fordert eine Lösung ein. Er gewährt danach aber die notwendige Zeit zum Nachdenken und erlaubt die entstehenden Wechselgespräche und Vorschläge der Spieler.

In diesem Ansatz wird kein Wert auf das „richtige Spielen von Anfang an“ gelegt, wie man dies in sonst üblichen Lern- und Trainingsprozessen gewohnt ist. Die gewünschte kognitive Dissonanz provoziert Zweifel, Fehler und Missverständnisse auf Seiten der Spieler, die speziell den Anfang schwer machen. Außerdem sind es die Spieler in der Regel nicht gewohnt, sich bei der Ausführung von Übungen und Kleinen Spielen derartig stark kognitiv zu engagieren. Dies rechtfertigt eine gewisse Zeitspanne für die Gewöhnung an die Anforderungen der Spielsituation. Der Trainer muss mit solchen Anpassungsfristen rechnen, in denen das Kombinationsspiel chaotisch umgesetzt wird.

\section{Zweikämpfe}

Das Spiel soll auf kollektiven Prinzipien aufgebaut werden. Die rechtzeitige Koordination der Handlungen aller Spieler und die gegenseitige Unterstützung der Mitspieler untereinander sind grundlegende Voraussetzungen für eine gute offensiv und defensiv Spielleistung. Allerdings sind in Situationen von Opposition und Kooperation die Einzelhandlungen vor allem dann entscheidend, wenn beide Mannschaften im Spiel gleich stark sind. Im Spiel sind in der Regel viele Situationen von „1 gegen 1“ vorhanden, in denen der Gewinner entscheidende Vorteile für seine Mannschaft erreichen kann. Die gewonnenen Zweikämpfe sind heutzutage ein bedeutender Anzeiger der Leistung einer Mannschaft.

Aus einer kybernetischen Perspektive identifizieren ACERO, und LAGO (2005, S. 32) drei Analyseebenen der Sportspiele. Die erste Ebene wird als Mikrosystem bezeichnet. Sie ist durch die Zweikämpfe zwischen einem Angreifer und einem Verteidiger charakterisiert. Die zweite Ebene wird als Mezosystem bezeichnet und wird durch die Handlungen von Opposition zwischen zwei Gruppen von Spielern gebildet. Die dritte Ebene ist das Makrosystem, das die Gesamtheit des Spiels zwischen zwei Mannschaften berücksichtigt. Die erste Ebene, das Mikrosystem, repräsentiert die Gegenüberstellung beider 
Mannschaften auf, sie wird als Zweikämpfe in vielfältigen partiellen Konfrontationen geäußert.

Der DEUTSCHE FUSSBALL-BUND (2006) empfiehlt das Training von Zweikampfsituationen in allen Altersklassen und spricht die Empfehlung aus: „Das individuelle Taktische muss in eine ganzheitliche Ausbildungskonzeption eingebettet sein." (S. 6) Die Spiele 1gegen-1 mit oder ohne Ball bilden die Grundlage in den Altersklassen F- (6-8 Jahre) und E-Junioren (8-10 Jahre). Sie bilden für ein späteres, spezifischeres und formelleres Training ab der Altersklasse der D-Junioren die Basis. Das Modell der Spielhandlungskompetenzen schließt das Training der individuellen taktischen Fertigkeiten ein. Es nutzt dazu 1-gegen-1-Situationen als Lernaufgaben und setzt für die jeweilige Altersklasse geeignete Spiele ein, um eine breite Vielfalt dieser Situationen zu gestalten. In jeder Trainingssitzung sollte ein festgelegter Prozentsatz der Trainingszeit auf diese Aspekte verwendet werden. LUCASSEN (2010, S. 14) stellt die grundlegenden Fähigkeiten und Fertigkeiten vor, die man durch die Ausübung der 1-gegen-1-Situationen erwerben kann:

- „Taktisches Können: Situationsgerechte Entscheidungen treffen und zielorientierte Anschlusshandlungen durchführen.

- Technische Grundlagen: Die Basistechniken auch in höchstem Tempo beherrschen und darauf aufbauend individuelle Besonderheiten herausbilden.

- Persönlichkeitseigenschaften: Siegermentalität und Selbstvertrauen, fester Glauben in das eigene Können - auch in extremen Drucksituationen.

- Fitness: höchstes Tempo am Ball und Durchsetzungsvermögen beim Zweikampf“.

\section{Selbstüben der Technischen Fertigkeiten}

Das Ziel dieser didaktischen Strategie besteht aus der Förderung des „Bewussten Übens“ („deliberate practice“ im englischen Sprachraum, ERICSSON, KRAMPE, \& TESCH-RÖMER, 1993, S. 363). Durch ein Selbstlernprogramm wird außerhalb der Trainingssitzung geübt. Dies gelingt insbesondere für das Lernen, Perfektionieren und Automatisieren der technischen Fertigkeiten. Innerhalb der Strategie des Bewussten Übens übergibt der Trainer die Entscheidung über Inhalte, Ort, Zeit und Häufigkeit des Übens an die Spieler selbst. Er stellt dazu Hilfen in Form von didaktischen Materialien bereit. Dies können zum Beispiel Bilder, Texte und Videos sein. Ebenso steht er zur Verfügung, wenn die Spieler Fragen zu Inhalten und Strukturen der Übung haben. Außerdem bleibt der Trainer in Kontakt, beobachtet, stellt gezielte Fragen und bespricht mit den Spielern die Fortschritte, Zweifel, Auswertungskriterien und jede Form von Unstimmigkeiten. Bei der didaktischen Strategie des Selbstlernens der Technik geht es somit nicht darum, die Spieler mit dieser Aufgabe alleine zu lassen und das gemeinsame Training zeitlich zu entlasten. Vielmehr steht im Mittelpunkt, die Fähigkeit des „Lernens zu Lernen“ zu fördern. Das Selbstlernen erfordert hohe Disziplin und Organisationsfähigkeiten von den Spielern. Als Gegenleistung erhält der einzelne Spieler die Freiheit zur Exploration, zum Entdecken und Analysieren. Er kann die Zeitschemata und Systeme zur Steigerung der Schwierigkeit der Übungen und die ihm zur Verfügung stehende Zeit selbst planen. Dabei soll er sich konkrete Ziele setzen, sich bemühen diese zu erreichen und einen Lernerfolg zu erzielen. Dass Gelingen dieser Aufgabe hängt größtenteils von der Motivation des Spielers ab. 
Durch die Zuweisung einer Aufgabe des Selbstlernens kann das Engagement der Spieler steigen. Nach KELLER (2000, S. 4) können viele der Theorien über Lernmotivation in drei Grundkonzepten zusammengefasst werden:

1. Interesse und Neugier werden angeregt und gehalten.

2. Die Relevanz und der Inhalt passen sich an den Bedarf und die Ziele des Lernenden an.

3. Die Erwartungen an die Möglichkeiten von Lernerfolg werden dem Lernenden bewusst.

Das Lernen der technischen Fertigkeiten wird durch eine angemessen hohe Motivation begünstigt. Diese wird gesteigert, wenn der Spieler für seinen Lernprozess selbst verantwortlich ist. Ebenfalls förderlich sind eine das Lernen unterstützende Umgebung, angemessene Herausforderungen beim Lernen und für den Spieler bedeutsame Lernziele.

In der Abbildung 17 werden die werden die didaktischen Strategien des Modellsdargestellt.

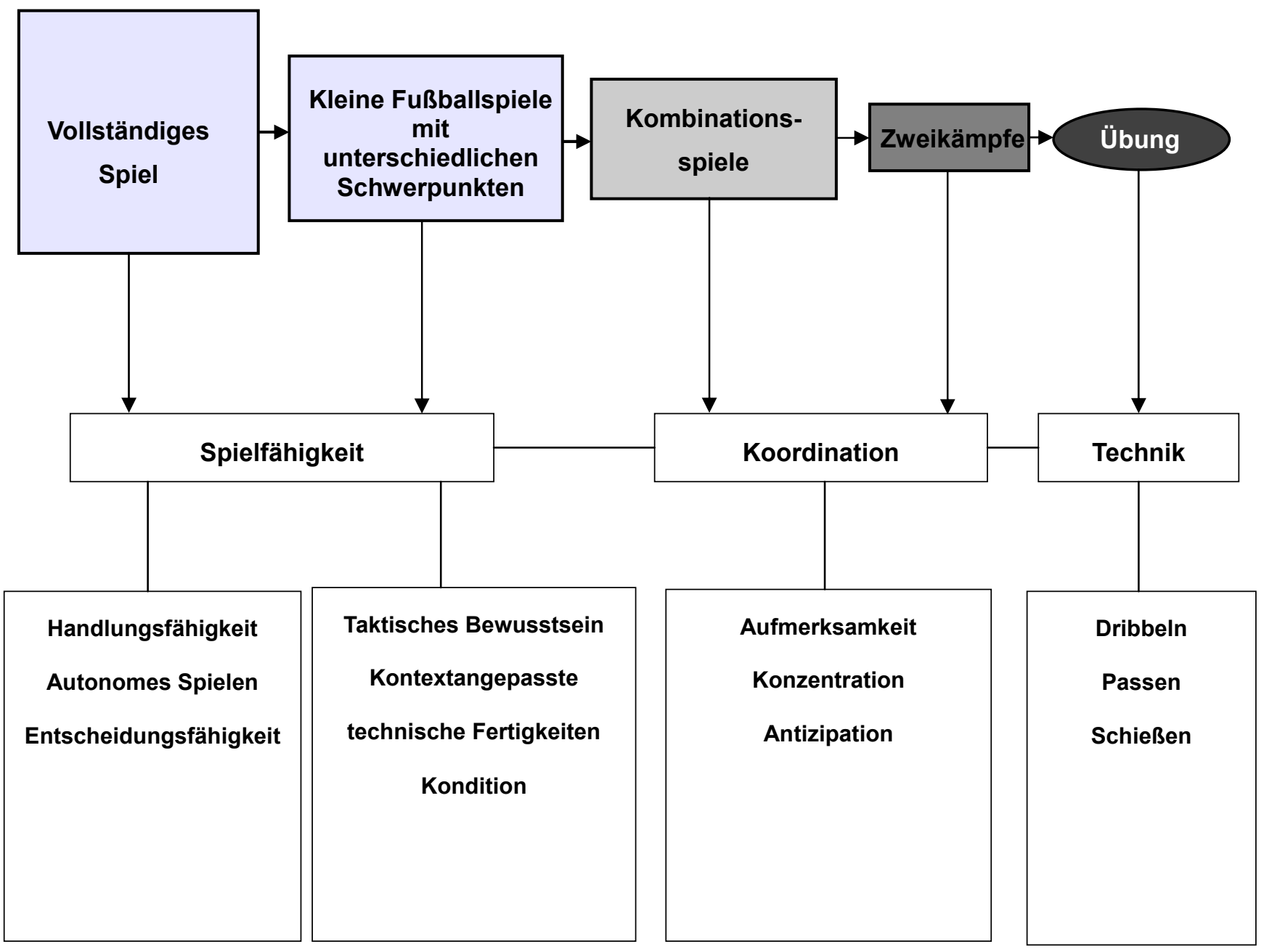

Abbildung 17: Inhalte des Handlungsspielkompetenzen-Modells 
Das vollständige Modell der Spielhandlungskompetenzen wird in Abbildung 18 dargestellt. Vier didaktische Strategien werden bei der Implementierung des Modells angewendet, sie tragen zur Entwicklung der Voraussetzungen für ein organisiertes und erfolgreiches Spielen und letztendlich zum Lernen der Spielhandlungskompetenzen bei.

Das große Spiel im Training erlaubt die Verbesserung der Entscheidungsfähigkeit, wenn durch eine problembasierte Lernmethode das autonome Spielen gefördert wird; dadurch verbessert sich die Spielhandlungsfähigkeit.

Die kleinen Fußballspiele mit unterschiedlichen Schwerpunkten üben, durch eine problembasierte Lernmethode zusammen mit einer Befragungsmethode, hauptsächlich die taktische Bewusstsein. Je nach Schwerpunkt werden auch im Spielkontext die technischen Fertigkeiten, die taktischen Fähigkeiten oder die Kondition geübt, die der Spielfähigkeit zugrunde liegen. Diese Fertigkeiten und Fähigkeiten beeinflussen sich gegenseitig.

Mit den Kombinationsspielen werden informationelle und kognitive Anforderungen an den Spieler gestellt, was das Üben der kognitiven Fähigkeiten fördert. Die Erfüllung der informationellen und kognitiven Anforderungen durch richtiges Handeln trägt zur fortschreitenden Steigerung der Koordination bei, dadurch verbessert sich auch die Fähigkeit des Zusammenspiels. Die Lernmethode des kooperativen Lernens erweist sich als besonders geeignet für die Anwendung dieser didaktischen Strategien.

Durch die didaktische Strategie der Zweikämpfe wird die individuelle Taktik gelernt und geübt. Durch eine Lernmethode der Partnerarbeit werden die individuellen offensiven und defensiven Handlungen geschult. Das Üben von Zweikämpfen trägt auch zur Förderung der Koordination bei.

Die technischen Fertigkeiten werden selbständig geübt. Die Technik, die hauptsächlich durch isolierte Übungen gelernt wird, braucht eine stufenweise Anpassung an den Spielkontext. Die Trainer sollen den Spielern eine geeignete Lernumwelt für die Förderung des selbständigen Lernens zur Verfügung stellen und technische Übungen als Hausaufgaben vorbereiten und empfehlen.

Die Implementierung der didaktischen Strategien wirkt auf die Gestaltung der Spielfähigkeit, Koordination und Technik.Mit diesen Ressourcen wird schrittweises Handlungswissen aufgebaut und als Spielkompetenz ausgedruckt.

Schlussendlich werden die Spieler wettbewerbsfähiger. 


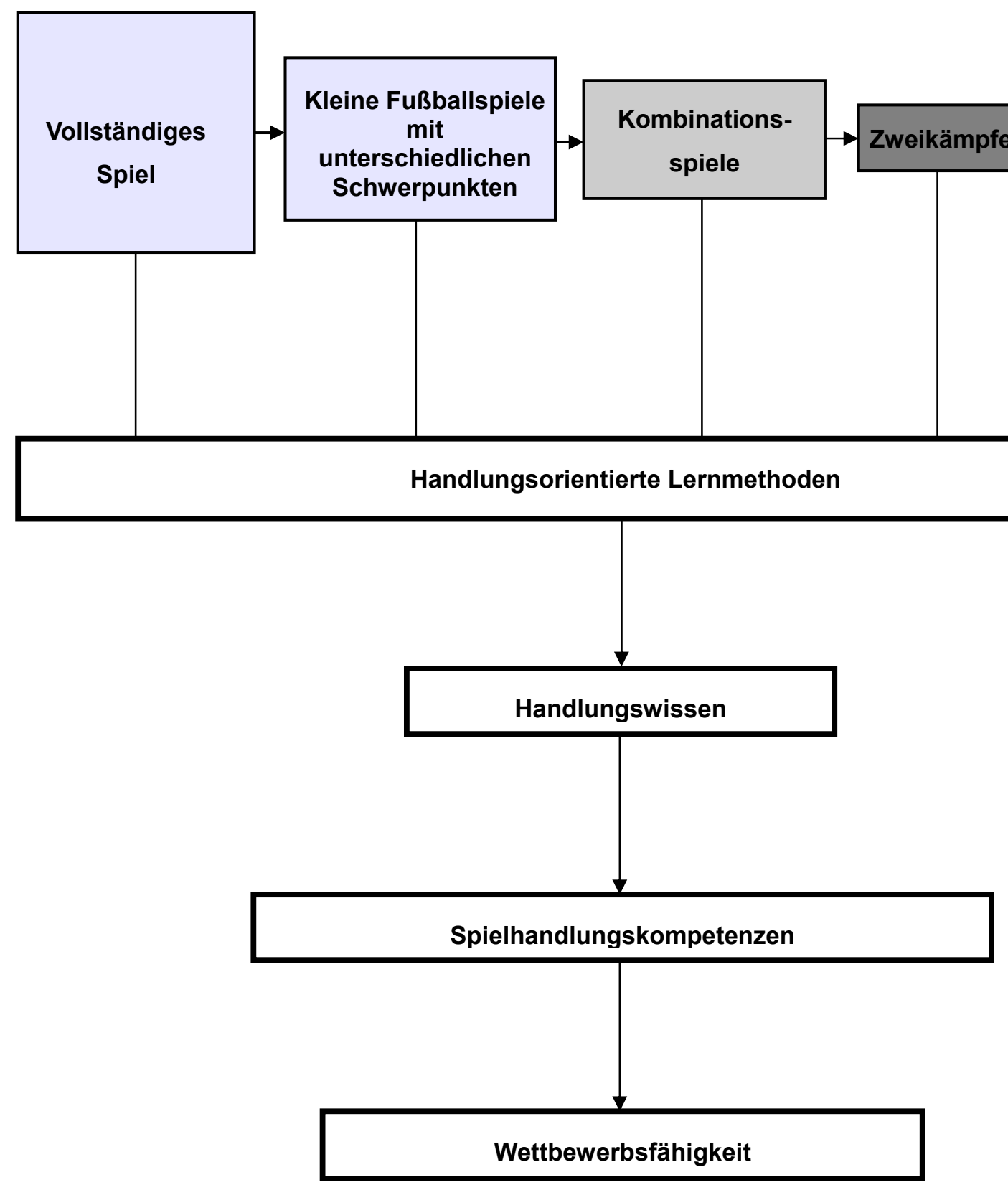

Abbildung 18: Das Modell der Spielhandlungskompetenzen

Bei der Implementierung des Ansatzes der Spielhandlungskompetenzen wird von einer Kombination von Spielsituationen ausgegangen, bei denen Bestandteile des am Anfang globalen Spieles schrittweise ausgegliedert werden. Es werden schließlich ausführliche Analysen der spezifischen Komponenten durchgeführt, die nach der Meinung des Trainers oder der Spieler bestimmter Entwicklungen und möglicher Korrekturen bedürfen oder festgestellte Defizite aufweisen. Die so entstehende Anwendungsstruktur des Modells lässt sich als eine rechteckige Form visualisieren.

Wie in der Abbildung 19 dargestellt ist, wird bei der Anwendung des Modells von einem globalen Spiel ausgegangen (1). Simplifizierte Formen werden als kleine Fußballspiele 
mit unterschiedlichen Schwerpunkten dargestellt (2), die Hauptmerkmale des Spiels, Mitspieler, Gegenspieler und Tore aufrechterhalten, aber die Sekundären Regeln werden zugunsten der Lernziele abgeändert. Bei weiterer Vereinfachung entfallen Tore und Gegenspieler, die kognitive Kompetenz wird durch Kombinationsspiele stimuliert (3), die Spieler werden als kleine oder große Gruppe organisiert. Die individuell taktische Kompetenz wird durch Zweikampfsituationen geübt (4), die Spieler agieren paarweise. Bei der stärksten Spielvereinfachung üben die Spieler selbständig die technische Fertigkeiten (5), dafür brauchen sie einen Ball und didaktisches Material in Form von verbalen Anweisungen und visuellen Anregungen auf Bildern und in Videos. Die Anwendung der kleinen Spiele mit unterschiedlichen Schwerpunkten, der Kombinationsspiele und der Zweikämpfe findet in jeder Trainingssitzung statt, die Abfolge kann beliebig sein. Entsprechende sportdidaktische Hinweise für jeden Bestandteil sind zu beachten. Das selbstständige Üben der technischen Fertigkeiten kann sowohl in das Training integriert als auch als „Hausaufgabe“ gestellt werden.

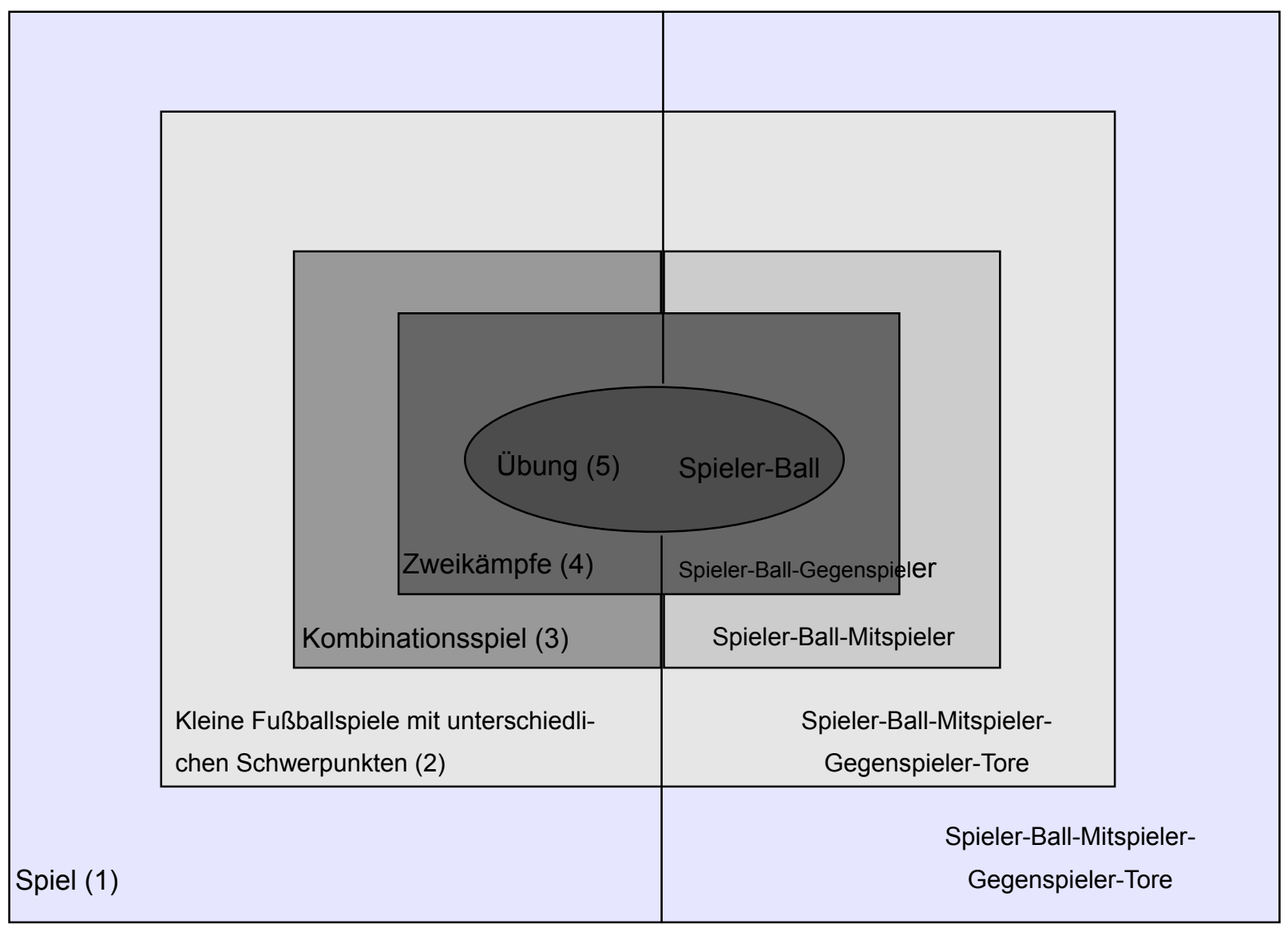

Abbildung 19: Anwendungsstruktur des Spielhandlungskompetenzen-Modells 


\subsubsection{Umsetzungsplan}

Der Trainer soll entscheiden, wie tief die Spieler den Lerninhalt verarbeiten sollen und welche Lernstufe sie je nach Alter, Erfahrungen, kognitiven Fähigkeiten, Zielen und Rahmenbedingungen erreichen sollen.

Der Lehrer oder der Trainer kann ein konzeptuelles System erarbeiten und dann die Methode der Lernmatrix nutzen, um den kognitiven Inhalt des Trainings auszuwählen und die Ziele für das Lernen zu erstellen. DiesesVerfahren wird anschließend dargestellt.

\section{Konzeptuelles System und Lernmatrix}

$\mathrm{Ab}$ einem bestimmten Lerninhalt gestaltet der Trainer ein konzeptuelles System. Die konzeptuellen Systeme stellen eine strukturierte und logische Integration von Fakten und Konzepten dar und bilden einen Zusammenhang von Konzepten zu einem bestimmten Thema. Dieser Zusammenhang dient als Bezugspunkt und stellt den Inhalt dar, den die Lernenden verarbeiten sollten. Bei Unstimmigkeiten zwischen verschiedenen Auffassungen wird nach dem ausgewählten konzeptuellen System eine Entscheidung getroffen; z.B. ein richtiger Pass könnte verschiedene Merkmale aufweisen, je nach berücksichtigtem konzeptuellem System. Innerhalb der konzeptuellen Systeme sind die Spielsituationen die Basis, auf denen die Konzepte aufgebaut werden. Mit Hilfe der sportwissenschaftlichen Kenntnisse werden die Ursachen und Effekte der Situationen analysiert und es wird auf die geeignete Handlung hingewiesen, um optimal auf eine Situation einwirken zu können.

Die folgende Abbildung stellt ein solches konzeptuelles System zum Thema „Passen beim Fußball“ dar. 


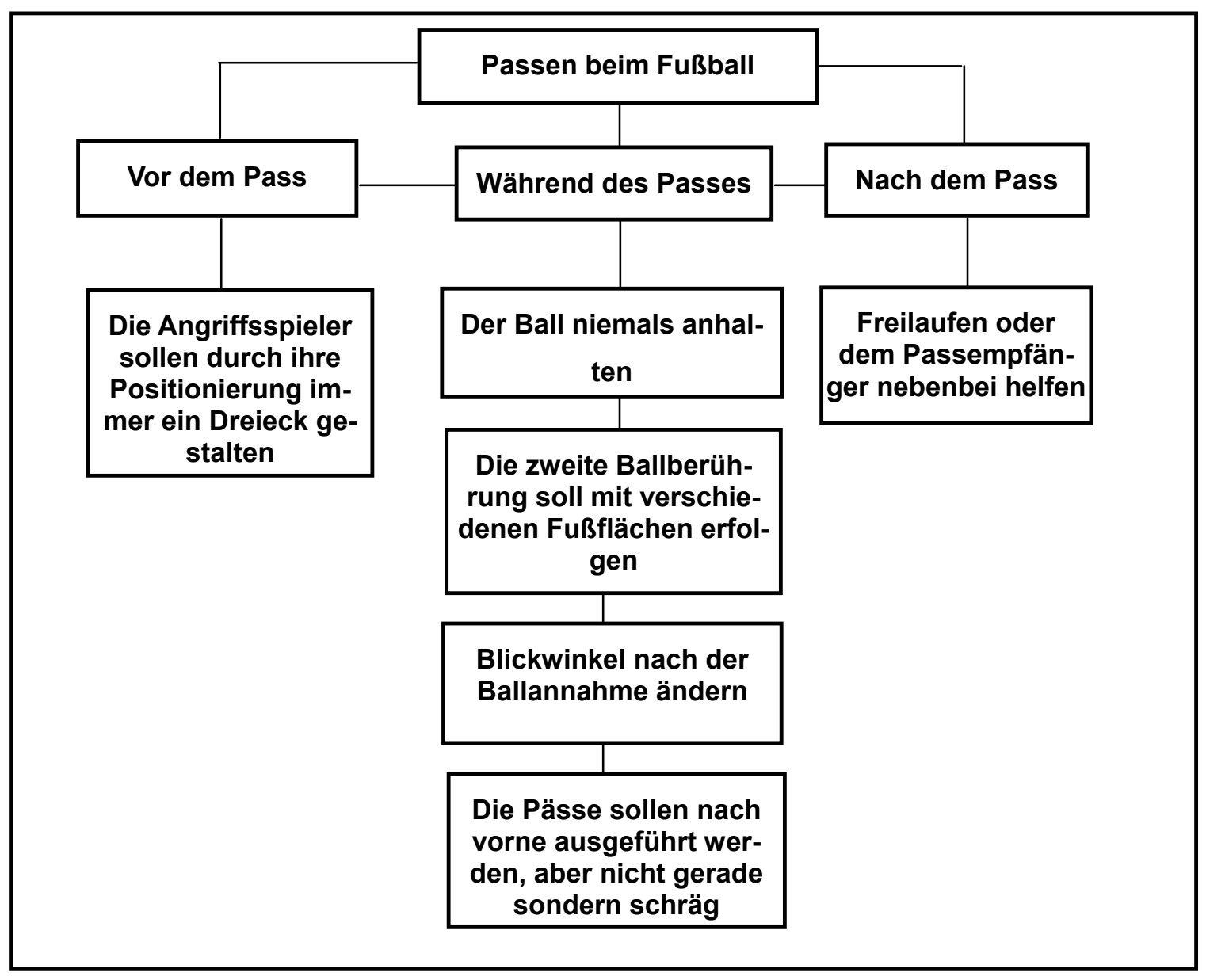

Abbildung 20: Konzeptuelles System über den Pass beim Fußball

Die Konzepte konstituieren die grundlegende Form, mit der das Denken operiert. Sie spiegeln die Essenz der dabei stattfindenden Phänomene und Prozesse und verallgemeinern ihre Eigenschaften. Sie sind die strukturelle Basis des rationalen Wissens, mit deren Hilfe man Objekte und Phänomene erkennen kann, da ihre bedeutendsten Merkmale generalisiert und abstrahiert werden. Die Konzepte sind „eine theoretische Verallgemeinerung des Denkens" (LÓPEZ \& MORENO, 2002a, S. 20). In unmittelbarer Beziehung zu den Konzepten liegen die Prinzipien, wie sie im Fall der Spielprinzipien auftreten.

Mit Hilfe eines konzeptuellen Systems wird dann die kognitive Kompetenz gefördert. Die kognitive Kompetenz besteht aus intellektuellen Operationen, die beim Denken die Informationen verarbeiten. Sie sind ein wichtiger Bestandteil des Lernens.

Die kognitive Kompetenz ist ein allen Arten von Wissen gemeinsames Merkmal. Sie ermöglicht dem Lernenden, sich Konzepte anzueignen und durch verschiedene Ebenen einer Wissenshierarchie zu verfolgen, je nachdem, welche Art der Bewältigung einer kognitiven Aufgabe auf der jeweiligen Ebene gefordert wird. So sind zum Beispiel Beobachten, Beschreiben, Vergleichen, Argumentieren, Verdeutlichen, Überlegen und 
Vorschlagen konsekutive und hierarchische Schritte, mit denen man die Intensität der Aneignung eines Konzeptes beschreiben kann.

Die kognitiven Funktionen liegen die kognitive Kompetenzen zugrunde und umfassen geistige Tätigkeiten und Leistungen wie z.B. Wahrnehmungsfähigkeit, abstraktes Denkvermögen und Handlungsplanung, diese Leistungen begünstigen eine hohe kognitive Unabhängigkeit des Spielers, während er Handlungen ausführt. Insbesondere diejenigen Handlungen, die einen aufwendigen mentalen Prozess erfordern und diejenigen, die auf vorher gelernten Konzepten basieren, profitieren davon. Solche Funktionen erlauben es dem Sportler, Selbstkontrolle zu üben und ein aktives und selbstregulierendes Element innerhalb des Lernprozesses zu werden. Ab der ersten Anwendung kognitiver Funktionen bei motorischen Aufgaben werden kognitive Kompetenzen erworben. Sie erleichtern z. B. die Identifizierung der Probleme in der Spielsituation, das Diskutieren über ihre Ursache und das Vorschlagen von zu erarbeitenden Lösungen. FEUERSTEIN (1978) schlägt eine Reihe von kognitiven Funktionen aus seiner Arbeit mit geistig behinderten Kindern vor, die Schwierigkeiten bei der Durchführung von Aufgaben haben. Tritt ein Problem an einer Stelle auf, die eine bestimmte kognitive Funktion erfordert, wäre dies ein Hinweis über die Unfähigkeit des jeweiligen Kindes, diese Funktion umzusetzen. Der Schluss auf das Vorliegen einer bestimmten Behinderung liegt nahe. IAFRANCESCO (2006) ordnet den kognitiven Kompetenzen kognitive Funktionen zu und nimmt an, dass eine gute Leistung bei der Durchführung dieser Aufgaben die Entwicklung der kognitiven Fähigkeiten beweisen könnte.

IAFRANCESCO (2006) unterscheidet drei Stufen innerhalb der kognitiven Kompetenz, die hierarchisch organisiert werden und für die Wissensaneignung stehen:

- Interpretieren: der Lernende identifiziert Merkmale und Situationen,

- Argumentieren: der Lernende kann seine Entscheidungen begründen,

- Lösungen vorschlagen: der Lernende ist in der Lage, Lösungen für neue problematische Situationen hervorzubringen.

Der Lerninhalt bei der Schulung der Spielkompetenz könnte dieser kognitiven Kompetenz zugeordnet und in Form einer Matrix organisiert werden, nach gewünschter Vertiefung des Inhalts. Bei der Wahl des Trainingsinhalts könnte der Trainer Lernaufgaben auswählen, die zum Erreichen der entsprechenden Kompetenz beitragen. In Tabelle 7 werden diese kognitiveKompetenzen und kognitive Funktionen aufgeführt. 
Tabelle 7: Kognitive Kompetenzen und die ihnen zugeordneten kognitiven Funktionen (IAFRANCESCO, 2006)

\begin{tabular}{|l|l|l|}
\hline \multicolumn{1}{|c|}{ Interpretative Competences } & Argumentative Competences & \multicolumn{1}{|c|}{ Propositive Competences } \\
\hline Relate & Analyze & Design \\
Interpret & Argue & Planning \\
Differentiate & Sustain & Evaluate \\
Distinguish & Demonstrate & Apply \\
Inferring & Verify & Develop \\
Determine & Integrate & Create \\
Set & Discriminate & Suggest \\
Select & Constitute & Formular \\
Classify & & Build \\
Sort & Structure \\
Express & Develop \\
Deduce & & Process \\
Choose & Decide \\
\hline
\end{tabular}

Dafür wählt der Trainer bestimmte Kompetenzen aus, die es zu lernen gilt und die anhand einer "Lernmatrix" ausweisen, ob die Spieler den Lerninhalt bis zum Identifizieren, Argumentieren oder bis auf eine kreative Stufe bringen sollen. Die Matrixmethode ermöglicht es, die Beziehung zwischen dem zu erwerbenden Wissen und der kognitiven Kompetenz zu veranschaulichen. Sie verdeutlicht und bestimmt auch, inwieweit die Spieler die Aneignung des Wissens nachweisen sollen.

In der folgenden Tabelle wird ein Beispiel einer solchen Matrix dargestellt. 
Tabelle 8: Matrix mit dem Thema „Passen bei Fußballspielen“

\begin{tabular}{|l|l|l|l|}
\hline \multicolumn{1}{|c|}{ Konzept } & Interpretieren & Argumentieren & Vorschlagen \\
\hline Den Ball niemals anhalten & & & \\
\cline { 1 - 2 } $\begin{array}{l}\text { Nach dem Pass frei laufen oder } \\
\text { dem Passempfänger helfen }\end{array}$ & & & \\
\cline { 1 - 2 } $\begin{array}{l}\text { Die zwei Ballberührungen immer } \\
\text { mit unterschiedlichen Fußflächen } \\
\text { machen }\end{array}$ & & & \\
\hline $\begin{array}{l}\text { Blickwinkel nach der Ballannahme } \\
\text { wechseln }\end{array}$ & & & \\
\hline $\begin{array}{l}\text { Die Pässe sollen nach vorne aus- } \\
\text { geführt werden, aber nicht gerade, } \\
\text { sondern diagonal }\end{array}$ & & & \\
\hline $\begin{array}{l}\text { Die Angriffsspieler sollen durch ihre } \\
\text { Positionierung immer ein Dreieck } \\
\text { gestalten }\end{array}$ & & & \\
\hline
\end{tabular}

Sind die konzeptuellen Systeme einmal auf die Matrix angepasst, ist es einfach, die Lernziele zu bestimmen und zu formulieren. In den Lernzielen werden die Konzepte und die zu übenden kognitiven Kompetenzen festgelegt. Darüber hinaus sollte man die konkrete Leistung bestimmen, die die Spieler liefern sollten und an der der Lernerfolg messbar wird.

In dieser Arbeit wird die Anwendung des Dargestellten bei Trainingsprozessen durch Kleine Fußballspielen mit unterschiedlichen Schwerpunkten (KFSS) vorgeschlagen (siehe eine Beschreibung der KFSS auf dem Abschnitt 3.3.1auf S. 102 ff.und im Anhang $\mathrm{N}$ und $\mathrm{O}$ ). Der Trainer stellt in diesen Spielen Probleme bereit, indem er sekundäre Regeln manipuliert. Die vom Trainer eingesetzte Befragungsmethode soll das Üben der kognitiven Kompetenz erlauben. Die Spieler werden aufgefordert, bestimmte UrsacheWirkung-Beziehungen in Spielsituationen mit eigener oder fremder Beteiligung zu bearbeiten. Diese Verarbeitung läuft mehrschrittig ab. Die Spieler müssen interpretieren (was passiert), argumentieren (welche Ursache für das Problem vorliegt) und vorschlagen (mögliche Lösungen nachdenken und äußern), indem sie klar und verständlich sich äußern.

Der Trainer entscheidet über die zu erreichende Lernstufe und plant eine Reihenfolge von Fragestellungen, problematischen Situationen und Lernaufgaben für das Engagement der Spieler. Der Plan soll die spezifischen zu lösenden Probleme enthalten und beschreiben, wie diese gestaltet werden sollen. Ebenso soll er ein Verzeichnis von Fra- 
gestellungen für die Zeit vor, während und nach der Tätigkeit der Spieler zur Verfügung stellen. Für den Lerninhalt „Feldstellung beim Angreifen“ beim Fußball könnte der Trainer z. B. Fragestellungen wie die folgenden vorbereiten:

- Warum spielt Deine Mannschaft so wenige Pässe?

- Ist dies die beste Weise, den Ball zum gegnerischen Tor zu bringen?

- In der Unterbrechung nach einem Spielzug: Wie kannst Du Deiner Mannschaft helfen, wenn Du das machst?

- Kannst Du mir zeigen, wo Du stehen sollst, wenn der Ball sich im Mittelfeld befindet?

\subsubsection{Auswahl der Inhalte und Rolle des Trainers}

Der Trainer macht eine Liste von taktischen Situationen und entwirft die Spielaufgaben, die den Spielern dabei helfen sollen, taktische Bewusstsein und Entscheidungsfähigkeit zu entwickeln.

Der Trainer soll geeignete anregende und kreative Lernaufgaben entwerfen und die Rahmenbedingungen stellen. Er soll auch die unterschiedlichen Lösungsformen kennen, die die Spieler anwenden könnten, um die in die Aufgaben eingeschlossen problematischen Situationen zu lösen. Das Wissen und die Vorgehens- und Verhaltensweise des Trainers sind in ihrer Funktion aber immer als indirekt zu betrachten und haben bei diesem Modell eine ganz andere Bedeutung als diejenigen, die bei Direktem Modell angewendet werden. Während des Aufgabenentwurfs stellt sich der Trainer die folgenden Fragen:

1. Welches sind die „Probleme“ die in jeder Aufgabe eingeschlossen sind? Welche kognitiven, motorischen und sozialen Anforderungen beinhaltet die Aufgabe?

2. Welche Leistungen oder Verhaltensweisen werden durch diese Aufgabe herausgefordert? Wie wird diese Leistung ausgewertet?

3. Welche Rolle spielt der Trainer, während die Spieler die Aufgabe lösen?

Der Trainer wählt die Mannschaften aus, stellt die Aufgabe vor und führt die Mannschaften zum Start. Hierfür ist die Fähigkeit erforderlich, den Spielern einerseits genug Informationen für die Durchführung der Aufgabe zu geben, ohne ihnen aber andererseits zu sagen, wie die Probleme gelöst werden sollen. 


\subsection{Aufgabenstellung}

\subsubsection{Die Lernaufgaben}

Innerhalb des Modells gibt es vier grundlegende Aufgaben, die zur Anwendung kommen: Kleine Fußballspiele mit unterschiedlichen Schwerpunkten, Kombinationsspiele, 1-gegen-1-Übungen und technische Fertigkeiten zum Selbstlernen. Jede Art der Aufgaben hat ihre eigene Struktur und Vorstellungsform.

Die Aufgabenvorstellung wird schnell ausgeführt, die Aufgabenstruktur wechselt mehrere Male, immer jedoch auf der vorhergehenden Struktur aufbauend. Die Aufgabenstellung enthält Fragestellungen, die Spieler werden zum „Denken und Bewegen“, also zur Suche nach der Antwort auf diese Fragen aufgefordert.

In jeder Trainingseinheit wird das Spiel neu strukturiert. Die Hauptbestandteile, und deshalb mit mehr zur Verfügung stehender Zeit ausgestattet, sind die KFSS. Das Spielen wird im Training immer vorhanden sein. Jeweils eine Gruppe wird aber aus dem Training „in vivo“ herausgenommen und in ein "Labor“ gestellt, wo sie die technischen Fertigkeiten und Gruppenhandlungen „in vitro“ üben, d. h. unter veränderten Bedingungen des Spiels: ohne Gegner und Zeitdruck und mit fortlaufenden Wiederholungen. Mit einer Gruppe von 13 Spielern beginnt das Training etwa mit einem 4 vs. 4 KFSS und die übrigen 5 Spieler üben außerhalb dieses Spiels. 3 praktizieren ein KS und 2 machen Übungen mit Ball für die Entwicklung der technischen Fertigkeiten (siehe Abbildung 21). Manchmal bekommen die Spieler "in vitro“ auch nur taktische Instruktion und/ oder beobachten das Spiel mit der Aufgabe, taktische Schlussfolgerungen zu ziehen (kognitive Kompetenzen und Wissenskompetenzen). 

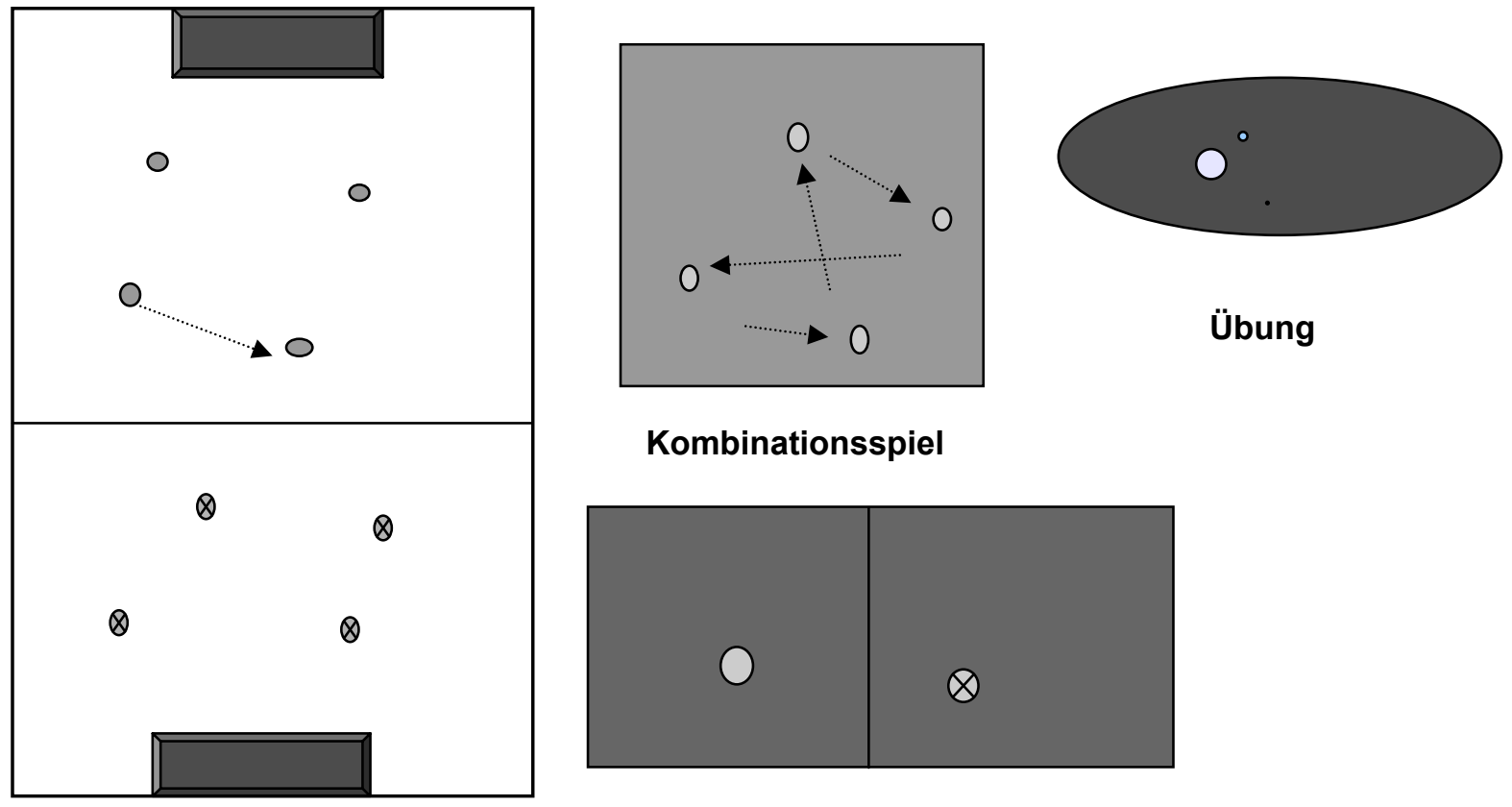

Kombinationsspiel

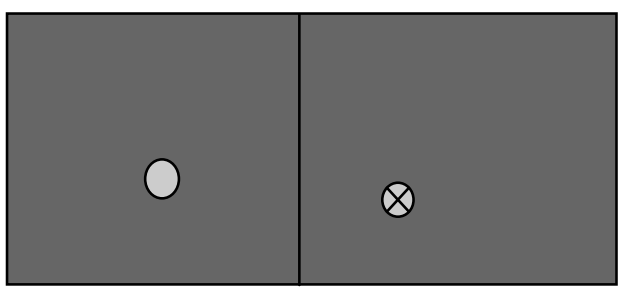

Zweikämpfe

KFSS

Abbildung 21: Beispiel Trainingssitzung mit Umsetzung des Spielhandlungskompetenzen-Modells

Die Umstrukturierung des Spiels ist für jeden Spieler verschieden, da er die Trainingseinheit entweder in der Situation „in vitro“ oder in der "in vivo“ Situation beginnen kann.

Die Aufgaben „in vitro“ sind nicht für alle Spieler gleich, jeder versucht jene Kompetenzen zu entwickeln, die nach individueller Auswertung bei inm noch schwach ausgebildet sind.

\subsubsection{Aufgabenpräsentation}

Die Vorstellung der Aufgabe wird abhängig vom Lerninhalt und Lernziel durchgeführt. Jede Aufgabenstellung hat unterschiedliche Ziele und führt zu unterschiedlichen Aufgabestrukturen, die Aufgabenstellung variiert innerhalb des Modells.

Beim Vorstellen der Aufgabe soll der Trainer nur so viele Informationen liefern, wie für das Begreifen der Aufgaben nötig sind. Er stellt auch die zu lösenden Probleme dar. Danach weist der Trainer den Spieler an, das „Bewegen und Denken“ zu beginnen. Die Aufgabe vorzustellen bedeutet, diese innerhalb eines Kontextes zu formulieren und den Spielern einen Anhaltspunkt zu geben. Man kann auch die Aufgabe modifizieren oder die Komplexität steigern. Der letzte Teil der Vorstellung der Aufgabe bezieht sich auf das Fragenstellen an die Spieler, um die kognitive oder motorische Aufgabe zu gestalten, die sie lösen sollen. 
Bei den Kleinen Spielen mit unterschiedlichen Schwerpunkten stellt der Trainer eine problematische Situation durch die Stellung von deduktiven Fragen dar. Die Spieler äußern Lösungsformen, bevor sie diese im Spiel einsetzen. Der Trainer erklärt das Spiel, erlaubt den Spieler aber, die Antworten für die taktischen Probleme aus dem Spiel selbst abzuleiten.

Bei den Kombinationsspielen stellt der Trainer das Ziel der Aufgabe dar und erklärt, wie die Grundaufstellungen, Positionen, Passrichtungen, Laufrichtungen und Laufstrecken aussehen. Die Spieler sollen dann selbst die Probleme lösen, die während der Ausführung auftreten. Wenn das Kombinationsspiel nicht wie gewünscht läuft, hält der Trainer das Spiel an, weist auf die Aufgabe hin und fordert eine vernünftige Problemlösung der Spieler ein.

Bei den Eins-gegen-Eins-Situationen stellt der Trainer das Ziel vor und gibt während und nach der Aufgabe korrigierende Hinweise.

Bei den technischen Übungen stellt der Trainer didaktische Materialien vor, diese sollen den Spieler beim Selbstlernprozess helfen und stufenweise Hinweise, Korrekturen und Auswertungsverfahren für die technischen Fertigkeiten andeuten. Bilder, Videos und Modelle sind dafür geeignet. 


\subsection{Validierung}

Zahlreiche vorliegende Forschungsergebnisse legen nahe, dass das Spielen selbst die beste Methode für das Lernen der Spielkompetenzen ist (z.B. TURNER \& MARTINEK, 1992, 1999; GRIFFIN, OSLIN \& MITCHELL, 1995); FRENCH et al., 1996, 1996A, 1996B; BLOMQVIST, LUHTANEN, \& LAASKO, 2001; HARVEY, 2006; TALLIR et al., 2007). Die Kleinen Spiele erlauben viele Variationen, um die gewünschten Lernziele bekräftigen zu können. Durch Spielen kann man sowohl taktische als auch technische und konditionelle Kompetenzen in ihrer Gesamtheit schulen, obwohl das Spiel nicht deutlich besser als die Direkte Methode für das Lernen einzelner bestimmter Aspekte ist, z. B. das Erlernen der technischen Kompetenzen.

Da eine plötzliche, vollständige Umwandlung der traditionellen Vermittlungsmodelle in ein neues, noch nicht voll überprüftes Modell als zweifelhaft bezeichnet werden könnte, wird versucht, die didaktischen Strategien unabhängig voneinander zu validieren und erst danach alle Bestandteile in ein gemeinsames Modell zu integrieren. Die didaktischen Strategien, die aus Selbstlernen und kooperativen didaktischen Modellen stammen, wurden aus vorliegenden Forschungsergebnissen abgeleitet, in denen diese Techniken bei mehreren Lerninhalten und Kontexten validiert wurden.

\subsubsection{Allgemeine Hypothesen}

\section{Prozedurale Spielkompetenz}

Wenn es das primäre Ziel des Trainings ist, das Spielen zu lernen oder die Spielfähigkeit zu verbessern, dann muss ein Großteil der Trainingszeit diesem Ziel gewidmet werden. Nach WEIN (2009, S. 15; vgl. WILLIAMS, 2008, S. 38) ist es der zentrale Bestandteil dieses Lernziels, dass die Spieler die Fähigkeit entwickeln, das Spiel „zu lesen“, um in der Folge mit Effizienz daran teilzunehmen. Diese Teilnahme geschieht durch eigene Handlungen. Sie wirken auf die Spielumwelt und erzeugen Änderungen der Spielsituationen. Die richtige Handlungsausführung ist dabei das Produkt von spezifischem Handlungswissen. Das weist darauf hin, dass Spieler mit einem größeren Repertoire von spielspezifischem Handlungswissen die Spielsituationen effizienter lösen können, sowohl im Angriff als auch in der Abwehr, entweder mit Ball oder ohne ihn. Diese Spieler suchen kontinuierlich nach günstigen Situationen, die sie zu ihrem Vorteil auszunutzen in der Lage sind. Auf diese Weise haben sie die Tendenz, das Spiel durch größere Beteiligung zu kontrollieren als andere Spieler mit weniger Handlungswissen dies tun.

Das Spielhandlungswissen ist ein kontextabhängiges Wissen, das man nur durch Spielen erwerben kann. Es kann nicht übermittelt werden (siehe hierzu die Wissenshierarchie auf Seite $71 \mathrm{ff}$.). Mit ihrer Teilnahme an Kleinen Fußballspielen mit unterschiedlichen Schwerpunkten können die Spieler dieses Spielhandlungswissen durch die aus dem Spiel herausgezogenen Erfahrungen aufbauen. Um die von einer Spielsituation implizierten, augenblicklichen und kontextbezogenen Probleme zu lösen, benutzen die Spieler das schon vorhandene Wissen für die Verarbeitung von Hypothesen über Lösungsalternativen, d. h., sie benutzen präskriptives Wissen. Nach der Handlungsausführung und dem Verarbeiten der sensorischen Wirkungen wird entsprechendes Feed- 
back $(\mathrm{KR} \text { und } \mathrm{KP})^{8}$ erzeugt, die Spieler erlangen interpretatives Wissen, das sukzessive ihre Handlungen verbessert und damit selbst zu neuem präskriptiven Wissen wird. Die Beziehung zwischen dem, was bereits erzielt ist (präskriptives Wissen) und den Wirkungen (interpretatives Wissen) führt zur Gestaltung von Aktionsregeln des Typs „wenndann" (vgl. ANDERSON, 1996, S. 202). Die generierten Lösungen tragen so zum Aufbau des Handlungswissens bei. Dieser Aufbauprozess steht im einen positiven Zusammenhangmit derLange der Zeitspanne in der die Spieler an Spielsituationen beteiligt sind. Es ist daher erstrebenswert, dass ein hoher Anteil kontextbezogenen Spielens erfolgt, d. h. unter Oppositionsbedingungen durch KFSS ein Wissensaufbau ermöglicht wird.

Die Hypothese hinsichtlich der prozeduralenSpielkompetenz lautet damit folgendermaßen:

H1. Ein Trainingsprogramm, das aus Kleinen Fußballspielen mit unterschiedlichen Schwerpunkten besteht und in dem durch eine Befragungsmethode das Fußballspiel geschult wird, verbessert die Effizienz der Spielhandlungen der Spieler und damit ihre Spielkompetenz.

Die Studie eins (ab Seite 125) behandelt diese Hypothese.

\section{Aufbau des präskriptiven Wissens durch eineBefragungsstrategie}

Sowohl das DIKW-Modell (Daten, Informationen, Wissen, Weisheit) aus der Informationstechnologie und dem Wissensmanagement und als auchdas Konstrukt derWissenshierarchie schlagen eine hierarchische Organisation des Wissens vor (ACKOFF, 1989, S. 3-9). Dieser Vorschlag entspricht den kognitiven Prozessen, die während des Lernens ablaufen (ENGELKAMP, 2006, S. 5). Die Spielhandlung wird im Rahmen dieser Arbeit als ein umfassenderes Konzept der Sportbewegung betrachtet. Dementsprechend sollte ein Sportspiellernansatz an der Wissenskonstruktion ausgerichtet sein, statt in Richtung des Wissenserwerbs oder der Informationsvermittlung zu tendieren. Die sensorischen Effekte der Handlungsausführung tragen zum Aufbau von Denkstrategien und kognitiven Strukturen zur Problemlösung in bestimmten Situationen bei (vgl. PAOUR, CĖBE, \& HAYWOOD, 2000, S. 9). Information allein ist demnach nicht genug für die Konstruktion von Wissen. Wenn dieses Wissen zur Lösung neuer Probleme bei der zukünftigen Ausführung der selben Spielhandlung bestimmt ist, ist es auch notwendig, die sensorischen Effekte zu berücksichtigen, die sich aus der Durchführung der Spielhandlung heraus ergeben, um den Aufbau des Spielhandlungswissens zu ermöglichen.Die Hypothese hinsichtlich der kognitiven Kompetenz und der Wissenskompetenz lautet folgendermaßen:

8 "Knowledge of Results (KR) refers to extrinsic information that tells learners something about the success of their actions with respect to the intended environmental goal."

"Knowledge of Performance (KP) augmented feedback that provides information about the quality of the movement (e.g., rhythmic, smooth, mechanically efficient, beautiful, etc.)".(SCHMIDT \& WRISBERG, 2008, S. 286-289) 
H2. Der durch Spielerfahrungen und Befragungen als indirekte Methode geförderte Aufbau des Wissens ist besser für die Lösung der kognitiven Aufgaben während des Spiels geeignet als die direkte Vermittlung von Informationen.

Die Hypothese wird in der Studie zwei (ab Seite 136) behandelt.

\section{Entwicklung der technischen Kompetenz in Sportspielen durch Kleine Fußballspiele mit unterschiedlichen Schwerpunkten (KFSS)}

Mit der Anwendung der Kleinen Spiele wird versucht, die Entwicklung der technischen Fertigkeiten als wichtigsten Bestandteil der Sportleistung zu berücksichtigen, der aber nicht der notwendigste für eine hohe Spielleistung ist. Die Ausführung der technischen Fertigkeiten in Sportspielen im Kontext, d. h. während des Spiels, setzt eine Integration mehrerer Bestandteile in die Handlung voraus. So setzt eine gelungene Handlung Intentionen, Motivationen und Entscheidungen voraus. Die in die Trainingssituation integrierte Ausführung von spielnahen Übungen der Kooperation und Opposition erzeugt den in Wettspielen auftretenden Situationen ähnliche Gelegenheiten, in denen das Gelernte angewendet werden soll. Im Vergleich mit Wettspielen bieten die KFSS die Möglichkeit, die Wiederholungszahl zu erhöhen (JONES \& DRUST, 2007). Außerdem erlauben sie Komplexitätsanpassungen durch die Variation von Spielbestandteilen wie Raum, Teilnehmerzahl und spezifischen Regeln.

Das Ziel des Lernens technischer Fertigkeiten ist nicht, hohe Präzision oder maximale biomechanische Effizienz zu erreichen, sondern die höchste Wirksamkeit beim Einsatz der Fertigkeiten im Spiel zu fördern. So ist es im Fall des Passens z. B. nicht notwendig, dass die Spieler einen isolierten Pass üben, bis sie die höchstmögliche Präzision erreichen. Vielmehr sollten die Spieler „erreichbare“ Pässe in vielfältigen Spielsituationen spielen können (BLOMQVIST, LUHTANEN \& LAASKO, 2001, S. 343).

Die einfachen Kleinen Spiele können durch die Forderung der vielfältigen Anwendung der Fertigkeiten bei Spielen zum zentralen Lernprozess der technischen Fertigkeiten beitragen. Studien zum "contextual interference“ (HALL, \& MAGILL, 1995; HALL, DOMINGUES, \& CAVAZOS, 1994; ARIAS \& JIMENEZ, 2004) haben nahegelegt, dass ein Lernprozess, in dem die Bewegungsfertigkeiten in einer zufälligen Weise variiert werden, ein beständigeres Lernen ermöglicht als ein Lernprozess, in dem Bewegungen in einer festen Reihenfolge wiederholt werden. Da keine Situation zufälliger angeordnet sein kann als ein Wettspiel, ist das Spiel eine geeignete Umsetzung für diesen Ansatz. Allerdings kann man aufgrund der Oppositionsbedingungen des Spiels keine technischen Fertigkeiten isolieren. Aus diesem Grund benötigt man mehr Zeit, um durch das reine Spielen die technischen Fertigkeiten als solche zu erlernen. Damit ist die Spielmethode nicht wirksamer als die direkte Methode, aber auch nicht weniger wirksam, $\mathrm{d}$. $\mathrm{h}$. das Lernen „in vitro“ wirkt über den Zugang der technischen Fertigkeiten ähnlich effizient, wie das Lernen „in vivo“ über den Spielzugang. Folglich lautet die Hypothese hinsichtlich des Lernens der technischen Fertigkeiten in dieser Arbeit folgendermaßen:

\section{H3: Die Kleinen Fußballspiele mit unterschiedlichen Schwerpunkten sind für das Erlernen der technischen Fertigkeiten des Fußballs ebenso wirksam wie das spe- zifisch technikorientierte Training.}

Diese Hypothese wird in der Studie drei (ab Seite 149) behandelt. 


\section{Förderung der kognitiven Fähigkeiten durch Kombinationsspiele}

Im Vergleich zu weniger qualifizierten Spielern sind erfahrene Spieler schneller und genauer bei der Erkennung und Erinnerung von Spielhandlungen. Sie können ihre Gegner besser antizipieren, wenden wirksamer und geeigneter visuelle Anhaltspunkte an und sind genauer in ihrer Erwartung dessen, was wahrscheinlich bei einer bestimmten Reihe von Umständen geschehen wird (WILLIAMS, 2008, S. 38). Einige kollektive Ballspiele ohne Opposition fördern die Entwicklung der kognitiven Fähigkeiten wie Wahrnehmung, Bewegung, Antizipation, Aufmerksamkeit und Konzentration (ROTH, 1993; ROTH, 1998; KRÖGER \& ROTH; 1999, S. 86 ff.; ROTH; KRÖGER \& MEMMERT, 2007, S. 21; MEMMERT \& ROTH, 2007). Dadurch kann man die Koordination der Aktionen innerhalb der Mannschaft verbessern und dadurch das Zusammenspiel fördern.

Im Rahmen dieser Arbeit wird eine Reihe von Spielen für die Förderung der kognitiven Fähigkeiten die informationelle und motorische Anforderungen stellen, als didaktische Strategie vorgeschlagen. Sie werden als "Kombinationsspiele“ bezeichnet. Es sind Spiele ohne Opposition, in denen die Teilnehmer Aufgaben von Wechselstellung, Antizipation und Passgenauigkeit absolvieren sollen, was hohe Aufmerksamkeit und Konzentration erfordert. Die Anwendung dieser Strategie bei der Spielerausbildung soll durch eine Steigerung des Zusammenspiels und Behaupten des Balls zugunsten des Angriffs zur Verbesserung der Mannschaftsspielfähigkeiten beitragen, wenn die Mannschaft sich in Ballbesitz befindet.

Die Hypothese für diese Lehrstrategie lautet folgendermaßen:

H4. Ein Trainingsprogramm, das aus einer Reihe von Kombinationsspielen besteht, verbessert die Fähigkeit des Zusammenspiels.Damit schaffen die Spieler wirksamere offensive Spielhandlungen, wenn sie sich in Ballbesitz befinden.

Diese Hypothese wird in der Studie vier (ab Seite 168) behandelt.

\subsubsection{Forschungsstrategien}

Die Spielmodellforschung ist weit davon entfernt, in der Sportpraxis anerkannt zu sein. Die Umsetzung eines innovativen und noch nicht bestätigten Modells könnte problematisch sein in einem Milieu, in dem, Eltern und Trainer traditionell ergebnisorientiert sind und das Direkte Modell immer noch als wirksam betrachtet wird. Noch schwieriger wäre es, dass Trainer, Eltern und Spieler auf die Kontrolle verzichten, die sie seit langer Zeit gewohnt sind. Die schlagartige Zunahme der kognitiven Anforderungen an die Spieler könnte auch auf Widerstand stoßen.

Unter Berücksichtigung der im Abschnitt 2.3.16 (siehe S. 62 ff.) beschriebenen Hinweise von KIRK (2005) und METZLER (2005b) bezüglich der Forschung über didaktische Modelle für Sportspiele wurden Strategien für die Überprüfung des Modells der Spielhandlungskompetenzen angewendet, die eine schrittweise Anpassung an die Vorstellungen und Usancen der Sportvereine anstrebten. Diese Strategien werden hier beschrieben:

- Es wird auf das sogenannte „Versus Paradigma“ verzichtet. Erst einmal wird die Wirksamkeit der methodischen Maßnahmen überprüft. Ein zukünftiger Vergleich 
mit anderen Modellen oder didaktischen Strategien hat als Voraussetzung, dass das Modell die Erwartungen an Kontextanpassung, Spielkompetenzentwicklung und Anwendungsfreundlichkeit erfüllt, bevor seine Überlegenheit über andere Modelle (insbesondere das Direkte Modell) getestet wird.

- Das Modell wird in seine didaktischen Strategien untergliedert, jede didaktische Strategie wird jeweils in einer eigenen Studie getestet.

- Bei der Anwendung jeder didaktischen Strategie wird nur ein Teil der Trainingszeit im Anspruch genommen, um die geplante Vorbereitung auf Wettkampfspiele nicht zu stören, da der Wettkampf bei Eltern und Trainern als unverzichtbar angenommen wird, sogar wenn es um kleine Kinder geht.

- Es werden Auswertungsmethoden entwickelt, um die spezifischen Erfolge der didaktischen Strategie testen zu können. Die vorhandenen Auswertungsmethoden im Bereich der Sportspiele sind momentan nicht ausreichend für die Auswertung der Spielkompetenzen.

- Der Forscher wird bei jeder Studie als Trainer während der Umsetzung der didaktischen Strategien agieren. Die Teilnahme der Trainer jeder Mannschaft hätte die entsprechende Schulung und vor allem die Überwindung der Widerstände gegenüber Änderungen zur Voraussetzung. Überzeugungsarbeit könnte nur erfolgreich geleistet werden, wenn die Wirksamkeit des Modells wenigstens einmal bestätigt würde.

- Die im Rahmen dieser Arbeit durchgeführten Studien werden als erster Schritt der Validierung des Modells betrachtet. Eine spätere Validierung in seiner Gesamtheit und der Vergleich mit anderen didaktischen Modellen unter Berücksichtigung der angestrebten Ziele wird als notwendig erachtet. Die vorliegende Arbeit reicht wegen ihrer Beschränkungen nicht aus, dieser Forderung gerecht zu werden. 


\section{Studie}

\subsection{Studie 1. Prozedurale Spielkompetenz}

\subsubsection{Einführung}

Während eines Fußballspiels berühren die Spieler den Ball nur eine sehr geringe Zeit. Eine Studie bei jungen Spielern auf Weltmeisterschafts-Ebene (GREENER, 1993; zitiert nach LIGHT, 2005a, S. 171) berichtet von 60 Minuten Spielzeit mit Ball in Bewegung, in denen jeder Spieler nur zwischen 2 und 3 Minuten Spielzeit mit dem Ball absolviert. Eine eigene Studie mit Kindern im Alter zwischen 10 und 12 Jahren (ARIAS \& JIMÉNEZ, 2007) legt nahe, dass in einem Fußballspiel der Ball nur 53\% der Gesamtzeit in Bewegung ist und jedes Kind inn im Durchschnitt nur 52 Sekunden lang berührt. Allerdings sind diese Augenblicke von Ballbesitz von entscheidender Wichtigkeit, denn innerhalb dieser geringen Zeit kann der Spieler auf das Spiel einwirken und durch seine individuelle Leistung mit Passen, Schießen, Dribbeln und Ballführung zur Gesamtleistung der Mannschaft beitragen.

Aus diesem Grund sollen die technischen Fertigkeiten gelernt, automatisiert und verfeinert werden, um eine optimale Leistung bei Handlungen mit Ball zu schaffen. Dieser Prozess von Lernen und Training dauert normalerweise viele Jahre. Trotz dieses zeitlichen Aufwands versuchen die Sportvereine geschickte Spieler auszubilden, die eine hohe Spielleistung durch die Beherrschung des Balls erreichen. Das Hauptziel der Ausbildungsprogramme ist das Lernen der technischen Fertigkeiten, ihm wird ein Großteil der Trainingszeit gewidmet (vgl. SAMMER, 2010, S. 12). Das Lernen der Handlungen ohne Ball in Angriffs- und Abwehrsituationen bekommt hingegen vergleichsweise wenig Aufmerksamkeit während der Instruktion. Das Lernen des Verhaltens ohne Ball wird als Teilgebiet der taktischen Ausbildung zugeordnet. Der Deutsche Fußball Bund empfiehlt in seinen Ausbildungsprogrammen die Schulung der Taktik erst ab der Altersklasse D Junioren im Alter von 11 und 12 Jahren.

In Deutschland nehmen Kinder unter 6 Jahren schon an organisiertem Training und an Wettspielen in der Altersklasse G (Bambini) teil. Bis zum 11. Lebensjahr sollten die jungen Fußballspieler laut Empfehlung nur eine Ausbildung der technischen Fertigkeiten bekommen, einetaktische Ausbildung ist nicht vorgesehen. Die Trainer/innen bemühen sich um ein organisiertes Zusammenspiel ihrer Mannschaften zu schaffen, ihre Arbeit in Bezug auf Handlungen ohne Ball innerhalb des Trainings und den Spielen wird jedoch auf Hinweise über das richtige Verhalten während des Spiels bei Situationen ohne Ballbesitz beschränkt. Die Trainer/innen bieten den Kindern Informationen an, damit versuchen sie, explizites Wissen über das richtige Verhalten ohne Ball während des Spiels zu vermitteln. Das soll ein explizites Lernen auslösen, d.h., bewusste Verhaltensmuster anregen. Einem Prozess des impliziten Lernens d.h. unbewusste Verhaltensmuster hervorzurufen(MANZUR \& STEINWECK-FLECKNER, 2006, S. 178), die zum Wissenserwerb hinsichtlich der Handlungen ohne Ballbesitz beitragen könnten, wird nicht angestrebt.

Dem Begriff „Taktik“ werden viele Bedeutungen zugewiesen (z. B. CARL, 1976, S. 312; DÖBLER, SCHNABEL \& THIEß, 1989, S. 190; WEINECK, 2004b, S. 605; HOHMANN, 
1994, S. 73; TSCHIENE \& BARTH, 1997, S. 88). LOY (2005, S. 192-193) unterscheidet einen weiten Sinn von einem engen Sinn des Begriffs „Sportliche Taktik“. Im weiten Sinn wird sportliche Taktik betrachtet als eine langfristige Vorbereitung der Sportler für das Erreichen der Leistung. Das beinhaltet alle Handlungen „die der Sportler vom Beginn bis zum Ende des sportlichen Kampfes ausführt, um optimale Ergebnisse zu erzielen oder den Sieg zu erringen“ (HARRE, 1982, S. 219). Häufig wird fälschlicherweise die Taktik nur aus der Perspektive des theoretischen Wissens betrachtet; dieses Wissen umfasst u.a. abstrakte Inhalte wie taktisches Verhalten in einer speziellen Spielposition, Wettkampforganisation und Spielsysteme. Tatsächlich wird manchmal die Taktik in eingeschränkter Sichtweise als Synonym für die Anwendung von Spielsystemen und komplexen mannschaftstaktischen Maßnahmen verstanden. Diese Vorstellung wird von Fußballexperten, die in den Massenmedien auftreten, häufig bekräftigt. Deswegen wird eine taktische Schulung abgelehnt, sie würde die Kinder zu einer strengen, leistungsorientierten Routine führen.

Im engeren Sinn versteht man unter Taktik „die kurzfristigen, situationsbedingten Problemlösungsprozesse, die die Athleten während der Wettkämpfe zu bewältigen haben“ (ROTH, 1989, S. 6). Nach dieser Auffassung ist die Taktik stets gefragt, wenn es problematische Situationen zu lösen gilt, wie dies in Sportspielen ständig der Fall ist.

Fußball ist ein Sport mit Kooperation und Opposition. Bei jeder Situation während des Spiels muss der Spieler Entscheidungen treffen. Auch wenn Kinder Fußball spielen, werden sie kontinuierlich zu Entscheidungen in Angriffs- und in Abwehrsituationen aufgefordert. Trotzdem erzeugt die natürliche Anziehungskraft des Balls als Spielobjekt eine übertriebene Hervorhebung der Schulung der technischen Fertigkeiten und ein vollständig auf den Ball zentriertes Spiel. Als Folge laufen die Kinder hin und her, ohne eine Idee darüber zu haben, was zu tun ist, wenn sie keinen Ballbesitz haben. Das mannschaftliche Zusammenspiel ohne Ball ist in den meisten Kinderwettkämpfen kaum vorhanden. Diese Stufe der Spielentwicklung wird von OLIVEIRA und GRACA $(2005,8)$ als „Phase des chaotischen Spiels“ charakterisiert. Die Autoren schlagen verschiedene „Phasen“ des Spiels vor. Das Lernen von bestimmten Spielregeln oder Prinzipien ermöglicht die Evolution des Spiels und das Erreichen einesbesseren Niveaus, in der das Spiel organisierter und vernünftiger erscheint. Vom „chaotischen Spiel“ ausgehend, kann man ein besseres Spiel erzeugen, wenn die Kinder die geeigneten Handlungen in Abwehr und Angriff und mit und ohne Ballbesitz auszuführen lernen.

Die Betonung von kognitiven Aspekten fördert auch den Spaß beim Spielen, da das Spiel nicht ausschließlich von den Spielern mit weiter fortgeschrittener physischer und technischer Entwicklung dominiert wird. Jedes Kind hat eine faire Gelegenheit, gemäß seiner kognitiven Entwicklung am Spiel teilzunehmen. Kurz gesagt: Die Leistung von Fußballspielern wird nicht nur durch physiologisch-konditionelle Fähigkeiten und technische Fertigkeiten determiniert, sondern auch von kognitiven Fähigkeiten mitbestimmt.

Häufig wird das Lernen in ein explizites und ein implizites Lernen unterteilt (CALÁBRIA, 2011, S. 24). Bei ersterem kann man die logischen Überlegungen, die zur Entscheidung führen, bewusst beschreiben, bei letzterem hingegen geht es um einen unbewussten Prozess. 
„...Unter implizitem Lernen werden Lernformen verstanden, die ohne Beteiligung des Bewusstseins stattfinden und die häufig (aber nicht immer) beiläufig und ohne gezielte Lernabsicht eintreten. Das zentrale Kriterium für implizites Lernen besteht in seiner unbewussten Qualität.

Da nicht jede der zahlreichen im Spiel auftretenden Spielkonstellationen während des Lernens dargestellt werden und ihre Lösung bewusst erlernt werden kann, werden die taktischen Fähigkeiten und Fertigkeiten am besten durch implizites Lernen gelernt.

ROTH und MEMMERT (2007, S. 203; vgl. ROTH, 1999; MEMMERT, 2000) haben festgestellt, dass Kinder taktische Verhaltensweisen lernen können, indem man reglementierte Spiele einsetzt, die das implizite Lernen fördern. Verschiedene didaktische Ansätze für die Sportspiel-Schulung, z. B. Teaching Games for Understanding (BUNKER \& THORPE, 1986; BUTLER \& McCAHAN, 2005), Play Practice (LAUNDER, 2001), Tactical Games (MITCHELL, OSLIN \& GRIFFIN 1997), Game Sense (LIGHT, 2005a,b), Fußballentwicklungsmodell (WEIN, 2009) und die Heidelberger Ballschule (HOSSNER \& ROTH, 2000) schlagen Lernprozesse für Sportspiele vor, die aus modifizierten Spielen bestehen und die kognitiven Fähigkeiten fördern.

Im Rahmen dieser Studie wird Taktik als die Gesamtheit der Aktionsregeln verstanden, die ein Spieler beherrschen soll, um in Spielsituationen die besten Entscheidungen zu treffen und die besten Handlungen ausführen zu können, d. h. Taktik wird als Handlungswissen betrachtet. Diese Aktionsregeln könnten besser gelernt werden, wenn ein Prozess stattfindet, in dem die kognitive Leistung gefördert wird. Kleine einfache Spiele, Spiele mit reduziertem Spielfeld und weniger Teilnehmerzahl pro Mannschaft als in Wettkampfspielen stellen taktische Probleme bereit, die die jungen Spieler während des Spiels lösen sollen. Dadurch sollen sie eine Entwicklung der Entscheidungsfähigkeit und auch eine bessere Spielhandlung hervorbringen. Die Kleinen Spiele scheinen auch ein geeignetes didaktisches Verfahren für das Lernen der Spielhandlungen ohne Ballbesitz zu sein.

Das implizite Lernen könnte auch durch ein explizites Lernen unterstützt werden. Das Reflektieren trägt zum „denkenden Handeln“ (RICHARD \& WALLIAN, 2005, S. 24) bei. Dies gelingt durch Fragen über das richtige oder falsche Handeln bei problematischen Situationen, die in den kleinen Fußballspiele vorkommen. Orientierungsfragen führen die Spieler auf den Weg der richtigen Lösungen (BUTLER, 1997, S. 43) und die Anwendung der Lösungen beim Spielen wird gefördert. Der Trainer wird nicht als Informationsvermittler sondern als „Lernerleichterer“ verstanden. Das Spielen selbst wird, wie in Zeiten des Straßenfußballs, durch Spielen gelernt - die Fragen ergänzen es jedoch durch kognitive Anreize, die ein denkendes Spielen schaffen sollen.

In dieser Studie wird ein Programm für das Lernen der Spielhandlungen durch kleine einfache Spiele in der Ausbildung von jungen Spielern eingeführt und ausprobiert. Mit dieser Studie wird versucht eine Antwort auf die folgende Frage zu finden.

- Ermöglicht ein Trainingsprogramm, das auf Kleinen Spielen und Fragen basiert ist, die Verbesserung der Spielkompetenzen mit und ohne Ballbesitz von Spielern im Alter von 6 - 8 Jahren?

Die folgenden Hypothesen wurden aus dieser Fragestellung abgeleitet: 
- Hypothese 1: Der Mittelwert richtiger Handlungen wird im Posttest nach Absolvieren eines Lernprogramms für das Lernen der Spielkompetenzen durch Spielen und Nachdenken größer als im Vortest sein.

- Hypothese 2: Der Mittelwert richtiger Handlungen wird im Retentionstest größer als im Vortest sein.

\subsubsection{Methoden}

\section{Teilnehmer}

In der Versuchsgruppe waren 16 Mädchen. Sie sind Fußballspielerinnen der Altersklasse F-Junioren des DFBs (6 - 8 Jahre alt), die zu einem Verein gehörten und am Wettspielbetrieb im Kreis Göttingen teilnahmen. Sie hatten jede zweite Woche ein „3Punkte“-Spiel. Alle Kinder hatten mindestens sechs Monaten Fußballausbildung und an mindestens vier offiziellen Spielen teilgenommen. Es wurden Genehmigungen der Probanden und ihrer Eltern beantragt und gewährleistet, dass die Bilder, Videos und Daten, die während der Evaluation und Trainingssitzungen gespeichert werden, nur für die Forschung benutzt und anonym behandelt werden. Die TrainerInnen und die LeiterIn der Fußballabteilungen des Vereins waren einverstanden mit der Forschungsarbeit.

\section{Kriteriumsaufgaben und -instrumente}

Es wurde eine Umstellung des Tests „GK3-3GK“ (COSTA, GARGANTA, GRECO \& MESQUITA, 2009) vorgenommen.Dieser wurde durch das Hinzufügen einer zusätzlichen Spielerin in ein „T4:T4“ erweitert, um die Handlungen der Spieler mit und ohne Ball bezüglich wesentlicher taktischer Prinzipien des Fußballs auszuwerten. Bei diesem Test nehmen die Spieler an einem Mini-Fußball-Spiel (4 gegen 4 mit Torwart) teil (WEIN \& SCHREINER, 2004, S. 9). Das Spiel findet in einem 32 Meter langen und 25 Meter breiten Fußballfeld in einer Sporthalle statt (siehe Anhang A). Alle Regeln des Fußballspiels, mit Ausnahme der Abseits- und Strafstoßregeln, werden eingehalten. Das Spiel dauert 10 Minuten.

Vor dem Test wurde den Spielern eine kurze Erklärung der Regeln und Ziele des Spiels gegeben. Die Mannschaften wurden zufällig ausgewählt. Trugen zwei oder mehr Spieler Sportkleidung mit ähnlichen Farben, wurden sie durch Leibchen kenntlich gemacht, um ihre Identifizierung zu erleichtern. Eine kurze Vor-Spielzeit von 30 Sekunden wurde zur Eingewöhnung in das Spiel gewährt.

Das Spiel wurde mit einer Videokamera JVC Everio S, GZ-MS90 aufgezeichnet. Dabei wurde die Videokamera an eine Ecke der Sporthalle auf eine obere Ebene gestellt, um das ganze Feld überblicken und alle 8 teilnehmenden Spielerinnen auf der Videoaufnahme ständig sichtbar zu machen. Die Spielerinnen, die als Torhüterin agierten, wurden beim nächsten Spiel als Feldspielerinnen eingesetzt. Es wurden 3 Spiele aufgenommen, damit konnte man eine 10-minütige Videoaufnahme von jeder Spielerin als Feldspielerin erhalten. Der Abbildung 22 stellt die Kriteriums-Aufgabe dar. 


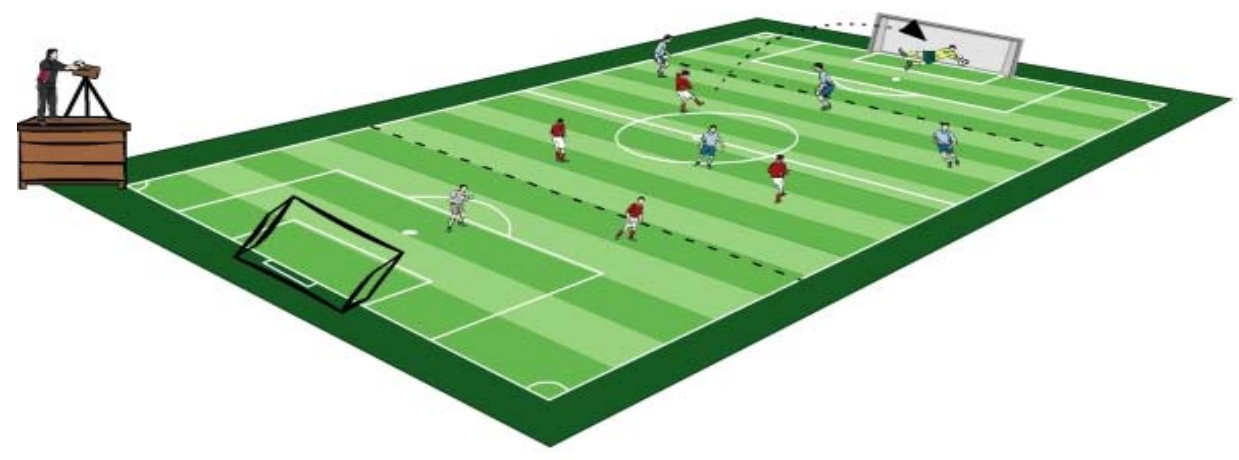

Abbildung 22: Kriteriums-Aufgabe: Das Mini-Spiel T4:T4

Die Videoaufnahmen wurden auf einen Laptop übertragen und mit Hilfe des Programms „Pinnacle VideoStudio 2.0“ in „avi“-Dateien umgewandelt und in Spielhandlungen geschnitten. Ein geschulter Beobachter führte eine Auswertung durch. Er beobachtete das in den Videoaufnahmen aufgezeichnete Spiel und beurteilte mit Hilfe von Formblättern (Anhang B und C) die Spielhandlungen, die die Spielerinnen jeweils mit Ball (Spieler mit Ball: SmB), als Mitspielerinnen (Spieler ohne Ball, Mannschaft am Ball: SoBMB) und als Verteidigerinnen (Spieler ohne Ball, Mannschaft ohne Ball: SoBMoB) ausführten. Der Auswerter beobachtete bei jeder Auswertung dieselbe Spielerin während des ganzen Spiels. Anschließend beobachtete er eine andere Spielerin und setzte dies fort, bis alle Teilnehmerinnen ausgewertet waren. Die Spielhandlungen jeder Spielerin wurden als geeignet (1) oder nicht geeignet (0) bewertet, und der Beobachter wies jeder Spielhandlung die entsprechende Nummer in dem dafür entworfenen Formblatt zu (siehe Anhang C). Die Betrachtung einer Spielhandlung als geeignet oder nicht geeignet erfolgte mit Hilfe der Parameter im Anhang B. Wenn z. B. eine Spielerin versuchte, den Ball zu erobern, obwohl die Ballbesitzerin eine Mitspielerin war, wurde diese Handlung nach dem Zahlzeichen 8 des Auswertungsblatts als nicht geeignet bewertet und auf dem Feld SoBMB im Formblatt dieser Spielerin wurde eine 0 eingetragen.

Das Auswertungsverfahren wurde zu drei Messzeitpunkten durchgeführt: Im einem Vortest vor Beginn der Intervention, im einem Posttest unmittelbar nach Ende der Lernperiode von 8 Sitzungen und im einem Retentionstest drei Wochen nach Ende der Intervention. Mit diesen Daten wurden verschiedene Leistungszahlen für die Spielprinzipien errechnet. Eine Spielhandlung wurde dann als Verhalten des Spielers betrachtet, wenn er oder andere Spieler im Ballbesitz waren. Da bei jedem Wechsel des Ballbesitzers der Spieler beobachten und eine Entscheidung treffen muss, um sich an eine neue Situation anzupassen, wurde ein neuer Ballbesitz als eine neue Spielhandlung betrachtet.

\section{Variablen}

Spielkompetenz wird in dieser Arbeit zuerkannt, wenn die teilnehmenden Spielerinnen die Spielsituationen, die während des Spiels stattfinden, durch geeignete Handlungen 
Iösen. Als Variable wurde die Anzahl der geeigneten Spielhandlungen innerhalb von drei Spielsituationen bestimmt:

- Spieler mit Ball (SmB): Die Spielerin besitzt den Ball und führt zunächst eine von drei möglichen Handlungen aus: Dribbeln, Passen oder zum Tor schießen.

- Spielerin ohne Ball, Mannschaft am Ball (SoBMB): Die Spielerin agiert momentan als Mitspielerin der Ballbesitzerin.

- Spielerin ohne Ball, Mannschaft ohne Ball (SoBMoB): Die Spielerin agiert momentan als Verteidigerin.

\section{Intervention}

DasInterventionsverfahren bestand aus einer Serie von 8 Trainingseinheiten. Innerhalb jeder Sitzung wurden Kleine Fußballspiele mit unterschiedlichen Schwerpunkten (KFSS) durchgeführt, die zur Schulung von Spielprinzipien (GARGANTA \& PINTO, 1994, S. 22) entworfen wurden. Die KFSS bestehen aus besonderen Regeln, die die Spieler zum Zusammenspielen auffordern. Die geringe Anzahl der Spieler bei jedem Spiel, immer weniger als bei Wettkampfspielen, fordert eine Beteiligung aller Teilnehmer an der entsprechenden Spielsituation in Angriff oder Abwehr. Die Überzahl im Angriff erleichtert die Aufgabe der Angreifer und die Unterzahl in der Abwehr verpflichtet die Spieler in der Verteidigung zu einem Kompromiss. Einige Spiele werden in einer Anpassung des Fußballspiels durchgeführt, in der die Spieler den Ball mit den Händen anfassen können. Dies erleichtert die technischen Anforderungen und erlaubt die Konzentration auf die taktischen Probleme.

Jedes KFSS stellt den Spielern ein Problem, das sie durch die Durchführung geeigneter Handlungen lösen sollen. Die Handlungen werden abhängig von der Spielrolle durchgeführt, die die Spieler momentan übernehmen, d. h. Spieler mit Ball (SmB), Spieler ohne Ball der Mannschaft am Ball (SoBMB) und Spieler ohne Ball der Mannschaft ohne Ball (SoBMoB). Die Regeln der Spiele fordern vom SmB zu passen, zum Tor zu schießen oder zu dribbeln, vom SoBMB sich freizulaufen oder anzubieten und vom SoBMoB den Weg des Angreifers mit Ball zu behindern oder seine Mitspieler zu decken. Die Abbildung 23 stellt ein Beispiel von einem kleinen Spiel mit Schwerpunkt (siehe auch Anhang $D$ und $E)$. 


\section{3 gegen $1+1$ Torwart.}

Eine Dreier-Mannschaft greift die beiden Tore auf jeder der beiden Grundlinien des Mini-

Feldes an. Die Tore werden von einem Abwehrspieler und von einem Torwart verteidigt.

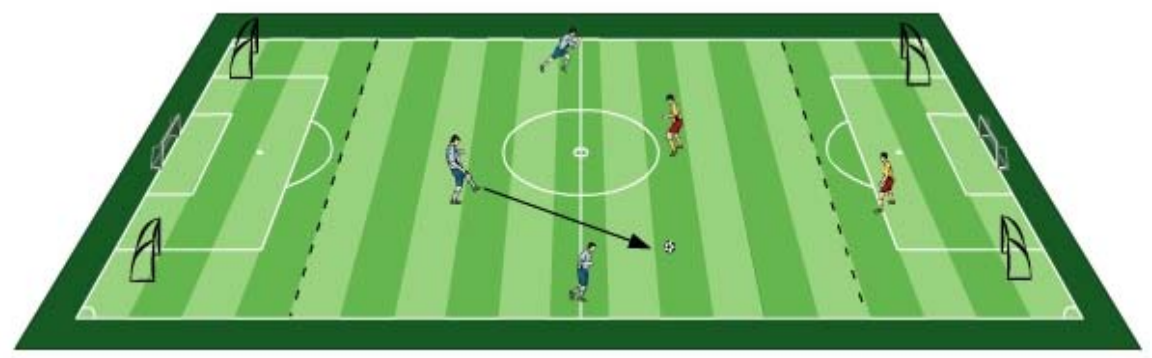

Der Ballbesitzende sollte vor seinem Pass nicht nur das Stellungsspiel des Verteidigers, sondern auch das des Torwartes berücksichtigen. Nach jedem Angriff wechselt ein Spieler von Mannschaft um, die Dreier-Mannschaft greift jetzt in die Gegenrichtung.

Es gewinnt die Dreier-Mannschaft, die bei einen Angriff 3 gegen 2 die geringste Zeit brauch, um ein Tor zu erzielen.

Dauer: 7 Min.

\section{Abbildung 23: Beispiel eines Kleinen Fußballspiels mit Unterschiedlichen Schwerpunkten}

Während des Spiels wurden Pausen eingefügt, in denen die Spielerinnen ihre momentane Position und Situation behalten sollten („eingefrorene Zeitpunkte“). Die Trainerin und der Co-Trainer stellten Fragen, die die Spielerinnen in Abhängigkeit ihrer jeweiligen Rollen zur Überlegung über geeignete Handlungen führten. Mit Hilfe von Bildern wurde den Spielern eine Spielsituation vorgestellt und Fragen über die auszuführende richtige Handlung bei dieser Situation wurden gestellt (siehe ein Beispiel in Anhang F). Die Bilder dienten dabei zu einer besseren Erklärung der Situation und erleichterten das Verständnis der Fragen. Die Bilder selbst stellten kognitive Anregungen dar, die auch zum Lernprozess beitragen (DAUGS \& BLISCHKE, 1993). Nach jedem KFSS fand während einer Trinkpause ein reflektierendes Gespräch mit den Spielerinnen statt.

Die Forscher bereiteten die Trainingseinheiten vor und stellten sie der Trainerin vor, um Meinungen darüber auszutauschen. Darauf basierend wurde jede Trainingseinheit unter Beachtung der Anregungen der Trainerin verarbeitet und eine übereinstimmende Vorbereitung wurde fertiggestellt. Die Trainerin wurde über alle Prozeduren informiert, die Ziele und ihre Rolle in der Studie wurden ihr erklärt. Die Anregungen der Trainerin wurden immer beachtet, allerdings ohne die Grundidee des „Spielenlernens durch das Spielen“ im Ansatz zu ändern, so dass in jedem Fall ausreichende Informationen über Auswer- 
tungs- und Trainingsverfahren zur Verfügung standen. Ein Forscher wurde als CoTrainer während der Interventionszeit eingesetzt.

In der Abbildung 24 wird den Untersuchungsablauf dargestellt.

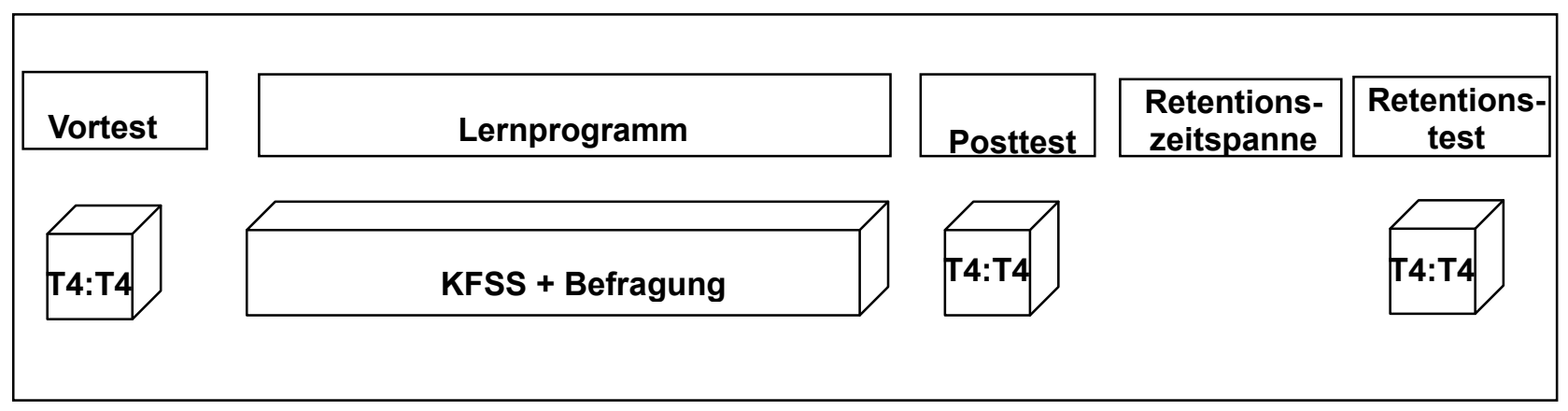

Abbildung 24: Untersuchungsablauf der Studie eins

\section{Statistische Analysen}

Die statistischen Verfahren wurden mit SPSS für Windows $®$, Version 17.0 durchgeführt. Deskriptive Analysen (Häufigkeit, Mittelwert und Standardabweichung) wurden berechnet, um die Stichprobe zu charakterisieren. Die Normalverteilung der Daten wurde durch den Test von Kolmogorov-Smirnov überprüft und die Homogenität der Varianzen wurde durch den F-Test gesichert. Eine einfaktorielle Varianzanalyse mit wiederholten Messungen wurde genutzt, um die Werte der Leistungszahlen über geeignete Handlungen zwischen Vor-, Post- und Retentionstest zu vergleichen.

Die statistischen Hypothesen gelten für alle Variablen:

H0: $\quad \mu 1=\mu 2 \quad$ bzw. $\quad \mu 1=\mu 3$

$\mathrm{H} 1: \quad \mu 1<\mu 2 \quad$ bzw. $\quad \mu 1<\mu 3$

wobei:

HO die Nullhypothese ist,

$\mu 1$ der Mittelwert des Vortests,

$\mu 2$ der Mittelwert des Posttests und

$\mu 3$ der Mittelwert des Retentionstests. 


\section{Auswertungsobjektivität}

Um die Auswertungsobjektivität festzustellen, beobachteten drei Sportwissenschaftler, zwei davon Fußballtrainer, das Video und beurteilten 294 von insgesamt 1470 Handlungen, die die teilnehmenden Spielerinnen bei den Messzeitpunkten ausführten; sie beobachteten damit 20\% der Videoaufnahme. Die Experten beobachteten zweimal denselben Videoausschnitt und dieselben Spieler und werteten daher unabhängig voneinander zweimal jede der 294 Spielhandlungen aus. Korrelationen zwischen beiden Auswertungen jedes Experten wurden durch das Pearson-product-moment-correlationVerfahren berechnet. Die Ergebnisse zeigen eine Intra-Experten-Korrelation jeweils von $r=.85(p=.004), r=.91(p=.01)$ und $r=.87(p=.045)$. Eine der zwei Auswertungen wurde jeweils von jedem Experten per Zufall ausgewählt, Korrelationen zwischen den drei ausgewählten Auswertungen wurden ebenfalls berechnet. Es wurden eine InterExperten-Korrelation jeweils von $r=.86(p=.005)$ zwischen den Experten 1 und $2, r=$ $.90(p=.010)$ zwischen den Experten 1 und 3 und $r=.87(p=.032)$ zwischen den Experten 2 und 3 gefunden. Einer der Experten war der Forscher selbst, dieser wertete die ganze Stichprobe aus.Anschließend wurden die Ergebnisse statistisch aufbereitet und analysiert.

\subsubsection{Ergebnisse}

Die Mittelwerte der richtigen Handlungen der „Spieler mit Ball (SmB)“ sind im Posttest stabil geblieben. Jedoch fand eine deutliche Steigerung der Werte im Retentionstest statt. Die Mittelwerte der Fehler zeigen eine leichte Verringerung im Postest, sind im Retentionstest jedoch angestiegen. Im Gegensatz dazu sind die Mittelwerte der „Spieler ohne Ball Mannschaft am Ball (SoBMB)" im Posttest gestiegen und beim Retentionstest leicht gesunken, die Mittelwerte der Fehler sind hier leicht gesunken. Ein ähnliches Verhalten findet sich in der Variable „Spieler ohne Ball der Mannschaft ohne Ball (SoBMoB)“. Auch wenn die Gesamtzahl der Handlungen berücksichtigt wird, zeigen sich Verbesserungen der Handlungen mit und ohne Ball im Posttest und leichte Verschlechterungen beim Retentionstest gleichzeitig mit einer Verminderung der Fehleranzahl (Tabelle 9), (siehe Gesamtdaten im Anhang G).

Tabelle 9: Mittelwerte der richtigen Handlungen (+), Fehler (-) und Standardabweichungen (in Klammern) bei Spielen. SmB: Speler mit Ball; SoBMB: Spieler ohne Ball, Mannschaft am Ball; SoBMoB: Spieler ohne Ball, Mannschaft ohne Ball $(n=16)$

\begin{tabular}{|c|c|c|c|c|c|c|c|c|} 
& \multicolumn{2}{|c|}{ SmB } & \multicolumn{2}{c|}{ SoBMB } & \multicolumn{2}{c|}{ SoBMoB } & \multicolumn{2}{c|}{ Gesamt } \\
& + & - & + & - & + & - & + & - \\
\hline Prätest & 2,67 & 3,25 & 5,33 & 5,67 & 10,33 & 11,92 & 18,33 & 20,83 \\
& $(1,61)$ & $(1,29)$ & $(1,72)$ & $(3,5)$ & $(3,39)$ & $(5,21)$ & $(4,96)$ & $(7,07)$ \\
\hline Posttest & 2,75 & 3,17 & 7,08 & 4,25 & 13,75 & 11,00 & 23,58 & 18,42 \\
& $(1,06)$ & $(1,47)$ & $(2,54)$ & $(1,06)$ & $(4,11)$ & $(3,57)$ & $(3,75)$ & $(4,23)$ \\
\hline $\begin{array}{c}\text { Retentions- } \\
\text { test }\end{array}$ & 3,67 & 3,83 & 6,58 & 4,25 & 12,25 & 10,75 & 22,5 & 18,83 \\
& $(1,67)$ & $(1,8)$ & $(2,57)$ & $(1,6)$ & $(2,6)$ & $(1,86)$ & $(4,15)$ & $(1,99)$ \\
\hline
\end{tabular}


Eine grafische Darstellung dieser Ergebnisse folgt in der Abbildung 25.

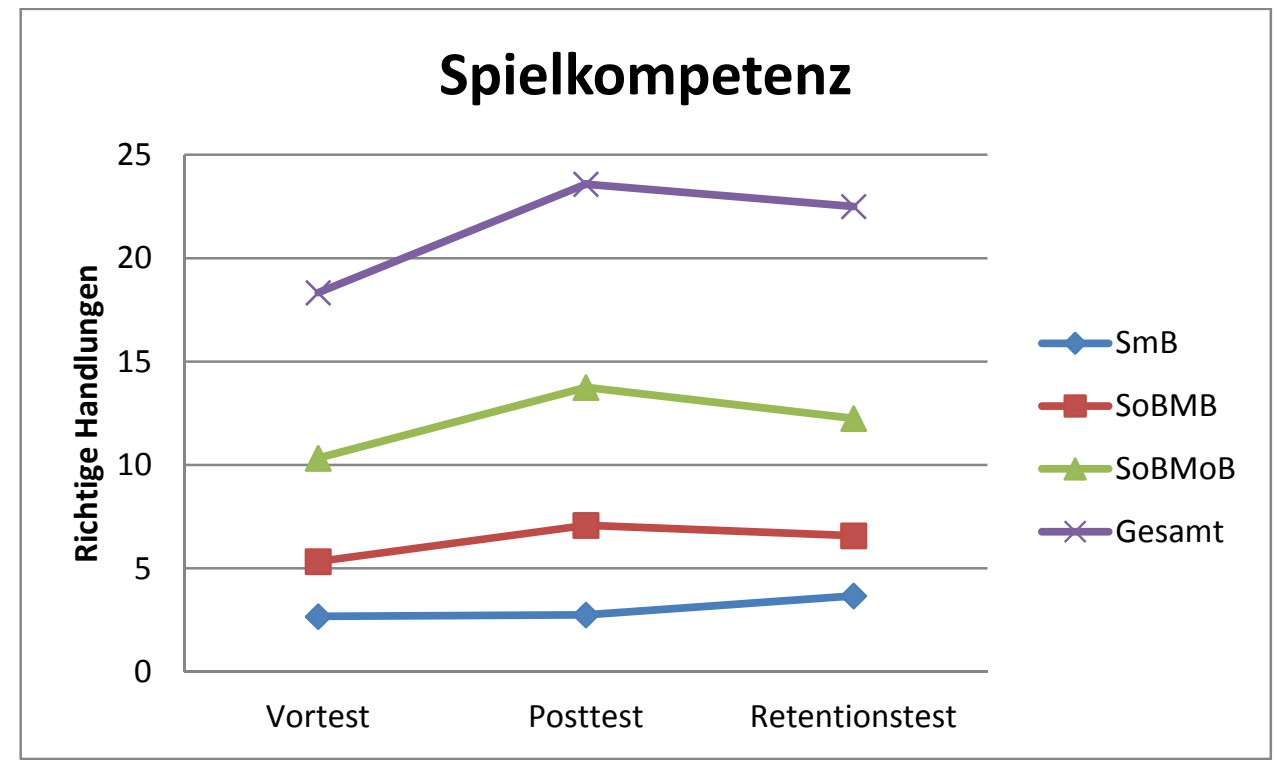

Abbildung 25: Entwicklung der richtigen Handlungen bei Variablen der Spielkompetenz: SmB: Speler mit Ball; SoBMB: Spieler ohne Ball, Mannschaft am Ball; SoBMoB: Spieler ohne Ball, Mannschaft ohne Ball

Prozentuale Mittelwerte der geeigneten Handlungen jeder Variable bei den drei Messzeitpunktenwurden berechnet (Tabelle 10), die Summe der geeignet und ungeeignete Handlungen wurde als $100 \%$ angenommen. Eine prozentuale Steigerung der Leistung der Spielerinnen nach der Anwendung eines Trainings-programms, das aus KFSS besteht, von fast 5\% für die Variable „Spieler mit Ball (SmB)“ wurde erreicht. 10\% ergeben sich für die Variable „Spieler ohne Ball Mannschaft am Ball (SoBMB)“, 8\% für „Spieler ohne Ball Mannschaft ohne Ball (SoBMoB)" und 10\% für die Gesamtzahl der Spielhandlungen. Im Retentionstest stieg der Mittelwert um zusätzliche $2 \%$ für die Gruppe SmB. Demgegenüber sind die Mittelwerte der anderen Variablen SoBMB und SoBMoB im Retentionstest nach eine Zeitspanne von drei Wochen ohne spezifische Anwendung des Lernprogramms durch KFSS leicht gesunken.

Tabelle 10: Prozentzahl der geeigneten Handlungen. SmB: Spieler mit Ball; SoBMB: Spieler ohne Ball, Mannschaft am Ball; SoBMoB: Spieler ohne Ball, Mannschaft ohne Ball $(n=16)$

\begin{tabular}{|l|c|c|c|c|} 
& SmB \% & SoBMB \% & SoBMoB \% & Gesamt \% \\
\hline Vortest & 42,89 & 52,35 & 47,34 & 46,80 \\
\hline Posttest & 47,69 & 62,54 & 55,36 & 56,27 \\
\hline Retentionstest & 49,25 & 59,98 & 53,02 & 54,12 \\
\hline
\end{tabular}


Die Annahme der Normalverteilung wurde durch den Kolmogorov-Smirnov-Test bestätigt. Die Ergebnisse widerlegen nicht die Normalverteilungsannahme $(p>.05)$ (siehe die Werte des Kolmogorov-Smirnov-Tests im Anhang H). Die Sphärizität wurde durch den Mauchly-Test getestet, sie wurde für die Variablen dieser Studie nicht verletzt $(p>.05)$ (siehe die Werte des Mauchly-Tests in Anhang $\mathrm{H}$ ). Wegen der geringen Anzahl der Stichproben wurde trotzdem der angenommenen Sphärizität die Greenhouse-GeisserKorrektur der Freiheitsgrade verwendet.

In einer einfaktoriellen Varianzanalyse mit wiederholter Messungen traten keine signifikanten Unterschiede zwischen Vor-, Post- und Retentiontest für die geeigneten Handlungen der Variable Spieler mit Ball $(\mathrm{SmB})(\mathrm{F}[1,94 ; 21,36]=.36, p=.70)$, der Variable Spieler ohne Ball Mannschaft am Ball (SoBMB) $(\mathrm{F}[1,60 ; 17,50]=2.54, \quad p=.12)$ und der Variable Spieler ohne Ball Mannschaft ohne Ball (SoBMoB) $(\mathrm{F}[1,76 ; 19,35]=1.71, p=$ .21), auf. Für die Mittelwert der Gesamtzahl der richtigen Handlungen wurde dagegen ein signifikanter Unterschied gefunden $(F[1,81 ; 19,97]=4.57, \quad p<.05)$. Die Post-Hoc Analyse zeigt, dass die Unterschiede zwischen Vortest und Posttest vorhanden sind (M $=18,33$ vs. $M=23,58, p<.05)$, nicht aber zwischen Vortest und Retentionstest $(M=$ $18,33$ vs. $M=22,50, p=>.05)$ und ebenfalls nicht zwischen Posttest und Retentionstest( $\mathrm{M}=22,50$ vs. 23,58, $p=>$.05) (siehe die Werte der Post Hoc Analyse im Anhang I).

\subsubsection{Diskussion und Schlussfolgerung}

Die Ergebnisse zeigen eine Tendenz zur Verbesserung des Verhaltens in Spielsituationen mit und ohne Ball. Die nachgewiesene Verbesserung wird jedoch nicht statistisch für alle Variablen signifikant. Werden hingegen alle Spielhandlungen mit und ohne Ball zusammengefasst, wird ein signifikanter Unterschied zwischen Vortest und Posttest deutlich. Dieses Ergebnis legt die Annahme von Auswirkungen der Kleinen Fußballspiele mit Unterschiedlichen Schwerpunkten (KFSS) auf das Lernen der Spielhandlungen mit und ohne Ball nahe. Der signifikante Unterschied wurde im Retentionstest allerdings nicht aufrechterhalten.

Die Ergebnisse dieser Studie legen nahe, dass Mädchen im Alter zwischen 6 und 8 Jahren auch ein implizites Lernen der Handlungen ohne Ball leisten können. Dies erlaubt ihnen eine bessere Orientierung auf dem Spielfeld. Sie haben außerdem gelernt, geeignete Entscheidungen zu treffen, wenn sie in Ballbesitz sind.

Der Erwerb von Handlungswissen scheint begünstigt zu werden, wenn die Spielerinnen die Gelegenheit erhalten, über ihre Handlungen nachzudenken (RICHARD \& WALLIAN, 2005, S. 24). Die Kleinen Spiele stellen problematische Situationen bereit, die die Spielerinnen lösen müssen. Die Fragen der Trainer fördern zusätzlich das Nachdenken. Auf diese Weise wird die Entscheidungsfähigkeit gefördert, und die Spielkompetenz verbessert sich.

Die Kleine Spiele mit unterschiedlichen Schwerpunkten und die Befragungsmethode haben sich als wirksam erwiesen, um als Unterstützung zum Lernen der technischen Fertigkeiten zu dienen. Das Verhalten ohne Ball sowohl im Angriff (SoBMB) als auch in Abwehr (SoBMoB) wurde durch das Üben dieser Spiele begünstigt. Weiterhin kann das Spiel in dieser Altersklasse effektiver werden und die jungen Spieler können mit Hilfe 
der KFSS die Phase des chaotischen Spiels (OLIVERA \& GRACA, 2005, S. 8) überwinden.

Die Ergebnisse dieser Studie stimmen mit denen von FRENCH und THOMAS (1987); MITCHELL, OSLIN und GRIFFIN (1995); GRIFFIN, OSLIN und MITCHELL (1995); MCPHERSON UND KERNODLE (2003); TALLIR, MUSCH, LENOIR, und VALCKE, M. (2004) und HARVEY (2006) überein. Sie legen nahe, dass die KFSS das Lernen von technischen Fertigkeiten auf der einen Seite und das Lernen des taktischen Verhaltens auf der anderen Seite fördern. Folglich sind das Spielen und das Nachdenken geeignete Maßnahmen für die Entwicklung der Spielkompetenzen. In Anlehnung an ROTH und MEMMERT (2007, S. 203) wird daher vorgeschlagen, dass Kinder die Aktionsregeln des Spiels durch modifizierte Spiele lernen können. Daher sind die KFSS eine geeignete didaktische Strategie für das Lernen der Fußballtaktik, wenn die Taktik als Handlungswissen betrachtet wird.

Die Ergebnisse zeigen keine statistisch signifikanten Unterschiede zwischen Vortest, Posttest und Retentionstest für die Variablen SmB; SoBM und SoBMoB. Vermutlich wurde die Anwendung des Trainingsprogramms durch die KFSS zu kurz durchgeführt, um große Änderungen der Spielfähigkeit und eine niedrigere Variabilität der Ergebnisse zu erreichen. Spätere Studien sollten daher eine Intervention über einen längeren Zeitraum anstreben, um eine Verbesserung der Spielkompetenzen zuverlässiger feststellen zu können. Die Auswertung unter Trainingsbedingungen ist von unzureichenden Anstrengungen und möglicher fehlender Motivation der Spieler mitbeeinflusst. Die Auswertungen der Spielkompetenzen sollten unter Wettkampfbedingungen erfolgen. Dadurch kann der Transfer des Lernens in die Zielsituation des Spiels nachgewiesen werden und gleichzeitig das Engagement der Spieler/innen gewährleistet werden.

In jedem Fall sollte bei zukünftigen Studien auch eine Kontrollgruppe eingerichtet werden, um die didaktische Strategie der Kleinen Spiele mit unterschiedlichen Schwerpunkten (KFSS) mit anderen Strategien zu vergleichen, z. B. mit dem traditionellen Bereitstellen von expliziten Informationen und direkter Instruktionen durch den Trainer. 


\subsection{Studie 2. Aufbau des präskriptiven Wissens durch eine Befragungsstrategie}

\subsubsection{Einführung}

Die Sportausübung sollte die spezifische kognitive Kompetenz fördern, indem sie geeignete Lösungen für Probleme ermöglicht, die im Wettkampf auftreten. Dieser Bereich der Kognition wird als "taktisches operatives Denken“ bezeichnet (MATVEEV, 1980, S. 147; MAHLO, 1974, S. 550). Außerdem sollte die Sportausbildung den Erwerb von theoretischem Wissen in unmittelbarem Bezug zu Sporthandlungen ermöglichen. Dies wird für die Prinzipien von integraler Ausbildung und rationaler und bewusster Beteiligung der Sportler an Trainingsprozessen untermauert, die auch auf die Leistungssteigerung wirken und zu einer strukturierten Vorbereitung auf ein sportliches Leben der Jugendlichen beitragen. Um diese Forderungen zu erfüllen, sollte die kognitive Schulung der Sportler auf zwei grundlegenden Prinzipien basieren (LOPEZ \& MORENO, 2002a, S. 19):

1 Die Verbindung der Theorie mit der Praxis. Das bedeutsamste Moment im Verlauf der intellektuellen Entwicklung tritt ein, wenn Rede und Praxis zusammenlaufen (VIGOTSKY, 1982, zitiert nach LÓPEZ \& MORENO, 2002a, S. 19).

2 Jedes neue generalisierte Wissen wird gemäß eines bereits vorliegenden Konzeptes durch die Sequenz "Wahrnehmung - Repräsentation - neues Konzept" abgewandelt. Die Bewegung reicht dabei von Wahrnehmung bis Konzept, das entspricht der Durchführung von Sensorischem und Konkretem bis zu Abstraktem (DAVYDOV, 1982, zitiert nach LÓPEZ \& MORENO, 2002a, S. 19).

Aus dieser Sicht geht das Lernen von Konzepten (theoretisches Wissen) von Fakten aus. Diese Fakten sind Ereignisse und Situationen, die in der Praxis ständig auftreten. Bei den Sportspielen beziehen sich diese Fakten auf kontextspezifische Situationen, die als Erlebnisse und Erfahrungen wahrgenommen werden und die sowohl individuelle als auch kollektive Erfahrungen sind.

Das Verständnis ist der Weg, der die Umwandlung von der wahrgenommenen Information in Handlungswissen ermöglicht. Dieser Wissensaufbau ist abgeschlossen, wenn das erworbene Wissen im Langzeitgedächtnis gespeichert ist. Auf dieser Annahme aufbauend, wird hier eine Methode vorgeschlagen die das Vorhanden von spezifisches Wissen in Langzeitgedächtnis nachweisen sollte. Das vorhandene Wissen in Langzeitgedächtnis trägt auf die Lösung von spielspezifischen Aufgaben bei. Eine richtige Lösung von theoretischen Spielsituationen könnte ein Hinweis für die Anwesenheit von Wissen und auch von kognitiven Kompetenzen sein.

Im Rahmen dieser Studie wird eine Befragungsmethode dargestellt und empirisch getestet, die eine Entwicklung der kognitiven Kompetenzen durch Fragen zum Ziel hat. Damit wird ein Verständnis der Konzepte angestrebt, die zur richtigen Lösung der Spielsituationen beitragen. Als motorisch-kognitive Aufgabe wurden die Kleinen Fußballspiele mit unterschiedlichen Schwerpunkten (KFSS) vorgeschlagen. Jedesdieser Spiele hat besondere Regeln, die einzelne Probleme darstellen. Die Spieler werden darin gefördert, diese Probleme durch eigene kognitive Aktivität zu lösen. Bei einer absichtlichen Spielunterbrechung oder während einer Spielpause stellte der Trainer Fragen, die zum 
Überlegen aufrufen und stellte die problematischen Situationen vor, die eine kognitive Lösung verlangen. Die Spieler schlugen dann Lösungen vor, der Trainer fördertet die Anwendung dieser Vorschläge im folgenden Spiel und ermöglichte genug Spielzeit, um die theoretischen Lösungen in praktische Spielhandlungen zu verwandeln.

Bei der Befragungsmethode leitet der Trainer die Fragen aus einem konzeptuellen System ab. Die konzeptuellen Systeme stellen eine strukturierte und logische Integration von Fakten und Konzepten dar und bilden einen Zusammenhang von Konzepten zu einem bestimmten Thema, sie entsprechen der Ziele und Inhalte des Trainings zur Förderung der kognitiven Fähigkeiten. Anhand der konzeptuellen Systeme und der Gestaltung einer "Lernmatrix" fertigt der Trainer eine Auswahl von „motorik-kognitiven Aufgaben" an (Erläuterungen über die konzeptuelle Systeme und die Lernmatrix befinden sich auf Seite 111 ff.).Diese Aufgaben sollen die konzeptuellen Systeme und kognitiven Kompetenzen integrieren. Im Training kann die Förderung der kognitiven Kompetenzen grundsätzlich innerhalb jedes Teils stattfinden. Bestimmte Abschnitte sind allerdings zu bevorzugen, um die Beziehungen zwischen den Konzepten und den motorischen Aufgaben zu gewährleisten. In den ersten drei bis fünf Minuten der Trainingssitzung oder bei Spielunterbrechungen beim „Einfrieren“ der Situation findet der Trainer genauso geeignete Momente vor wie beim Ausklang des Trainings. Hier werden idealerweise die Fragen gestellt, durch die der Wissensaufbau und die Konsolidierung hervorgerufen werden.

Zusammen mit den Kleinen Fußballspielen mit unterschiedlichen Schwerpunkten trägt auch der Einsatz von visuellem Lehrmaterial zur Förderung der gewünschten Lernziele bei. Bilder, Karten, Zeichnungen, kleine schriftliche Informationen und Hinweise erleichtern das Verständnis der Fragen, fördern das Nachdenken und motivieren die Diskussion über die problematischen Situationen, mögliche Ursachen und Lösungsansätze.

Gemäß LÓPEZ und MORENO (2002a, S. 26), soll die Auswertung der kognitiven Kompetenz folgende praktische Prinzipien beachten:

- Die kognitive Kompetenz sollte in Übereinstimmung mit der motorischen Aktion stehen und entsprechend der Trainingsziele ausgewertet werden.

- Das Verständnis des Sportlers der Fakten und Konzepte sollte bewertet werden, nicht das bloße mechanische Auswendiglernen.

Die Auswertung der kognitiven Kompetenz sollte ein interaktiver Prozess sein. Somit sollen die Sportler durch problematische Situationen, Fragestellungen und systematische Beobachtungen ihr Wissen in engem Zusammenhang mit der Praxis beweisen.

Der Zweck dieser Studie ist es, zu beobachten, welche Effekte ein Trainingsprogramm auf den Erwerb von Wissen hat, das aus einer Befragungsstrategie ohne ausdrückliche vorherige Anweisungen besteht, gleichzeitig aber genügend Zeit für Überlegungen gewährt, die durch Leitfragen nach dem „sokratischen Ansatz für Spiellehren“ (BUTTLER, 1997) gestaltet sind. Die Effekte wurden im Vergleich mit einem traditionellen Trainingsprogramm (Direkte Instruktion) bewertet, in dem eine Reihe von Befehlen und Anweisungen an die Spieler gegeben werden, bevor sie in die Spielpraxis eintreten. Die Studie strebt eine Antwort auf die folgenden Fragen an:

1. Welche der beiden Formen des Trainings erzeugt mehr Handlungswissen - die Direkte Instruktion oder die Reflexive Instruktion?

2. Welche der beiden Formen des Trainings erzeugt ein nachhaltigeres Lernen? 
Die folgenden Hypothesen wurden für diese Studie formuliert:

- Hypothese 1: Eine Spielergruppe, die durch Spielen und Reflektieren lernt (Reflexive Instruktion), beweist einen signifikant größeren Umfang von Handlungswissen durch eine höhere Anzahl an richtig beantworteten Fragen über Spielsituationen des Fußballs als eine Spielergruppe, die durch Hinweise auf richtige Handlungen und richtiges Spielen lernt (Direkte Instruktion).

- Hypothese 2: Der Umfang von Handlungswissen wird nach einer Zeitspanne ohne Training für eine Spielergruppe, die durch Spielen und Reflektieren lernt $(\mathrm{Re}-$ flexive Instruktion), signifikant größer als für eine Spielergruppe, die durch Hinweise auf richtige Handlungen und richtiges Spielen lernt (Direkte Instruktion).

\subsubsection{Methode}

\section{Teilnehmer}

Es wurden 30 junge Fußballspieler (Alter 11-12 Jahre) aus zwei verschiedenen Vereinen beobachtet. Alle nahmen regelmäßig an Wettkampfspielen teil. Jede Mannschaft stellte eine Untersuchungsgruppe dar. Drei Variablen, die das Lernen beeinflussen können, wurde vor Beginn des Lernprozesses überprüft. So wurde sichergestellt, dass die zwei Gruppen so homogen wie möglich waren. Folgende Variablen wurden geprüft:

- Alter

- Schulklasse

- Vorerfahrung im Fußball

\section{Kriteriumsaufgaben und -instrumente}

Für die Auswertung der kognitiven Kompetenzen wurde eine selbst entwickelte Methode eingesetzt. Die "Auswertungsmethode der kognitiven Spielkompetenz (AMKS)" besteht aus der Darstellung problematischer Situationen in Beziehung mit dem Spielkontext und Fragen zur geeigneten Lösung für die dargestellte Situation. Von insgesamt 30 Fragen über das konzeptuelle System (siehe eine Definition von konzeptuellem System auf Seite 112 ff.) wurden 10 vor der Intervention im Vortest, 10 weitere im Posttest und die restlichen 10 im Retentionstest gestellt. Bei jedem der Messzeitpunkte hatte sowohl die Experimentalgruppe als auch die Kontrollgruppe dieselben Fragen zu beantworten.

\section{Auswertungsmethode der kognitiven Spielkompetenzen (AMKS)}

Der Spieler sitzt vor einen Computer. Es wird ein zehnsekündiger Videoclip gezeigt, der eine Spielsituation in Beziehung mit einem bestimmten konzeptuellen System darstellt. Bei dieser Studie wurden die Videoclips mit Hilfe des Videospiels FIFA 10 (@2009 Electronic Arts $\mathrm{GmbH}$ ) hergestellt. Die gewünschte Spielsituation wurde zuerst am Computer erarbeitet und mit einer Videokamera aufgenommen. Nach der Darstellung des Videoclips wird der Testperson eine Reihe von Fragen gestellt. Jede Frage fordert die Ausführung bestimmter intellektueller Operationen und damit eine bestimmte kognitive Kompe- 
tenz. Die Fragen werden durch ein Computerprogramm erarbeitet, das die Genauigkeit der Antwort bewertet. Der Fragentyp ist eine Mehrfachoption mit nur einer richtigen Antwort. Jede Frage besteht aus eine Darlegung von vier Antwortoptionen. Sie werden mit der Buchstaben A, B, C, D bezeichnet.

Die folgenden Bemerkungen werden bei der Erarbeitung der Frage beachtet (ARCOFADER, 2007):

Bei der Darlegung und dem Kontext

a) Die Klarheit, Sachbezogenheit und Genauigkeit ist zu gewährleisten.

b) Sehr allgemeine oder sehr spezifische Fragen sind zu vermeiden, Fragen dürfen immer nur über relevante Aspekte gestellt werden.

c) Nur die Information ist einzufügen, die als notwendig für die Lösung der Fragestellungen erachtet werden.

d) Jedes Mal darf nur eine Frage oder Situation gestellt werden.

Bei den Antwortoptionen

a) Alle Antwortoptionen sollen dieselbe Wortlänge, Fachsprache und denselben konzeptuellen Fokus und identische Kohärenz bezüglich der Darlegung erzielen.

b) Bei der Darlegung der Frage benutzte, bedeutsame Worte sind in den Antwortoptionen zu vermeiden.

c) Es wird keine Antwortoption benutzt, die sich auf andere bezieht wie z. B. „alle oder keine der Optionen sind richtig“.

d) Die Reihenfolge der Antwortoptionen sollte keinen Hinweis und keine Anregung für die richtige Antwort geben.

e) Die Antwortoptionen sind auf eine Weise zu verfassen, die den gezielten Kompetenzstufen entspricht, d.h. die zum Identifizieren, Argumentieren oder Vorschlagen auffordern.

Bei den Fragen im Allgemeinen

a) Nummern für die Fragestellungen und Buchstaben für die Antwortoptionen benutzen.

b) Keine Verneinung in der Darlegung oder in der Antwortoption einfügen.

c) Klare, eindeutige und bezogen auf die Bildungsstufe der Probanden geeignete Sprachen benutzen.

d) Immer sicherzustellen, dass eine Antwort für die Frage in den Antwortoptionen vorliegt.

e) Man soll vermeiden, Probanden nach der Meinung zu fragen.

f) Die Unabhängigkeit zwischen Fragestellungen anstreben, d. h. die Darlegung oder Antwortoptionen einer Frage sollten keine Hinweise für die richtige Antwort anderer Fragen darstellen. 
g) Bei der richtigen Antwort ist sicherzustellen, dass es keine besonderen Häufungen gibt, z. B. dass in der Mehrzahl der Fälle die richtige Lösung „C“ ist.

Antwortoptionen können aus verschiedenen konzeptuellen Systemen für dasselbe Problem gebildet werden. Aus diesem Grund sollte die richtige Antwort aus einem bestimmten konzeptuellen System gewählt werden, um Widersprüche zu vermeiden. Die Antwortoptionen, die auf andere konzeptuelle Systeme passen, sollen ausgeschlossen werden. Es ist ferner zweckmäßig, dass die Fragestellungen die drei Gruppen von Kompetenzen beinhalten. Auch soll auf die Anpassung an die konzeptuellen Systeme und auf die Balance zwischen Fragen von niedriger, mittlerer und hoher Komplexität Wert gelegt werden. Im Folgenden wird ein Beispiel für die Auswertung des konzeptuellen Systems „Passen beim Fußball“ dargestellt:

Ein Videoclip mit einem Angriffsspielzug beim Angriff wird dem Spieler dargestellt.

1. Was war falsch in der Spielhandlung?
A. Der Spieler hielt den
Ball an.
C. Der Spieler hat nicht den
Blickwinkel gewechselt.
B. Der Spieler bleibt nach
D. Der Pass war zu kurz. dem Pass stehen.

2. Warum sollen die Spieler als ein Dreieck stehen?
A. Um das eigene Tor bei
Ballverlust zu verteidigen.
C. Um das Spielsystem der Mann- schaft aufrechtzuerhalten
B. Um Passmöglichkeiten anzubieten.
D. Um die Gegenspieler zu verunsichern.

3. Du stehst dem letzten Verteidiger gegenüber. Er ist schneller als Du, Deine Mitspieler befinden sich genau hinter Dir. Was machst du?
A. Den Gegenspieler zu überdribbeln
C. Ins Tor schießen. versuchen.
B. Den Ball zur Seite führen und auf Unterstützung warten.
D. Einen Pass nach hinten spielen.

\section{Variablen}

Die kognitive Kompetenz eines Fußballspielers kann man aus dem Erfolg oder Misserfolg schließen, den der Spieler bei der Lösung von kognitiven Aufgaben erreicht, die mit dem Handlungswissen des Fußballspiels verbunden sind. In dieser Studie wurde daher 
als abhängige Variable die Anzahl der richtigen Antworten auf die Fragen über Spielsituationen bestimmt. Als unabhängige Variable wurde die Zugehörigkeit zur Kontrollgruppe (DIG) oder zur Experimentalgruppe (RIG) betrachtet.

\section{Intervention}

Eine Gruppe wurde als Direkte Instruktionsgruppe (DIG) bezeichnet. Diese Gruppe nahm an einem Lernprozess teil, der aus Kleinen Spielen mit Schwerpunkt bestand. Ein theoretischer Inhalt wurde durch eine direkte Instruktion unterrichtet, d. h. bevor die Spieler zum Spiel kamen, hielt der Trainer einen 10 Minuten-Vortrag über die Spielprinzipien, die während verschiedener Spielhandlungen beachtet werden sollen.

Zum Beispiel wurde am Anfang einer Trainingseinheit bei dem DIG ein zehnminütiger Vortrag mit dem folgenden Inhalt gehalten:

Die Spieler einer Dreiermannschaft sollen immer ein Dreieck auf dem Feld bilden.

Eine Aufstellung im Dreieck bietet mehr Passmöglichkeiten.

Wenn die Drei Feldbereiche besetzt werden, wird die gegnerische Abwehr auseinandergezogen.

Die Gegner haben weniger Möglichkeiten zu zweit einen Angreifer erfolgreich zu decken das erleichtert die aktive An- und Mitnahme des Balls und ein direktes Weiterspielen.

Wenn der Verteidiger außen angreift, bleibt der mittlere Angreifer eher zurück oder bietet sich für einen Pass hinter dem Verteidiger an. Der gegenüberliegende Außenstürmer besetzt den mittleren Feldbereich und nimmt die Rolle eines mittleren Angreifers ohne Ball ein.

Anschließend wurde den Spielern ein 3:3 auf vier Tore-Spiel, bei dem den Spielern nicht mehr als zwei Ballkontakte erlaubt sind, gestellt und Anweisungen in Bezug auf die Dreieck-Formation während des Spiels gegeben.

Die zweite Gruppe wurde als Reflexive Instruktionsgruppe (RIG) bezeichnet. Diese Gruppe nahm ebenfalls am Lernprozess mit Kleinen Fußballspielen mit unterschiedlichen Schwerpunkten teil. Der theoretischer Inhalt wurde mittels einer Befragungsstrategie unterrichtet, d. h. durch Fragestellungen von einem konzeptuellen System abgeleitet, das ein reflektiertes Denken während des Spielens fördert. Zu bestimmten Zeitpunkten wurde das Spiel angehalten und den Spielern Fragen in Beziehung auf die Anwendung der Spielprinzipien bei der momentanen Spielsituation gestellt. Die Fragen ent- 
sprachen den konzeptuelle „Systeme Passen bei Fußballspielen“ und „Erfolgreiche Lösung der Zweikämpfe“.

Zum Beispiel wurde am Anfang einer Lernsitzung bei der RIG den Spielern zunächst ein T3:T3-Spiel aufgegeben. Die Spieler haben sofort zu spielen begonnen. Wenn es der Trainer für erforderlich erachtete, hielt er das Spiel an, forderte die Spieler auf, auf ihrer Feldstellung zu verharren („eingefrorene Zeitpunkte“) und stellte innen zur momentanen Spielsituation folgende Fragen.

Wenn der Spieler in der Mitte den Ball besitzt:

wo sollten sich die anderen Spieler aufstellen?

Wenn die Angreifer sich nicht anbieten:

warum sollt Ihr in einer Dreieck-Formation spielen?

Wenn die Angreifer leicht zu decken sind:

Welche Schwierigkeiten haben die Abwehrspieler, wenn die Angreifer sich in DreieckFormation aufstellen?

Wenn zwei Spieler auf derselben Seite stehen:

Wie viele Möglichkeiten hat der Ballbesitzer, wenn beide Mitspieler auf derselben Feldseite stehen?

Warum sollten alle drei Angriffspositionen immer besetzt sein?

Wenn ein Spieler auf der Seite den Ball besitzt:

Wo sollte sich der mittlere Angreifer aufhalten, wenn der Ball bei einem Außenstürmer ist? Wo sollte sich der andere Außenstürmer aufhalten?

Bei den Antworten wurde auch nachgefragt; damit sollten das Nachdenken und das Vertreten verschiedener Meinungen gefördert werden. Anschließend wurde weitergespielt.

Beide Gruppen hatten die gleichen Spiele und die gleiche Anzahl von Lerneinheiten. Der Prozess wurde unter ähnlichen Wetterbedingungen durchgeführt und erfolgte mit den gleichen didaktischen Materialien. Jede Lernsitzung dauerte 30 Minuten.

Vor Beginn der Intervention wurde den AMKS-Test für die Bewertung des Niveaus des Wissens bezüglich der Kompetenzen und Fähigkeiten im Passspiel durchgeführt. Die Ergebnisse wurden zum Nachweis der Homogenität der Gruppe verglichen. Der gleiche 
Test wurde unmittelbar nach Abschluss der Ausbildung durchgeführt. Nach einem zeitlichen Abstand von ungefähr zwei Wochen nach Abschluss des Treatments, in dem sichergestellt wurde, dass im Training keine Informationen oder Anweisungen zum Passspiel an die Spieler erfolgten, wurde der Retentionstest erneut eingesetzt, um Daten über den Lernerfolg zu erhalten.In Abbildung 26 wird denUntersuchungsablauf dieser Studie dargestellt.

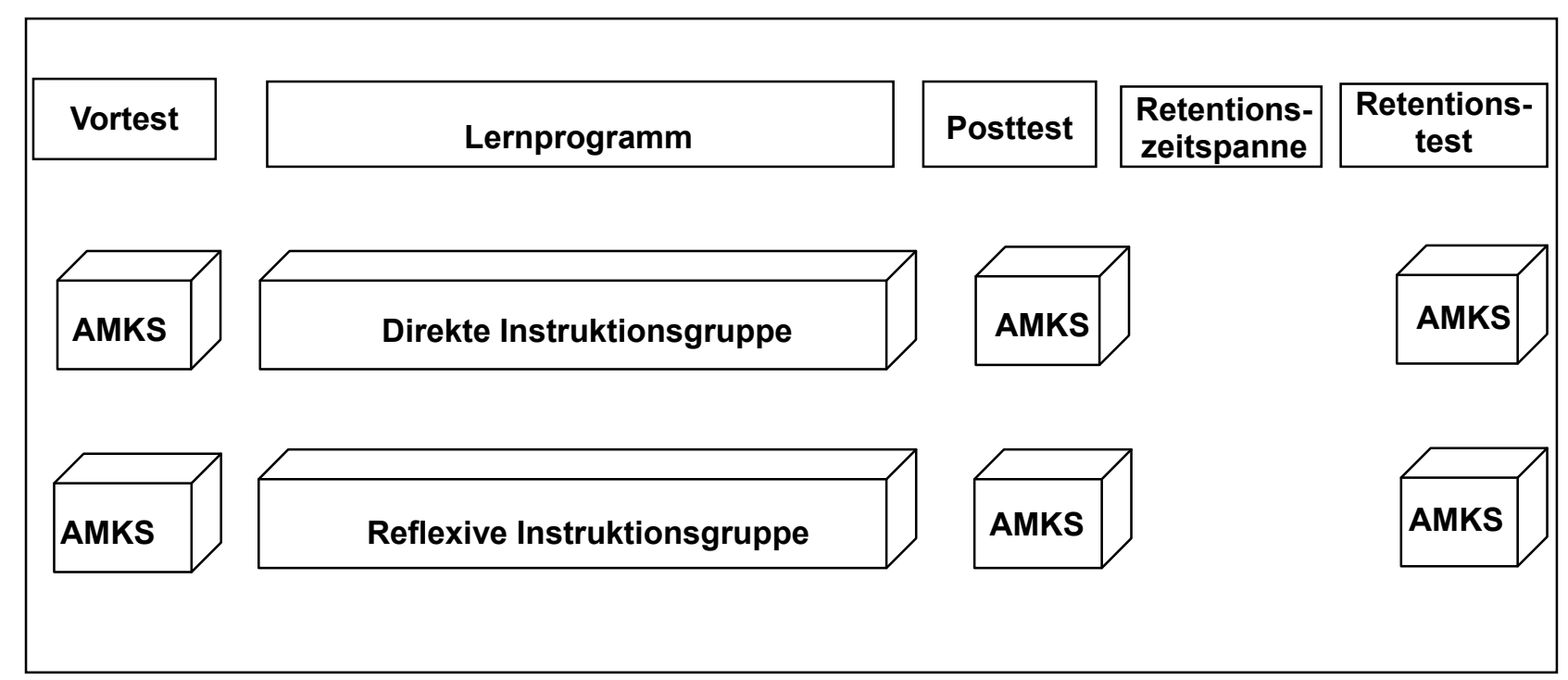

Abbildung 26: Untersuchungsablauf der Studie zwei

\section{Statistische Analysen}

Die statistischen Verfahren wurden mit SPSS für Windows $®$, Version 17.0 durchgeführt. Deskriptive Analysen (Häufigkeit, Mittelwert und Standardabweichung) wurden durchgeführt, um die Stichprobe zu charakterisieren. Es lag keine Normalverteilung der Variable dieser Studie im Vortest der DIG und im Posttest der RIG vor $(p<.05)$ (siehe Ergebnisse des Kolmogorov-Smirnov-Tests in Anhang K), auf diesem Grund wurden parameterfreie Tests angewendet um signifikante Unterschiede zu bestimmen. Ein Friedman-Test wurde gerechnet, um die richtig beantworteten Fragen jeder Gruppe in der AMKS zwischen Vor-, Post- und Retentionstest zu vergleichen. Außerdem wurde ein U-Test nach Mann und Whitney berechnet, um die Leistungen der Gruppen nach dem Lernprogramm zu vergleichen.

Die folgenden statistischen Hypothesen wurden getestet:

Statistisch gilt für die AMKS Variable: 
Intra-Gruppe:

H0: $\quad \mu 1=\mu 2 \quad$ bzw. $\quad \mu 1=\mu 3$

H1: $\quad \mu 1<\mu 2 \quad$ bzw. $\quad \mu 1<\mu 3$

Inter-Gruppe:

H0: $\quad \mu 2$ DIG $=\mu 2$ RIG $\quad$ bzw. $\quad \mu 3$ DIG $=\mu 3$ RIG

H1: $\quad \mu 2$ DIG $<\mu 2$ RIG bzw. $\quad \mu 3$ DIG $<\mu 3$ RIG

wobei:

$\mathrm{HO}$ die Nullhypothese ist,

$\mu 1$ der Mittelwert des Vortests,

$\mu 2$ der Mittelwert des Posttests und

43 der Mittelwert des Retentionstests.

\subsubsection{Ergebnisse}

In Tabelle 11 sind die Mittelwerte der Ergebnisse dargestellt, die beide Gruppen während der drei Messzeitpunkte erreicht haben (siehe Gesamtdaten im Anhang J). 
Tabelle 11: Mittelwerte (M) und Standardabweichung (SD) der richtigen Antworten auf die Fragen beim Vortest, Posttest und Retentionstest in der Direkten Instruktionsgruppe (DIG) (n=13) und der Reflexiven Instruktionsgruppe (RIG) (n=14)

\begin{tabular}{|c|c|c|c|c|c|c|}
\hline & \multicolumn{2}{|c|}{ Vortest } & \multicolumn{2}{c|}{ Posttest } & \multicolumn{2}{c|}{ Retentionstest } \\
\hline & M & SD & M & SD & M & SD \\
\hline DIG & 3,46 & 1,45 & 4,54 & 1,27 & 4,23 & 1,48 \\
\hline RIG & 3,71 & 1,2 & 7,57 & 1,28 & 8,00 & 1,36 \\
\hline
\end{tabular}

Tabelle 12 zeigt, dass die Experimentalgruppe (RIG) ihre Leistung um mehr als 40\% steigern konnte, da die Spieler doppelt so viele richtige Antworten im Posttest gegeben haben. Die Kontrollgruppe hingegen erhöhte ihre Leistung lediglich um ca. $10 \%$.

Tabelle 12: Prozentzahl der richtigen Antworten auf die Fragen beim Prätest, Posttest und Retentionstest in der Direkten Instruktionsgruppe (DIG) $(n=13)$ und der Reflexiven Instruktionsgruppe (RIG) (n=14)

\begin{tabular}{|l|c|c|c|} 
& Vortest \% & Posttest \% & Retentionstest \% \\
\hline DIG & 34,62 & 45,38 & 42,31 \\
\hline RIG & 37,14 & 75,71 & 80 \\
\hline
\end{tabular}

Im Vortest wurden keine signifikanten Unterschiede zwischen den Gruppen gefunden wie ein U-Test nach Mann und Whitney festgestellt hat $(U=-0.50 ; p>.05)$. Nach dem Lernprogramm hat sich die Kontrollgruppe (DIG) leicht verbessert. Diese Gruppe bekam dem Lerninhalt als direkte Information vom Trainer präsentiert. Die erreichte Verbesserung ist aber nicht signifikant, der Friedman-Test zeigt keine signifikanten Unterschiede zwischen Vor-, Post- und Retentionstest $\left(X^{2}[2]=2.93 ; p>\right.$.05).

Die Experimentalgruppe (RIG), die durch eine Befragungsstrategie unterrichtet wurde, hat sich hingegen stark verbessert. Der Friedman-Test zeigt signifikante Unterschiede für diese Gruppe zwischen Vor-, Post-, und Retentionstest $\left(X^{2}[2]=23.10 ; p<.05\right)$. Die Verbesserung zwischen Vor- und Posttest, bestätigt durch ein Wilcoxon-Test mit Bonferroni Korrektur $(T=-3.31 ; p<.01)$, wurde auch zwischen Vortest und Retentionstest $(T=-3.32 ; p<.01)$ aufrechterhalten. Dieser erfolgte nach zwei Wochen ohne spezifisches Trainingsprogramm oder Informationen über das Konzeptuelle System. Dieses Ergebnis weist auf ein langfristig stabiles Lernen hin. 
Ein weiterer U-Test nach Mann und Whitney zeigt einen signifikanten Unterschied zwischen der Experimentalgruppe (RIG) und der Kontrollgruppe (DIG) im Posttest (U = $4.05 ; p<.05)$ und auch im Retentionstest $(U=-4.16 ; p<.05)$.

In Abbildung 27 ist die Lernentwicklung beider Gruppen dargestellt. Zu Beginn des Lernprogramms hatten die zwei Gruppen eine ähnliche Leistung. Nach dem Training hat die Gruppe von Spielern der Reflexiven Instruktionsgruppe (RIG) deutliche Leistungsvorteile.Diese Spieler haben ihre Leistung in einer Testaufgabe verbessert, die deklaratives Wissen erfordert.

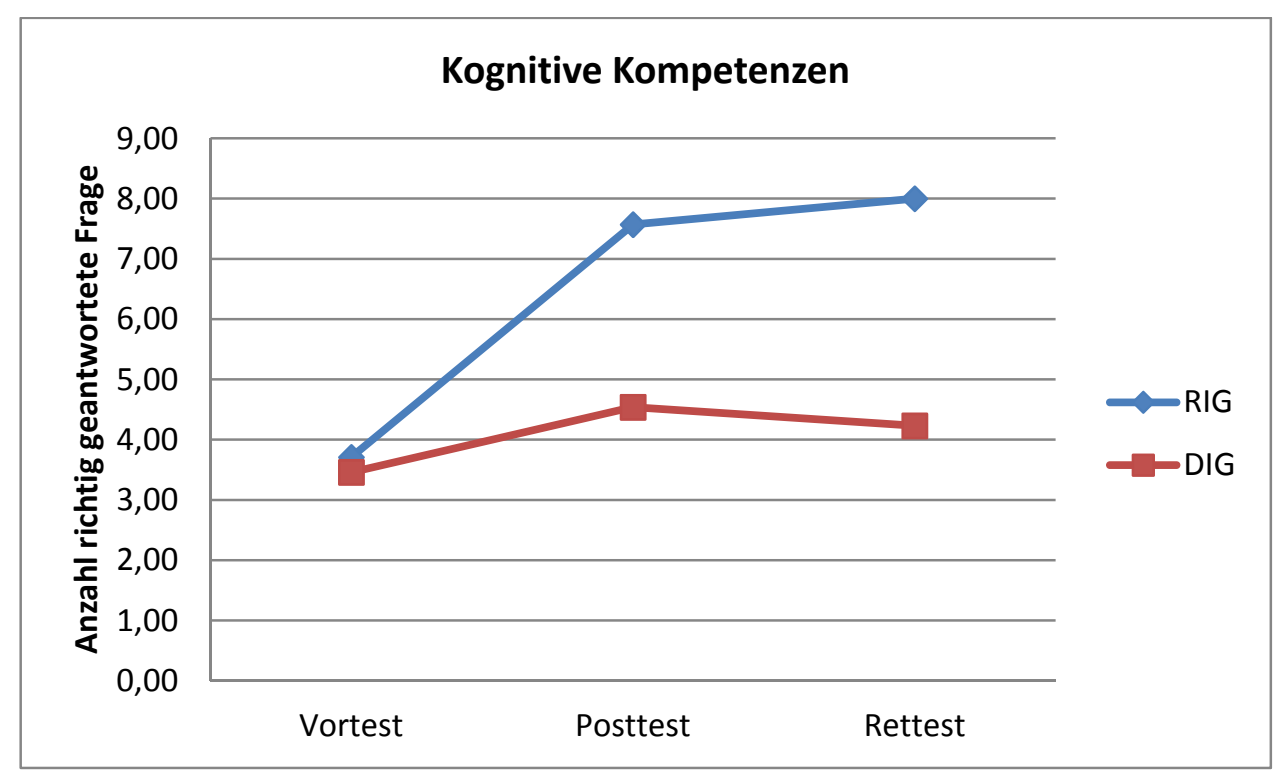

Abbildung 27: Entwicklung des Lernens kognitiver Kompetenzen im Fußball in der Direkten Instruktionsgruppe (DIG) und der Reflexiven Instruktionsgruppe (RIG)

\subsubsection{Diskussion und Schlussfolgerung}

In der Sportspielausbildung wird traditionell versucht, das Lernen von deklarativem Wissen als Grundlage für das Lernen und die Verbesserung der Spielhandlungen zu fördern. Es wird davon ausgegangen, dass das theoretische oder deklarative Wissen das Erlernen von prozeduralem Wissen begünstigt (TURNER, ALLISON \& PISSANOS, 2001, S. 38). Deswegen bemühen sich Trainer und Sportlehrer, die notwendigen Kenntnisse zu vermitteln, um eine geeignete Ausbildung der Spieler zu erreichen. Besonders beim Lehren von taktischen Kompetenzen wird häufig auf die Vermittlung von theoretischem Wissen zurückgegriffen.

In den traditionellen Sportspielvermittlungsmodellen, wie z. B. dem Direkten Modell, versucht der Trainer, die zu vermittelnden Information als Hinweise, Empfehlungen und Ratschläge an die Spieler zu übermitteln. Er versucht auch, den Spieler auf die im Spiel möglichen problematischen Situationen vorzubereiten und Lösungsmöglichkeiten dafür 
zu lehren. Während und nach dem Spiel ist der Trainer häufig damit beschäftigt, Rückmeldungen zu den Spielhandlungen und vor allem über die Fehler der Spieler zu geben. Er äußert Auswertungen und gibt Korrekturen, die die die Spieler beherzigen sollen, um ihre Spielkompetenz bei späteren Spielen zu verbessern.

Dabei wird vernachlässigt, dass Wissen im Gegensatz zur Information nicht direkt vermittelt werden kann (vgl. DEUTSCH, 1990, S. 187). Der Trainer kann den Spielern durch Hinweise, Ratschläge und Befehle lediglich Informationen anbieten. Viel eher benötigen die Spieler aber zum erfolgreichen Lernen einen Prozess des Erlebens, Überdenkens und der kognitiven Verarbeitung von Spielsituationen, um entsprechendes Handlungswissen aufbauen zu können. Das Wissen muss Stück für Stück aufgebaut werden, es entsteht aus dem Nachdenken über die Erfahrungen und Erlebnisse, aus den Ursache-Wirkung-Beziehungen. Auf diesem Weg werden „wenn-dann-Regeln“ aufgebaut (SEEL, 2003, S. 218). Das neue Wissen soll bedeutende Beziehungen zu den schon im Langzeitgedächtnis gespeicherten Wissensbeständen haben, damit es besser im Langzeitgedächtnis gespeichert werden kann (WIEMEYER, 1994, S. 12). Einmal angelegt, kann das Wissen sowohl als präskriptives, interpretatives und auch als prozedurales Wissen bei Spiel- oder Testaufgaben angewendet werden. Die Unterscheidung von deklarativem und prozeduralen Wissen ist im Fall der Sportspielausbildung nicht unbedingt hilfreich, da beide Wissensarten notwendige und untrennbare Bestandteile der Spielkompetenz sind. Beide zusammen bilden das „Spielkönnen“ der Spieler (HOTZ, 1995, S. 181; ANDERSON, 2005, S. 106). Aus einer konstruktivistischen Sicht (REICH, 2006) ist es nicht möglich, Wissen zu vermitteln. Es muss vielmehr durch einen Prozess von Planen, Ausführen und Nachdenken aufgebaut werden. Aus diesem Prozess entsteht Wissen in Form von Schlussfolgerungen.

In dieser Studie wurden gegenüber einer traditionellen Konzeption der Wissensvermittlung bessere Ergebnisse mit einem Lernprogramm erreicht, das auf der Konstruktivistischen Theorie basiert. Das eingesetzte Lernprogramm geht vom Entwurf eines konzeptuellen Systems aus und stellt die Lernziele mit Hilfe einer Matrixmethode dar. Das Lernprogramm wurde durchgeführt, ohne Informationen an die Spieler abzugeben, nutzte aber eine der sokratischen Methode ähnliche Befragungsstrategie für das Lernen der Sportspiele (BUTLER, 1997; LOIBL, 2001). Die Daten weisen darauf hin, dass ein gleichermaßen wirksames Lernen nicht mit einer traditionellen Methode gelingt, in der das prozedurale Wissen als Information und in Form von korrektiven Rückmeldungen vermittelt wird. Das durch die Befragungsmethode aufgebaute Wissen zeigte sich darüber hinaus auch als über die Zeit stabileres Lernergebnis, da die getesteten Spieler auch nach einigen Wochen ohne spezifisches Lernprogramm dieselbe Leistung wie unmittelbar nach dem Programm erreichten.

Die Ergebnisse der Studie bestätigen die Ergebnisse früherer Studien (z. B. LAWTON, 1989; ALLISON \& THORPE, 1997; TURNER \& MARTINEK, 1999; BLOMQVIST, LUHTANEN, LAAKSO \& KESKINEN, 2000; TURNER, 2003; TALLIR, MUSCH, LENOIR \& VALCKE, 2004). In allen genannten Studien gelangt man mit Hilfe einer Konstruktivistischen Theorie zu besseren Lernergebnissen. In den Studien wurden die kognitiven Kompetenzen allerdings lediglich als theoretisches Wissen betrachtet, das durch richtige Antworten auf abstrakte theoretische Fragen bestätigt werden konnte. In dieser Studie wurde jedoch keine rein theoretische Testaufgabe angewendet, sondern eine Auswertungsmethode der kognitiven Kompetenzen vorgeschlagen, in der auch die notwendigen Beziehungen zwischen deklarativem und prozeduralen Wissen berücksichtigt 
wurden. Die angewandte Auswertungsmethode könnte geeigneter sein als andere Auswertungsmethode, die auf Papier basierten Fragebögen (ALISON \& THORPE, 1997; FRENCH, WERNER, TAYLOR, HUSSEY \& JONES, 1996A; 1996B; JONES \& FARROW, 1999; TURNER UND MARTINEK, 1992, 1999) oder auf verbalen Protokollen (McPHERSON \& THOMAS, 1989; HARVEY, 2006) einsetzen. In diesen werden Probanden nach abstraktem Wissen gefragt und sollen dabei grundsätzlich theoretisches Wissen anwenden, um die Fragen zu beantworten. Das Abstrahieren ist nicht immer möglich, wenn es um prozedurales Wissen geht. BLOMQVIST, LUHTANEN, LAAKSO und KESKINEN (2000) setzten bei ihrer Studie außer papierbasierten Fragebögen auch Videoclips als Auswertungsmethode ein. Obwohl diese zunächst als geeignetere Auswertungsmethode für die Auswertung von kognitiven Kompetenzen erscheinen, berücksichtigen sie nicht die unterschiedlichen Entwicklungsstufen der kognitiven Kompetenzen. Ferner sind sie nicht aus einem konkreten konzeptuellen System abgeleitet.

Weitere Studien werden notwendig sein, um die Wirkungen der Befragungsmethode als wirksame Methode für das Lehren und Training der Sportspiele zu bestätigen. Die hier entworfene und angewandte Auswertungsmethode der kognitiven Kompetenzen sollte dabei auch in weiteren Studien validiert werden. Eine Verifizierung der Wirksamkeit des deklarativen Wissens auf die Spielleistung wäre ebenso wünschenswert. 


\subsection{Studie 3. Entwicklung der technischen Kompetenz in Sportspielen durch Kleine Fußballspiele mit unterschiedlichen Schwerpunkten (KFSS)}

\subsubsection{Einführung}

Die Variabilität des Übens (variability of practice) ist im Rahmen der Forschung zum motorischen Lernen ausgiebig untersucht worden (LIN, 2006; SCHMIDT \& LEE, 2006; STOKES, LAI, HOLTZ, RIGSBEE \& CHERRICK, 2008). Der allgemeine Konsens besagt, dass das variable Üben während derersten Phase des Lernens nicht so effektiv wirkt wie das konstante Üben. Bei der Anwendung von gelernten Bewegungen nach einiger Zeit erweist es sich aber als effizienter, das heißt, die Lerneffekte bleiben länger im Langzeitgedächtnis. Insbesondere für offene Bewegungsfertigkeiten, wie z. B. die technischen Fertigkeiten der Sportspiele, scheint das variable Üben wirksamer als das konstante Üben zu sein.

Eine besondere Form der Variation der Übung ist das zufällige Üben (random practice im englischen Sprachraum) (SCHMIDT \& WRISBERG, 2008, S. 275). Bei dieser Art der Organisation des Übens wird mehr als eine Bewegung verwendet, um eine Wirkungsinterferenz oder kontextuelle Interferenz $(\mathrm{KI})$ zwischen verschiedenen Arten von Bewegungen zu erzeugen. Streng genommen ist es keine Variation einzelner Bewegungen, sondern die Kombination von verschiedenen Bewegungen in einer Übungseinheit. Diese Kombination wird nach dem Zufallsprinzip angeordnet. Das Üben von verschieden technischen Fertigkeiten nach dem Zufallsprinzip erweist sich in vielen Studien als effizienter für das Erlernen als das Üben einer einzelnen Bewegung oder das Üben der verschiedenen Bewegungen in serieller Form (z.B. SHEA \& MORGAN, 1979; PORTER \& MAGILL, 2004; SEKIYA, 2006; SIMON, 2007). Mehrere Hypothesen sind zur Erklärung dieses Phänomens formuliert worden. Die meisten gehen davon aus, dass die KI eine tiefere kognitive Verarbeitung und daher ein wirksameres Lernen erzeugt.

Jede technische Fertigkeit kann als Lösung eines Problems betrachtet werden. Während des wiederholten Übens von Versuchen in Blöcken löst der Ausführende das Problem und wiederholt sofort in aufeinander folgenden Aufführungen die gegebene Lösung. Wird das Üben hingegen nach dem Zufallsprinzip angeordnet, muss der Ausführende bei jeder Ausführung ein neues Problem lösen. Diese Probleme erscheinen in zufälliger Reihenfolge. Während des Übens mit KI werden stetig kognitive Prozesse wie Wahrnehmung, Entscheidung und Planung der Bewegungen ausgeübt, um das Handeln an den Kontext anzupassen und die Bewegungsprobleme richtig zu lösen. Allerdings werden die meisten Studien mit $\mathrm{KI}$ unter Laborbedingungen durchgeführt (vgl. HILLEBRECHT, 1998, S. 159; LIN, 2006 , S 11). Die Probanden müssen künstlich erscheinende Bewegungen durchführen, die zwar menschliche Fähigkeiten wie Antizipation und Genauigkeit erfordern, sich motorisch aber meistens auf einen Knopfdruck beschränken. Eine Transferierbarkeit dieser Studien auf reale Bewegungssituationen im Sport ist allerdings wegen der niedrigen externen bzw. ökologischen Validität (vgl. LIN, 2006, S. 14) nicht gewährleistet. In mehreren Studien wurde versucht, eine ökologische Validierung der Ergebnisse der Laborstudien durchzuführen. Solche Studien nutzen technische Fertigkeiten von Sportbewegungen bei Lernsituationen auf Sportfeldern und mit Gruppen von Probanden, Schülern oder Sportlern, wie sie in normalen Alltagslernsituationen der Fall sind. 
LIN (2006) analysierte bei einer Metaanalyse 236 Studien mit KI und stellte dabei fest, dass die große Mehrheit der Studien bei Retention und Transfertests bessere Lerneffekte für die zufällige Übung aufweisen, als für die konstante Übung. Weiterhin zeigen die Studien unter Feldbedingungen im Gegensatz zu den Studien unter Laborbedingungen keine niedrigeren Lerneffekte für zufällige Übung während der Erwerbsphase. Die Lerneffekte scheinen für erwachsene Probanden stärker zu sein und steigen proportional zur Übungserfahrung, d. h. der Gesamtzahl der Versuche. Außerdem wurden keine Unterschiede zwischen den Geschlechtern festgestellt.

BARREIROS, FIGUEREDO und GODINHO (2007) analysierten 27 Studien und stellten fest, dass $60 \%$ keine positiven Effekte fürs Lernen hatten, wenn das Üben als ein kontextuelle-Interferenz-Ansatz organisiert wurde. Mögliche Erklärungen berücksichtigen die Merkmale von Aufgaben: Größere Effekte in seriellen Aufgaben; die Geschwindigkeit der Ausführung: Größere Effekte auf die zeitaufwändigeren Aufgaben; das Zeitintervall zwischen jeder Ausführung: Kurze Intervalle zwischen den einzelnen Ausführungen sind schwer zu gewährleisten.

Das zufällige Üben ist aufgrund der Komplexität seiner Organisation schwer umzusetzen, denn es müssen mehrere Aufgaben gleichzeitig von mehreren Lernenden ausgeführt werden, da diese nach jeder Ausführung sofort eine andere Bewegung ausführen sollen.

In einer eigenen Studie (ARIAS \&, JIMENEZ, 2004) konnten wir feststellen, dass das Erlernen der Fußballtechniken durch die Organisation des Übens in einer zufälligen Reihenfolge gefördert wird. Dennoch zeigen Beobachtungen von PIERON (1998), dass auch in Lern- und Trainingseinheiten für Mannschaftssportarten die am weitesten verbreitete Art der Übung das Üben in Blöcken in statischen Situationen ist. Aufgrund der viel weniger aufwendigen Organisationsvoraussetzungen der Übung in Blöcken wird diese Art des Übens häufiger eingesetzt als die zufällige Übung, deren Durchführung in den Bildungsprozessen des Sports wegen der hohen Anforderungen an Material, Personal und Einrichtungen schwierig ist. Andererseits weisen viele Spielvermittlungsmodelle (LOIBL, 2001; BUTLER \& McCAHAN, 2005; GRIFFIN, MITCHELL, \& OSLIN, 2003; LAUNDER, 2001 ROTH, 2001) darauf hin, dass der wichtigste Bestandteil des Lerninhalts bei Sportspielen das Spielen selbst ist. SAMMER $(2008$, S.8) empfiehlt die höchste Prozentzahl der Trainingszeit dem Spielen zu widmen.Allerdings wird immer dann auf das Üben in Blöcken zurückgegriffen, wenn es um das Lernen der technischen Fertigkeiten geht. Jedoch ist das eigentliche Spiel ganz anders als eine Übungsanordnung in Blöcken. Zum Beispiel werfen Basketballspieler in vielen Trainingseinheiten immer wieder in Reihe von der gleichen Feldposition aus auf den Korb. Während des Spiels findet das Werfen jedoch selten zweimal in Reihe oder aus derselben Feldposition statt. Die Technik wird vielmehr nach einem Zeitraum von abwechslungsreichem Spiel ausgeführt, wo sie mit verschiedenen anderen Basketballtechniken, wie Passen, Dribbeln, usw. kombiniert wird.

Die Kleinen Spiele, die sogenannten Small Sided Games im englischen Sprachraum (SNOW, 2005), sind Oppositionsspiele, die zwischen zwei Mannschaften stattfinden und die ähnliche Grundregeln wie der Wettkampf haben. Es werden jedoch sekundäre Regeln variiert, um bestimmte Trainingsziele zu erreichen. So behält ein Fußballspiel 4:4 in einem 20 x 30 Meter großen Feld dieselben Regeln und Merkmale wie das 11:11Spiel, so zum Beispiel Ballführung mit dem Fuß, Abwehren und Angreifen, Tore, identische technische Fertigkeiten usw. Das kleine Feld und die geringe Spieleranzahl bieten 
jedoch die Möglichkeit, viele Wiederholungen der technischen Fertigkeiten während der Spielpraxis durchzuführen (JONES \& DRUST, 2007). Ihre Anwendung im Training kann durch besondere Regeln und Spielbedingungen als Schwerpunkt für eine bestimmte technische Fertigkeit zusätzlich betont werden.

Es wird bei dieser Studie angenommen, dass das Spiel selbst die höchste Variabilität in der Praxis bietet und dass die Spielpraxis damit die idealen Bedingungen für das Üben der technischen Fertigkeiten in einem kontextuelle-Interferenz-Ansatz bietet. Darüber hinaus erfolgt die Trainingseinheit, wenn sie in Form von Spielen organisiert wird, in einem ähnlichen Kontext wie die konkrete Umsetzung von dem, was man gelernt hat. Das Spiel hat auch Auswirkungen auf kognitive Prozesse, wie die Fähigkeit gute Entscheidungen zu treffen (BANYARD, 1995, S. 203).

Aus der traditionellen Sicht der Spielvermittlung müssen die Kinder die technischen Fertigkeiten außerhalb des Spiels lernen, um mit Erfolg am Spiel teilnehmen zu können. Technisches Training wird als Voraussetzung für das Spiel betrachtet. Gerade bei Kindern besteht eine übliche Trainingseinheit noch häufig aus Bewegungslernen außerhalb des Spielkontextes. Taktisches Lernen wird kaum thematisiert und das Lernen der Spielfähigkeit wird unterschätzt. Das technische Lernen wird noch als Übung organisiert, die meisten didaktischen Handbücher für das Lernen der technischen Fertigkeiten bei Sportspielen bestehen heute aus ausführliche Beschreibungen und "geometrischen Übungen" (DOUCET, 2006, S. 15).

Wenn es Anzeichen dafür gibt, dass das Üben im Rahmen des Kleine-Spiele-Ansatzes einen Effekt auf das Lernen von Bewegungen ähnlich dem Effekt des zufälligen Übens hat - oder zumindest nicht weniger wirksam als das Üben in Blöcken ist - könnte dieser Ansatz als der ideale Weg für die Übung der technischen Fertigkeiten bei Sportspielen betrachtet werden, da durch das Spielen auch die Taktik und die kognitiven Fähigkeiten und letztendlich die zentrale Spielfähigkeit verbessert werden. Das Ziel dieser Studie ist es daher, zu beobachten, welche Wirkung ein Programm für die Schulung von Bewegungen hat, das ausschließlich aus Kleinen Spielen besteht. Dieses ist dem Erlernen von Bewegungen in den Sportspielen mittels eines Programms mit herkömmlichen organisatorischen Formen für das Üben gegenüber zu stellen, d. h. dem Üben in Blöcken.

Die Studie sucht damit eine Antwort auf folgende Fragen:

- Welche der zwei Formen der Organisation des Übens (Blöcke oder Spiel) produziert das bessere Lernen von Bewegungsfertigkeiten imFußball?

- Welche der zwei Formen der Organisation des Übens erzeugt den stabileren Lerneffekt?

Die Hypothesen für diese Studie werden wie folgt formuliert:

- Hypothese 1: Eine Gruppe junger Fußballspieler, die Kleine Fußballspiele mit unterschiedlichen Schwerpunkten (KFSS) übt (Spielgruppe), erreicht eine höhere Leistung bei der Ausführung einer technischen Fertigkeit des Fußballs sowohl bei Spiel- als auch bei standardisierten Situationen außerhalb des Spiels als eine Gruppe die geometrische Übungen übt - d.h., die Übungen werden blockweise organisiert (Übungsgruppe). 
- Hypothese 2: Die höhere Passgenauigkeit der Spielgruppe wird nach einer Zeitspanne ohne Üben aufrechterhalten. Die Passgenauigkeit der Übungsgruppe wird nicht aufrechterhalten.

\subsubsection{Methoden}

\section{Teilnehmer}

Die Stichprobe bestand aus 25 jungen Fußballspielern der Altersklasse D-Junioren, 12 Jahre alt (S.D. 0,1), die in einem Sportverein regelmäßig Fußball spielten und ein oder zwei Trainingseinheiten und ein Wettkampfspiel pro Woche absolvierten. 16 dieser Spieler gehörten zur D-3-Mannschaft und wurden als Spielgruppe (Experimentalgruppe) bezeichnet, 9 Spieler der D-4-Mannschaft wurden als Übungsgruppe (Kontrollgruppe) bezeichnet. Beide Gruppen nahmen an 5 Trainingseinheiten teil, in denen jeweils über 30 Minuten die technische Fußballbewegung des Passes während des Spiels oder als Übung praktiziert wurde. Die Spieler beider Gruppe absolvierten also insgesamt 150 Minuten technische Fertigkeiten, 6 Spieler der Spielgruppe und 1 Spieler der Übungsgruppe nahmen nicht an allen drei Tests teil und wurden deshalb aus der Stichprobe ausgeschlossen.

\section{Kriteriumsaufgaben und- instrumente}

Die Auswertung des Passes in einem Spielkontext erfolgt mit einem Spieltest (4:4 plus Torwart): Zwei 4er-Mannschaften plus Torwart spielen ein zehnminütiges Spiel auf einem 32 × 25 Meter großen Feld (siehe Anhang A). Bei der Auswertung wird jeder Spieler während der ganzen 10 Minuten des Spiels beobachtet. Wenn der beobachtete Spieler den Ball berührte, wird auf die Passhandlungen, die er anschließend ausführt, geachtet. Passt er den Ball erfolgreich zu einem Mitspieler, wird diese Handlung als erfolgreicher Pass auf einem Formblatt vermerkt.Passt er den Ball zu einem Mitspieler, aber der bekommt den Ball nicht, wird diese Handlung als ein nicht erfolgreicher Pass registriert.Passt der Spieler den Ball nicht und führt irgendeine andere Handlung aus, wird diese nur als Kontakt eingetragen (siehe Anhang L). Für den Unterschied zwischen einem erfolgreichen und einem nicht erfolgreichen Pass wird die folgende Definition von KUHN und MAIER (1978, S. 32) berücksichtigt:

„Erfolgreicher (bzw. nicht erfolgreicher) Pass: Jedes Zuspiel (darin eingeschlossen sind Abstoß, Abschlag, Abwurf und Einwurf), das bei einem Mitspieler ankommt. Als nicht erfolgreich wird ein Pass bezeichnet, der keinen Mitspieler erreicht oder ins Aus geht. Um Ermessensentscheidungen aus dem Wege zu gehen, haben wir festgelegt, dass ein Fehlpass grundsätzlich dem Passgeber angerechnet wird, auch dann, wenn offensichtlich ein Fehlverhalten des Passempfängers vorliegt. (Fehl-)Pässe sind unter Störeinfluss möglich“.

Das Spiel wurde als Video aufgenommen und später am Computer mit Hilfe des Programms „Pinnacle VideoSpin 2.0“ analysiert. Wenn die zehn Minuten des Videoausschnitts abgelaufen waren, wurden die Kontakte und erfolgreiche und nicht erfolgreiche Pässe gezählt und aufgeschrieben.Anschließend wurde dasselbe Video erneut beobachtet und die Kontakte und Pässe der anderen Spieler eingetragen. 
Die Auswertung des Passes bei standardisierten Bedingungen erfolgte mit dem Loughborough-Soccer-Pass-Test (LSPT) (McGREGOR, HULSE, \& STRUDWICK, 2002; ALI, WILLIAMS, HULSE, STRUDWICK, REDDIN, \& HOWARTH, 2007). Während dieses Tests sollen die Probanden auf einer $1 \times 1$ Meter großen, viereckigen Fläche stehen und mit einem Ball durch einen Pass mit dem Fuß vier 0,50-Meter große Ziele treffen. Die Ziele sind in unterschiedlicher Entfernung und in vier unterschiedlichen Richtungen aufgestellt. Die Probanden sollen zweimal die vier Ziele treffen und die Gesamtzeit wird als Ergebnis genommen. Fehler werden mit Strafzeit belegt und zur Gesamtzeit hinzugerechnet. Für „nicht Treffen des Ziels“: plus 5 Sekunden, Berührung von einem der vier Gegenstände, die das Viereck markieren durch den Ball: plus 2 Sekunden, den Ball außerhalb der Vierecke annehmen oder passen: plus 2 Sekunden. Die Ausführung des Tests wurde als Video angenommen und später am Computer bezüglich der Fehler und der aufgewandten Zeit ausgerechnet (siehe den LSPT im Anhang M).

\section{Variablen}

Aus der Messung der benötigten Zeit und der beobachteten Fehler bei dem Loughborugh-Pass-Test und der Ballkontakte sowie der erfolgreichen und nicht erfolgreichen Pässe bei dem Spieltest wurden Hauptvariablen und Nebenvariablen für diese Studie abgeleitet.

Als Hauptvariablen wurden aufgrund ihrer Aussagekraft die Gesamtzeit, die die Spieler für den Louborough-Pass-Test benötigten und der Index EP/P ausgewählt. Letzterer wird durch Dividieren der Anzahl der erfolgreichen Pässe durch die Gesamtzahl der Pässe berechnet:

\section{Variable 1:}

$$
\begin{aligned}
\text { Gesamtzeit }= & \text { Zeit }+(\text { Anzahl der Zielvermisse } * 5 \text { Sekunden })+(\text { Anzahl der Fehler } \\
& * 2 \text { Sekunden })
\end{aligned}
$$

Variable 2:

$$
\text { Index } E P /_{P}=\frac{\text { Anzahl der erfolgreichen Pässe }}{\text { gesamte Anzahl der Pässe }}
$$

Als Neben-Variablen wurden die folgende Indizes berechnet: 
Variable 3:

$$
\text { Index }^{P} / B K=\frac{\text { gesamte Anzahl der Pässe }}{\text { gesamt Anzahl der Ballkontakte }}
$$

Variable 4:

$$
\text { Index }^{E P} / B K=\frac{\text { Anzahl der erfolgreichen } P \text { ässe }}{\text { gesamte Anzahl der Ballkontakte }}
$$

Variable 5:

$$
\text { Index NEP } / B K=\frac{\text { Anzahl der nicht erfolgreichen Pässe }}{\text { gesamte Anzahl der Ballkontakte }}
$$

Variable 6:

$$
\text { Index }{ }^{N E P} / P=\frac{\text { Anzahl der nicht erfolgreichen Pässe }}{\text { gesamte Anzahl der Pässe }}
$$

Variable 7:

$$
\text { Index } E P / N E P=\frac{\text { Anzahl der erfolgreichen } P \text { ässe }}{\text { Anzahl der nicht erfolgreichen Pässe }}
$$

\section{Auswertungsobjektivität}

Es wurde ein Expertenrating durchgeführt, um Unstimmigkeiten bei der Auswertung des Passes während des Spiels zu vermeiden. Drei Sportwissenschaftler, zwei davon Fußballtrainer, beobachteten 7 Spieler, die insgesamt 156 Ballberührungen ausführten, und beurteilten dabei, welche dieser Ballberührungen in einen erfolgreichen Pass verwandelt wurden; sie beobachteten damit 17,23\% der Stichprobe (156 Ballberührungen von insgesamt 905 der drei Messzeitpunkte Vor-, Post- und Retentionstest). Die Experten beobachteten zweimal denselben Videoausschnitt und dieselben Spieler und werteten daher zweimal unabhängig voneinander dieselben 156 Ballberührungen aus. Korrelationen zwischen beiden Auswertungen jedes Experten wurden durch das Pearsonproduct-moment-correlation-Verfahren berechnet. Die Ergebnisse zeigen eine IntraExperten-Korrelation von $r=.95(p<.001), r=.97(p<.001)$ und $r=.94(p<.001)$. Eine der zwei Auswertungen wurde jeweils von jedem Experten per Zufall ausgewählt, Korrelationen zwischen den drei ausgewählten Auswertungen wurden ebenfalls berechneteine Inter-Experten-Korrelation jeweils von $r=.87(p=.009)$ zwischen den Experten 1 und 2, $r=.86(p=.011) z$ wischen den Experten 1 und 3 und $r=.91(p=.004)$ zwischen den Ex- 
perten 2 und 3 wurden gefunden. Einer der Experten war der Forscher selbst, dieser wertete die ganze Stichprobe aus; anschließen wurden die Ergebnisse statistisch aufbereitet und analysiert.

\section{Intervention}

Mit jeder Gruppe wurde ein Lernprogramm zur Verbesserung der technischen Fußballfertigkeit „Pass“ durchgeführt. Die Experimentalgruppe wurde als Spielgruppe genannt, diese Experimentalgruppe übte die technische Fertigkeit des Fußballpasses einschließlich im Fußballspiel selbst. In der Spielgruppe wurden die Spieler in Mannschaften geteilt und unterschiedliche Kleine Fußballspiele organisiert. Jedes dieser Spiele hatte besondere Regeln für die häufige Verwendung von Pässen, z. B. begrenzte Ballführung und Ballkontakte, verschiedene Torzahl und Torgröße usw. Häufig wurden Spiele verwendet, in denen die Mannschaft in Ballbesitz in Überzahl spielte. Dadurch konnte man den Druck verringern, um die Ausführung von mehr und genaueren Pässe zu fördern. Die Spiele fanden in zwei Spielfelder einer Sporthalle statt. Auf diese Weise konnten alle Spieler gleichzeitig aktiv sein und es gab keine Wartezeiten. Als Tore wurden dicke Matten, Bänke und Kästen benutzt (siehe ein Beispiel einer Trainingssitzung in Anhang N).

Ein Beispiel einer Aufgabe für die Spielgruppe wird in der Abbildung 28 beschrieben.

Tor nach 5 Pässen

\section{Durchlauf}

4 gegen 4 auf vier Tore, ein Tor kann nur erzielt werden wenn nach innerhalb der angreifenden Mannschaft 5 Pässe in einer Reihe gemacht werden haben.

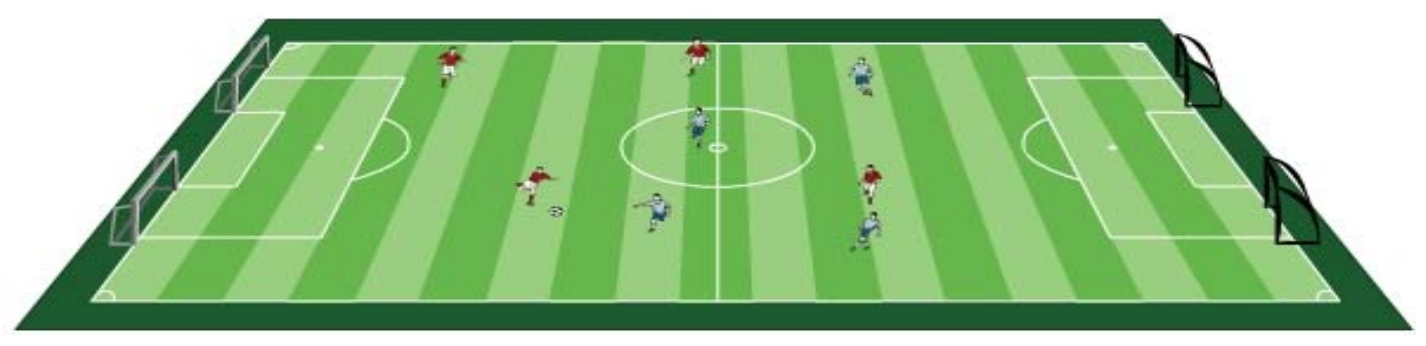

\section{Hinweise}

Es wird nicht erlaubt, den Ball zurückpassen. Ein Ballbesitz endet mit einem Tor, mit Ball-aus oder mit dem Ballberühren durch einen Verteidiger. Dauer: 4 Minuten.

Abbildung 28: Beispiel eines Kleinen Fußballspiels mit unterschiedlichen Schwerpunkten als Aufgabe der Spielgruppe 
In der Übungsgruppe wurden dagegen übliche „geometrische Übungen“ (DOUCET, 2006, S. 15) für das Lernen und Üben des Passes benutzt. Bei solchen Übungen sollen die Spieler bestimmte Grundstellungen auf dem Feld einnehmen und Pässe zum selben Partner während einer bestimmten Zeit oder in bestimmter Wiederholungsanzahl ausführen. Das Üben wurde in Blöcken organisiert. Die Übungen wurden abwechslungsreich gestaltet, es wurde aber nie während der Passschulung gespielt (siehe ein Beispiel einer Trainingssitzung in Anhang $\mathrm{O}$ ).

Ein Beispiel einer Aufgabe für die Übungsgruppe wird in Abbildung 29 beschrieben.

\section{Passaufgabe}

Zwei Gruppen stehen sich 10 Meter gegenüber und passen sich den Ball zu. Nach Ballabgabe läuft der Spieler um die Markierung und stellt sich wieder hinten an.

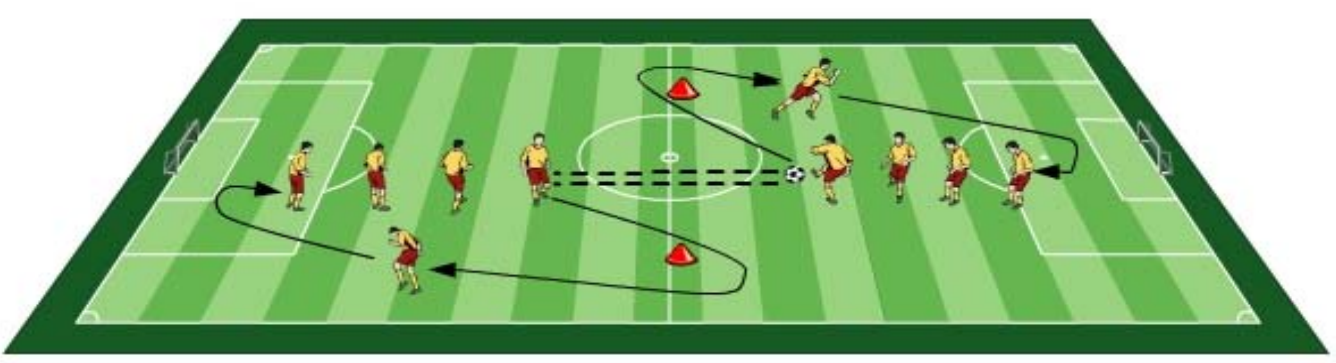

\section{Hinweise}

Direktes und genaues Passspiel.

Materialien: 2 Gruppen mit je max. 6 Spieler, 1 Ball, Markierungen.

Dauer: 4 Minuten

Abbildung 29: Beispiel einer „geometrischen Übung“ als Aufgabe der Übungsgruppe

Die beiden Gruppen übten in derselben Sporthalle, hatten die gleiche Anzahl von Lerneinheiten und der Prozess wurde unter ähnlichen Bedingungen durchgeführt. Jede Sitzung dauerte 30 Minuten. Die anderen Variablen, die das Lernen beeinflussen könnten, wurden vor Beginn des Lernprozesses überprüft, um sicherzustellen, dass die Gruppen so homogen wie möglich waren. Die folgenden Variablen wurden geprüft: frühere Erfahrungen, Lebensstil und zusätzliche Fußballpraxis außerhalb des Trainings. Es wurde ein 
unstrukturiertes Interview durchgeführt, um Informationen über die Kontrollvariablen zu erheben. Darin wurden an die teilnehmenden Spieler die folgenden Fragen gestellt:

- Seit wann Spielst du Fußball bei einem Sportverein?

- Hast du Unterbrechungen deiner Sportpraxis gehabt?

- Trainierst du systematisch eine andere Sportart?

- Was für eine Schule besuchst du?

- Was machst du in deiner Freizeit?

- Wie oft spielst du Fußball außerhalb des Trainings und der Wettkampfspiele?

Alle Kinder hatten ähnliche Erfahrungen und Lebensstile und alle berichteten von gelegentlichem, spontanem Fußballspielen in der Schule. Keines hatte ein systematisches Lernprogramm außerhalb des Trainings absolviert. Alle Spieler trainieren systematisch Fußball seit mindestens drei Jahren bei einem Verein. Keiner hatte eine Unterbrechung von mehr als 6 Monaten. 15 Spieler besuchten ein Gymnasium und acht eine Gesamtschule, drei besuchten eine Hauptschule. Nur zwei Kinder trainierten systematisch andere Sportarten und zwar Handball und Reiten. Die Spieler verbrachten ihre Freizeit mit ihrem Alter adäquaten Beschäftigungen: Freunde treffen, Fernsehen, Videospiele spielen, Musik hören, Einkaufen, Lesen, im Internet surfen usw.

Die beiden Tests für die Bewertung der Leistung bei der Passfertigkeit wurden vor dem Start der zwei Lernprogramme durchgeführt. Die Ergebnisse wurden verglichen und damit zum Nachweis des Startpunkts (Vortest, Baseline) der Gruppen. Die gleichen Tests wurden unmittelbar nach Abschluss der Lernprogramme eingesetzt (Posttest). Nach drei Wochen Schul- und- Vereinsferien ohne systematische Trainingseinheit wurden die beiden Tests noch einmal durchgeführt, um die Nachhaltigkeit des Lernens zu prüfen (Retentiontest). Die folgende Abbildung stellt den Untersuchungsablauf dar:

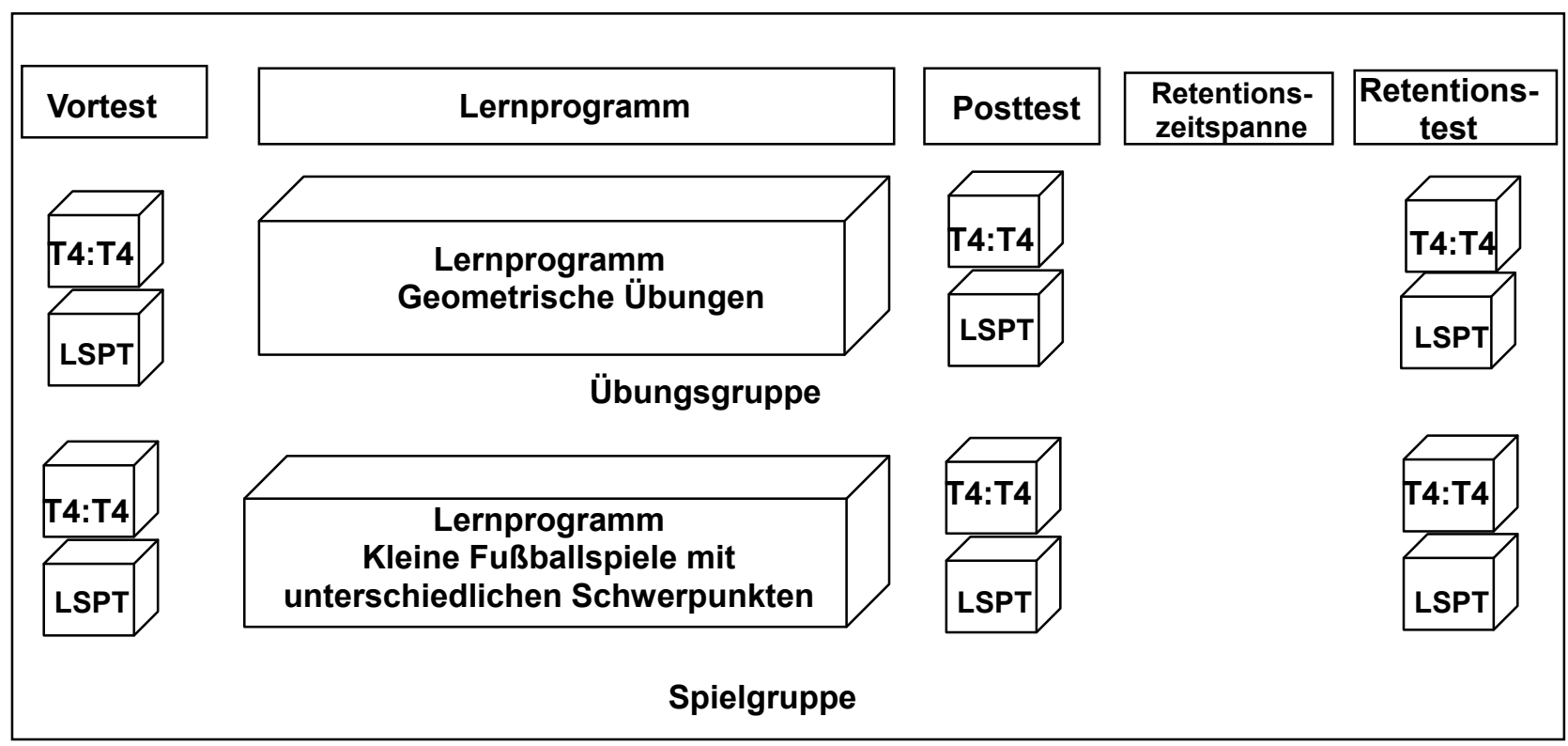

Abbildung 30: Untersuchungsablauf Studie drei 
Die statistische Analyse wurde mit der Software Statgraphics XI und SPSS 17.0. durchgeführt. Beide Gruppen führten dreimal die zwei Tests durch: Vor-, Post- und Retentionstest. Für jede Gruppe wurden Maße der zentralen Tendenz und Streuung berechnet. Die normale Verteilung der Stichprobe wurde mit einem Kolmogorov-Smirnov-Test bestätigt. Durch ein F-Ratio-Verfahren wurde die Homogenität der Varianzen von Mittelwerten bestätigt. Wenn kein signifikanter Unterschied zwischen den Varianzen beider Mittelwerte entstand, wurde eine zweifaktoriellen Varianzanalyse mit Messwiederholung auf einem Faktor und auch einent-Test durchgeführt, um Unterschiede innerhalb der Gruppen während der drei Auswertungszeitpunkte festzustellen. Es wurden auch Unterschiede zwischen den Gruppen in jeder Phase bestimmt.

Die statistischen Hypothesen wurden grundsätzlich für die Hauptvariablen formuliert, sie sind jedoch auch für die Nebenvariablen umsetzbar:

Für den Vergleich der Intra-Gruppe gilt:

$\begin{array}{llll}\text { H0: } & \mu 1=\mu 2 & \text { bzw. } & \mu 1=\mu 3 \\ \text { H1: } & \mu 1<\mu 2 & \text { bzw. } & \mu 1<\mu 3\end{array}$

Für den Vergleich der Zwischengruppen gilt:
H0: $\quad \mu 2 \ddot{U G}=\mu 2 \mathrm{SG}$
bzw.
$\mu 3 \ddot{U G}=\mu 3 \ddot{U G}$
H1: $\quad \mu 2$ ÜG $<\mu 2 S G$
bzw.
$\mu 3$ ÜG $<\mu 3$ SG

wobei:
$\mathrm{HO}$ die Nullhypothese ist,
$\mu 1$ der Mittelwert des Vortests,
$\mu 2$ der Mittelwert des Posttests und
$\mathrm{3}$ der Mittelwert des Retentionstests.

\subsubsection{Ergebnisse}

In der Tabelle 13 werden die Mittelwerte des Spieltests dargestellt (Siehe Gesamtdaten im Anhang P). Die Anzahl der Berührungen des Balls sowie erfolgreiche und nicht erfolgreiche Pässe jedes Spielers wurden gezählt. Die Anzahl der Ballberührungen und Pässe blieb über die drei Zeitpunkte von Vor-, Post- und Retentionstest unverändert in der Übungsgruppe und auch in der Spielgruppe. Jedoch ist nach dem Treatment der Mittelwert der Anzahl erfolgreicher Pässe in der Übungsgruppe um 0,87 und in der Spielgruppe um 2,3 erhöht und die Anzahl nicht-erfolgreicher Pässe um 1,5 in der Übungsgruppe und um 1,4 in der Spielgruppe gesunken. Diese Verbesserung der Leistung wurde in keiner der zwei Gruppen für den Retentionstest festgestellt. Die Ergeb- 
nisse weisen eine Steigerung der Leistung bei Pässen während des Spiels beider Gruppe aus. Aus den beobachteten Parametern „Ballberührung“, „erfolgreicher und nicht-erfolgreicher Pass“ wurden einige Indizes abgeleitet, um die Auswirkungen des Treatments näher zu betrachten. In keiner der drei Auswertungen wurde eine Änderung der Anzahl der Ballberührungen gefunden. Grundsätzlich spielten die teilnehmenden jungen Fußballspieler einen Pass aus jeder zweiten Ballberührung, nach 50 \% der Ballberührungen verloren die Spieler den Ball entweder oder schossen auf das Tor. Eine spätere Studie könnte über die Auszählung der Torschüsse genauere Hinweise über die Anzahl der verlorenen Bälle eines Spieler oder einer Mannschaft während des Spiels gewinnen.

Die Anzahl der erfolgreichen Pässe relativ zur Anzahl von Ballkontakten (EP/BK) wurde beim Post-Test von 0,27 bis 0,35 in der Übungsgruppe und von 0,30 bis 0,43 in der Spielgruppe leicht erhöht, dieselbe Erhöhung wurde aber bei keiner der beiden Gruppen im Retentionstest erreicht. Ähnlich ist die Anzahl von nicht-erfolgreichen Pässen relativ zur Anzahl von Ballkontakten (NEP/BK) im Post-Test leicht abgesunken, nicht jedoch im Retentionstest.

Der bedeutendste Index für die Leistung der Spieler beim Passen während des Spiels ist die Anzahl der erfolgreichen Pässe relativ zu ihrer Anzahl (EP/P). Die Übungsgruppe erhöhte diesen Index um 21\% und die Spielgruppe um 18\% im Post-Test, allerdings erreichten die Spieler beim Retentionstest nach drei Wochen ohne Training wieder Werte, die in der Nähe des Vortests liegen. Die Anzahl der nicht-erfolgreichen Pässe relativ zur Anzahl der Pässe (NEP/P) sank um 20\% in der Übungsgruppe und $19 \%$ in der Spielgruppe, die Anzahl der nicht-erfolgreichen Pässe wurde jedoch beim Retentionstest erhöht. Das Verhältnis erfolgreiche Pässe/nicht-erfolgreiche Pässe (EP/NEP) zeigte dieselben Änderungen wie die anderen. 
Tabelle 13: Mittelwerte der Variablen in Beziehung zum Pass im Vor-, Post- und Retentionstest bei der Übungsgruppe $(n=8)$ und Spielgruppe ( $n=10$. P/BK: Pässe/Ballkontakte, EP/BK: erfolgreiche Pässe/Ballkontakte, NEP/BK: nicht-erfolgreiche Pässe/Ballkontakte, E/P: erfolgreiche Pässe/Pässe, NEP/P: nicht-erfolgreiche Pässe/Pässe, EP/NEP: erfolgreiche Pässe/ nicht-erfolgreiche Pässe

\begin{tabular}{|c|c|c|c|c|c|c|}
\hline & \multicolumn{2}{|c|}{$\begin{array}{l}\text { Mittelwerte } \\
\text { Vor-Test }\end{array}$} & \multicolumn{2}{|c|}{$\begin{array}{c}\text { Mittelwerte } \\
\text { Post-Test }\end{array}$} & \multicolumn{2}{|c|}{$\begin{array}{c}\text { Mittelwerte } \\
\text { Retentions-Test }\end{array}$} \\
\hline & $\begin{array}{l}\text { Übungs- } \\
\text { gruppe }\end{array}$ & $\begin{array}{l}\text { Spiel- } \\
\text { Gruppe }\end{array}$ & $\begin{array}{l}\text { Übungs- } \\
\text { gruppe }\end{array}$ & $\begin{array}{l}\text { Spiel- } \\
\text { gruppe }\end{array}$ & $\begin{array}{l}\text { Übungs- } \\
\text { gruppe }\end{array}$ & $\begin{array}{l}\text { Spiel- } \\
\text { gruppe }\end{array}$ \\
\hline Ballkontakte & 15,50 & 15,70 & 14,63 & 16,80 & 19 & 18,7 \\
\hline Pässe & 7,75 & 7,60 & 7 & 8,60 & 10,13 & 9,30 \\
\hline Erfolgreiche Pässe & 4,13 & 4,60 & 5 & 6,90 & 6,38 & 6 \\
\hline $\begin{array}{l}\text { Nicht-erfolgreiche } \\
\text { Pässe }\end{array}$ & 3,50 & 3,10 & 2 & 1,70 & 3,88 & 3,30 \\
\hline P/BK & 0,51 & 0,50 & 0,48 & 0,53 & 0,53 & 0,48 \\
\hline E/BK & 0,27 & 0,30 & 0,35 & 0,43 & 0,34 & 0,30 \\
\hline NEP/BK & 0,22 & 0,20 & 0,14 & 0,10 & 0,19 & 0,17 \\
\hline EP/P & 0,53 & 0,61 & 0,74 & 0,79 & 0,65 & 0,63 \\
\hline NEP/P & 0,46 & 0,40 & 0,26 & 0,21 & 0,36 & 0,37 \\
\hline EP/NEP & 1,82 & 1,48 & 2,78 & 4,73 & 2,51 & 2,02 \\
\hline
\end{tabular}

Eine grafische Darstellung der Ergebnisse des Spieltests folgt in den Abbildung 31. 


\section{Spiel Test}

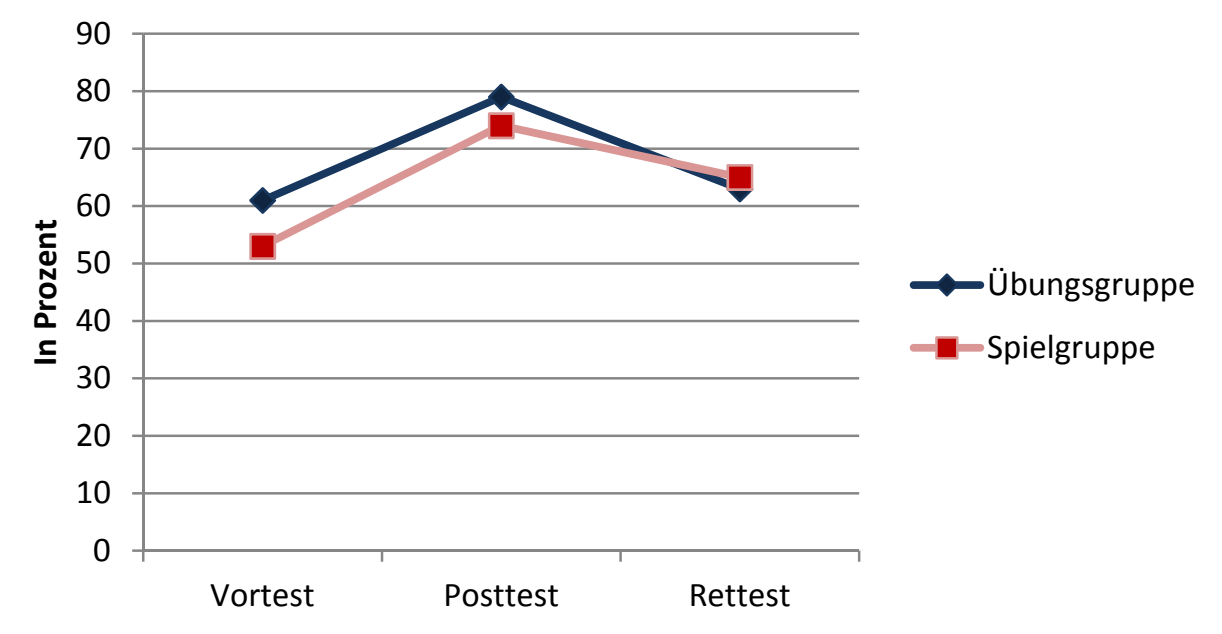

Abbildung 31: Prozent der erfolgreichen Pässe der zwei Gruppen im Vor-, Post- und Retentionstest des Spiel-Tests

Die Tabelle 14 zeigt die Ergebnisse, die die Spieler zum jeweiligen Zeitpunkt des Loughborough-Pass-Tests erreichten (siehe Gesamtdaten im Anhang Q). Die benötigte Gesamtzeit wurde bis zu 6,5 Sekunden abgesenkt. In beiden Gruppen geschah dies grundsätzlich wegen der Steigerung der Genauigkeit und Verringerung der Fehleranzahl während der Ausführung, die Ausführungszeit selbst wurde nur in der Übungsgruppe leicht verringert. Im Gegensatz zum Spieltest wurden die Verbesserungen beider Gruppen im Retentionstest aufrechterhalten.

Tabelle 14: Ergebnisse in Vor-, Post-und Retentions-Test beim Loughborough-Pass-Test der Übungsgruppe $(n=8)$ und Spielgruppe $(n=10)$

\begin{tabular}{|l|c|c|c|c|c|c|} 
& \multicolumn{2}{|c|}{$\begin{array}{c}\text { Mittelwerte } \\
\text { Vor-Test }\end{array}$} & \multicolumn{2}{c|}{$\begin{array}{c}\text { Mittelwerte } \\
\text { Post-Test }\end{array}$} & \multicolumn{2}{c|}{$\begin{array}{c}\text { Mittelwerte } \\
\text { Retentions-Test }\end{array}$} \\
\hline & $\begin{array}{c}\text { Übungs- } \\
\text { gruppe }\end{array}$ & $\begin{array}{c}\text { Spiel- } \\
\text { gruppe }\end{array}$ & $\begin{array}{c}\text { Übungs- } \\
\text { gruppe }\end{array}$ & $\begin{array}{c}\text { Spiel- } \\
\text { gruppe }\end{array}$ & $\begin{array}{c}\text { Übungs- } \\
\text { gruppe }\end{array}$ & $\begin{array}{c}\text { Spiel- } \\
\text { gruppe }\end{array}$ \\
\hline Zeit & 28,88 & 28,90 & 26,00 & 28,70 & 25,63 & 28,20 \\
\hline Ziel nicht getroffen & 1,88 & 1,70 & 1,13 & 0,60 & 1,63 & 1,00 \\
\hline Fehler & 4,00 & 3,30 & 3,38 & 3,50 & 3,00 & 2,50 \\
\hline Gesamtzeit & 46,25 & 44,00 & 38,38 & 38,70 & 39,75 & 38,20 \\
\hline
\end{tabular}

Eine grafische Darstellung der Ergebnisse des Loughborough-Pass-Tests folgt in der Abbildung 32. 


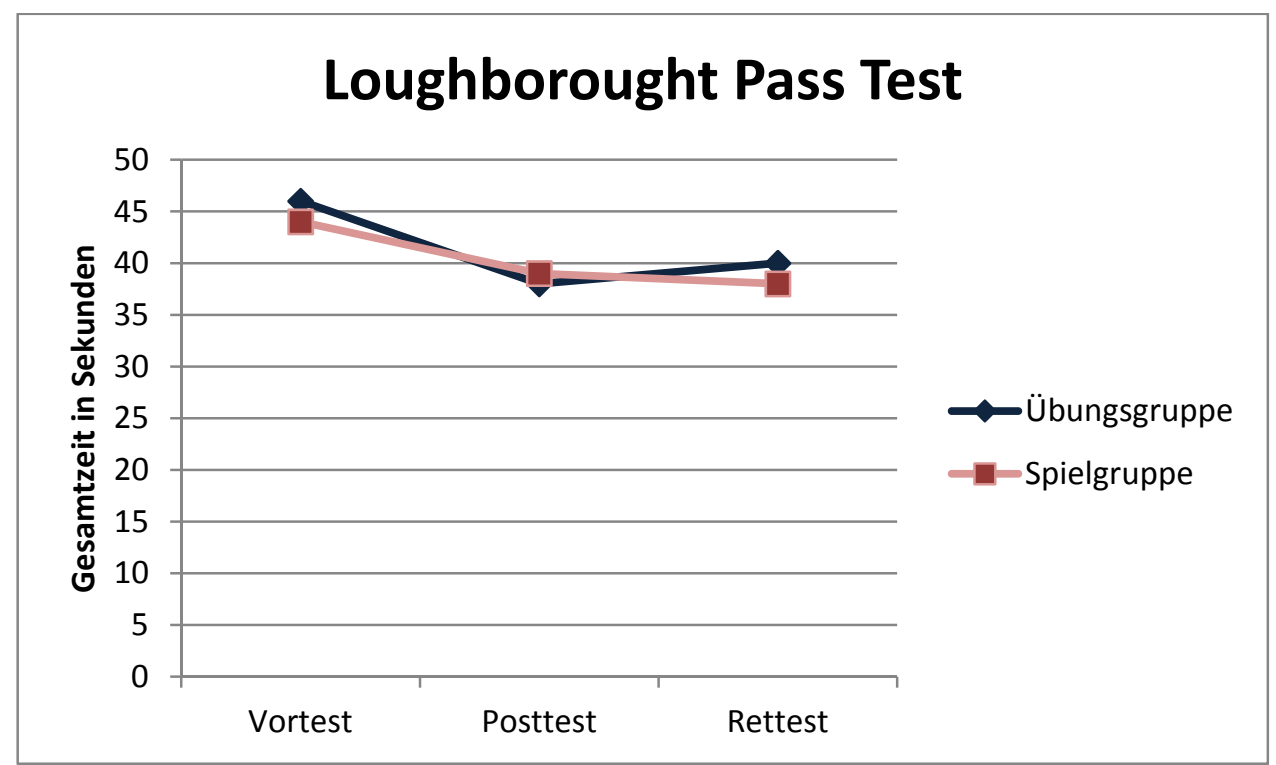

\begin{abstract}
Abbildung 32: Ergebnisse in Gesamtzeit der zwei Gruppen im Vor-, Post-und Retention des Loughborough-Pass-Tests
\end{abstract}

Die Annahme der Normalverteilung der Daten wurde nicht durch den KolmogorovSmirnov-Test abgelehnt $(p>$.5) (siehe die Werte des Kolmogorov-Smirnov-Tests im Anhang R) und der Homogenität der Varianzen wurde durch die F-Test bestätigt. Einezweifaktorielle Varianzanalyse mit Messwiederholung auf einem Faktor wurde angewendet, um signifikante Unterschiede zwischen den Daten der drei Zeitmesspunkte und zwischen den Gruppen festzustellen. Da eine Verletzung der Sphärizität wurde bei einigen Variablen durch den Mauchly Test bestätigt (EP: W[2] = 0,694; $p<.05$; NEP/BK: $\mathrm{W}[2]=0,497 ; p<.01$ und NEP/P: $\mathrm{W}[2]=0,024 ; p<.01$ ) (siehe die Werte des MauchliyTests im Anhang R) undauch wegen der kleinen Stichprobe wurde die GreenhouseGeisser Korrektur der Freiheitsgrade verwendet. Wie die Tabelle 15 zeigt, wurden die nicht-erfolgreichen Pässe $(\mathrm{F}[1,53 ; 24,50]=4,44 ; p<.05)$ und der Index der nichterfolgreichen Pässe in Bezug auf die Gesamtzahl der Pässe $(F[1,01 ; 16,20]=12,94 ; p<$ .01 ) auf beide Gruppe signifikant verringert. Die Indizes der erfolgreichen Pässe in Bezug auf die Gesamtzahl der Pässe $(F[1,70 ; 27,33]=6,71 ; p<.05)$ und auf die nichterfolgreichen Pässe $(F[1,72 ; 27,46]=4,84 ; p<.05)$ wurden hingegen signifikant erhöht. Kein signifikanter Unterschied zwischen den Gruppenund keinsignifikanterInteraktionseffektvon Messzeitpunkt x Gruppe wurde festgestellt. 
Tabelle 15: Ergebnisse der zweifaktoriellen Varianzanalyse mit Messwiederholung auf einem Faktor der Variablen ausgewertet mit dem Spieltest. P/BK: Pässe/Ballkontakten, EP/BK: erfolgreiche Pässe/Ballkontakten, NEP/BK nicht-erfolgreiche Pässe/Ballkontakten, EP/P: erfolgreiche Pässe/Pässe, NEP/P: nicht-erfolgreiche Pässe/Pässe, EP/NEP: erfolgreiche Pässe/ nicht-erfolgreiche Pässe

\begin{tabular}{|l|c|c|c|c|c|c|c|c|c|}
\hline & \multicolumn{3}{|c|}{ Messzeitpunkt } & \multicolumn{3}{c|}{ Gruppe } & \multicolumn{3}{c|}{$\begin{array}{c}\text { Interaktion Messzeit- } \\
\text { punkt x Gruppe }\end{array}$} \\
& $\begin{array}{c}\text { Korrigierte } \\
\text { d.f. }\end{array}$ & $F$ & $p$ & d.f & $F$ & $p$ & d.f & $F$ & $p$ \\
\hline Ballkontakte & $(1,83 ; 29,22)$ & 2,68 & 0,09 & $(1,16)$ & 0,21 & 0,66 & $(1,16)$ & 0,30 & 0,87 \\
\hline Pässe & $(1,64 ; 26,33)$ & 1,92 & 0,17 & $(1,16)$ & 0,08 & 0,78 & $(1,16)$ & 0,84 & 0,77 \\
\hline Erfolgreiche & $(1,39 ; 22,23)$ & 2,69 & 0,11 & $(1,16)$ & 0,73 & 0,41 & $(1,16)$ & 0,29 & 0,60 \\
Pässe & & & & & & & & & \\
\hline $\begin{array}{l}\text { Nicht-erfolgreiche } \\
\text { Pässe }\end{array}$ & $(1,53 ; 24,50)$ & 4,44 & $0,03^{*}$ & $(1,16)$ & 0,54 & 0,47 & $(1,16)$ & 0,13 & 0,91 \\
\hline P/BK & $(1,91 ; 30,64)$ & 0,01 & 0,99 & $(1,16)$ & 0,02 & 0,91 & $(1,16)$ & 0,22 & 0,64 \\
\hline EP/BK & $(1,90 ; 30,30)$ & 2,21 & 0,13 & $(1,16)$ & 0,42 & 0,53 & $(1,16)$ & 0,61 & 0,45 \\
\hline NEP/BK & $(1,33 ; 21,23)$ & 3,33 & 0,07 & $(1,16)$ & 0,55 & 0,47 & $(1,16)$ & 0,00 & 0,98 \\
\hline EP/P & $(1,70 ; 27,33)$ & 6,71 & $0,01^{*}$ & $(1,16)$ & 0 & 0,98 & $(1,16)$ & 0,67 & 0,42 \\
\hline NEP/P & $(1,01 ; 16,20)$ & 12,94 & $0,00^{*}$ & $(1,16)$ & 0,42 & 0,53 & $(1,16)$ & 0,45 & 0,51 \\
\hline EP/NEP & $(1,72 ; 27,46)$ & 4,84 & $0,02^{*}$ & $(1,16)$ & 1,25 & 0,28 & $(1,16)$ & 1,06 & 0,32 \\
\hline
\end{tabular}

${ }^{*} p<0,05$

Die Tabelle 16 zeigt die Ergebnisse der Varianzanalyse für den Loughboroug-Pass-Test angewendet. Die Anzahl der Fehler wegen verfehlter Ziele wurde signifikant verringert, d.h. die Genauigkeit wurde erhöht $(F[1,81 ; 29,02]=5,85 ; p<.05)$ und auch die Gesamtzeit $(F[1,73 ; 27,63]=5,83 ; p<.05)$ wurde signifikant verringert. Solche statistisch signifikanten Verbesserungen kamen in beiden Gruppen vor.Kein signifikanter Interaktionseffekt Messzeitpunkt x Gruppe wurde festgestellt. 
Tabelle 16: Ergebnisse der zweifaktoriellen Varianzanalyse mit Messwiederholung auf einem Faktor für den Loughborough-Pass-Test

\begin{tabular}{|l|c|c|c|c|c|c|c|c|c|} 
& \multicolumn{4}{|c|}{ Messzeitpunkt } & \multicolumn{4}{c|}{ Gruppe } & \multicolumn{2}{c|}{$\begin{array}{c}\text { Interaktion Messzeit- } \\
\text { punkt x Gruppe }\end{array}$} \\
\hline & $\begin{array}{c}\text { Korrigierte } \\
\text { Freiheitsgrade }\end{array}$ & $\mathrm{F}$ & $p$ & $\begin{array}{c}\text { Frei- } \\
\text { heitsgr } \\
\text { ade }\end{array}$ & $\mathrm{F}$ & $p$ & $\begin{array}{c}\text { Frei- } \\
\text { heitsgr } \\
\text { ade }\end{array}$ & $\mathrm{F}$ & $p$ \\
\hline Zeit & $(1,71 ; 27,32)$ & 1,92 & 0,17 & $(1,16)$ & 2,72 & 0,12 & $(1,16)$ & 1,71 & 0,21 \\
\hline Ziel nicht getroffen & $(1,81 ; 29,02)$ & 5,85 & $0,01^{*}$ & $(1,16)$ & 4,46 & 0,05 & $(1,16)$ & 0,56 & 0,46 \\
\hline Fehler & $(1,95 ; 31,17)$ & 2,24 & 0,13 & $(1,16)$ & 1,33 & 0,27 & $(1,16)$ & 0,55 & 0,82 \\
\hline Gesamtzeit & $(1,73 ; 27,63)$ & 5,83 & $0,01^{*}$ & $(1,16)$ & 0,69 & 0,42 & $(1,16)$ & 0,34 & 0,86 \\
\hline
\end{tabular}

$p<.05$

Die Tabelle 17 zeigt die Ergebnisse vont-Tests für abhängige Stichproben, die verwendet wurden, um die Änderungen der Mittelwerte zwischen den drei Auswertungen innerhalb jeder Gruppe zu verdeutlichen. Bei der Evaluation der Technik des Passes mit dem Loughborough-Pass-Test wurde eine signifikante Änderung der Variablen Gesamtzeit zwischen Vor- und Posttest der Übungsgruppe festgestellt $(t[2 ; 16]=2,45 ; p<.05)$. Keine Änderung wurde hingegen in der Spielgruppe gefunden. Zwischen Vor- und Retentions-Test wurde eine signifikante Änderung in der Spielgruppe gefunden $(t[2 ; 16]=$ 2,97; $p<.01$ ) und die Übungsgruppe erreicht nicht mehr die Leistung des Posttests.

Tabelle 17: t-Test für jede Gruppe eines Vergleichs der Mittelwerte im Vor-, Post-und Retentionstest der Variablen Gesamtzeit beim Loughborough-Pass-Test und der Variable EP/P beim SpielTest

\begin{tabular}{|l|c|c|c|c|c|c|c|c|}
\hline \multirow{4}{*}{} & \multicolumn{9}{|c|}{ Differenzen } \\
\cline { 2 - 9 } & \multicolumn{3}{|c|}{ LOUGHBOROUGH-PASS-TEST } & \multicolumn{3}{c|}{ SPIEL-TEST } \\
\cline { 2 - 9 } & \multicolumn{2}{|c|}{ Vor-Post } & \multicolumn{2}{c|}{ Vor-Ret. } & \multicolumn{2}{c|}{ Vor-Post } & \multicolumn{2}{c|}{ Vor-Ret. } \\
\hline & $T$ & $P$ & $t$ & $p$ & $t$ & $p$ & $T$ & $P$ \\
\hline Übungsgruppe & 2,45 & ${ }^{*} 0,02$ & 1,80 & 0,09 & $-2,28$ & ${ }^{*} 0,03$ & $-1,25$ & 0,23 \\
\hline Spielgruppe & 1,91 & 0,07 & 2,97 & ${ }^{*} 0,00$ & $-2,40$ & ${ }^{*} 0,02$ & $-0,23$ & 0,82 \\
\hline
\end{tabular}

${ }^{*} p<0,05$

Im Spieltest wurden signifikante Änderungen der Mittelwerte der Variable „erfolgreiche Pässe/Pässe" (EP/P) beider Gruppen beim Posttest festgestellt $(t[2 ; 16]=-2,28 ; p<.05$ und $(t[2 ; 16]=2,40 ; p<.05))$. Das weist darauf hin, dass sich sowohl die Übungen als auch das Spiel positiv auf das Lernen der technischen Fertigkeiten des Fußballs auswirken. Andererseits waren die Lerneffekte nach einer Zeit ohne Übung schnell verschwunden. Dies widerspricht den Ergebnissen von vielen Studien zu kontextueller Interferenz, in denen die Probanden, die unter diesen Bedingungen übten, ein langfristig stabiles Lernen erreichten als die Probanden, die unter standardisierten Bedingungen, d. h., durch geblocktes Üben lernten. 


\subsubsection{Diskussion und Schlussfolgerung}

Nach der Schema-Theorie (SCHMIDT, 1975) ist die Variabilität des Übens eines der wichtigsten Forschungsthemen des motorischen Lernens geworden. Die Lernenden sollten die Lernaufgabe variieren, wenn sie versuchen, eine technische Fertigkeit zu erlernen (STOKES, LAI, HOLTZ, RIGSBEE \& CHERRICK, 2008, S. 654). Nach den Studien von SHEA und MORGAN im Jahr 1979 haben die meisten Studien (LIN, 2006) die Vorteile des zufälligen Übens gegenüber dem Üben in Blöcken bestätigt. Allerdings wurden die meisten dieser Studien im Labor durchgeführt und fanden mit Bewegungen statt, die gar nichts mit den technischen Fertigkeiten des Sports zu tun haben (HILLEBRECHT, 1998, S. 159). In unserer Studie verwendeten wir eine reale Situation des Sports. Der Pass ist eine sehr wichtige technische Fertigkeit im Fußball. Die Ergebnisse früherer Studien (ARIAS \& JIMENEZ, 2004, 2007) bestätigen die Vorteile des zufälligen Übens auch für diese Technik. Auch wenn die Verwendung der Blockübung in Form von „geometrischen“ Übungen der Passtechnik verbessert werden konnte, waren die Auswirkungen der Spielpraxis ähnlich. So scheint es, dass das zufällige Üben in Form von Spielpraxis sich für die Lehre sportlicher Bewegungen eignet.

Gegenwärtig konzentrieren sich viele Forscher auf die Untersuchung der menschlichen Bewegung aus kognitiver Sicht. Die Variabilität des Übens stellt ein beliebtes Forschungsfeld für das Studium der Grundlagen der Entwicklung der kognitiven Fähigkeiten und Fertigkeiten durch das Üben dar (vgl. IMMINK \& WRIGHT, 2001, S. 423). Unklar ist bislang, wie zufälliges Üben in der Sportpraxis umgesetzt werden kann. Laboruntersuchungen verwenden Geräte und Einrichtungen, um die Praxis der motorischen Aufgaben nach dem Zufallsprinzip zu organisieren (NEWELL \& RUSSELL, 2007, S. 20). In Studien über kontextuelle Interferenz beim Unterrichten von technischen Fertigkeiten des Fußballs (ARIAS \& JIMENEZ, 2004, 2007) haben wir die Schwierigkeiten zur Kenntnis genommen, die bei der Organisation der Lernbedingungen in zufälliger Form auftreten. Normalerweise braucht diese Art des Übens viele Materialien, große Sportplätze und Hilfe von mehreren Trainern, die zusammen arbeiten müssen, um die zufälligen Bedingungen zu gewährleisten. Dies gilt speziell in Situationen des Unterrichts von Mannschaftssportarten, in denen es notwendig ist, bewegte Objekte wie Bälle, Hütchen, Tore usw. einzusetzen. Besonders wenn es sich um große Gruppen handelt, kann die Umsetzung des zufälligen Übens chaotisch werden. Wenn das zufällige Üben hingegen auch als Spiel umsetzbar ist, gibt es keine Notwendigkeit für eine große Anzahl von Materialien. Die Regeln des Spiels bestimmen die Organisation und die Lehrer oder Trainer haben mehr Zeit, um den Lernprozess zu beobachten und zu steuern.

Die dargestellten Ergebnisse bestätigen die Wirksamkeit der zwei Arten von Übung für die Verbesserung der Technik des Passes im Fußball mit Jungen zwischen 10 und 12 Jahren. Die erzielten Verbesserungen konnten jedoch nach einer Zeitspanne von drei Woche ohne Training nicht aufrechterhalten werden.

Die vorliegende Studie hat positive Auswirkungen des Spiels auf den Lernprozess der technischen Fertigkeiten gezeigt.Diese Effekte sind ähnlich denen der "geometrischen“ Übungen. Das Üben in Form von gezielten Übungen impliziert die Anordnung der Teilnehmer in einer festen Reihenfolge, in Kreisen und ordnungsgemäßen Situationen und die Festlegung bestimmter Standorte, was die Kontrolle der Tätigkeit erleichtert. Die Anordnung wird nur am Ende der Übung geändert. Sportlehrer und Trainer erkennen 
die Vorteile des zufälligen Übens, sie wenden aber noch immer Übungen in geblockten Folgen an, um die Kontrolle aufrecht zu erhalten (PIERON, 1998). BUNKERund THORPE (1986a, S. 9) förderten die Idee, dass Spiele besser beim Spielen zu lernen sind und bekräftigten die Vorteile der Anwendung des Spiels für die Entwicklung des taktischen Bewusstseins. Daher müssen die Spiele, die während des Trainings verwendet werden, angepasst werden. Darüber hinaus sind die Kleinen Spiele spezifisch für das Training innerhalb des Spielkontextes geeignet. Das ermöglicht ein situatives Lernen.

Die "KleinenFußballspiele mit unterschiedlichen Schwerpunkten" fördern die häufige Verwendung des Passes. Die Spielanforderungen zwingen die Spieler, gute Pässe zu spielen um das Abfangen des Balles durch den Gegner zu vermeiden. Somit führen die Kleinen Spiele zur Verbesserung dieser Fertigkeit. Die sich ständig ändernden Umstände gewährleisten genug Variabilität. Immer dann, wenn ein Spieler sich im Besitz des Balles befindet, muss er eine Entscheidung treffen. Dies trägt zur Verbesserung der Entscheidungsfähigkeit der Kinder bei.

Die Ergebnisse dieser Studie könnten als Bestätigung der Effizienz der „geometrischen“ Übungen interpretiert werden, wo viele Wiederholungen der Bewegungen außerhalb der Spielsituation stattfinden. Wie frühere Studien nahelegen (vgl. FARROW \& MASCHETTE, 1997; MEIRA \& TANI, 2003; PEREZ, MEIRA \& TANI, 2005; PORTER \& MAGILL, 2004), gingen die Verbesserungen nach einiger Zeit ohne Praxis wieder verloren. Die signifikanten Unterschiede zwischen Post- und Retentionstest in der Spielgruppe, nicht aber zwischen Vor- und Posttest, wurden auch in früheren Studien gefunden (z.B. ARIAS \& JIMÉNEZ, 2004). Bei diesen Studien mit Üben unter Interferenz erscheint das Lernen der Probanden, die dem zufälligen Üben zugeordnet wurden, zu einem späteren Zeitpunkt aufzutreten, nämlich erst beim Retentionstest. Allerdings könnten die Änderungen in der Leistung bei dem Test auch dem Effekt der Anpassung an die Aufgabe des Tests zugerechnet werden. Die Spieler könnten bei der zweiten oder dritten Ausführung derselben Aufgabe etwas aus den ersten Ausführungen gelernt haben oder einfach aufmerksamer, konzentrierter und bemühter zu Werke gegangen sein. Dies steigert die Genauigkeit und die Anzahl der Fehler sinkt. Weitere Studien werden daher notwendig sein, in denen die Gewöhnung an den Test verhindert wird.

Mehrere Forschungen (LIN, 2006) in Bezug auf kontextuelle Interferenz legen nahe, dass es am besten ist, zufälliges Üben zu nutzen, um die Variabilität zu fördern. In dieser Studie wurden die Spiele hingegen als ebenso effektiv wie das klassische Üben bewertet. Daher könnte das Konzept der Kleinen Spiele am besten an die Anforderungen der Variabilität des Übens anzupassen sein, denn das Spiel ist in den meisten Fällen die variabelste und zufälligste der Praktiken.Zusammenfassend kann man aus dieser Studie die folgenden Schlussfolgerungen ziehen:

Die zwei Arten des Übens, klassisches Üben und Spiel, wirken gleichwertig auf das Erlernen der technischen Fertigkeiten desFußballs. Dies gilt sowohl bei einem standardisierten Test als auch bei einem Spieltest.

Die festgestellten Auswirkungen wurden jedoch nicht bis in die Behaltensphase stabil beibehalten.

Der Spielansatz führt zu ähnlichen Effekten wie der Übungsansatz, dennoch ermöglichen Spiele auf kleinen Feld ein spielnäheres Lernen und erfordern weniger didaktische Materialien und Lehreinrichtungen. 
Die Verwendung eines Spielansatzes mit "Kleinen Fußballspielen mit unterschiedlichen Schwerpunkten" beim Lernen und beim Training der technischen Fertigkeiten der Sportspiele ist sinnvoll. Die Vorteile dieser Art von Lernstrategie überwiegen die des traditionellen Übungsansatzes.

Es sind weitere Studien erforderlich, um die Nachhaltigkeit dieser didaktischen Strategien zu erforschen. 


\subsection{Studie 4. Förderung der kognitiven Fähigkeiten durch Kombinationsspiele}

\subsubsection{Einführung}

Sportspiele sind durch große Handlungsvariabilität gekennzeichnet. Während des Spiels ist ein andauerndes und schnelles Wechseln von Spielobjekt, Mitspielern, Gegenspielern und Spielsituationen vorhanden. Das erfordert von den Spielern eine hohe Entwicklungsstufe der kognitiven Fähigkeiten. Sie sollen die Spielhandlungen richtig wahrnehmen, die erforderlichen Aufgaben erkennen und die möglichen Lösungen situationsadäquat abwägen können (MAHLO, 1974 zitiert nach HARRE, 1985, S. 222, ROTH, 1989, S. 6, MARTIN, CARL \& LEHNERTZ, 1991, S. 61, SCHNABEL, HARRE \& BORDEN, 1997, S. 298).

Die Fähigkeiten, das Spiel zu „Iesen“ (WEIN, 2009, S. 15; WILLIAMS, 2008, S. 38) ist entscheidend für die Leistung bei Sportspielen. Die geschicktesten Spieler können Spielmuster erkennen und erinnern, sie können basierend auf visuellen Hinweisen die Handlungen des Gegners antizipieren (z. B anhand ihrer Feldaufstellung und Orientierung) und sie sind präzise in ihren Erwartungen dessen, was bei besonderen Spielumständen im Anschluss geschehen wird (WILLIAMS, 2008, S. 38).

Wenn der Prozess der Ausbildung der Spieler nicht nur von außen betrachtet wird, sondern das Zentrum der methodischen Maßnahmen den Spieler selbst und seine Beeinflussung des Spielgeschehens betrifft, dann wird auf das Konzept der Kompetenzen zurückgegriffen. Kompetenzen sind hypothetische Konstrukte, die aus dem Zusammenspiel von Umweltanforderungen (Spielsituationen) und den dem Spieler zur Verfügung stehenden Ressourcen bestehen. Sie werden in der Spielhandlung selbst beobachtet und in dieser Arbeit als der Ausdruck des Handlungswissens betrachtet. Die Entwicklung der Kompetenzen ist letztendlich das Hauptziel jedes Ausbildungsprozesses.

Für ein erfolgreiches Handeln sollen die Spieler nach KONZAG (1992, S. 11) die folgenden kognitive Kompetenzen haben:

1. „Handlungsrelevante Informationen aus der Spielsituation annehmen.

2. Die beabsichtigten Handlungen der Gegner und Mitspieler bzw. der Bewegungen des Balles sowie eigener Handlungen und ihrer Folgen vorausnehmen.

3. Gedankliche Informationen, Vorstellungen und Erfahrungen, die schließlich in eine richtige Entscheidung münden, verarbeiten.

4. Die zielgerichteten Handlungsausführungen im Vergleich mit der Zielvorstellung ständig kontrollieren.

5. Die Ergebnisse der gewonnenen Erfahrungen auswerten und wiederverwenden.

6. Ständig situationsangepasste Aufmerksamkeit in wechselnden Richtungen, zwischen hoher und geringer Intensität, zwischen verteilter und konzentrierter Aufmerksamkeit variieren".

Auf Basis der Analysen der Sportpraxis und der Integration bisheriger Konzepte über Kognition und Leistungsvorrausetzungen im Sport stellen NEUMAIER und MECHLING (1995, S. 15-16) einen Strukturierungsvorschlag der Anforderungen des Sports vor. Dabei geht es in den Sportspielen nicht nur um die Bestimmung der Informationsanforderungen unter entsprechenden Druckbedingungen sowie deren Variation in typischen Handlungssituationen (NEUMAIER, 1999, S. 150). Die Systematisierung der Druckanforderungen bei Bewegungsaufgaben nach Präzisions-, Zeit-, Komplexitäts-, Organisa- 
tions-, Belastungs- und Variabilitätsdruck (vgl. ROTH, 1999, S. 21) stimmen mit den koordinativen Anforderungen des Sports überein (BÜSCH, 2001, S. 55-59).

Die perzeptiven und kognitiven Anforderungen jeder Spielhandlung schließen eine Informationsflut von großem Umfang ein, die sowohl bewusst als auch unbewusst verarbeitet werden soll. Damit trägt das Koordinationstraining in besonderem Maße zur Schulung der selektiven Wahrnehmung bei (GLASAUER \& NIEBER, 2002, S. 191-194). Kognitive Ausbildung in Sportspielen bedeutet somit auch Koordinationsschulung und training.

\begin{abstract}
„Insbesondere wäre stärker zu berücksichtigen, daß die Bewältigung von Bewegungsaufgaben wie auch das Bewegungslernen selbst für den Handelnden zunächst einmal ein kognitiv zu strukturierendes Problem darstellt. Training müsste deshalb auch verstärkt darauf abgestellt werden, nicht nur Bewegungsabfolgen einzuschleifen, sondern kognitive Strukturen zu optimieren, d. h., unangemessene Strukturen zu ändern und angemessenere aufzubauen".
\end{abstract}

(NITSCH \& MUNZERT 1997, 170)

Kurz dargestellt, bestehen die Spielkompetenzen aus den koordinativen Ressourcen, über die die Spieler verfügen.Solche koordinativen Ressourcen stützen sich auf kognitiven Fähigkeiten wie z.B. Wahrnehmen, Antizipieren und Entscheiden. Nach GLASSAUER (2003, S. 38) sind die kognitiven Prozesse entscheidend für eine motorisch flüssige und gut koordinierte Bewegung; besonders gilt das für die Handlungsantizipation, den Schutz der Intentionen gegenüber Störungen und Ablenkungen während der Realisationsphase und die Interpretation der Ergebnisse (vgl. NITSCH \& MUNZERT, 1997, S. 124-125).

Für die Koordinationsschulung im Training wird seit den achtziger Jahren das Modell der koordinativen Fähigkeiten als das brauchbarste für die Praxis angesehen. BLUME (1981), identifizierte sieben koordinative Fähigkeiten, die auf den Vorschlägen von HIRTZ (1964, zitiert nach GLASAUER \& NIEBER, 2000, S. 32) über koordinative Voraussetzungen des Sports basieren. Mehrere weitere Autoren haben zur Etablierung dieses Modells beigetragen (vgl. ROTH 1989, 1993; ZIMMERMANN \& BLUME, 1998). Es erscheint bis heute in vielen Lehrbüchern der Sportdidaktik und ist die allgemeinste Konzeption der Koordinationsschulung. ZIMMERMAN und BLUME (1998, S. 221) schlagen in Anlehnung an das Modell eine Schwerpunktlegung im Koordinationstraining vor. Sie beinhaltet die Variation der Bewegungsausführung, die Veränderung der äußeren Bedingungen, das Kombinieren von Bewegungsfertigkeiten, das Üben unter erhöhten Genauigkeitsanforderungen und mit maximalem Tempo, die Variation der Informationsaufnahme und das Üben unter Vorbelastung. Dieser Vorschlag wird heute in den meisten Sportvereinen als Basis für die Koordinationsschulung und das Koordinationstraining angewendet. Allerdings werden seit den neunziger Jahren Zweifel am Nutzeffekt des Konzepts der koordinativen Fähigkeiten als Grundlage einer sportartspezifischen Koordinationsschulung geäußert (HIRTZ, 1994; MECHLING, 1999; NEUMAIER \& MECHLING, 1994). Dies gilt insbesondere für seinen Nutzen bei der Schulung und im Training der Sportspiele. „Der hohe Komplexitätsgrad koordinativer Fähigkeiten, die Differenzierungsproblematik und der Generalitätsanspruch scheinen die Grenzen dieses Modells offen zu legen“" (GLASAUER, 2000, S. 33).

DieSchulung der koordinativen Fähigkeiten stellt die Bedingung für eine sportartübergreifende allgemeine Grundausbildung dar. Bei späteren Lern- und- Trainingsprozessen wird aber eine spezifischere Koordinationsschulung benötigt, die weit über die allge- 
meinen koordinativen Fähigkeiten hinausgeht und insbesondere den jeweiligen kognitiven und koordinativen Anforderungen des Sports entspricht (vgl. HIRTZ, 1998, S. 1415). Daher wird nach NIEBER und GLASAUER (2000, S. 33) in den Sportspielen mit ihrem hohen Grad an koordinativen und kognitiven Anforderungen ein Paradigmenwechsel nötig. Ein allgemeines Konzept für ein spezifisches Koordinationstraining in den Sportspielen ist zwar noch nicht vorhanden, jedoch wird das variierte Üben (HIRTZ, 1989, S. 30) als die Hauptmethode für das Training der koordinativen Fähigkeiten vorgeschlagen. ROTH (1993, S. 90) schlägt dabei vor, das wichtigste Prinzip der Koordinationsschulung sei: „Einfache Bewegungen und erschwerte Bedingungen“.

HOSSNER (1995) betrachtet die Bewegung als einen aktiven Konstruktionsprozess, der abhängig von spezifischen Situationsbedingungen ist. Er bezeichnet alle an Bewegungen beteiligten Prozesse als Module. Diese Module werden als vertikale Fähigkeiten betrachtet. Dazu gehören Wahrnehmung, Planung, Entscheidung und Kontrolle. Im Gegensatz dazu werden die koordinativen Fähigkeiten von HIRTZ und BLUME als horizontale Fähigkeiten eingeordnet. HOSSNER schlägt damit einen „modularen Ansatz“ vor und entwirft nach diesem Modell einen Baukasten mit Technik- und Taktikbausteinen für das Volleyballtraining. NEUMAIER (1999) sieht den modularen Ansatz als ein systematisches und strukturiertes Training der koordinativen Leistungsvoraussetzungen des Sportspiels an. Ähnlich wie die motorischen Schemata von SCHMIDT (1991, SCHMIDT \& WRISBERG, 2008) und die Handlungsprogramme von MUNZERT (1987) sind die Module damit Systeme, die spezifische Operationen durchführen. Sie übersetzen die Stimuli und verarbeiten äußere Informationen in verschiedenen Bereichen des kognitiven Apparats. Die entstehenden Antworten äußern sich als Bewegungen.

GLASAUER und NIEBER (2000) identifizieren charakteristische Spielsituationen (Taktikbausteine) im Basketball, die konkrete Aufgaben für dieses Spiel beschreiben und innen typische Handlungen für die Aufgabenlösung zuordnen. NAGEL und SPRECKLES (2010) wenden eine ähnliche Konzeption bei der Schulung des Tennisspiels an. KRÖGER und ROTH (1999) gehen von einem „fertigkeitsorientierten Zugang“ aus und schlagen ein Modell für das Üben der Ballkoordination vor. Dieser fertigkeitsorientierte Ansatz fasst das Lernen von übergreifenden Ballfähigkeiten und Ballfertigkeiten mit offensiven und defensiven Taktikbausteinen zusammen. Es sollen die verallgemeinerten Spielfähigkeiten und Spielkompetenzen durch eigenständige Spielformen geschult werden.Die sogenannte Ballschule versucht damit ein spielerisches, inzidentelles Lernen zu erzeugen. Die taktischen Fähigkeiten (ROTH, 1989, 1998) werden durch spielerisches Handeln geschult. Dadurch entstehen situativ ausgelöste Muster und mentale Schemata (PIAGET, 1975). Bei schnellen Spielen werden diese abgerufen, um mit den Entscheidungsanforderungen im Augenblick des Geschehens zusammen zu passen (ROSENDAHL, 1999, S. 4).

In der hier vorgestellten Studie wurden Spielaufgaben für das Training der Koordination und der Spielkompetenzen entworfen und empirisch überprüft. In Anlehnung an den modularen Ansatz von HOSSNER (1995) und das inzidentelle Lernen von KRÖGER und ROTH (1999) wurden charakteristische Spielsituationen für das Sportspiel Fußball identifiziert, die typische Aufgaben beinhalten und deren Bewältigung durch koordinative Handlungen möglich ist. Davon ausgehend wurden die sogenannten „Kombinationsspiele" entworfen. Diese sollen unter variierenden Informationsanforderungen und Zeitdruckbedingungen die kognitiven Fähigkeiten und damit die spezifische Koordination 
trainieren. Durch die Kombinationsspiele wird besondere Aufmerksamkeit auf die individuellen Handlungen und ihren Einfluss auf die kollektiven Handlungen gerichtet.

Bei den Kombinationsspielen sollen die Teilnehmer bestimmte Grundaufstellungen einnehmen. Das Spiel beginnt mit einer einfachen Durchführung einer Passaufgabe. Die Spieler sollen nach jedem Pass die Feldaufstellung wechseln, sich neu positionieren und auf den nächsten Pass achten. Die Pässe erfolgen durch Orientierungswechsel, Antizipation der Stellung der passannehmenden Mitspieler und mit der richtigen Dosierung der Kraft und Schnelligkeit des Passspiels. Mit der Einfügung von zwei und mehr Bällen wird der Komplexitätsgrad weiter angehoben, da alle Bälle und alle Spieler gleichzeitig und ständig in Bewegung sein sollen. Dies stellt hohe Anforderungen an die Aufmerksamkeit, Konzentration und Antizipation der Spieler.

Der Einsatz von Kombinationsspielen ist eine didaktische Strategie, die sowohl die Entwicklung kognitiver Fähigkeiten wie Wahrnehmung, Bewegungsantizipation, Aufmerksamkeit und Konzentration als auch die Koordination von individuellen und kollektiven Handlungen fördert.

Bei der Schulung der technischen Fertigkeiten und taktischen Fähigkeiten der Sportspiele spielt normalerweise der Trainer die führende Rolle. Als Experte und Wissensträger stellt er die Lösungen für alle Spielsituationen bereit und legt das korrekte Ausführungstempo fest. Er fordert auch eine von inm festgelegte, bestimmte Anzahl von Wiederholungen der Aufgabe ein. Bei den Kombinationsspielen werden hingegen psychosoziale Prozesse berücksichtigt. Der einzelne Spieler ist nicht nur Objekt der Trainingssteuerung, sondern Subjekt des Trainingsprozesses (vgl. GLASAUER \& NIEBER, 2003, S. 54). Der Trainingsakzent wird damit nicht so sehr auf den ausgesprochenen Forderungen der Trainer liegen, sondern vielmehr auf der flüssigen Durchführung der Aufgabe als Verantwortungsbereich der teilnehmenden Spieler. Das Gelingen hängt von einer kollektiven Verarbeitung ab. Die spielende Gruppe muss die erwünschte Spielstruktur selbständig umsetzen, die Probleme bei der Ausführung identifizieren und eine gemeinsame Lösung finden. Der Trainer stellt lediglich die Aufgabe und die Erfolgskriterien aber keine fertige Lösung vor, der Akzent wird vielmehr auf die Optimierung der selbstständigen Bewältigung von Anforderungs- und Aufforderungssituationen durch den Spieler gelegt (NITSCH \& MUNZERT, 1997, S. 50), kooperatives Lernen wird damit angestrebt (vgl. METZLER, 2005a).

Die Wirksamkeit der Kombinationsspiele auf das Fußballspiel zu untersuchen war der Inhalt dieser Studie. Durch die Anwendung als Teil der Trainingsinhalte einer Jungendmannschaft wurden Antworten auf die folgenden Fragen gesucht:

- Wirken die Kombinationsspiele auf die Verbesserung der Spielkompetenzen der jungen Fußballspieler?

- Trägt die Schulung der kognitiven Fähigkeiten durch die didaktische Strategie der Kombinationsspiele dazu bei, mehr und wirksamere offensive Spielzüge der ballbesitzenden Mannschaft und mehr Ballgewinne in defensiven Situationen zu erzeugen?

- Ist das kooperative Lernen eine geeignete Lernmethode für die Spielkompetenzen im Fußball?

Daraus wurden die folgenden Hypothesen abgeleitet: 
- Hypothese 1: Wenn eine didaktische Strategie, die auf Kombinationsspielen basiert, als Teil des Trainings eingeführt wird, dann werden die jungen Fußballspieler mit dem Ball erfolgreichere Spielhandlungen ausführen.

- Hypothese 2: Wenn die didaktische Strategie der Kombinationsspiele nicht mehr angewendet wird, wird die erhöhte Häufigkeit der richtigen Handlungen mit dem Ball während mindestens drei Wochen aufrechterhalten.

\subsubsection{Methode}

\section{Stichprobe}

Die untersuchte Stichprobe bestand aus 12 jungen Fußballspielern der Altersklasse DJunioren, 12 Jahre alt (S.D. 0,1), die bei einem Sportverein regelmäßig Fußball spielten und zwei Trainingseinheiten und ein Wettkampfspiel pro Woche absolvierten. Die Spieler nahmen an acht Trainingseinheiten teil, in denen jeweils über 30 Minuten verschiedene Kombinationsspiele gelernt und geübt wurden. Die Kombinationsspiele erfordern die Anwendung kognitiver Fähigkeiten und wurden immer unmittelbar nach dem Aufwärmen und vor dem Hauptteil der Trainingssitzung durchgeführt.

\section{Kriteriumsaufgaben und -instrumente}

Der TSAP (Team Sport Assessment Procedure) (GREHAIGNE, GODBOUT, \& BOUTHIER, 1997) wurde angewendet um die Leistungssteigerung der Spieler auszuwerten. Der TSAP ist ein Beobachtungsinstrument, mit dessen Hilfe die Balleroberung und der Einsatz des Balles während des Spiels registriert und ein Effizienzindex (Efficency Index) sowie ein Index des Spielvolumens (volume of play index or played balls) berechnet wird (siehe Anhang Q). Die Validitätsindizes des TSAP sind hoch bis sehr hoch (übereinstimmende Validität (.74), Interbeobachter-Reliabilität (>.90), Stabilität der Ausführung (.88), inhaltliche und ökologische Validität). Sie wurden von den Autoren selbst und anderen berechnet und diskutiert (GREHAIGNE, GODBOUT \& BOUTHIER 1997; RICHARD, GODBOUT \& GREHAIGNE, 2000; PILL, 2008).

Die Spieler nahmen an einem Mini-Fußball-Spiel (4 gegen 4 mit Torwart) (WEIN \& SCHREINER, 2004, S. 9; WATSON, 2010) teil. Das Spiel fand in einem 32 Meter langen und 25 Meter breiten Fußballfeld statt (Anhang A). Alle Regeln des Fußballspiels mit Ausnahme der Abseits- und Strafstoßregeln wurden eingehalten. Vor dem Test wurden den Spielern eine kurze Erklärung der Regeln und eine Erläuterung der Ziele des Spiels gegeben. Die Mannschaften wurden zufällig ausgewählt. Trugen zwei oder mehr Spieler Sportkleidung mit ähnlichen Farben, wurden sie durch Leibchen differenziert, um ihre Identifizierung zu erleichtern. Eine kurze Vor-Spielzeit von 30 Sekunden wurde zur Eingewöhnung in das Spiel durchgeführt.

Das Spiel wurde mit einer Videokamera JVC Everio S, GZ-MS90 auf Video aufgezeichnet. Die Videoaufnahmen wurden zu einem Laptop transferiert und mit Hilfe des Programms „Pinnacle VideoStudio 2.0“ in „avi“-Dateien umgewandelt. Ein trainierter Be- 
obachter beobachtete unabhängig voneinander das Spiel. Mit Hilfe von Formblättern (Anhang S) zeichnete er jede Handlung der Spieler mit Ball auf.

\section{Variablen}

Der TSAP erlaubt die Beobachtung und Auswertung der folgenden Variablen:

- Conquered Balls (CB): Ein Spieler erobert den Ball, der im Besitz der gegnerischen Mannschaft ist.

- Received Balls (RB): Der Spieler erhält den Ball von einem Mitspieler und verliert nicht die Kontrolle darüber.

- Neutral Ball: Der Spieler übergibt den Ball einem Mitspieler seitwärts oder rückwärts, eine klare Absicht zum Angreifen ist nicht erkennbar.

- Lost Balls(LB): Der Gegner nimmt dem Spieler den Ball ab.

- Offensive Balls (OB): Ein Pass zum einem Mitspieler, der vorn ist und in der Lage, den Angriff fortzusetzen.

- Succesful Shots: (SS): Ein Schuss wird als Erfolg betrachtet, wenn die angreifende Mannschaft ein Tor schießt oder in Ballbesitz bleibt.

- Attack Balls (AB): Die Summe der OB und der SS.

- Played Balls (PB): Anzahl der Situationen, wo der Spieler den Ball annimmt oder erobert. Das PB wird durch die Aufsummierung von CB und RB berechnet.

- Efficiency Index, er wird wie folgt berechnet:

$$
\text { Efficiency Index }=\frac{(C B+A B)}{(10+L B)}
$$

\section{Intervention}

Die Interventionsverfahren bestanden aus einer Reihe von acht Trainingseinheiten. Innerhalb jeder Sitzung wurden Kombinationsspiele durchgeführt. Die Kombinationsspiele sind Spiele mit Kooperation, aber ohne Opposition. Sie fördern die kognitiven Fähigkeiten und durch ihre Komplexität auch die taktischen Fähigkeiten. Das Ziel der Spiele ist es, Kombinationsmuster von kollektiven Sportspielen zu erreichen, in denen die Spieler schnelle Pässe ausführen müssen sowie Pass- und Laufrichtungen wechseln und die Handlungen der Mitspieler antizipieren müssen. Auch die Gleichzeitigkeit der Handlungen von mehreren Spielern und die kognitiven Anforderungen erzeugen Ausführungsprobleme, die die Spieler selbstständig lösen müssen. Das Spiel beginnt jeweils mit einfachen Situationen, die zunehmend komplexer werden, wenn die Spieler das Kombinationsziel erreichen und der Trainer zusätzliche Elemente einführt, die den Schwierigkeitsgrad steigern. Der Trainer stellt die Spiele und die zu erreichenden Ausführungsmuster vor, äußert aber keine Lösung für die aufgetretenen Probleme, sondern fordert die Lösungen von den Spielern. Wenn das gewünschte Kombinationsmuster geschafft wird, steigert der Trainer die Komplexität durch das Hinzufügen von mehr Bällen und durch Änderungen der Richtungen, in denen die Bälle zu passen sind und die Spieler 
laufen sollen. Die ganze Gruppe der Spieler nimmt gleichzeitig am Spiel teil und sucht gemeinsame Lösungen für die Ausführungsprobleme. Der Trainer gewährt Zeit zum Nachdenken, die Diskussion und die Erprobung der vorgeschlagenen Lösungen. Nachdem das Spiel korrekt durchgeführt wurde und die Möglichkeiten für die Schwierigkeitssteigerung erschöpft sind, wird ein neues Spiel durch den Trainer vorgestellt, was zu einem Prozess der progressiven Steigerung der Komplexität führt (siehe ein Beispiel einer Trainingssitzung in Anhang T).

Die Kombinationsspiele wurden unmittelbar nach dem Aufwärmen umgesetzt. Die Dauer eines Spiels kann man nicht voraussehen, sie hängt von der Bearbeitungszeit ab, die die Spieler brauchen, um das Spiel richtig ausführen zu können. Allerdings wurden im Rahmen dieser Studie 30 Minuten einschließlich Aufgabestellung und Erholungspausen von jeder Trainingseinheit den Kombinationsspielen gewidmet. Wenn das Zielspiel nicht erreicht wurde, wurde dasselbe Spiel in der nächsten Trainingseinheit wiederholt. Die teilnehmenden Spieler brauchten zwischen einer und drei 30-minütigen Sitzungen, um die höchste Komplexitätsebene eines Spiels zu erreichen. Ein Beispiel eines Kombinationsspieles, das während dreiTrainingseinheiten gelehrt wurde, wird in der Abbildung Nummer 33 dargestellt. 


\section{Die Uhr}

\section{Grundaufstellung}

So viele Markierungshütchen wie anwesende Spieler werden mit $2 \mathrm{~m}$. Abstand zwischen ihnen im Kreis gestellt, eins von diesen Hütchen wird in der Mitte des Kreises gestellt. Jeder Spieler stellt sich vor jeweils einem Hütchen mit Sicht zum Hütchen in der Mitte. Ein beliebiger Spieler besitzt einen Ball, der Spieler neben dem Hütchen in der Mitte orientiert sich zum Ballbesitzer.

\section{Verlauf}

Der Ballbesitzer (A) passt zum Spieler in der Mitte (B), läuft dem Pass hinterher und stellt sich vor dem Hütchen in die Mitte. Der Spieler in der Mitte (B) nimmt den Ball an, dreht sich kurz nach rechts, passt zum nächsten Spieler im Kreis (C), läuft den Pass hinterher und stellt sich vor dem Hütchen, wo der annehmende Spieler stand. Der Spieler (C) passt zu (A) und läuft den Ball hinterher. Dieser Pass- und- Stellungswechsel wird fortgesetzt mit allen anderen Spielern in dem Kreis.

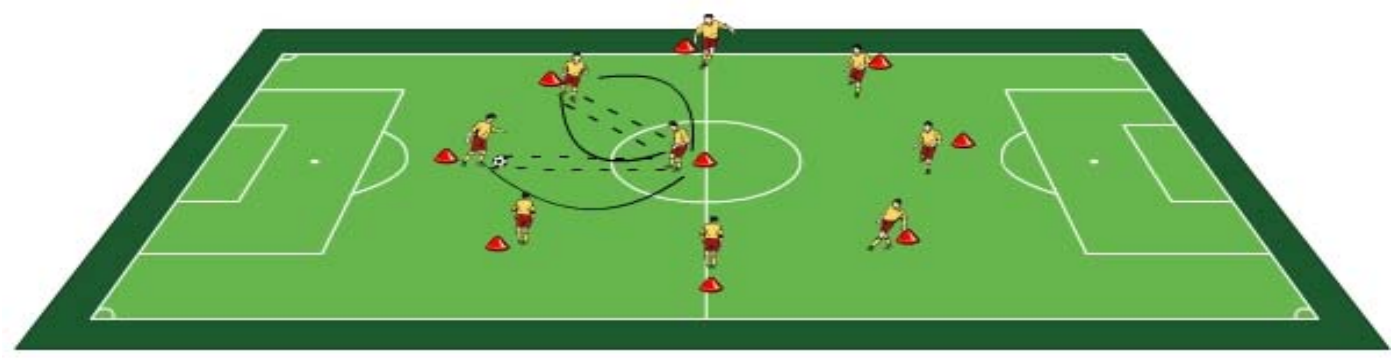

\section{Komplexitätssteigerung}

Wenn der Ball flüssig umläuft und die Spieler fehlerfrei ihre Feldstellungen wechseln, werden die Markierungshütchen ausgezogen und ein weiterer Ball eingefügt. Das Spiel beginnt mit Ballbesitz von zwei gegenüberstellenden Spielern im Kreis und zwei Spielern in der Mitte, die sich jeweils auf einen Ball orientieren. Bei Fehler werden die zwei Bälle gehalten und wieder gleichzeitig wie am Anfang gespielt.

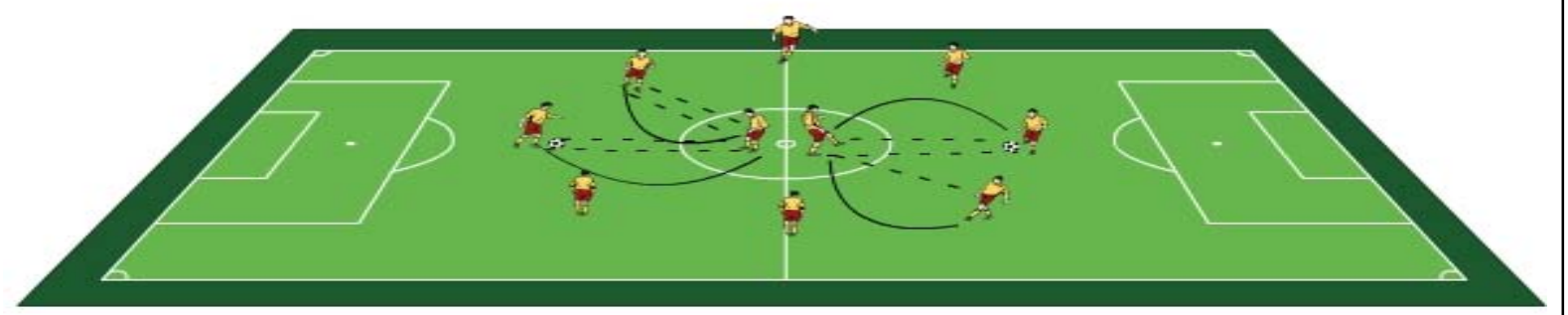

\section{Hinweise}

Wenn die Spieler die Aufgabe beherrschen, werden weitere Bälle eingefügt. Die Steigerung der Komplexität wird schrittweise durch Einfügen eines Balls gesteigert bis zu einem Verhältnis von einem Ball je drei teilnehmende Spieler. Bei Fehler mit dem Umlauf eines Balles werden die andere Bälle nicht gehalten. Der Umlauf soll aber wieder anfangen ohne die Umläufe der anderen Bälle zu stören.

\section{Abbildung 33: Beispiel eines Kombinationsspiels}




\section{Statistische Analysen}

Die statistische Analyse wurde mit der Software Statgraphics XI und SPSS 17.0 durchgeführt. Die teilnehmenden Spieler führten den Test dreimal an drei Messzeitpunkten durch: Vor-, Post- und Retentionstest. Für jede Variable wurden Maße der zentralen Tendenz und Streuung berechnet. Die normale Verteilung der Stichprobe wurde mit einem KolmogorovSmirnov-Test ausgewertet (siehe die Werte des Kolmogorov-SmirnovTests im Anhang V). Da nicht alle die Variablen eine Normalverteilung belegten, wurden parameterfreie Tests angewendet. Um Unterschiede zwischen den drei Messzeitpunkten festzustellen wurde ein Friedman-Test durchgeführt. Um die Richtung der gefundenen Unterschiede herauszufinden wurde ein Wilcoxon-Test mit Bonferroni-Korrektur angewendet.

\section{Auswertungsobjektivität}

Das Zählen der Variablen in der Videoaufnahme des Minifußballspiels durch den TSAP wurde vom Forscher selbst ausgeführt. Dieser Sportwissenschaftler und Fußballtrainer verfügt über hinreichende Ausbildung und Erfahrung bei der Anwendung des Berechnungsinstruments. Durch ein Expertenrating wurde zusätzlich die Objektivität der Berechnung sichergestellt. Zwei weitere Sportwissenschaftler zählten je zweimal 20 \% der Handlungen. Die zwei Zählungen jedes Experten wurden verglichen, anschließend wurde eine der zwei Zählungen per Zufall ausgewählt und damit der Hauptbeobachter verglichen. Ein Pearson-product-moment-correlation-Verfahren wurde damit berechnet. Eine Korrelation von $r=.91$ für den Hauptbeobachter $(p=.02), r=.90(p=<.001)$ für den zweiten Experten und $r=.97(p=.01)$ für den dritten Experten wurde abgeschätzt. Die Interexperten-Korrelationenwaren $r=.89(p=<.001)$ zwischen dem Hauptbeobachter und dem zweiten Experten; $r=.92(p=.02)$ zwischen dem Hauptbeobachter und dem dritten Experten und $r=.94(p=.03)$ zwischen dem zweiten und dem dritten Experten.

\subsubsection{Ergebnisse}

Es wurden folgende Variablen durch die Team Sport Assessment Procedure (TSAP) erhoben: gewonnene Bälle (Conquered Balls, CB), angenommene Bälle (Received Balls, RB), neutrale Pässe (Neutral Balls, NB), verlorene Bälle (Lost Balls, LB), angreifende Pässe (Offensive Ball, OB), erfolgreiche Schüsse (Sucessfull Shots, SS), Angriffsbälle (Attack Balls, AB), gespielte Bälle (Played Balls, PB). Auch ein Ausführungsindex (Execution Index, El) wurde berechnet.

In der Tabelle 18 sind die Ergebnisse der Anwendung der Messprozedur zu den drei Zeitpunkten Vor-, Post- und Retentionstest dargestellt (siehe Gesamtdaten im Anhang $U)$. Verbesserungen sind sowohl in den offensiven (OB) als auch in defensiven Variablen (CB) vorhanden. Die Anzahl der Fehler (LB) wurde leicht verringert, ebenso die der neutralen Pässe (NB). Die Indizes, die für die beobachteten Variablen berechnet wurden (Angriffsbälle (AB), gespielte Bälle (PB) und Ausführungsindex (EI)), verbesserten sich beim Posttest, die Werte verringern sich aber leicht für den Retentionstest nach eine Zeitspanne von drei Wochen ohne Training der Kombinationsspiele. Die Werte 
beim Retentionstest sind jedoch trotzdem höher als die am Anfang der Studie im Vortest beobachteten.

Tabelle 18: Mittelwert (M) und Standardabweichungen (S.D.) der Variablen des TSAP : CB: Conquered Balls, RB: Received Balls, NB: Neutral Balls, LB: Lost Balls, OB: Offensive Balls, SS: Sucessfull Shots, AB: Attack Balls, PB: Played Balls, El: Efficency Index beim Vor-, Post-, undRetentionstest $(n=12)$

\begin{tabular}{|c|c|c|c|c|c|c|c|c|c|c|c|c|c|c|c|c|c|c|c|}
\hline & \multicolumn{2}{|c}{ CB } & \multicolumn{2}{|c|}{ RB } & \multicolumn{2}{c|}{ NB } & \multicolumn{2}{c|}{ LB } & \multicolumn{2}{c|}{ OB } & \multicolumn{2}{c|}{ SS } & \multicolumn{2}{c|}{ AB } & \multicolumn{2}{c|}{ PB } & \multicolumn{2}{c|}{ EI } \\
\hline & M & SD & M & SD & M & SD & M & SD & M & SD & M & SD & M & SD & M & SD & M & SD \\
\hline Vor & 4,50 & 2,97 & 7,17 & 3,01 & 2,42 & 1,38 & 3,75 & 2,22 & 3,33 & 2,10 & 1,33 & 1,23 & 4,67 & 2,35 & 11,67 & 4,54 & 0,64 & 0,23 \\
\hline Post & 5,25 & 2,67 & 7,50 & 2,84 & 2,00 & 1,41 & 3,17 & 1,75 & 5,50 & 2,35 & 1,92 & 1,56 & 7,42 & 3,00 & 12,75 & 4,67 & 0,96 & 0,34 \\
\hline Ret & 5,08 & 2,39 & 7,92 & 3,42 & 1,67 & 1,07 & 3,75 & 1,71 & 5,08 & 3,34 & 1,58 & 1,08 & 6,67 & 3,5 & 13,00 & 4,73 & 0,85 & 0,36 \\
\hline
\end{tabular}

In den Abbildungen 34, 35und 36ist die Entwicklung der Variablen der Spielkompetenzen nach der Anwendung der didaktischen Strategie aus Kombinationsspielen graphisch veranschaulicht. Auffällig ist die Steigerung der Indizes für Played Balls (PB) und den Efficiency Index (EI).

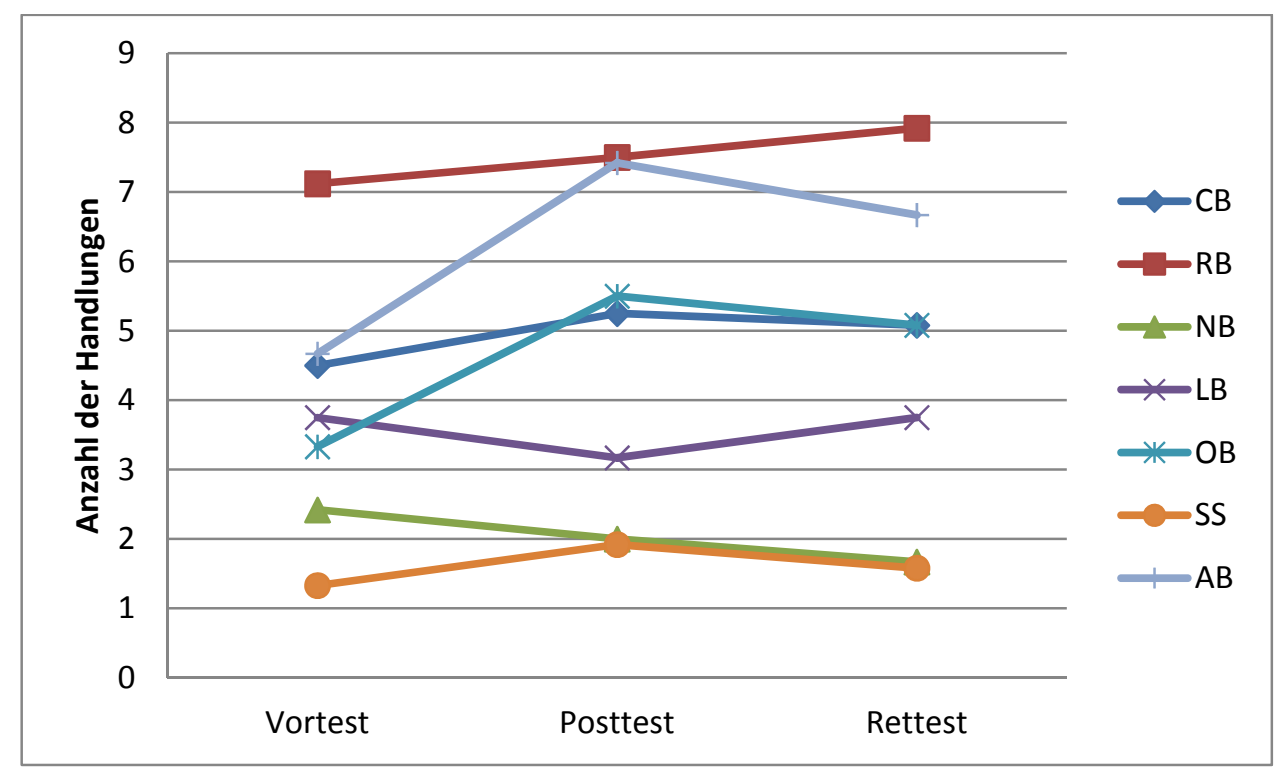

Abbildung 34: Mittelwerte der Variablen des TSAP : CB: Conquered Balls, RB: Received Balls, NB: Neutral Balls, LB: Lost Balls, OB: Offensive Balls, SS: Sucessfull Shots, AB: Attack Balls, PB: Played Balls, El: Efficency Index beim Vor-, Post-, und- Retentionstest $(n=12)$ 


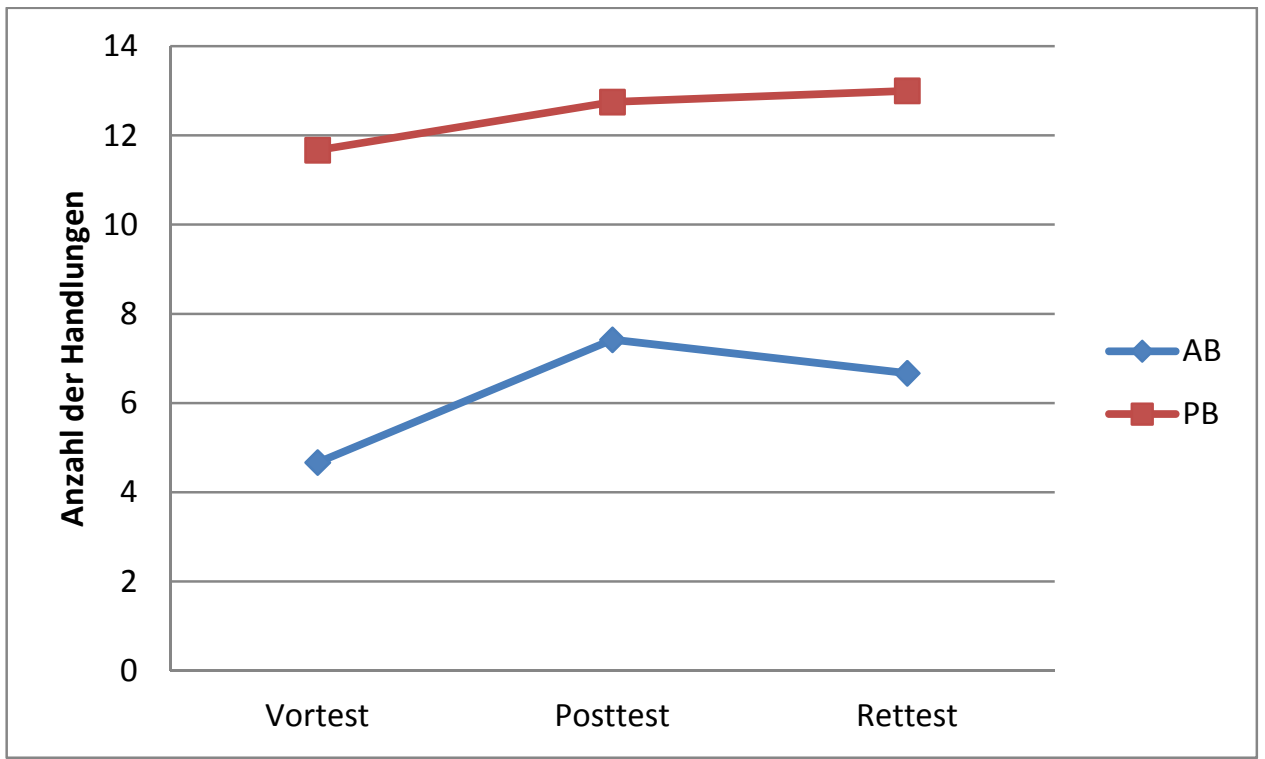

Abbildung 35: Ergebnisse der errechneten Variablen des TSAP. AB: Attack Balls, PB: Played Balls (volume of play) beim Vor, Post- und Retentionstest

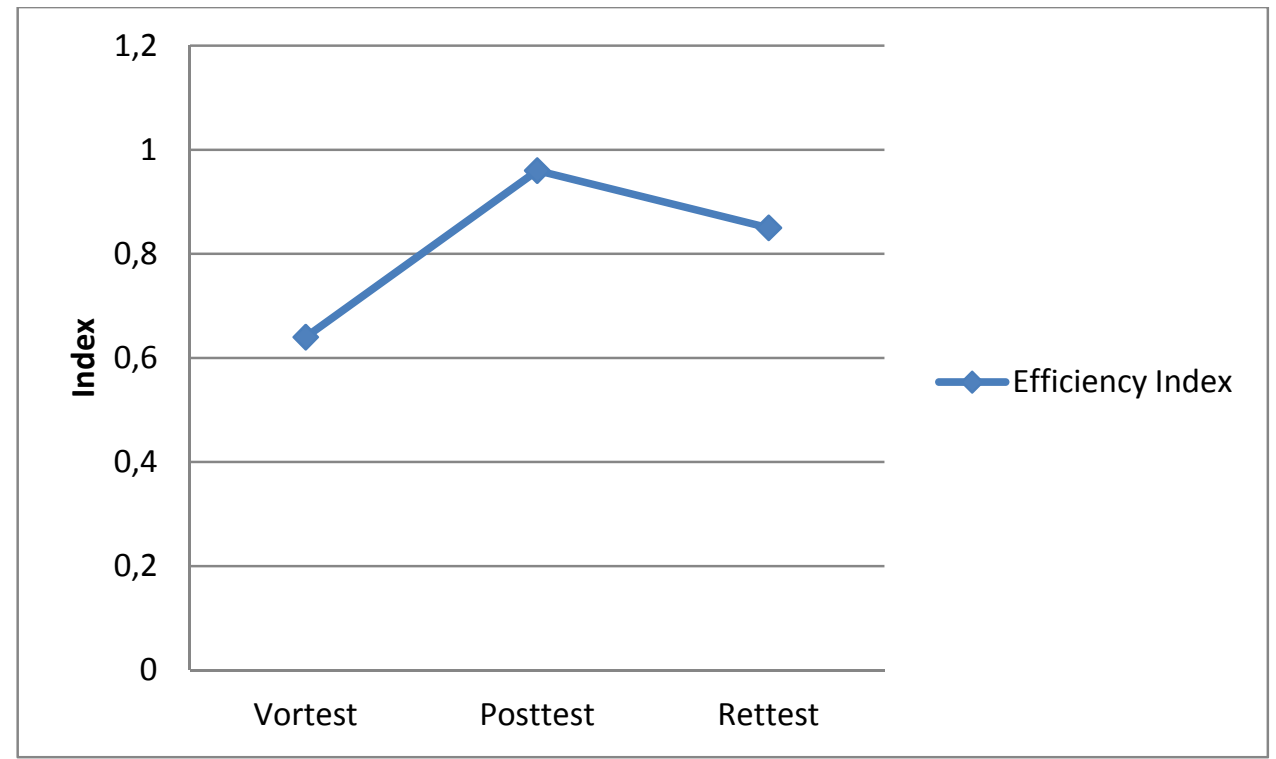

Abbildung 36: Ergebnisse der errechneten Variablen des TSAP. El: Efficiency Index beim Vor, Postund Retentionstest

Die Annahme der Normalverteilung wurde durch den Kolmogorov-Smirnov Test überprüft. Die Ergebnisse widerlegen die Normalverteilungsannahme der Mittelwerte einiger der Variable bei bestimmten Messzeitpunkte (Mittelwert des Posttests NB $p<.05$; Retentionstest LB $p<.05$; Retentionstest SS $p<.05$ und Retentionstest El $p<.05)$. Deswegen wurden parameterfreie Tests für den Unterschied der Mittelwerte aller Variablen bei den drei Messzeitpunkten angewendet. 
Ein Friedman-Test weist nur signifikante Unterschiede für den berechneten Effizienz Index (EI) hin (Tabelle 19). Die Analysen durch ein Wilcoxon-Test mit BonferroniKorrektur geben signifikante Unterschied zwischen Vortest und Postest $(T=-2.31$; $\mathrm{k}<$ $.05)$ an, aber weder zwischen Vortest und Retentionstest $(T=-1.81 ; k>.05)$, noch zwischen Posttest und Retentionstest ( $T=-1.02 ; \mathrm{k}>.05)$.

Tabelle 19: Ergebnisse des Friedman-Test $($ d.f. $=2)$ der Variablen des TSAP: CB: Conquered Balls, RB: Received Balls, NB: Neutral Balls, LB: Lost Balls, OB: Offensive Balls, SS: Sucessfull Shots, AB: Attack Balls, PB: Played Balls, El: Efficency Index beim Vor-, Post-, und- Retentionstest (n=12)

\begin{tabular}{|l|c|c|} 
Variablen & $\boldsymbol{X}^{\mathbf{2}}$ & Signifikanz \\
\hline CB & .93 & $p>.05$ \\
\hline RB & .93 & $p>.05$ \\
\hline NB & 1.64 & $p>.05$ \\
\hline LB & 1.10 & $p>.05$ \\
\hline OB & 5.16 & $p>.05$ \\
\hline SS & 2.53 & $p>.05$ \\
\hline AB & 5.15 & $p>.05$ \\
\hline PB & .43 & $p>.05$ \\
\hline EI & 6.00 & $p<.05^{*}$ \\
\hline
\end{tabular}

Aus einer deskriptiven Perspektive kann man prozentuale Unterschiede der beobachteten Variablen und ihre Änderungen zwischen Vortest und Posttest feststellen. Aus defensiver Sicht wurde die Quote der gewonnenen Bälle (CB) um drei Prozent verbessert und die neutralen Bälle (NB) und die Quote der verlorenen Bälle (LB) wurden mit dem Trainingsprogramm um jeweils fünf Prozent reduziert. Die angreifenden Pässe (OB) und der Index der Angriffe (AB) haben sich um 14 Prozent und 17 Prozent erhöht.

Die Indizes zeigen nur prozentuale jedoch nicht statistisch signifikante Unterschiede zwischen Vortest und Posttest für die Variablen dieser Studie. Aus dieser Sicht kann man sagen, dass sich die Spielkompetenzen nach der Anwendung eines Trainingsprogramms mit der didaktischen Strategie der Kombinationsspiele nicht signifikant verbessern, eine Tendenz zur Verbesserung kann eindeutig identifiziert werden. Wahrscheinlich ist auch, dass die didaktische Strategie in einer kurzen Interventionszeit eine leichte Verbesserung erreichen kann, aber wenige oder keine statistische signifikante Unterschiede in den Variablen aufzeigen kann.

\subsubsection{Diskussion und Schlussfolgerung}

Die Spielkompetenzen der Fußballspieler sind hauptsächlich kognitiver Art, da die wirksame Teilnahme am Spiel besondere Kompetenzen erfordert, eine richtige Aufstellung im Feld einzunehmen, schnelle und effektive Entscheidungen zu treffen, wenn man 
amBall ist, und sich richtig zu verhalten, wenn man den Ball nicht hat (FRENCH \& THOMAS, 1987, S. 17).

Die Entwicklung der Spielkompetenzen steht in Beziehung zu den kognitiven Fähigkeiten, die durch Koordinationsschulung zu fördern sind. Allerdings werden in der Mehrheit der Ausbildungsprozesse der Spieler Aufgaben und Übungen gestellt, die aus dem Ansatz der koordinativen Fähigkeiten abgeleitet wurden. Nach HIRTZ (1998, S. 14-15) wird dadurch die allgemeine Koordination gefördert. Dies reicht jedoch nicht für eine spezifische Koordinationsschulung bei Spielsportarten.Die Entwicklung der koordinativen Fähigkeiten scheint demnach nicht genug zu sein, um wirksam auf die hohen kognitiven Forderungen dieser Sportarten einwirken zu können. Spezifische didaktische Strategien für die Entwicklung der Koordination durch Aufgaben mit hohem kognitiven Inhalt wurden für Volleyball (HOSSNER, 1993), Basketball (GLASAUER, 2003) und in der Heidelberger Ballschule entwickelt und vorgestellt (KRÖGER \& ROTH, 1999).

Da ein Messinstrument für die Auswertung der kognitiven Fähigkeiten noch nicht entwickelt worden ist, wurde es notwendig, die kognitiven Fähigkeiten als die Leistungen der Spieler in Spielsituationen zu operationalisieren. Bei dieser Studie wurde eine didaktische Strategie angewendet und empirisch überprüft, die die Entwicklung der kognitiven Fähigkeiten und spezifischen Koordination als offensiv und defensiv wirksame Handlungen gestaltet und die Umsetzung im Fußballspiel anstrebt. Solche Strategien fördern außerdem das kooperative Lernen (METZLER, 2005a). Damit wird versucht, die Anwendung von kognitiven Prozessen während des Spielens seitens der Spieler zu stimulieren und auch zu provozieren, so werden die Leistungsanforderungen durch das Spiel selbst bestimmt, wie dies auch im Wettkampf der Fall ist. In einem traditionellen Trainingsansatz bestimmt hingegen der Trainer „künstlich“ die Anforderungen. In den Kombinationsspielen sollen die Probleme, die bei der kollektiven Ausführung der Aufgabe auftreten, von den Teilnehmern selbst unabhängig von der Hilfe der Informationen und der Vorgabe der Lösung eines Trainers bewältigt werden.

Das kooperative Lernen könnte eine wirksame Lernmethode der Spielkompetenzen sein. Bei dieser Lernmethode übernehmen die Spieler selbst Verantwortung für ihren Ausbildungsprozess und bemühen sich um Leistung bei einer Aufgabe, die selektive Aufmerksamkeit, Wahrnehmung und Antizipation der Laufwege anderer Spieler und des Balls, kollektive Ausführung und kollektive Verständigung für die Lösung der auftretenden Probleme erfordert. Die Anforderungen der Aufgabe und der Sozialdruck der Gruppe fördern die Anstrengung bei der Ausführung und auf diesem Weg das Lernen.

Die Ergebnisse dieser Studie zeigen eine leichte Tendenz zur Steigerung der Werte für die Variablen des offensiven und defensiven Spiels. Die Anzahl der zurückeroberten Bälle wurde leicht erhöht. Das offensive Spiel wurde deutlich verbessert, die Anzahl der Pässe „nach vorne“ steigerte sich bemerkenswert. Der Angriff verbesserte sich nach dem Trainingsprogramm. Die Verbesserungen sanken aber nach einer Zeitspanne von drei Wochen ohne die Anwendung der Kombinationsspiele im Training wieder ab. Mit der didaktischen Strategie der Kombinationsspiele wurden Verbesserungen im Spielvolumen (PB) und im Wirksamkeitsindex (EI) erreicht. Die Unterschiede zwischen Vortest, Posttest und Retentionstest werden allerdings nicht statistisch signifikant. Deswegen ist es noch nicht möglich, die Wirksamkeit der Kombinationsspiele auf die Spielkompetenzen zu bestätigen. 
Nach METZLER (2005b, S. 190) hat der Vergleich zweier didaktischer Strategien keinen Sinn, da sie schon im Ansatz verschiedene Lernziele anstreben. Aus diesem Grund wurde bei dieser Studie keine Kontrollgruppe genutzt. In traditionellen Spielvermittlungsmodellen wird der Fokus auf die Entwicklung der koordinative Fähigkeiten und technischen Fertigkeiten gelegt. Daher werden die Lernergebnisse auf diesen Bereich beschränkt. Wird hingegen der Schulung der Koordination als eine Kompetenz mit hohen kognitiven Inhalten, die hauptsächlich bei kollektiven Handlungen gebraucht wird, mehr Aufmerksamkeit geschenkt, werden die Lernergebnisse ganz andere sein, als dies durch das Konzept der koordinativen Fähigkeiten zu erwarten ist. Der Vergleich der Ausbildung eines Ballkünstlers, der hohes technisches Können erwerben soll, mit der Ausbildung eines Fußballers, der kognitive Fähigkeiten entwickeln und kollektives Können erwerben soll, hätte keinen Sinn. Jedoch könnte es bei späteren Studien empfehlenswert sein, den Einsatz einer Kontrollgruppe in Erwägung zu ziehen, um die Verbesserungen auszuschließen, die durch andere Trainingsinhalte erworben wurden. Weitere Studien sind erforderlich, um die Wirksamkeit der Kombinationsspiele als didaktische Strategie für das Lernen und Training der Spielkompetenzen bestätigen zu können. 


\section{Zusammenfassung}

Aus der Expertiseforschung kann man den Schluss ziehen, dass die Sportfähigkeiten eher gelernt als vererbt werden. Der Unterschied zwischen einem Experten und einem Novizen wird durch den größeren Wissensumfang des Experten repräsentiert, d.h., zwischen dem Experten und dem Novizen steht ein Ausbildungsprozess. Dieser Ausbildungsprozess wird jedoch durch viele Stunden von absichtsvoller Übung ergänzt, wie durch Studien der Lebensgeschichte vieler Experten in verschiedenen Bereichen bestätigt wird. Bei den Sportspielen wird auch ein größerer Umfang absichtsvoller Übung der prominenten Spieler festgestellt, wenn man ihre Ausbildungsgeschichte betrachtet.

Die wissenschaftliche Weiterentwicklung der Sportpädagogik und der Sportdidaktik hat viel zur Sportspieldidaktik beigetragen, sie hat Methoden und Strategien eingeführt, die sich als wirksamer für das Erlernen der prozeduralen, kognitiven und emotionalen Kompetenzen der Lernenden ausweisen. Diese Beiträge sind allerdings nur begrenzt auf dem Schulsportbereich übertragbar. Bei Ausbildungsprozessen innerhalb der Sportvereine wird aber immer noch das Direkte Didaktische Modell angewandt.

Das Direkte Modell für die Schulung der Sportspiele ist auf die behavioristische Theorie des Lernens ausgerichtet. Bei diesem Modell wird angenommen, dass der Trainer Kenntnisse besitzt, die er den Spielern vermitteln soll und die Spieler sollen ihr Verhalten auf dem Spielfeld nach den Anweisungen des Trainers gestalten. Erstmals sollen sie die technischen Fertigkeiten lernen, bevor taktische Schulung in Frage kommt, bis dahin sollen die Spieler während des Hauptteils der Trainingseinheit die technischen Fertigkeiten üben und nur am Ende spielen. Wenn es um die taktische Schulung geht, versucht der Trainer, sein taktisches Wissen verbal durch Vorträge, Beratung, Empfehlungen oder Befehle zu vermitteln.

Vertreter anderer Stellungnahme haben vorgeschlagen, das Training sollte eher auf das Spiel und auf die Spieler fokussiert werden als auf das Verhalten des Trainers. Es wird dabei angenommen, dass die Sportspiele mehr als eine biomechanisch vollständige Ausführung der Bewegungen sind und taktische Lösungen der problematischen Spielsituationen erfordern. Es wurden alternative didaktische Modelle für die Schulung der Sportspiele vorgeschlagen, z.B. die auf das Spiel und die Spieler zentrierten Modelle (Game and Player Centered Approaches, GPCA). Solche Modelle betrachten nicht die Technik als den entscheidenden Bestandteil in der Spielerausbildung, sondern die Integration technischer, kognitiver und emotionaler Fähigkeiten und Fertigkeiten. Bei der Mehrheit der didaktischen Modelle die unter dieser Betrachtungsweise vorgeschlagen wurden, steht das Spielen-lassen als überwiegende didaktische Maßnahme, da man durch das Spielen besser die Spielkompetenzen lernen kann und das Lernen in einem Spielkontext stattfindet.

Im Rahmen des Game and Player Centered Approach (GPCA) sind vielfältige didaktische Modelle für das Lernen der Sportspiele vorgeschlagen worden. Diese zielen auf die Entwicklung kognitiver und emotionaler Fähigkeiten und das Lernen technischer und taktischer Fertigkeiten während des Spielens ab. Die empirische Forschung, insbesondere bei dem TGfU Modell, stellt bisher keinen eindeutigen Vorteil der alternativen Modelle über das Direkte Modell für das Lernen der technischen Fertigkeiten und Spiel- 
kompetenzen fest.Allerdings wird auf eine eindeutige Tendenz in den wissenschaftlichen Studien zur Erreichung besserer Ergebnisse für das Lernen kognitiver und emotionaler Kompetenzen hingewiesen, d.h. die alternativen Modelle scheinen besser geeignet für das Verständnis und taktische Bewusstheit zu sein als das Direkte Modell. Jedoch werden die auf Spiel und Spieler zentrierten didaktischen Modelle auf den Schulportkontext beschränkt. Die leistungsorientierten und kontrollorientierten Bedingungen im Wettkampfsport machen eine Umsetzung der sportdidaktischer Innovationen schwer. Die üblichen Widerstände gegen das Neue, die Angst vor dem Kontrollverlust und Unvertrauheit mit den Alternativen könnten die wichtigsten Faktoren sein, die eine Umsetzung des auf das Spiel und die Spieler zentrierten Modells im Wettkampfsport behindern.

Einige Autoren (z.B. METZLER, 2005b, S. 190; KIRK, 2005, S. 216) sind der Meinung, dass die Modellvergleichung keinen Sinn habe, da jedes Modell auf die Erreichung verschiedener Kompetenzen orientiert sei. Sie schlagen deshalb vor, jedes Modell aus unterschiedlichen Perspektiven zu betrachten, ihre Anwendungsmöglichkeiten in verschiedenen Kontexten und ihre Auswirkungen auf das Verhalten des Trainers/Lehrers zu studieren.

Die alternativen didaktischen Modelle, die bisher entwickelt worden sind, werden ausschließlich im Schulsportkontext angewendet; dort finden wir etwas andere Lernziele als im Wettkampfsport. Die Schule kann nur eine Einführung der Schüler in das Sportspiel anstreben: es steht keine Zeit zur Verfügung für eine tiefe Einführung in den Wettkampfsport.

In den Sportvereinen dagegen treiben Kinder seit frühem Alter ein spezifisches Sportspiel, es wird ein tieferes Lernen eines ausgewählten Spiels angestrebt und der Transfer auf andere Spiele wird nicht berücksichtigt. Die Leistung ist eine der wichtigsten Ziele der Sportvereine. Obwohl die soziale und emotionale Ausbildung auch ein wichtiges Ziel ist, wünschen sich die Kinder und auch die Eltern und Trainer Erfolge bei Wettkämpfen . Trotz der theoretischen und empirischen Entwicklungen der Sportdidaktik, sind diese nur im Schulsportkontext berücksichtigt, im Wettkampfsportkontext wird immer noch das Direkte Modell für das Lernen der Sportspiele angewendet. LOIBL (2001, S. 25) ist der Ansicht, dass dieses Modell sich gegen die Entwicklungen der Sportspieldidaktik und die neuen alternativen Modelle immunisiert hat.

Im Modell der Spielhandlungskompetenzen werden Wissen und Information deutlich unterschieden. Information kann vermittelt werden, Information allein ist aber nicht ausrreichend für das Lernen. Wissen hingegen kann nicht wie Information vermittelt werden, sondern wird durch einen Prozess von Teilnahme und Nachdenken aufgebaut . Das Wissen das für Sportspielen aufgebaut werden soll, bezeichnet man als Handlungswissen, und wird in theoretisches, präskriptives, prozedurales und interpretatives Wissen unterschieden. Das Konzept der Wissenstreppe erleichtert theoretische Begriffe über Wissensaufbau, Wissensanwendung und Wissensauswertung. Das Modell der Spielhandlungskompetenzen wird darauf bezogen.

Das Modell nimmt an, dass die Spieler ihre eigenes Lernen regulieren können. Der Trainer ist eher Helfer und Organisator, das Hauptziel ist die Entwicklung der kognitiven Fähigkeiten der Spieler für das erfolgreiche Spielen. Durch indirekte Lernstrategien wird den Spielern die Gelegenheit gegeben, über ihre Handlungen nachzudenken und auf 
Grund dieses Nachdenkes Schlussfolgerungen zu ziehen, die auch auf zukünftige Spielsituationen tranferiert werden könnten;

Das Modell der Spielhandlungskompetenzen besteht aus vier didaktischen Strategien: Kleinen Spielen mit Schwerpunkt, Kombinationsspielen, Zweikämpfen und Selbstlernen der technischen Fertigkeiten. Diese vier Strategien wurden durch empirische Studien getestet, die keine vollständige Umwandlung der Trainingsgewohnheiten der untersuchten Mannschaften erforderten. Die didaktischen Strategien wurden nicht zusammen, sondern isoliert in verschiedenen Spielergruppen umgesetzt. So wurde versucht, die Effektivität der Strategie zu testen ohne Widerstand der Spieler, Trainern und Eltern auszulösen.

Mit der Durchführung von 4 Studien wurde die Wirksamkeit des entworfenen Modells auf das Spiellernen untersucht.

In der ersten Studien wurde das Lernen der Spielprinzipien des Fußballs überprüft. Die Teilnehmerinnen waren 6- bis 8-jährige Mädchen, die regelmäßig Fußball trainierten. Durch Kleine Fußballspiele mit unterschiedlichen Schwerpunkten und Befragung wurde das implizite und auch das explizite Lernen gefördert. Die Spielerinnen bekamen keine technische Schulung und keine Hinweise über das richtige Verhalten in Spielsituationen. Dabei wurden Kleine Spiele angewendet, die durch bestimmte spezifische Regeln die Anwendung der Spielprinzipien erlaubten. Während der Spielpausen und wenn der Trainer das Spiel anhielt, stellte der Trainer den Spielerinnen Fragen, die an der Förderung des Nachdenkens orientiert waren. Die Spielerinnen sollten so ihre Entscheidungen anhand der Spielprinzipien treffen und deren Tauglichkeit begründen.

Die Spielsituationen wurden je nach Ballbesitzerin zugeordnet. Wenn die beobachtete Spielerin in Ballbesitz war, wurde diese Situation als Spieler mit Ball bezeichnet (SmB). War es eine Mitspielerin, wurde die Situation als „Spieler ohne Ball Mannschaft mit Ball“ (SoBMB) zugeordnet; wenn eine gegnerische Spielerin in Ballbesitz war, wurde diese Situation als „Spieler ohne Ball Mannschaft ohne Ball“ (SoBMoB) bezeichnet. Die Tauglichkeit der Spielhandlungen der Spielerinnen in diesen Situationen wurde durch ein hierfür entworfenes Beobachtungsinstrument bestimmt.

Der Vergleich der Ergebnisse der Auswertungsverfahren vor und nach der Intervention zeigt in den 3 Spielsituationen eine prozentuale Leistungssteigerung zwischen 5 und 10 $\%$. Allerdings wurde statistisch kein signifikanter Unterschied erkannt. Als alle tauglichen Handlungen aufsummiert waren, konnte man einen statistisch signifikanten Unterschied zwischen der Gesamtzahl der tauglichen Handlungen vor dem Interventionsprogramm und der Gesamtzahl der tauglichen Handlungen nach Interventionsende erkennen. Nach einer gewissen Zeitspanne ohne Intervention wurde aber bei einem Retentionstest eine leichte Abnahme der prozentualen Verbesserungen beobachtet. Der Unterschied war bei der Gesamtzahl der Handlungen im Retentionstest nicht mehr statistisch signifikant.

Die didaktische Strategie der kleinen Fußballspiele mit unterschiedlichen Schwerpunkten wurde als nicht wirksam für das implizite Lernen der Spielprinzipien bestätigt; allerdings weist eine Tendenz zur Leistungsverbesserung durch das Interventionsverfahren darauf hin, dass bei späteren Studien mit längeren Interventionszeiten statistisch signifikante Unterschiede festgestellt werden könnten.

In der Studie 2 wurde eine didaktische Strategie für die Förderung des theoretischen Wissens beim Fußballspiel erprobt. Eine Gruppe junger Fußballspieler wurde durch di- 
rekte Instruktion über das Spielverhalten geschult. Eine andere Gruppe wurde durch eine Befragungsmethode geschult, die das Nachdenken und kognitive Beteiligung fördert. In beiden Gruppen wurde das theoretische Wissen durch ein für dieses Vorhaben entworfene Auswertungsverfahren abgefragt, darin wird durch ein konzeptuelles System und durch eine Matrixmethode eine Fragestellung erzeugt.

Die Ergebnisse des Vergleichs der Gruppen zeigen, dass theoretisches Wissen beim Fußballspiel durch die Befragungsmethode wirksamer erlangt wird als durch die direkte Instruktion. Die Gruppe, die zum Nachdenken angeregt wurde, erreichte mehr richtige Antworten auf die Fragestellung als die Gruppe, die direkte Hinweise auf das richtige Verhalten in Spielsituationen bekam. Die bessere Leistung der reflexiven Gruppe wurde nach einer Zeitspanne von zwei Wochen ohne Anwendung der Befragungsmethode aufrechterhalten, was auf ein nachhaltiges Lernen hindeutet. Nach der Theorie der Wissenshierarchie, die das Modell der Spielhandlungskompetenzen untermauert, liefert die direkte Instruktion vorwiegend Informationen. Durch reine Information und ohne Nachdenken ist es aber schwer, Wissen zu erwerben. Die Befragungsmethode hingegen lädt die Spieler zum Nachdenken ein und fördert den Wissensaufbau. Damit verarbeiten die Spieler die kognitiven Produktionsregeln, die das Verhalten während der Spielsituationen steuern.

Es erscheint notwendig, in nachfolgenden Studien die Wirksamkeit der Befragungsmethode auf die Spielwissenskompetenz weiter nachzuweisen. Auch sollte der Effekt der Methode auf unterschiedliche kognitive Funktionen untersucht werden. Zudem sollte die Validität und Reliabilität des Auswertungsverfahrens überprüft werden.

Die dritte Studie untersuchte die Möglichkeit, das Spiel selbst als eine Art zufälligen Übens der technischen Fertigkeiten des Fußballspiels zu betrachten. Das Üben in einer zufällig organisierten Reihenfolge hat sich für das Techniklernen als wirksamer als das Üben in einer in Blöcken organisierten Reihenfolge erwiesen, allerdings gestaltet sich seine Implementierung besonders schwierig bei Lernprozessen eines Sportspiels. Die didaktische Strategie der kleinen Fußballspiele mit unterschiedlichen Schwerpunkten könnte eine wirksame Methode sein, das zufällige Üben in das Sportspiellernen zu implementieren.

Eine Gruppe junger Fußballspieler, die die Passtechnik des Fußballs durch Kleine Fußballspiele mit unterschiedlichen Schwerpunkten übte, wurde mit einer Gruppe, die dieselbe Passtechnik nur durch „geometrische“ Übungen übte, verglichen. Die Leistungen beider Gruppen wurden sowohl in Spielsituationen als auch in standardisierten Situationen außerhalb des Spiels unter Anwendung einer Beobachtungsprozedur bzw. eines standardisierten Tests ausgewertet.

In beiden Gruppen erhöhte sich die Rate gut gespielter Pässe, auch verbesserten sich die Leistungen im standardisierten Test. Die kleinen Fußballspiele mit unterschiedlichen Schwerpunkten sind bei zwischen 10 und 12 Jahre alten Fußballern gleich wirksam wie die Übungen für das Techniklernen. Entsprechend diesen Ergebnissen könnte die didaktische Strategie der kleinen Spiele erfolgreich für die Schulung der Technik angewendet werden, da das Spiel gleichzeitig ein zufälliges Üben ist. Durch das Spielen kann man weitere Spielkompetenzen fördern, etwa die Entscheidungsfähigkeit und die taktischen Fertigkeiten; diese könnten durch ein Situiertes Lernen zusammen mit den technischen Fertigkeiten gelernt werden. Die kleinen Fußballspiele mit unterschiedli- 
chen Schwerpunkten erweisen sich demnach als eine geeignete didaktische Strategie für die Schulung von Sportspielen.

Die didaktische Strategie der Kombinationsspiele wurde in der Studie 4 erprobt. Mit dieser Strategie wird angestrebt, durch kooperatives Lernen die Entwicklung der kognitiven Kompetenz zu fördern, insbesondere wenn es um die Koordination diverser Spielsituationen geht. Es geht um Gruppenspiele, worin die Spieler ständig ihre Feldstellung wechseln und den Ball in ständig wechselnde Richtungen passen müssen. Die Spieler müssen sich an die Stellungs- und Richtungswechsel anpassen und dürfen keinen Fehler machen. Ein individueller Fehler unterbricht den Spielverlauf, damit wird das gemeinsame Handeln der ganzen Gruppe gestört. Solche Koordinationsspiele verlangen von den Spielern Aufmerksamkeit, richtige Wahrnehmung, Konzentration und nicht zuletzt Koordination.

Eine Gruppe junger Fußballspieler übte die Kombinationsspiele als Teil ihres normalen Trainings während acht Wochen. Vor der Einführung wurden die Spielleistungen der Teilnehmer ausgewertet. Mittels der Beobachtungsprozedur „Team Sport Assessment Procedure" (TSAP) kann man die Effizienz der Spielhandlungen bei Ballbesitz bestimmen. Dieselbe Prozedur wurde zum Ende der Interventionszeitspanne angewendet, um die Leistungssteigerung der Spieler festzustellen.

Die Ergebnisse weisen eine Tendenz zur Leistungssteigerung bei der beobachteten Variablen Ballgewinn und bei Offensivhandlungen auf. Bei der kalkulierten Variablen Spielumfang (Play Volume) und beim Ausführungsindex (Execution Index) wurden ebenfalls proportionale Verbesserungen festgestellt. Allerdings wurden nur beim Ausführungsindex statistisch signifikante Unterschiede bestätigt, wenn die Ergebnisse vor der Intervention mit denen danach verglichen wurden. Dieser signifikante Unterschied wurde jedoch nicht mehr zwischen dem Vortest und einem Retentionstest drei Wochen nach dem Interventionsende festgestellt.

In der vierten Studie konnte man nur teilweise die Wirksamkeit der didaktischen Strategie der Kombinationsspiele auf die offensive Spielleistung nachweisen. Es sind daher weitere Studien erforderlich, in denen die teilnehmenden Spieler während einer längeren Zeitspanne Kombinationsspiele üben, um die Wirksamkeit dieser Strategie bestätigen zu können.

Die Studien wiesen auf die Wirksamkeit des Modells für das Lernen der Spielkompetenzen hin, wenn die vier didaktischen Strategien getrennt getestet wurden. Allerdings weist die Studie methodische Beschränkungen auf, die bei späteren Studien behoben werden sollten. Mit der partielle Umsetzung der didaktischen Strategien bei unterschiedlichen Gruppen wurde es möglich, die Effektivität des Modells zu bestätigen, diese Vorgehensweise beschränkt aber auch seine Anwendbarkeit; das Modell wurde noch nicht als Gesamtheit getestet. Die kurze Zeit der Umsetzung und die Anwendung von neuen noch nicht validierten Messinstrumenten sind andere methodische Beschränkung, die es noch nicht erlaubt, die Wirksamkeit des Modells bei der Ausbildung junger Fußballspieler zu bestätigen. Es ist daher notwendig, die vollständige Umsetzung des Modells während eines langfristigen Lernprogramms und mit ständiger Leistungskontrolle durchzuführen.

Wegen des Mangels von Messinstrumenten für die Auswertung einiger der untersuchten Variablen (z.B. das Spiel ohne Ball und die kognitiven Kompetenzen) ist es notwendig, weitere Auswertungsmethoden für diese besonderen Variablen zu entwerfen. 
Das Modell der Spielhandlungskompetenzen strebt nicht an, andere Modelle zu übertreffen. Es wird vielmehr als eine Alternative betrachtet, die die Trainer einsetzen könnten, um mit Schwerpunkt die Spielkompetenzen auszubilden, statt lediglich Kenntnisse zu vermitteln. Das Modell ist in diesem Sinn kein Sportspielvermittlungsmodell, sondern ein didaktisches Modell für das Lernen der Sportspielkompetenzen. Wenn das Modell bei späteren Studien als wirksam bestätigt würde, sollten weitere praktische Umsetzungsmöglichkeiten der didaktischen Strategien entwickelt werden. Das Modell der Spielhandlungskompetenzen könnte eine Alternativ zum traditionellen Direkten Modell werden: Seine Anwendung in Sportvereinen sollte die jungen Spieler durch die Wissenstreppe führen und sie befähigen, im sportlichen Wettkampf zu bestehen. 


\section{Literaturverzeichnis}

ACERO, R. \& LAGO, C. (2005). Deportes de equipo: comprender la complejidad para elevar el rendimiento.Barcelona: Inde.

ABERNETHY, B.\& SPARROW, W. (1992).The rise and fall of dominant paradigms in motor behaviour research.In J.J. Summers (Ed.).Approaches to the study of motor control and learning (S.3-45). Amsterdam: Elsevier Science.

ACKOFF, R. L. (1989). From Data to Wisdom, Journal of Applied Systems Analysis, 16, 3-9

ALI, A.; ELDERED, J.; HIRST, M.; HULSE, M.; MCGREGOR, S.; STRUDWICK, A.; \& WILLIAMS, C. (2003). The reliability and validity of the modified Loughborough Soccer Passing Test. J. Sports Sci., 21, 258.

ALI A.; WILLIAMS C.; HULSE M.; STRUDWICK A.; REDDIN J. \& HOWARTH L. (2007). Reliability and validity of two tests of soccer skill.J. Sports Sci., 25, 1461-1470.

ALISON, S., \& THORPE, R. (1997). A comparison of the effectiveness of two approaches to teaching games within physical education. A skills approach versus a games for understanding approach. The British Journal of Physical Education, 28(3), 9-13.

ALMOND, L. (1986a).Reflecting on themes: a games clasification.In R. THORPE; D. BUNKER\& L. ALMOND(Eds.), Rethinking games teaching (pp. 71-72). Lougborough: University of Technology Loughborough.

ALMOND, L. (1986b).Primary and secundary rules in games.In R. THORPE; D. BUNKE \& L. ALMOND(Eds.), Rethinking games teaching (pp. 73-74). Lougborough: University of Technology Loughborough.

ANDERSON, R.J. (1996). Kognitive Psychologie (2. Aufl.). Heidelberg: Spektrum.

ARCOFADER, Asociación Red Colombiana de Facultades de Educación Física y Deporte (2007). Lineamientos para la evaluación de competencias en los programas de Educación Física. Bogotá: Servicio Nacional de Aprendizaje.

ARIAS, E. \& JIMENEZ, J. O. (2004). Comparación entre la práctica aleatoria y la práctica en bloques en dos fundamentos técnicos del fútbol en niños de 6 a 8 años. Revista Educación Física y Deporte. Universidad de Antioquia. Instituto Universitario de Educación Física y Deporte,23(2), 97-108.

ARIAS, E. \& JIMENEZ, J.O. (2007). Tiempo de pausa y tiempo de Juego en los partidos de fútbol. En Arias, E.; Jimenez, J. O.; Olaya, Z.D. \& Ramon, G.,Características del Fútbol y del futbolista infantil; (p. 31-43). Medellín: Funánbulos Editores.

ARNOLD, R.; GÓMEZ, T.; CLAUDIA, J. \& KAMMERER, J. (2001). Selbstlernkompetenzen. Pädagogische Materialien der Universität Kaiserslautern, Heft 12. 
AUGUSTIN, D. (1998). Zum Techniktraining im Fußball - Ausgewählte Grundlage, Ziele Methoden und Inhalte. In G. Bäumler; g. Bauer (Hrsg.),Sportwissenschaft rund um den Fußball(S. 9-28). Hamburg: Czawalina.

AUSUBEL, D. P. (1968).Educational psichology: a cognitive view. New York: Holt, Rinehart \& Wiston.

BANDURA, A. (1977). Social learning theory.New York: Prentice Hall.

BANYARD, P. (1995).Introducción a los procesos cognitivos. Barcelona: Ariel, S.A.

BARREIROS, J.; FIGUEREDO, T. \& GODINHO,M. (2007). The contextual interference effect in applied settings.European Physical Education Review,13(2),195-208.

BECKERMANN, A. (2001). Zur Inkohärenz und Irrelevanz des Wissensbegriffs. Plädoyer für eine neue Agenda in der Erkenntnistheorie. Zeitschrift für Philosophische Forschung, 55, 571-593. Verfügbar unter http://wwwedit.uni-bielefeld.de/philosophie/personen/beckermann/wnutz ww.pdf[07.10.2008]

BECKMANN, J. (1984). Kognitive Dissonanz: eine handlungstheoretische Perspektive.Berlin: SpringerVerlag.

BELLINGER,G. (2004).Knowledge Management: Emerging perspectives. Available under http://www.systems-thinking.org/kmgmt/kmgmt.htm[03.11.2008]

BIETZ, J. (1994). Die spielgemäße Vermittlung des Handballspiels. Sportunterricht, 43(9), 372-381.

BISANZ, G.\& GERISCH, G. (1996). Fußball. Reinbeck: Rowohlt Taschenbuch.

BISANZ, G. \& GERISCH, G. (2008). Fußball: Kondition, Technik, Taktik und Coaching. Aachen: Meyer \& Meyer.

BLASER, P.; STUCKE, CH. \& WITTE, K. (Hrsg.) (1992). Steuer- und Regelvorgänge der menschlichen Motorik. Sankt Augustin: Akademia.

BLÁZQUEZ, D. (1992). Evaluar en Educación Física. Barcelona: Inde.

BLOMQVIST, M.; LUHTANEN, P.; LAAKSO, L. \& KESKINEN, E. (2000). Validation of a videogameunderstanding test procedure in badminton.Journal of Teaching in Physical Education, 19, 325-337.

BLOMQVIST, M.; LUHTANEN, P. \& LAASKO, L. (2001). Comparison of two types ofinstruction in badminton.European Journal of Physical Education, 6, 139-155.

BLOMQVIST, M.; VANTTINEN, T. \& LUHTANEN, P. (2005). Assessment of secondary schoolstudents decision-making and game-play ability in soccer. Physical Education \& Sport Pedagogy, 10(2), 107-113.

BLUME, D.D. (1981). Kennzeichnung koordinativer Fähigkeiten und Möglichkeiten ihrer Herausbildung im Trainingsprozeß. Wissenschaftliche Zeitschrift der Deutschen Hochschule für Körperkultur Leipzig, 22, 3, 17-41. 
BORGGREFE, C.; THIEL, A. \& CACHAY, K. (2006). Sozialkompetenz von Trainerinnen und Trainern in Spitzensport. Köln: Strauß.

BREMEN, D. (1987). Handlungsorientierte Spielmethodik. In DVS- Protokolle Nr. 29, Beiträge und Analyse zum Fußballspiel (S. 46-77): Clausthal-Zellerfeld.

Brick Lane FilmsLtd. (2005).Urban Freestyler DVD.(Lehrprogramm auf DVD), Internet-Ressource verfügbar unter: http://www.urbanfreestyler.com/index1.html [23.11.2010].

BROPHY, J. \& GOOD, T. (1986).Teacher behavior and student achievement.In M.C. WITTROCK (Ed.), Handbook of research on teaching (3.Ed., S. 360-364). New York: McMillan.

BRÜGGEMANN, D. \& ALBRECHT, D. (1982). Fußballhandbuch: modernes Fußballtraining.Schondorf: Hofmann.

BRUNER, J. S. (1961). The act of discovery. Harvard Educational Review, 31, 21-32.

BUNKER, D. \& THORPE, R. (1982).A model for the teaching of games in the secondaryschool.Bulletin of Physical Education, 10, 9-16.

BUNKER, D. \& THORPE, R. (1986a).The curriculum model.In R. THORPE; D. BUNKER\& L. ALMOND(Eds.), Rethinking games teaching (pp.7-9). Lougborough: University of Technology Loughborough.

BUNKER, D. \& THORPE, R. (1986b).From theory to practice.In R. THORPE; D. BUNKER\& L. ALMOND(Eds.), Rethinking games teaching (pp. 11-16). Lougborough: University of Technology Loughborough.

BUNKER, D. \& THORPE, R. (1986c).is there a need to reflect on our games teaching? In R. THORPE; D. BUNKER\& L. ALMOND (Eds.), Rethinking games teaching (pp. 25-34).Lougborough: University of Technology Loughborough.

BÜSCH, D. (2001). Funktioniert das Analyseraster für koordinative Anforderungen ?.Spektrum, 1, 54-69.

BUTLER, J. I. (1996). Teacher responses to teaching games for understanding. Journal of Physical Education, Recreation and Dance, 67(9), 17-20.

BUTLER, J. I. (1997). How would Socrates teach games?: a constructivist approach. Journal of Physical Education, Recreation \& Dance, 68(9), 42-47.

BUTLER, J. I. (2002). Teaching Games for Understanding: Aholistic alternative in games education. In B. Lombardo (Ed.).Sport in the twenty fist centruy: alternatives for the new millenium. Boston: Pearson Education.

BUTLER, J. I., \& MCCAHAN, B. J. (2005). Teaching games for understanding as a curriculum model. In L. L. GRIFFIN\& J. I. BUTLER (Eds.), Teaching games for understanding. Theory, research and practice (p. 33-55). Champaign, IL: Human Kinetics.

CALABRIA, M. (2011). Wirksamkeit von impliziten und expliziten Lernprozessen, Aneignung taktischer Kompetenzen und motorischer Fertigkeiten im Basketball.Dissertation Universität Heidelberg. Verfügbar 
unter:

http://archiv.ub.uniheidelberg.de/volltextserver/volltexte/2011/12026/pdf/Doktorarbeit Versionlll endguelti ge.pdf [05.05.2012]

CARL, K. (1976). Taktik. In P. Röthig, Sportwissenschaftliches Lexikon.Schorndorf: Hofman.

CARO, A.; RODRÍGUEZ, A.; CALERO, C.; FERNÁNDEZ-MEDINA, E.\& PIATTINI, M. (2005). Análisis y revisión de la literatura en el contexto de proyectos de fin de carrera: una propuesta. VIII Congreso Chileno de Educación Superior en Computación.

CHANDLER, T. \& MITCHELL, S. (1990). Reflections on models of games education.Journal of Physical Education. Recreation, and Dance, 61(8), 19-21.

CHOW, J,I.; DAVIDS, K.; BUTTON, C.; SHUTLEWORTH, R.; RENSHAW, I. \& ARAÚJO, D. (2007). The Role of Nonlinear Pedagogy in Physical Education.Review of Educational Research, 77(3), 251-278.

CLANCEY, W.J. (1995). A tutorial on situated learning proceedings of the international conference on computers and education (Taiwan).Self, J. (ED). Charlottesville: VAAACE, 49-70.

COBB, P. (1986). Making mathematics, children's learning and constructivst tradition.Harvard Educational Review, 56, 301-306.

COOLEY, M. (1987).Architect or Bee?The human price of technology.London: The Hogarth Press.

COSTA, I.; GARGANTA, J.; GRECO, P.J. \& MESQUITA, I. (2009). Avaliação do desempenho tático no futebol: concepção e desenvolvimento da grelha de observação do teste "gr3-3gr. R. Min. Educ. Fís., Viçosa, v. 17, n. 2, pp. 36-64.

COSTA, I.; GARGANTA, J.; GRECO, P.; MESQUITA, I.; CASTELÃO, D.; MÜLLER, E.; SILVA, B.; REBELO, A. \& SEABRA, A. (2009). Analysis of Tactical Performance of Youth Football Players. CD-ROM Published in the Proceedings of 1st International Symposium of Sports Performance: Performance Enhanced by Bridging the Gap between Theory and Practice, Vila Real, Portugal, 2009.

DAUGS, R. \& BLISCHKE,K. (Hrsg.) (1993). Aufmerksamkeit und Automatisierung in der Sportmotorik. Sankt Augustin: Academia.

COTE, J.; BAKER, J. \& ABERNETHY, B. (2003).From play to practice. In J.L. STARKES \& K.A. ERICSSON (Eds), Expert Performance in Sport (p. 115-135). Champaign, IL: Human Kinetics.

DEN DUYN, N. (1997). Game Sense: Developing Thinking Players. Canberra:Australian Sports Commission.

DEUTSCH, K. (1990). Wissen und Macht: Wirtschaftliche und soziale Bedeutung von Wissen. In H.SCHEIDGEN; P. STRITTMATTER \& W. TACK (Hrsg.), Information ist noch kein Wissen (S.37-54). Basel: Beltz.

DEUTSCHE FUSSBALL-BUND (2005). Fußball von Morgen Band 1 Kinderfußball. Münster: PhilippkaSportverlag. 
DEUTSCHE FUSSBALL-BUND (2006). Trainings des 1 gegen 1 in der Defensive: Tipps für die Vereinstrainer. DFB- Talentförderungsprogramm: Info-Abende für Vereinstrainer 7. Münster: PhilippkaSportverlag.

DIETRICH, K. (1984). Fußball: Spielgemäß lernen - Spielgemäß üben. Schorndorf: Hofmann.

DIETRICH, K. (1985). Sportspiele. Reinbek bei Hamburg: Rowohlt Taschenbuch.

DIETRICH, K. (2007). Dürrwächter, G. \& Schaller, H-J. Die Großen Spiele (6. Auf.). Aachen: Meyer \& Meyer Verlag.

DODDS, P.; GRIFFIN, L. L. \& PLACEK, J. H. (2001).A selected review of the literature on development of learners' domain-specific knowledge.Journal of Teaching in Physical Education, 20, 301-313.

DÖBLER, H.; SCHNABEL, G. \& THIEß, G. (1989). Grundbegriffe der. Sportspiele Berlin: Sportverlag.

DOOLITTLE, S. (1995). Teaching net games to skilled students: A teaching games for understanding approach.Journal of Physical Education, Recreation \& Dance, 66(7), 18-23.

DOUCET, C. (2006). Fussball: Taktik perfektionieren. Leer: Onli Verlag.

DYSON, B.; GRIFFIN, L. L. \& HASTIE, P. (2004). Sport Education, Tactical Games, and Cooperative Learning. Theoretical and Pedagogical Considerations. Quest, 56, 22-24.

EBERSPÄCHER, H. (1987). Handlexikon Sportwissenschaft. Reinbek bei Hamburg: Rowohlt Taschenbuch.

EMRICH, A. (2007). Spielen Handball lernen (5. Auf.). Wiebelsheim: Limpert.

ENGELKAMP, J. (2006). Kognitionspsychologie. Heidelberg: Spektrum.

ERICSSON, K.A.; KRAMPE, R.T. \& TESCH-RÖMER, C. (1993). The role of deliberate practice in the adquisition of expert performance. Psychological Review, 100, 363-406.

ERICSSON, K. A. \& SMITH, J. (1991). Prospects and limits of the empirical study of expertise: An introduction. In K. A. ERICSSON \& J. SMITH (Eds.), Toward a general theory of expertise: Prospects and limits (pp. 1-38). Cambridge, England: Cambridge University Press.

ERICSSON, K.A. \& CHARNESS, N. (1994). Expert performance: Its structure and acquisition. Am Psychol, 49,725-747.

ERICSSON, K.A. (1996). The acquisition of expert performance: An introduction to some of the issues. In K.A. ERICSSON (Ed.), The road to excellence: the acquisition of expert performance in the arts and sciences, sports, and games (pp.1-50). Mahwah, NJ: Erlbaum.

ERICSSON, K.A. (2003). Development of elite performance and deliberate practice: an update from the perspective of the expert performance approach. In JL.STARKES\& KA. ERICSSON (Eds.),Expert performance in sports: advances in research on sport expertise (pp. 49-82). Champaign, IL: Human Kinetics. 
EYSENCK, M.W. \& KEANE, M.T. (2000).Cognitive Psychology: A Student's Handbook London: Psychology Press.

FARROW, D.\& MASCHETTE, W. (1997).The effects of contextual interference on children learning forehand tennis groundstrokes.Journal of Human Movement Studies,33, 47-67.

FENOGLIO, R. (2003). The Manchester United 4 V 4 Pilot Scheme for Under 9's: Part II, The Analysis. Insight : The Football Association Coaches Association Magazine. 6(4), 21-24.

FEUERSTEIN, R. (1978). The ontogeny of learning.In M. Brazier. (Ed). Brain Mechanisms in Memory and learning. New York : Raven Press.

FILMHAUS WIESBADEN (2010).Street Skills: King style (Lehrprogramm auf DVD). Wiesbaden: SchröderMedia.

FORBES, C. (1992). The Polgar sisters: Training or genius? New York: Henry Holt.

FRENCH, K. E. \& THOMAS, J.P. (1987).The relation of knowledge to children's basketball performance.Journal of Sport Psychology, 8, 15-32.

FRENCH, K.E. \& McPHERSON, S.L. (1999). Adaptations in response selection processes used during sport competition with increasing age and expertise. Int J Sport Psychol, 30,173-193.

FRENCH, K. E.; NEVETT, M. E.; SPURGEON, J. H.; GRAHAM, K. C.; RINK, J. E. \& MCPHERSON,S. E. (1996). Knowledge representation and problem solutions in expert and novice youth baseball players.Research Quarterly for Exercise and Sport, 67(4), 386-395.

FRENCH, K. E.; WERNER, P., TAYLOR, K.; HUSSEY, K. \& JONES, J. (1996). The effects of a 6-week unit of tactical, skill or combined tactical and skill instruction of badminton performance of ninth grade students. Journal of Teaching in Physical Education,15(4), 439-463.

FRENCH, K. E.; WERNER, P.; RINK, J. E.; TAYLOR, K. \& HUSSEY, K. (1996). The effects of a3-week unit of tactical, skill or combined tactical and skill instruction on badminton performance of ninth grade students. Journal of Teaching in Physical Education, 15(4), 418-438.

GARGANTA, J. \& PINTO, J. (1994). O ensino do futebol. In A. Graça \& E. J. Oliveira (Ed.), O ensino dos jogos desportivos I (p.95-136). Porto: Faculdade de Ciências do Desporto e de Educação Física da Universidade do Porto: Rainho \& Neves Lda.

GARRISON, D. R. (1993). A Cognitive Constructivist View of Distance Education: An Analysis of Teaching-Learning Assumptions. Distance Education, 14, (2), 199-211.

GLASAUER, G. J. \& NIEBER, L. (2000). Systematisches Koordinationstraining im Basketball, theoretische Grundlagen. co@cher Corner, Verfügbarkeit unter

http://www.bbcoach.de/element.php?name=content\&pa=printpage\&pid=104 [03.11.2010]

GLASAUER, G.J. \& NIEBER, L. (2002). Zur Theorie eines trainingsbegleitenden akzentuierten Koordinationstrainings im Basketball. In K. FERGER; N. GISSEL \& J. SCHWIER (Hrsg.), Sportspiele erleben, vermitteln, trainieren. (S.187-202) Sportspiel-Symposium der dvs vom 4.-6.10.2000 in Gießen. Hamburg: Czawalina. 
GLASAUER, G. J. (2003). Koordinationstraining im Basketball.Hamburg: Dr Kovac.

GRACA, A.; MUSCH, E.; MERTENS, B.; TIMMERS, E.; MERTENS, T.; TABORSKY, F.; REMY, C.; DE CLERCQ, D.; MULTAEL, M.\& VONDERLYNCK, V.. (2003). O modelo de competência nos jogos de invasão: Proposta metodológica para o ensino e aprendizagem dos jogos desportivos. Presented at "II Congreso Ibérico de Baloncesto Caceres, Spain, 27-29 Noviembre

GRANT, B. (1992). Integrating sport into the physical education curriculum in New Zealand secondary schools.Quest, 44(3), 304-316.

GRANTHAM, N. (2008). Sports psychology: the theory of the decision training model in sport. In Peak Peformance. London: P2P Publishing Ltd. Avaliable under: http://www.pponline.co.uk/encyc/sportspsychology-the-theory-of-the-decision-training-model-in-sport-42359. [08.12.2010]

GREEN, A. J. K. \& GILHOOLY, K. J. (1992). Empirical advances in expertise research. In K. J. GILHOOLY AND M. T. G. KEANE (Eds.), Advances in the Psychology of Thinking, Vol. 1. (pp.45-70). UK: Harvester Wheatsheaf.

GRÉHAIGNE, J-F.\& GODBOUT, P. (1995). Tactical knowledge in team sports from a constructivist and cognitivist perspective. Quest, 47, 490-505.

GRÉHAIGNE, J-F.; GODBOUT, P.\& BOUTHIER, D. (1997).Performance assessment in team sports.Journal of Teaching in Physical Education, 16, 500-516.

GRÉHAIGNE, J-F.;\& GODBOUT, P. (1998).Formative assessment in team sports.Journal of Physical Education, Recreation \& Dance, 69(1), 46-51.

GRÉHAIGNE, J-F.; GODBOUT, P.\& BOUTHIER, D. (1999).The foundations of tactics and strategy in team sports.Journal of Teaching in Physical Eduaction, 47, 490-505.

GRÉHAIGNE, J-F.; GODBOUT, P. \& BOUTHIER, D. (1997).Performance Assessment in Team Sports.Journal of Teaching in Physical Education, 16, 500-516

GRÉHAIGNE, J. F.; RICHARD, J. F. \& GRIFFIN, L. (2005).Teaching and Learning Team Sports and Games. New York: Routledge.

GRIFFIN, L. L.; OSLIN, J. L. \& MITCHELL, S. A. (1995).An analysis of two instructionalapproaches to teaching net games.Research Quarterly for Exercise and Sport, March, Supplement, A-64.

GRIFFIN, L,L.; MITCHELL, S, A. \& OSLIN, J.L. (2003). Sport Foundations for Elementary Physical Education: A Tactical Games Approach. Champaign, IL: Human Kinetics.

GRIFFIN, L.L.; PATTON, K.(2005). Two decades of teaching games for understanding: looking at the past presente and future. In L. L. GRIFFIN \& J. I. BUTLER (Eds.), Teaching games for understanding. Theory, research and practice. Champaign, IL: Human Kinetics. 1-17.

GRÖBEN, B. (2000). Einheitenbildung im Bewegungshandeln: zur phänomenalen Struktur des sportbezogenen Bewegungslernens. Schorndorf: Hofmann. 
GROEBEN, N. (2000).Handeln, Tun, Verhalten als Einheiten einer verstehend-erklärenden Psychologie. Tübingen: Francke.

GRUBER, H. (2004). Kompetenzen von Lehrerinnen und Lehrern: ein Blick aus der Expertiseforschung (Forschungsbericht Nr. 13). Regensburg: Universität Regensburg, Lehrstuhl für Lehr-Lern-Forschung. In A. HARTINGER \& M. FÖLLING-ALBERS (Hrsg.), Lehrerkompetenzen für den Sachunterricht.Bad Heilbrunn: Klinkhardt.

HALL, K.G.; DOMINGUES, D.A. \& CAVAZOS, R. (1994). Contextual interference effects with skilled baseball players. Percept Mot Skills, 78, 835-41.

HALL, K.G. \& MAGILL, R. A. (1995).Variability of practice and contextual interference in motor skill learning.Journal of motor behaviour, 27(4), 299-309.

HARRE, D. (1982).Trainingslehre. Berlin: Sportverlag.

HARRISON, J.M.; BLAKEMORE, C.L.; RICHARDS, R.P.; OLIVER, J.; WILKINSON, C. \& FELLINGHAM, G.W. (1998). The effects of two instructional models on skill development, knowledge, self-efficacy, game play and student perceptions in volleyball.Research Quarterly for Exercise and Sport.March, Supplement, 93-94.

HARVEY, S. (n.y.). Using the Game Performance Assessment Instrument. Teaching Games for Understanding: U. K. Available under

Http://www.conferences.unimelb.edu.au/sport/proceedings/PROCEEDINGS\%20-

\%20harvey.pdf[02.05.2009]

HARVEY, S.; (2006).Effects of Teaching Games for Understanding on Game Performance in Middle School Physical Education. Oregon State University: Dissertation. Available under

http://ir.library.oregonstate.edu/xmlui/bitstream/handle/1957/3010/A\%20Study\%20of\%20the\%20Effects\% 20of\%20TGFU\%20in\%20a\%20Middle\%20School\%20Setting\%20-\%20FINAL.pdf?sequence=1

[23.07.2008]

HASTIE, P. A. \& SHARPE, T. (1999).Effects of a Sport Education curriculum on the positive social behavior of at-risk rural adolescent boys.Journal of Education for Students Placed at Risk,4, 417-430.

HELSEN, W.F. \& STARKES, J. (1999). Amultidimensional approach to skill perception and performance in sport.Applied cognitive psichology, 13, 1-27.

HERBST, D. (2000). Erfolgsfaktor Wissensmanagement. Berlin: Cornelsen.

HERNANDEZ, J. (2005). Fundamentos del deporte. Análisis de las estructuras del juego deportivo (3 Ed). Barcelona: Inde.

HERNANDEZ, J. \& JIMENEZ, J. (2000). Los contenidos deportivos en la educacion fisica escolar desde la praxiologia motriz (I). Revista digital Lecturas Educacion fisica y Deportes, 5, 19. Accesible en http//:www.efdeportes.com/efdiga/praxll.htm

HEIL, F. (1970). Mit kleine Spielen zum großen Spiel. Die Leibeserziehung, 10. 
HILLEBRECHT, N. (1998). Wie gestaltet man Variabilitätstraining? Instruierte und resultative Variabilität. Dissertation Universität Georg-August, Göttingen. Hamburg: Dr. Kovac.

HIRTZ, P. (1989). Phänomen koordinative Fähigkeiten. Theorie und Praxis der Körperkultur, 38, 2, 30-33.

HIRTZ, P. (1994). Arten und Funktionen des Koordinationstrainings. Trainingswissenschaft, 313.

HIRTZ, P. (1998). Koordinative Fähigkeiten: Gewandtheit, motorische Kompetenz. In J. ROSTOCK; K. \&ZIMMERMANN (Hrsg.), Bericht zum Kolloquium „Theorie und Empirie sportmotorischer Fähigkeiten.Werdau: Fachgebiet Sport der TU Chemnitz, (S. 12-19).

HOFF,J. (2002). Soccer specific aerobic endurance training.British Journal of Sports Medicine,36(3), 218221.

HOFFMANN, J. (1993). Vorhersage und Erkenntnis. Göttingen: Hogrefe.

HOHMANN, A. (1994). Grundlagen der Trainingssteuerung im Sportspiel.Hamburg: Czawalina.

HOHMANN, A; LAMES, M. \& LETZELTER, M. (2002). Einführung in die Trainingswissenschaft. Wiesbaden: Limpert.

HOLT, N.L.; STREAN, W.,B. \& BENGOECHEA, E. G. (2002). Expanding the teaching games for understanding model: New avenues for future research and practice.Journal of Teaching in Physical Education, 21, 177-192.

HOLT, J.; WARD, P.\& WALLHEAD, T. (2006). The transfer of learning from play practices to game play in young adult soccer players. Physical Education and Sport Pedagogy,11(2), 101-118.

HOOPER, T. \& LIGHT, R. (2008).Scientific work on TGfU. Available under http://www.tgfu.org/articles/ReferencesTGfU2007.pdf[12.10.2007]

HOSSNER, E-J. (1993). Beim Fertigkeitslehren im Sport: keine Angst vor Überforderungen ! Sportsychologie, 7, 2, 17-20.

HOSSNER, E-J. (1995). Module der Motorik. Schondorf: Hofmann.

HOSSNER, E.-J. \& KORTMANN, O. (1995). „Stein auf Stein“ - Techniktraining nach dem Baukastenprinzip. In F. Dannenmann (Hrsg.), Neue Aspekte des Volleyballspiels (S. 40-58). Hamburg: Czwalina.

HOSSNER, E.-J. \& KORTMANN, O. (1996). Techniktraining im Spitzenbereich: ein Baukasten wird gefüllt. In F. Dannenmann (Hrsg.), Volleyball ‘95. Das Spiel im Jubiläumsjahr (S. 9-18). Hamburg: Czwalina.

HOSSNER, E.J. \& ROTH, K. (2000).Sportspiele vermitteln. In FERGER, K.; GISSEL, N. \& SCHWIER, J. (Hrsg.),Sportspiele erleben, vermitteln, trainieren. Band 124 (S. 111-124). Hamburg: Czwalina.

HOTZ, A. (1995). Bewegung kennen und können: zur Didaktik einer „Bewegung-verstehenden“ Sportlehrer(innen) Ausbildung (S. 181-197). In PROHL, R. \& SEEWALD, J. (Hrsg). Bewegung verstehen Schorndorf: Hofmann. 
HOWARTH, C. (2005). Introducing the Teaching Games for Understanding model in teacher education programs. In L. GRIFFIN \& J. BUTLER (Eds) Teaching Games for Understanding: Theory, Research and Practice (pp. 91-106), Champaign, IL: Human Kinetics.

IAFRANCESCO, G. (2006). Unveröffentlichtes pdf-Dokument.

IGLESIAS, D.; GARCÍA-GONZÁLEZ, L.; GARCÍA-CALVO, T.; LEÓN, B. \& DEL VILLAR F. (2010). Expertise development in sport: contributions under cognitive psychology perspective. J. Hum. Sport Exerc. 5(3), pp. 462-475.

IMMINK, M. A. \& WRIGHT, D. L. (2001). Motor programming during practice conditions high and low in contextual interference. Journal of Experimental Psychology: Human Perception and Performance, 27(2), 423-437.

IMPELLIZZERI, F.M.; RAMPININI, E.; MAFFIULETTI, N.A.; CASTAGNA, C.; BIZZINI, M. \& WISLOFF, U. (2008).Effects of aerobic training on the exercise-induced decline in short-passing ability in junior soccer players.Applied Physiology, Nutrition, and Metabolism [Electronic Version] Dec, 2008.

INTERNATIONAL ICE HOCKEY FEDERATION. (2007). Coaching Manual: Introduction to coaching. Zürich: International Ice Hockey Federation.

JANALIK, H. (1982). Mannschaftsspiel Handball: Alle wollen mitspielen. Sportpädagogik, 6, 48-51.

JANELLE, C. M.\& HILLMAN, C. H. (2003). Expert performance in sport: Current Perspectives and Critical Issues. In K.A. ERICSSON \& J. STARKES (Eds.), Recent Advances in Research on Sport Expertise (p. 19-47). Human Kinetics: Champaign, IL.

JONES, S. \& DRUST, B. (2007).Physiological and technical demands of $4 \vee 4$ and $8 \vee 8$ games in elite youth soccer players. Kinesiology, 39(2), 150-156.

JONES, C. \& FARROW, D. (1999). The transfer of strategic knowledge: A test of the games classification curriculum model. The Bulletin of Physical Education, 25(2), 103-124.

JANK, W. \& MEYER, H. (1991) Didaktische Modelle (5. Auf.) Berlin: Cornelsen.

KEIL, R. \& PELLEGRINO, J. (1988). Menschliche Intelligenz. Heidelberg: Spektrum der Wissenschaft.

KELSO, J. A. (1995). Dynamic patterns: the selforganization of brain and behavior. Cambridge, MA: MIT Press.

KELLER, J. M. (2008). How to integrate learner motivation planning into lesson planning: The ARCS model approach. Paper presented at VII Semanario, Santiago, Cuba, avalaible under:

http://mailer.fsu.edu/ jkeller/Articles/Keller\%202000\%20ARCS\%20Lesson\%20Planning.pdf [24.01.2011]

KERN, U. \& SÖLL, W. (2005). Praxis und Methodik der Shulsportarten (3. Auf). Schorndorf: Hofmann.

KIRK, D. (2003). Model-Based Teaching and Assessment in Physical Education: The Tactical GamesModel. In GREEN, K. \& HARDMAN, K. (Eds.),An Essential Reader in Physical Education. London: Sage. 
KIRK, D.\& MCPHAIL, A. (2002). Teaching Games for Understanding and Situated Learning: Rethinking the Bunker-Thorpe Model. Journal of Teaching in Physical Education, 21, 177-192.

KIRK, D. (2005). Future prospects for Teaching Games for Understanding. In GRIFFIN, L.L. \& BUTTLER, J.I. (Eds.).Teaching Games for Understanding: Theorie Research and Practice (p. 213-227). Champaign, IL.: Human Kinetics.

KEELE, S. (1968).Movement control in skilled motor competence.Psychological Bulletin, 70, 370-403.

KERN, U. \& SÖLL, W. (2005). Praxis und Methoden der Schulsportarten.(3. Ed.). Schondorf: Hofmann.

KIELLER, T. (2002).Michael Jordan, above the rim.Unitet Athletes. The North American Sport Magazine, Sept. 11, [Electronic version] Available under: http://www.unitedathletes.com/english/profiles/mjordan.html [13.02.2011]

KIRCHNER, G. \& PÖHLMANN, R. (2005). Lehrbuch der Sportmotorik. Kassel: Zimmermann + Kaul $\mathrm{GbR}$.

KONZAG, G. (1992). Komplexität der Sportspielleistung: Eine Herausforderung an die Sportpsychologie. In G. HAGEDORN \& N. HEYMEN, N. (Hrsg.), Methodologie der Sportspielforschung (S. 10-22). 9. Internationales Berliner Sportspiel-Symposion. Ahrensburg bei Hamburg.

KRÖGER, C.\& ROTH, K. (1999). Ballschule: Ein ABC für Spielanfänger.Schondorf: Hofmann.

KUGLER, P.N.; KELSO, J. A.; TURVEY, M. T. (1980). On the concept of coordinative structures as dissipative structures: I. Theoretical lines of convergence. In G.E. STELMACH\& J. REQIUN (Eds.), (pp. 1-49), Tutorials in motor behavior. New York: North-Holland.

KUGLER, P.N.; KELSO, J. A.; TURVEY, M. T. (1982). On the control and coordination of naturally developing systems.In J.A. KELSO\&J.E. CLARK. (Ed.), The development of movement control and coordination, (pp. 5-78). New York: Wiley.

KUHLMAN, D. (1993). Wie führt man Spiele ein?. In Bielefelder Sportpädagogen (Hrsg.), Methoden im Sportunterricht (S. 117-130). Schorndorf: Hofmann.

KUHN, W. \& MAIER, W. (1978). Beiträge zur Analyse des Fußballspiels. Schriftenreihe des Bundesinstituts für Sportwissenschaft (Band 16). Schorndorf: Hoffmann.

LANDIS, J.R. \& KOCH, G.C. (1977). The measurement of observer agreement for categorical data.Biometrics 33, 1089-91.

LASIERRA, G. (1990). Aproximación a una propuesta de aprendizaje de los elementos tácticos individuales en los deportes de equipo. Apunts: Educacio Fisica i Esports, 24, 59-68.

LAVE, J. \& WENGER, E. (1991). Situated learning: Legitimate peripheral participation. New York: Cambridge University Press.

LAUNDER, A.G. (2001).Play Practice: the games approach to teaching and coaching sports. Champaign, IL: Human Kinetics. 
LAUNDER, A. \& PILTZ, W. (2006). Beyond 'Understanding' to Skilful Play in Games, through Play Practice.Journal of Physical Education New Zealand, 39(1), 47-57.

LAWTON, J. (1989). Comparison of two teaching methods in games.Bulletin of Physical Education, 25(1), 35-38.

LEMLECH, J.K. (1998). Curriculum and instructional mehtods for elementary and middle school. Upper Saddle River, NJ.: Prenctice-Hall.

LEE, M.A. (2004). Generalization of supporting movement in tag rugby from practice to games in 7 th and 8th grade physical education.Dissertation. The Ohio State University. Available under: http://etd.ohiolink.edu/send-pdf.cgi/Lee\%20MyungAh.pdf?osu1086195558 [23. 07.2010].

LIGHT, R. (2005a). Making sense of the chaos: Australian coaches talk about game sense. In L. L. GRIFFIN \& J. I. BUTLER (Eds.), Teaching games for understanding: Theory, research and practice (pp. 169-182). Champaign, IL: Human Kinetics.

LIGHT, R. (Ed.). (2005b). An international perspective on Teaching Games for Understanding, special issue of Physical Education and Sport Pedagogy,10(3), 241-254..

LIGHT, R. \& GEORGAKIS, S. (2005). Can 'Game Sense' make a difference? Australian pre-service primary school teachers' responses to 'Game Sense' pedagogy in two teacher education programs. Available under http://www.aare.edu.au/05pap/geo05240.pdf[02.02.2011]

LIGHT, R. (2006). The games approach to coaching. Journal of Physical Education New Zealand, 39(1).

LIN, H. (2006). A Meta-analysis of Contextual Interference Effects on Motor Learning.Master's Thesis. Graduate Institute of Sport and Leisure Education, China.

LOIBL, J. (1991). Erfahrungsorientiertes Lehren und Lernen im Sportspiel. In S. REDL; R. SOBOTKA \& A. RUSS (Hrsg.), Sport im der Wende (S. 191-199). Wien: Österreichischer Bundesverlag.

LOIBL, J. (2001). Basketball: genetisches Lehren und Lernen. Schorndorf: Hofmann.

LÓPEZ, R. A. \& MORENO, J.A. (2002a). Aprendizaje de hechos y conceptos en Educación Física: Una propuesta metodológica. Apunts: Educación Física y Deportes, 69, 18-26.

LÓPEZ, R. A. \& MORENO, J.A. (2002b). Integralidad, variabilidad y diversidad en Educación Física. Revista digital Lecturas: Educación Física y Deportes, 19, disponible en www.efdeportes.com.

LOY, R. (2005). Zur Diagnostik Taktischer Leistungen im Sportspiel. Dissertation zum Erwerb des akademischen Grades Dr. phil. im Fachbereich 2 Erziehungswissenschaft, Psychologie, Sport- und Bewegungswissenschaft Universität Duisburg-Essen.

LUCASSEN, M. (2010). Das 1 gegen 1 als Basis individueller Qualität. Fußballtraining, 1 + 2, 15.

MANDIGO, J. L. \& HOLT, N. L. (2004). Reading the game: Introducing the notion of games literacy. Physical \& Health Education Journal, 70(3), 4-10. 
MANDIGO, J.; BUTLER, J.I.\& HOPPER, T. (2007). What is teaching games for understanding? A Canadian perspective. The PHE, 73(2), 14-20.

MANZUR, J. E. \& STEINWECK-FLECKNER, E. (2006). Lernen und Verhalten (6. Auf.). München: Pearson Studium.

MARTIN, D. (1977). Grundlagen der Trainingslehre. Teil I. Schondorf: Hofmann.

MARTIN, D.; CARL, K. \& LEHNERTZ, K. (1991). Handbuch Trainings/ehre. Schorndorf.

MAHLO, F. (1974). Die Ausbildung von Spielfähigkeiten im Sportunterricht. Körpererziehung, 24, 12, 550558.

MATVEEV, L. (1980). Fundamentos del entrenamiento deportivo. Moscú: Raduga.

MCGREGOR, S. J.; HULSE, M. \& STRUDWICK, A. (2002). The reliability and validity of two test of soccer skills. In W. SPINKS;T. REILLY\& A. MURPHY (Eds.),Science and Football IV. London: Routledge.

MCMORRIS, T. (1998). Teaching games for understanding: Its contribution to the knowledge of skillacquisition from a motor learning perspective. European Journal of Physical Education, 3(3), 65-74.

McPHERSON, S.(1999). Tactical differences in problem representations and solutions in collegiate varsity and beginner female tennis players. Research Quarterly for Exercise and Sport, 70(4), 369-384.

McPHERSON, S. \& THOMAS, J. (1989). Relation of knowledge and performance in boys' tennis: age and expertise. Journal of experimental child psychology, 48, 190-211.

McPHERSON, S.\& FRENCH, K. E. (1991).Changes in cognitive strategies and motor skill in tennis. Journal of Sport and Exercise Psychology, 13, 26-41.

McPHERSON, S. \& KERNODLE, M. W. (2003). Tactics, the neglected attribute of expertise: Problem representations and performance skills in tennis. In J. L. STARKES \& K. A. ERICSSON (Eds.), Expert performance in sports: Advances in research on sport expertise (pp. 137-168). Champaign, IL: Human Kinetics.

MECHLING, H. (1984). Bewegungswissenschaft. In K. Carl, H. Kayser, H. Mechling \& W. Preising (Hrsg.),Handbuch Sport: Wissenschaftliche Grundlagen von Unterricht und Training. Bd. 1 (S. 83-134) Düsseldorf: Schwann.

MECHLING, H. (1999). Fähigkeit - Fertigkeit: Generalität versus Spezifität im Techniktraining. In J. WIEMEYER (Hrsg.), Techniktraining im Sport (S. 31-46). Darmstadt: IFS/TUD.

MECHLING, H. (2005). Zur Vereinbarkeit von Erkenntnisfortschritt: Praxisanforderungen und universitären Entwicklungen in der „Sportmotorik“. In K. BLISCHKE; D. BÜSCH; C. IGEL; F. MARSCHALL\& H. MÜLLER (Hrsg.), Sportmotorik. Verfügbar unter http://www.sportmotorik2005.de/pdf/abstract mechling.pdf [25.09.2008]

MEINEL, K. \& SCHNABEL,G. (1998). Bewegungslehre - Sportmotorik. Berlin: Sportverlag. 
MEIRA, C. M. JR. \& TANI, G. (2003).Contextual interference effects assessed by extended transfer trials in the acquisition of the volleyball serve. Journal of Human Movement Studies, 45(5), 449-468.

MEMMERT, D. (2000). Sportspielübergreifende Schulung von Technik- und Taktikbausteinen. Handballtraining, 3/4, 2-6.

MEMMERT, D. \& ROTH, K. (2003). Individualtaktische Leistungsdiagnostik im Sportspiel. Spektrum der Sportwissenschaft,15(1), 44-70.

MEMMERT, D. \& ROTH, K. (2007). Förderung von spielerischer Kreativität mit dem sportspielübergreifenden Konzept der Ballschule Heidelberg. In R. NAUL \& U. WICK (Hrsg.), 20 Jahre dvs-Kommission Fußball Herausforderung für den Fußballsport in Schule und Sportverein Beiträge und Analysen zum Fußballsport XVI. Schriften der Deutschen Vereinigung für Sportwissenschaft, Band 193. Hamburg: Czawalina.

MERTENS, B. \& MUSCH, E. (1990). A methodological sport game concept applied to basketball.Paper presented at the AIESEP World Convention: Moving towards excellence, Loughborough, UK.

METZLER, M. W. (2005a). Instructional models for physical education (1st Ed.). Boston, MA: Allyn and Bacon.

METZLER, M. W. (2005b). Implications of model based intruction for research on teaching: a focus on teaching games for understanding. In L.L. GRIFFIN \& J.I. BUTLER (Eds.), Teaching Games for Understanding (pp. 183-192), Champaign, IL.: Human Kinetics.

MILLER, P. (1993). Theorien der Entwicklungspsychologie.Heidelberg: Spektrum.

MITCHELL, S. A. (2005). Teaching and learning games at the elementary level. In L. GRIFFIN \& J.I. BUTLER (Eds), Teaching Games for Understanding: Theory, Research and Practice (pp. 55-70), Champaign, IL: Human Kinetics.

MITCHELL, S. A.; OSLIN, J. L. \& GRIFFIN, L. L. (1995). The effects of two instructional approaches on GP.Pedagogy in practice: teaching and coaching in physical education and sports, 1(1), 36-48.

MITCHELL, S.A.; GRIFFIN, L.L. \& OSLIN,J.L. (1995). An analysis of two instructional approaches to teaching invasion games. Research Quarterly for Exercise and Sport, 66, A-65.

MITCHELL, S. A.; OSLIN, J. L. \& GRIFFIN, L. L. (2006).Teaching sport concepts and skills: A tactical games approach (2nd Ed.). Champaign: Human Kinetics.

MUSCH, E. \& MERTENS, B. (1991). L'enseignement des sports collectifs: Une conception elaborée a l'isep de l'université de gand. Revue de l'Education Physique,31(1): 7-20.

MUSCH, E.; MERTENS, B.; TIMMERS, E.; MERTENS, T.; GRAÇA, A.; TABORSKY, F.; REMY, C.; DE CLERCQ, D.; MULTAEL, M. \& VONDERLYNCK, V. (2002). An innovative didactical invasion games model to teach basketball and handball, presented on cd. Paper presented at the 7th Annual Congress of the European College of Sport Science, Athens. Greece.

MUNZ, F. (2008).Fußball Trix. 3. Auf. München: Streetspot. 
MUNZERT, J.(1987). Schema-Repräsentation bei der sensomotorischen Regulation. Sportwissenschaft, $17,411-422$

MUNZERT, J. (1989). Flexibilität des Handels: Theoretische Überlegungen und experimentelle Untersuchungen zum Konzept des Motorikschemas. Köln: bps.

MUNZERT, J. (1995). Bewegung als Handlung verstehen. In R. PROHL \& J. SEEWALD (Hrsg),Bewegung verstehen, (S. 77-95). Schorndorf: Hofmann.

MUNZERT, J. (1997). Sprache und Bewegungsorganisation. Schorndorf: Hofmann.

NAGEL, V. \& SPRECKLESLS, C. (2010). Mit Ballspielen zum Tennis (2. Auf.). Aachen: Meyer \& Meyer.

NEUMAIER, A. (1999). Koordinatives Anforderungsprofil und Koordinationstraining: Grundlagen, Analyse, Methodik. Köln: Sport und Buch Strauss.

NEUMAIER, A. \& MECHLING, H. (1994). Taugt das Konzept der koordinativen Fähigkeiten als Grundlage für sportartspezifisches Koordinationstraining ? In P. BLASER; K. WITTE \& CHR. STUCKE (Hrsg.), Steuer- und Regelvorgänge der menschlichen Motorik, (S. 207-212) 2. Symposium der dvs-Sektion Sportmotorik vom 27.-29.01.1994 in Magdeburg. St. Augustin.

NEUMAIER, A. \& MECHLING, H. (1995). Allgemeines oder sportartspezifisches Koordinationstraining. Leistungssport, 5, 14-18.

NEWELL, K.M. \& RUSELL, D.M. (2007). How persistent and general is the contextual interference effect? Research Quarterly of Exercise and Sport, 78, 18-27.

NIEBER, L. \& GLASAUER, G.J. (2000).Zur Methodik eines theoriegeleiteten Koordinationstrainings im Basketball. Leistungssport, 30, 6, 39-49.

NIEDLICH, D. \& SCHMIDT, D. J. (1996). Taktiktraining: Grundlagen. In G. HAGEDORN; D. NIEDLICH\& D.J. SCHMIDT (Hrsg.),Das Basketball Handbuch. Reinbek: Rowohlt.

NITSCH, J. R. (2000). Handlungstheoretische Grundlagen der Sportpsychologie. In H. GABLER; J. R. NITSCH\& R. SINGER (Hrsg.), Einführung in die Sportpsychologie. Teil 1: Grundthemen. (Bd. 2, 3. Aufl., S. 43-164). Schorndorf: Hofmann.

NITSCH, J. R. \& MUNZERT, J. (1997). Handlungstheoretische Aspekte des Techniktrainings - Ansätze zu einem integrativen Modell. In J. R. NITSCH; A. NEUMAIER; H. DE MARÉES \& J. MESTER (Hrsg.), Techniktraining. Beiträge zu einem interdisziplinären Ansatz (S. 50-71). Schorndorf: Hofmann.

NORTH, K. (1998). Wissensorientierte Unternehmensführung. 4. Auf. Wiesbaden: Gabler.

OLIVEIRA, J. \& GRAÇA, A. (2005). O Ensino dos Jogos Desportivos. Porto: Faculdade de Ciencias Desportivas Educação Física.

OSLIN, J. L. (2005). The role of assessment in teaching games for understanding. In L. L. GRIFFIN \& J. I. BUTLER (Eds.), Teaching games for understanding: Theory, research and practice (pp. 125-136). Champaign, IL: Human Kinetics. 
OSLIN, J. L.; MITCHELL, S. A. \& GRIFFIN, L. L. (1998). The GP Assessment Instrument (GPAI): Development and preliminary validation. Journal of Teaching in Physical Education, 17(2), 231-243.

OSLIN, J.L. \& MITCHELL, S, A. (1998). An investigation of tactical transfer in net games.Research Quarterly for Exercise and Sport.66, Supplement, A-98.

OSLIN, J. L\& MITCHELL, S. (2005). Game centred approaches to teaching physical education In D. KIRK, D. MACDONALD \& M. O'SULLIVAN (Eds.), Handbook of Physical Education. London: Sage Publications.

OWEN, A.; TWIST, C. \& FORD, P. (2004). Small Sided games: The physiological and technical effect of altering pitch size and player numbers. Insight, The Football Association Coaches Association Magazine, $7(2), 50-53$.

O'DONOVAN, T. M. (2003). A changing culture?.Interrogating the dynamics of peer affiliations over the course of a sport education season.European Physical Education Review, 9(3), 237-251.

PAOUR, J.-L.; CĖBE, S. \& HAYWOOD, H.C. (2000). Learning to Learn in Preschool Education. Journal of Cognitive Education and Psychology, 1, No. 1.

PELE \& FISH, R.L. (2007). My life and the beautiful game: the autobiography of soccer's greatest star. New York: Skyhorse Publishing, Inc.

PEREZ, C. R.; MEIRA, J. \& TANI, G. (2005). Does the contextual interference effect last over extended transfer trials? Perceptual and Motor Skills, 100(1), 58-60.

PEYRÓ, R. \& SANPEDRO, J. (1986). Pedagogía del baloncesto.Valladolid: Miñon.

PIAGET, J. (1972). Intellectual evolution from adolescence to adulthood.Human Development, 15(1), 112.

PIAGET, J. (1975). Nachahmung Spiel und Traum. Gesammelte Werke (Band 5). Stuttgart: Klett.

PIERON, J.P. (1998). Para una enseñanza eficaz de las actividades físicas. Sevilla: Wanceulen.

PILL, S. (2008). Involving students in the assessment of game performance in physical education. Paper presented at the Flinders University Play to Educate Sport in Education Conference, January 21. Available under http://caef.flinders.edu.au/assets/files/Shane Pill Game Assessment.pdf[28.04.2010]

PORTER, J. M. \& MAGILL, R. A. (2004). The effects of practicing a golf putting task moving along the contextual interference continuum. Journal of Sport \& Exercise Psychology (supplement), 26, S151.

PRATT, D. D. (2002). Good teaching: one size fits all?.In An Up-date on Teaching Theory, Jovita RossGordon (Ed.), San Francisco: Jossey-Bass.

PROBST, G.; RAUB, S. \& ROMHARDT, K. (1998).Wissen managen: wie Unternehmen ihre wertvollste Ressource optimal nutzen (2. Aufl.). Frankfurt am Main/Wiesbaden: Gabler. 
RAAB, M. (2000). SMART Techniken des Taktiktrainings - Taktiken des Techniktrainings. Köln: Sport und Buch Strauss.

RAAB, M. (2001). SMART: Ein dynamisches Zwei-Prozess-Modell zur Erklärung von Entscheidungsverhalten im Sport. In J. R. NITSCH \& H. ALLMER (Hrsg.), Denken, Sprechen, Bewegen (S. 215-220). Köln: bps.

RAAB, M. (2007). Think SMART, not hard: a review of teaching decision making in sport from an ecological rationality perspective, Physical Education and Sport Pedagogy,12(1), pp. 1-22.

REICH, K. (2006). Konstruktivistische Didaktik: ein Lehr- und Studienbuch inklusive Methodenpool auf $C D$. Weinheim: Beltz.

REICH,K. (o.J.a). Einführung in der interationistische Konstruktivismus. Verfügbar unter http://www.unikoeln.de/hf/konstrukt/texte/download/einfuehrung.pdf [28.08.2011]

REICH, K. (o.J.b). Konstruktivistische Didaktik auf dem Weg, die Didaktik neu zu erfinden. Verfügbar unter http://www.uni-koeln.de/hf/konstrukt/reich works/aufsatze/reich 47.pdf [28.08.2011].

REICH, K. (2007). Unterrichtsmethoden im konstruktiven und systemischen Methodenpool. Verfügbar unter

http://methodenpool.uni-koeln.de/index.html [28.08.2011].

REILLY, T. (2005).Training Specificity for Soccer.International Journal of Applied Sports Sciences,17(2), 17-25.

REINMANN-ROTHMEIER, G. \& MANDL, H. (2001). Wissen. In Lexikon der Neurowissenschaften (Band 3, S. 466), Heidelberg: Spektrum.

RICHARD, J-F.; GODBOUT, P. \& GREHAIGNE, J.F. (2000).Students' precision and interobserver reliability of performance assessment in team sports. Research Quarterly for Exercise and Sport. 71:1. 8591.

RICHARD, G-F.\& WALLIAN, N. (2005).Emphasizing Student Engagement in the Construction of Game Performance.In L. GRIFFIN \& J. BUTLEr (Ed.).Teaching Games for Understanding: Theory, Research and Practice. Champaign, IL: Human Kinetics.

RINK, J. E. R.; FRENCH, K,E. \&GRAHAM, K.C. (1996). Implications for practice and research.Journal of Teaching in Physical Education, 15(4), 490-502.

RINK, J. E. R.; FRENCH, K,E. \& TJEERDSMA, B. L. (1996). Foundations for the learning and instruction of sport and games.Journal of Teaching in Physical Education, 15(4), 399-417.

ROSENDAHL, J. (1999). Taktiktraining im Kindes- und Jugendalter am Beispiel Basketball. Seminararbeit für die Vorlesung Kinder- und Jugendtraining. Verfügbar unter

http://www.bbcoach.de/TaktiktrainingSS99.pdf[09.12.2009]

ROTH, K. (1989). Taktik im Sportspiel. Schorndorf: Hofman. 
ROTH, K. (1993). Wie verbessert man die koordinativen Fähigkeiten?. In Bielefelder Sportpädagogen: Methoden im Sportunterricht, ein Lehrbuch in 13 Lektionen (S. 85-97). Schorndorf: Hofmann.

ROTH, K. (1998). Wie verbessert man die koordinativen Fähigkeiten?. In Bielefelder Sportpädagogen (Hrsg.), Methoden im Sportunterricht (S. 84-101). Schorndorf: Hofmann.

ROTH, K. (1999). Das ABC des Spielens: Technik- und Taktiktraining im Anfängerbereich. In WIEMEYER (Hrsg.), Techniktraining im Sport. 13. Darmstädter Sport-Forum. Darmstadt: Institut für Sportwissenschaft der Technischen Universität.

ROTH, K. (2000). Die Straßenspielhypothese oder das Modell der inzidentellen Inkubation: ein Erklärungsansatz für die Kreativitätsentwicklung im Sportspiel. In W. SCHMIDT (Hrsg.), Sportspielforschung: Gestern - heute - morgen. Hamburg: Czwalina.

ROTH, K.; KRÖGER, C. \& MEMMER, D. (2007). Ballschule Rückschlagspiele,2. Auf. Schorndorf: Hofmann.

ROTH, K.; MEMMERT, D. \& SCHUBERT, R. (2006). Ballschule Wurfspiele. Schorndorf: Hofmann

ROTH, K. \& MEMMERT, D. (2007). Ballschule Heidelberg: spielerische, vielseitige und implizite Förderung der taktischen Kreativität, Motorik, 30, 202-209.

ROVEGNO, I.; NEVETT, M. \& BABIARZ, M. (2001).Chapter 5. Learning and teaching invasion-game tactics in 4th grade: Introduction and theoretical perspective. Journal of teaching in physical education [Monograph], 20, 341-351.

ROWLEY, J. (2007). The wisdom hierarchy: representations of the DIKW hierarchy. [Electronic Version] Journal of Information Science, 33, 163-180.

SAMMER, M. (2008). DFB Ausbildungskonzeption: die Kinder frei spielen lassen. Fußballtraining, $1+2$.

SANCHEZ, B. F. (1986). Bases para una didáctica de la Educación Física y el Deporte.Madrid: Gymnos.

SEILER, R. (1995). Kognitive Organisation von Bewegungshandlungen: Empirische Untersuchungen mit dem Inversionsprinzip. Köln: Deutschen Sporthochschule.

SEEL, N. (2003). Psyichologie des Wissens: Lehrbuch für Pädagogen und Psychologen. München: Ernst Reinhardt.

SCHACK, T. (2002). Zur kognitiven Architektur von Bewegungshandlungen:modelltheoretischer Zugang und experimentelle Untersuchungen [Habilitation Thesis]. Köln: DSHS Köln.

SCHACK, T. (2007). Repräsentation und Bewegungssteuerung: die kognitiv-perzeptuelle Perspektive. Zeitschrift für Sportpsychologie, 14(3), 104-113.

SCHAUB, H. (1993). Modellierung der Handlungsorganisation. Bern: Hueber.

SCHMIDT, R. (1975). A Schema Theory of Discrete Motor Skill Learning.Sciences Reviews,82(4), 225260. 
SCHMIDT, R. (1988). Motor Control and Learning: a Behavioral Approach. Champaign, IL.: Human Kinetics.

SCHMIDT, R. (1991). Motor Learning and Performance: From principles to practice. Champaign, IL.: Human Kinetic Books.

SCHMIDT, R. \& WRISBERG, C. A. (2008).Motor Control and Learning: a Behavioral Approach (4 th Ed.). Champaign, IL.: Human Kinetics.

SCHNABEL, G.; HARRE, D. \& BORDE, A. (1994).Trainingswissenschaft: Leistung - Training Wettkampf: Berlin: Sportverlag Berlin.

SCHNABEL, G.; HARRE, H.-D. \& KRUG, J. (2009).Trainingslehre -Trainingswissenschaft. Aachen: Meyer \& Meyer.

SCHRÖDER, J. (2001). Didaktisches Wörterbuch (3. Aufl.). Oldenburg: Wissenschaftsverlag.

SCHULZ, W. \& ELSÄßER, A. (2010). Didaktisch-Methodische Vorüberlegungen zum VolleyballSpielkonzept. Verfügbar unter http//:www.volleyball-trainieren.de/download/spielkonzept.pdf [12.12.2010].

SEILER, R. (1995). Kognitive Organisation von Bewegungshandlungen.Sankt Augustin: Academia.

SEKIYA, H. (2006). Contextual interference in implicit and explicit motor learning.Percept Mot Skills,103(2), 333-43.

SHARMA, N. (2008). The Origin of the Data Information Knowledge Wisdom Hierarchy.Verfügbar unter http://www-personal.si.umich.edu/ nsharma/dikw origin.htm[04.07.2008].

SHEA, J. \& MORGAN, R.L. (1979).Contextual Interference Effects on the Acquisition, Retention, and Transfer of a Motor Skill.Journal of Experimental Psychology: Human Learning and Memory. 5(2) 5, 179187.

SHUELL, T. J. (1987).Cognitive perspectives of learning.Review of Educational Research, 56 (4), 411 436.

SIEDENTOP, D. (1994). Sport education: Quality PE through positive sport experiences. Champaign, IL.: Human Kinetics.

SIEDENTOP, D. (1996). Physical education and educational reform: The case of sport education. In S. SILVERMAN \& C. ENNIS (Eds.), Student learning in physical education (pp. 247-267). Champaign, IL.: Human Kinetics,

SIEDENTOP, D. (1994). Sport education: quality P. E. through positive sport experiences. Champaign, IL: Human Kinetics.

SIEDENTOP, D.; HASTIE, P.A. \& VAN DER MARS, H. (2004). Complete guide to sport education. Champaign, IL.: Human Kinetics. 
SILVERMAN, S. (1997). Is the tactical approach to teaching games better than a skills approach?.JOPERD, 68(7), 5 .

SIMON, D.A. (2007). Contextual interference effects with two tasks. Percept Mot Skills,105(1), 177-83.

SIMON, H. A. \& CHASE, W. G. (1973).Skill in chess.American Scientist, 61, 394-403.

SINNIG, I. (2008). Ist die Ballschule ein überschätztes Konzept?.Sportpädagogie, 1, 44-46.

SNOW, S. \& THOMAS, J. (2005).Small Sided Games Manual.Us Youth Soccer Coaching Education Department. Available under http://www.usyouthsoccer.org/doc lib/small sided games manual.pdf[03.07.2009]

SOLSO, R. (2005). Kognitive Psychologie. Heidelberg: Springer.

STENBERG, R.J. (1984). A theory of knowledge acquisition in the development of verbal concepts.Develomental Review, 4, 113-138.

STIEHLER, G.; KONZAG, I. \& DÖBLER, H. (1988). Theorie und Methode der Sporspiele: Basketball, Fußball, Handball, Volleyball. Berlin: Sportverlag.

STOKES, P. D.; LAI, B.; HOLTZ, D.; RIGSBEE, E.\& CHERRICK, D. (2008).Effects of practice on variability, effects of variability on transfer.Journal of Experimental Psychology: Human Perception and Performance, Vol 34(3), 640-659.

Strand, B.N. \& Wilson, R. (1993).Assessing sport skills. Champaign, IL: Human kinetics.

TALLIR, I.; MUSCH, E.; LENOIR, M. \& VALCKE, M. (2004).Assessment of game play in Basketball. In Proceedings of the $2^{\text {nd }}$ international conference: teaching Sport and physical education for understanding. Available under

http://www.conferences.unimelb.edu.au/sport/proceedings/PROCEEDINGS\%20-\%20tallir\%201.pdf [07.12.2009]

TALLIR, I.B.; LENOIR, M.; VALCKE, M. \& MUSCH, E. (2007). Do alternative instructional approaches result in different game performance learning outcomes?.Authentic assessment in varying game conditions.International Journal of Sport Psychology, 38(3), 263-282.

TSCHIENE, P. \& BARTH, B. (1997). Die Wettkampfvorbereitung. In G. THIESS ;P. TSCHIENE\& H. NICKEL, Der sportliche Wettkampf (S. 60-111). Münster: Philippka-Sportverlag.

THOMAS, K. T. \& THOMAS, J. R. (1994).Developing expertise in sport: The relation of knowledge andperformance.International Journal of Sport Psychology, 25(3), 295-312.

THORPE, R. (1989). A changing focus on teaching games for understanding. In L. ALMOND (Ed.),The place of physical education in schools. (pp.42-71). London: Kogan Page.

THORPE, R. (1990). New directions in games teaching. In N. ARMSTRONG (Eds.), New directions in P.E.: Vol. 1 (pp. 79-100). Champaign, IL: Human Kinetics. 
THORPE, R. (2001). Rod Thorpe on teaching games for understanding. In L. KIDMAN (Ed.),Developing decision makers: An empowerment approach to coaching (pp.22-36). Christchruch: Innovate Print Communication.

THORPE, R. \& BUNKER, D. (1986). Is there a need to reflect on our games teaching?.In R.THORPE; D. BUNKER \& L. ALMOND (Eds.),Rethinking games teaching (pp.25-34). Louborough: University of Technology, Department of Physical Education and Sport Science.

TIMMERS, E.; MASSINK, M.; MEERSTENS, T.; ROODE, D. \& SCHUSTER, H. (2009). Aktives und selbstständiges Lernen eines Sportspiels. Sportunterricht, 8(58), 240-241.

TSG 1899 HOFFENHEIM (2008). Ausbildungsphilosophie: Anpfiff ins Leben. Hoffenheim: TSG 1899 Hoffenheim Fußball-Spielbetrieb GmbH.

TURNER, A. P. (2003). A comparative analysis of two approaches for teaching tennis: games for understanding approach versus the technique approach (Abstract). In 2nd International conference: teaching sport and physical education for understanding, Melbourne: Vic., University of Melbourne.

TURNER, A. P. \& MARTINEK, T. J. (1992).A comparative analysis of two models for teaching games (technique approach and game centered (tactical focus) approach).International Journal of Physical Education,29(4), 15-31.

TURNER, A. P. (1996a). Teaching for Understanding: Myth or Reality?.Journal of Physical Education, Recreation \& Dance, 67(4), 46-48.

TURNER, A. P. (1996b). Teachers' perceptions of technical and tactical models of instruction.Research Quarterly for Exercise and Sport.March, Supplement, A-90.

TURNER, A. P. \& MARTINEK, T,J. (1995). Teaching for Understanding: A model for improving decision making during game play. Quest,47, 44-63.

TURNER, A. P., \& MARTINEK, T. J. (1999). An investigation into teaching games for understanding: Effects on skill, knowledge, and game play. Research Quarterly for Exercise and Sport, 70(3), 286-296.

TURNER, A. P.; ALLISON, P.\& PISSANOS, B. (2001). Constructing a Concept of Skilfulness in Invasion Games Within a Games for Understanding Context (abstract). Physical Education \& Sport Pedagogy, $6(1), 38-54$.

VICKERS, J. N.; REEVES, M. A., CHAMBERS, K. L. \& MARTELL, S. (2004). Decision training.Cognitive strategies for enhancing motor performance.In A. M. WILLIAMS \& N. J. HODGES (Eds), Skill acquisition in sport. Research theory and practice (pp. 103-120). London: Routledge.

VICKERS, J.N. (2007). Perception, Cognition, and Decision Training: The Quiet Eye in Action.Champaign, III: Human Kinetics.

VOLLMAR, G. (2004). Das Wissen steckt in den Köpfen. Wissensmanagement, 6, 20-22.

WATSON, S. (2010). Small Side Games, why 4 vs.4?.Forum und praktische Ideen online für Fußballtrainern, Available under www.footy4you.com[23.11.2009] 
WAGENSCHEIN, M. (1991). Verstehen lehren. Weinheim und Basel: Beltz.

WALDE, A. (1965). Lateinisches etymologisches Wörterbuch (5. Aufl.). Heidelberg: Winter.

WALLHEAD, T. L. \& NTOUMANIS, N. (2004).Effects of a sport education intervention on students' motivational responses in physical education.Journal of Teaching in Physical Education, 23, 4-18.

WALLHEAD, T. L. \& O'SULLIVAN, M. (2005).Sport Education: physical education for the new millennium.Physical Education and Sport Pedagogy, 10 (2), 181-211.

WEBB, P. \& PEARSON, P. (2008).An integrated approach to teaching games for understanding (TGfU). A paper presented at the 1st Asia Pacific Sport in Education Conference, Adelaide. Available under: http://ro.uow.edu.au/cgi/viewcontent.cgi?article=1053\&context=edupapers\&seiredir=1\#search=\%22Webb + +\&+Pearson, $+2008 \% 29 . \% 22[31.10 .2010]$.

WEIN, H. \& SCHREINER, P. (2004). Entwicklung der Spielintelligenz im Fußball. Heinsberg: CarolusSportverlag.

WEIN, H. (2009). Fußball kindgemäß trainieren.Aachen: Meyer \& Meyer.

WEINECK, J. (2004a). Optimales Fußballtraining (4. Auf.). Balingen: Spitta.

WEINECK, J. (2004b). Optimales Training (4. Auf.). Balingen: Spitta.

WERNER, P., THORPE, R., \& BUNKER, D. (1996).Teaching games for understanding: Evolution of a model. Journal of Physical Education, Recreation and Dance,67(1), 28-33.

WICKSTROM, R. L. (1990). Patrones Motores Básicos. Madrid: Alianza Deporte.

WIEMEYER, J. (1994). Interne Bewegungsrepräsentation. Köln: bps.

WILSON, T. D. (2002). The nonsense of knowledge management.Information Research,8(1), 144-54.

WILLIAMS, M. (2008). Learning Football Skills Effectively: Challenging Tradition. Conference: Development, Enhancement and Sustainability of Expert Performance in Sport. November 13 -14, 2008, The United States Olympic Committee. Available Under:

http://www.thefa.com/GetIntoFootball/FALearning/FALearningPages/ /media/Files/PDF/Get\%20into\%20F ootball/FA Learning YouthModule2/Learning\%20Football\%20Skills\%20Effectively.ashx/Learning\%20Foo tball\%20Skills\%20Effectively.pdf [22.12.2009].

WINKEL, S.; PETERMANN, F. \& PETERMANN, U. (2006). Lernpsychologie.Paderborn: Schöningh.

WOLLNY, R. (1991). Stabilität und Variabilität im motorischen Verhalten, Unveröffentlichte Dissertation, Bielefeld.

WONG, J. A. \& MATSUMOTO, E.D. (2008).Cognitive motor learning for teaching surgical skill: how are surgical skills taught and assessed?.Nature Clinical Practice Urology, 5, 47-54 
WRIGHT, D.; MAGNUSON, E. \& BLACK, B. (2005).Programming and Reprogramming Sequence Timing Following High and Low Contextual Interference Practice.Research Quarterly for Exercise and Sport, 3(76), 258.

ZAAL, A. (2005). Zur Bedeutung der Koordination im niederlandischen Nachwuchsfußball.(Examensarbeit) Nordersedt: Grin.

ZELENY, M. (1987). Management Support Systems: Towards Integrated Knowledge Management. Human Systems Management,7(1), 59-70.

ZHANG, P. (2008). The effects of play practice on teaching table tennis forehand skills. Dissertation: Graduate School Ohio State University. Available under http://etd.ohiolink.edu/sendpdf.cgi/Zhang\%20Peng.pdf?osu1211575204[23.08.2010]

ZIMMER, A. (1991). Kognitive Repräsentationen und Techniktraining. In R. DAUGS; H. MECHLING; K. BLISCHKE \& N. OLIVIER (Hrsg.), Sportmotorisches Lernen und Techniktraining (Bd. 1, S. 191-2002). Schorndorf: Hofmann.

ZIMMERMANN, K. \& BLUME, D. (1998). Koordinative Fähigkeiten und Beweglichkeit. In K. MEINEL \& G. SCHNABEI (Hrsg.), Bewegungslehre: Sportmotorik (S.206-236) Berlin: Sportverlag.

ZIMMER, A. \& KÖRNDLE, H. (1988).A model for hierarchically ordered schemata in the control of skilled motor action.Gestalt Theory, 10, 85-102. 


\section{Abbildungsverzeichnis}

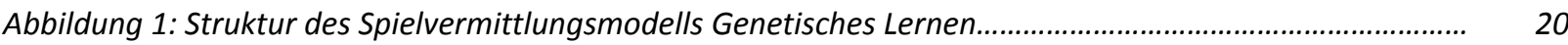

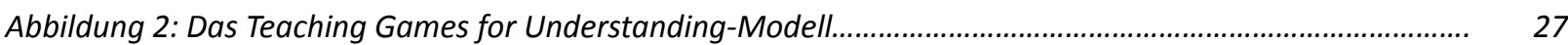

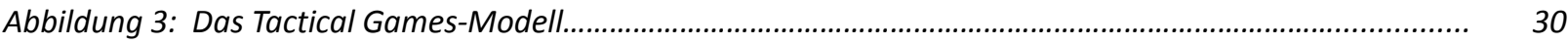

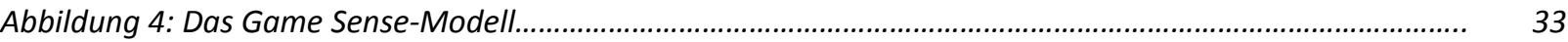

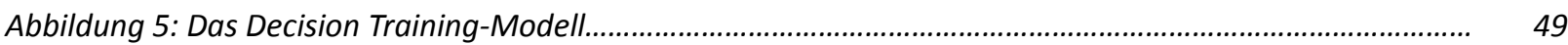

Abbildung 6: SMART: Situation Model for Anticipated Response consequences of Tactical training.................. 51

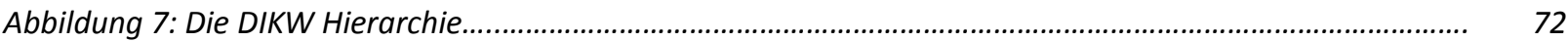

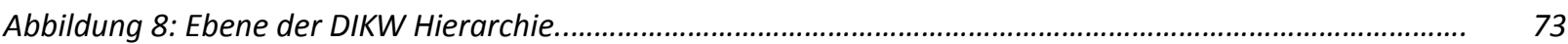

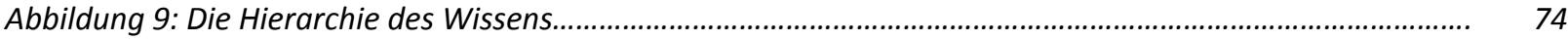

Abbildung 10: Die Hierarchie des Wissens und ihre entsprechenden kognitiven Prozesse..................................... 76

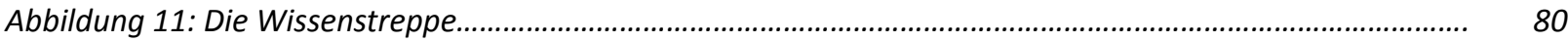

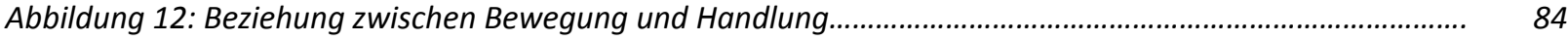

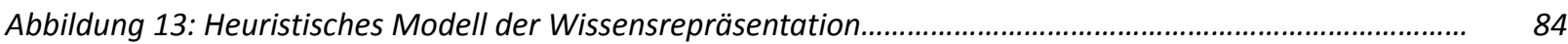

Abbildung 14: Flussdiagramm zur Darstellung der Entstehung und Bestandteile des Handlungswissens

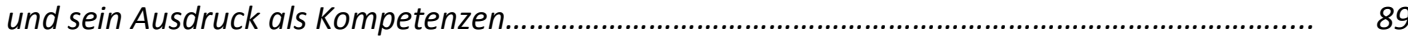

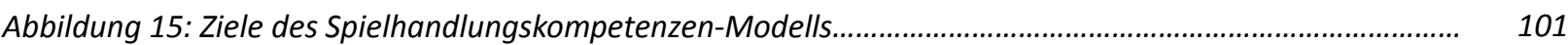

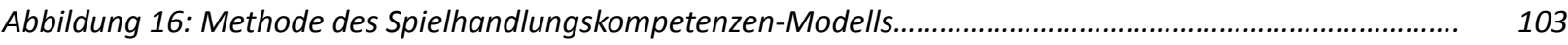

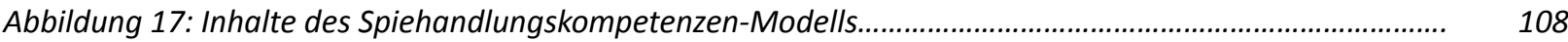

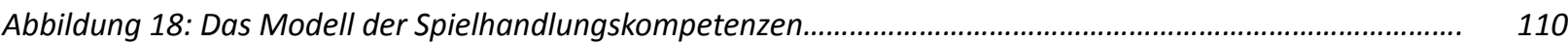

Abbildung 19: Anwendungsstruktur des Modells der Spielhandlungskompetenzen..........................................112

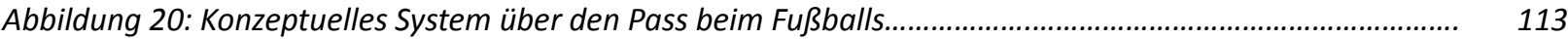

Abbildung 21: Beispiel Trainingssitzung mit Umsetzung des Spielhandlungskompetenzen-Modells.

Abbildung 22: Kriteriums-Aufgabe: Das Mini-Spiel T4:T4.

Abbildung 23: Beispiel eines Kleinen Fußballspiel mit unterschiedlichen Schwerpunkten.

Abbildung 24: Untersuchungsauflauf der Studie eins. 
Abbildung 26: Untersuchungsablauf der Studie zwei.

Abbildung 27: Entwicklung der Lernens kognitiver Kompetenzen im Fußball.

Abbildung 28: Beispiel eines Kleinen Fußballspiels mit unterschiedlichen Schwerpunkten als Aufgabe der

Spielgruppe.

Abbildung 29: Beispiel einer „geometrischen Übung" als Aufgabe der Übungsgruppe.

Abbildung 30: Untersuchungsablauf Studie vier.

Abbildung 31: Prozent der erfolgreichen Pässe der zwei Gruppen im vor-, Post- und Retentionstest des

Spiel-test.

Abbildung 32: Ergebnisse in Gesamtzeit der zwei Gruppen im Vor, Post- und Retention des

Loughborough-Pass-Test.

Abbildung 33: Beispiel eines Kombinationsspiels

Abbildung 34: Mittelwerte der Variablen des TSAP.

Abbildung 35: Ergebnisse der errechneten Variablen des TSAP. AB: Attack Balls, PB: Played Balls

Abbildung 36: Ergebnisse der errechneten Variablen des TSAP. El: Efficiency Index beim Vor, Post- und Retentionstest 


\section{Tabellenverzeichnis}

Tabelle 1: Gestaltung eines Übungsspiels bei der Situativen Spieldidaktik

Tabelle 2: Struktur des integrativen Modell, Alternativen der didaktischen Reduktion......

Tabelle 3: Stufe des Fußballentwicklungsmodells .....

Tabelle 4: Zugang, Ziele, Inhalte und Methoden der allgemeinen Ballschule.

Tabelle 5: Synoptische Darstellung der Spielvermittlungsmodelle.

Tabelle 6: Schritte zur Beschreibung des Modells der Spielhandlungskompetenzen.

Tabelle 7: Kognitive Kompetenzen und die ihnen zugeordneten kognitiven Operationen.

Tabelle 8: Matrix mit dem Thema „Passen bei Fußballspielen“.

Tabelle 9: Mittelwerte der richtigen Handlungen bei Spielen

Tabelle 10: Prozentzahl der geeigneten Handlungen bei Spielen..

Tabelle 11: Mittelwerte der richtigen Antworten auf die Fragen beim Vortest, Posttest und Retentionstest in der Direkten Instruktionsgruppe (DIG) und der Reflexiven Instruktionsgruppe (RIG)...

Tabelle 12: Prozentzahl der richtigen Antworten auf die Fragen beim Vortest, Posttest und Retentionstest in der Direkten Instruktionsgruppe (DIG) und der Reflexiven Instruktionsgruppe (RIG)......

Tabelle 13: Mittelwerte und Standardabweichung der Variablen in Beziehung zum Pass im Vor-, Post- und Retentionstest bei der Übungsgruppe und Spielgruppe.

Tabelle 14: Ergebnisse in Vor-, Post-und Retentions-Test beim Loughborough-Pass-Test der Übungsgruppe und Spielgruppe.

Tabelle 15: Ergebnisse der zweifaktoriellen Varianzanalyse mit Messwiederholung auf einem Faktor der Variablen ausgewertet mit dem Spieltest.

Tabelle 16: Ergebnisse der zweifaktoriellen Varianzanalyse mit Messwiederholung auf einem Faktor der Variablen ausgewertet mit dem Loughborough-Pass-Test.

Tabelle 17: T-Test für jede Gruppe eines Vergleichs der Mittelwerte im Vor-, Post-und Retentionstest der Variablen Gesamtzeit beim Loughborough-Pass-Test und der Variable EP/P beim Spiel-Test.

Tabelle 18: Mittelwert und Standardabweichungen der Variablen des TSAP...

Tabelle 19: Ergebnisse des Friedman-Test der Variablen des TSAP. 
Anhang 
Anhang A: der Minifußball-Test

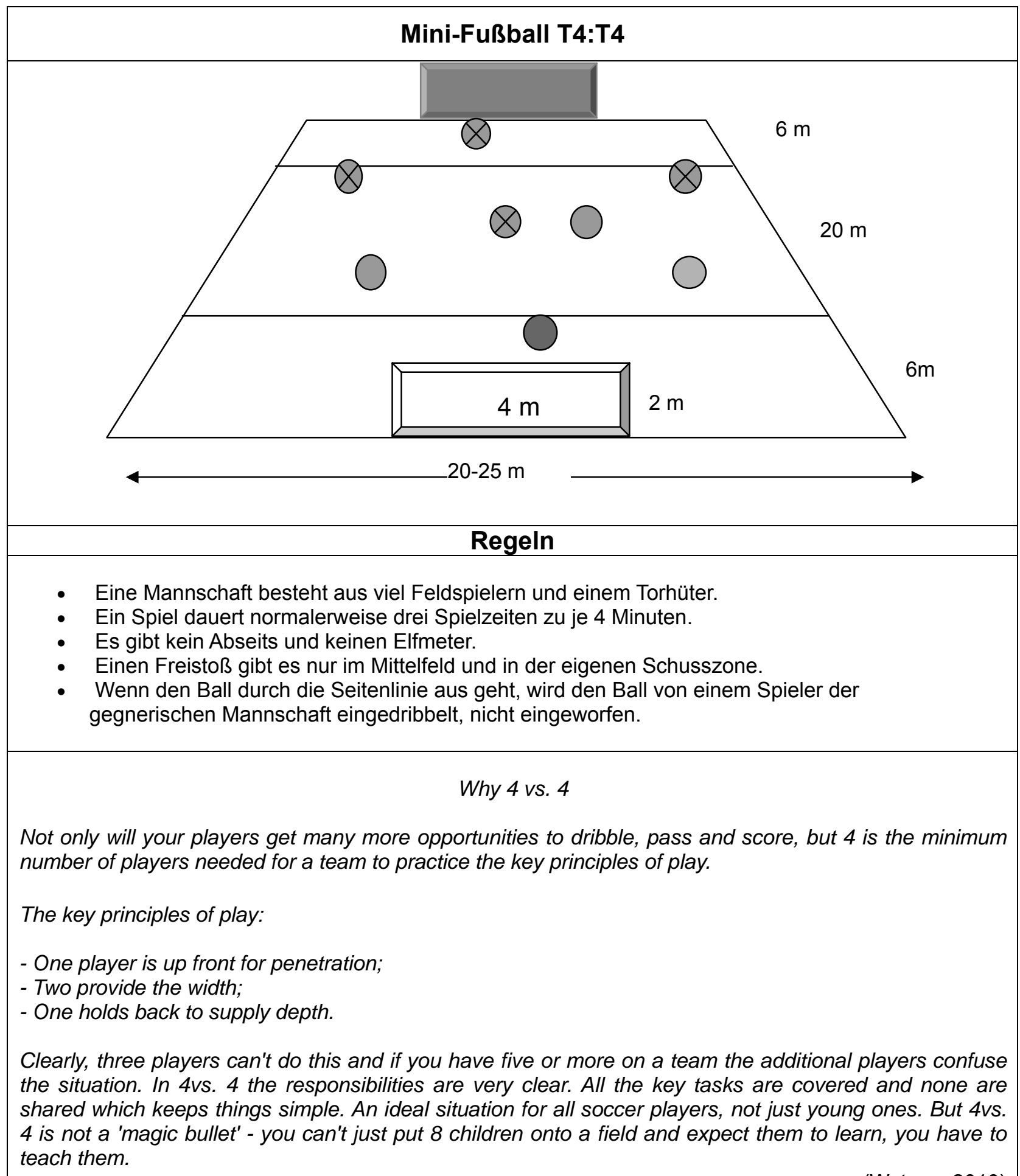

(Watson, 2010) 


\section{Anhang B: Hinweise für die Auswertung der Spielhandlungen}

\begin{tabular}{|c|c|c|}
\hline \multicolumn{3}{|c|}{ Spieler mit Ball (SB) } \\
\hline Handlungen & Geeignet (1) & Nicht Geeignet (0) \\
\hline Passen & $\begin{array}{l}\text { 1. Ein Mitspieler ist frei und hat genug } \\
\text { Raum, um weiter zu Spielen. }\end{array}$ & $\begin{array}{l}\text { 2. Der Mitspieler ist hinter dem Gegner, hat } \\
\text { nicht genug Raum, um weiter zu Spielen oder } \\
\text { ist gedeckt. }\end{array}$ \\
\hline Ball Führen & $\begin{array}{l}\text { 3. Es gibt freien Raum, es gibt keinen bes- } \\
\text { ser platzierten oder freien Mitspieler. }\end{array}$ & $\begin{array}{l}\text { 4. Es gibt einen Mitspieler frei, es gibt keinen } \\
\text { freien Raum. }\end{array}$ \\
\hline Schießen & $\begin{array}{l}\text { 5. Das Tor ist in der Nähe, es gibt genug } \\
\text { Raum und es gibt keine besser platzierten } \\
\text { Mitspieler. }\end{array}$ & $\begin{array}{l}\text { 6. Das Tor ist weit, es gibt nicht genug Raum, } \\
\text { es gibt einen besser platzierten Mitspieler. }\end{array}$ \\
\hline \multicolumn{3}{|c|}{ Spieler ohne Ball Mannschaft mit Ballbesitz (SoBMB) } \\
\hline Position & Geeignet (1) & Nicht Geeignet $(0)$ \\
\hline \multirow[t]{2}{*}{$\begin{array}{l}\text { In der Nähe } \\
\text { des Ballbesit- } \\
\text { zers }\end{array}$} & $\begin{array}{l}\text { 7. Der Spieler läuft frei aus dem Ballbesit- } \\
\text { zer und sucht freie Räume. }\end{array}$ & $\begin{array}{l}\text { 8. Der Spieler läuft zum Ball und versucht, } \\
\text { inn zu erobern. }\end{array}$ \\
\hline & $\begin{array}{l}\text { 9. Der Spieler bietet Stützung zum Ballbe- } \\
\text { sitzer an. }\end{array}$ & $\begin{array}{l}\text { 10. Der Spieler bleibt stehen und nimmt nicht } \\
\text { an der Handlung teil. }\end{array}$ \\
\hline \multirow[t]{2}{*}{$\begin{array}{l}\text { Weit vom Ball- } \\
\text { besitzers }\end{array}$} & $\begin{array}{l}\text { 11. Der Spieler sucht freien Raum und } \\
\text { verlangt den Ball oder bietet sich an. }\end{array}$ & 12. Der Spieler steht hinter dem Gegner. \\
\hline & $\begin{array}{l}\text { 13. Der Spieler nähert sich dem Ballbesit- } \\
\text { zer und sucht Position. }\end{array}$ & $\begin{array}{l}\text { 14. Der Spieler bleibt stehen oder läuft zu } \\
\text { langsam. }\end{array}$ \\
\hline \multicolumn{3}{|c|}{ Spieler ohne Ball Mannschaft ohne Ballbesitz (SoBMoB) } \\
\hline Position & Geeignet (1) & Nicht Geeignet (0) \\
\hline \multirow[t]{3}{*}{$\begin{array}{l}\text { In der Nähe des } \\
\text { Ballbesitzers }\end{array}$} & $\begin{array}{l}\text { 15. Befindet sich zwischen Ballbesitzer und } \\
\text { eigenem Tor. }\end{array}$ & $\begin{array}{l}\text { 16. Befindet sich seitlich oder hinter dem } \\
\text { Ballbesitzer. }\end{array}$ \\
\hline & 17. Reduziert den Raum des Ballbesitzers. & $\begin{array}{l}\text { 18. Geht zurück, ohne den Raum des Ball- } \\
\text { besitzers zu reduzieren. }\end{array}$ \\
\hline & $\begin{array}{l}\text { 19. Wartet auf die bessere Gelegenheit, } \\
\text { um den Ballbesitzer zu attackieren. }\end{array}$ & $\begin{array}{l}\text { 20. Attackiert den Ballbesitzer, ohne auf die } \\
\text { bessere Möglichkeit zu warten. }\end{array}$ \\
\hline \multirow[t]{2}{*}{$\begin{array}{l}\text { Weit des Ball- } \\
\text { besitzers }\end{array}$} & $\begin{array}{l}\text { 21. Läuft in Richtung eigenes Tor und ver- } \\
\text { sucht, die Unterzahl zu verhindern. }\end{array}$ & $\begin{array}{l}\text { 22. Der Spieler bleibt stehen oder läuft zu } \\
\text { langsam. }\end{array}$ \\
\hline & $\begin{array}{l}\text { 23. Deckt einen Gegenspieler, hilft einem } \\
\text { Mitspieler in Abwehr. }\end{array}$ & $\begin{array}{l}\text { 24. Kein Versuch, den Gegenspieler zu de- } \\
\text { cken. }\end{array}$ \\
\hline
\end{tabular}


Anhang C: Formblatt: Auswertung der Spielhandlungen

\begin{tabular}{|l|l|}
\hline Spieler: \\
\hline SB & \\
\hline SoBMB & \\
& \\
\hline SoBMoB & \\
& \\
\hline
\end{tabular}

\begin{tabular}{|l|l|}
\hline Spieler: \\
\hline SB & \\
\hline SoBMB & \\
\hline SoBMoB & \\
& \\
\hline
\end{tabular}

\begin{tabular}{|l|l|}
\hline Spieler: \\
\hline SB & \\
\hline SoBMB & \\
\hline SoBMOB & \\
\hline
\end{tabular}




\section{Anhang D: Beispiel Trainingseinheit für die Schulung der Spielkompetenzen: Abwehr}

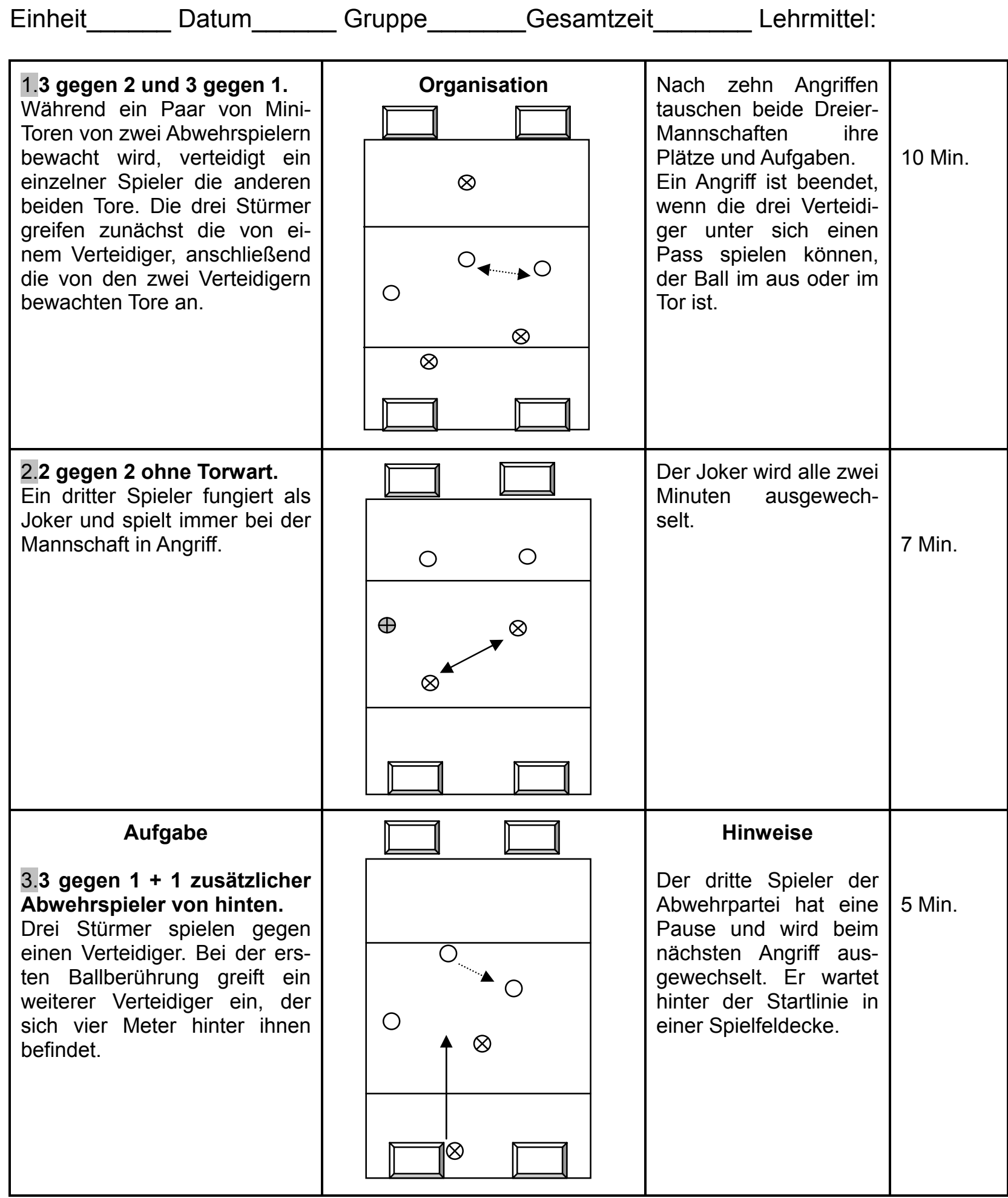




\section{Anhang E: Beispiel Trainingseinheit für die Schulung der Spielkompetenzen: Angriff}

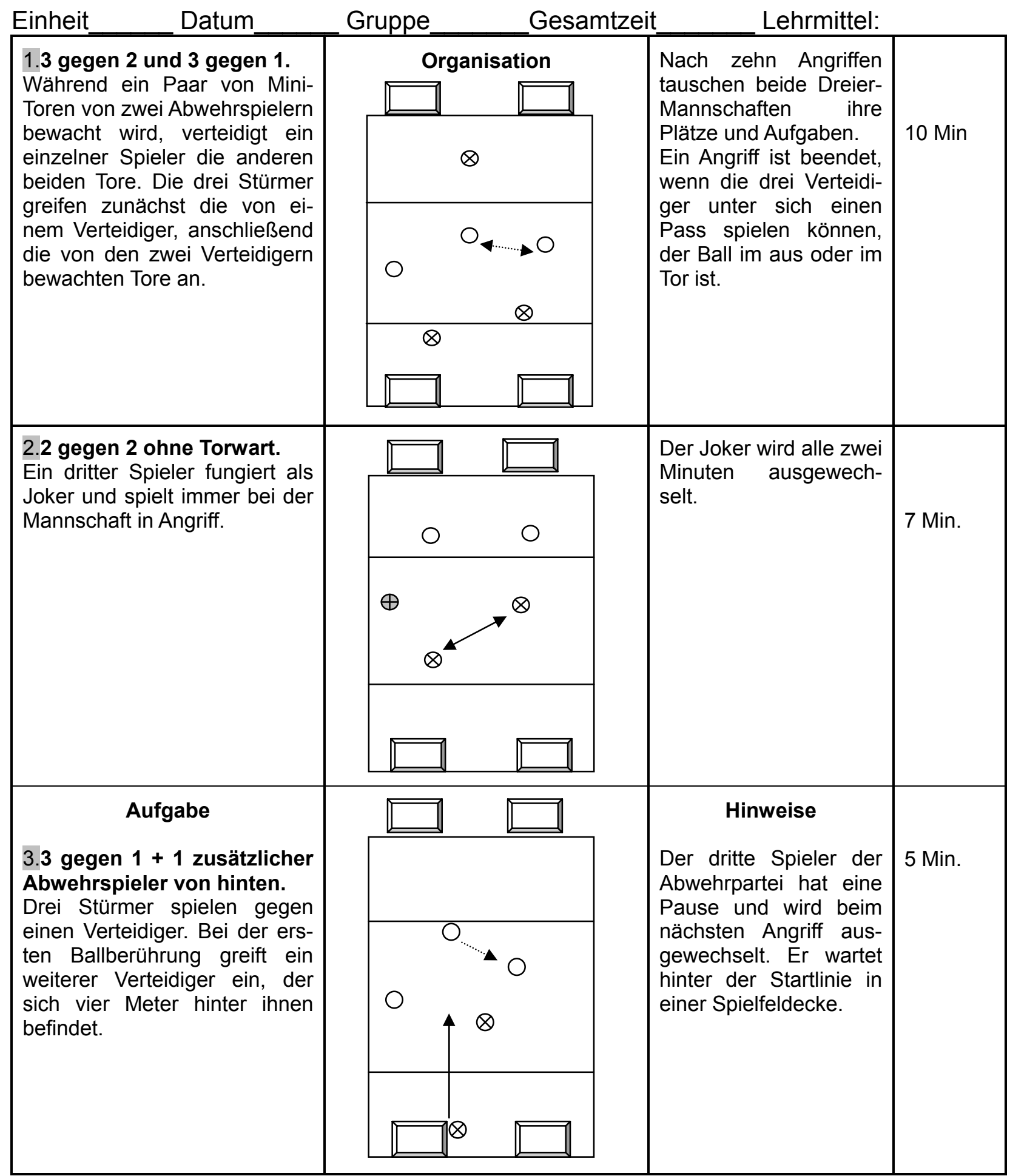




\section{Anhang F: Schulung der Spielkompetenzen, Beispiel Fragen zum Reflektieren}

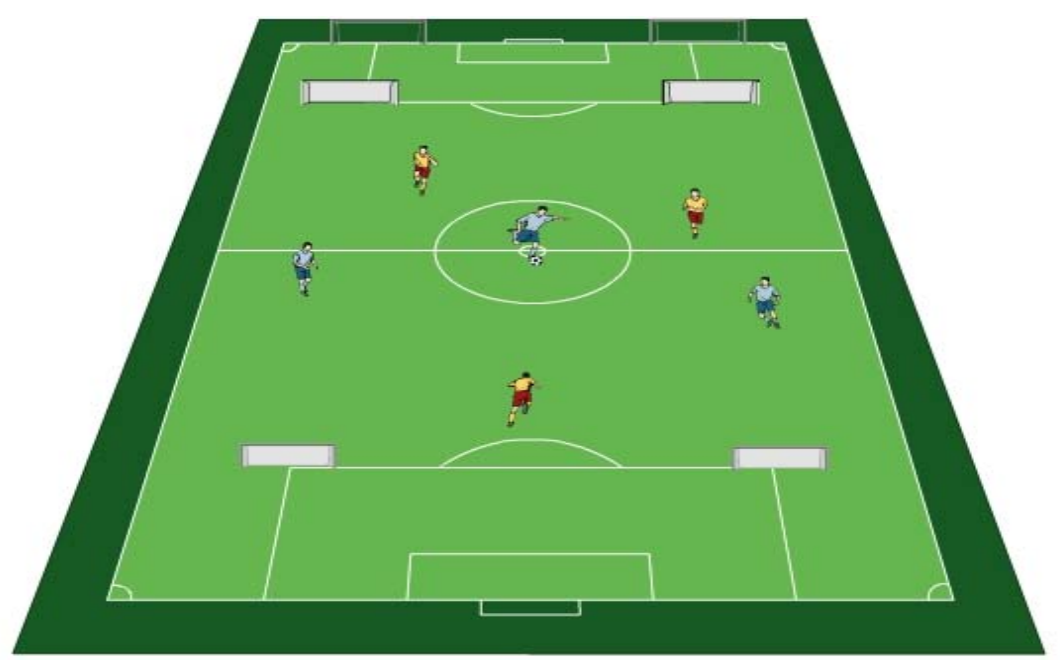

\section{Situation:}

Kleine Spiel 3:3 mit vier Toren. Zwei Spieler haben bei einem Torversuch den Ball verloren, die gegnerische Mannschaft stellt sich nach Ballgewinn für den Konterangriff auf. Die folgenden Fragen kommen dazu:

\section{Angriff}

Wie müssen sich die Mitspieler ohne Ball verhalten?

A: Sie sollten sich immer außerhalb des Aktionsradius des Verteidigers in einer optimalen Position vor einem der beiden Tore anbieten. Die Art und Weise, wie und wen der Verteidiger gerade angreift, bestimmt ihr Spiel ohne Ball.

Welcher Angreifer hat zu Beginn des Angriffs die besten Möglichkeiten, zum Torerfolg zu kommen?

A: Derjenige Stürmer, der im Augenblick eines Abspiels am Weitesten von dem Verteidiger entfernt ist.

\section{Abwehr}

\section{Wie sollte sich der einzige Verteidiger am besten verhalten?}

A: Er sollte den mittleren Angreifer so früh wie möglich zum Abspiel des Balls zu einem Außenstürmer verleiten, da er an der Seitenlinie, wo das Spiel des Außenstürmer beschränkt ist, bessere Abwehrmöglichkeiten besitzt als in der Mitte des Feldes. Er sollte nur dann eingreifen, wenn der Ball für einen Augenblick zu weit vom Fuß des Angreifers entfernt ist. 
Greifen die von hinten kommenden Verteidiger den ballführenden Spieler an oder machen sie besser seine Passwege zu?

A: Während der erste Verteidiger versucht, den individuellen Angriff des mittleren Angreifers zu verzögern, eilen seine beiden Mitverteidiger herbei, um Passwege zu schließen. Die drei Abwehrspieler sollten lernen, sich vor dem Angriff abzustimmen. 
Anhang G: Ergebnisse Test der Spielhandlungen

\section{Pretest}

\begin{tabular}{|c|c|c|c|c|c|c|c|c|c|c|c|c|c|}
\hline $\begin{array}{c}\text { Spie- } \\
\text { lerin }\end{array}$ & \multicolumn{3}{|c|}{ SmB } & \multicolumn{3}{|c|}{ SoBMmB } & \multicolumn{3}{c|}{ SoBMoB } & \multicolumn{3}{c|}{ Gesamt } & HdI. \\
\hline & + & - & Indiz & + & - & Indiz & + & - & Indiz & + & - & Indiz & $\Sigma$ \\
\hline 1 & 4 & 2 & 66,7 & 3 & 2 & 60 & 6 & 6 & 50 & 13 & 10 & 56,5 & 23 \\
\hline 2 & 3 & 5 & 37,5 & 6 & 4 & 60 & 11 & 20 & 35,5 & 20 & 29 & 40,8 & 49 \\
\hline 3 & 2 & 3 & 40 & 8 & 7 & 53,3 & 11 & 18 & 37,9 & 21 & 28 & 42,9 & 49 \\
\hline 4 & 5 & 2 & 71,4 & 6 & 12 & 33,3 & 13 & 12 & 52 & 24 & 26 & 48 & 50 \\
\hline 5 & 5 & 4 & 55,6 & 5 & 3 & 62,5 & 10 & 8 & 55,6 & 20 & 15 & 57,1 & 35 \\
\hline 6 & 3 & 1 & 75 & 3 & 7 & 30 & 4 & 12 & 25 & 10 & 20 & 33,3 & 30 \\
\hline 7 & 2 & 2 & 50 & 4 & 3 & 57,1 & 8 & 5 & 61,5 & 14 & 10 & 58,3 & 24 \\
\hline 8 & 4 & 4 & 50 & 7 & 10 & 41,2 & 13 & 9 & 59,1 & 24 & 23 & 51,1 & 47 \\
\hline 9 & 2 & 5 & 28,6 & 7 & 3 & 70 & 13 & 21 & 38,2 & 22 & 29 & 43,1 & 51 \\
\hline 10 & 1 & 4 & 20 & 4 & 9 & 30,8 & 15 & 12 & 55,6 & 20 & 25 & 44,4 & 45 \\
\hline 11 & 0 & 3 & 0 & 4 & 1 & 80 & 7 & 10 & 41,2 & 11 & 14 & 44 & 25 \\
\hline 12 & 1 & 4 & 20 & 7 & 7 & 50 & 13 & 10 & 56,5 & 21 & 21 & 50 & 42 \\
\hline M & 2,67 & 3,25 & 42,89 & 5,33 & 5,67 & 52,35 & 10,33 & 11,92 & 47,34 & 18,33 & 20,83 & 46,8 & 39,17 \\
\hline S.A. & 1,61 & 1,29 & 22,97 & 1,72 & 3,5 & 15,89 & 3,39 & 5,21 & 11,43 & 4,96 & 7,07 & 7,5 & 11,07 \\
\hline
\end{tabular}




\section{Posttest}

\begin{tabular}{|c|c|c|c|c|c|c|c|c|c|c|c|c|c|}
\hline $\begin{array}{c}\text { Spie } \\
\text { lerin }\end{array}$ & \multicolumn{3}{|c|}{ SmB } & \multicolumn{3}{|c|}{ SoBMmB } & \multicolumn{3}{c|}{ SoBMoB } & \multicolumn{3}{c|}{ Gesamt } & HdI. \\
\hline & + & - & Indiz & + & - & Indiz & + & - & Indiz & + & - & Indiz & $\Sigma$ \\
\hline 1 & 1 & 2 & 33,3 & 6 & 4 & 60 & 15 & 10 & 60 & 22 & 16 & 57,9 & 38 \\
\hline 2 & 2 & 3 & 40 & 6 & 5 & 54,5 & 12 & 12 & 50 & 20 & 20 & 50 & 40 \\
\hline 3 & 3 & 4 & 42,9 & 12 & 5 & 70,6 & 10 & 9 & 52 & 25 & 18 & 58,1 & 43 \\
\hline 4 & 2 & 3 & 40 & 5 & 4 & 55,6 & 16 & 12 & 57,1 & 23 & 19 & 54,8 & 42 \\
\hline 5 & 3 & 1 & 75 & 8 & 7 & 53,3 & 10 & 12 & 45,5 & 21 & 20 & 51,2 & 41 \\
\hline 6 & 3 & 3 & 50 & 10 & 2 & 83,3 & 14 & 13 & 51,9 & 27 & 18 & 60 & 45 \\
\hline 7 & 2 & 5 & 28,6 & 9 & 4 & 69,2 & 12 & 8 & 60 & 23 & 17 & 57,5 & 40 \\
\hline 8 & 5 & 3 & 62,5 & 4 & 4 & 50 & 20 & 5 & 80 & 29 & 12 & 70,7 & 41 \\
\hline 9 & 3 & 1 & 75 & 7 & 6 & 53,8 & 13 & 10 & 56,5 & 23 & 17 & 57,5 & 40 \\
\hline 10 & 3 & 3 & 50 & 7 & 5 & 58,3 & 19 & 10 & 65,5 & 29 & 18 & 61,7 & 47 \\
\hline 11 & 4 & 4 & 50 & 3 & 1 & 75 & 18 & 11 & 62,1 & 25 & 16 & 61 & 41 \\
\hline 12 & 2 & 6 & 25 & 8 & 4 & 66,7 & 6 & 20 & 23,1 & 16 & 30 & 34,8 & 46 \\
\hline M & 2,75 & 3,17 & 47,69 & 7,08 & 4,25 & 62,54 & 13,75 & 11 & 55,36 & 23,58 & 18,42 & 56,27 & 42 \\
\hline S.A. & 1,06 & 1,47 & 16,39 & 2,54 & 1,6 & 10,31 & 4,11 & 3,57 & 13,46 & 3,75 & 4,23 & 8,6 & 2,73 \\
\hline
\end{tabular}
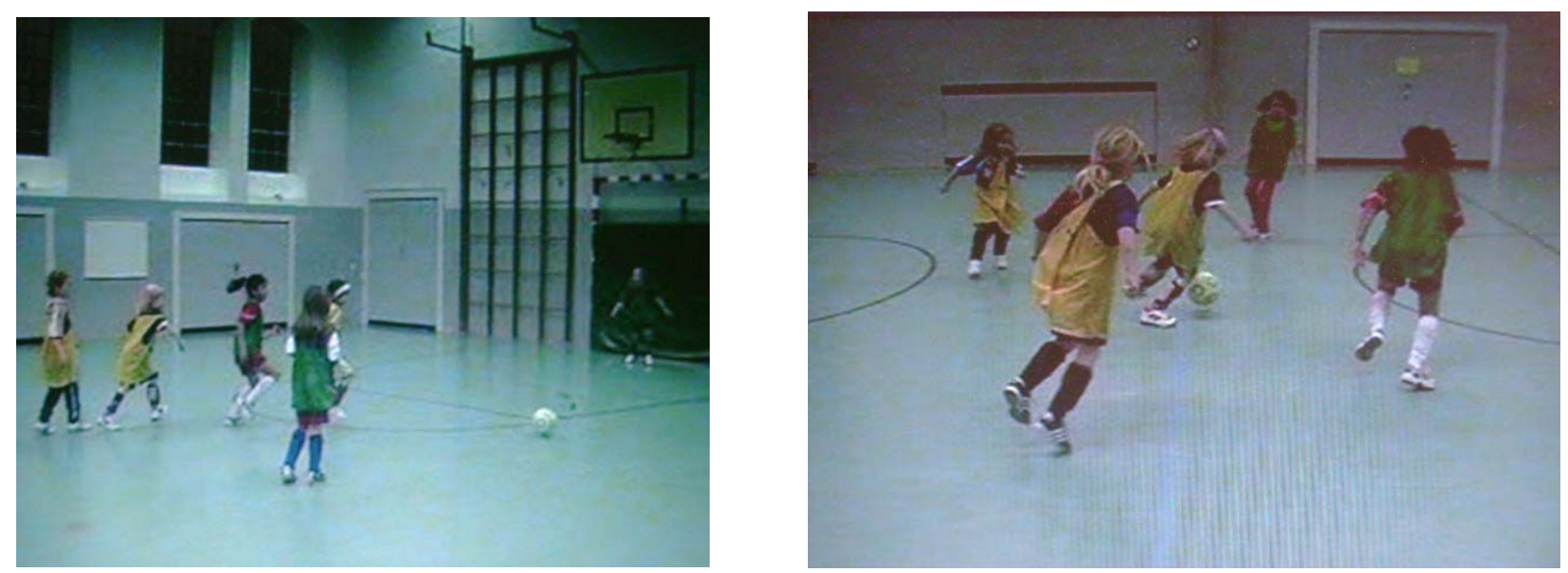
Rettest

\begin{tabular}{|c|c|c|c|c|c|c|c|c|c|c|c|c|c|}
\hline $\begin{array}{c}\text { Spie } \\
\text { lerin }\end{array}$ & \multicolumn{3}{|c|}{ SmB } & \multicolumn{3}{|c|}{ SoBMmB } & \multicolumn{3}{c|}{ SoBMoB } & \multicolumn{3}{c|}{ Gesamt } & HdI. \\
\hline & + & - & Indiz & + & - & Indiz & + & - & Indiz & + & - & Indiz & $\Sigma$ \\
\hline 1 & 2 & 8 & 20 & 5 & 4 & 55,6 & 14 & 10 & 58,3 & 21 & 22 & 48,8 & 43 \\
\hline 2 & 3 & 2 & 60 & 10 & 6 & 62,5 & 17 & 10 & 63 & 30 & 18 & 62,5 & 48 \\
\hline 3 & 6 & 4 & 60 & 4 & 3 & 57,1 & 11 & 11 & 50 & 21 & 18 & 53,8 & 39 \\
\hline 4 & 4 & 3 & 57,1 & 4 & 4 & 50 & 12 & 13 & 48 & 20 & 20 & 50 & 40 \\
\hline 5 & 3 & 6 & 33,3 & 9 & 4 & 69,2 & 13 & 10 & 56,5 & 25 & 20 & 55,6 & 45 \\
\hline 6 & 4 & 2 & 66,7 & 8 & 6 & 57,1 & 11 & 9 & 55 & 23 & 17 & 57,5 & 40 \\
\hline 7 & 7 & 5 & 58,3 & 10 & 3 & 76,9 & 10 & 9 & 52,6 & 27 & 17 & 61,4 & 44 \\
\hline 8 & 2 & 3 & 40 & 2 & 2 & 50 & 11 & 10 & 52,4 & 15 & 15 & 50 & 30 \\
\hline 9 & 2 & 3 & 40 & 7 & 7 & 50 & 15 & 10 & 60 & 24 & 20 & 54,5 & 44 \\
\hline 10 & 2 & 2 & 50 & 8 & 5 & 61,5 & 12 & 14 & 46,2 & 22 & 21 & 51,2 & 43 \\
\hline 11 & 4 & 4 & 50 & 7 & 5 & 58,3 & 14 & 9 & 60,9 & 25 & 18 & 58,1 & 43 \\
\hline 12 & 5 & 4 & 55,6 & 5 & 2 & 71,4 & 7 & 14 & 33,3 & 17 & 20 & 45,9 & 37 \\
\hline M & 3,67 & 3,83 & 49,25 & 6,58 & 4,25 & 59,98 & 12,25 & 10,75 & 53,02 & 22,5 & 18,83 & 54,12 & 41,33 \\
\hline S.A. & 1,67 & 1,8 & 13,48 & 2,57 & 1,6 & 8,79 & 2,6 & 1,86 & 8,11 & 4,15 & 1,99 & 5,13 & 4,64 \\
\hline
\end{tabular}
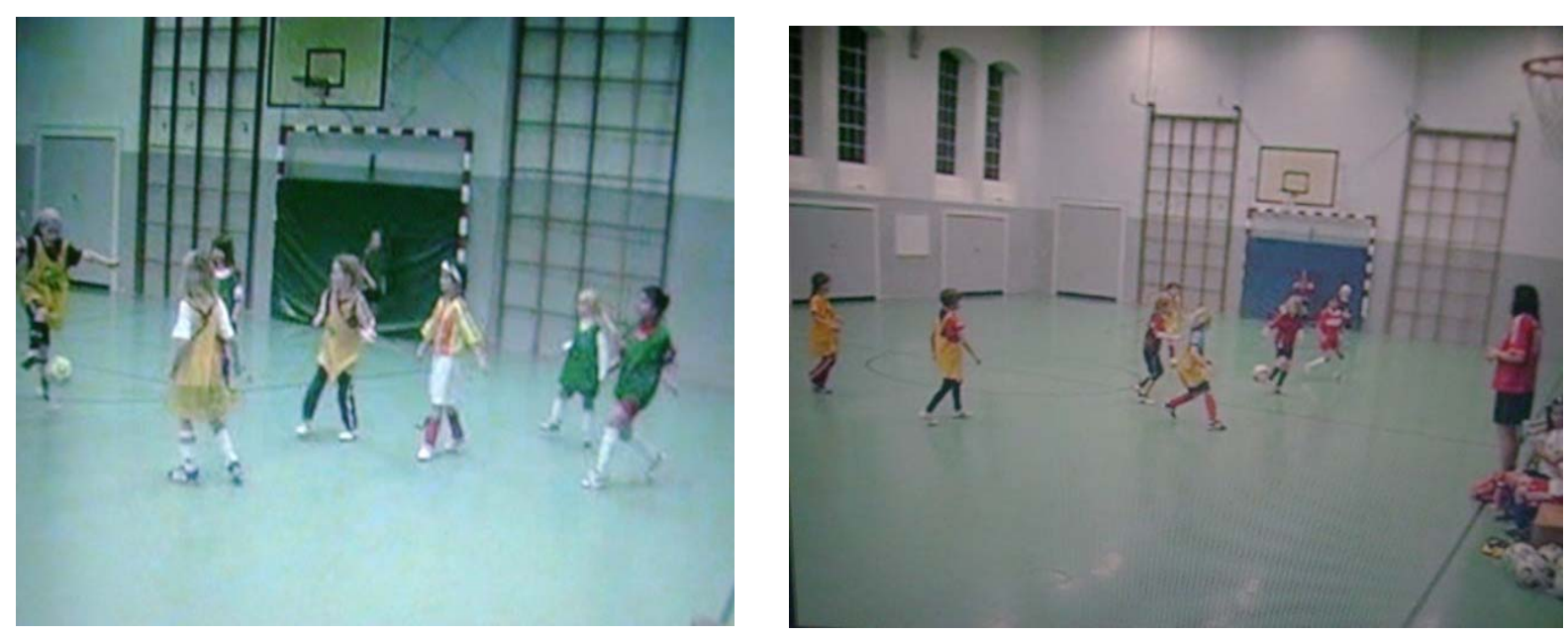
Anhang H: Kolmogorov-Smirnov-Test und Mauchly-Test für die Variablen der Studie 1

\begin{tabular}{|l|c|c|c|c|c|c|c|c|c|}
\hline Variable & \multicolumn{3}{|c|}{ Vortest } & \multicolumn{3}{c|}{ Posttest } & \multicolumn{3}{c|}{ Retentionstest } \\
\hline & $\begin{array}{c}\text { Diffe- } \\
\text { renz }\end{array}$ & $\begin{array}{c}\text { Kolmogorov } \\
- \text {-Smirnov-z }\end{array}$ & $p$ & $\begin{array}{c}\text { Diffe- } \\
\text { renz }\end{array}$ & $\begin{array}{c}\text { Kolmogorov } \\
- \text {-Smirnov-z }\end{array}$ & $p$ & $\begin{array}{c}\text { Diffe- } \\
\text { renz }\end{array}$ & $\begin{array}{c}\text { Kolmogorov } \\
- \text { Smirnov-z }\end{array}$ & $p$ \\
\hline SmB & 0,121 & 0,421 & 0,994 & 0,194 & 0,672 & 0,757 & 0,189 & 0,654 & 0,786 \\
\hline SoBMmB & 0,135 & 0,467 & 0,981 & 0,180 & 0,624 & 0,831 & 0,159 & 0,549 & 0,923 \\
\hline SoBMoB & 0,182 & 0,629 & 0,823 & 0,180 & 0,623 & 0,832 & 0,136 & 0,472 & 0,979 \\
\hline Gesamt & 0,158 & 0,549 & 0,924 & 0,224 & 0,775 & 0,585 & 0,131 & 0,453 & 0,987 \\
\hline
\end{tabular}

\section{Mauchly-Test}

\begin{tabular}{|l|c|c|c|}
\hline & W von Mauchly & d.f. & $p$ \\
\hline SmB & 0,970 & 2 & 0,860 \\
\hline SoBMmB & 0,746 & 2 & 0,231 \\
\hline SoBMoB & 0,863 & 2 & 0,478 \\
\hline Gesamt & 0,898 & 2 & 0,585 \\
\hline
\end{tabular}


Anhang I: Post-Hoc Analyse: Bonferroni-Test für die Variablen der Studie 1

\begin{tabular}{|c|c|c|c|c|}
\hline Variable & \multicolumn{2}{|c|}{ Messzeitpunkt } & \multirow{2}{*}{$\begin{array}{c}\text { Diferenz } \\
-4,7917\end{array}$} & \multirow{2}{*}{$\begin{array}{c}p \\
1,000\end{array}$} \\
\hline \multirow{6}{*}{ SmB } & \multirow[t]{2}{*}{ Vortest } & Posttest & & \\
\hline & & Retentionstest & $-6,3500$ & 1,000 \\
\hline & \multirow[t]{2}{*}{ Posttest } & Vortest & 4,7917 & 1,000 \\
\hline & & Retentionstest & $-1,5583$ & 1,000 \\
\hline & \multirow[t]{2}{*}{ Retentionstest } & Vortest & 6,3500 & 1,000 \\
\hline & & Posttest & 1,5583 & 1,000 \\
\hline \multirow{6}{*}{ SoBMmB } & \multirow[t]{2}{*}{ Vortest } & Posttest & $-10,1750$ & 0,140 \\
\hline & & Retentionstest & $-7,6167$ & 0,393 \\
\hline & \multirow[t]{2}{*}{ Posttest } & Vortest & 10,1750 & 0,140 \\
\hline & & Retentionstest & 2,5583 & 1,000 \\
\hline & \multirow[t]{2}{*}{ Retentiostest } & Vortest & 7,6167 & 0,393 \\
\hline & & Posttest & $-2,5583$ & 1,000 \\
\hline \multirow{6}{*}{ SoBMoB } & \multirow[t]{2}{*}{ Vortest } & Posttest & $-7,9667$ & 0,274 \\
\hline & & Retentionstest & $-5,6750$ & 0,672 \\
\hline & \multirow[t]{2}{*}{ Posttest } & Vortest & 7,9667 & 0,274 \\
\hline & & Retentionstest & 2,2917 & 1,000 \\
\hline & \multirow[t]{2}{*}{ Retentionstest } & Vortest & 5,6750 & 0,672 \\
\hline & & Posttest & $-2,2917$ & 1,000 \\
\hline
\end{tabular}




\begin{tabular}{|l|l|l|c|c|}
\hline \multirow{2}{*}{ Gesamt } & Vortest & Posttest & 8,8083 & $0,016^{*}$ \\
\cline { 3 - 5 } & & Retentionstest & 2,1583 & 1,000 \\
\cline { 3 - 5 } & Posttest & Vortest & $-8,8083$ & $0,016^{*}$ \\
\cline { 3 - 5 } & Retentionstest & $-6,6500$ & 0,92 \\
\cline { 2 - 5 } & Retentionstest & Vortest & 2,1583 & 1,000 \\
\cline { 3 - 5 } & Posttest & 6,6500 & 0,92 \\
\hline
\end{tabular}


Anhang J: Ergebnisse Auswertungsmethode der kognitiven Spielkompetenzen Kontrollgruppe (DIG)

\begin{tabular}{|c|c|c|c|}
\hline Spieler & Prätest & Posttest & Rettest \\
\hline 1 & 2 & 6 & 5 \\
\hline 2 & 3 & 5 & 6 \\
\hline 3 & 5 & 4 & 4 \\
\hline 4 & 3 & 3 & 3 \\
\hline 5 & 5 & 5 & 7 \\
\hline 6 & 3 & 4 & 4 \\
\hline 7 & 2 & 6 & 3 \\
\hline 8 & 3 & 3 & 5 \\
\hline 9 & 5 & 7 & 2 \\
\hline 10 & 4 & 3 & 6 \\
\hline 11 & 1 & 4 & 3 \\
\hline 12 & 6 & 5 & 4 \\
\hline 13 & 3 & 4 & 3 \\
\hline Mittelwert & 3,46 & 4,54 & 4,23 \\
\hline S.A. & 1,45 & 1,27 & 1,48 \\
\hline
\end{tabular}

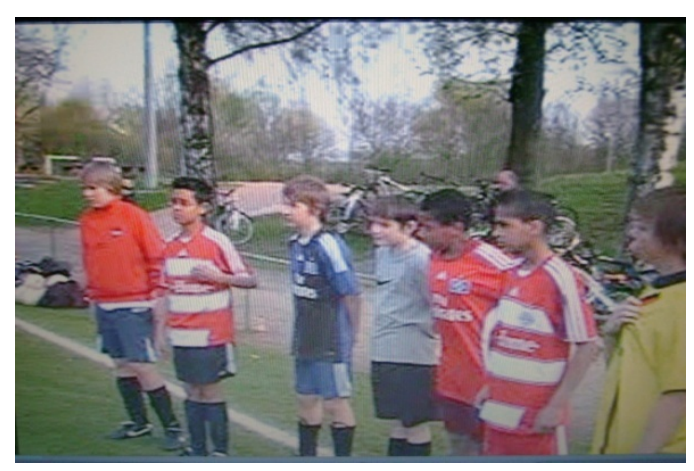




\section{Experimentalgruppe (RIG)}

\begin{tabular}{|c|c|c|c|}
\hline Spieler & Prätest & Posttest & Rettest \\
\hline 1 & 3 & 6 & 6 \\
\hline 2 & 5 & 7 & 7 \\
\hline 3 & 4 & 7 & 7 \\
\hline 4 & 6 & 8 & 9 \\
\hline 5 & 3 & 9 & 10 \\
\hline 6 & 2 & 10 & 9 \\
\hline 7 & 5 & 9 & 8 \\
\hline 8 & 4 & 7 & 7 \\
\hline 9 & 3 & 6 & 10 \\
\hline 10 & 4 & 8 & 8 \\
\hline 11 & 3 & 9 & 7 \\
\hline 12 & 5 & 7 & 9 \\
\hline 13 & 3 & 6 & 9 \\
\hline 14 & 2 & 7 & 6 \\
\hline Mittelwert & 3,71 & 7,57 & 8 \\
\hline S.A. & 1,2 & 1,28 & 1,36 \\
\hline
\end{tabular}




\section{Anhang K: Kolmogorov-Smirnov-Test für die Variablen der Studie 2}

Kolmogorov-Smirnov-Test:

\begin{tabular}{|c|c|c|c|c|c|c|c|c|c|}
\hline \multirow{2}{*}{$\begin{array}{l}\text { Varia- } \\
\text { ble }\end{array}$} & \multicolumn{3}{|c|}{ Vortest } & \multicolumn{3}{|c|}{ Posttest } & \multicolumn{3}{|c|}{ Retentionstest } \\
\hline & Differenz & $\begin{array}{c}\text { Kolmogorov- } \\
\text { Smirnov-Z }\end{array}$ & $\boldsymbol{P}$ & Differenz & $\begin{array}{c}\text { Kolmogorov- } \\
\text { Smirnov-Z }\end{array}$ & $p$ & Differenz & $\begin{array}{c}\text { Kolmogorov- } \\
\text { Smirnov-Z }\end{array}$ & $p$ \\
\hline $\begin{array}{l}\text { Direkte } \\
\text { Instruktion } \\
\text { Gruppe } \\
\text { (DIG) }\end{array}$ & 0,240 & 0,866 & $0,039 *$ & 0,203 & 0,733 & 0,146 & 0,182 & 0,655 & 0,784 \\
\hline $\begin{array}{l}\text { Reflexive } \\
\text { Instruktion } \\
\text { Gruppe } \\
\text { (RIG) }\end{array}$ & 0,223 & 0,836 & 0,056 & 0,243 & 0,910 & $0,024 *$ & 0,198 & 0,740 & 0,143 \\
\hline
\end{tabular}


Anhang L: Formblatt Auswertung der Passgenauigkeit bei dem Minifußball-Test

Ballkontakt: X

Guter Pass: O

Schlechter Pass: I

\begin{tabular}{|c|}
\hline \hline Ballkontakte: \\
\hline Pässe: \\
\hline IIIII III 0100 II \\
\hline
\end{tabular}

\section{Spieler Nr.2}

Ballkontakte:

$X X X X X X X X X X X X X X X X X X X X$

Pässe:

II O IIIII II O I

Spieler Nr.3

Ballkontakte:

$X X X X X X X X X X X X X X X X X X X X X X X X X X X$

Pässe:

0 II 00 IIIII I O II OO I

\section{Spieler Nr.4}

Ballkontakte:

$X X X X X X X X X X X X X X X X X X X$

Pässe:

I 01100011111 
Anhang M: The Loughborough-Soccer-Pass-Test (LSPT)

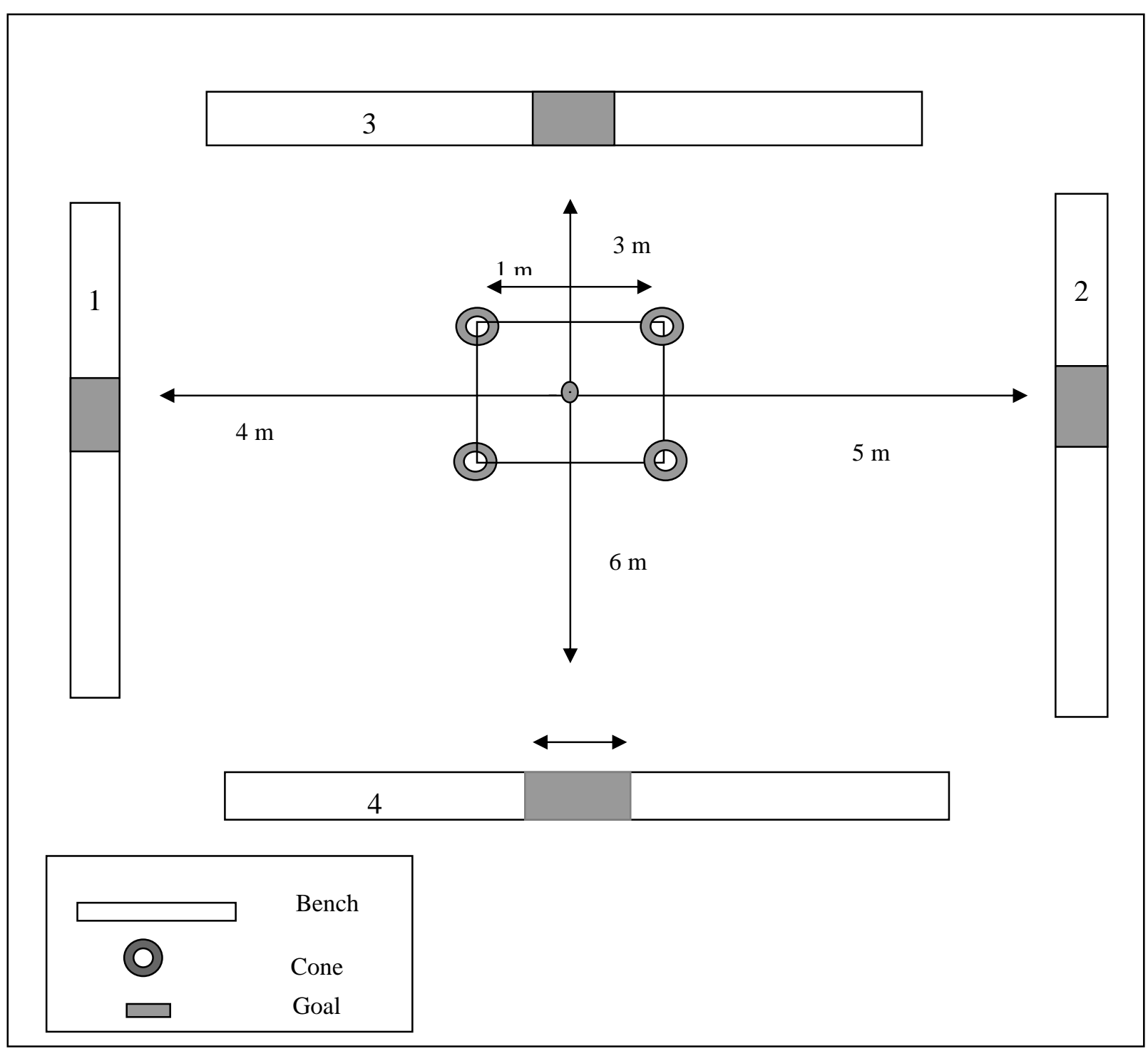

The LSPT involves the performance 16 passes made from a central zone to four $0.5 \mathrm{~m}$ targets that are marked onto benches. The benches are used to allow the ball to rebound and the targets are colour-coded for identification. Small disc cones are placed around the central zone. The passes are randomly selected and called out to the players by the test examiner. The time to complete the test is recorded using a stop watch. If the marked areas are missed, or one of the cones is hit during the test, additional „penalty seconds" are added. If the player ventured out of the outer zone, missed the $0.5-\mathrm{m}$ target or the player hit a cone, 2 seconds are added. If the bench is missed then a 5-s penalty is added. The LSPT is shown below. 


\section{Anhang N: Beispiel Trainingseinheit Passschulung durch Spielen}

Einheit 2 Datum_01.03.2010 Gruppe D3

Gesamtzeit_30 Min. Lehrmittel: Bälle, Hütchen, Leibchen, Kasten

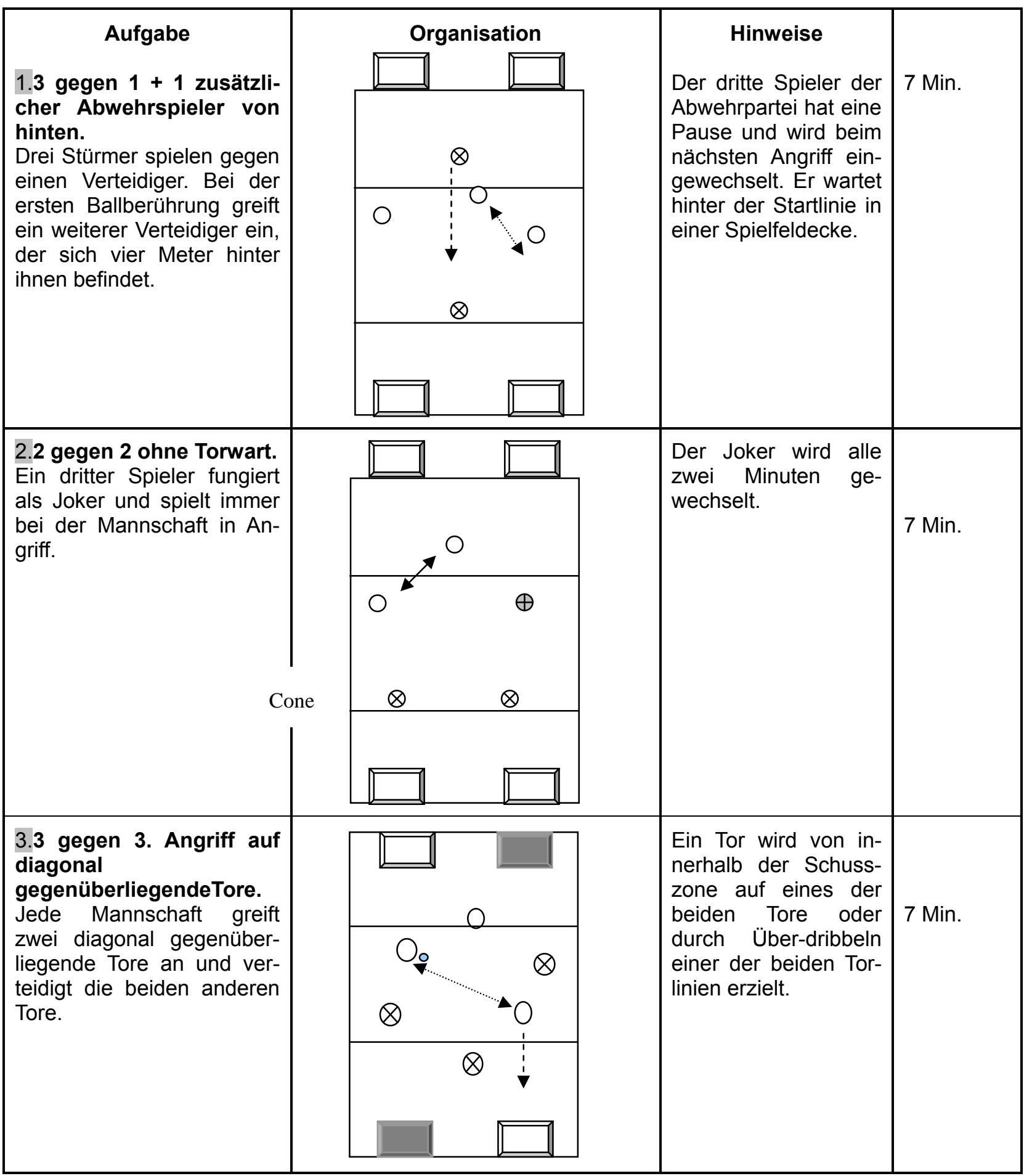




\section{Anhang O: Beispiel Trainingseinheit Passschulung durch Üben}

Einheit Datum Gruppe Gesamtzeit Lehrmittel:

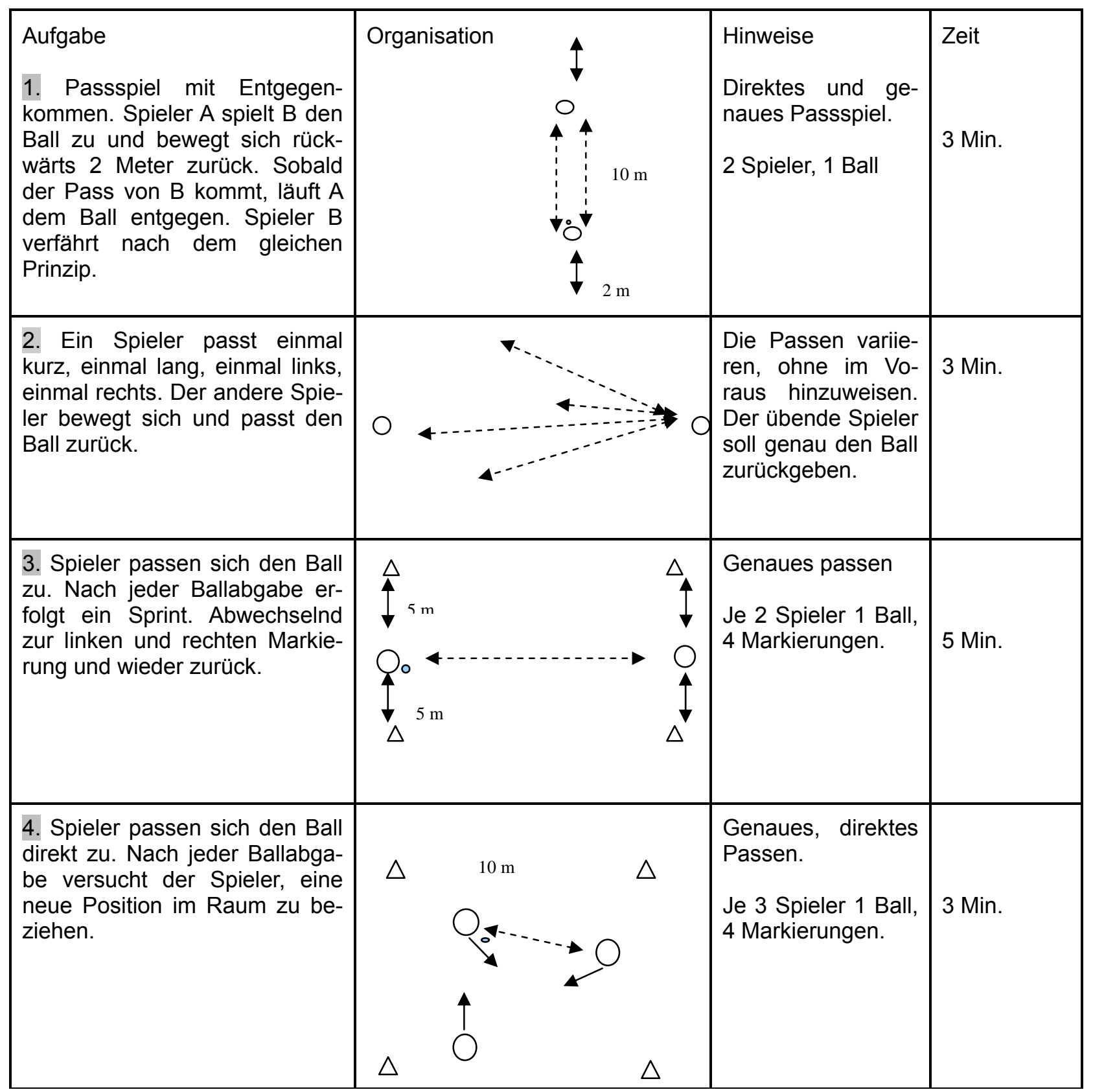




\begin{tabular}{|l|l|l|l|l|}
\hline $\begin{array}{l}\text { 4. Ein Ball für drei Spieler. 1 } \\
\text { passt auf 2, der einen Doppel- } \\
\text { pass mit 3 spielt. 2 spielt zu 1 } \\
\text { zurück. Nach jedem Durchgang } \\
\text { tauschen 2 und 3 ihre Rollen. }\end{array}$ & $\begin{array}{l}\text { Alle Pässe werden } \\
\text { flach gespielt. 2 } \\
\text { und 3 spielen mit } \\
\text { nur einer Ballbe- } \\
\text { rührung. }\end{array}$ & 3 Min. \\
\hline $\begin{array}{l}\text { 5. 2 Spieler passen im Lauf den } \\
\text { Ball hin und her. Am Ziel folgt } \\
\text { die Rückkehr beider Spieler auf } \\
\text { der Außenseite. }\end{array}$ & $\triangle$ & & &
\end{tabular}




\section{Anhang P: Ergebnisse Minifußball-Test}

\section{Vortest}

\section{Ergebnisse Spielgruppe}

\begin{tabular}{|c|c|c|c|c|c|c|c|c|c|c|}
\hline Spieler & $\begin{array}{c}\text { Ball- } \\
\text { Kontakte }\end{array}$ & Pässe & E. Pässe & $\begin{array}{c}\text { N. E. } \\
\text { Pässe }\end{array}$ & P/BK & EP/BK & NEP/BK & EP/P & NE/P & EP/NEP \\
\hline 1 & 9 & 6 & 1 & 5 & 0,67 & 0,11 & 0,56 & 0,17 & 0,83 & 0,2 \\
\hline 2 & 13 & 6 & 3 & 3 & 0,46 & 0,23 & 0,23 & 0,5 & 0,5 & 1 \\
\hline 3 & 11 & 8 & 7 & 1 & 0,73 & 0,64 & 0,09 & 0,88 & 0,13 & 7 \\
\hline 8 & 13 & 3 & 2 & 1 & 0,23 & 0,15 & 0,08 & 0,67 & 0,33 & 2 \\
\hline 9 & 16 & 7 & 3 & 4 & 0,44 & 0,19 & 0,25 & 0,43 & 0,57 & 0,75 \\
\hline 11 & 18 & 10 & 7 & 3 & 0,56 & 0,39 & 0,17 & 0,7 & 0,3 & 2,33 \\
\hline 12 & 26 & 14 & 8 & 7 & 0,54 & 0,31 & 0,27 & 0,57 & 0,5 & 1,14 \\
\hline 13 & 20 & 4 & 3 & 1 & 0,2 & 0,15 & 0,05 & 0,75 & 0,25 & 3 \\
\hline 15 & 13 & 7 & 6 & 1 & 0,54 & 0,46 & 0,08 & 0,86 & 0,14 & 6 \\
\hline 16 & 18 & 11 & 6 & 5 & 0,61 & 0,33 & 0,28 & 0,55 & 0,45 & 1,2 \\
\hline Mittelwert & 15,7 & 7,6 & 4,6 & 3,1 & 0,5 & 0,3 & 0,2 & 0,61 & 0,4 & 1,48 \\
\hline S.A. & 4,99 & 3,31 & 2,46 & 2,13 & 0,17 & 0,17 & 0,15 & 0,21 & 0,22 & 2,29 \\
\hline
\end{tabular}

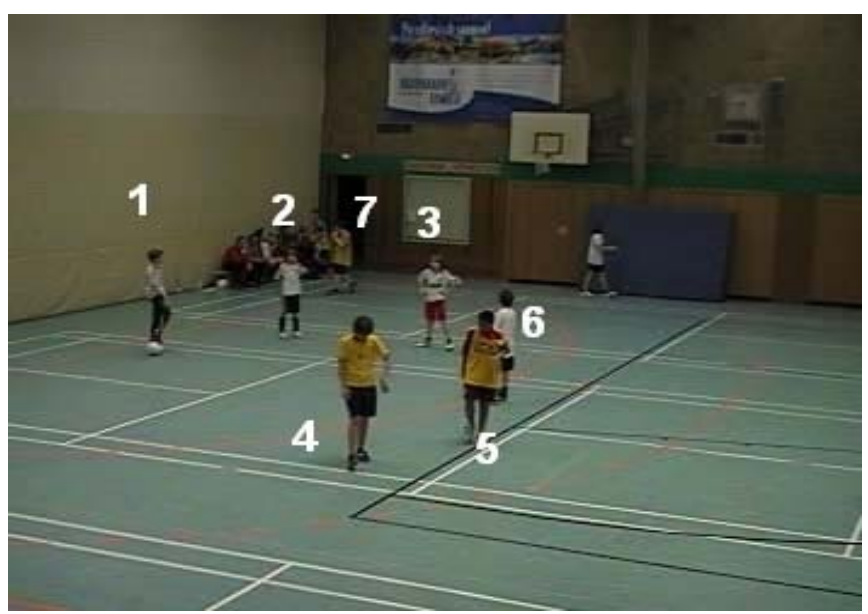


Posttest

\begin{tabular}{|c|c|c|c|c|c|c|c|c|c|c|}
\hline Spieler & $\begin{array}{c}\text { Ball- } \\
\text { Kontakte }\end{array}$ & Pässe & E. Pässe & $\begin{array}{c}\text { N. E. } \\
\text { Pässe }\end{array}$ & P/BK & EP/BK & NEP/BK & EP/P & NE/P & EP/NEP \\
\hline 1 & 14 & 8 & 6 & 2 & 0,57 & 0,43 & 0,14 & 0,75 & 0,25 & 3 \\
\hline 2 & 7 & 3 & 2 & 1 & 0,43 & 0,29 & 0,14 & 0,67 & 0,33 & 2 \\
\hline 3 & 21 & 9 & 9 & 0 & 0,43 & 0,43 & 0 & 1 & 0 & 9 \\
\hline 8 & 11 & 9 & 8 & 1 & 0,82 & 0,73 & 0,09 & 0,89 & 0,11 & 8 \\
\hline 9 & 12 & 8 & 7 & 1 & 0,67 & 0,58 & 0,08 & 0,88 & 0,13 & 7 \\
\hline 11 & 24 & 10 & 7 & 3 & 0,42 & 0,29 & 0,13 & 0,7 & 0,3 & 2,33 \\
\hline 12 & 27 & 10 & 7 & 3 & 0,37 & 0,26 & 0,11 & 0,7 & 0,3 & 2,33 \\
\hline 13 & 15 & 7 & 6 & 1 & 0,47 & 0,4 & 0,07 & 0,86 & 0,14 & 6 \\
\hline 15 & 21 & 14 & 12 & 2 & 0,67 & 0,57 & 0,1 & 0,86 & 0,14 & 6 \\
\hline 16 & 16 & 8 & 5 & 3 & 0,5 & 0,31 & 0,19 & 0,63 & 0,38 & 1,67 \\
\hline Mittelwert & 16,8 & 8,6 & 6,9 & 1,7 & 0,53 & 0,43 & 0,1 & 0,79 & 0,21 & 4,73 \\
\hline S.A. & 6,29 & 2,76 & 2,6 & 1,06 & 0,14 & 0,15 & 0,05 & 0,12 & 0,12 & 2,76 \\
\hline
\end{tabular}




\section{Retentionstest}

\begin{tabular}{|c|c|c|c|c|c|c|c|c|c|c|}
\hline Spieler & $\begin{array}{c}\text { Ball- } \\
\text { Kontakte }\end{array}$ & Pässe & E. Pässe & $\begin{array}{l}\text { N. E. } \\
\text { Pässe }\end{array}$ & P/BK & EP/BK & NEP/BK & EP/P & NE/P & EP/NEP \\
\hline 1 & 16 & 8 & 6 & 2 & 0,5 & 0,38 & 0,13 & 0,75 & 0,25 & 3 \\
\hline 2 & 31 & 21 & 13 & 8 & 0,68 & 0,42 & 0,26 & 0,62 & 0,38 & 1,63 \\
\hline 3 & 21 & 9 & 7 & 2 & 0,43 & 0,33 & 0,1 & 0,78 & 0,22 & 3,5 \\
\hline 8 & 12 & 4 & 2 & 2 & 0,33 & 0,17 & 0,17 & 0,5 & 0,5 & 1 \\
\hline 9 & 13 & 5 & 3 & 2 & 0,38 & 0,23 & 0,15 & 0,6 & 0,4 & 1,5 \\
\hline 11 & 19 & 8 & 4 & 4 & 0,42 & 0,21 & 0,21 & 0,5 & 0,5 & 1 \\
\hline 12 & 22 & 14 & 10 & 4 & 0,64 & 0,45 & 0,18 & 0,71 & 0,29 & 2,5 \\
\hline 13 & 14 & 7 & 3 & 4 & 0,5 & 0,21 & 0,29 & 0,43 & 0,57 & 0,75 \\
\hline 15 & 20 & 7 & 4 & 3 & 0,35 & 0,2 & 0,15 & 0,57 & 0,43 & 1,33 \\
\hline 16 & 19 & 10 & 8 & 2 & 0,53 & 0,42 & 0,11 & 0,8 & 0,2 & 4 \\
\hline Mittelwert & 18,7 & 9,3 & 6 & 3,3 & 0,48 & 0,3 & 0,17 & 0,63 & 0,37 & 2,02 \\
\hline S.A. & 5,54 & 4,95 & 3,53 & 1,89 & 0,12 & 0,11 & 0,06 & 0,13 & 0,13 & 1,15 \\
\hline
\end{tabular}

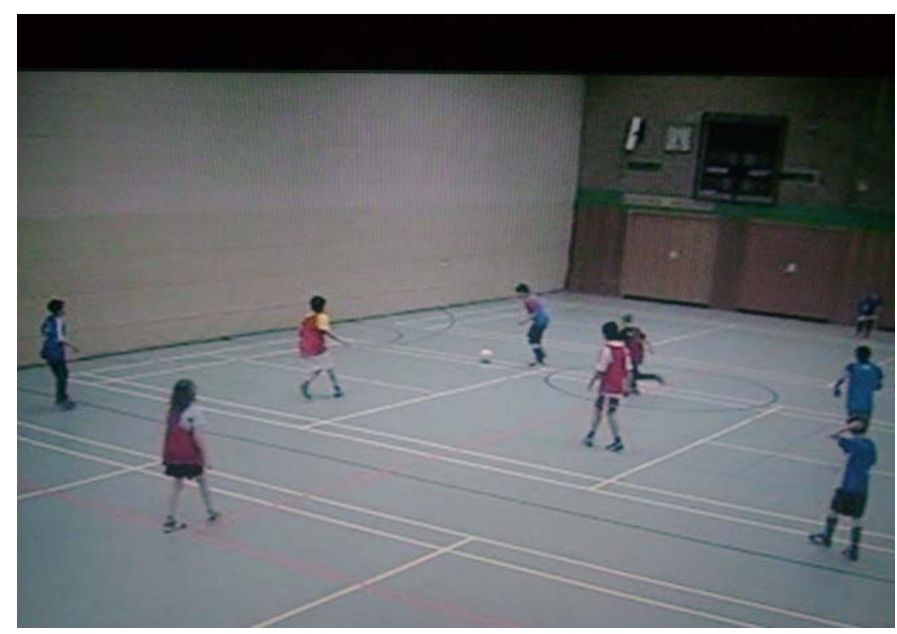




\section{Ergebnisse Übungsgruppe}

\section{Vortest}

\begin{tabular}{|c|c|c|c|c|c|c|c|c|c|c|}
\hline Spieler & $\begin{array}{c}\text { Ball- } \\
\text { Kontakte }\end{array}$ & Pässe & E. Pässe & $\begin{array}{c}\text { N. E. } \\
\text { Pässe }\end{array}$ & P/BK & EP/BK & NEP/BK & EP/P & NE/P & EP/NEP \\
\hline 18 & 10 & 4 & 2 & 2 & 0,4 & 0,2 & 0,2 & 0,5 & 0,5 & 1 \\
\hline 19 & 22 & 9 & 4 & 5 & 0,41 & 0,18 & 0,23 & 0,44 & 0,56 & 0,8 \\
\hline 20 & 16 & 5 & 4 & 1 & 0,31 & 0,25 & 0,06 & 0,8 & 0,2 & 4 \\
\hline 21 & 16 & 5 & 1 & 4 & 0,31 & 0,06 & 0,25 & 0,2 & 0,8 & 0,25 \\
\hline 22 & 15 & 10 & 2 & 8 & 0,67 & 0,13 & 0,53 & 0,2 & 0,8 & 0,25 \\
\hline 23 & 18 & 11 & 7 & 4 & 0,61 & 0,39 & 0,22 & 0,64 & 0,36 & 1,75 \\
\hline 24 & 14 & 10 & 8 & 2 & 0,71 & 0,57 & 0,14 & 0,8 & 0,2 & 4 \\
\hline 25 & 13 & 8 & 5 & 2 & 0,62 & 0,38 & 0,15 & 0,63 & 0,25 & 2,5 \\
\hline Mittelwert & 15,5 & 7,75 & 4,13 & 3,5 & 0,51 & 0,27 & 0,22 & 0,53 & 0,46 & 1,82 \\
\hline S.A. & 3,55 & 2,71 & 2,47 & 2,27 & 0,16 & 0,17 & 0,14 & 0,24 & 0,25 & 1,54 \\
\hline
\end{tabular}


Posttest

\begin{tabular}{|c|c|c|c|c|c|c|c|c|c|c|}
\hline Spieler & $\begin{array}{c}\text { Ball- } \\
\text { Kontakte }\end{array}$ & Pässe & E. Pässe & $\begin{array}{c}\text { N. E. } \\
\text { Pässe }\end{array}$ & P/BK & EP/BK & NEP/BK & EP/P & NE/P & EP/NEP \\
\hline 18 & 11 & 6 & 5 & 1 & 0,55 & 0,45 & 0,09 & 0,83 & 0,17 & 5 \\
\hline 19 & 10 & 3 & 2 & 1 & 0,3 & 0,2 & 0,1 & 0,67 & 0,33 & 2 \\
\hline 20 & 21 & 5 & 4 & 1 & 0,24 & 0,19 & 0,05 & 0,8 & 0,2 & 4 \\
\hline 21 & 11 & 9 & 5 & 4 & 0,82 & 0,45 & 0,36 & 0,56 & 0,44 & 1,25 \\
\hline 22 & 11 & 3 & 3 & 0 & 0,27 & 0,27 & 0 & 1 & 0 & 3 \\
\hline 23 & 16 & 9 & 6 & 3 & 0,56 & 0,38 & 0,19 & 0,67 & 0,33 & 2 \\
\hline 24 & 18 & 11 & 8 & 3 & 0,61 & 0,44 & 0,17 & 0,73 & 0,27 & 2,67 \\
\hline 25 & 19 & 10 & 7 & 3 & 0,53 & 0,37 & 0,16 & 0,7 & 0,3 & 2,33 \\
\hline Mittelwert & 14,63 & 7 & 5 & 2 & 0,48 & 0,35 & 0,14 & 0,74 & 0,26 & 2,78 \\
\hline S.A. & 4,37 & 3,16 & 2 & 1,41 & 0,2 & 0,11 & 0,11 & 0,13 & 0,13 & 1,21 \\
\hline
\end{tabular}

Retentionstest

\begin{tabular}{|c|c|c|c|c|c|c|c|c|c|c|}
\hline Spieler & $\begin{array}{c}\text { Ball- } \\
\text { Kontakte }\end{array}$ & Pässe & E. Pässe & $\begin{array}{c}\text { N. E. } \\
\text { Pässe }\end{array}$ & P/BK & EP/BK & NEP/BK & EP/P & NE/P & EP/NEP \\
\hline 18 & 16 & 7 & 3 & 4 & 0,44 & 0,19 & 0,25 & 0,43 & 0,57 & 0,75 \\
\hline 19 & 16 & 11 & 9 & 2 & 0,69 & 0,56 & 0,13 & 0,82 & 0,18 & 4,5 \\
\hline 20 & 18 & 10 & 7 & 3 & 0,56 & 0,39 & 0,17 & 0,7 & 0,3 & 2,33 \\
\hline 21 & 26 & 14 & 8 & 7 & 0,54 & 0,31 & 0,27 & 0,57 & 0,5 & 1,14 \\
\hline 22 & 20 & 4 & 3 & 1 & 0,2 & 0,15 & 0,05 & 0,75 & 0,25 & 3 \\
\hline 23 & 25 & 17 & 9 & 8 & 0,68 & 0,36 & 0,32 & 0,53 & 0,47 & 1,13 \\
\hline 24 & 13 & 7 & 6 & 1 & 0,54 & 0,46 & 0,08 & 0,86 & 0,14 & 6 \\
\hline 25 & 18 & 11 & 6 & 5 & 0,61 & 0,33 & 0,28 & 0,55 & 0,45 & 1,2 \\
\hline Mittelwert & 19 & 10,13 & 6,38 & 3,88 & 0,53 & 0,34 & 0,19 & 0,65 & 0,36 & 2,51 \\
\hline S.A. & 4,5 & 4,16 & 2,39 & 2,64 & 0,16 & 0,13 & 0,1 & 0,15 & 0,16 & 1,89 \\
\hline
\end{tabular}


Anhang Q: ErgebnisseLoughborough-Soccer-Pass-Test

Vortest
\begin{tabular}{|c|c|c|c|c|}
\hline Spieler & Zeit & $\begin{array}{c}\text { Fehler: Ziel nicht } \\
\text { getroffen }\end{array}$ & $\begin{array}{c}\text { Fehler: Ball au- } \\
\text { Ben der Vierecke }\end{array}$ & Gesamte Zeit \\
\hline 1 & 29 & 1 & 4 & 42 \\
\hline 2 & 30 & 0 & 3 & 36 \\
\hline 3 & 28 & 2 & 1 & 40 \\
\hline 8 & 29 & 2 & 3 & 45 \\
\hline 9 & 29 & 2 & 2 & 43 \\
\hline 11 & 26 & 2 & 6 & 48 \\
\hline 12 & 28 & 3 & 4 & 40 \\
\hline 13 & 25 & 1 & 5 & 46 \\
\hline 15 & 37 & 1 & 2 & 49 \\
\hline 16 & 28 & 3 & 3 & 44 \\
\hline Mittelwert & 28,9 & 1,7 & 3,3 & 4,67 \\
\hline S.A. & 3,21 & 0,95 & 1,49 & 51 \\
\hline
\end{tabular}




\section{Posttest}

\begin{tabular}{|c|c|c|c|c|}
\hline Spieler & Zeit & $\begin{array}{c}\text { Fehler: Ziel nicht } \\
\text { getroffen }\end{array}$ & $\begin{array}{c}\text { Fehler: Ball au- } \\
\text { Ben der Vierecke }\end{array}$ & Gesamte Zeit \\
\hline 1 & 24 & 0 & 2 & 28 \\
\hline 2 & 27 & 1 & 5 & 42 \\
\hline 3 & 34 & 1 & 5 & 41 \\
\hline 8 & 35 & 0 & 3 & 49 \\
\hline 9 & 29 & 2 & 5 & 34 \\
\hline 11 & 23 & 1 & 3 & 43 \\
\hline 12 & 32 & 1 & 3 & 37 \\
\hline 13 & 29 & 0 & 2 & 35 \\
\hline 15 & 25 & 0 & 3 & 38,7 \\
\hline 16 & 29 & 0 & 3,5 & 7,41 \\
\hline Mittelwert & 28,7 & 0,6 & 1,18 & 29 \\
\hline S.A. & 4,08 & 0,7 & & 49 \\
\hline
\end{tabular}

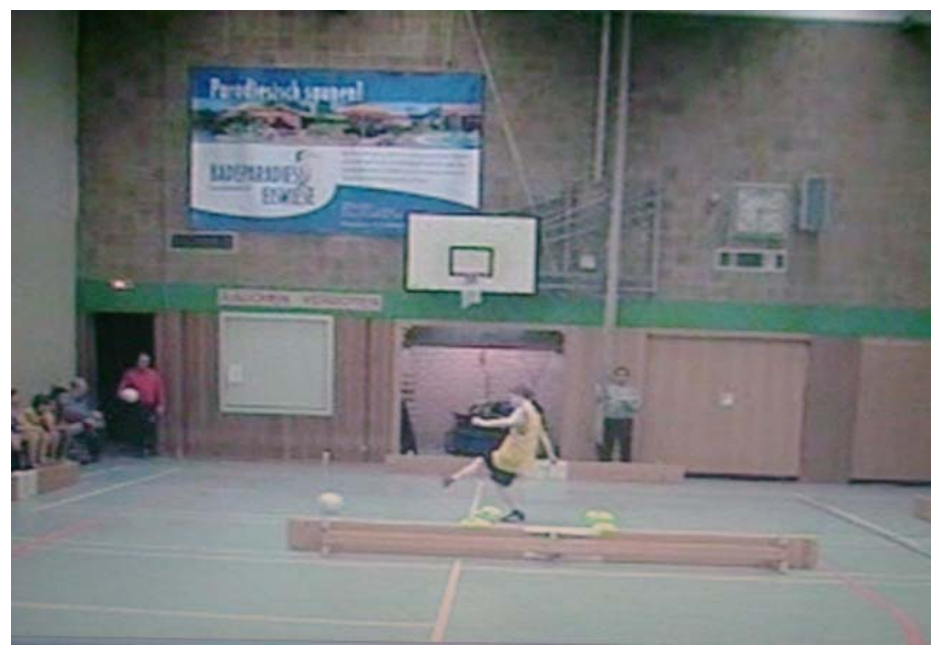


Retentionstest

\begin{tabular}{|c|c|c|c|c|}
\hline Spieler & Zeit & $\begin{array}{c}\text { Fehler: Ziel nicht } \\
\text { getroffen }\end{array}$ & $\begin{array}{c}\text { Fehler: Ball au- } \\
\text { Ben der Vierecke }\end{array}$ & Gesamte Zeit \\
\hline 1 & 25 & 2 & 3 & 41 \\
\hline 2 & 28 & 0 & 2 & 32 \\
\hline 3 & 29 & 0 & 3 & 41 \\
\hline 8 & 32 & 1 & 2 & 41 \\
\hline 9 & 29 & 2 & 3 & 35 \\
\hline 11 & 24 & 1 & 4 & 43 \\
\hline 12 & 30 & 1 & 2 & 37 \\
\hline 13 & 28 & 1 & 1 & 43 \\
\hline 15 & 27 & 1 & 4 & 38,2 \\
\hline 16 & 30 & 1 & 2,5 & 4,05 \\
\hline Mittelwert & 28,2 & 1 & 1,08 & 34 \\
\hline S.A. & 2,39 & 0,67 & & 43 \\
\hline
\end{tabular}


Vortest

Ergebnisse Übungsgruppe

\begin{tabular}{|c|c|c|c|c|}
\hline Spieler & Zeit & $\begin{array}{c}\text { Fehler: Ziel nicht } \\
\text { getroffen }\end{array}$ & $\begin{array}{c}\text { Fehler: Ball au- } \\
\text { Ben der Vierecke }\end{array}$ & Gesamte Zeit \\
\hline 18 & 27 & 3 & 4 & 50 \\
\hline 19 & 22 & 1 & 5 & 37 \\
\hline 20 & 28 & 2 & 4 & 46 \\
\hline 21 & 28 & 1 & 3 & 39 \\
\hline 22 & 29 & 2 & 3 & 45 \\
\hline 23 & 35 & 1 & 2 & 62 \\
\hline 24 & 32 & 4 & 5 & 47 \\
\hline 25 & 30 & 1 & 6 & 46,25 \\
\hline Mittelwert & 2,88 & 1,88 & 4 & 7,63 \\
\hline S.A. & 3,8 & 1,13 & 1,31 & \\
\hline
\end{tabular}


Posttest

\begin{tabular}{|c|c|c|c|c|}
\hline Spieler & Zeit & $\begin{array}{c}\text { Fehler: Ziel nicht } \\
\text { getroffen }\end{array}$ & $\begin{array}{c}\text { Fehler: Ball au- } \\
\text { Ben der Vierecke }\end{array}$ & Gesamte Zeit \\
\hline 18 & 22 & 1 & 5 & 37 \\
\hline 19 & 29 & 1 & 4 & 42 \\
\hline 20 & 26 & 1 & 3 & 37 \\
\hline 21 & 28 & 2 & 4 & 36 \\
\hline 22 & 23 & 1 & 3 & 43 \\
\hline 23 & 32 & 1 & 3 & 31 \\
\hline 24 & 24 & 1 & 1 & 37 \\
\hline 25 & 24 & 1 & 4 & 38,38 \\
\hline Mittelwert & 26 & 1,13 & 3,38 & 4,96 \\
\hline S.A. & 3,42 & 0,35 & 1,19 & \\
\hline
\end{tabular}

\section{Retentionstest}

\begin{tabular}{|c|c|c|c|c|}
\hline Spieler & Zeit & $\begin{array}{c}\text { Fehler: Ziel nicht } \\
\text { getroffen }\end{array}$ & $\begin{array}{c}\text { Fehler: Ball au- } \\
\text { Ben der Vierecke }\end{array}$ & Gesamte Zeit \\
\hline 18 & 23 & 1 & 1 & 30 \\
\hline 19 & 21 & 1 & 3 & 32 \\
\hline 20 & 25 & 2 & 5 & 45 \\
\hline 21 & 30 & 1 & 4 & 43 \\
\hline 22 & 31 & 3 & 2 & 50 \\
\hline 23 & 25 & 2 & 4 & 36 \\
\hline 24 & 27 & 1 & 2 & 39 \\
\hline 25 & 23 & 2 & 3 & 39,75 \\
\hline Mittelwert & 25,63 & 1,63 & 3 & 6,8 \\
\hline S.A. & 3,5 & 0,74 & 1,31 & \\
\hline
\end{tabular}


Anhang R: Kolmogorov-Smirnov-Test und Mauchly-Test für die Variablen der Studie 3

Spielgruppe

\begin{tabular}{|c|c|c|c|c|c|c|c|c|c|}
\hline \multirow[t]{2}{*}{ Variable } & \multicolumn{3}{|c|}{ Vortest } & \multicolumn{3}{|c|}{ Posttest } & \multicolumn{3}{|c|}{ Retentionstest } \\
\hline & $\begin{array}{l}\text { Diffe- } \\
\text { renz }\end{array}$ & $\begin{array}{l}\text { Kolmogorov- } \\
\text { Smirnov-Z }\end{array}$ & $p$ & $\begin{array}{l}\text { Diffe- } \\
\text { renz }\end{array}$ & $\begin{array}{l}\text { Kolmogorov- } \\
\text { Smirnov-Z }\end{array}$ & $p$ & $\begin{array}{l}\text { Diffe- } \\
\text { renz }\end{array}$ & $\begin{array}{l}\text { Kolmogorov- } \\
\text { Smirnov-Z }\end{array}$ & $p$ \\
\hline Ballkontakte & 0,206 & 0,651 & 0,791 & 0,151 & 0,476 & 0,977 & 0,176 & 0,555 & 0,917 \\
\hline Pässe & 0,172 & 0,544 & 0,929 & 0,214 & 0,676 & 0,750 & 0,244 & 0,771 & 0,592 \\
\hline Erfolgreiche Pässe & 0,242 & 0,767 & 0,599 & 0,185 & 0,584 & 0,885 & 0,215 & 0,679 & 0,746 \\
\hline $\begin{array}{l}\text { Nicht erfolgreiche } \\
\text { Pässe }\end{array}$ & 0,238 & 0,752 & 0,624 & 0,246 & 0,777 & 0,582 & 0,246 & 0,808 & 0,532 \\
\hline Pässe/Ballkontakte & 0,196 & 0,620 & 0,837 & 0,196 & 0,620 & 0,837 & 0,152 & 0,482 & 0,975 \\
\hline $\begin{array}{l}\text { Erfolgreiche Päs- } \\
\text { se/Ballkontakte }\end{array}$ & 0,154 & 0,488 & 0,971 & 0,197 & 0,624 & 0,831 & 0,246 & 0,778 & 0,581 \\
\hline $\begin{array}{l}\text { Nicht erfolgreiche } \\
\text { Pässe/Ballkontakte }\end{array}$ & 0,213 & 0,675 & 0,753 & 0,146 & 0,463 & 0,983 & 0,168 & 0,531 & 0,940 \\
\hline $\begin{array}{l}\text { Erfolgreiche Päs- } \\
\text { se/Pässe }\end{array}$ & 0,114 & 0,361 & 0,999 & 0,209 & 0,660 & 0,776 & 0,142 & 0,449 & 0,988 \\
\hline $\begin{array}{l}\text { Nicht erfolgreiche } \\
\text { Pässe/Pässe }\end{array}$ & 0,351 & 1,164 & 0,133 & 0,213 & 0,674 & 0,753 & 0,142 & 0,449 & 0,988 \\
\hline $\begin{array}{l}\text { Erfolgreiche Päs- } \\
\text { se/nicht erfolgrei- } \\
\text { che Pässe }\end{array}$ & 0,223 & 0,705 & 0,703 & 0,235 & 0,743 & 0,640 & 0,233 & 0,737 & 0,649 \\
\hline
\end{tabular}




\section{Übungsgruppe}

\begin{tabular}{|c|c|c|c|c|c|c|c|c|c|}
\hline \multirow[t]{2}{*}{ Variable } & \multicolumn{3}{|c|}{ Vortest } & \multicolumn{3}{|c|}{ Posttest } & \multicolumn{3}{|c|}{ Retentionstest } \\
\hline & Differenz & $\begin{array}{c}\text { Kolmogor } \\
\text { ov- } \\
\text { Smirnov- } \\
\text { Z }\end{array}$ & $p$ & $\begin{array}{l}\text { Diffe- } \\
\text { renz }\end{array}$ & $\begin{array}{c}\text { Kolmogor } \\
\text { ov- } \\
\text { Smirnov- } \\
\text { Z }\end{array}$ & $p$ & $\begin{array}{l}\text { Diffe- } \\
\text { renz }\end{array}$ & $\begin{array}{c}\text { Kolmogor } \\
\text { ov- } \\
\text { Smirnov- } \\
\text { Z }\end{array}$ & $p$ \\
\hline Ballkontakte & 0,194 & 0,549 & 0,924 & 0,296 & 0,838 & 0,483 & 0,213 & 0,602 & 0,862 \\
\hline Pässe & 0,220 & 0,621 & 0,835 & 0,236 & 0,669 & 0,762 & 0,167 & 0,471 & 0,979 \\
\hline $\begin{array}{l}\text { Erfolgreiche } \\
\text { Pässe }\end{array}$ & 0,180 & 0,508 & 0,958 & 0,125 & 0,354 & 1,000 & 0,188 & 0,531 & 0,941 \\
\hline $\begin{array}{l}\text { Nicht erfolgrei- } \\
\text { che Pässe }\end{array}$ & 0,246 & 0,695 & 0,719 & 0,260 & 0,736 & 0,651 & 0,138 & 0,391 & 0,998 \\
\hline $\begin{array}{l}\text { Päs- } \\
\text { se/Ballkontakte }\end{array}$ & 0,238 & 0,674 & 0,754 & 0,214 & 0,605 & 0,857 & 0,269 & 0,761 & 0,609 \\
\hline $\begin{array}{l}\text { Erfolgreiche } \\
\text { Päs- } \\
\text { se/Ballkontakte }\end{array}$ & 0,173 & 0,489 & 0,971 & 0,220 & 0,622 & 0,834 & 0,150 & 0,425 & 0,994 \\
\hline $\begin{array}{l}\text { Nicht erfolgrei- } \\
\text { che Päs- } \\
\text { se/Ballkontakte }\end{array}$ & 0,296 & 0,838 & 0,484 & 0,199 & 0,563 & 0,909 & 0,212 & 0,601 & 0,864 \\
\hline $\begin{array}{l}\text { Erfolgreiche } \\
\text { Pässe/Pässe }\end{array}$ & 0,169 & 0,478 & 0,976 & 0,170 & 0,481 & 0,975 & 0,202 & 0,571 & 0,900 \\
\hline $\begin{array}{l}\text { Nicht erfolgrei- } \\
\text { che Päs- } \\
\text { se/Pässe }\end{array}$ & 0,175 & 0,494 & 0,968 & 0,170 & 0,481 & 0,975 & 0,218 & 0,616 & 0,842 \\
\hline $\begin{array}{l}\text { Erfolgreiche } \\
\text { Pässe/nicht } \\
\text { erfolgreiche } \\
\text { Pässe }\end{array}$ & 0,202 & 0,573 & 0,898 & 0,178 & 0,504 & 0,961 & 0,255 & 0,722 & 0,674 \\
\hline
\end{tabular}


Mauchly-Test für die Variablen des Spieltests

\begin{tabular}{|l|c|c|c|}
\hline & W von Mauchly & d.f. & p \\
\hline Ballkontakte & & 2 & 0,472 \\
\hline Pässe & 0,785 & 2 & 0,162 \\
\hline Erfolgreiche Pässe & 0,560 & 2 & $0,013^{*}$ \\
\hline Nicht erfolgreiche Pässe & 0,694 & 2 & 0,065 \\
\hline Pässe/Ballkontakte & 0,955 & 2 & 0,711 \\
\hline $\begin{array}{l}\text { Erfolgreiche Päs- } \\
\text { se/Ballkontakte }\end{array}$ & 0,944 & 2 & 0,648 \\
\hline $\begin{array}{l}\text { Nicht erfolgreiche Päs- } \\
\text { se/Ballkontakte }\end{array}$ & 0,497 & 2 & $0,005^{*}$ \\
\hline Erfolgreiche Pässe/Pässe & 0,829 & 2 & 0,246 \\
\hline $\begin{array}{l}\text { Nicht erfolgreiche Päs- } \\
\text { se/Pässe }\end{array}$ & 0,024 & & 0,258 \\
\hline $\begin{array}{l}\text { Erfolgreiche Pässe/nicht } \\
\text { erfolgreiche Pässe }\end{array}$ & 0,835 & 2 & \\
\hline
\end{tabular}

Mauchly-Test für die Variablen des Loughborugh-Pass-Tests

\begin{tabular}{|l|c|c|c|}
\hline & W von Mauchly & d.f. & $\boldsymbol{P}$ \\
\hline Zeit & 0,829 & 2 & 0,244 \\
\hline Ziel nicht getroffen & 0,897 & 2 & 0,444 \\
\hline Fehler & 0,974 & 2 & 0,818 \\
\hline Gesamtzeit & 0,842 & 2 & 0,275 \\
\hline
\end{tabular}




\section{Anhang S: Formblatt: Auswertung des Team-Sport-Assessment- Procedure (TSAP)}

\section{Observational Grid for Collecting Raw Data}

Observer: Player No: Match:

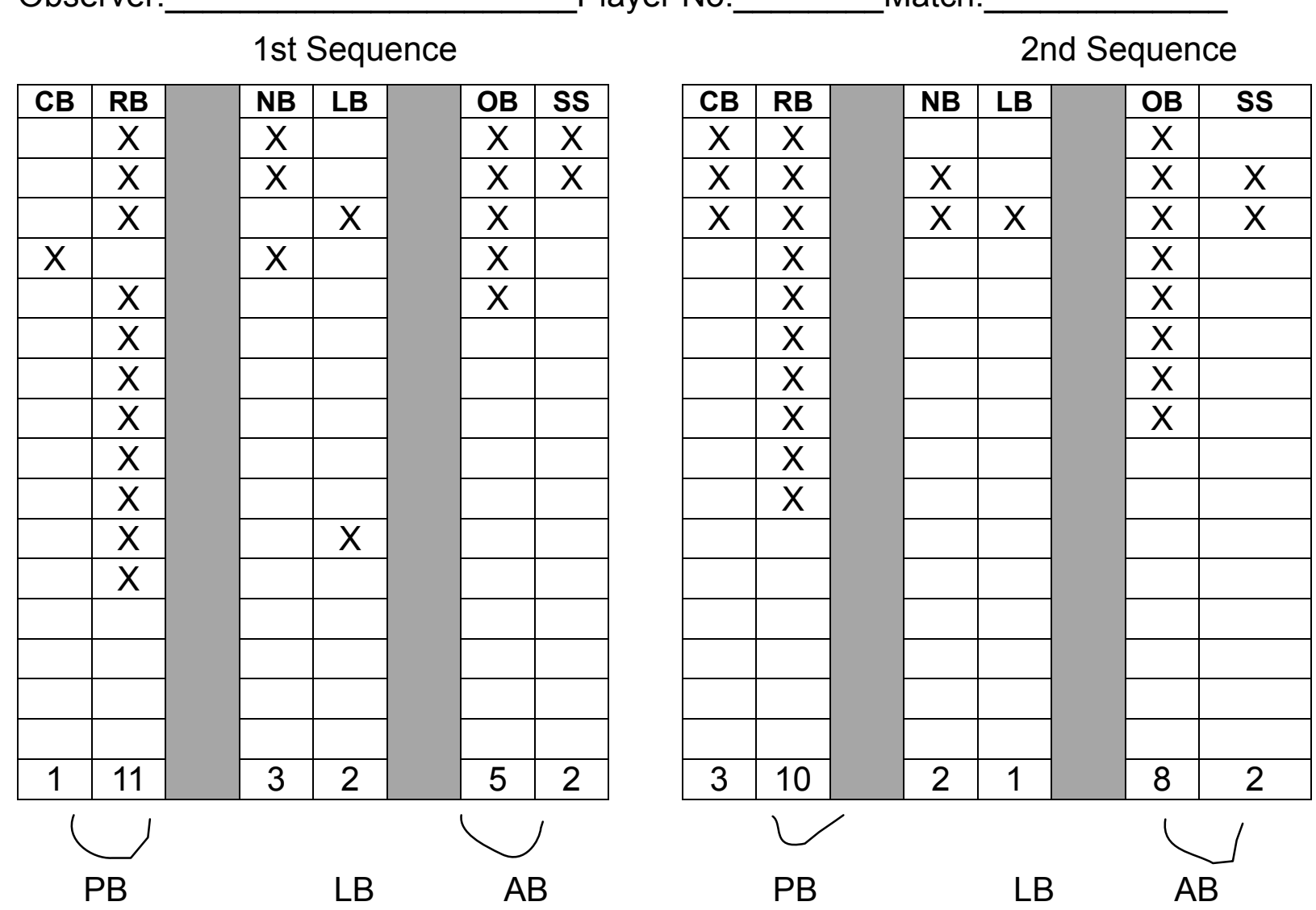

CB: Conquered Balls; RB: Received Balls; NB: Neutral Balls; LB: Lost Balls; OB. Offensive Balls; SS: Sucessfull Shots; PB: Played Balls; AB: Attack Balls. 


\section{Anhang T: Beispiele Trainingseinheiten Kombinationsspiele}

Einheit Datum_26.04.2010 Gruppe_D3

Gesamtzeit 20 Min.

Lehrmittel: Bälle, Hütchen.

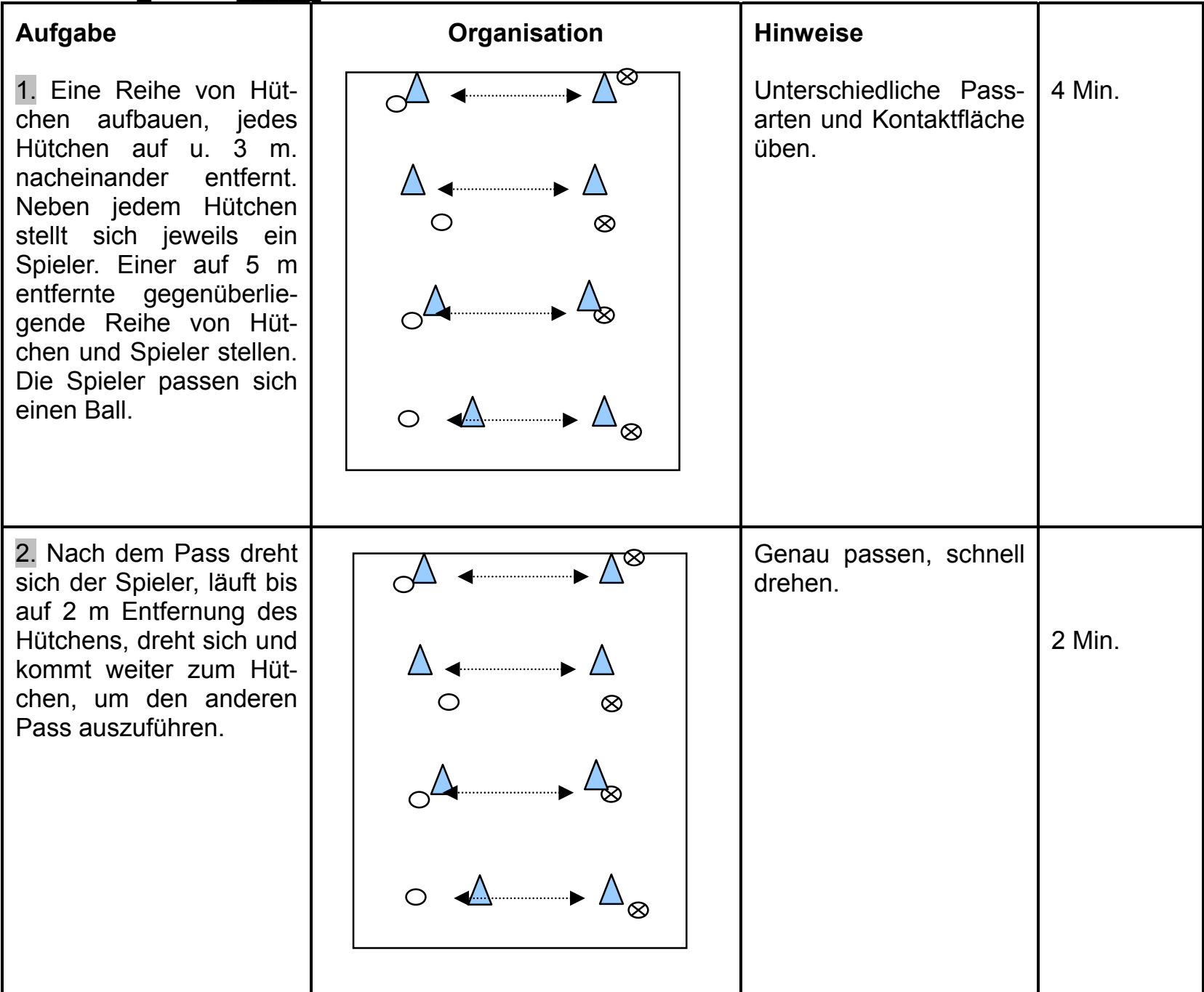




\begin{tabular}{|l|l|l|l|l|}
\hline $\begin{array}{l}\text { 3. Nach dem Pass läuft } \\
\text { jeder Spieler bis zum } \\
\text { rechts stehenden nächs- } \\
\text { ten Hütchen, dreht sich } \\
\text { und kommt zurück, past } \\
\text { wieder und läuft weiter } \\
\text { bis zum rechts stehen- } \\
\text { den nächsten Hütchen. }\end{array}$ & $\begin{array}{l}\text { Wenn ein Spieler einen } \\
\text { Pass mach und sein } \\
\text { Passpartner befindet } \\
\text { sich noch nicht auf } \\
\text { Hütchenebene, soll der } \\
\text { Passgeber den Pass } \\
\text { zum Hütchen machen } \\
\text { und nicht zum Spieler, } \\
\text { der unterwegs ist. }\end{array}$ & 2 \\
\end{tabular}
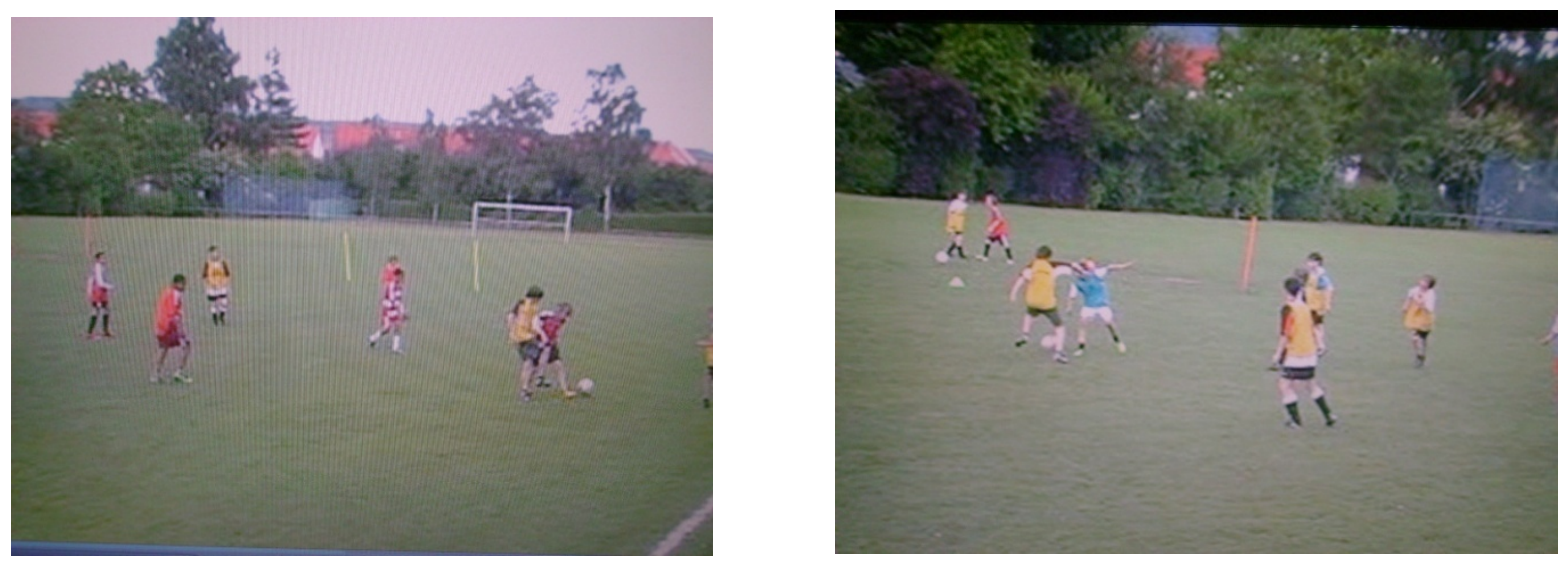
Einheit_2_ Datum_28.04.2010__ Gruppe_D3

Gesamtzeit_20 Min. Lehrmittel: Bälle, Hütchen.

Aufgabe
4.Passkette. Alle Bälle
befinden sich auf einer
Reihe, deren Spieler die
Abspielstationen sind. Die
Spieler ohne Ball nehmen
den Pass an, passen
zurück und laufen bis
zum rechts stehenden
nächsten Hütchen, neh-
men dort einen Pass an,
passen zurück und laufen
bis zum nächsten Hüt-
chen.

Organisation

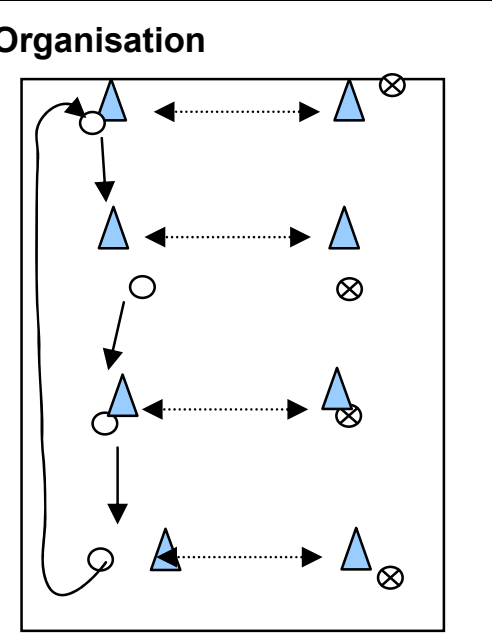

5. Doppelpasskette. Beide Seiten der Kette (Spieler-Reihe) bewegen sich auf gegenteiligen Richtungen. Jeder Spieler passt zum gegenüberliegenden Hütchen, wo ein Spieler kommt, nimmt den Ball an, passt wieder zum Hütchen und läuft weiter.

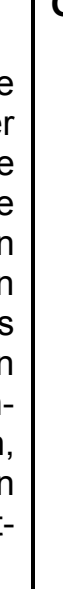

\section{Hinweise}

Die Abspielstationen bleiben auf dem Platz stehen und passen immer zu einem neuen kommenden Spieler. Die laufenden Spieler laufen zurück bis zum Anfang der Kette, wenn sie an das Ende der Reihe kommen.
5 Min.

2 Min.
- Richtung der Ketten wechseln.

- Abstand der Pässe erweitern.

- Annahme mit einem Fuß, Pass mit dem anderen.

- Dem Ball gegenüber laufen, Pass annehmen und weiter passen.

- Ohne Annehmen passen.
6. Beide Seiten der Kette bewegen sich auf gegenteiligen Richtungen, die Spieler passen zum Hütchen und der nächste Spieler bekommt den Ball und passt wieder zum gegenüberliegenden Hütchen. Am Ende der Kette wechseln die Spieler zur anderen Kette/SpielerReihe.
Wenn ein Spieler einen Pass macht und sein Passpartner befindet sich noch nicht auf 2 Hütchenebene, soll der Min. Passgeber den Pass zum Hütchen machen und nicht zum Spieler, der unterwegs ist. 


\section{Anhang U: Ergebnisse TSAP}

\section{Vortest}

\begin{tabular}{|c|c|c|c|c|c|c|c|c|c|}
\hline $\begin{array}{c}\text { Spie- } \\
\text { ler }\end{array}$ & CB & RB & NB & LB & OB & SS & AB & PB & E Index \\
\hline 1 & 6 & 8 & 0 & 6 & 2 & 3 & 5 & 14 & 0,69 \\
\hline 2 & 6 & 4 & 4 & 3 & 1 & 0 & 1 & 10 & 0,54 \\
\hline 3 & 0 & 6 & 2 & 1 & 2 & 1 & 3 & 6 & 0,27 \\
\hline 4 & 9 & 9 & 5 & 7 & 6 & 0 & 6 & 18 & 0,88 \\
\hline 5 & 6 & 12 & 3 & 5 & 3 & 3 & 6 & 18 & 0,8 \\
\hline 6 & 9 & 7 & 3 & 7 & 5 & 2 & 7 & 16 & 0,94 \\
\hline 7 & 2 & 5 & 3 & 4 & 3 & 0 & 3 & 7 & 0,36 \\
\hline 8 & 4 & 1 & 1 & 2 & 1 & 0 & 1 & 5 & 0,42 \\
\hline 9 & 5 & 6 & 3 & 2 & 2 & 3 & 5 & 11 & 0,83 \\
\hline 10 & 3 & 10 & 2 & 2 & 4 & 1 & 5 & 13 & 0,67 \\
\hline 11 & 0 & 8 & 1 & 1 & 3 & 2 & 5 & 8 & 0,45 \\
\hline 12 & 4 & 10 & 2 & 5 & 8 & 1 & 9 & 14 & 0,87 \\
\hline $\begin{array}{c}\text { Mittel- } \\
\text { wert }\end{array}$ & 4,5 & 7,17 & 2,42 & 3,75 & 3,33 & 1,33 & 4,67 & 11,67 & 0,64 \\
\hline S.A. & 2,97 & 3,01 & 1,38 & 2,22 & 2,1 & 1,23 & 2,35 & 4,54 & 0,23 \\
\hline
\end{tabular}


Posttest

\begin{tabular}{|c|c|c|c|c|c|c|c|c|c|}
\hline Spieler & CB & RB & NB & LB & OB & SS & AB & PB & E Index \\
\hline 1 & 8 & 6 & 0 & 3 & 7 & 2 & 9 & 14 & 1,31 \\
\hline 2 & 6 & 9 & 1 & 4 & 6 & 2 & 8 & 15 & 1 \\
\hline 3 & 5 & 9 & 4 & 1 & 3 & 4 & 7 & 14 & 1,09 \\
\hline 4 & 6 & 7 & 1 & 3 & 7 & 0 & 7 & 13 & 1 \\
\hline 5 & 5 & 10 & 3 & 3 & 7 & 5 & 12 & 15 & 1,31 \\
\hline 6 & 11 & 13 & 2 & 7 & 10 & 3 & 13 & 24 & 1,41 \\
\hline 7 & 4 & 10 & 5 & 5 & 7 & 1 & 8 & 14 & 0,80 \\
\hline 8 & 4 & 5 & 2 & 1 & 5 & 1 & 6 & 9 & 0,91 \\
\hline 9 & 2 & 7 & 1 & 3 & 5 & 0 & 5 & 9 & 0,54 \\
\hline 10 & 1 & 4 & 2 & 4 & 1 & 1 & 2 & 5 & 0,21 \\
\hline 11 & 4 & 7 & 1 & 3 & 4 & 3 & 7 & 11 & 0,85 \\
\hline 12 & 7 & 3 & 2 & 1 & 4 & 1 & 5 & 10 & 1,09 \\
\hline $\begin{array}{c}\text { Mittel- } \\
\text { wert }\end{array}$ & 2,25 & 7,5 & 2 & 3,17 & 5,5 & 1,92 & 7,42 & 12,75 & 0,96 \\
\hline S.A. & 2,67 & 2,84 & 1,41 & 1,75 & 2,35 & 1,56 & 3 & 4,67 & 0,34 \\
\hline
\end{tabular}

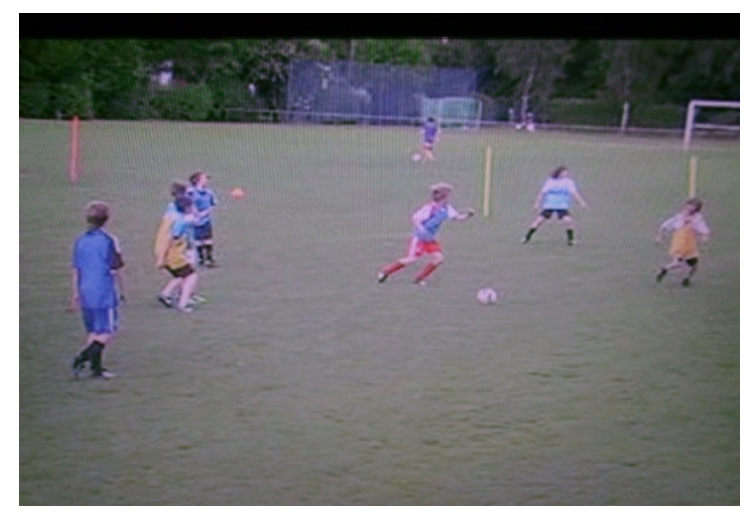


Rettest

\begin{tabular}{|c|c|c|c|c|c|c|c|c|c|}
\hline $\begin{array}{c}\text { Spie- } \\
\text { ler }\end{array}$ & CB & RB & NB & LB & OB & SS & AB & PB & E Index \\
\hline 1 & 7 & 6 & 2 & 3 & 5 & 2 & 7 & 13 & 1,08 \\
\hline 2 & 7 & 10 & 1 & 5 & 8 & 4 & 12 & 17 & 1,27 \\
\hline 3 & 5 & 7 & 3 & 3 & 2 & 2 & 4 & 12 & 0,69 \\
\hline 4 & 6 & 9 & 1 & 4 & 10 & 0 & 10 & 15 & 1,14 \\
\hline 5 & 9 & 7 & 2 & 5 & 7 & 1 & 8 & 16 & 1,13 \\
\hline 6 & 8 & 15 & 1 & 7 & 10 & 2 & 12 & 23 & 1,18 \\
\hline 7 & 4 & 11 & 4 & 3 & 3 & 2 & 5 & 15 & 0,69 \\
\hline 8 & 5 & 6 & 2 & 2 & 8 & 1 & 9 & 11 & 1,17 \\
\hline 9 & 2 & 4 & 1 & 3 & 2 & 0 & 2 & 6 & 0,31 \\
\hline 10 & 2 & 10 & 2 & 3 & 2 & 2 & 4 & 12 & 0,46 \\
\hline 11 & 2 & 8 & 1 & 6 & 1 & 2 & 3 & 10 & 0,31 \\
\hline 12 & 4 & 2 & 0 & 1 & 3 & 1 & 4 & 6 & 0,73 \\
\hline M. & 5,08 & 7,92 & 1,67 & 3,75 & 5,08 & 1,58 & 6,67 & 13 & 0,85 \\
\hline S.A. & 2,39 & 3,42 & 1,07 & 1,71 & 3,34 & 1,08 & 3,5 & 4,73 & 0,36 \\
\hline
\end{tabular}




\section{Anhang V: Komogorov-Smirnov-Test für die Variablen der Studie 4}

\begin{tabular}{|c|c|c|c|c|c|c|c|c|c|}
\hline \multirow[t]{2}{*}{ Variable } & \multicolumn{3}{|c|}{ Vortest } & \multicolumn{3}{|c|}{ Posttest } & \multicolumn{3}{|c|}{ Retentionstest } \\
\hline & Differenz & $\begin{array}{c}\text { Kolmogorov- } \\
\text { Smirnov-Z }\end{array}$ & $p$ & Differenz & $\begin{array}{c}\text { Kolmogorov- } \\
\text { Smirnov-Z }\end{array}$ & $p$ & Differenz & $\begin{array}{l}\text { Kolmogorov- } \\
\text { Smirnov-Z }\end{array}$ & $p$ \\
\hline CB & 0,140 & 0,485 & 0,973 & 0,153 & 0,530 & 0,941 & 0,151 & 0,524 & 0,946 \\
\hline RB & 0,109 & 0,378 & 0,999 & 0,153 & 0,530 & 0,941 & 0,121 & 0,420 & 0,995 \\
\hline NB & 0,169 & 0,587 & 0,881 & 0,250 & 0,866 & $0,037^{*}$ & 0,233 & 0,806 & 0,534 \\
\hline LB & 0,201 & 0,697 & 0,716 & 0,212 & 0,735 & 0,653 & 0,253 & 0,875 & $0,033^{*}$ \\
\hline OB & 0,230 & 0,795 & 0,552 & 0,179 & 0,619 & 0,838 & 0,233 & 0,809 & 0,530 \\
\hline SS & 0,194 & 0,672 & 0,757 & 0,221 & 0,766 & 0,601 & 0,267 & 0,925 & $0,018^{*}$ \\
\hline$A B$ & 0,223 & 0,773 & 0,589 & 0,173 & 0,599 & 0,866 & 0,194 & 0,671 & 0,759 \\
\hline PB & 0,124 & 0,429 & 0,993 & 0,232 & 0,803 & 0,539 & 0,115 & 0,400 & 0,997 \\
\hline E Index & 0,170 & 0,589 & 0,879 & 0,152 & 0,526 & 0,945 & 0,243 & 0,842 & $0,048 *$ \\
\hline
\end{tabular}




\section{Versicherung an Eides statt}

Ich versichere, dass ich die eingereichte Dissertation Aufbau von Handlungswissen und Kompetenzlernen bei Sportspielen, ein didaktisches Modell der Spielhandlungskompetenzen selbstständig und ohne unerlaubte Hilfsmittel verfasst habe. Anderer als der von mir angegebenen Hilfsmittel und Schriften habe ich mich nicht bedient. Alle wörtlich oder sinngemäß den Schriften anderer Autoren entnommenen Stellen habe ich kenntlich gemacht.

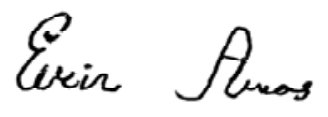

Wien, den12.6.2012

Elkin Arias 


\section{Danksagung}

Mein besonderer Dank gilt meinem Doktorvater Herrn Prof. Dr. Herbert Hopf für die geduldige und umfangreiche Betreuung während meiner Promotionszeit und die wertvolle Hilfe während meines Aufenthalts in Deutschland. Seiner Bereitschaft, Freundschaft und seinem Beistand ist es zuzuschreiben, dass ich die Dissertation vorlegen kann.

Prof. Dr. Andreas Bund als zweiter Gutachter der Dissertation hat mir wertvolle Hinweise gegeben.

Ich danke dem Instituto de Educación Física y Deportes der Universidad de Antioquia in Medellín, Kolumbien, für die Möglichkeit, meine wissenschaftliche Ausbildung in Deutschland absolvieren zu können.

Zwei Institutionen ermöglichten aus finanzieller Sicht meinen Aufenthalt in Deutschland und das Promotionsstudium, zum ersten das Alßan-Programm mit dem Stipendium EO7D402883CO, zum anderen der DAAD mit dem Stipendium A/06/31491 im Rahmen des Programms ALECOL. Meine Dankbarkeit gilt allen, die mich hinsichtlich der Finanzierung des Studiums beraten und unterstützt haben.

Ralf Tarant, sportlicher Leiter im Basisbereich des Vereins RSV Göttingen 05, hat mir die Mannschaften, Mittel und Zeit für den empirischen Teil der Arbeit zur Verfügung gestellt gestellt. Er hat mich während dieses Teils mit Rat und Tat begleitet und unterstützt. Seine Erfahrung, Bereitschaft und Hilfe ermöglichten die Umsetzung meiner Gedanken in der Praxis.

Die Kinderfußballspieler des RSV Göttingen 05 haben begeistert und aufgeschlossen an den empirischen Studien teilgenommen.

Meine Frau Olga am Studienort und meine Geschwister und Familie in Kolumbien haben mich stets begleitet bzw. motiviert.

Meine Freunde in Göttingen und in Kolumbien haben mir durch ihre wertvollen Ratschläge geholfen, so manche Hürde zu nehmen.

Die deutsche Gesellschaft hat mich ohne Vorbehalte in ihrer Mitte aufgenommen; durch den Aufenthalt in Deutschland habe ich meinen Erfahrungshorizont erweitert und an Lebenserfahrung gewonnen. Auch hierfür möchte ich Dank sagen. 


\section{Lebenslauf}

\section{Persönliche Daten:}

Name: $\quad$ Elkin Alberto Arias Arias

Geboren am: $\quad 02.11 .1973$

Geburtsort: Medellín, Kolumbien

Staatsangehörigkeit: Kolumbianer

Bildungsweg:

$\begin{array}{ll}1980-1984 & \text { Grundschule Contranal, Medellín } \\ 1985-1990 & \text { Gymnasium Ricardo Rendon Bravo, } \\ 1992-1996 & \begin{array}{l}\text { Studium der Sportwissenschaft an der Universidad de } \\ \text { Antioquia, Medellín }\end{array} \\ 1996-1998 & \text { Spezialisierung in Sporttraining an der Universidad de } \\ & \text { Antioquia }\end{array}$

Beruflicher Werdegang:

$\begin{array}{ll}\text { 1997-2001 } & \text { Lehrtätigkeit an Politecnico Colombiano Jaime Isaza } \\ & \text { Cadavid,Medellín, Kolumbien } \\ & \text { Dozent an der Universidad de Caldas, Manizales, } \\ & \text { Kolumbien }\end{array}$


Seit 2004

Dozent am Instituto de Educación Física y Deportes

Universidad de Antioquia 